DEPARTMENT OF THE INTERIOR

U.S. GEOLOGICAL SURVEY

Temperature, Thermal-Conductivity, and Heat-Flux Data: Raft River Area, Cassia County, Idaho (1974-1976)

by

T. C. Urban', W. H. Diment ${ }^{1}$, Manuel Nathenson ${ }^{2}$, E. P. Smith ${ }^{2}$, J. P. Ziagos, ${ }^{2}$ and M. H. Shaeffer ${ }^{2}$

Open-File Report 86-123

This report is preliminary and has not been reviewed for conformity with U.S. Geological Survey editorial standards and stratigraphic nomenclature. Any use of trade names is for descriptive purposes only and does not imply endorsement by the USGS.

${ }^{1}$ U.S. Geological Survey Denver, Colorado
${ }^{2}$ U.S. Geological Survey Menlo Park, California 


\section{CONTENTS}

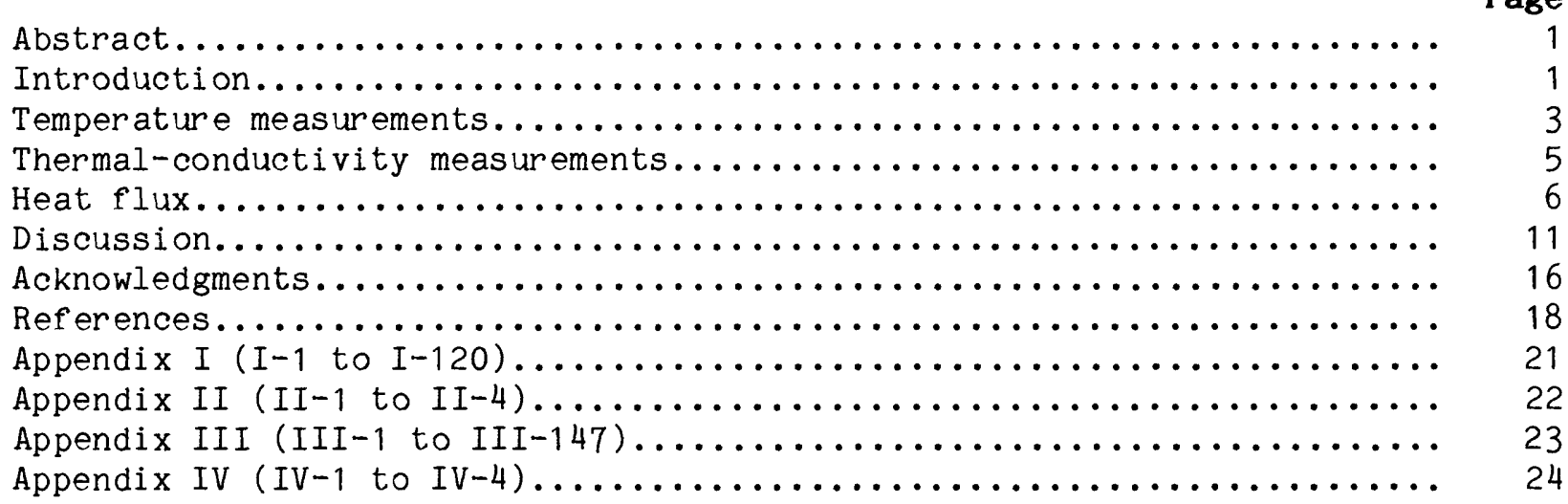

\section{ILLUSTRATIONS}

Page

Figure 1. Map showing location of intermediate and deep wells in the

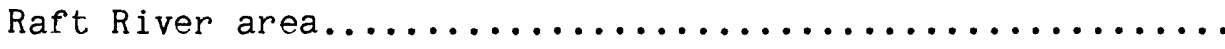

2. Map showing location of shallow auger holes drilled near

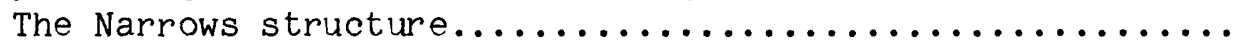

3. Map of contoured heat flux of intermediate and deep holes

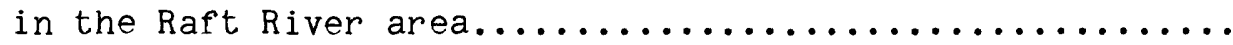

4. Map of contoured heat flux of shallow auger holes near the

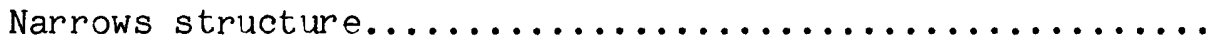

\section{TABLES}

Page

Table 1. Heat flux for intermediate and deep holes.............. 7

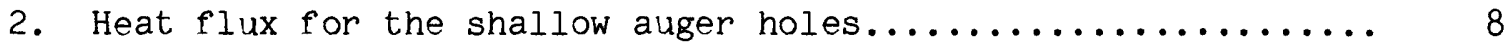

3. Drill holes logged in the Raft River area, Cassia County,

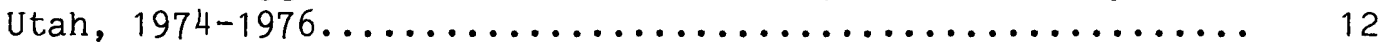

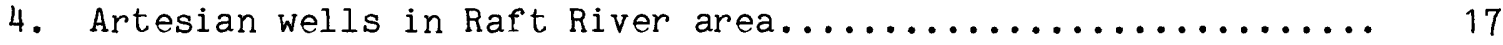




\title{
TEMPERATURE, THERMAL-CONDUCTIVITY, AND HEAT-FLUX DATA: RAFT RIVER AREA, CASSIA COUNTY, IDAHO (1974-1976)
}

By T. C. Urban, W. H. Diment, Manuel Nathenson, E. P. Smith, J. P. Ziagos, and M. H. Shaeffer

\begin{abstract}
The Energy Research and Development Administration (ERDA) selected the southern Raft River Valley as a site for geothermal exploration and development. The basic data presented here are a part of those collected by the U.S. Geological Survey under a cooperative program with ERDA.

Temperatures were measured at discrete intervals in shallow $(30 \mathrm{~m} ; 100$ $\mathrm{ft}$ ), intermediate $(100-500 \mathrm{~m} ; 300-1,500 \mathrm{ft})$, and deep holes $(1,500+\mathrm{m} ; 4,500+$ $f t)$ in the Raft River area, Cassia County, Idaho, on several occasions during 1974-1976 in order to define the size and nature of the geothermal system. The results of these measurements indicate that the system is intermediate in temperature $\left(-150{ }^{\circ} \mathrm{C}\right.$ at $\left.1.5 \mathrm{~km}\right)$. Water of this temperature is only marginally useful for electric-power production with present technology. Thermal conductivity measurements, by the needle-probe method on 241 core samples from four of the intermediate-depth drill holes, yielded values that ranged from 0.8 to $2.5 \mathrm{~W} / \mathrm{m} \cdot \mathrm{K}$ (Watts per meter.Kelvin).

Calculations indicate anomalously high heat flows $\left(0.3+\mathrm{W} / \mathrm{m}^{2}\right)$ as would be expected in a geothermal system. Drill hole I.D. 5 is located in an area considered outside the system and has a heat-flow value of about $0.1 \mathrm{~W} / \mathrm{m}^{2}$, similar to that obtained in other parts of the Basin and Range Province.

\section{INTRODUCTION}

The Raft River Valley is located in south-central Idaho near the Utah border (fig. 1). It is bounded on the west by the Jim Sage Mountains, on the south by the Raft River Range, and on the east by the Black Pine and Sublett Ranges. The Raft River drains to the north into the Snake River. The valley is approximately $20-24 \mathrm{~km}$ wide and $60 \mathrm{~km}$ long, although the geothermal area is confined to the southern $20 \mathrm{~km}$ or so. It is a late Cenozoic downwarp bounded by faults on the west, south, and east. The average elevation of the valley is about $1,400 \mathrm{~m}$.

The Raft River basin is filled with Pleistocene alluvium and MiocenePliocene tuffaceous sediments, conglomerate, and felsic volcanic rocks to a depth of about $2 \mathrm{~km}$ (Williams and others, 1976). Faulted Pennsylvanian and Permian sedimentary rocks comprise much of the Sublett and Black Pine Ranges on the east. On the western side of the valley the Jim Sage and Cotterel Mountains consist of Tertiary rhyolites and tuffaceous sediments. On the south, the Raft River Range forms one of the few east-west-trending mountain ranges in the North American Cordillera. The Raft River Range consists of gneiss-dome complexes of Precambrian quartz monzonite, mantled by Precambrian and lower Paleozoic metasedimentary rocks and upper Paleozoic sedimentary
\end{abstract}


으

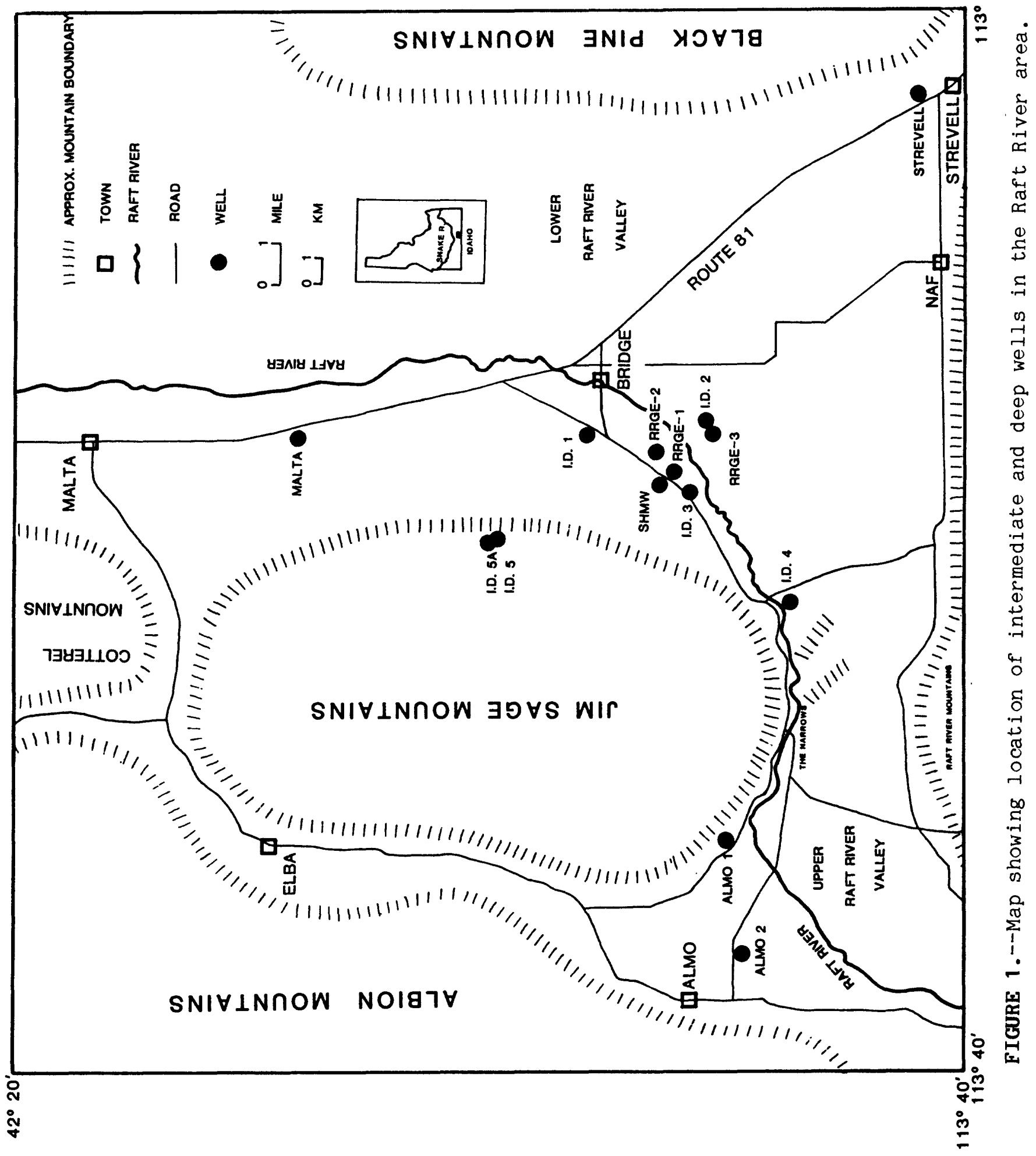


rocks. The Raft River flows into the valley from the southwest through a gap known as "The Narrows," which is formed by the boundary of the Raft River Range on the south and the southern terminous of the Jim Sage Mountains on the north. A discussion of the geology and geophysics of the area can be found in Ackermann (1979), Williams and others (1976), Mabey and others (1978), Covington (1977a, b, c, d), Crosthwaite (1974, 1976), Oriel and others (1978), and Nathenson and others (1979, 1980, 1982).

The Energy Research and Development Administration (ERDA) selected the southern Raft River Valley as a potential site for a demonstration plant for the production of electricity from intermediate-temperature $\left(-150^{\circ} \mathrm{C}\right)$ water using an experimental binary-fluid geothermal powerplant. In 1973 ERDA, now part of the Department of Energy (DOE), and the U.S. Geological Survey began a cooperative program to investigate the geothermal system and to determine if it was adequate to support the proposed demonstration plant. The data reported here were obtained from the series of wells that were drilled during this period (1974-1976) as part of that program or from preexisting wells in the region. The location of the intermediate and deep holes is shown in figure 1. Auger holes, $100 \mathrm{ft}$ deep or less, were only drilled near The Narrows structure (fig. 2).

\section{TEMPERATURE MEASUREMENTS}

Borehole termperatures were obtained during the period from 1974 through 1976 in 43 wells in the Raft River area. These measurements were made at $0.3-$ to $1.5-\mathrm{m}$ ( 1 - to $5-\mathrm{ft}$ ) intervals using single-bead thermistors, $4.86-\mathrm{mm}-$ diameter 4-conductor cables, and 5/2-digit multimeters. The digital output of the multimeter was recorded on punched-paper tape for later computer analysis. In general, the logs were made while the probe was lowered down the well at a steady, continuous rate of $4.6 \mathrm{~m} / \mathrm{min}$. The time constant of the probes in stirred water is less than 2 seconds. A few of the shallow holes were logged using a 3-mm-diameter, 4-conductor portable cable. In this case, the probe was lowered to the depth at which the temperature was to be measured and the resulting reading recorded from the multimeter by hand after the reading had "stabilized."

The temperature gradients in most of the holes exceed the critical gradient for convection. The range in temperature at a given depth caused by the convecting liquid is approximately equal to the temperature gradient times the radius of the hole times the aspect ratio of the convective motion. The aspect ratio (height/radius of the convection "cell") ranges from 4 to 20 (for example, Urban and Diment, 1985). In the holes considered here, errors in temperatures caused by this effect are on the order of $0.01-0.1{ }^{\circ} \mathrm{C}$.

The shallowest temperatures tabulated or plotted in the appendices are usually within one-depth increment of the water level, as independently determined with an electrical conductivity meter. Occasionally, temperatures were measured from the surface. The temperatures in the air-filled part of a hole are meaningless; the time constant of the probe in air is too long to give reliable temperatures at the logging rates used. 


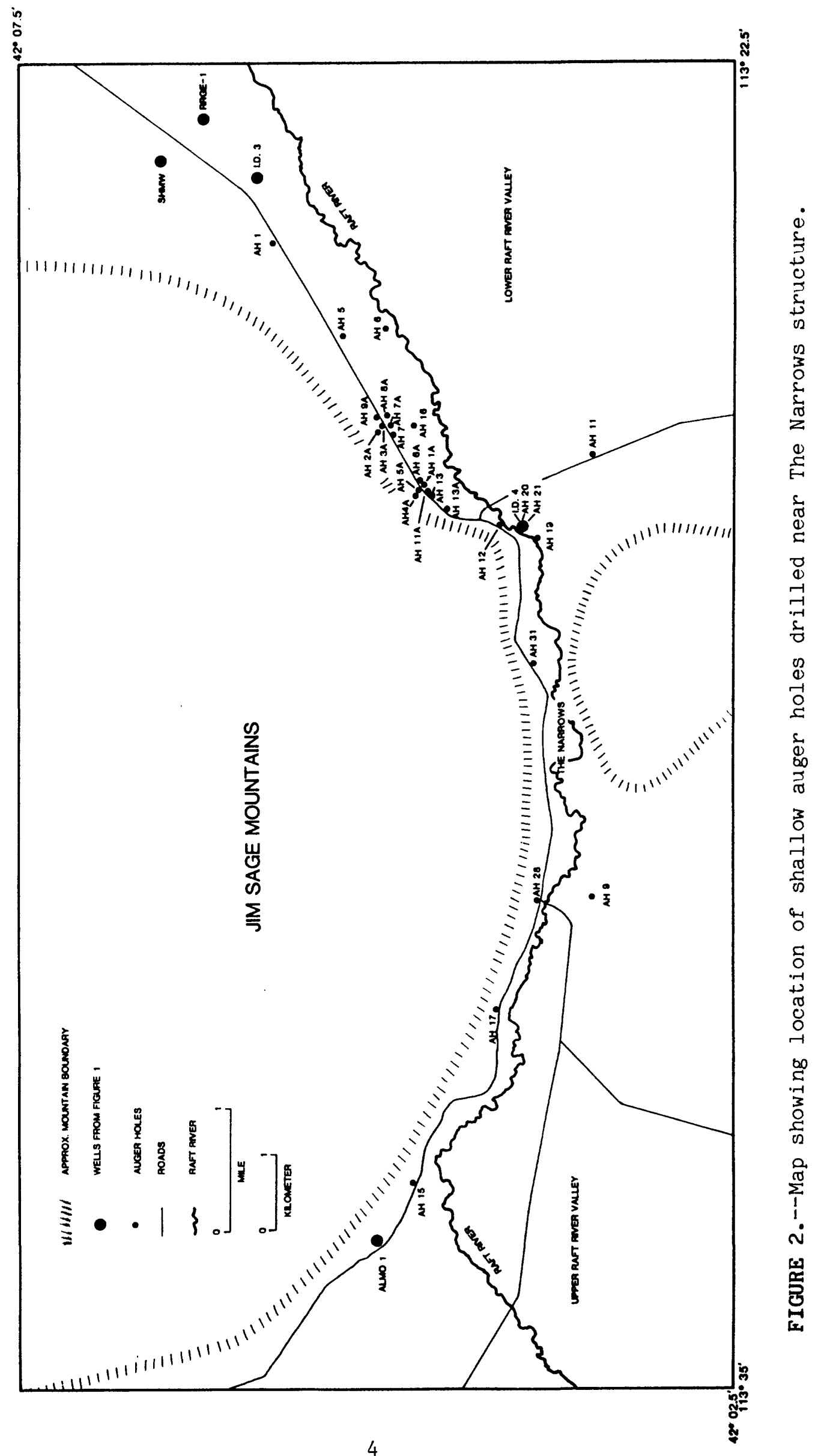


The thermistors were calibrated against a digital quartz-thermometer (Hewlett-Packard model 2801A) with calibrations traceable to the National Bureau of Standards. The thermistor resistances and the corresponding quartzthermometer temperatures were used to generate constants by a general leastsquares fit to the constants of the following equation:

$$
\mathrm{T}=\frac{\mathrm{C} 1+C 2 * \ln (R)}{\mathrm{C} 3+\ln (\mathrm{R})} \text {, }
$$

where $\mathrm{C} 1, \mathrm{C} 2$, and $\mathrm{C} 3$ are the generated constants, $\mathrm{R}$ is the thermistor resistance, $T$ is the temperature, and $I n$ is the natural logarithm. This equation is equation (4) of Robertson and others (1966, p. B6), where we have solved for $\mathrm{T}$ and combined several constant expressions into single constants. Robertson and others (1966) also discuss the effect of pressure on thermistors and thermistor drift, which is usually on the order of 0.001-0.005 ${ }^{\circ} \mathrm{C}$ per month. The absolute precision of the calibration is $\pm 0.02{ }^{\circ} \mathrm{C}$, and the relative accuracy is $\pm 0.002{ }^{\circ} \mathrm{C}$.

\section{THERMAL-CONDUCTIVITY MEASUREMENTS}

Samples of the cores from I.D. 1, 2, 3, and 5 were waxed to preserve the moisture content. Due to the friability of much of the core, thermalconductivity measurements were conducted by E. P. Smith using the needle-probe technique (Von Herzen and Maxwell, 1959; Lachenbruch and Marshall, 1966; Sass and others, 1984). This entails drilling a small hole in the core and inserting a needle containing a heater wire along its length and a thermistor half-way along its length. A constant current is supplied to the heater wire, and the temperature as a function of time is measured by the thermistor. The mathematical solution for this case is that of a line source in an infinite medium. The temperature should be a linear function of $10 g$ time. Deviations from linearity arise due to initial transient heating and at long times when the finite size of the core invalidates the assumption of an inf inite medium.

Although this is a relatively simple and straightforward technique, in most rocks there tends to be considerable scatter in comparison to conductivities measured by the divided bar apparatus. There are several possible causes of the high variation (for example, Sass and others, 1984). First, when the divided bar apparatus (Birch, 1950) is used to determine the thermal conductivity, a rock sample is machined into a $r$ ight circular cylinder with its axis oriented along the vertical or $Z$ direction in the earth. The thermal conductivity is measured along the direction of this axis. Since it is the vertical component of the heat flux out of the surface of the earth that is desired, a measurement of the thermal conductivity in this direction gives an unambiguous result. The needle probe, however, measures the thermal conductivity in the plane perpendicular to the axis of the probe. If the axis of the probe is oriented perpendicular to the vertical or $Z$ direction in the core and the rock thermal conductivity is isotropic, then the divided bar and the needle probe should yield the same value. However, if the rock is thermally anisotropic, then the resulting measurement yields a value which is the geometric mean of the other two principal conductivities (Simmons, 1961). For example, if the $x$ and $y$ axes are in the horizontal plane, the $z$ axis is vertical, the principal conductivities $\mathrm{K}_{\mathrm{x}}=\mathrm{K}_{\mathrm{y}} \neq \mathrm{K}_{\mathrm{z}}$, and the needle 
probe is oriented along the $\mathrm{y}$ axis, then the measured conductivity is the geometric mean of $\mathrm{K}_{\mathrm{x}}$ and $\mathrm{K}_{\mathrm{z}}$. Hence, the magnitude of $\mathrm{K}_{\mathrm{x}}$ will determine whether the effective conductivity is greater or less than the desired vertical conductivity, $\mathrm{K}_{\mathrm{z}}$. Even if $\mathrm{K}_{\mathrm{z}}$ is constant as measured by the divided bar apparatus, variations in $K_{x}$ will cause variations in the effective conductivity as measured by the needle probe and, thus, a scatter in the apparent thermal conductivity. Due to time constraints when the measurements were made, only a single conductivity determination was made on the available core samples. The conductivities were measured with the needle probe oriented perpendicular to the $z$ or vertical axis of the core. Second, this technique works best in rocks that are fine grained. In rocks which contain large crystals or pebbles, the measured conductivity is dominated by the effect of the nearby crystal or pebble and does not represent the overall rock conductivity. Finally, although the waxing procedure tends to preserve the moisture content of the core sample, there is some water loss. Moreover, the measurements were made at atmospheric pressure and some disaggregation probably occurred. The replacement of water by air, which has a significantly lower thermal conductivity, tends to lower the observed, apparent conductivity of the sample. All of these factors tend to contribute to the scatter observed with this kind of measurement as opposed to that of the divided-bar apparatus. The average conductivities are probably a bit low.

\section{HEAT-FLUX}

The vertical heat flux out of the Earth is the product of the thermal conductivity and the thermal gradient over some interval in the well that can be reasonably assumed to be undisturbed by water flows, terrain effects, the annual temperature wave, and other disturbances. In the case of the measurements reported here, many of the wells undoubtedly are affected by these disturbances. However, since the intent is to examine the geothermal system for anomalous conditions, the size of these disturbances probably is small in comparison to the effects of the movement of geothermal fluids throughout the system. Two methods were used to calculate the heat flux out of the surface. The first method used was the product of the thermal gradient over some interval and the average thermal conductivity in that interval or assumed for that interval, based on the thermal conductivity in nearby holes. The second method was suggested by Bullard (1939):

$$
Q=\left(T-T_{0}\right) / \Sigma\left(\Delta z_{i} / K_{i}\right)
$$

where $T$ is the temperature as a function of depth, $T_{0}$ is a constant, $K_{i}$ is the thermal conductivity in the $\Delta z_{i}$ interval, and $Q$ is the heat flux. The advantage of the Bullard method is that it allows for beds of varying thickness and conductivity in the analysis. The disadvantages are that the conductivities for the various intervals are not of uniform accuracy, the vertical component of the conductivity in the interval may be overestimated or underestimated, as discussed above, and systematic errors may arise from nonideal sampling of the core.

The results of these calculations are tabulated in tables 1 and 2 and are plotted and contoured in figures 3 and 4 . The contour interval in these plots is nonuniform due to the large range in values and the high concentration of holes near The Narrows. Several observations are apparent from an examination 
TABLE 1.--Heat flux for intermediate and deep holes by Bullard resistivity integral and gradient times conductivity product

[ \pm is the error in calculating the heat flux (slope of the line in the resistivity integral)]

\begin{tabular}{|c|c|c|c|c|c|c|}
\hline \multirow{2}{*}{$\begin{array}{l}\text { Well } \\
\text { No. } \\
\text { I.D. } 1\end{array}$} & \multirow{2}{*}{$\begin{array}{c}\begin{array}{c}\text { Bullard } \\
\text { resistivity } \\
\text { interval } \\
\text { (meters) }\end{array} \\
20\end{array}$} & \multicolumn{2}{|c|}{$\begin{array}{l}\text { Range } \\
\text { of } \\
\text { calculation } \\
\text { (meters) }\end{array}$} & \multicolumn{2}{|c|}{$\begin{array}{c}\text { Heat flux } \\
\text { (watts per } \\
\text { meter } \\
\text { squared) }\end{array}$} & $\begin{array}{l}\text { Conductivity } \\
\text { used in } \\
\text { well } 1^{2}\end{array}$ \\
\hline & & 90 & -170 & 0.132 & \pm 0.004 & $\cdots$ \\
\hline I.D. 2 & 20 & 80 & -180 & .302 & \pm .009 & $m-n$ \\
\hline I.D. 3 & 20 & 160 & -300 & .333 & .027 & $\ldots-$ \\
\hline I.D. 4 & $\left({ }^{3}\right)$ & & $7-13.4$ & .835 & & I.D. $1^{4}$ \\
\hline I.D 5 & 20 & 60 & -160 & .122 & .006 & $-\cdots$ \\
\hline RRGE-1 & 20 & 100 & -220 & .374 & .016 & -..- \\
\hline RRGE-2 & 20 & 80 & -400 & .150 & .004 & I.D. 3 \\
\hline RRGE-3 & 20 & 80 & -180 & .545 & .017 & I.D. 2 \\
\hline MALTA & 20 & 60 & -160 & .198 & .010 & I.D. 5 \\
\hline SHMW & 5 & 65 & -80 & .669 & .027 & I.D. 3 \\
\hline ALMO 1 & $\left({ }^{3}\right)$ & & $1-25.0$ & 1.317 & & I.D. $1^{4}$ \\
\hline ALMO 2 & $\left({ }^{3}\right)$ & 30. & $5-170.7$ & .057 & & I.D. $2^{5}$ \\
\hline STREVEII & $\left({ }^{3}\right)$ & 62. & $5-221.0$ & .116 & & I:D. $5^{6}$ \\
\hline
\end{tabular}

'Interval over which the resistivity integral was calculated, or the interval over which the gradient was calculated for use with the average thermal conductivity (see footnotes 4,5 , and 6 ).

${ }^{2}$ Leaders (--.-) indicate thermal conductivities were available from the indicated well.

${ }^{3}$ Gradient times conductivity method was used.

${ }^{4}$ Average conductivity from I.D. $1=1.08 \mathrm{~W} / \mathrm{m} \cdot \mathrm{K}$.

${ }^{5}$ Average conductivity from I.D. $2=1.30 \mathrm{~W} / \mathrm{m} \cdot \mathrm{K}$.

${ }^{6}$ Average conductivity from I.D. $5=2.10 \mathrm{~W} / \mathrm{m} \cdot \mathrm{K}$. 
TABLE 2.--Surface heat flux for the shallow auger holes by gradient times conductivity product

\begin{tabular}{|c|c|c|c|c|}
\hline $\begin{array}{l}\text { Well } \\
\text { No. }\end{array}$ & $\begin{array}{l}\text { Interval } \\
\text { depth } \\
\text { (meters) }\end{array}$ & $\begin{array}{l}\text { Thermal } \\
\text { gradient } \\
\text { (degrees } \\
\text { Celsius } \\
\text { per } \\
\text { kilometer) }\end{array}$ & $\begin{array}{l}\text { Date } \\
\text { of } \\
\text { temperature } \\
\text { logging }\end{array}$ & $\begin{array}{c}\text { Heat flux } \\
\text { (Watts } \\
\text { per } \\
\text { meter } \\
\text { squared) }\end{array}$ \\
\hline $\mathrm{AH} 1 \mathrm{~S}$ & $10.4-28.7$ & 207 & $2 / 09 / 76$ & 0.22 \\
\hline $\mathrm{AH} 1 \mathrm{~A}$ & $4.9-6.7$ & 5,102 & $2 / 09 / 76$ & 5.5 \\
\hline $\mathrm{AH} 2 \mathrm{~A}$ & $5.5-6.7$ & 149 & $1 / 16 / 76$ & .16 \\
\hline $\mathrm{AH} 3 \mathrm{~A}$ & $7 \cdot 3-11 \cdot 3$ & 946 & $8 / 16 / 76$ & 1.0 \\
\hline $\mathrm{AH} 4 \mathrm{~A}$ & $6.7-8.8$ & 133 & $2 / 09 / 76$ & .14 \\
\hline AH 5 & $.3-7.6$ & 43 & $8 / 16 / 76$ & .046 \\
\hline $\mathrm{AH} 5 \mathrm{~A}$ & $4.0-9.4$ & 1,033 & $2 / 09 / 76$ & 1.1 \\
\hline $\mathrm{AH} 6$ & $9.4-28.3$ & 75 & $2 / 09 / 76$ & .080 \\
\hline $\mathrm{AH} 6 \mathrm{~A}$ & $5.2-12.8$ & 3,450 & $8 / 16 / 76$ & 3.7 \\
\hline AH $7-N$ & $8.5-9.8$ & 17,713 & $8 / 16 / 76$ & 19 \\
\hline $\mathrm{AH} \quad 7-\mathrm{S}$ & $.9-4.9$ & 4,763 & $1 / 16 / 76$ & 5.1 \\
\hline $\mathrm{AH} 7 \mathrm{~A}$ & $1.8-6.4$ & 3,623 & $1 / 16 / 76$ & 3.9 \\
\hline $\mathrm{AH} 8 \mathrm{~A}$ & $1.5-5.8$ & 3,416 & $2 / 09 / 76$ & 3.7 \\
\hline $\mathrm{AH} 9$ & $7.6-14.3$ & 51 & $2 / 09 / 76$ & .055 \\
\hline $\mathrm{AH} 9 \mathrm{~A}$ & $2.1-10.1$ & 1,107 & $1 / 16 / 76$ & 1.2 \\
\hline AH 11 & $29.0-30.2$ & 77 & $2 / 08 / 76$ & .083 \\
\hline $\mathrm{AH} 11 \mathrm{~A}$ & $5.5-8.8$ & 4,987 & $1 / 16 / 76$ & 5.4 \\
\hline $\mathrm{AH} 12$ & $3.4-6.7$ & 726 & $2 / 08 / 76$ & .78 \\
\hline $\mathrm{AH} 13-\mathrm{N}$ & $7.0-10.1$ & 4,388 & $8 / 16 / 76$ & 4.7 \\
\hline AH $13-S$ & $3.0-10.1$ & 4,954 & $1 / 17 / 76$ & 5.4 \\
\hline $\mathrm{AH} 13 \mathrm{~A}$ & $6.4-13.1$ & 244 & $1 / 17 / 76$ & .26 \\
\hline $\mathrm{AH} 15$ & $5.2-8.5$ & 302 & $2 / 09 / 76$ & .33 \\
\hline $\mathrm{AH} 16$ & $4.6-5.8$ & 440 & $2 / 09 / 76$ & .48 \\
\hline AH 17 & $7.9-13.4$ & 143 & $8 / 15 / 76$ & .15 \\
\hline $\mathrm{AH} 19$ & $3.0-6.7$ & 531 & $2 / 08 / 76$ & .57 \\
\hline AH 20 & $5.2-8.2$ & 580 & $8 / 08 / 76$ & .63 \\
\hline AH 21 & $5.5-10.4$ & 630 & $8 / 08 / 76$ & .68 \\
\hline AH 28 & $9.1-11.3$ & 464 & $8 / 16 / 76$ & .50 \\
\hline $\mathrm{AH} 31$ & $5.5-7.3$ & 160 & $2 / 09 / 76$ & .17 \\
\hline
\end{tabular}

"Interval depths are the end points of the "best" straight line as determined by placing a straight edge on the temperature plots of the auger holes in appendix I. Using the temperatures associated with these end points, the thermal gradients were calculated.

${ }^{2}$ An average thermal conductivity of $1.08 \mathrm{~W} / \mathrm{m} \cdot \mathrm{K}$ from I.D. 1 was used to calculate the heat flux. Heat-flux values have been rounded to two significant figures. 


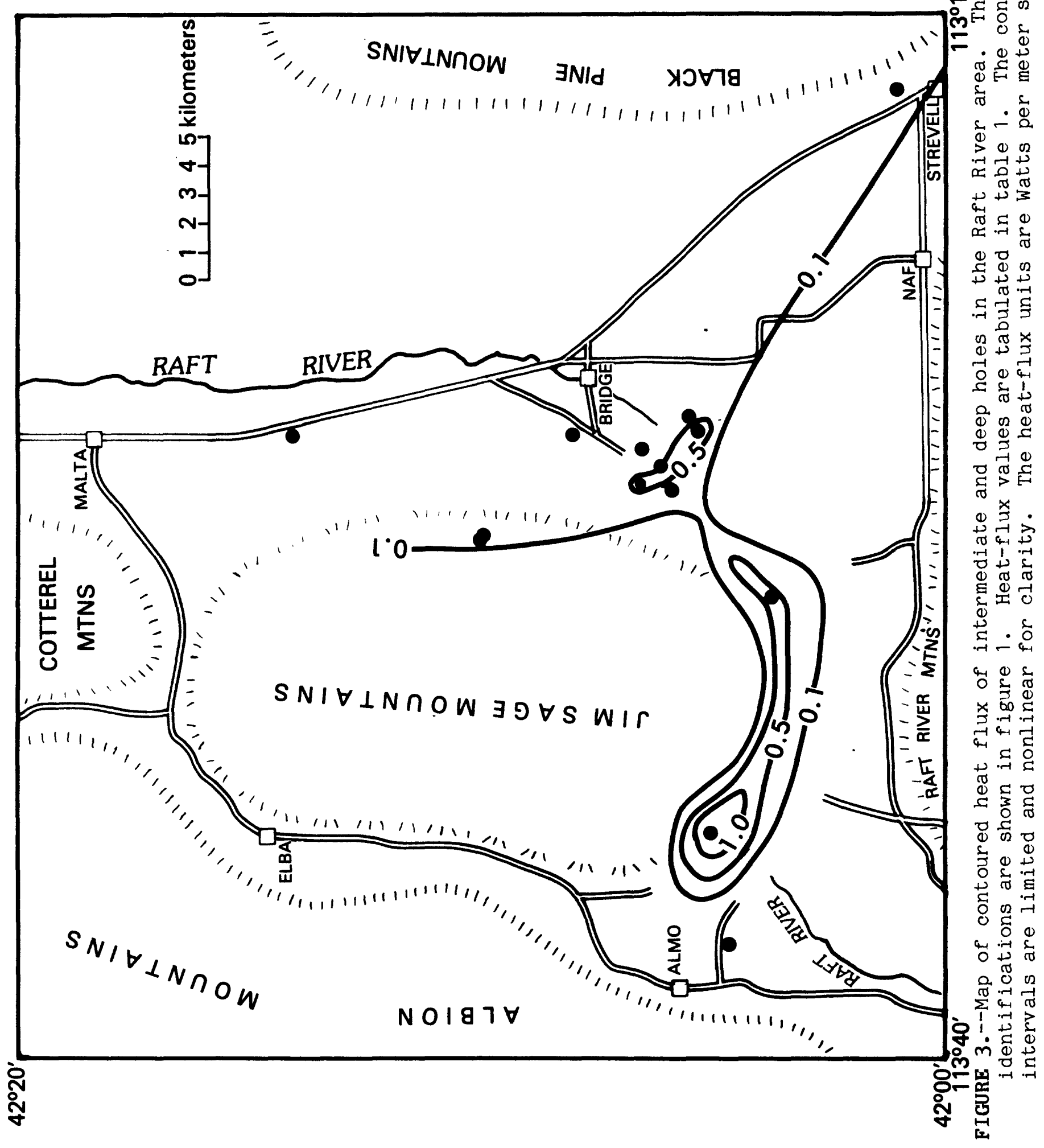




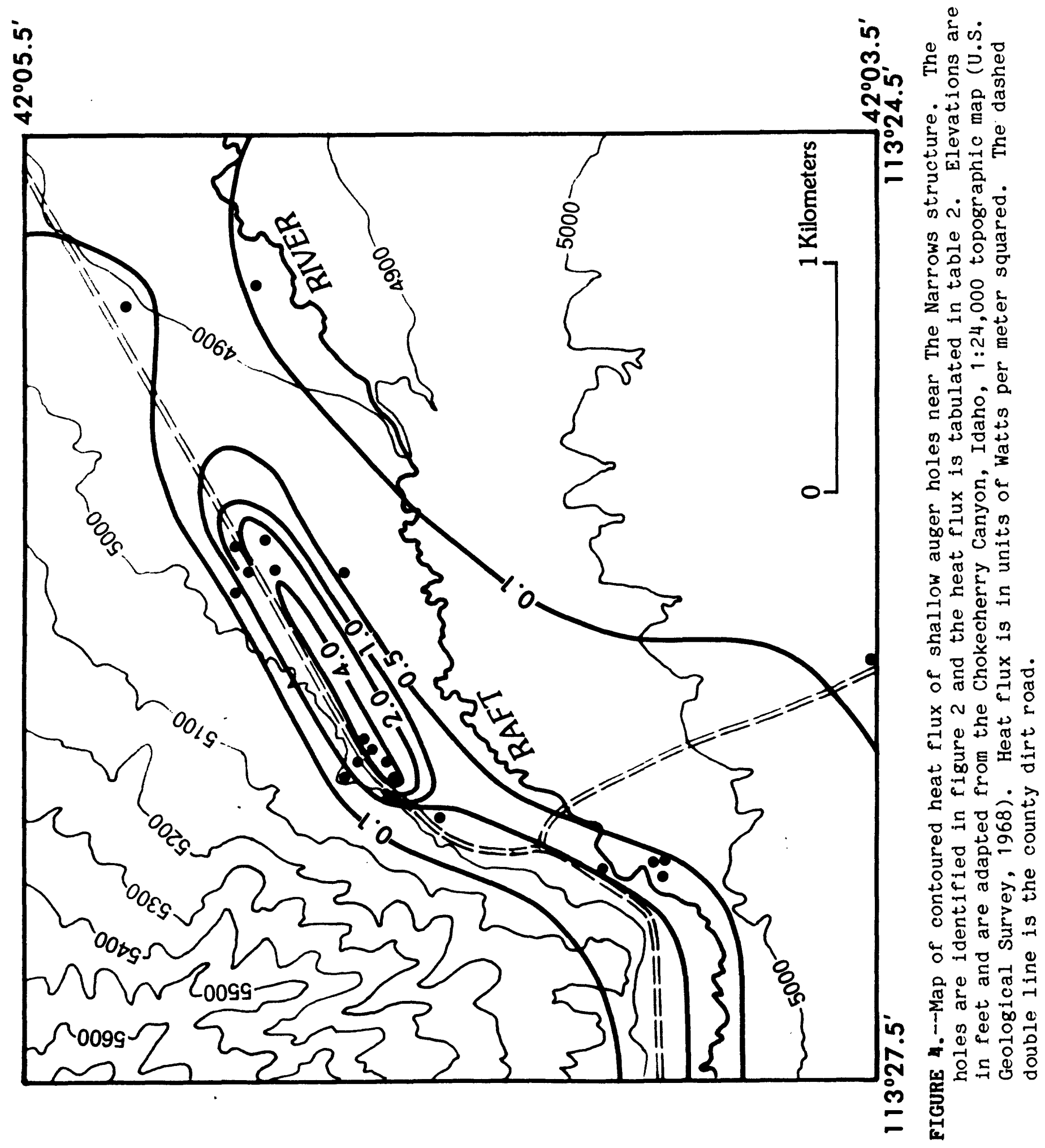


of figures 3 and 4. In figure 3, only the intermediate and deep holes are shown (see fig. 1 for identification). The roads and part of Raft River have been left out for clarity. The contours wrap around the southern boundary of the Jim Sage Mountains and in part are associated with The Narrows structure (Williams and others, 1976), a northeast-trending linear feature most easily seen in the Bouguer gravity anomaly map of the southern Raft River Valley (Mabey and others, 1978). The 0.5 contour associated with RRGE-1 and RRGE-2 is roughly constrained by the Bridge fault and The Narrows structure in this area. The extension of the 0.1 contour to the vicinity of Malta and Strevell (fig. 3) would be questionable due to the lack of wells. In the area east of The Narrows, a considerable number of shallow auger holes were drilled. These holes are entirely in the alluvium and subject to disturbances caused by the annual temperature wave and near-surface water movement.

The calculation of the heat flux for the auger holes was based on an average thermal conductivity for I.D. 1 and the "best" thermal gradient in the hole. The use of this value for the thermal conductivity was determined from the examination of the available conductivity information for all the wells in the area. Since the auger holes were entirely in alluvium, the effective thermal conductivity probably is quite low, and probably not too different from the conductivity in I.D. 1. An increase in the conductivity value used in figure 4 would increase the heat-flux values but would not change the appearance of the anomaly significantly, since the gradients associated with the anomaly are quite large.

The thermal anomalies (figs. 3 and 4) in the vicinity of The Narrows have no surface expression except for The Narrows spring near I.D. 4 at the edge of Raft River. This spring has a temperature of about $38{ }^{\circ} \mathrm{C}$, which is not large in comparison to the temperatures observed in many of the auger holes. The anomaly of figure 4 is confined almost entirely to the south of the road as is the thermal infrared anomaly of Watson $(1974,1975)$. Since the holes north of the road are cooler, this would tend to imply that the hot water does not move out from under the Jim Sage Mountains (Nathenson and others, 1980). However, this anomaly is based entirely on the shallow auger holes and deeper drilling might change this conclusion. For example, the anomaly parallels the contours rather nicely in figure 4, suggesting that there is some topographic relationship. This may, however, be fortuitous since the holes were drilled along a nearly linear trend paralleling the road.

\section{DISCUSSION}

Temperature measurements in shallow, intermediate, and deep holes show the thermal regime in the Raft $\mathrm{River}$ area is complex. The maximum temperatures and geochemistry indicate that the geothermal system is a mediumtemperature system (approximately $150^{\circ} \mathrm{C}$ ). Water of this temperature is only marginally useful for electric-power production with present technology. A discussion of the temperatures and geochemistry of the wells reported in this paper are contained in Nathenson and others (1980). Additional background information on the ground-water resources of the valley may be found in Nace and others (1961) and Crosthwaite (1974). The geology and geophysics are discussed in various publications 1 isted in the references.

The bulk of this report contains plots (app. I) and listings (app. III) of the temperature data obtained on the various dates shown in table 3 . Some 


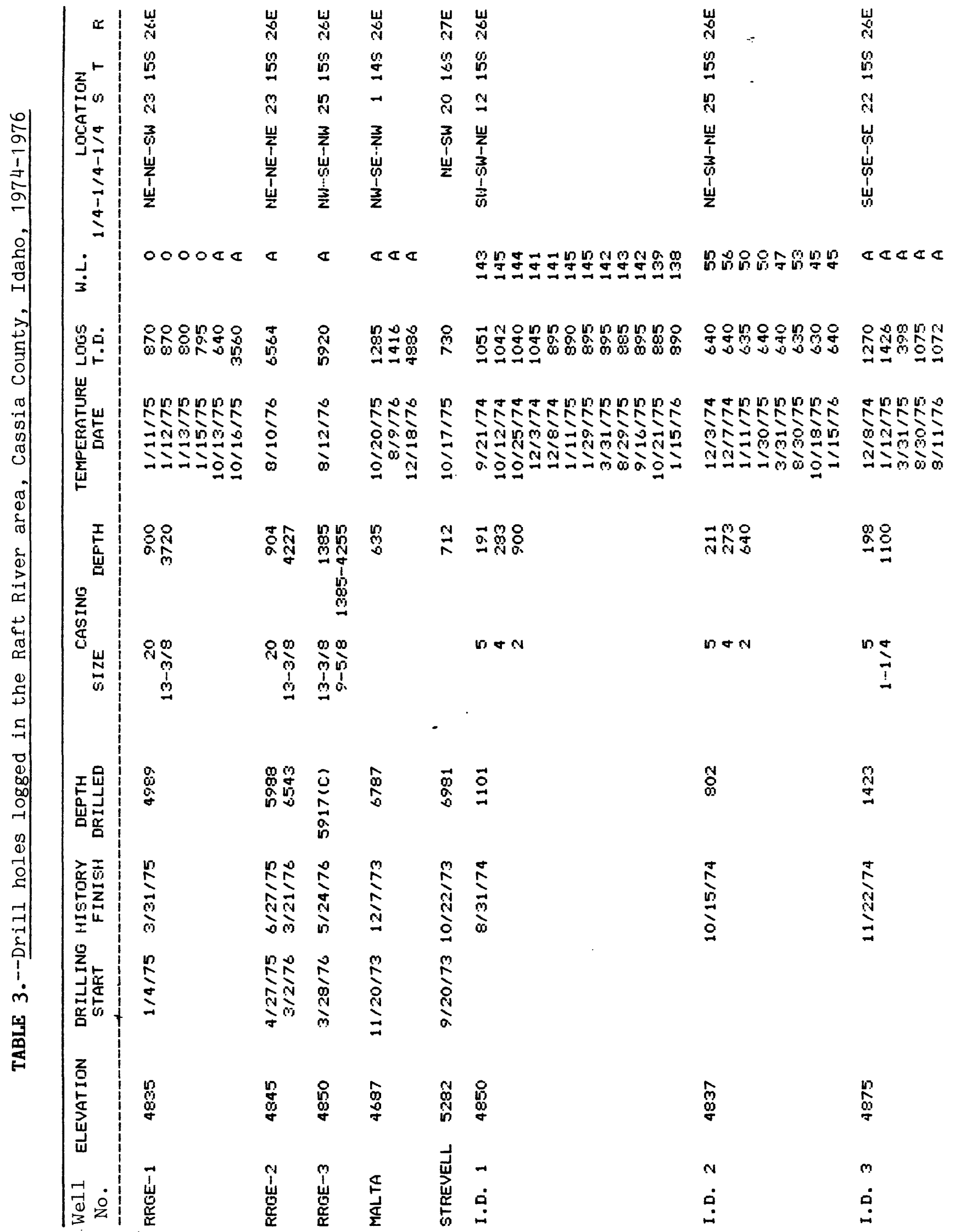




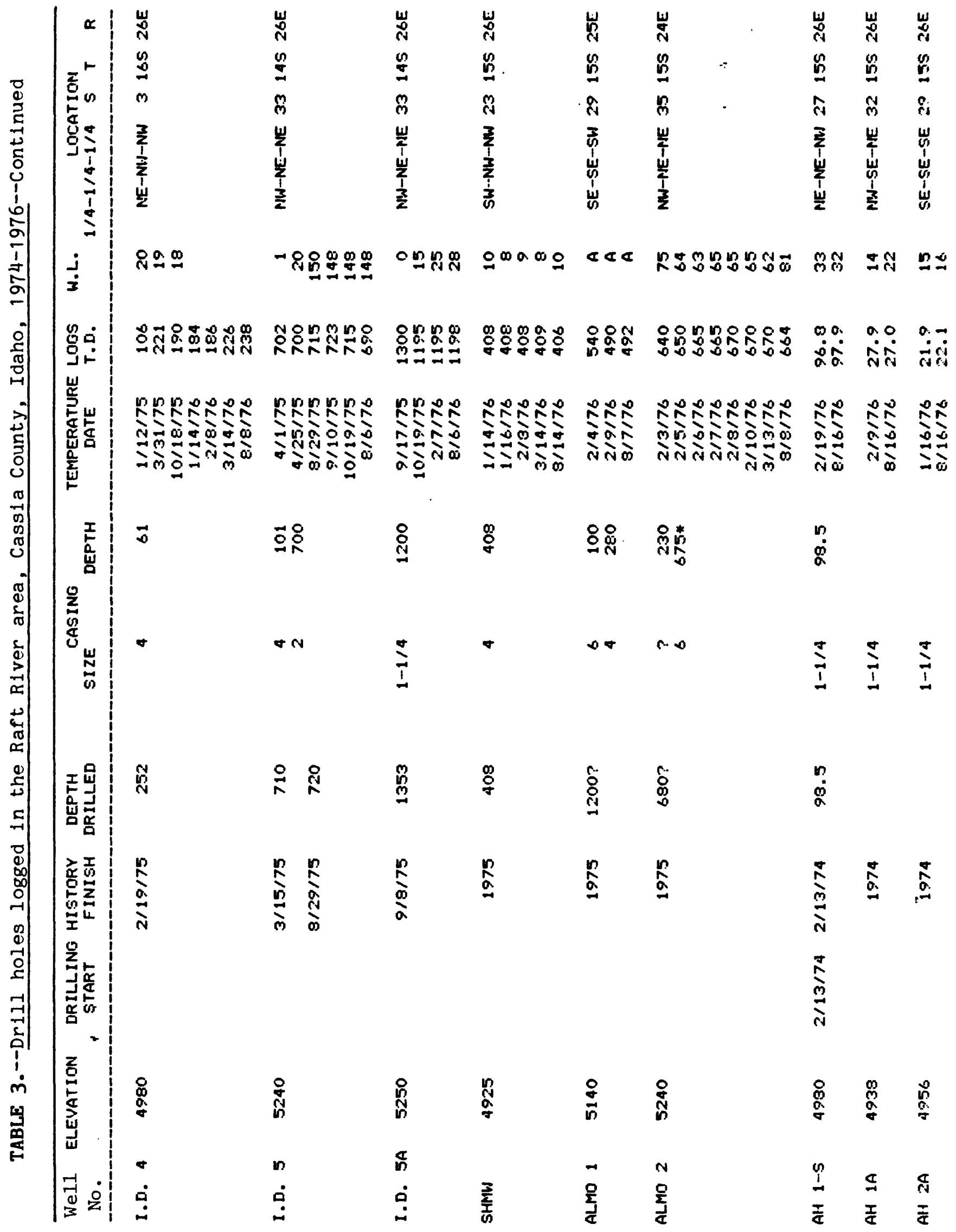




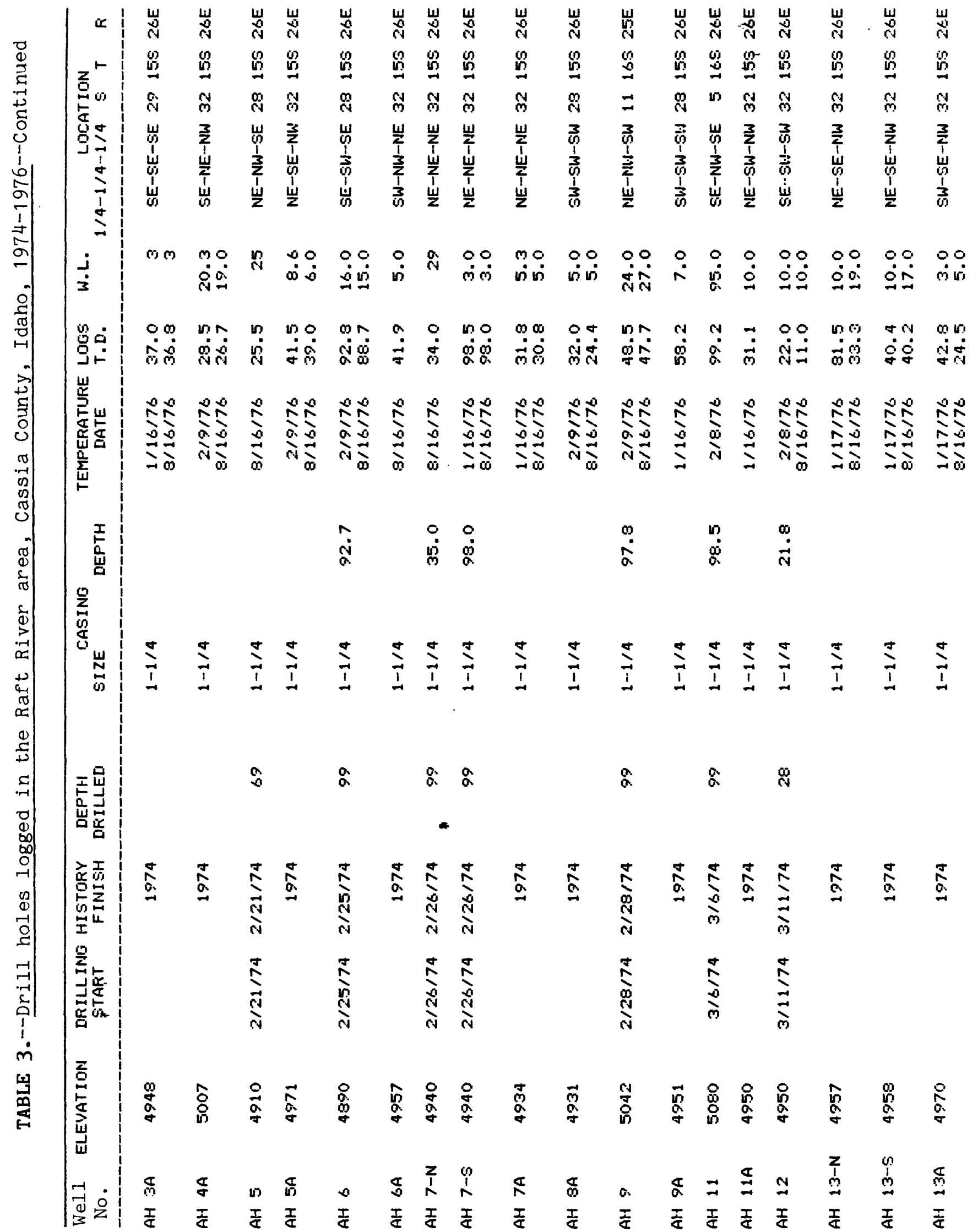




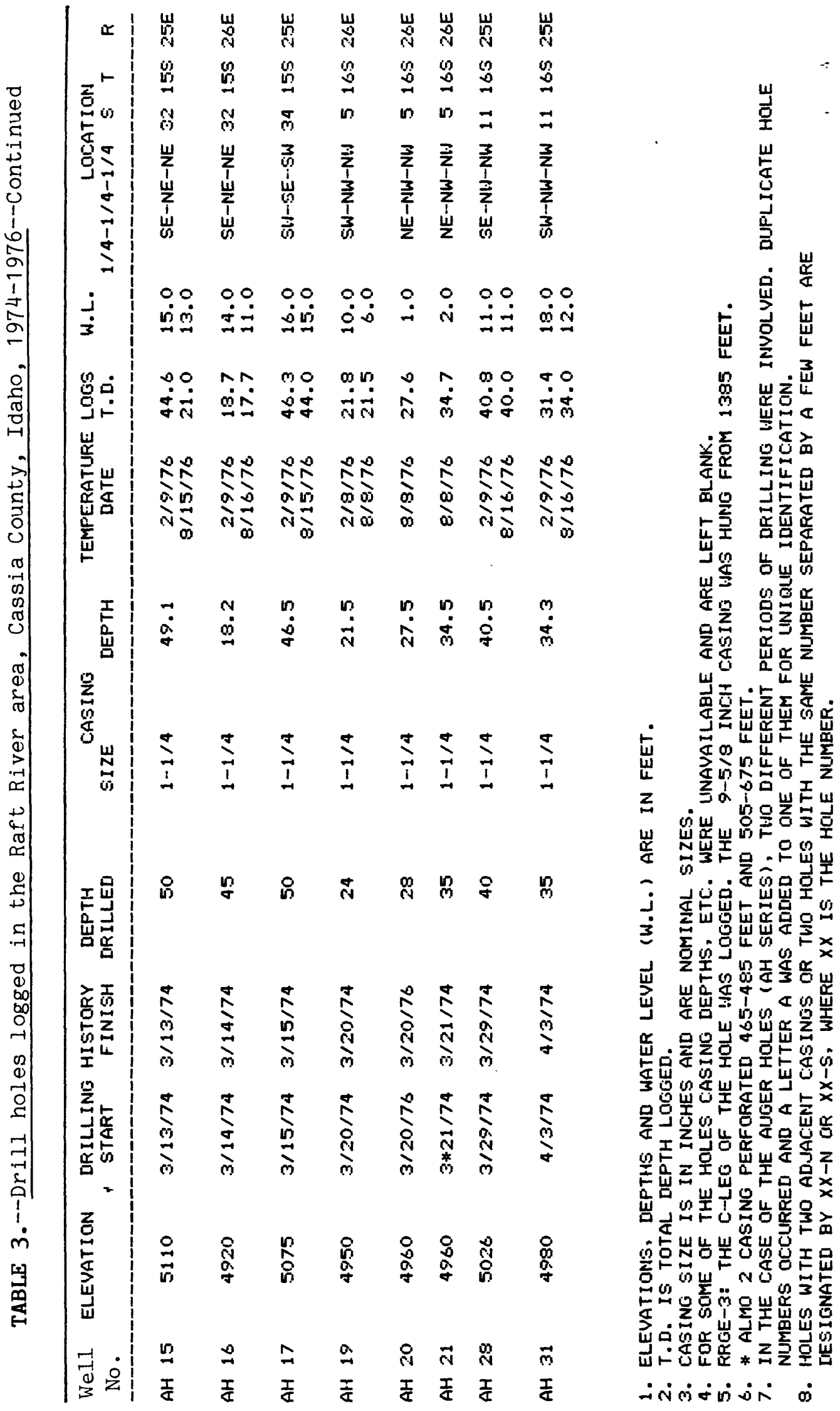


of the wells are artesian (table 4) and were logged under pressure through a lubricator. Thermal conductivity data (app. IV) and plots (app. II) are given where available. Calculated heat flows (Urban and Diment, 1975) are as high or higher than most of those in the Basin and Range Province to the south (Blackwell, 1978; Lachenbruch and Sass, 1978) and indicate an anomalous thermal regime in the valley. Although some irrigation does occur in the Raft River Valley, most of it is to the north of the geothermal system. What little irrigation that does occur in the southern part of the valley is a considerable distance from the holes considered in this report and the irrigation wells are too shallow (100 m or less) to affect the temperature profiles in the deep holes.

\section{ACKNOWLEDGMENTS}

We wish to thank the Department of Energy and, in particular, Roger Stoker of EG\&G-Idaho, Inc., and Idaho National Engineering Laboratory (INEL), for making the deep holes in the Raft River area available for temperature logging. 
TABLE 4.--Artesian wells in Raft River area

[Well-head pressure: C, cold; H, hot, from Kunze and Miller (1977). Leaders (--) indicate no data available]

\begin{tabular}{lcccc}
\hline Well No. & $\begin{array}{l}\text { Well-head } \\
\text { pressure } \\
\text { (PSIG) }\end{array}$ & $\begin{array}{c}\text { Flow } \\
\text { (gal/min) }\end{array}$ & $\begin{array}{c}\text { Maximum } \\
\text { temperature } \\
\left({ }^{\circ} \mathrm{C}\right)\end{array}$ & $\begin{array}{c}\text { Solids } \\
\text { in } \\
\text { water } \\
(\mathrm{mg} / \mathrm{l})\end{array}$ \\
\hline RRGE-1 & $50(\mathrm{C})-175(\mathrm{H})$ & 600 & 146 & 1,700 \\
RRGE-2 & $60(\mathrm{C})-165(\mathrm{H})$ & 780 & 148 & 1,800 \\
RRGE-3 & $40(\mathrm{C})-140(\mathrm{H})$ & 350 & 146 & 4,600 \\
Malta & 55 & 20 & 94.7 & $-\ldots$ \\
I.D. 3 & 9 & 10 & 98.7 & $-\ldots$ \\
Almo 1 & 11.5 & .04 & 73.5 & $-\ldots$ \\
\hline
\end{tabular}

1 For additional data on chemistry, permeability, porosity, and density of the RRGE wells, see Stoker and others (1977). 


\section{REFERENCES}

Ackermann, H. D., 1979, Seismic refraction study of the Raft River geothermal area, Idaho: Geophysics, v. 44, p. 216-225.

Birch, Francis, 1950, Flow of heat in the Front Range, Colorado: Geological Society of America Bulletin, v. 61, p. 567-630.

Blackwell, D. D., 1978, Heat flow and energy loss in the Western United States, in Smith, R. B., and Eaton, G. P., eds., Cenozoic tectonics and regional geophysics of the western Cordillera: Geological Society of America Memoir 152, p. 175-208.

Bullard, E. C., 1939, Heat flow in South Africa: Proceedings of the Royal Society of London, Series A, v. 173, p. 474-502.

Covington, H. R., 1977a, Deep drilling data, Raft River geothermal area, Idaho--Raft River Geothermal Exploration Well No. 1: U.S. Geological Survey Open-File Report 77-226, 1 sheet.

1977b, Deep drilling data, Raft River geothermal area, Idaho-Raft River Geothermal Exploration Well No. 2: U.S. Geological Survey Open-File Report 77-243, 1 sheet.

1977c, Deep drilling data, Raft River geothermal area, Idaho-Raft River Geothermal Exploration Well No. 3: U.S. Geological Survey Open-File Report 77-616, 1 sheet.

1977d, Deep drilling data, Raft River Geothermal Area, Idaho--Raft River Geothermal Exploration Well No. 3 sidetrack-C: U.S. Geological Survey Open-File Report 77-883, 1 sheet.

Crosthwaite, E. G., 1974, Preliminary data for (34) test-wells augered in the Raft River Valley, February 13-March 8, 1974: U.S. Geological Survey Open-File Report, 20 p., available from U.S. Geological Survey, Federal Bldg., Boise, Idaho 83843.

1976, Basic data from five core holes in the Raft River geothermal area, Cassia County, Idaho: U.S. Geological Survey Open-File Report 76-665, $12 \mathrm{p}$.

Kunze, J. F., and Miller, L. G., 1977, Experiments and testing at Raft River, in Kunze, J. F., ed., Geothermal R\&D project report for period October 1, 1976 to March 31, 1977: EG\&G-Idaho, Inc., Idaho National Engineering Laboratory, TREE-1134, Idaho Falls, Idaho 83401, p. 9-21.

Lachenbruch, A. H., and Marshall, B. V., 1966, Heat flow through the Arctic Ocean floor: The Canada Basin-Alpha Rise Boundary: Journal of Geophysical Research, v. 71, p. 1223-1248.

Lachenbruch, A. H., and Sass, J. H., 1978, Models of an extending lithosphere and heat flow in the Basin and Range Province, in Smith R. B., and Eaton, G. P., eds., Cenozoic tectonics and regional geophysics of the western Cordillera: Geological Society of America Memoir 152, p. 209-250. 
Mabey, D. R., Hoover, D. B., O'Donnell, J. E., and Wilson, C. W., 1978, Reconnaissance geophysical studies of the geothermal system in southern Raft River Valley, Idaho: Geophysics, v. 43, p. 1470-1484.

Nace, R. L. and others, 1961, Water resources of the Raft River Basin IdahoUtah: U.S. Geological Survey Water-Supply Paper 1587, $138 \mathrm{p}$.

Nathenson, Manuel, Nehring, N. L., Crosthwaite, E. G., Harmon, R. S., Janik, C., and Borthwick, J., 1982, Chemical and light-stable isotope characteristics of waters from the Raft River geothermal area and environs, Cassia County, Idaho; Box Elder County, Utah: Geothermics, v. 2 , p. $215-237$.

Nathenson, Manuel, Urban, T. C., and Diment, W. H., 1979, Approximate solution for the temperature distribution caused by flow up a fault and its application to temperatures measured in a drillhole at Raft River geothermal area, Cassia County, Idaho: Geothermal Resources Council Transactions, v. 3, p. 477-480.

Nathenson, Manuel, Urban, T. C., Diment, W. H., and Nehring, N. L., 1980, Temperatures, heat flow, and water chemistry from drill holes in the Raft River geothermal system, Cassia County, Idaho: U.S. Geological Survey Open-File Report 80-2001, 29 p.

Oriel, S. S., Williams, P. L., Covington, H. R., Keys, W. S., and Shaver, K. C., 1978, Deep drilling data, Raft River geothermal area, Idaho: Standard American Oil Co., Malta, Nafe, and Strevell Petroleum test boreholes: U.S. Geological Survey Open-File Report 78-361, 2 pls.

Robertson, E. C., Raspet, R., Swartz, J. H., and Lillard, M. E., 1966, Properties of thermistors used in geothermal investigations: U.S. Geological Survey Bulletin 1203-B, 34 p.

Sass, J. H., Kennelly, J. P., Jr., Smith, E. P., and Wendt, W. E., 1984, Laboratory line-source methods for the measurement of thermal conductivity of rocks near room temperature: U.S. Geological Survey Open-File Report 84-91, 21 p.

Simmons, Gene, 1961, Anisotropic thermal conductivity: Journal of Geophysical Research, v. 66, p. 2269-2270.

Stoker, R. C., Goldman, D., and Kunze, J. F., 1977, Reservoir engineering, in Kunze, J. F., ed., Geothermal R\&D project report for period October 1, 1976 to March 31, 1977: EG \& G-Idaho, Inc., Idaho National Engineering Laboratory, TREE-1134, Idaho Falls, Idaho 83401, p. 22-38.

Urban, T. C., and Diment, W. H., 1975, Heat flow on the south flank of the Snake River Rift [abs.]: Geological Society of America Abstracts with Programs, v. 7, p. 648 . 
1985. Convection in boreholes: Limits on interpretation of temperature logs and methods for determining anomalous fluid flow, in NWW Conference on Surface and Borehole Geophysical Methods in Ground water Investigations: National Water Well Association, Worthington, Ohio, p. 399-414.

Von Herzen, R. and Maxwell, A. E., 1959, The measurement of thermal conductivity of deep-sea sediments by the needle-probe method: Journal of Geophsical Research, v. 64, p. 1557-1563.

Watson, Kenneth, 1974, Geothermal reconnaissance from quantitative analysis of thermal infrared images: International Symposium on Remote Sensing of the Environment, 9th, Ann Arbor, Michigan, 1974, Proceedings, v. 3, p. 1919-1932.

1975, Geothermal reconnaissance from quantitative analysis of thermal infrared images: Institute of Electrical and Electronics Engineers, Proceedings, New York, N.Y., p. 128-137.

Williams, P. L., Mabey, D. R., Zohdy, A. A. R., Ackerman, H., Hoover, D. B., Pierce, K. L., and Oriel, S. S., 1976, Geology and geophysics of the southern Raft River Valley geothermal area, Idaho, USA: Second United Nations Symposium on the Development and Use of Geothermal Resources, San Francisco, California, 1975, Proceedings, v. 2, p. 1273-1282. 


\section{APPENDIX I}

TEMPERATURES PLOTTED AS A FUNCTION OF DEPTH FOR WELLS LOGGED IN THE RAFT RIVER AREA, CASSIA COUNTY, IDAHO (1974-1976)

\section{CONTENTS}

Well No.

Page

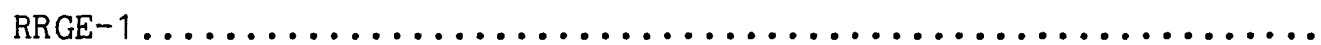

$I-1$

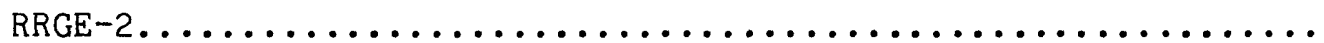

$I-7$

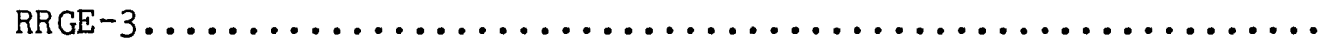

$I-8$

Malta ....................................

Strevell .................................... $I-12$

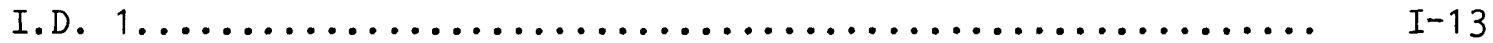

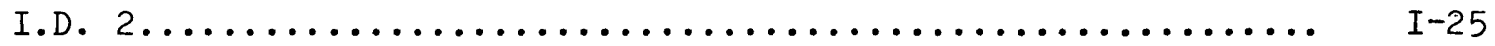

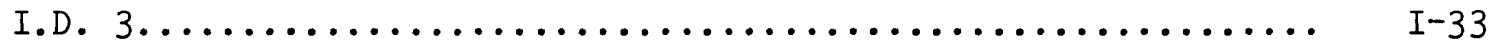

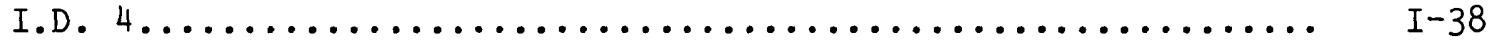

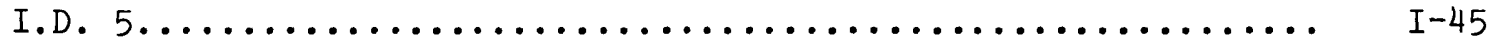

I.D. 5 A. ........................................

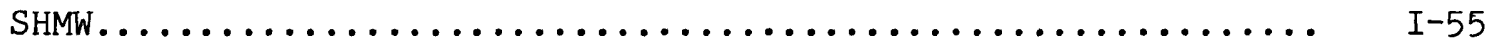

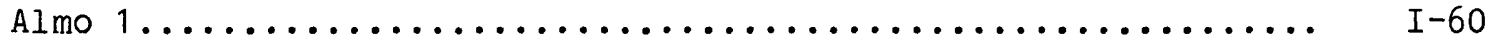

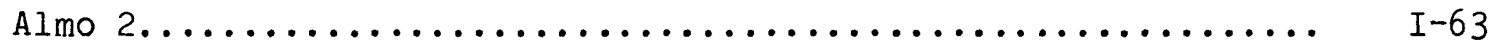

$\mathrm{AH} 1-\mathrm{S} \ldots \ldots \ldots \ldots \ldots \ldots \ldots \ldots \ldots \ldots \ldots \ldots \ldots \ldots \ldots \ldots \ldots \ldots \ldots \ldots \ldots \ldots$

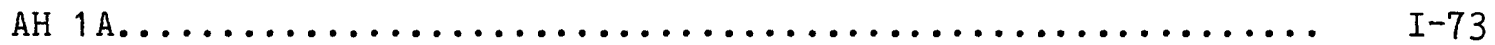

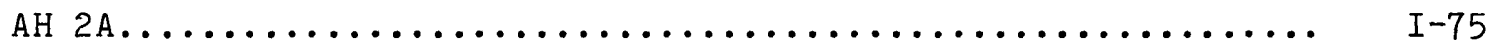

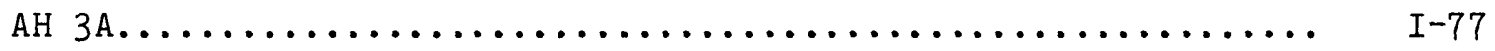

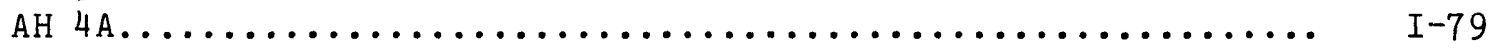

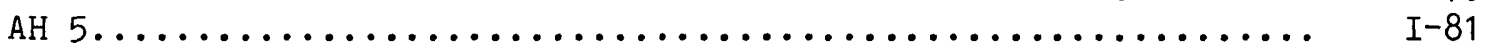

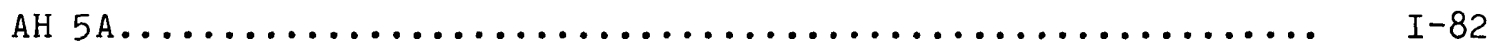

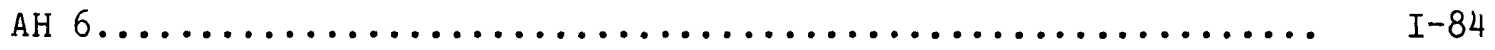

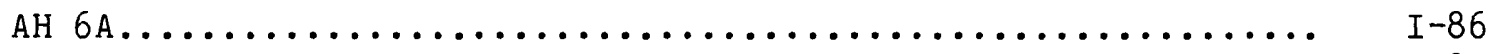

AH $7-N \ldots \ldots \ldots \ldots \ldots \ldots \ldots \ldots \ldots \ldots \ldots \ldots \ldots \ldots \ldots \ldots \ldots \ldots \ldots \ldots \ldots \ldots \ldots \ldots$

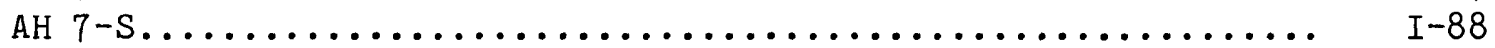

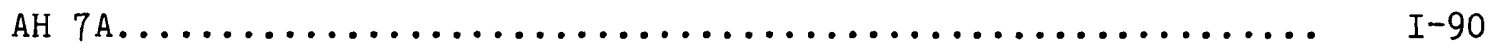

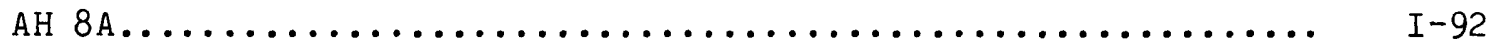

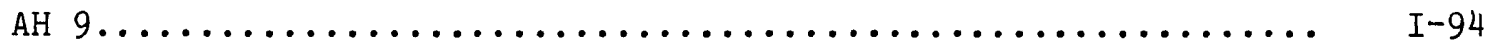

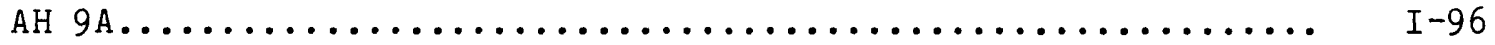

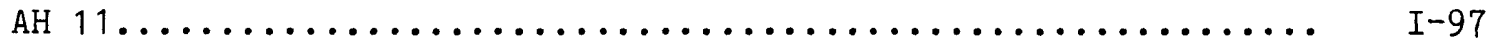

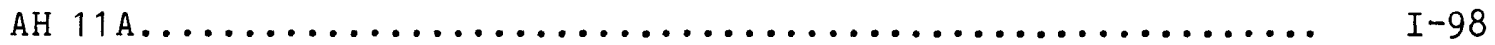

AH $12 \ldots \ldots \ldots \ldots \ldots \ldots \ldots \ldots \ldots \ldots \ldots \ldots \ldots \ldots \ldots \ldots \ldots \ldots \ldots \ldots \ldots \ldots \ldots \ldots \ldots$

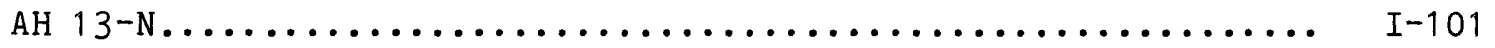

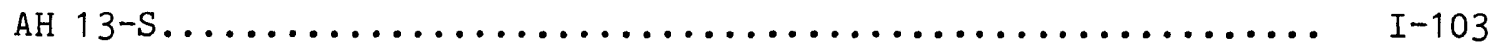

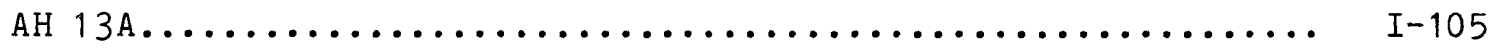

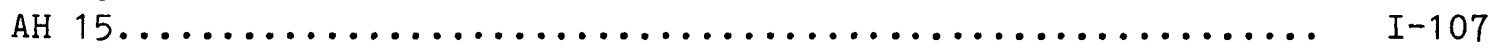

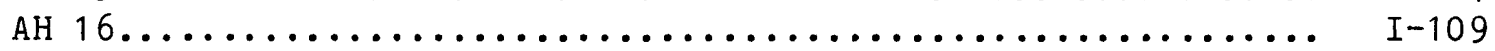

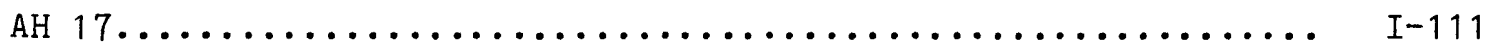

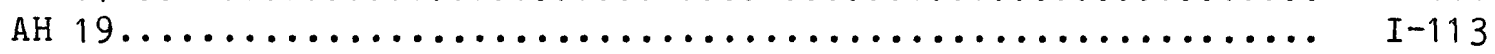

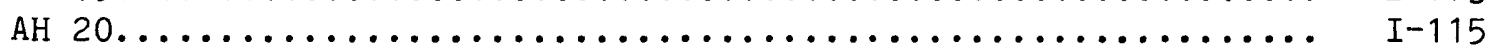

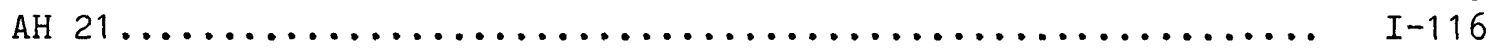

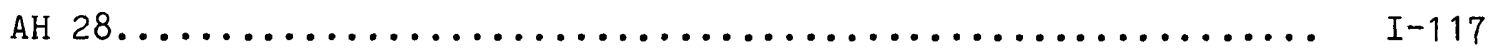

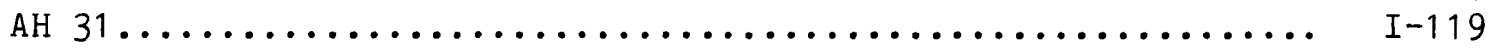




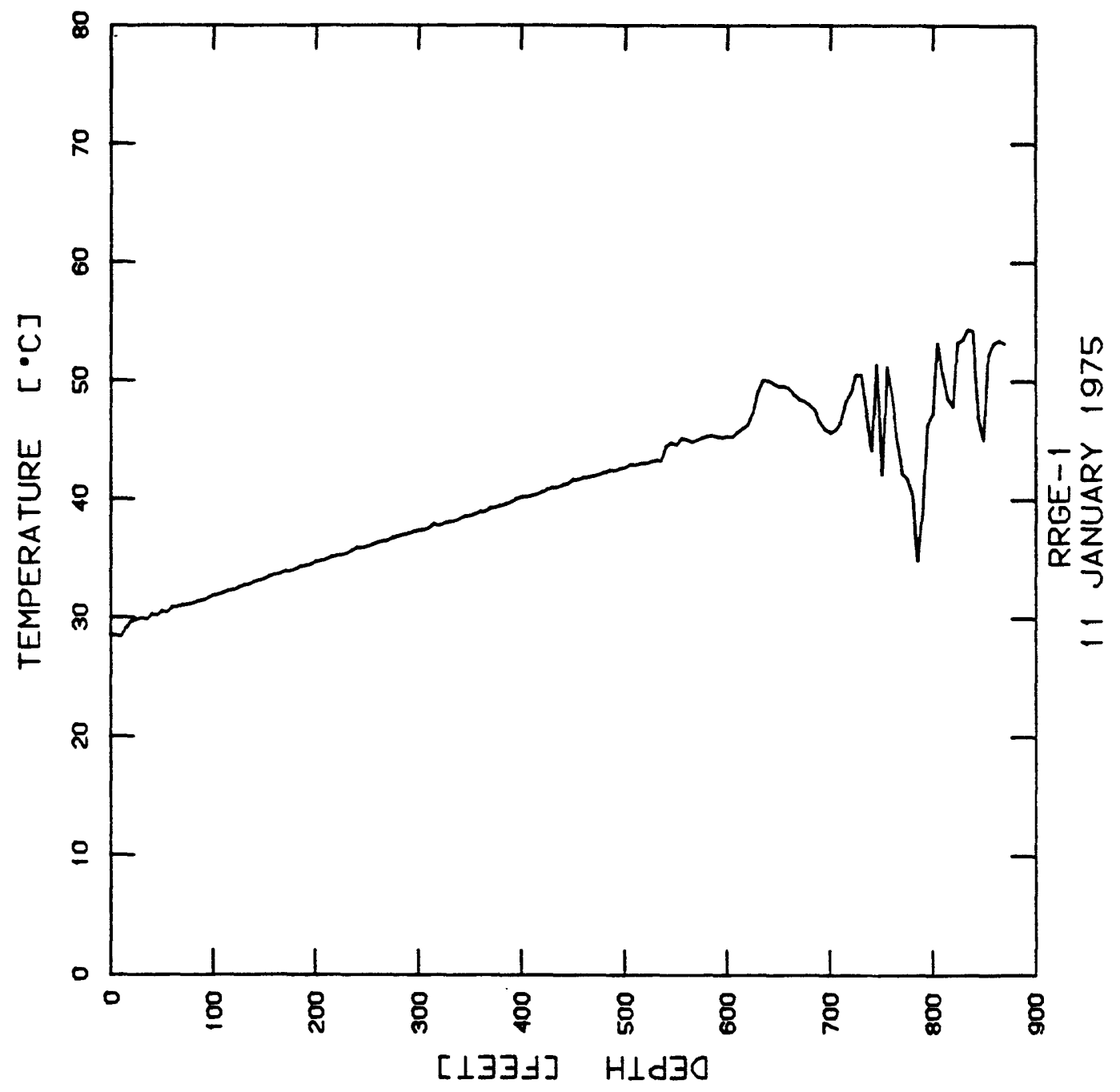

$I-1$ 


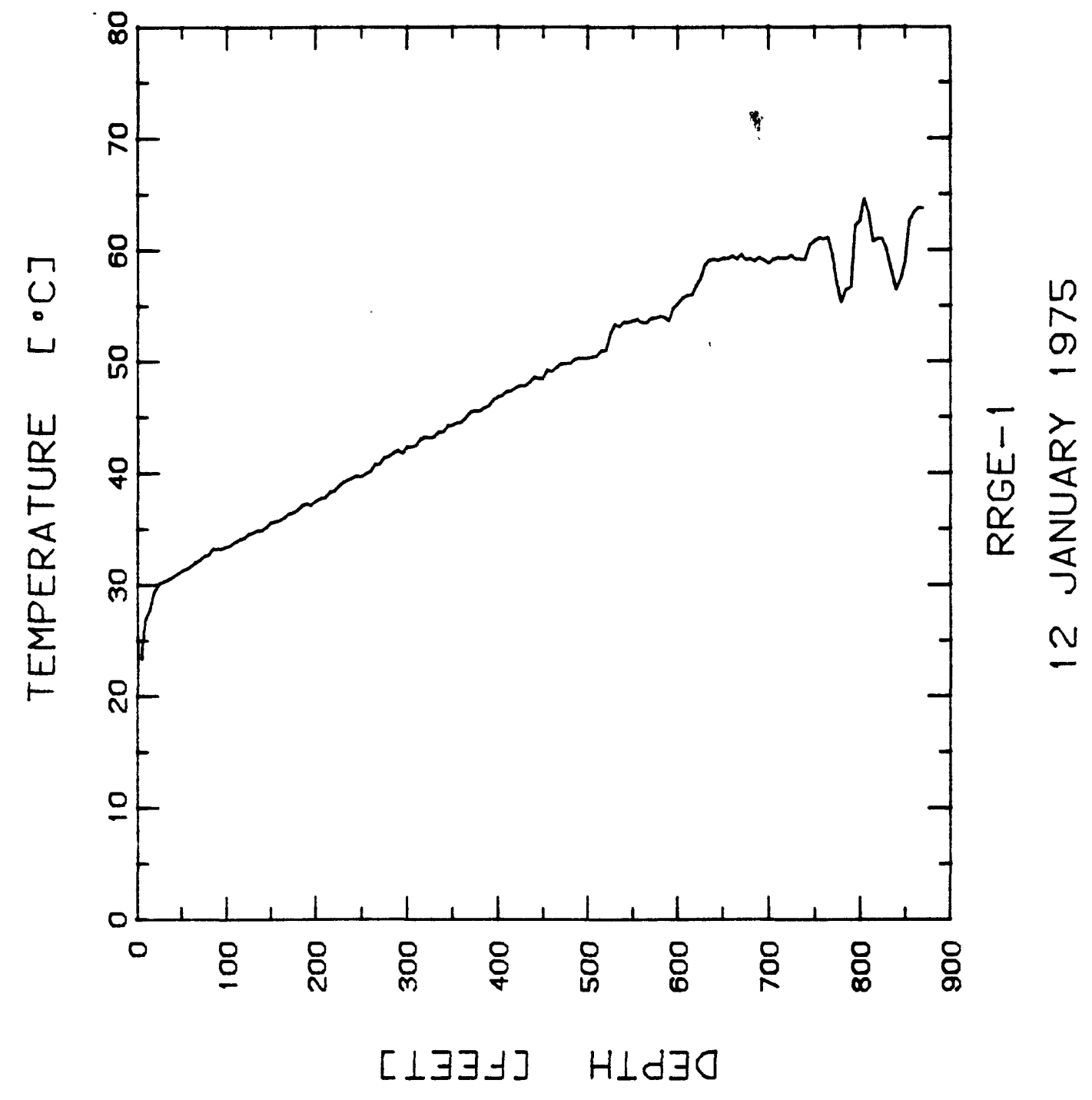

$I-2$ 


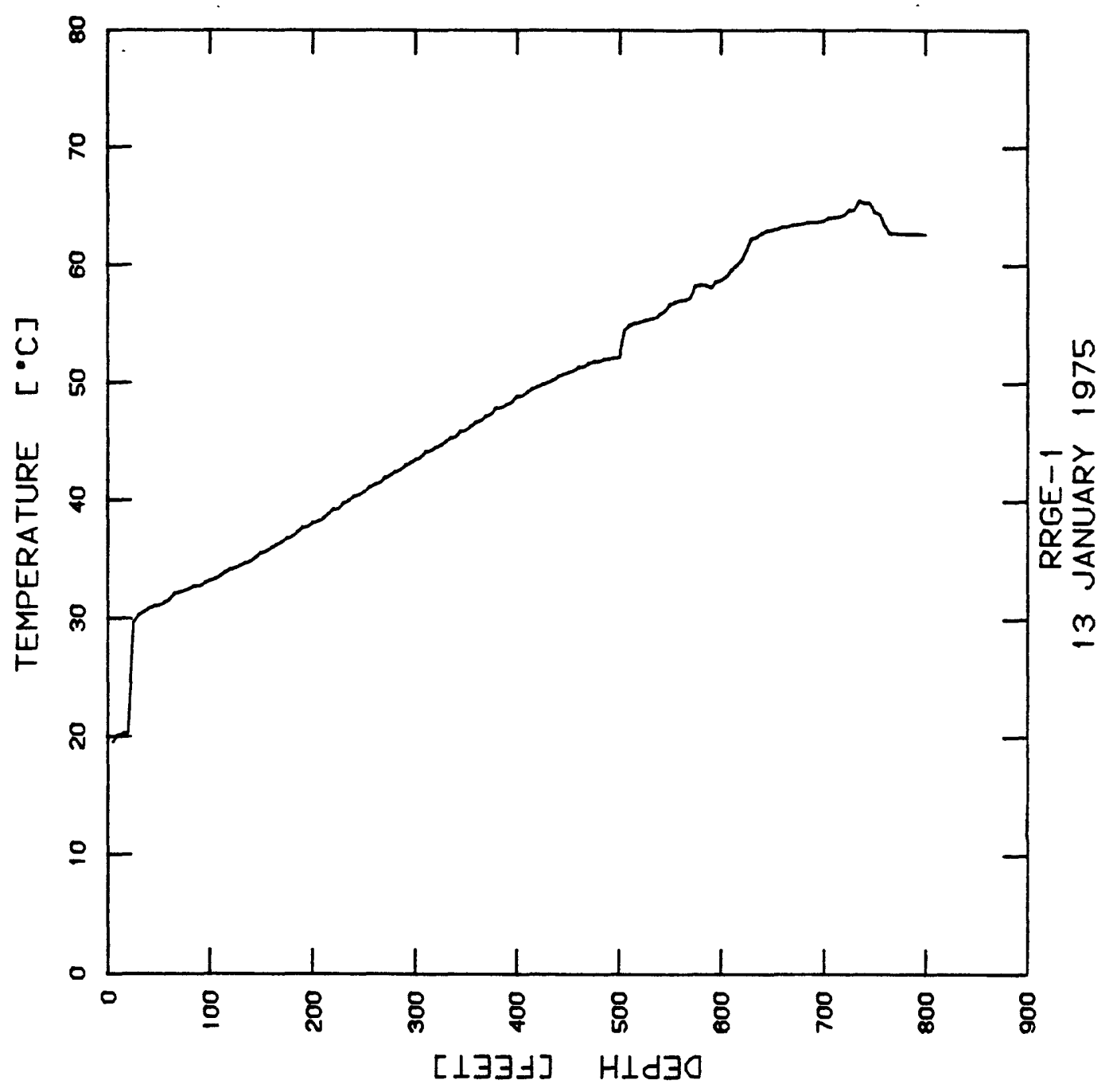

I-3 


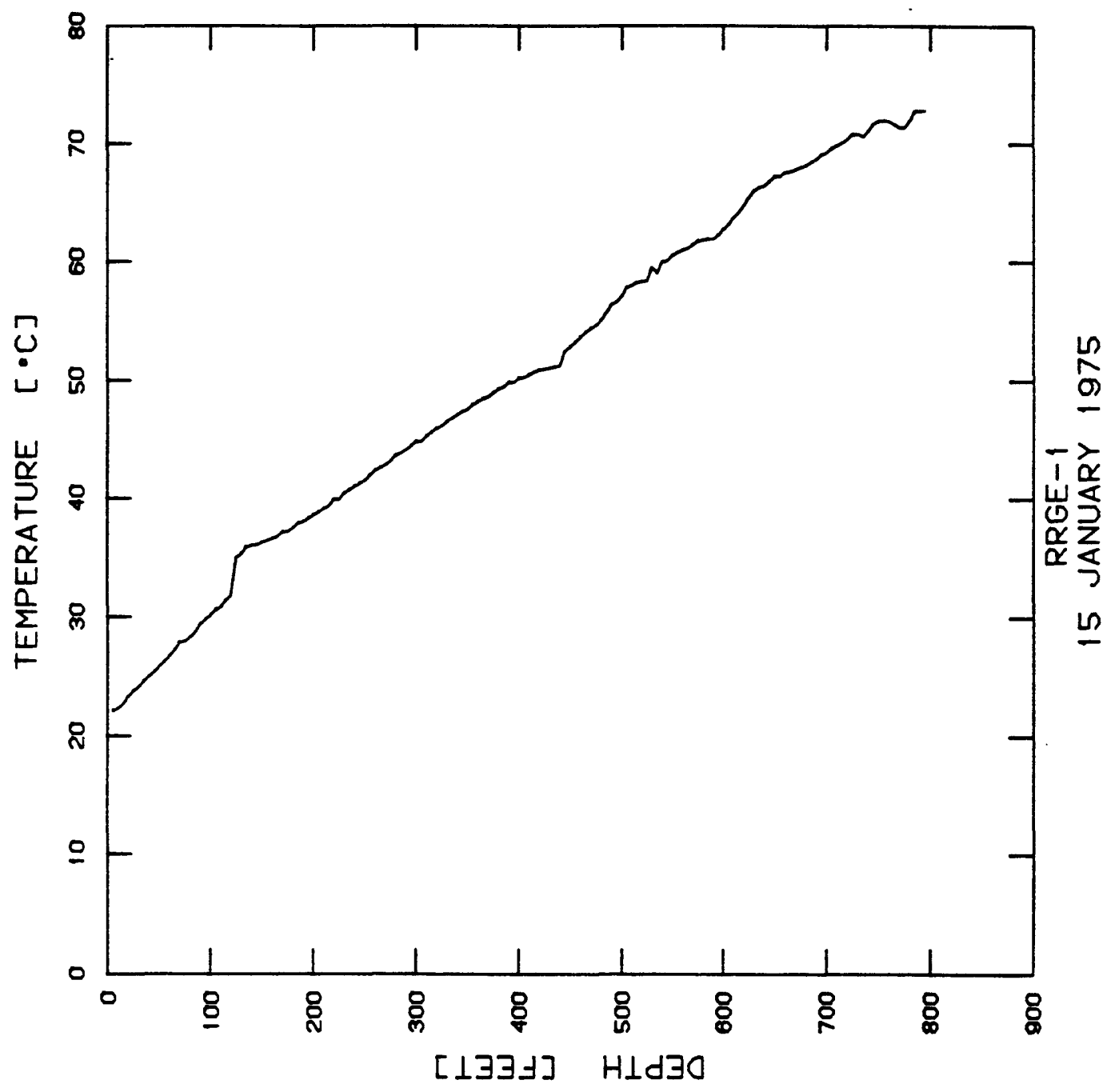

$$
I-4
$$




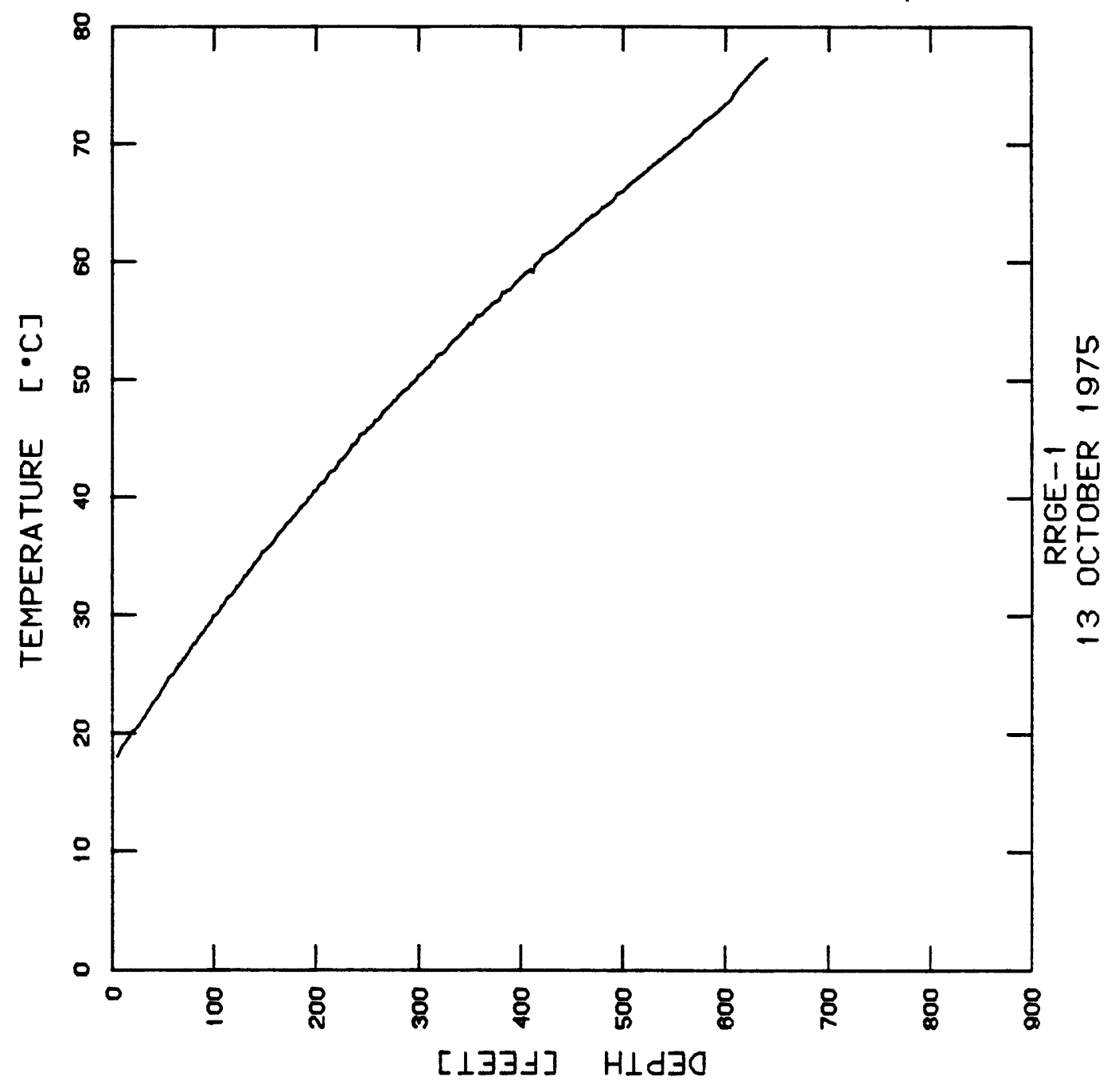

$I-5$ 


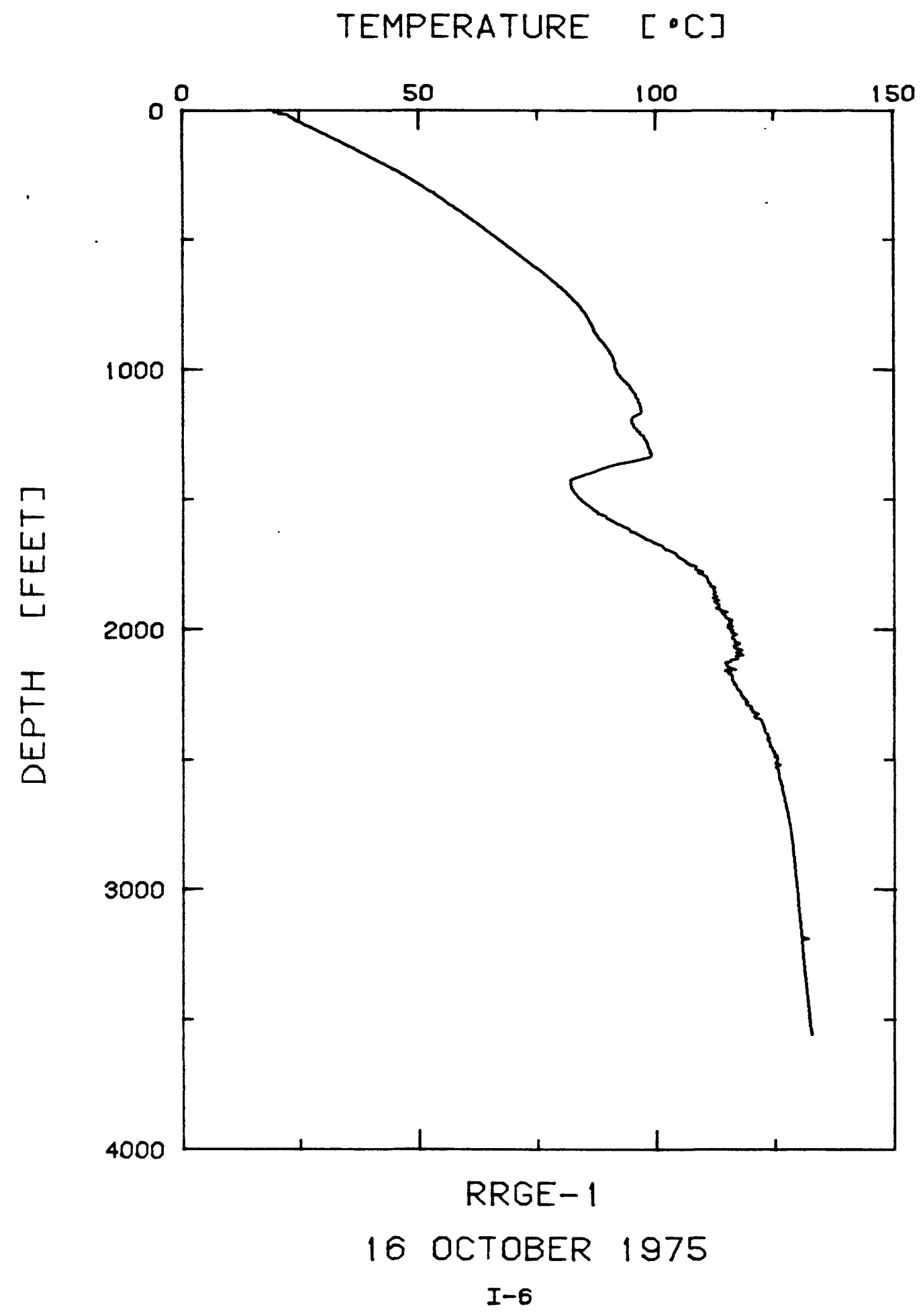




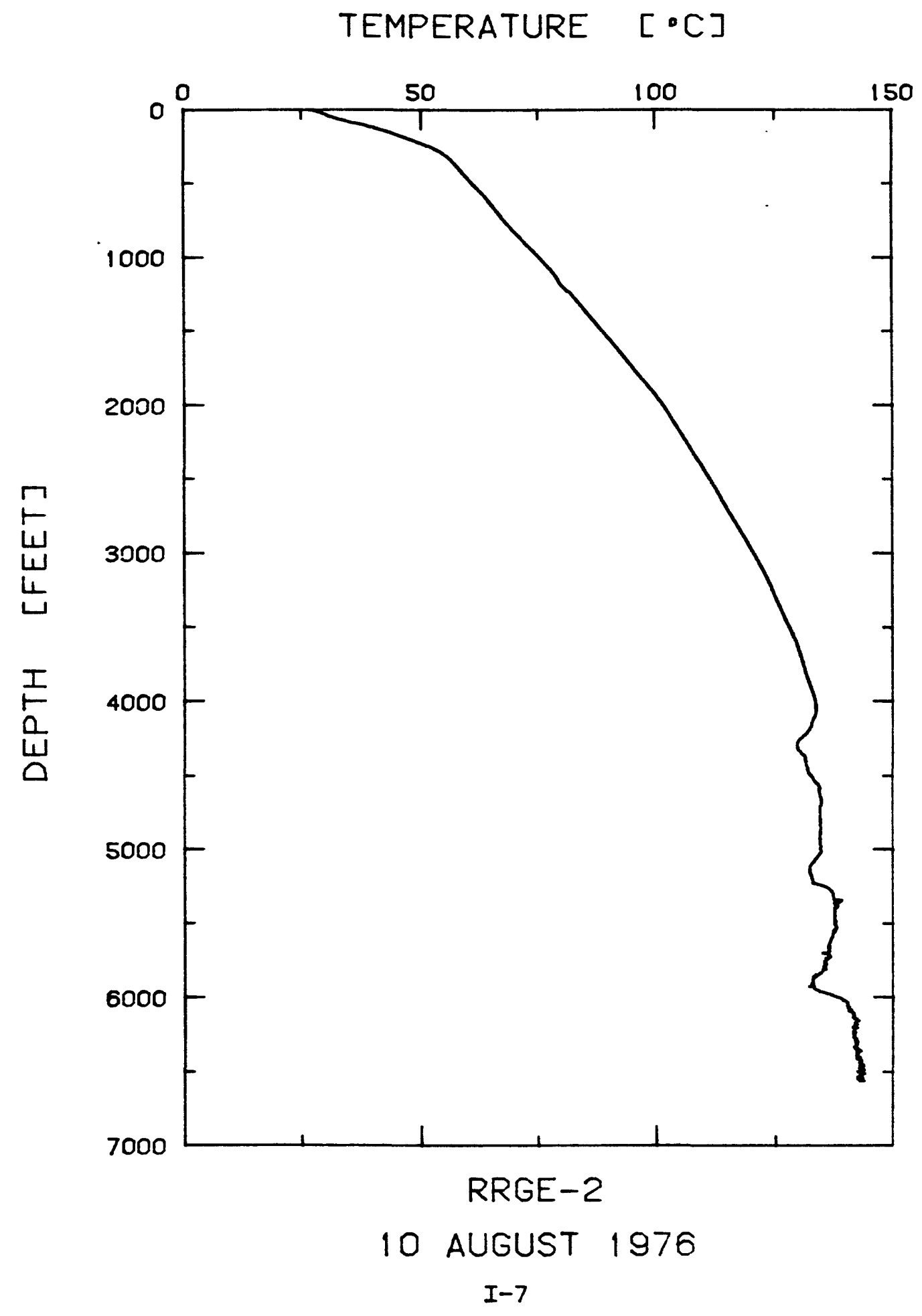




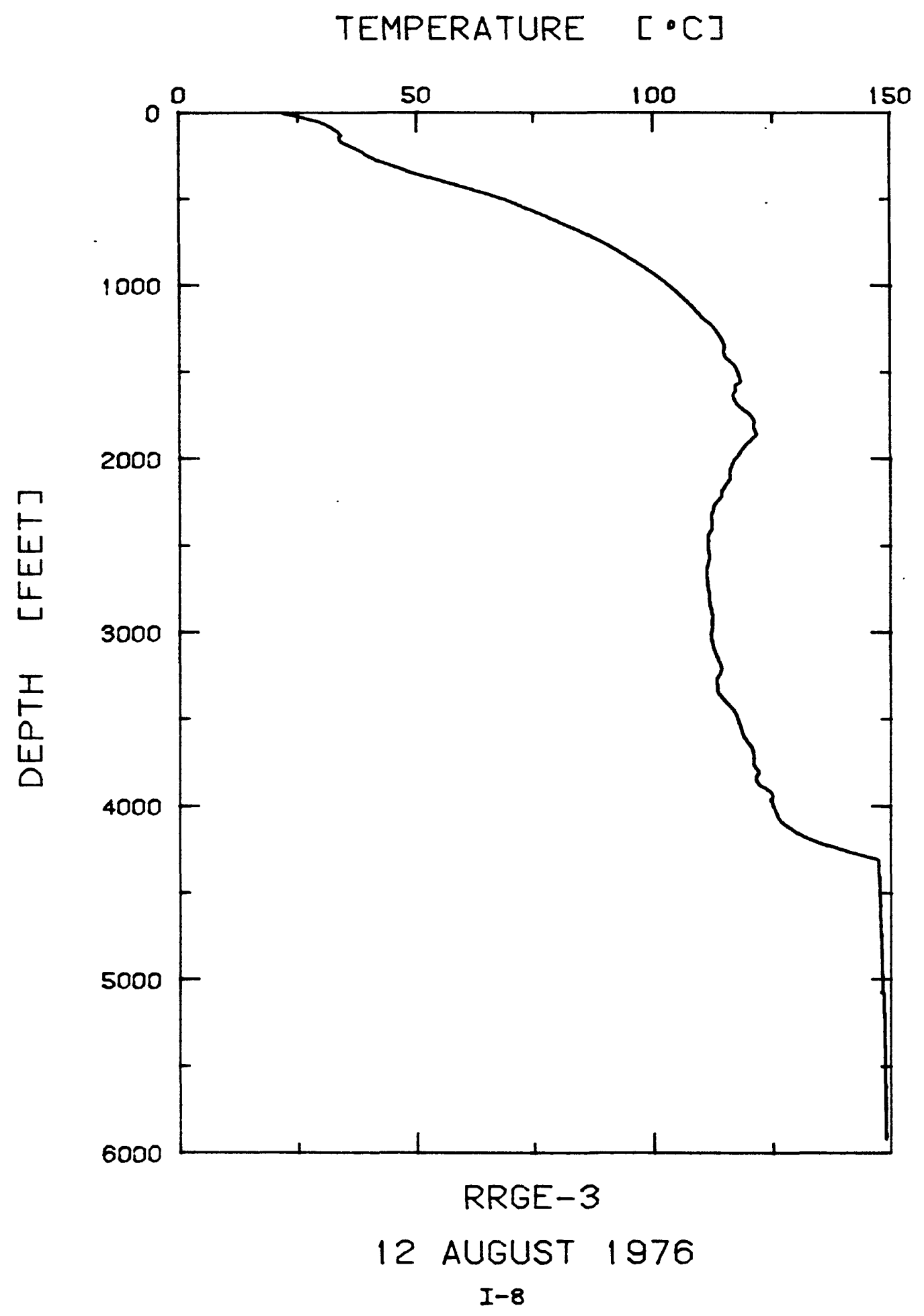




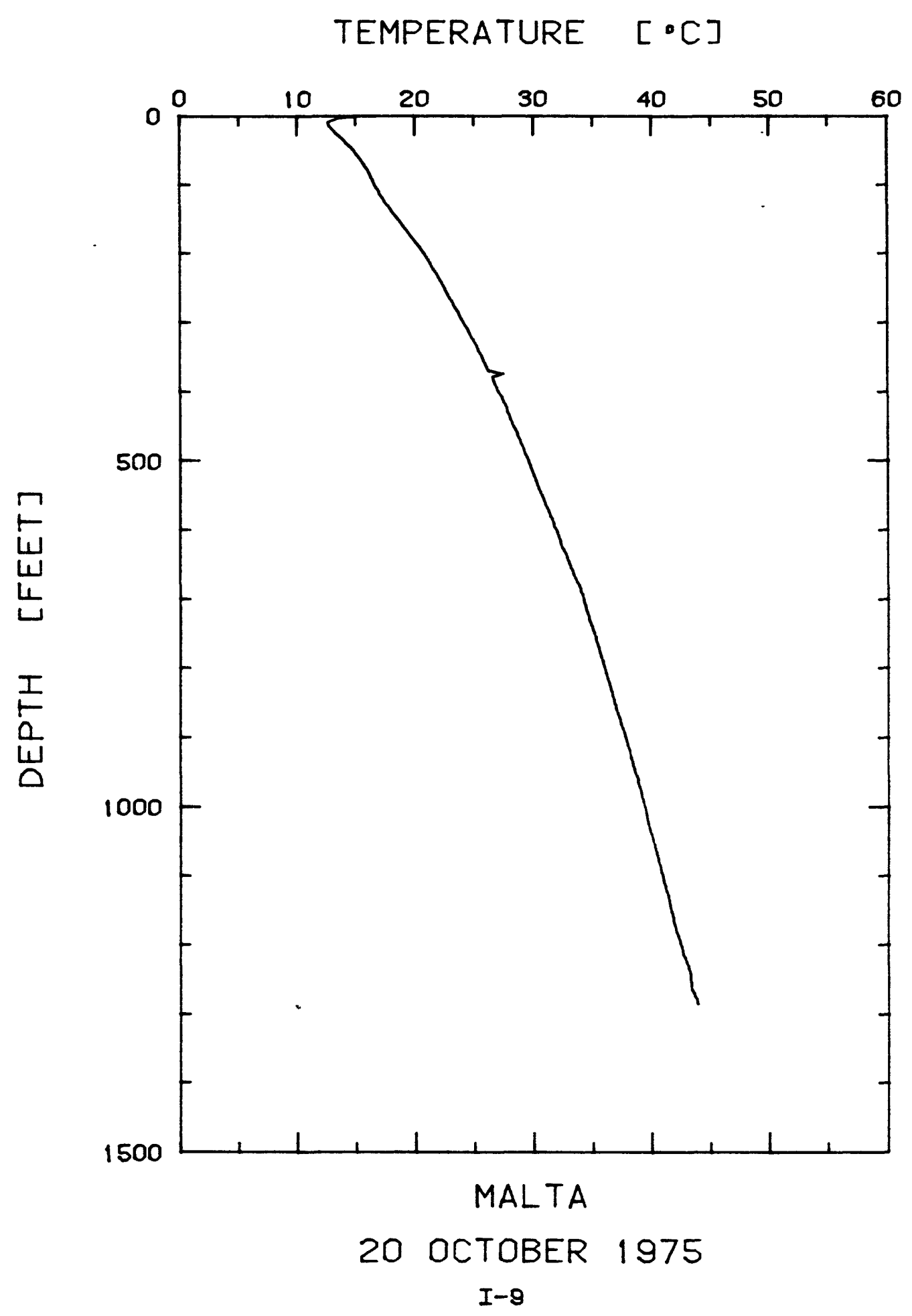




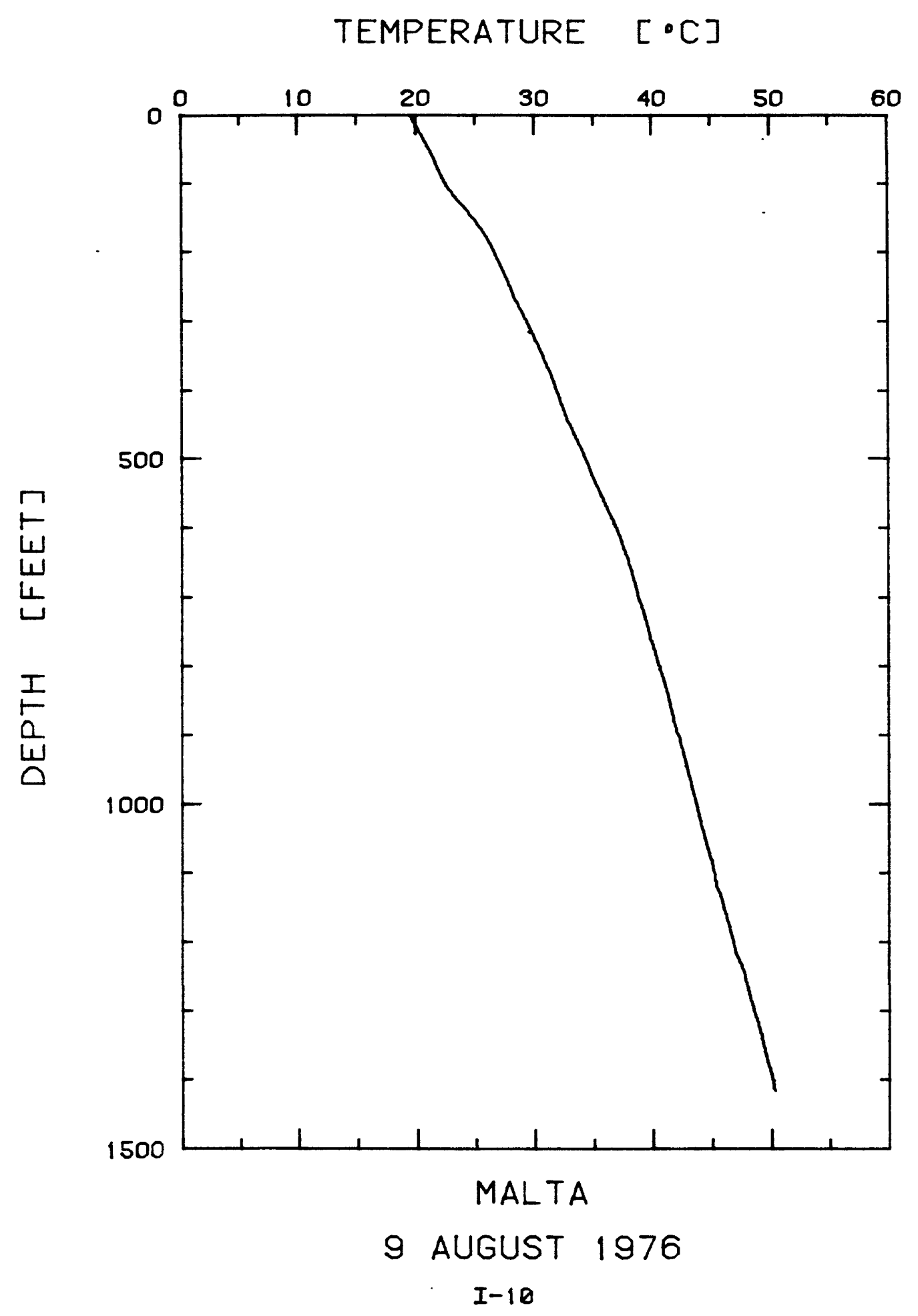




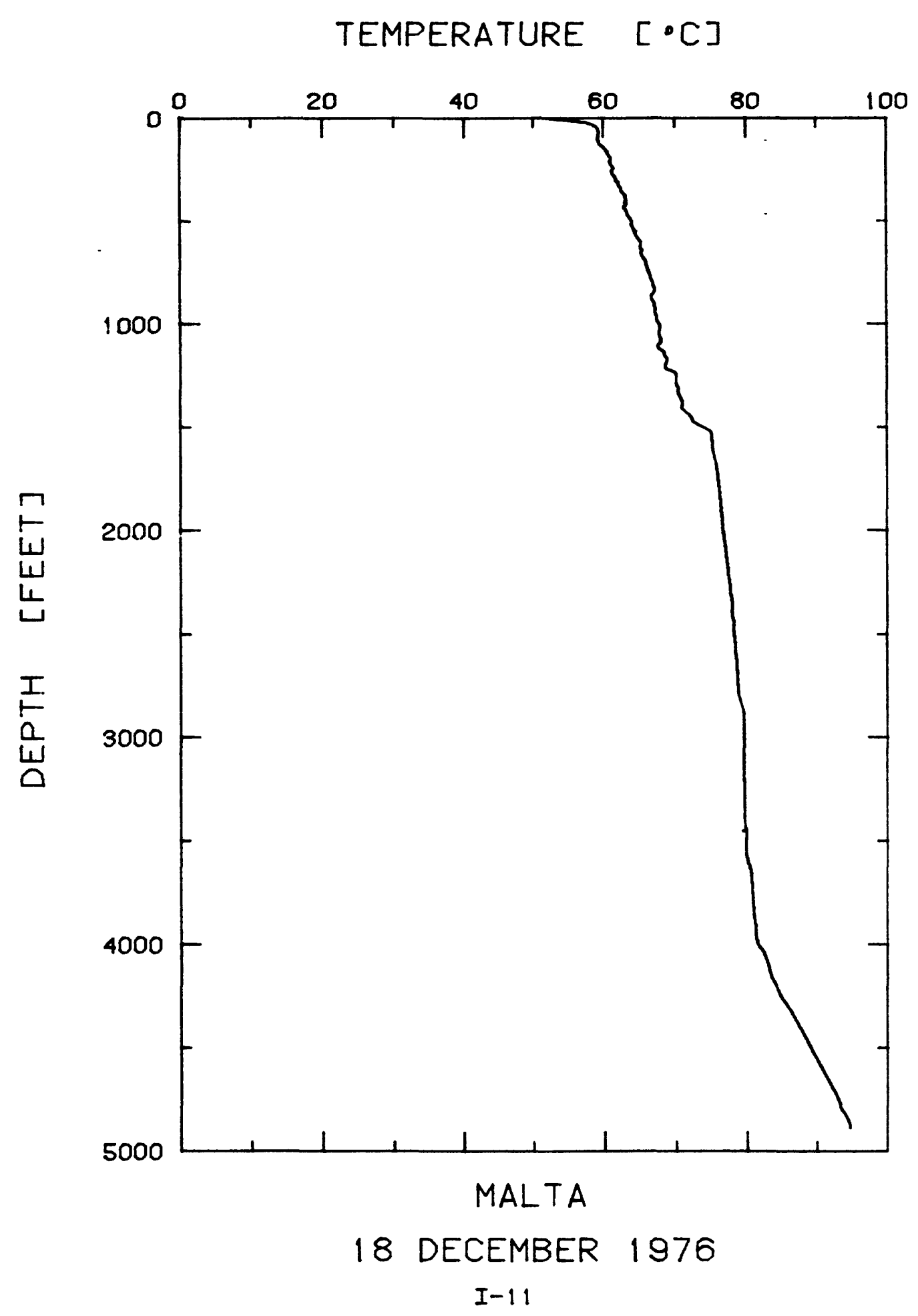




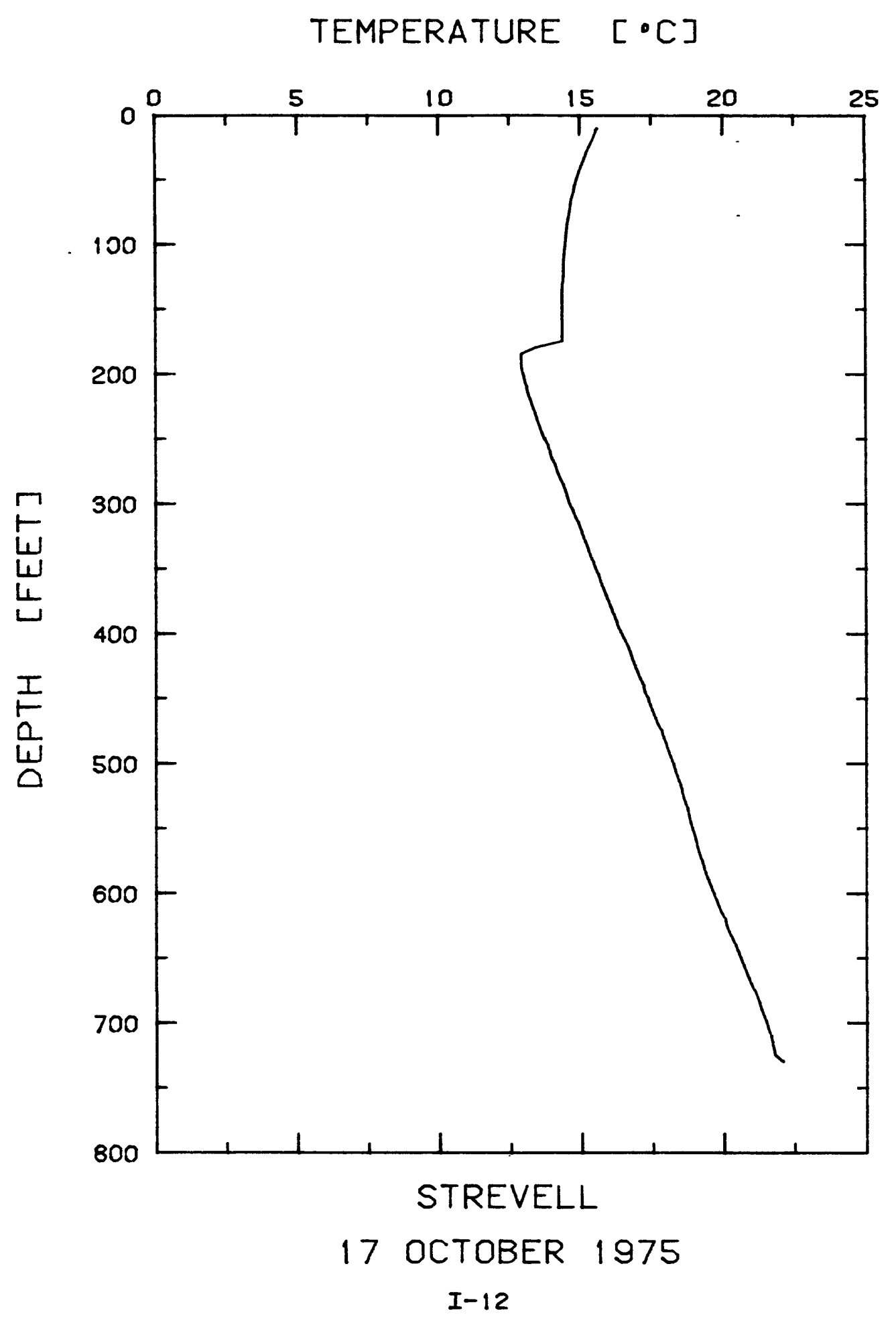




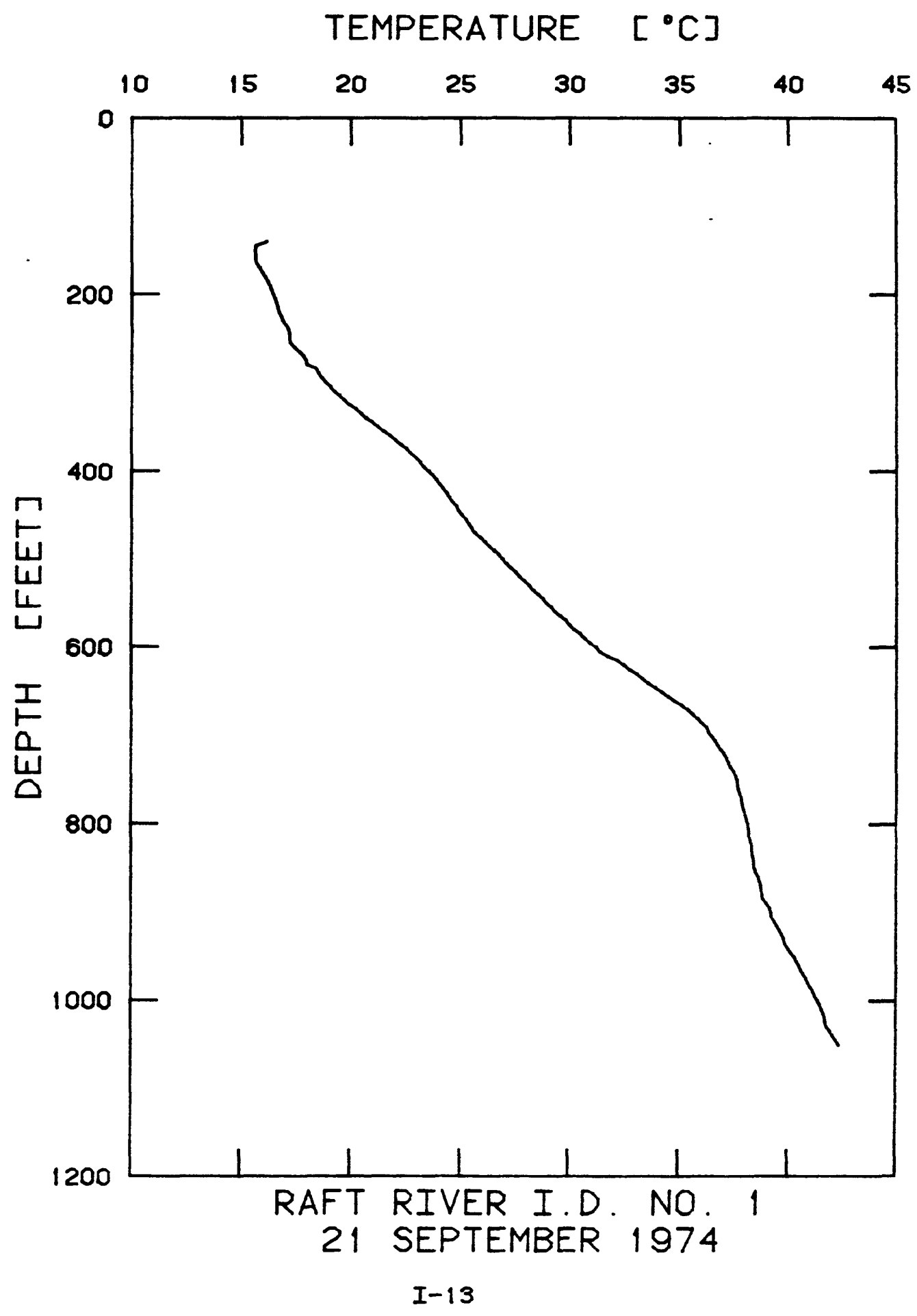




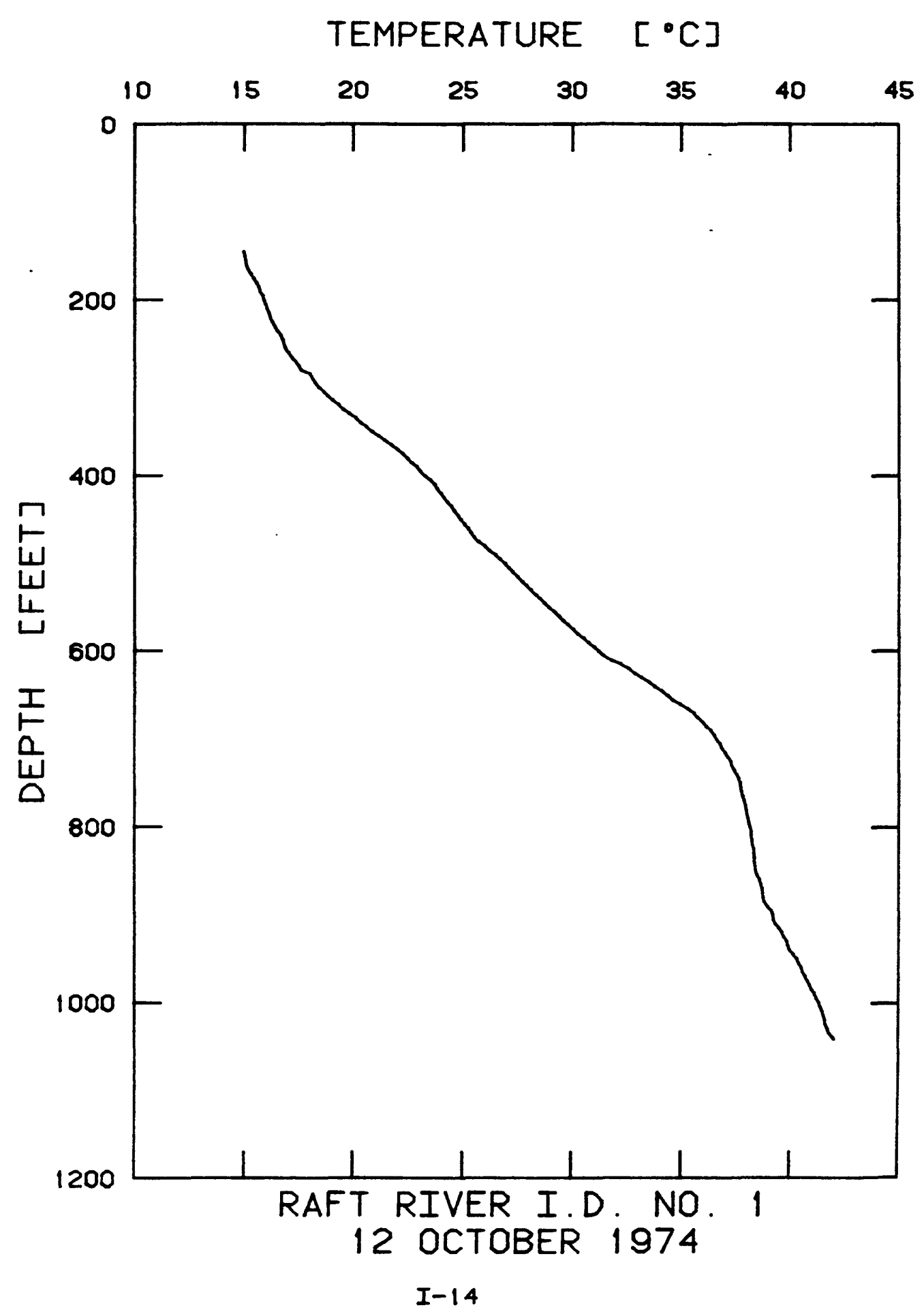




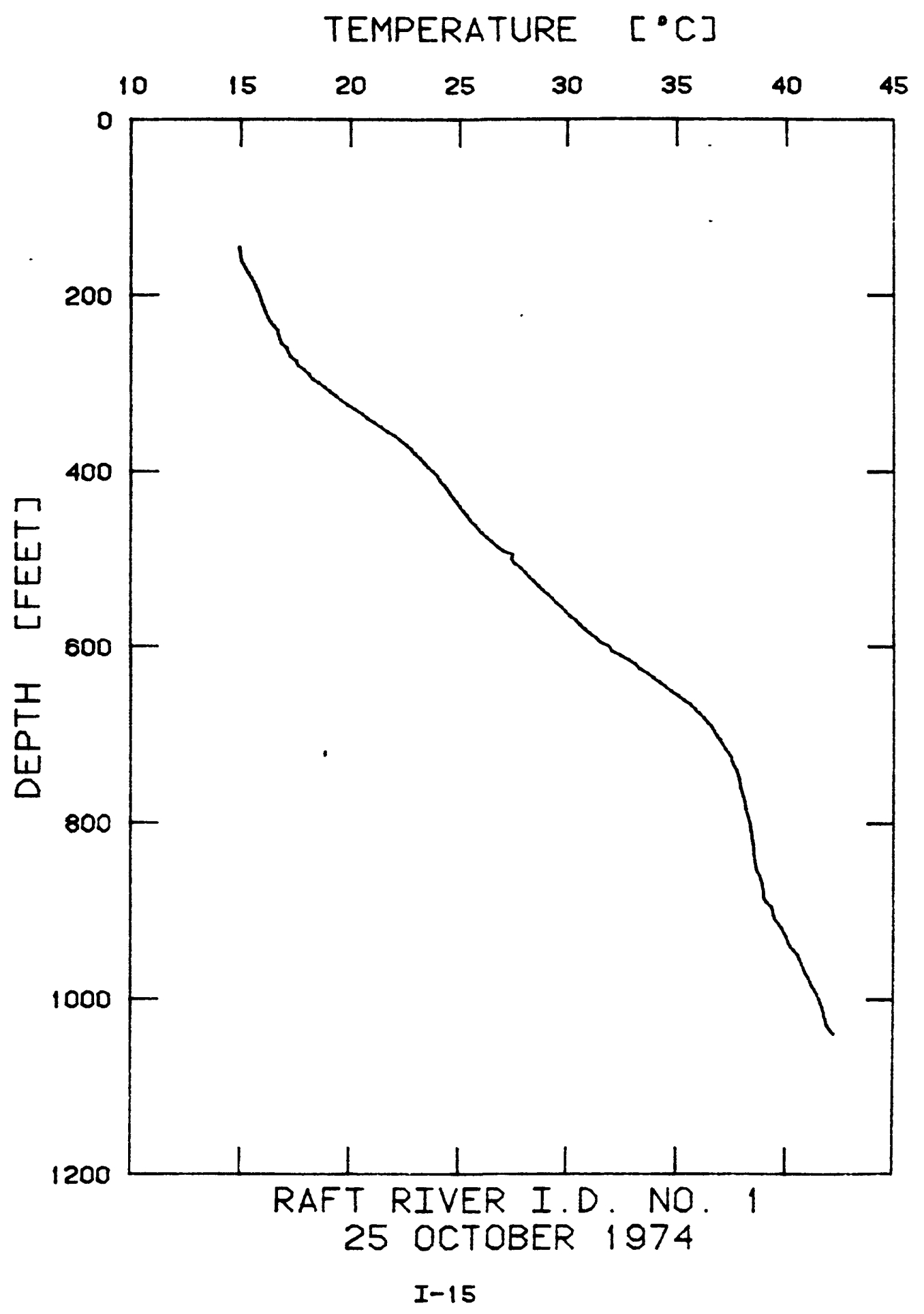




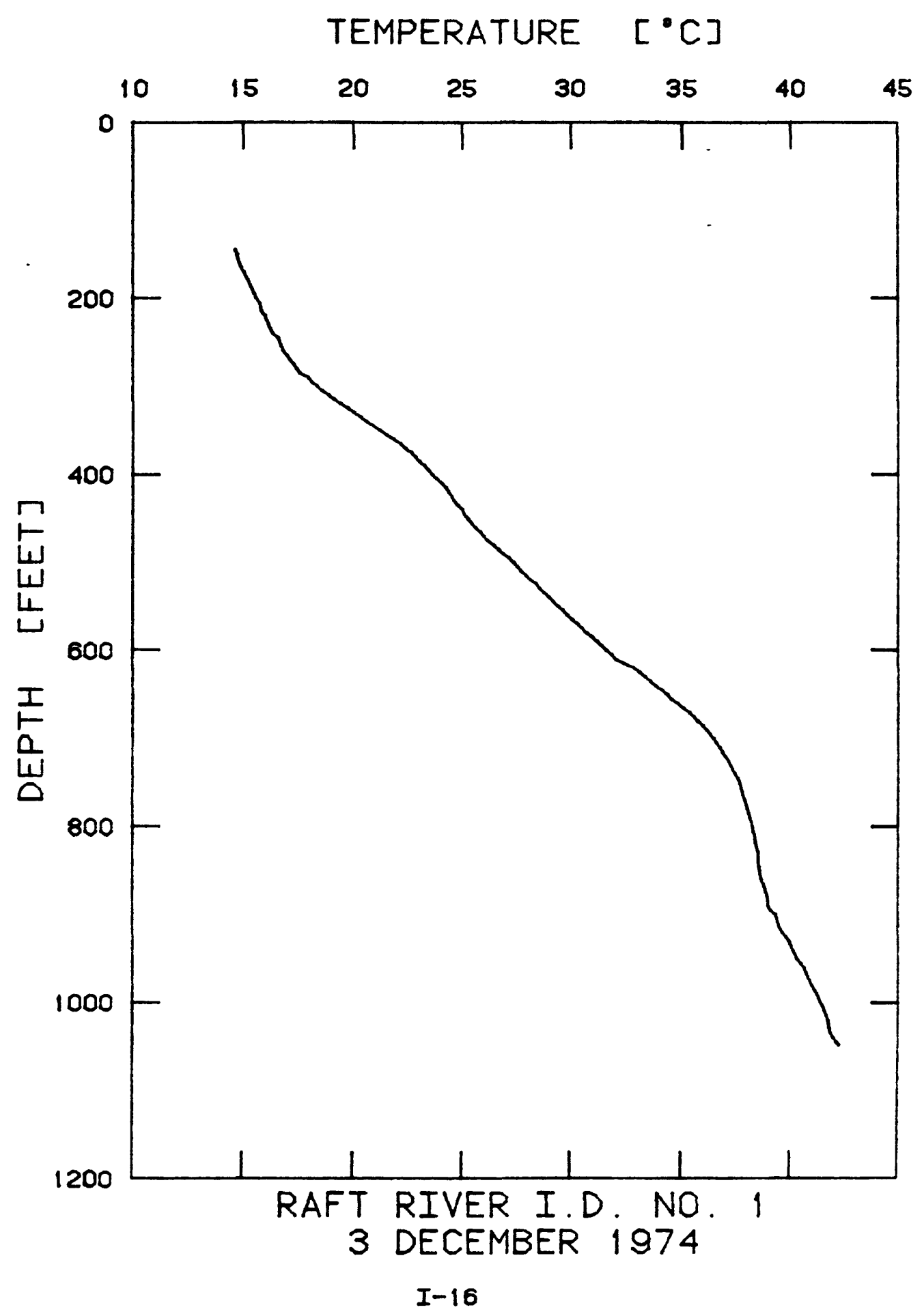




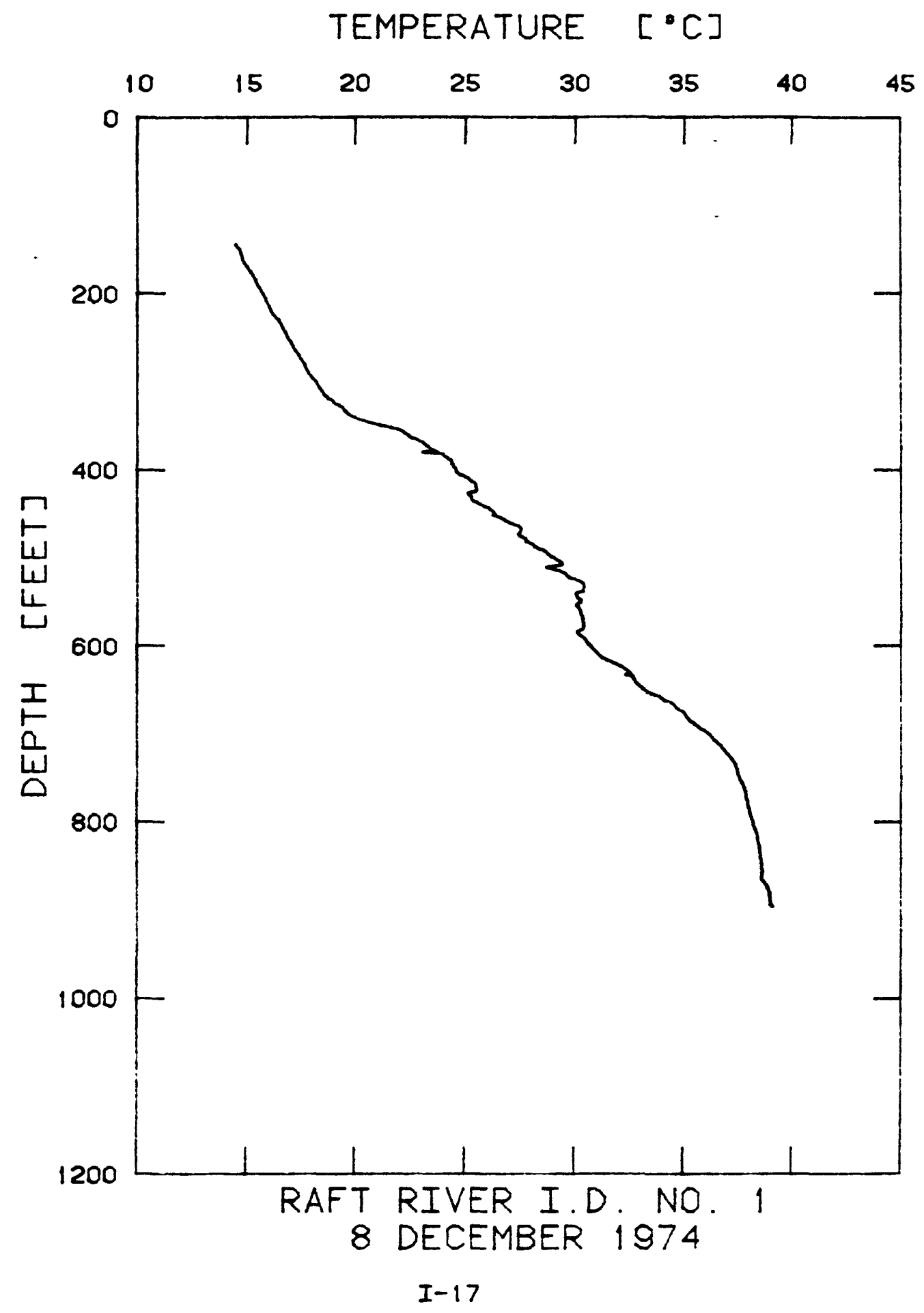




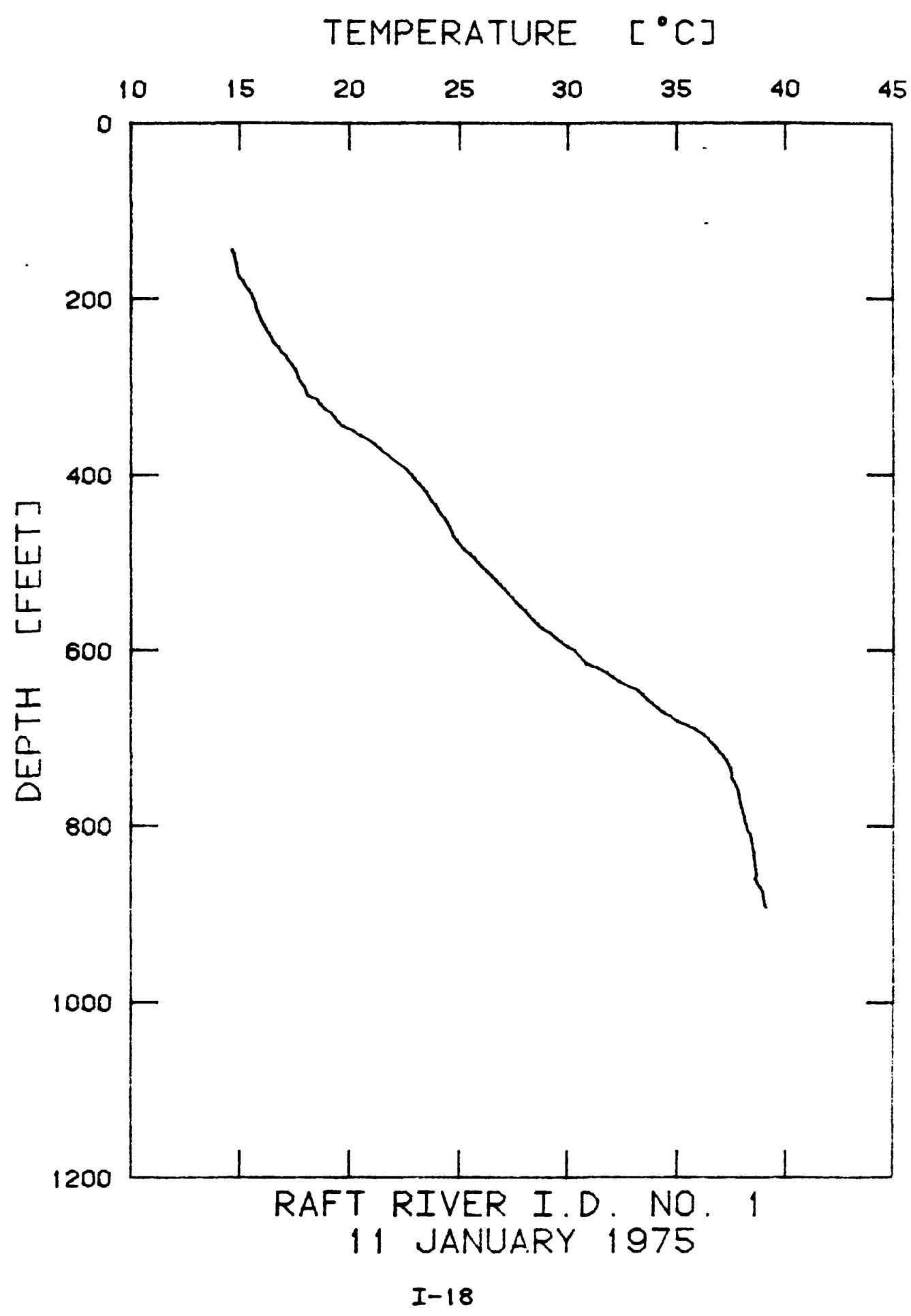




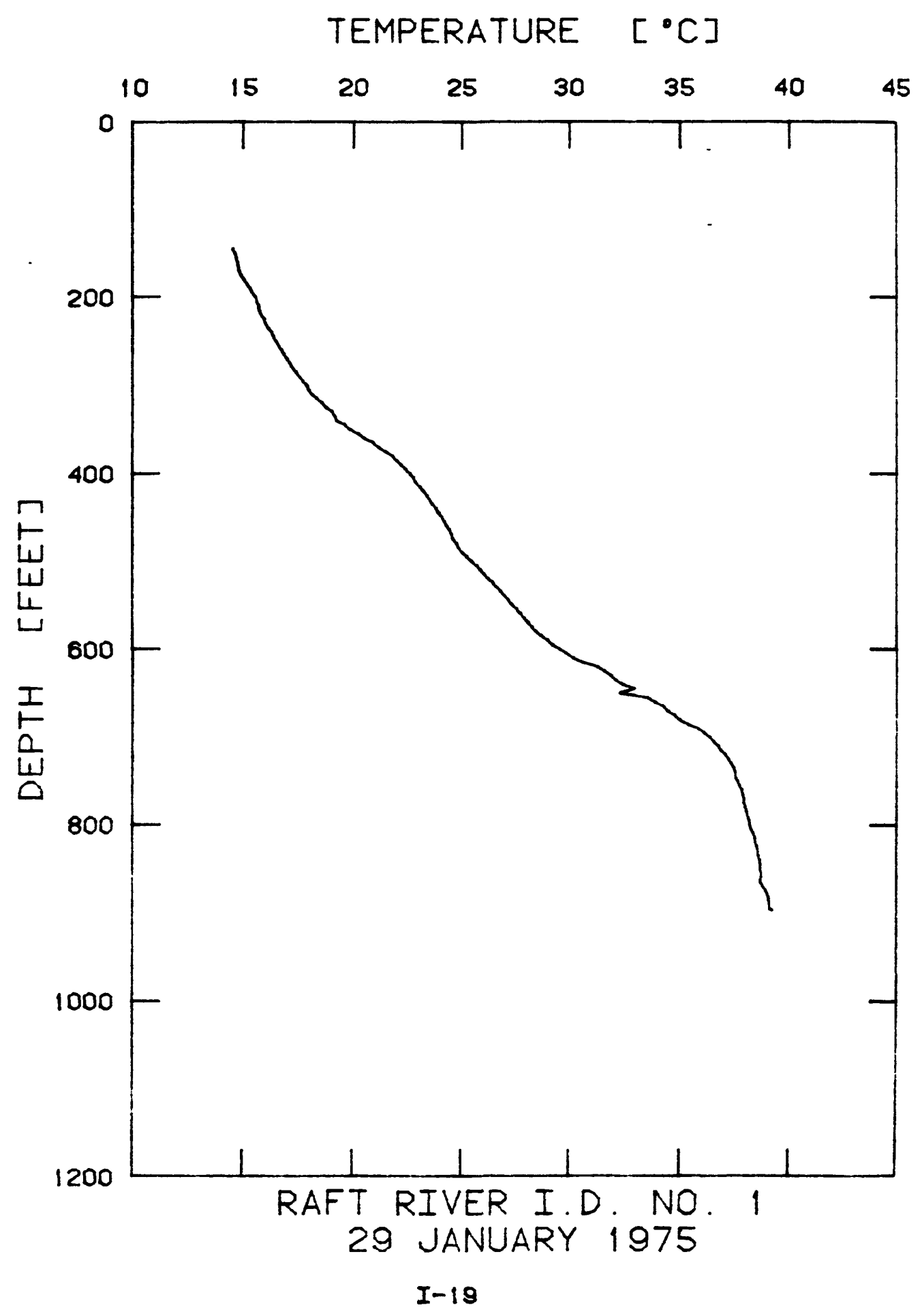




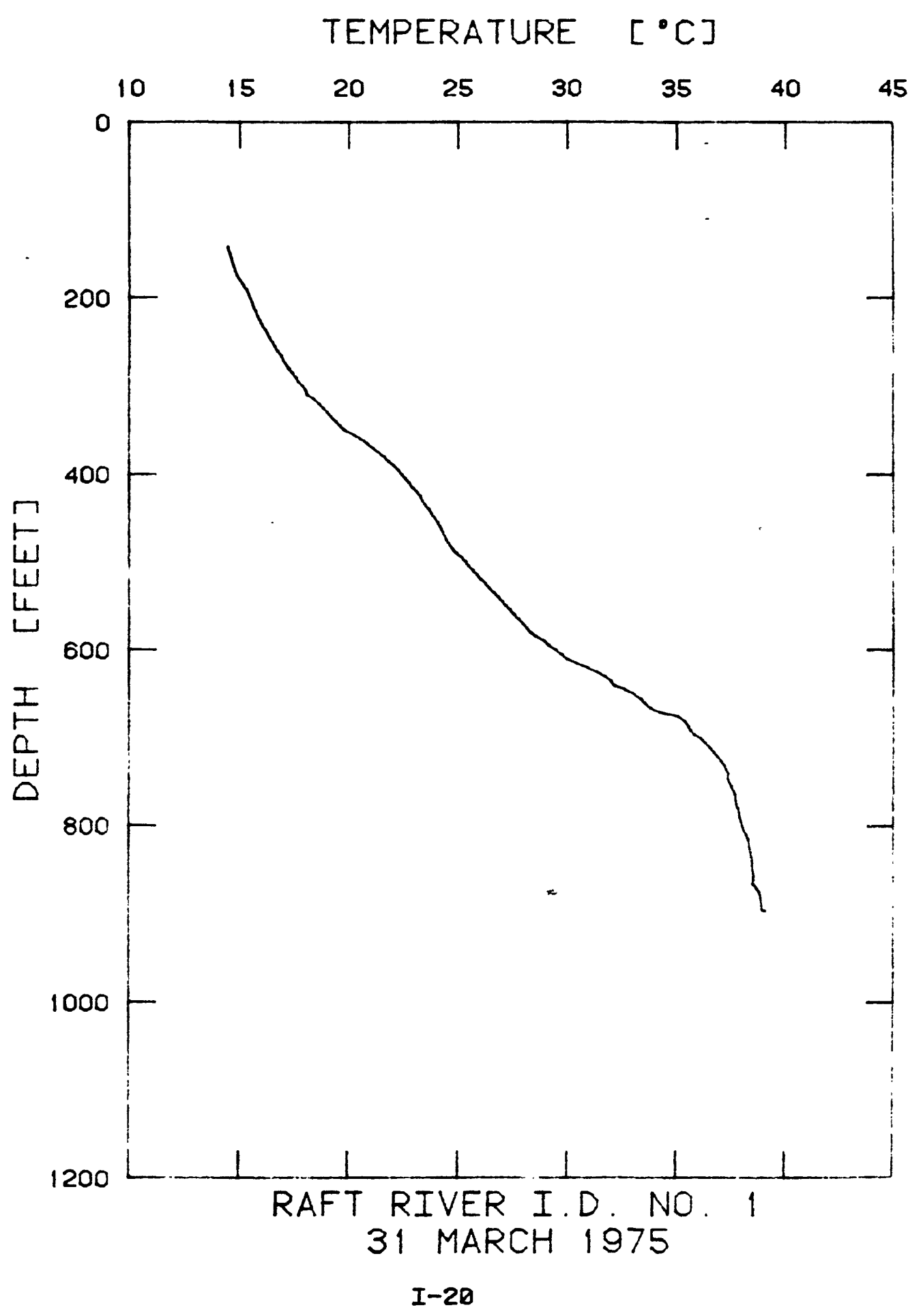




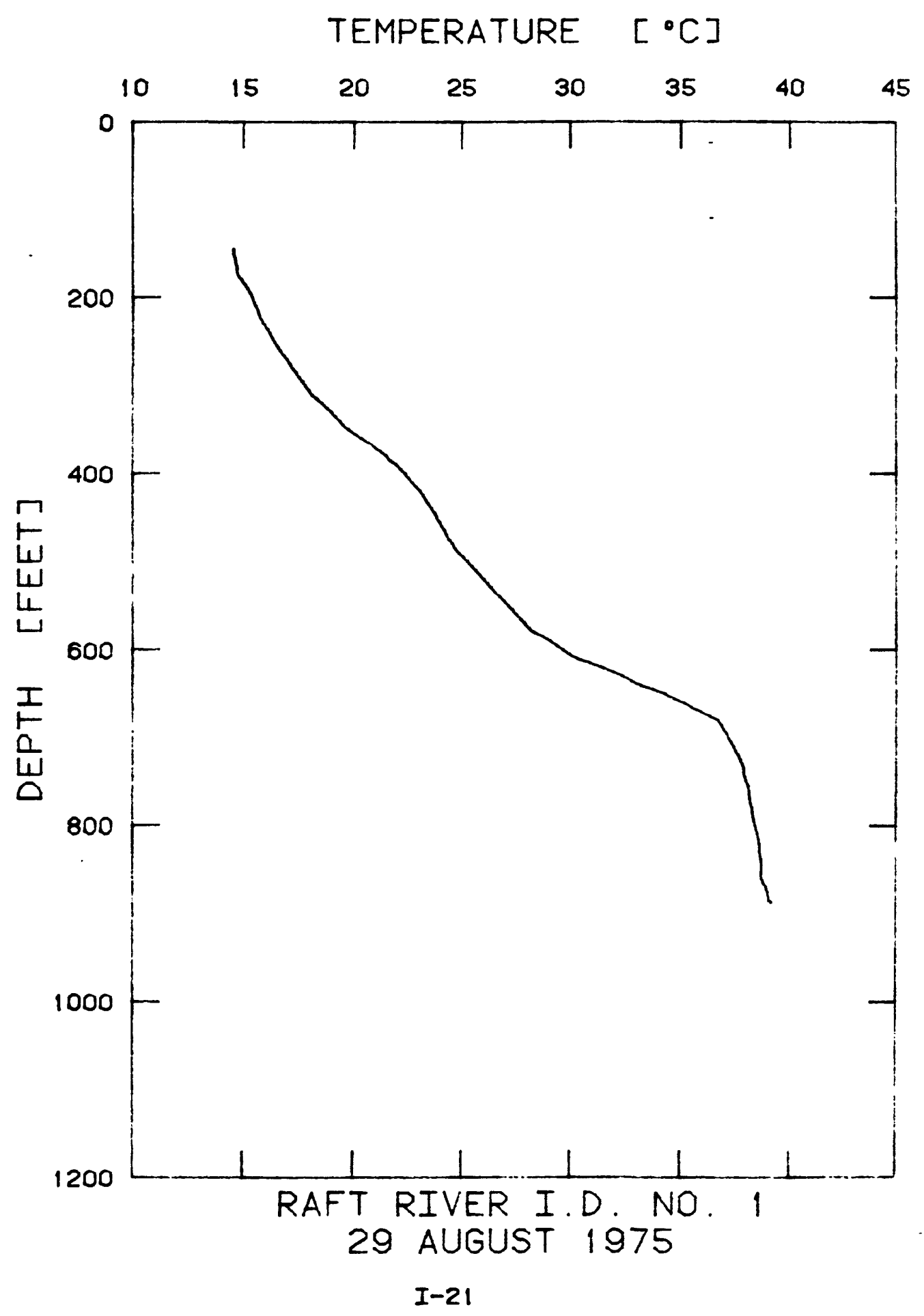




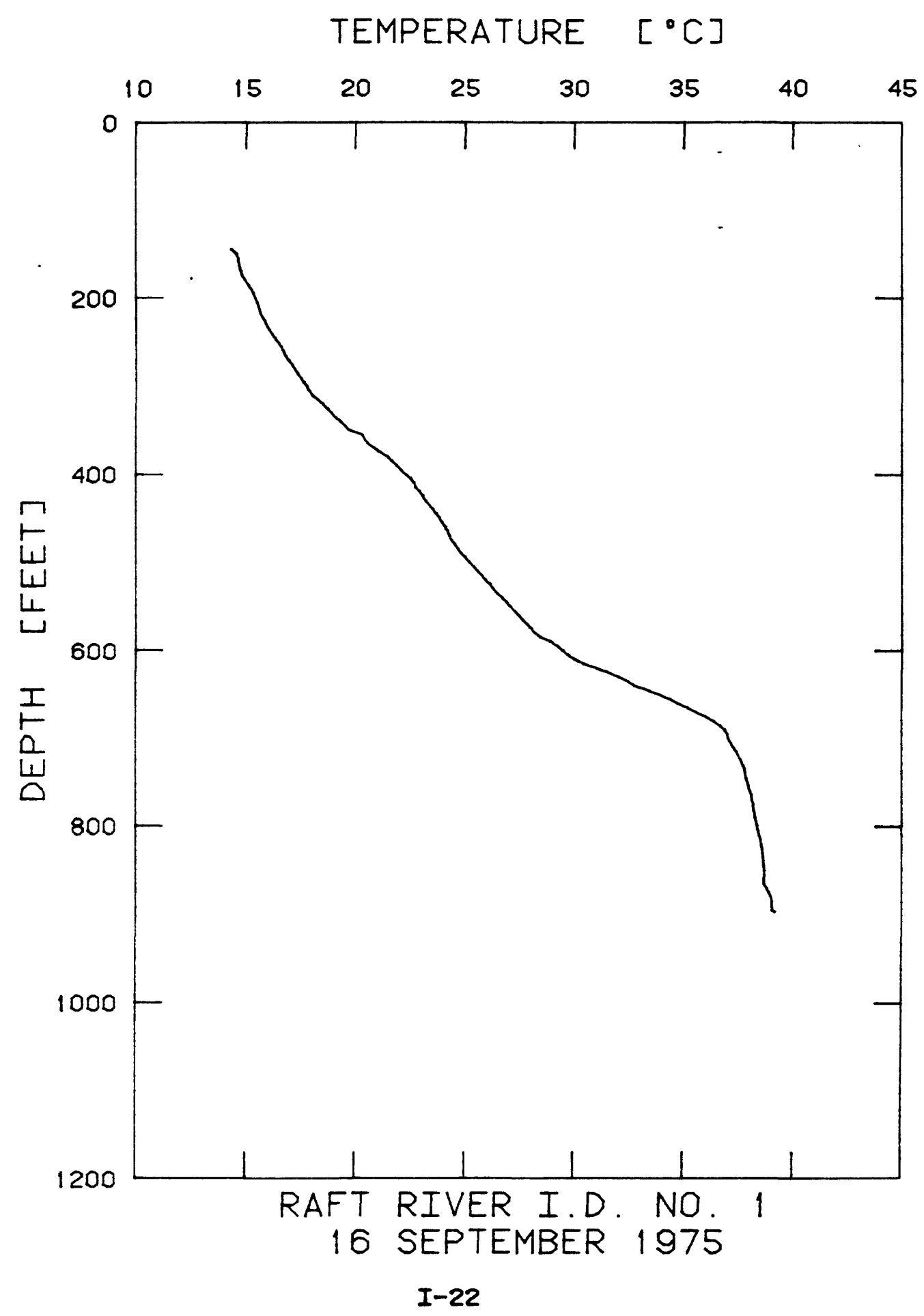




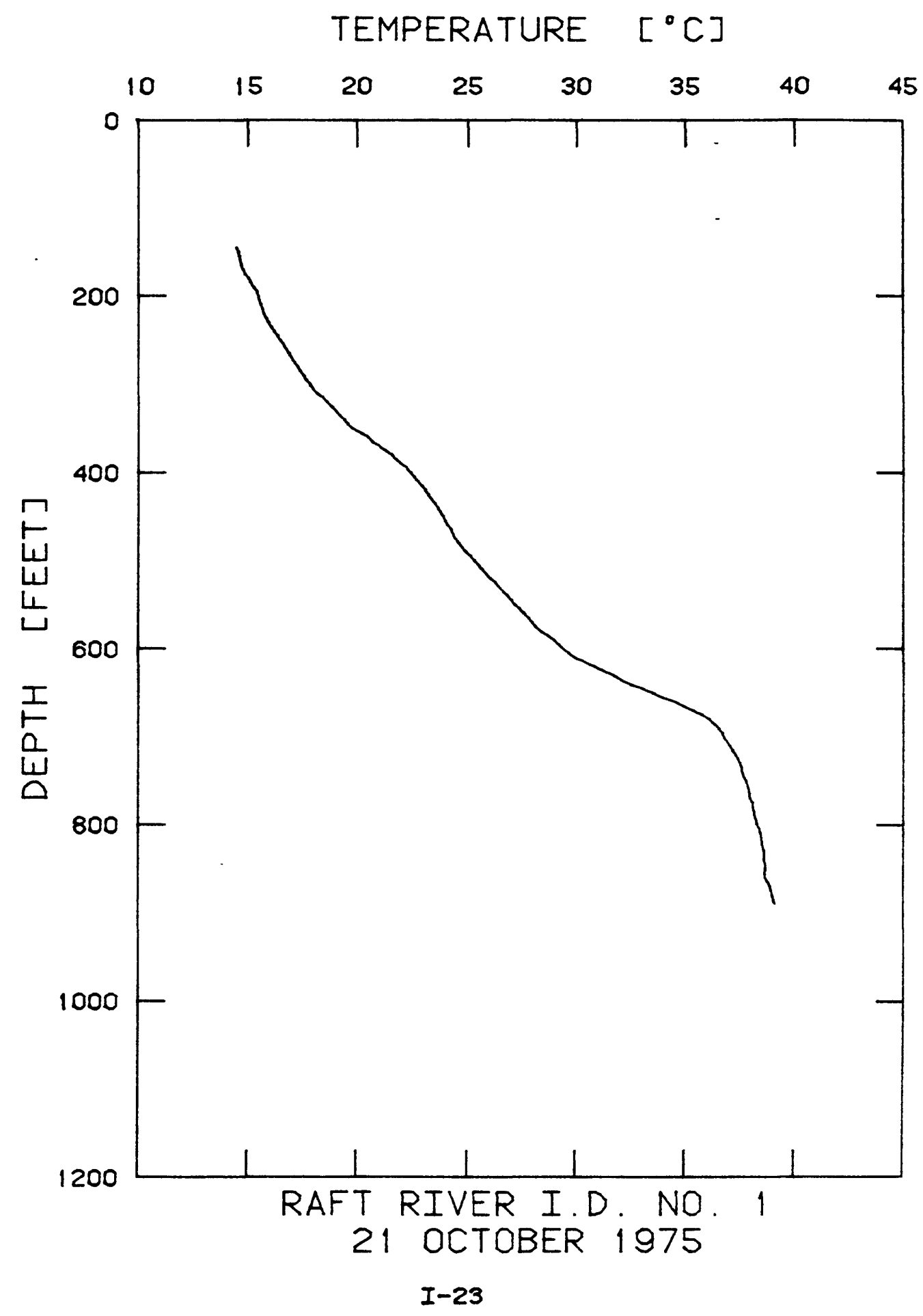




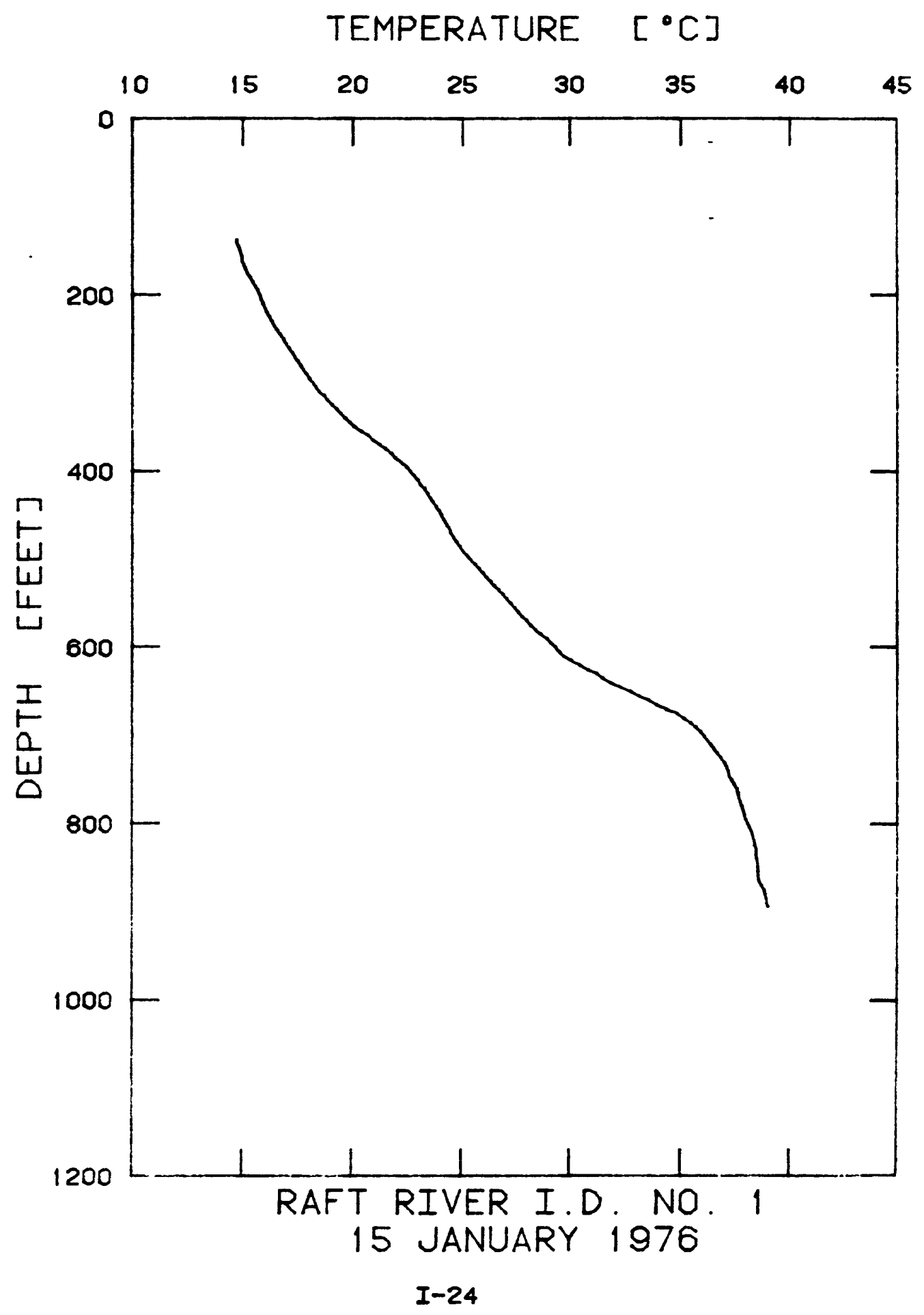




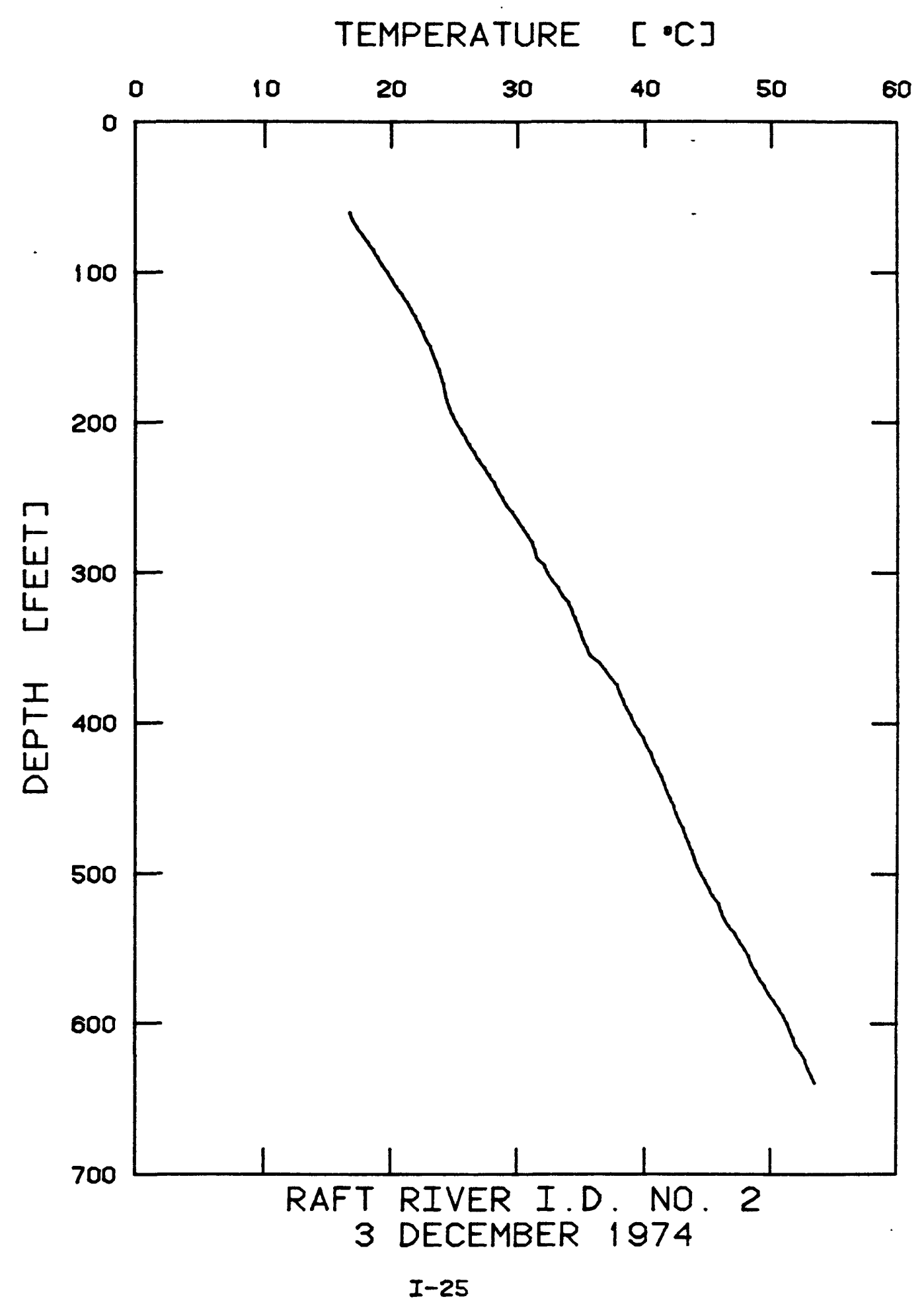




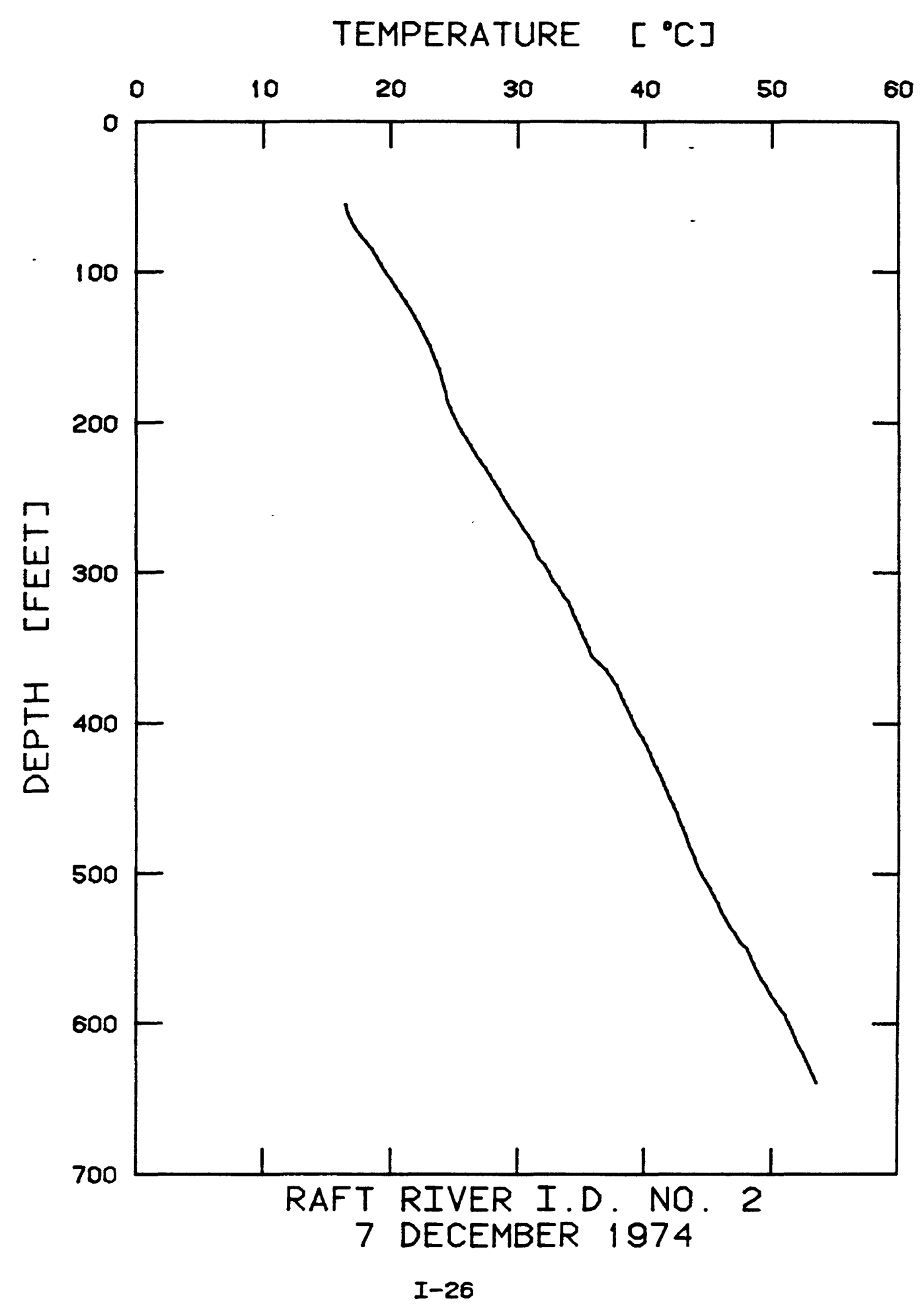




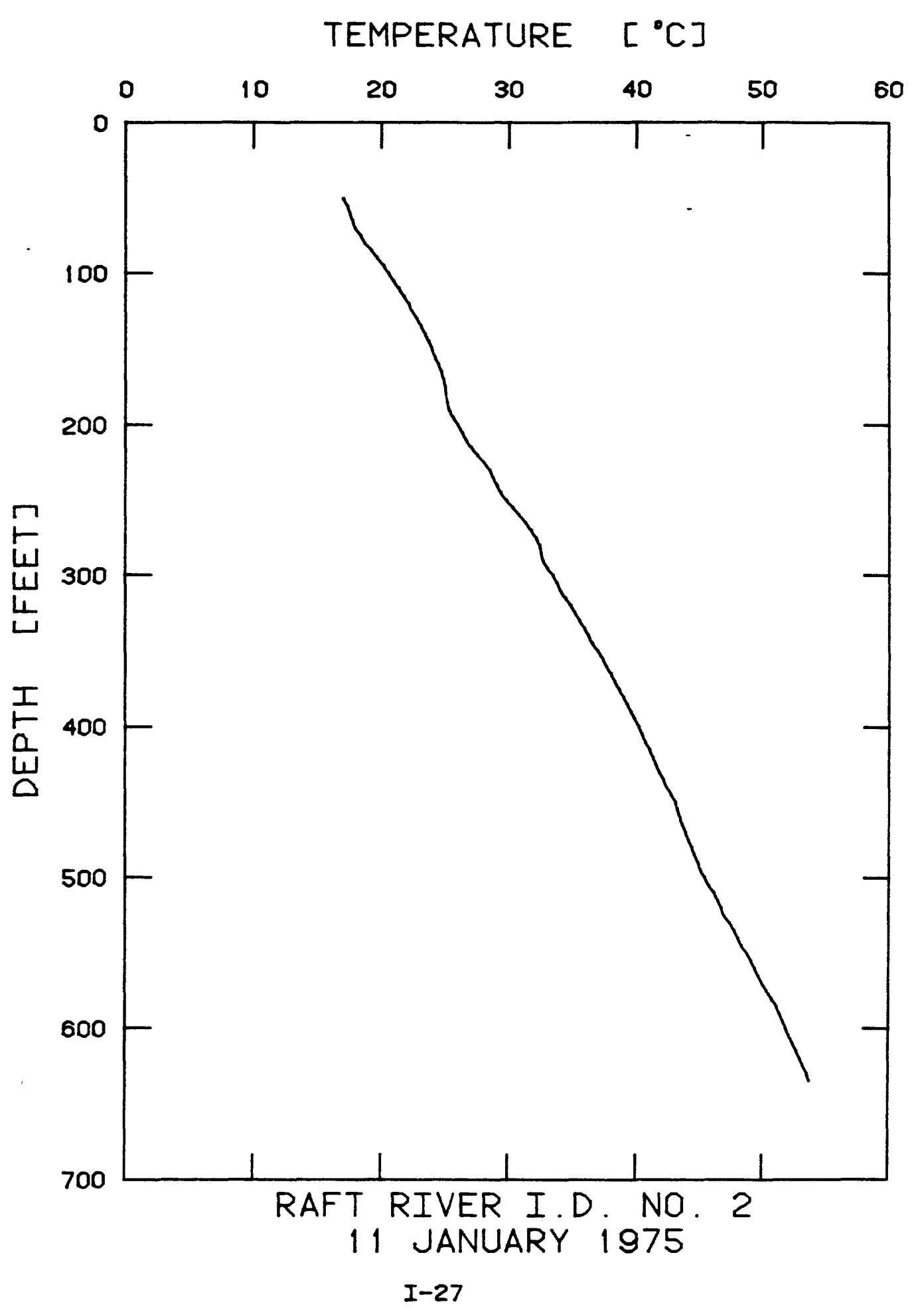




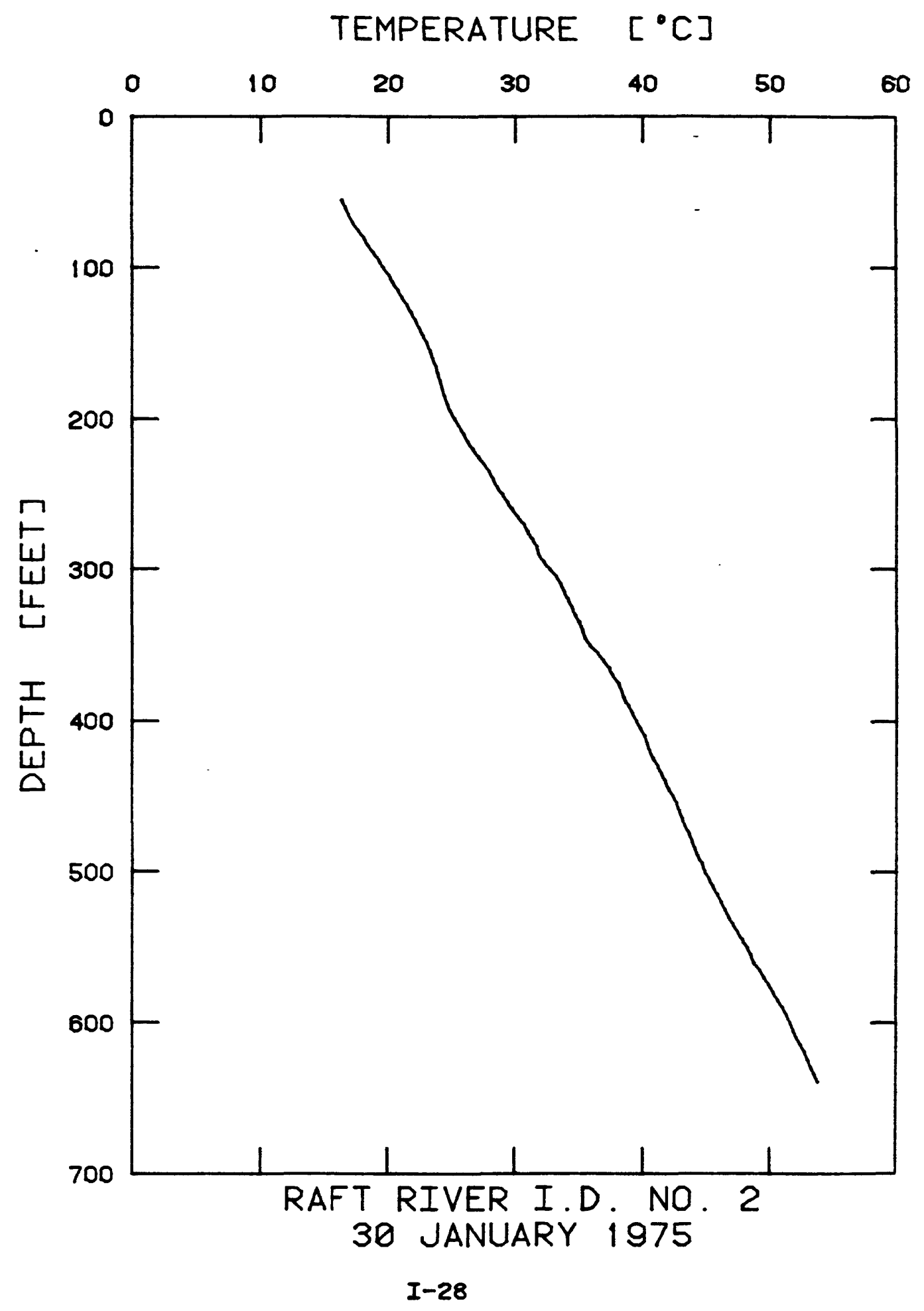




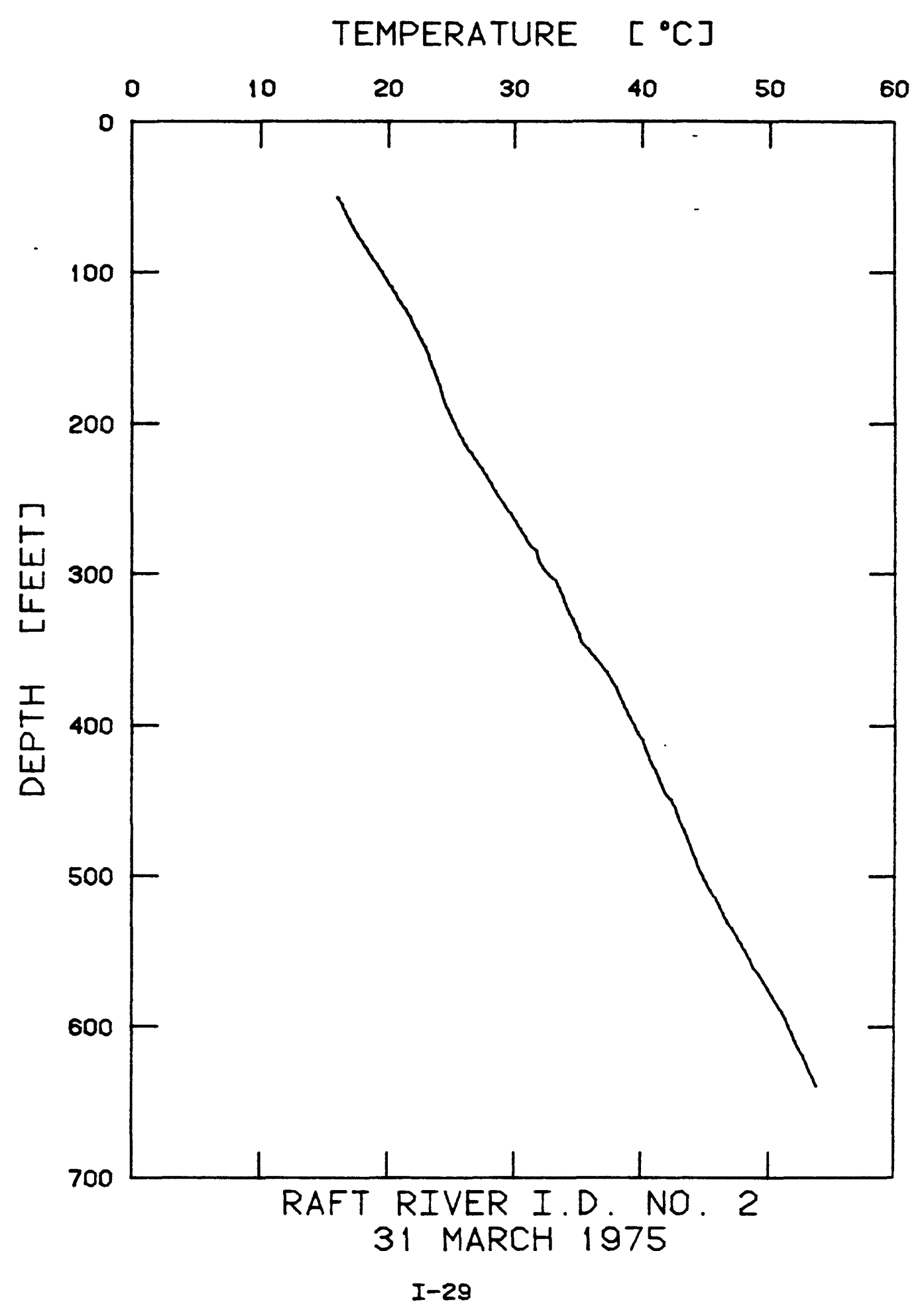




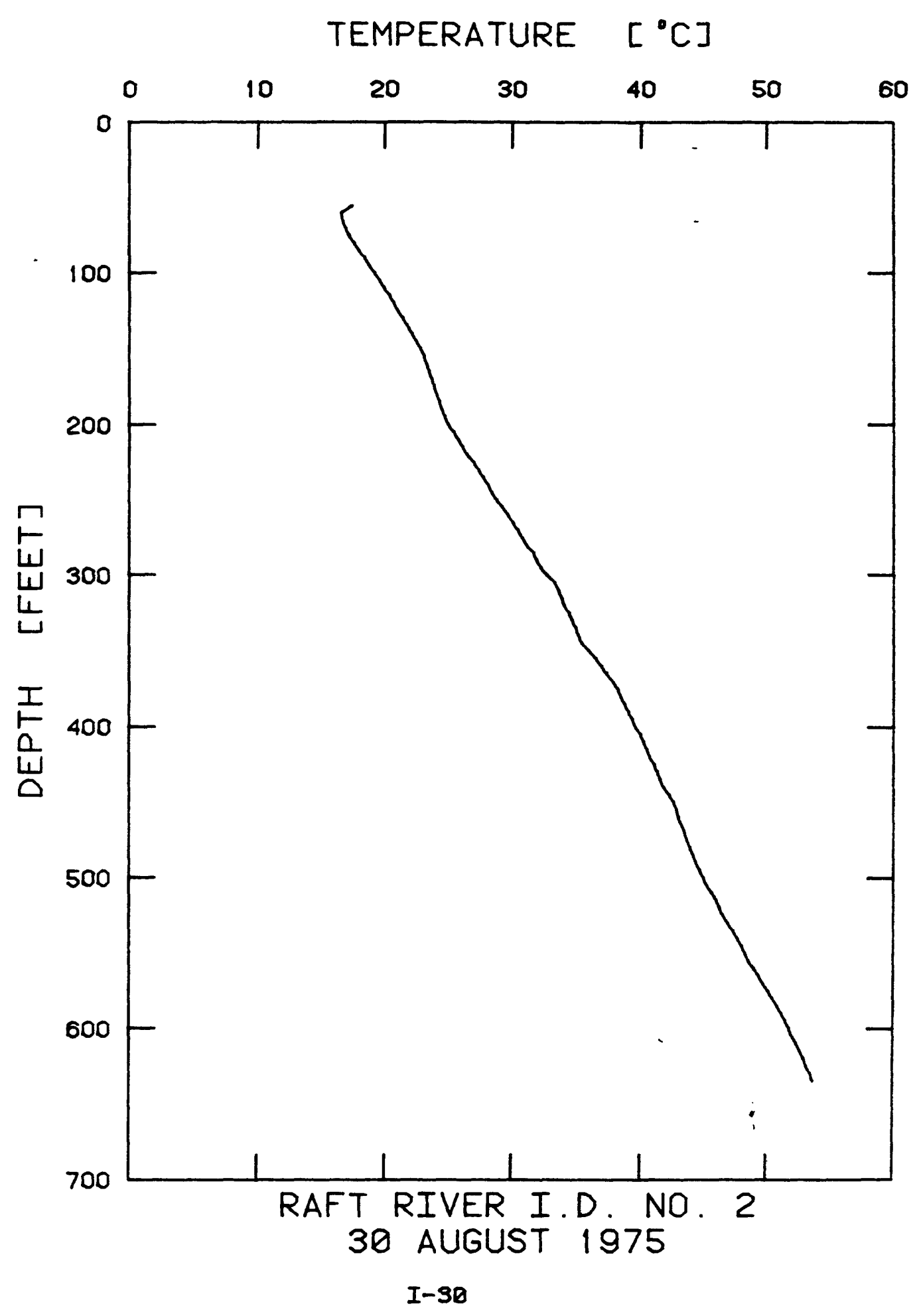




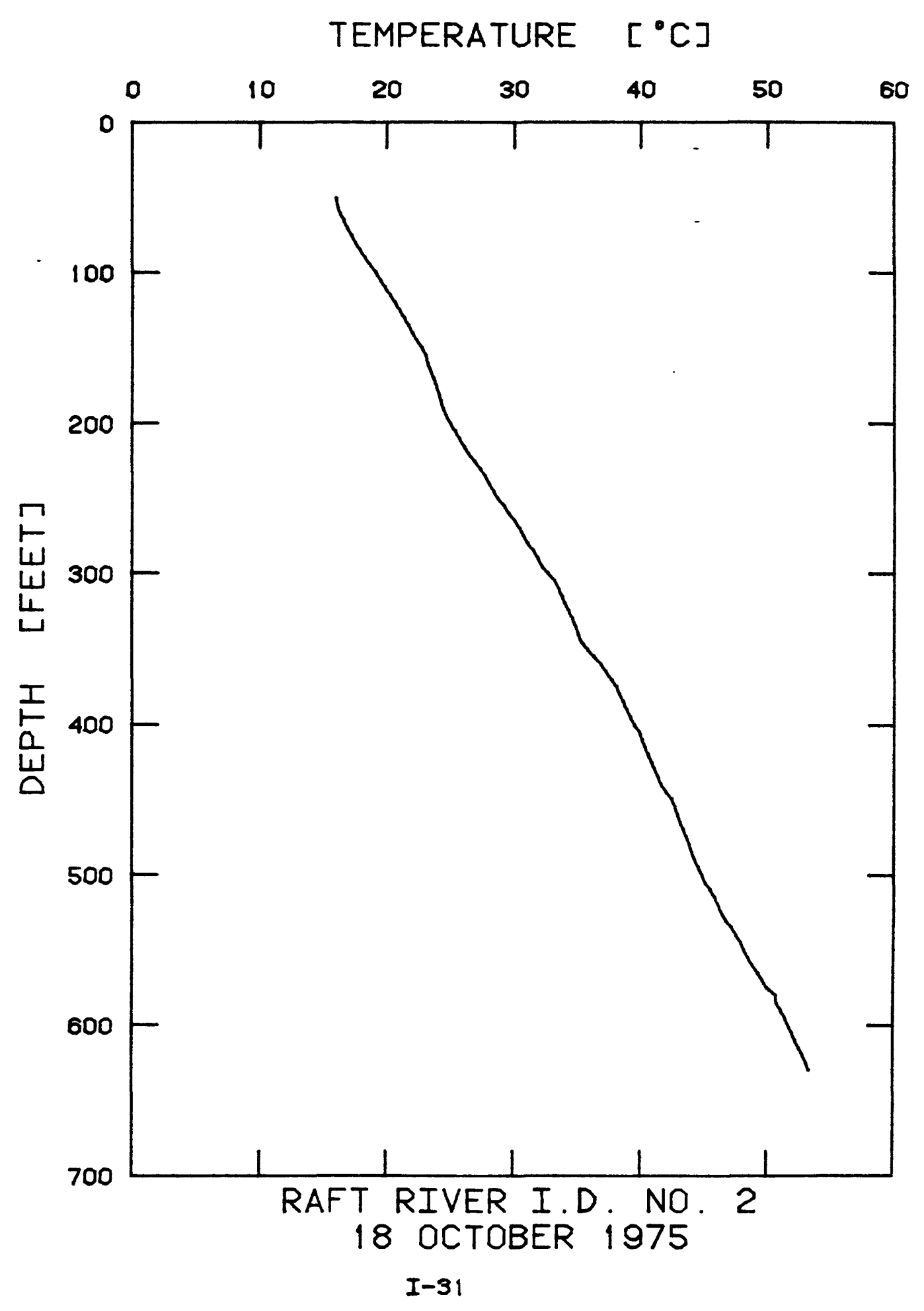




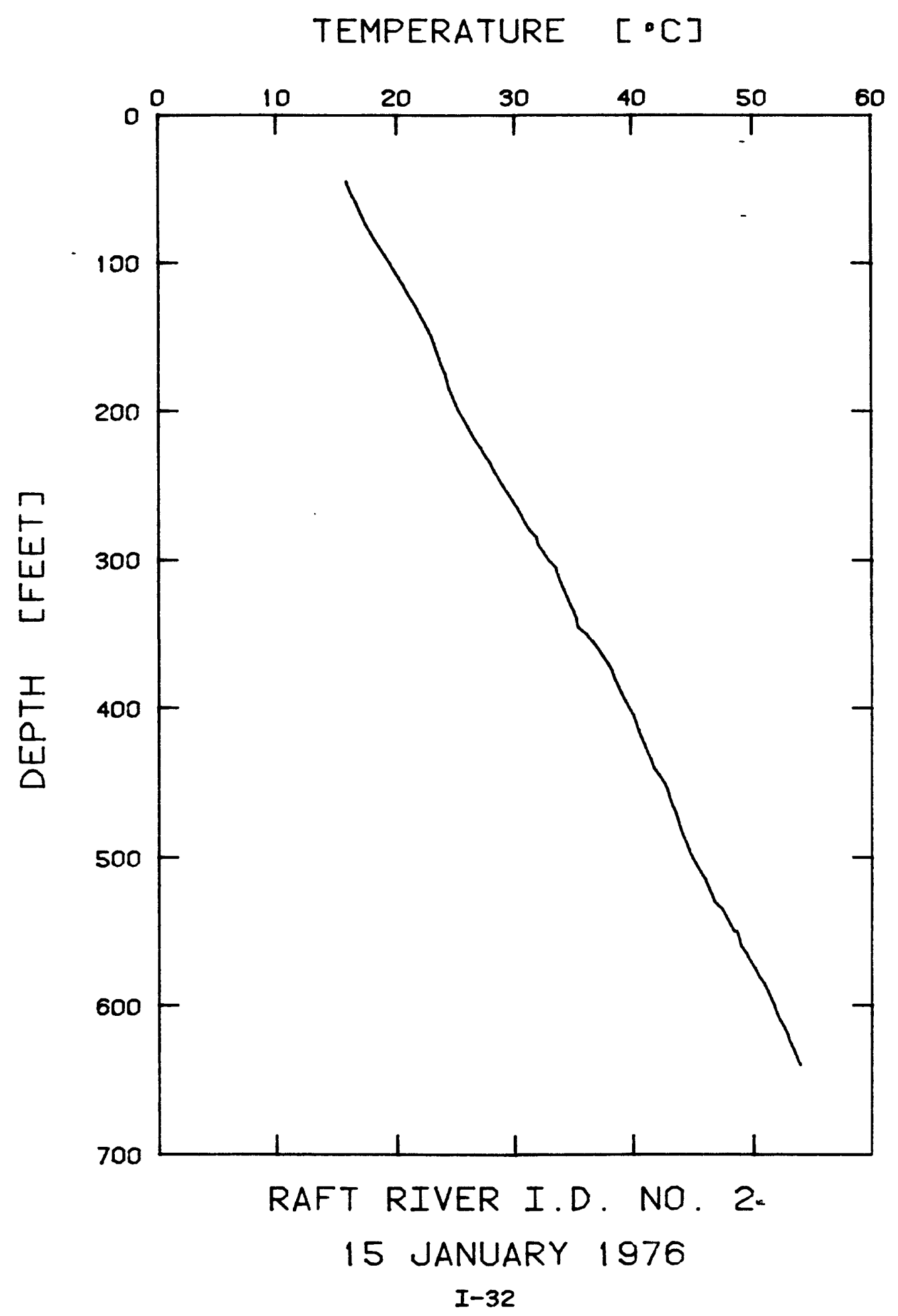




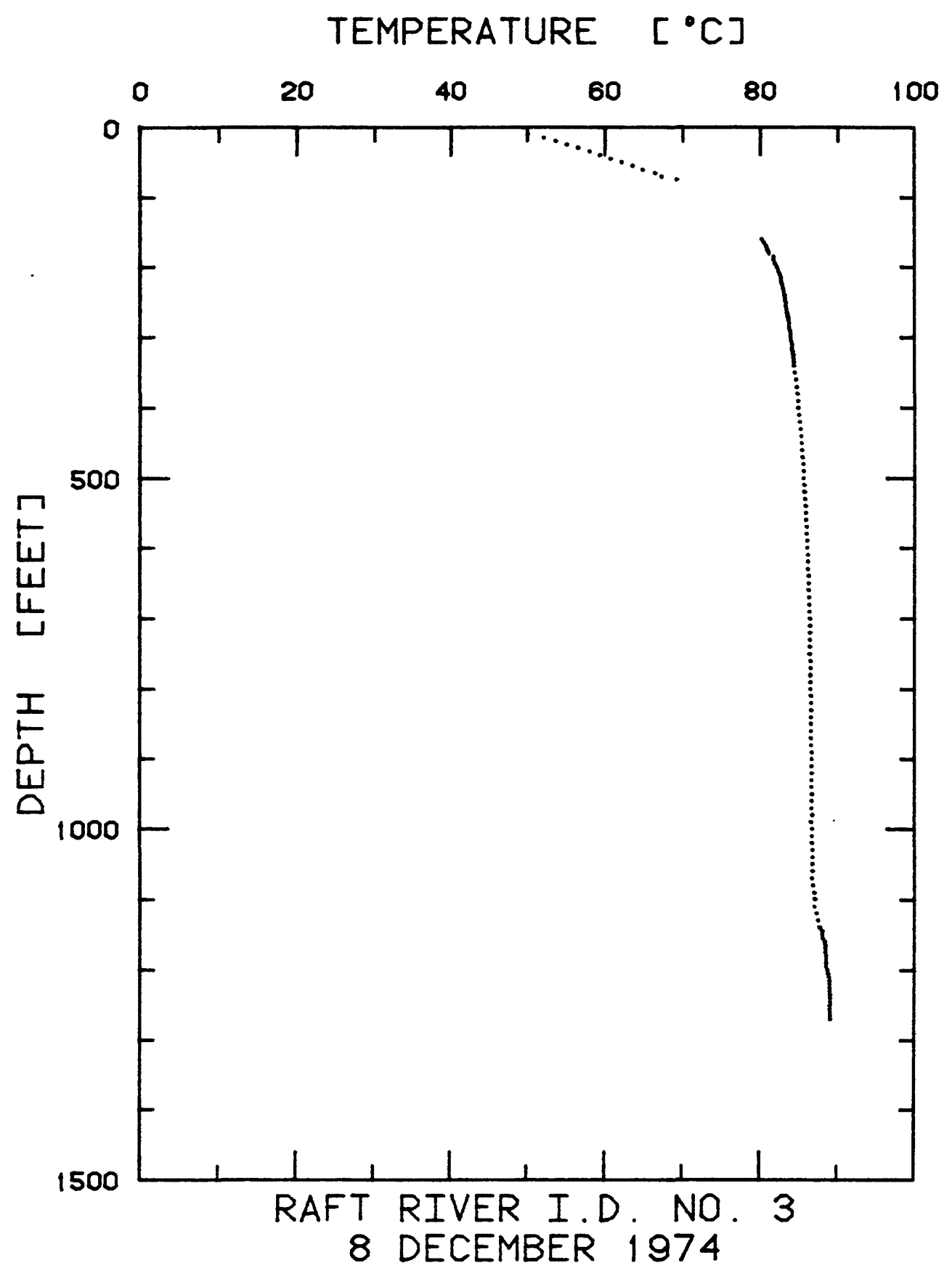

$I-33$ 


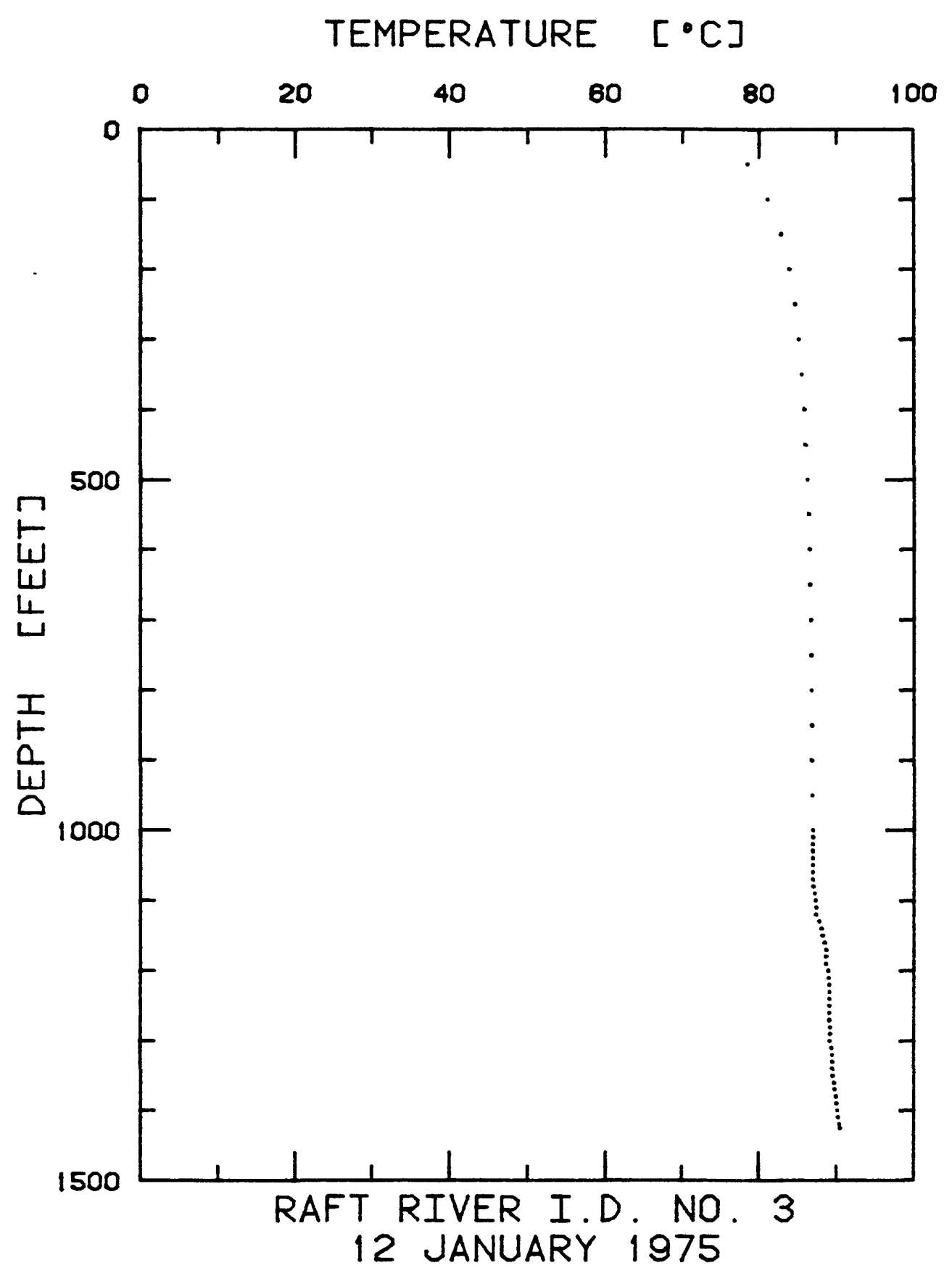




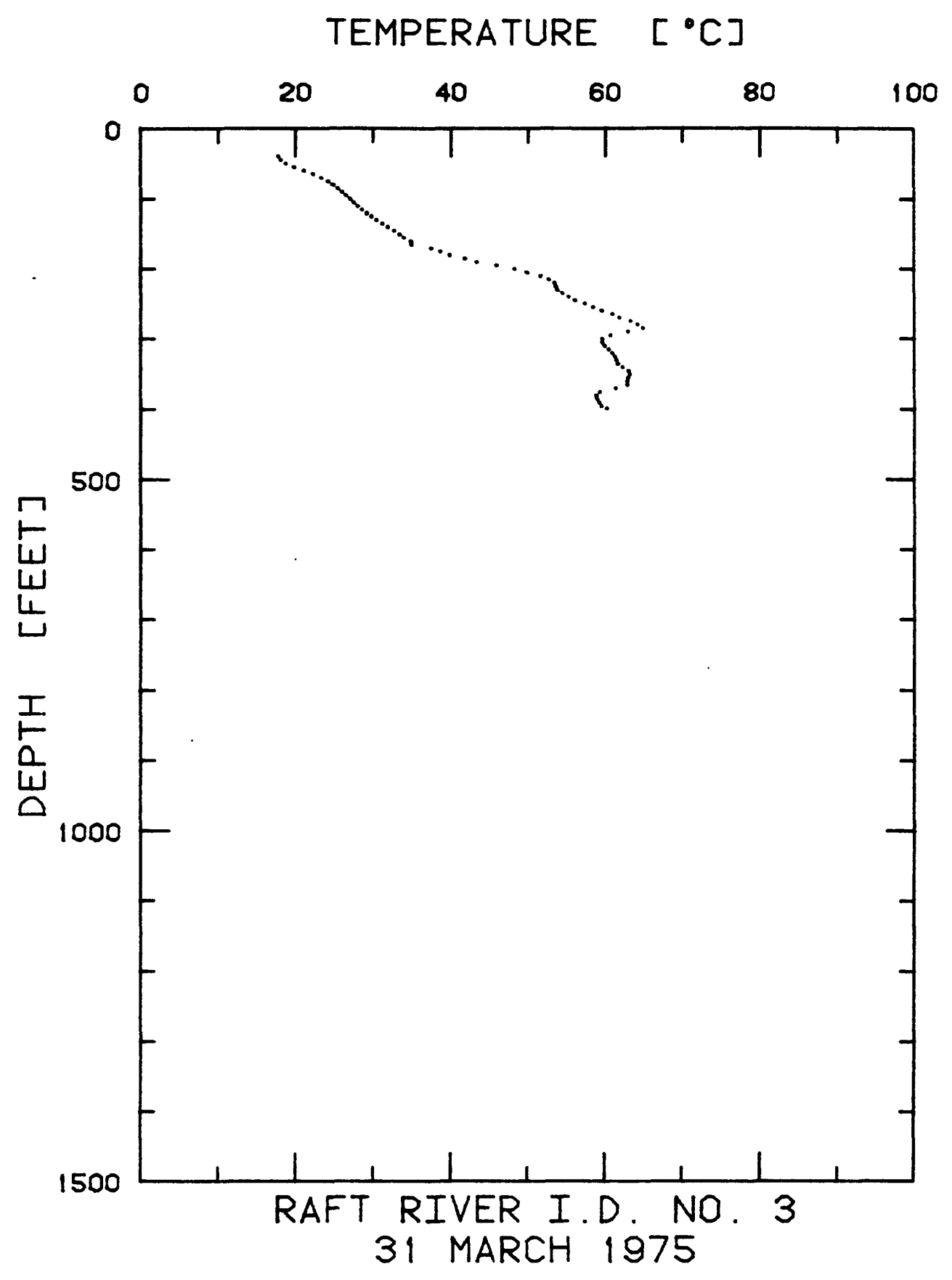

$I-35$ 


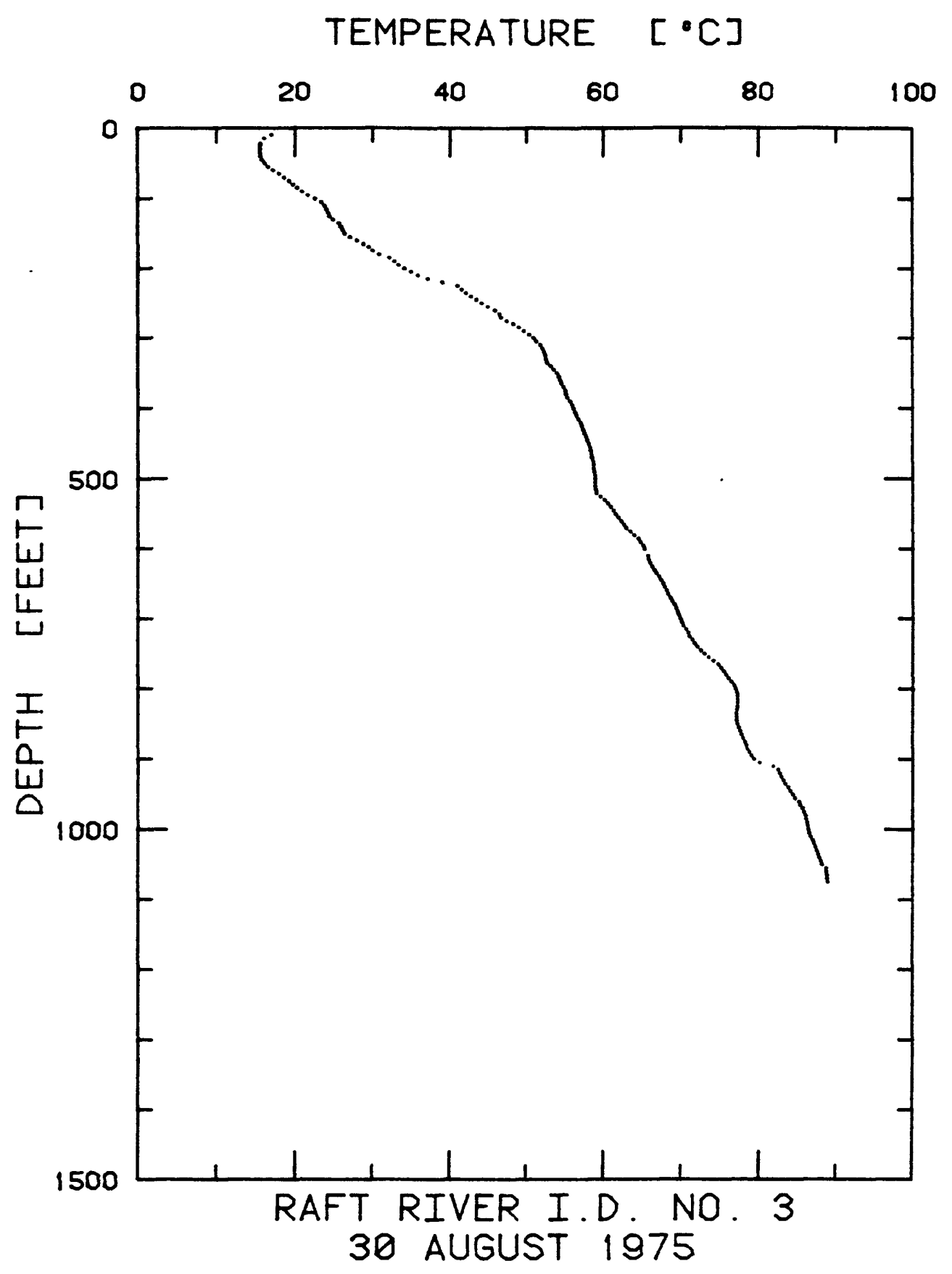

I-96 


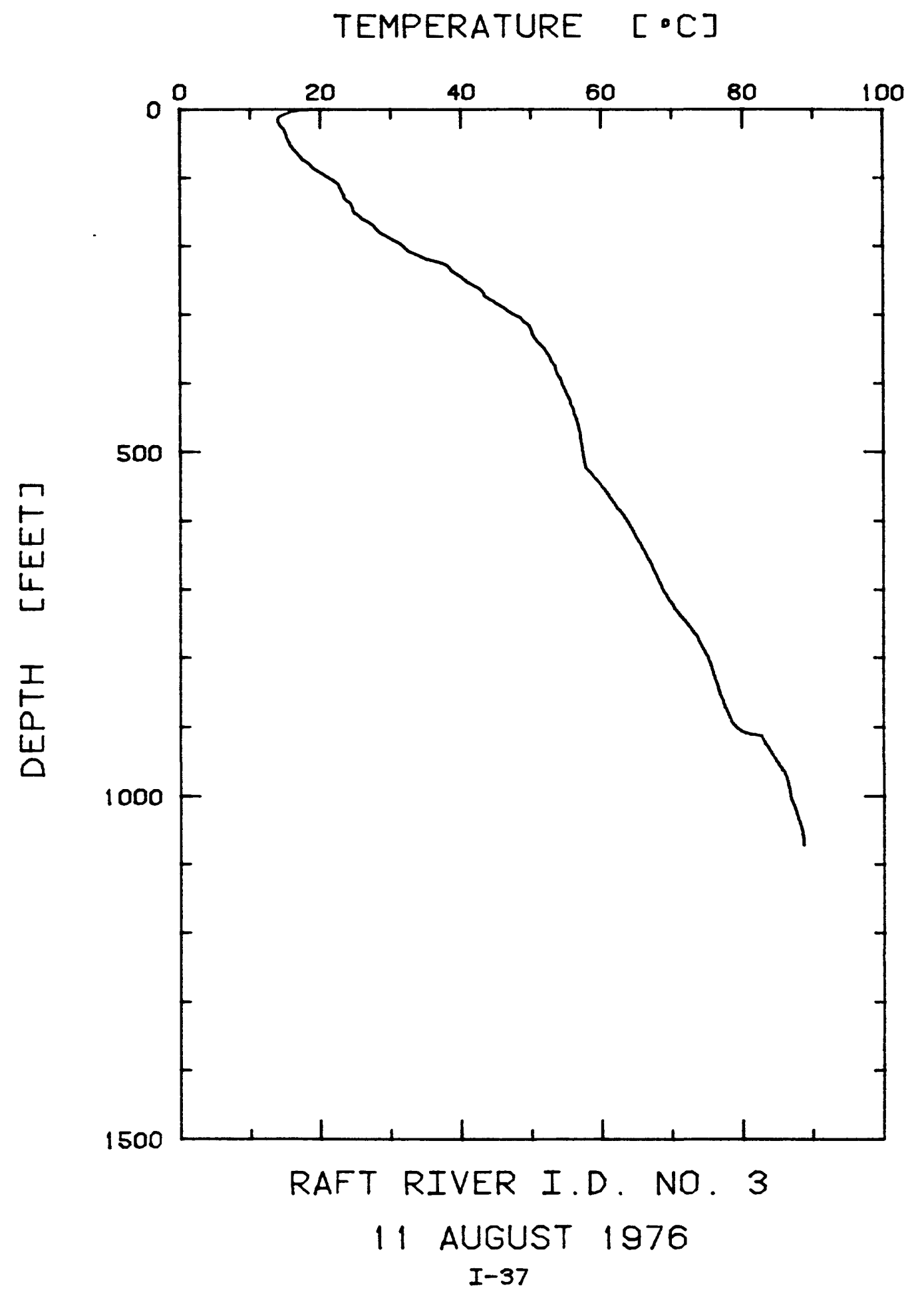




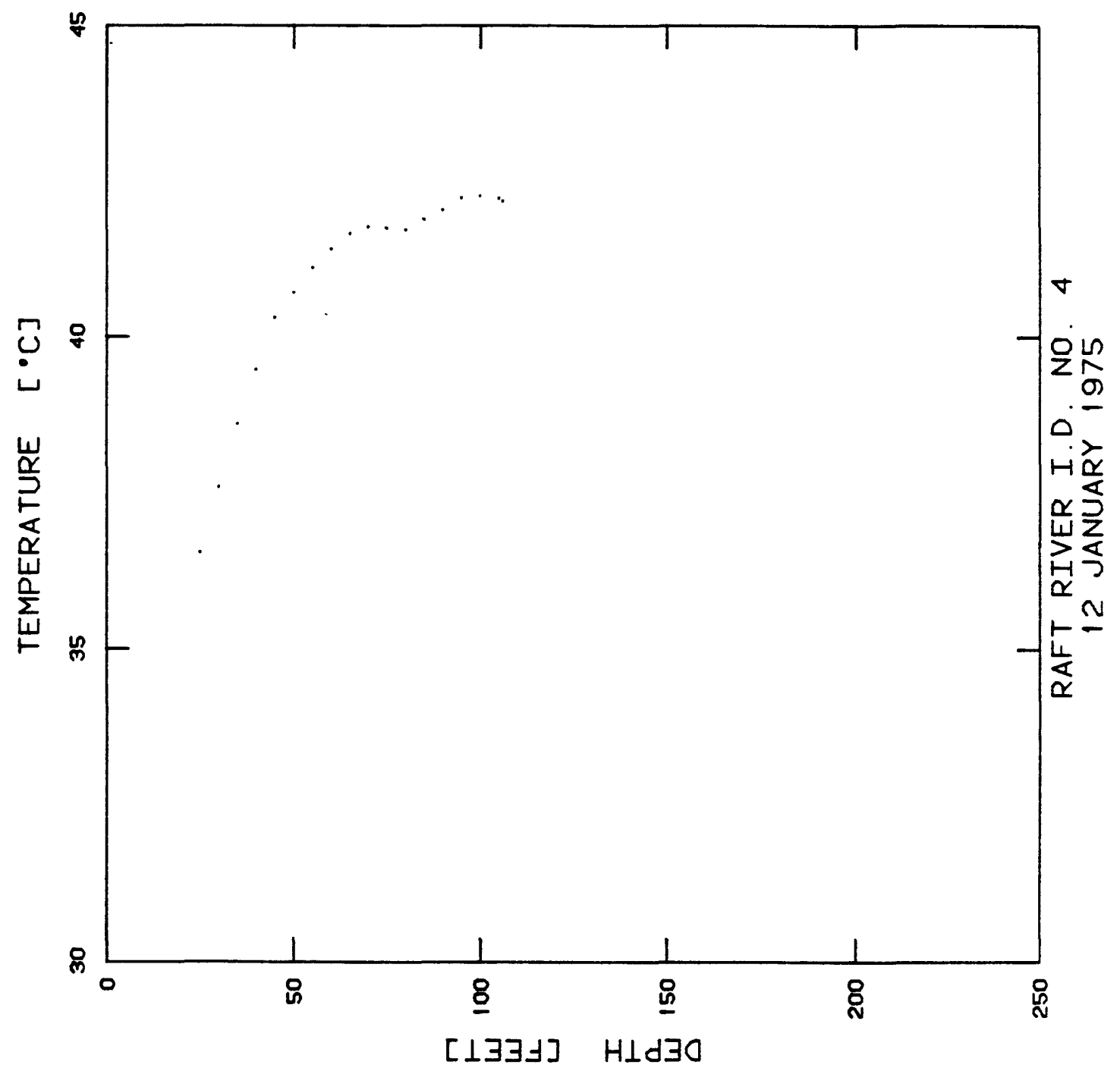

$I-38$ 


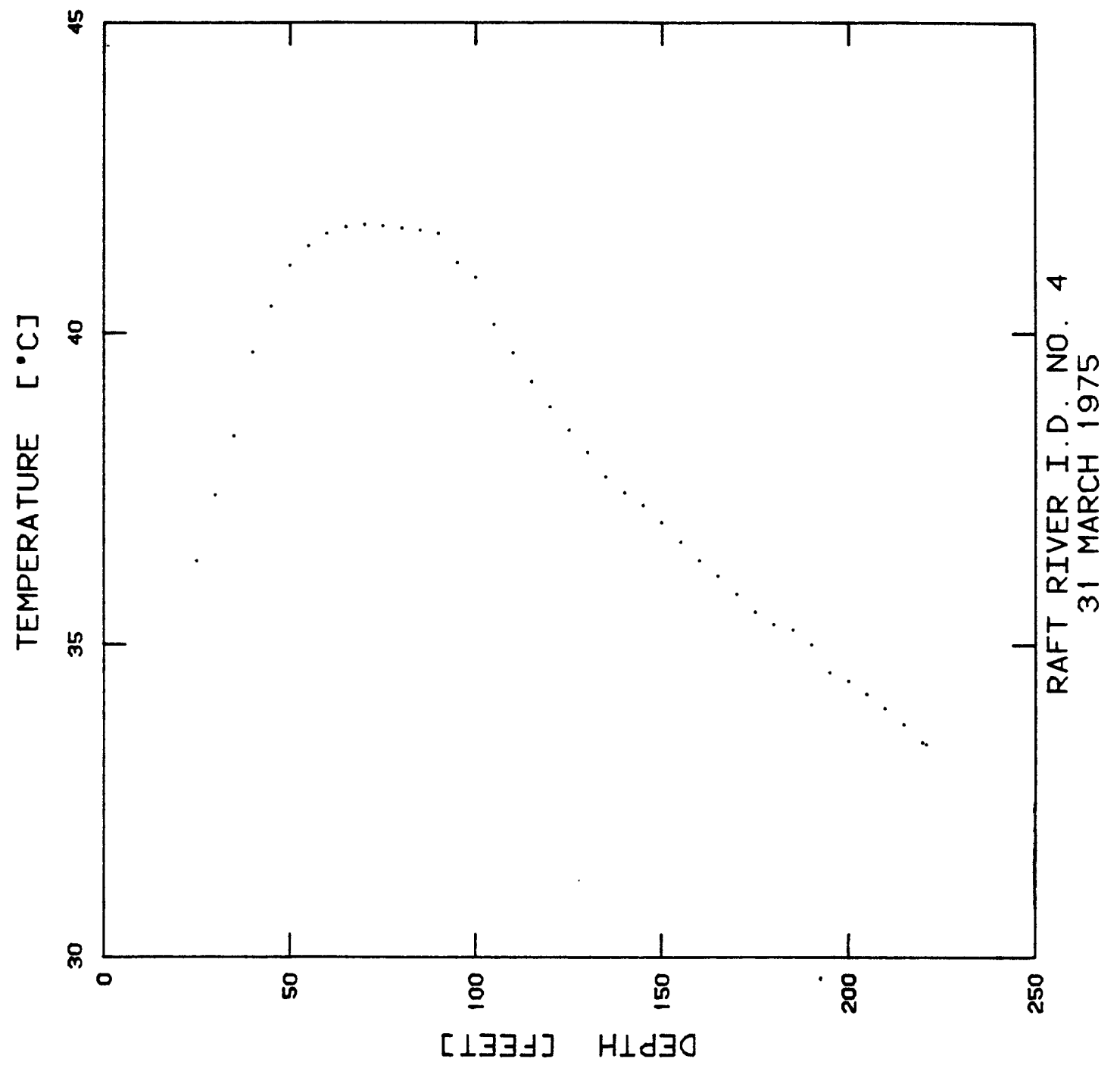

$I-39$ 


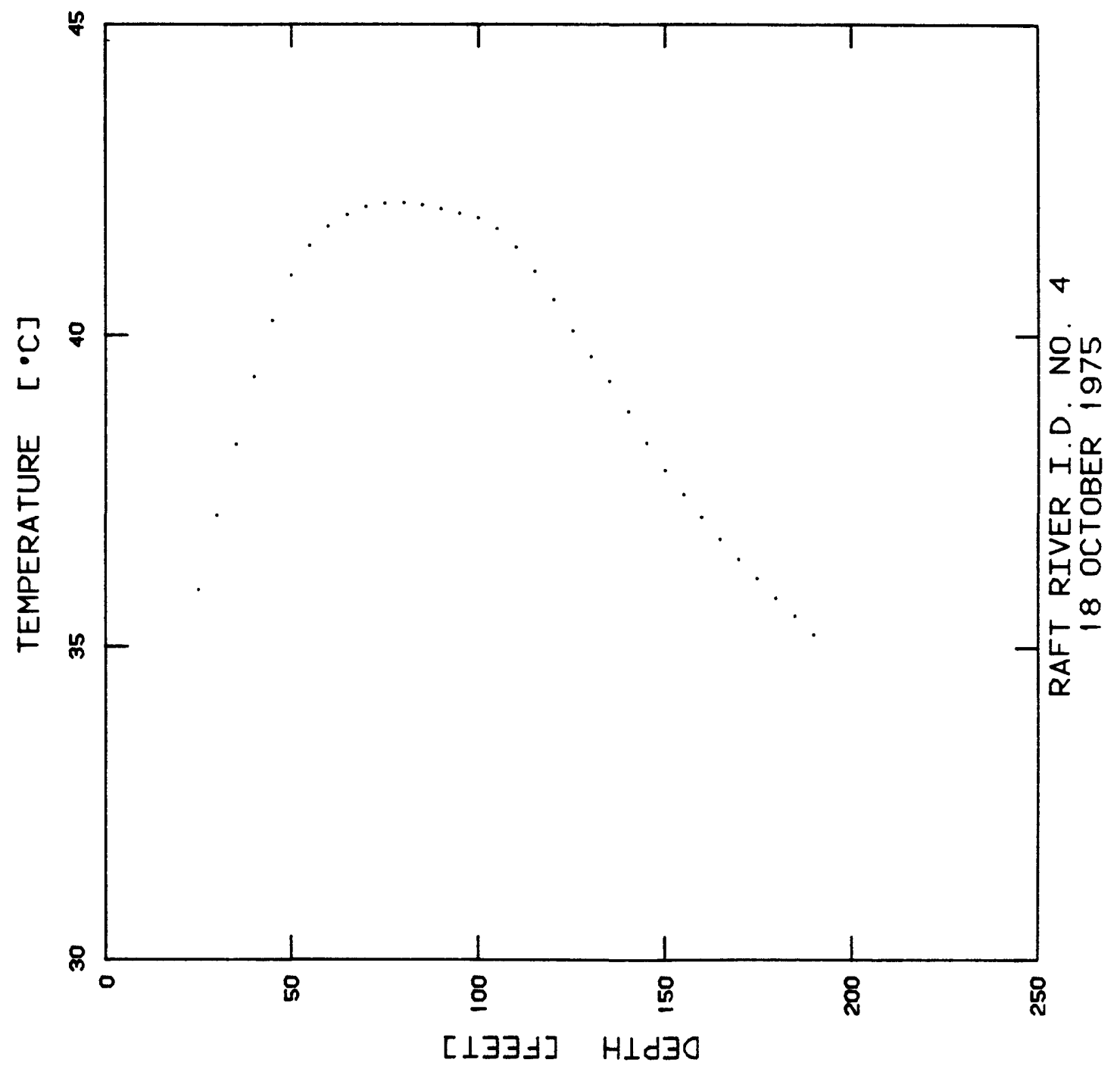




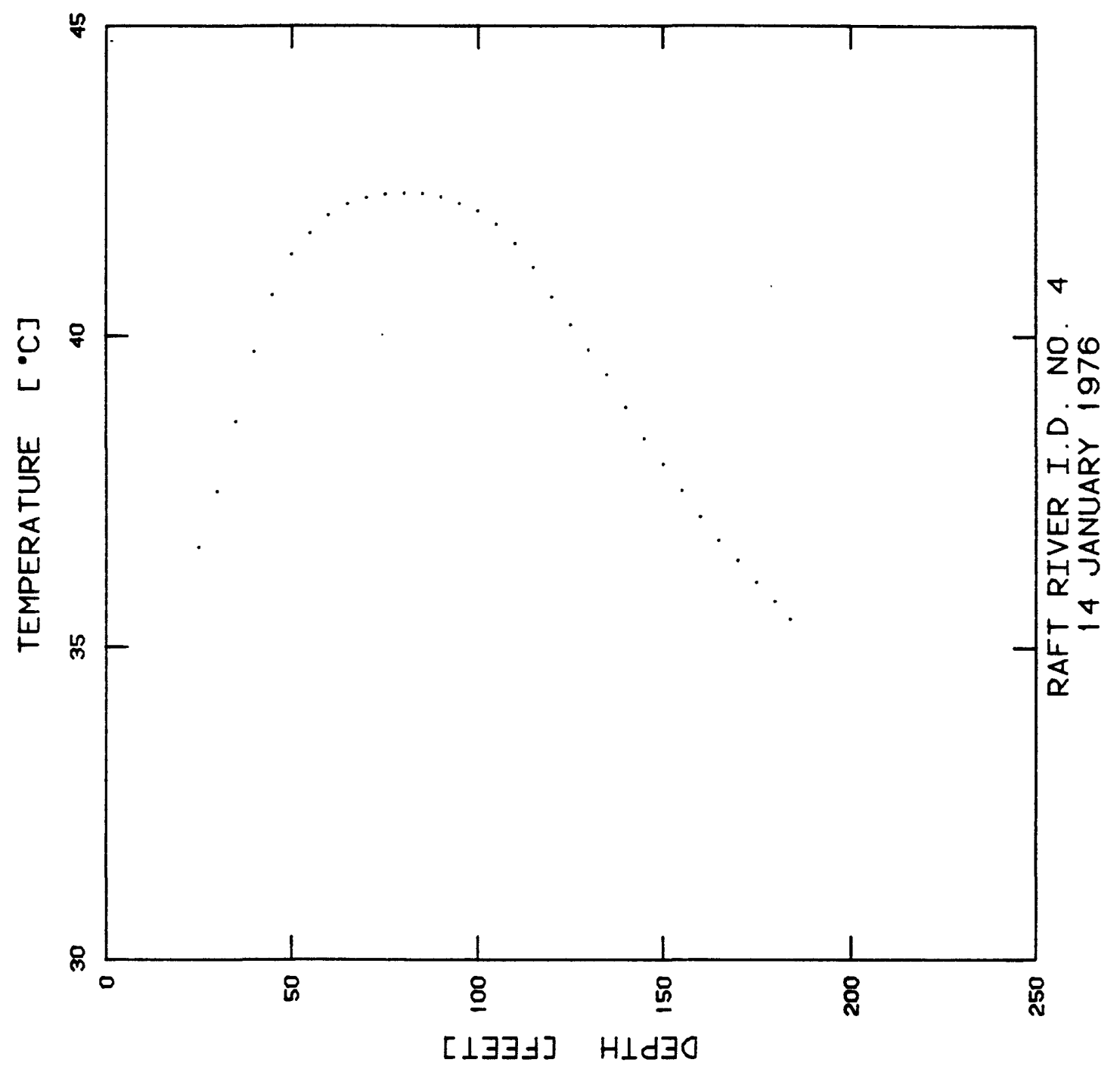

$I-41$ 


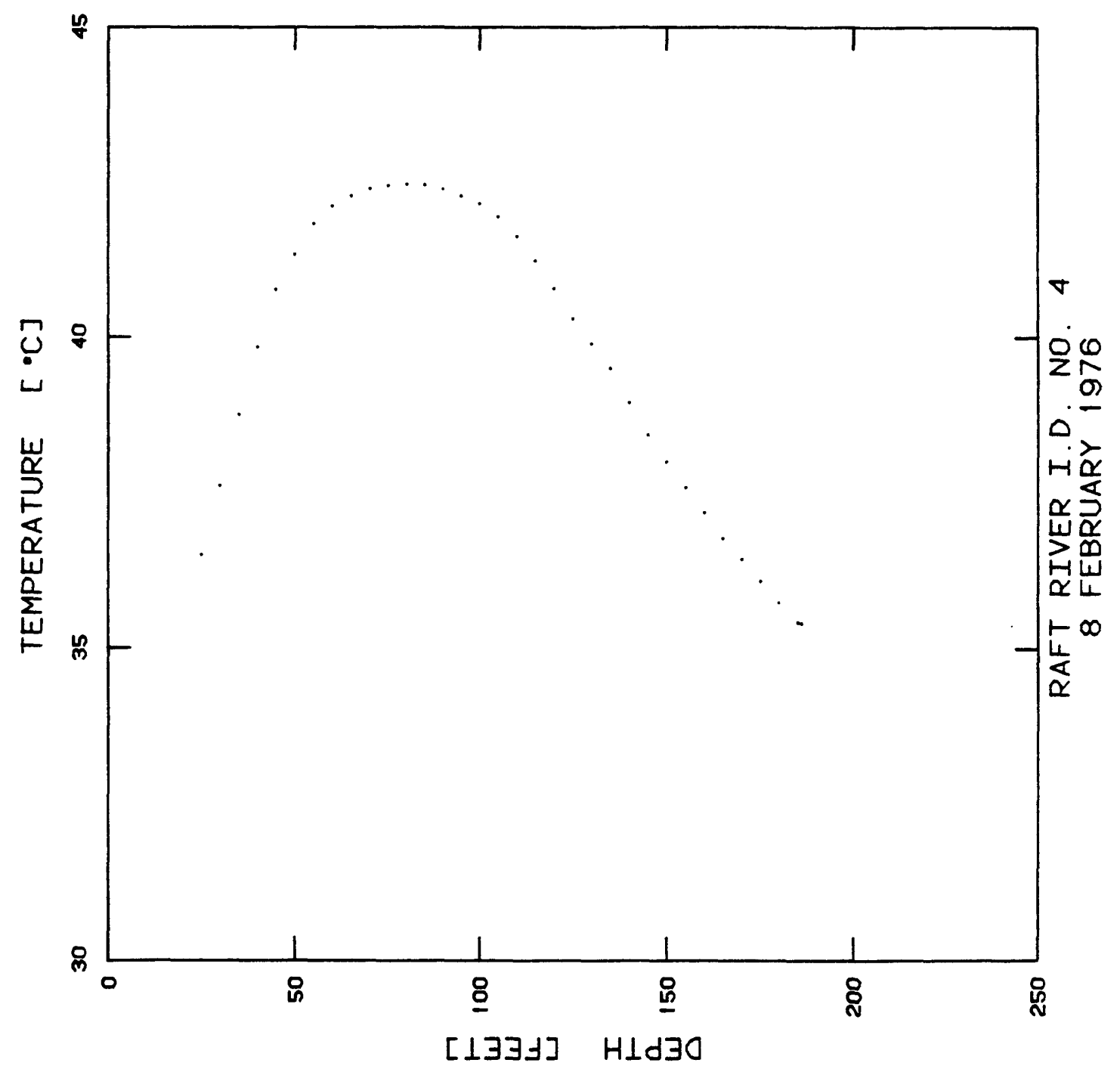

$I-42$ 


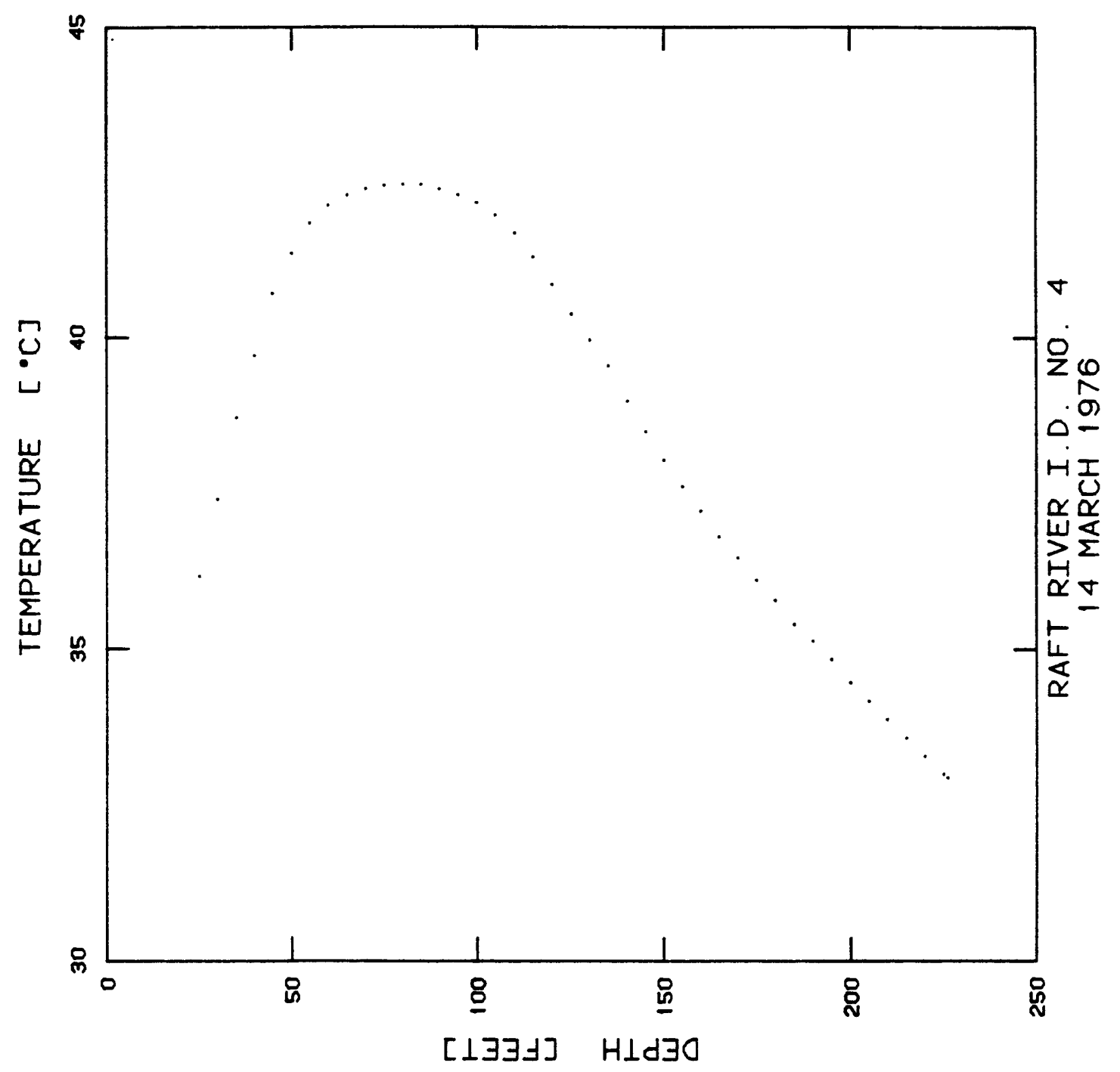




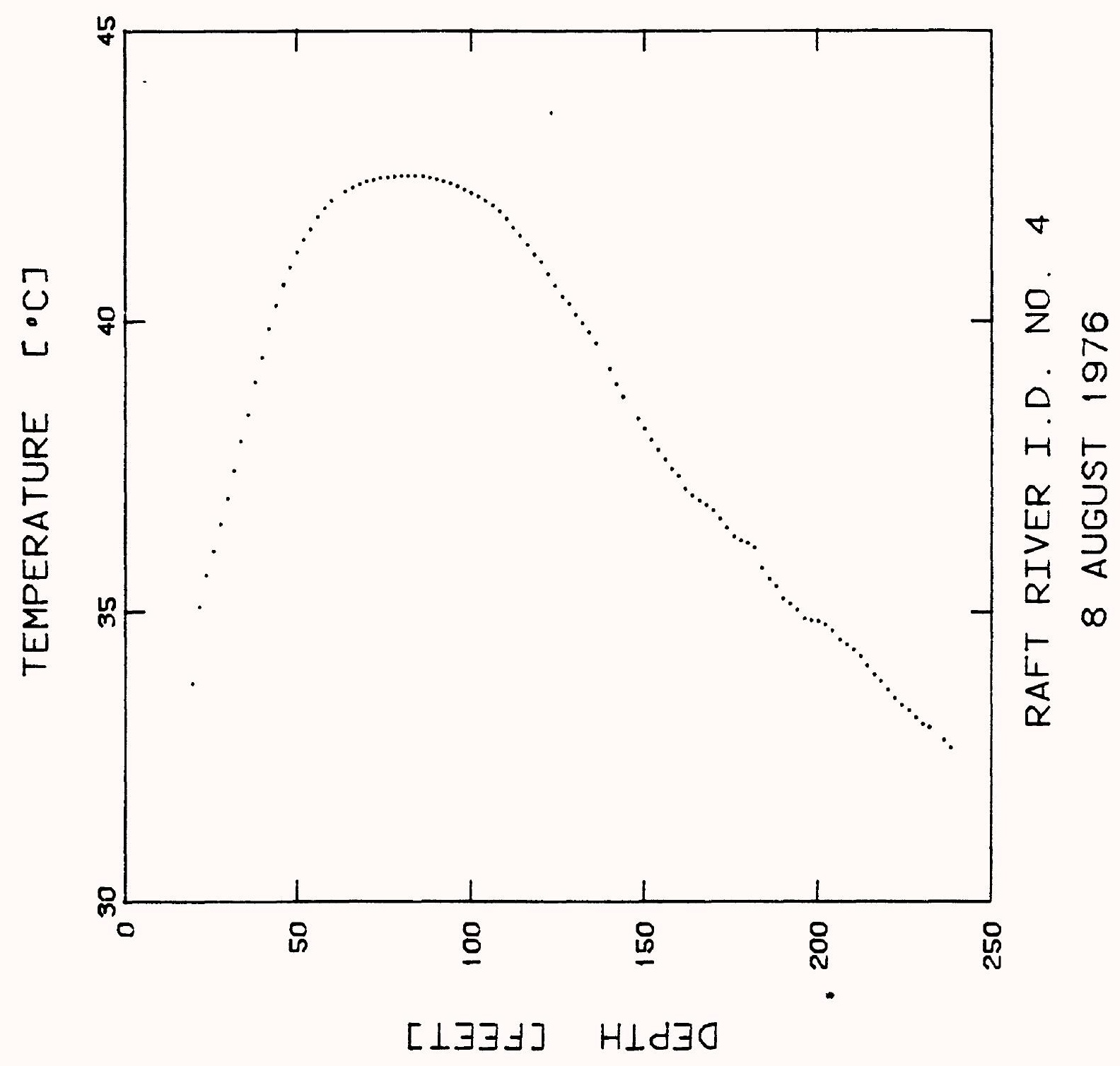




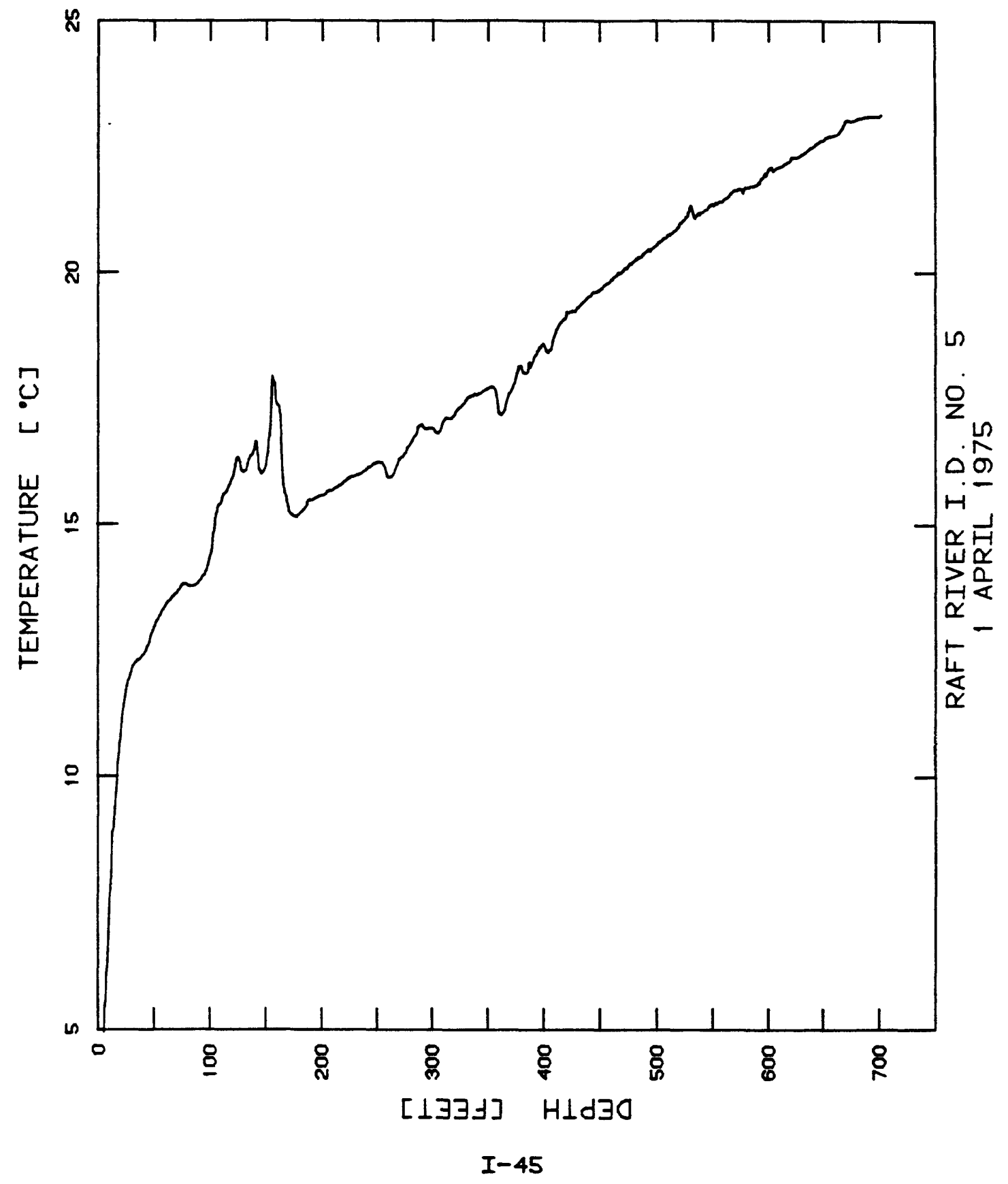




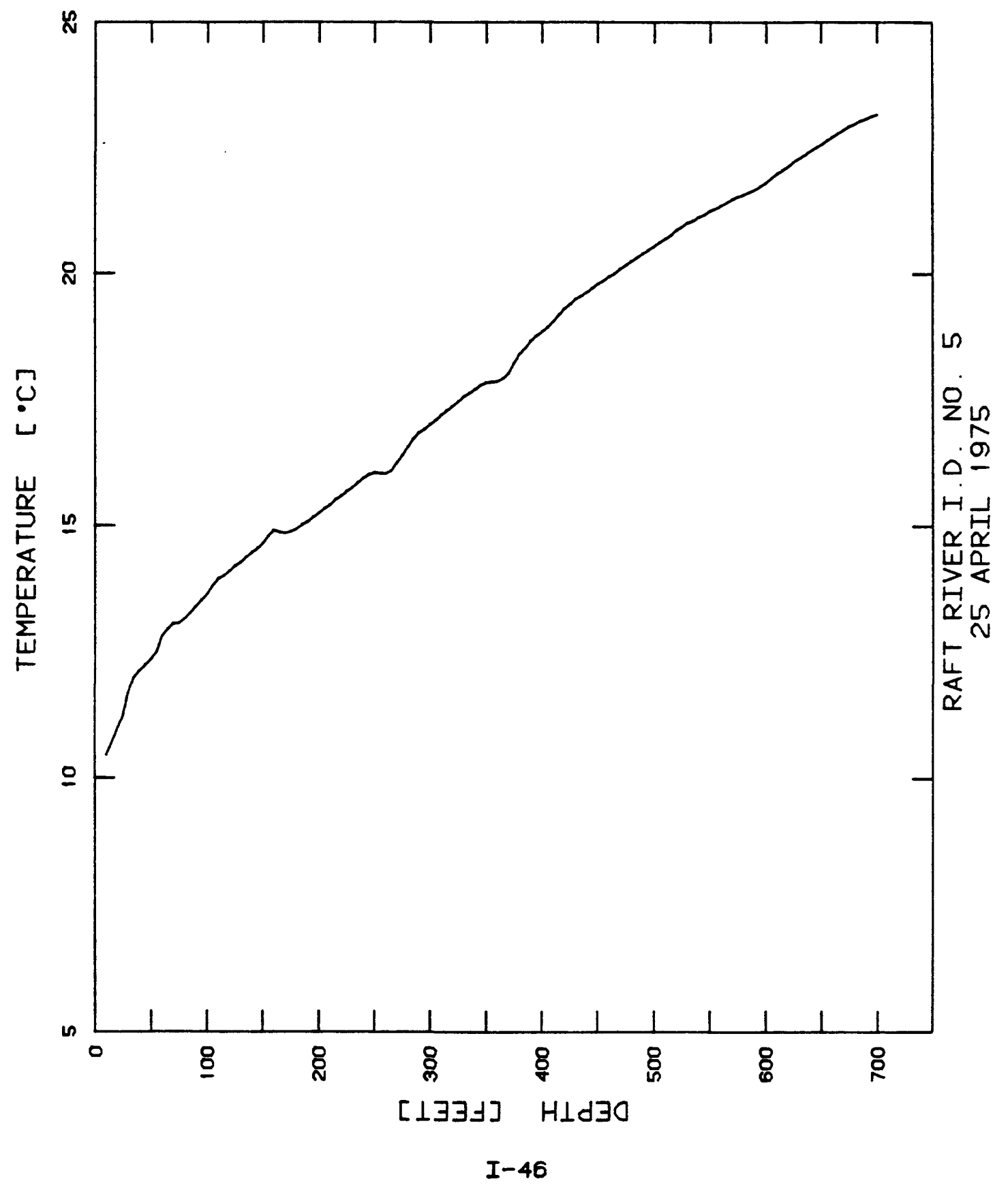




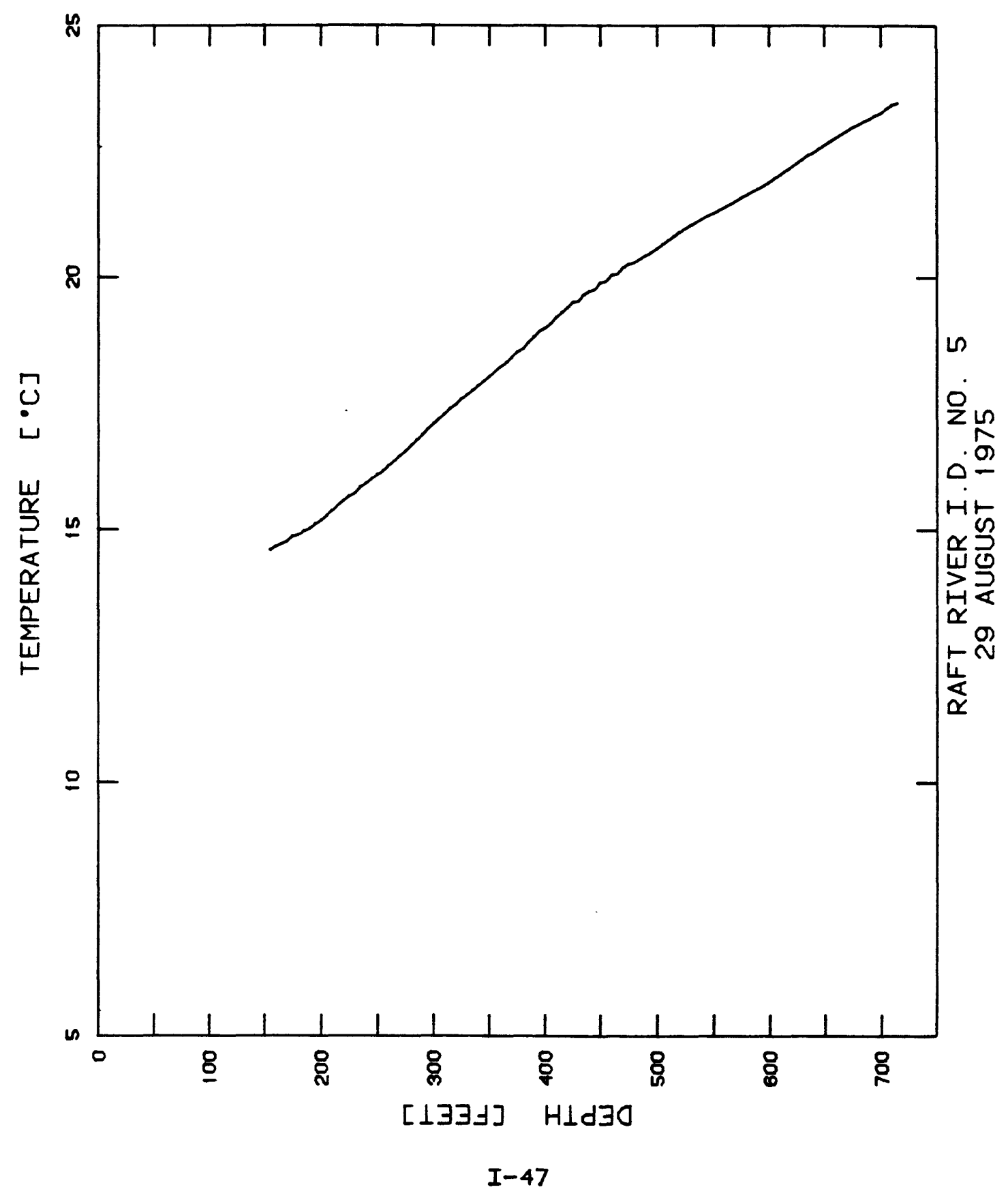




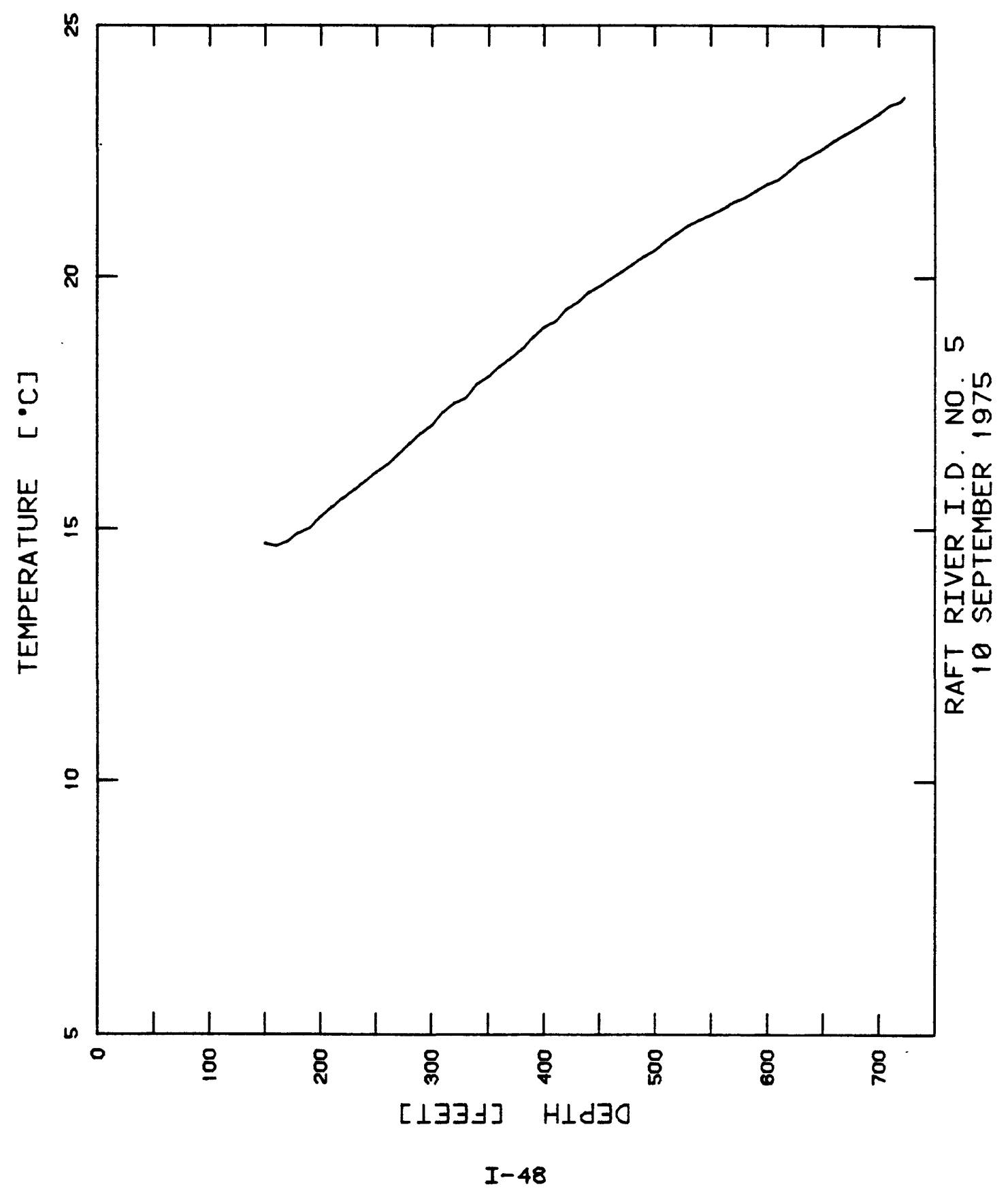




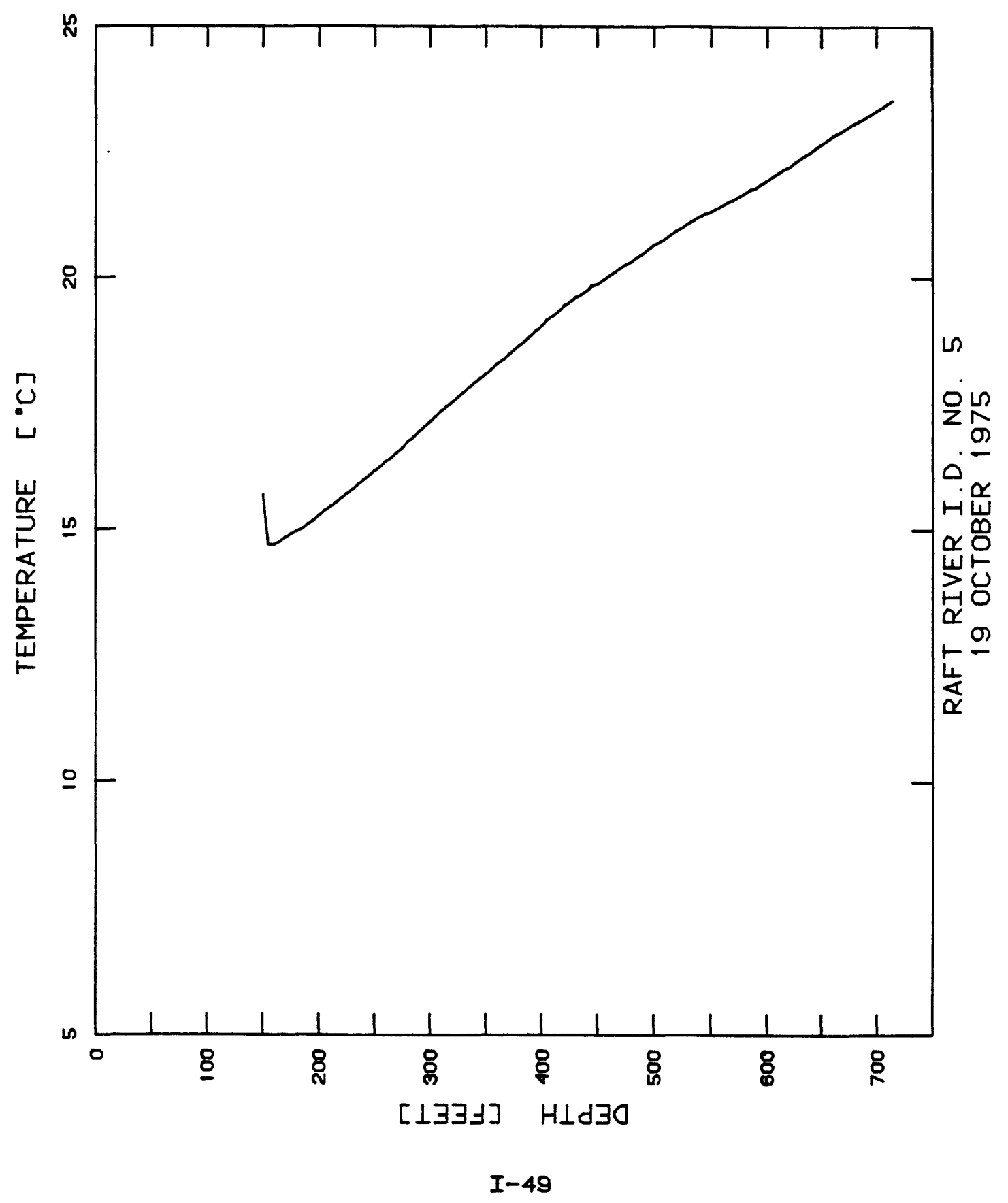




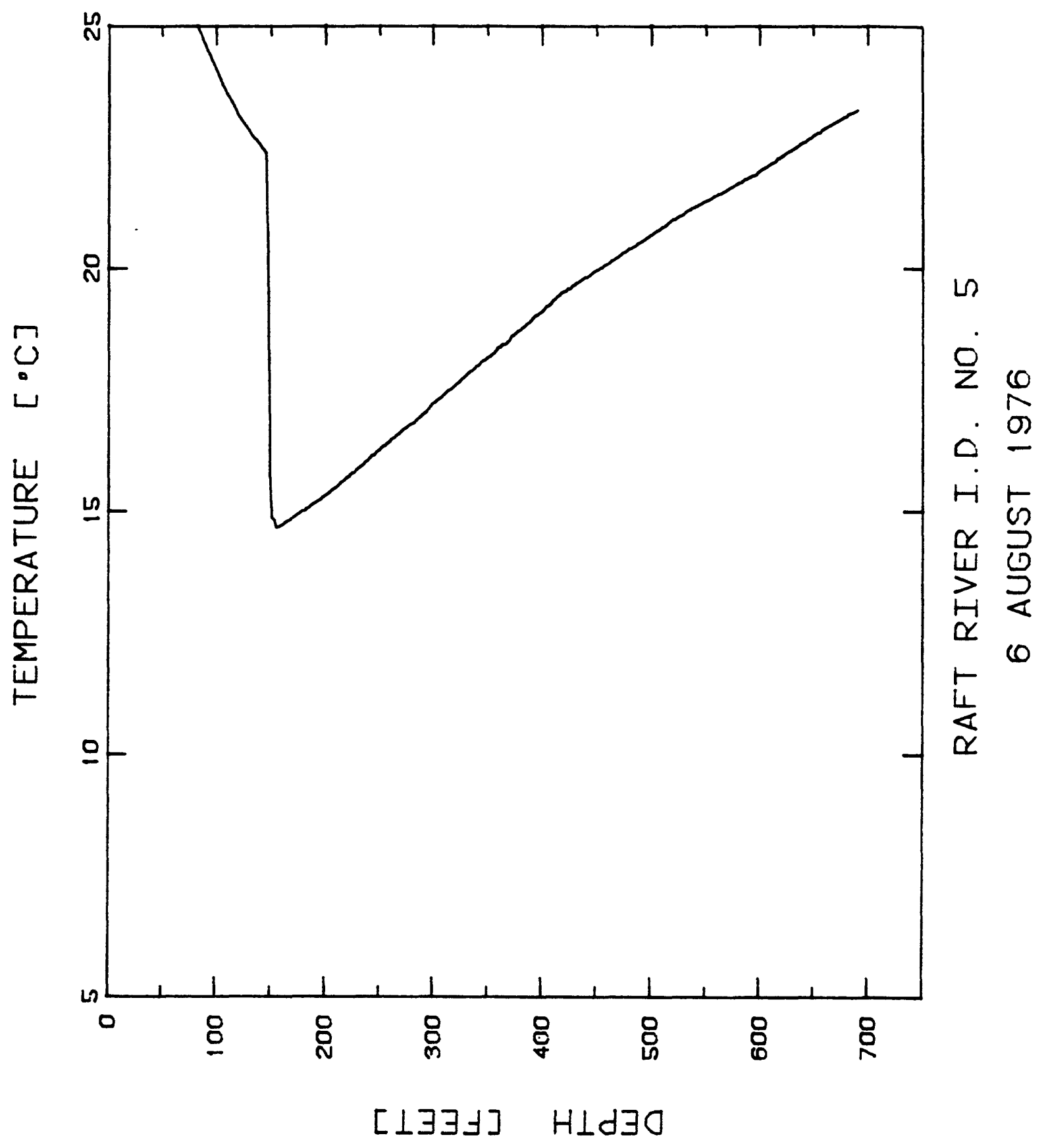

$I-50$ 


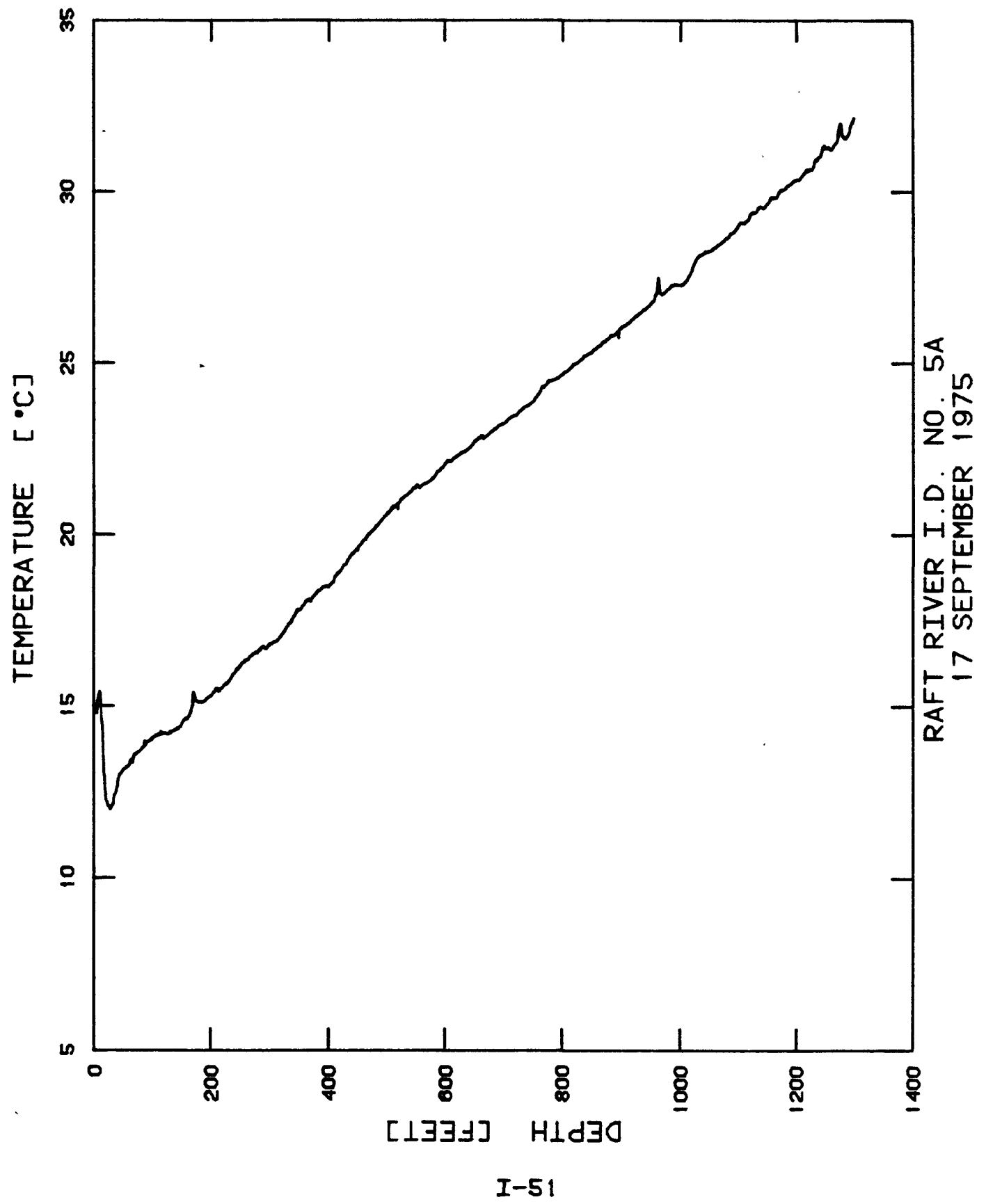




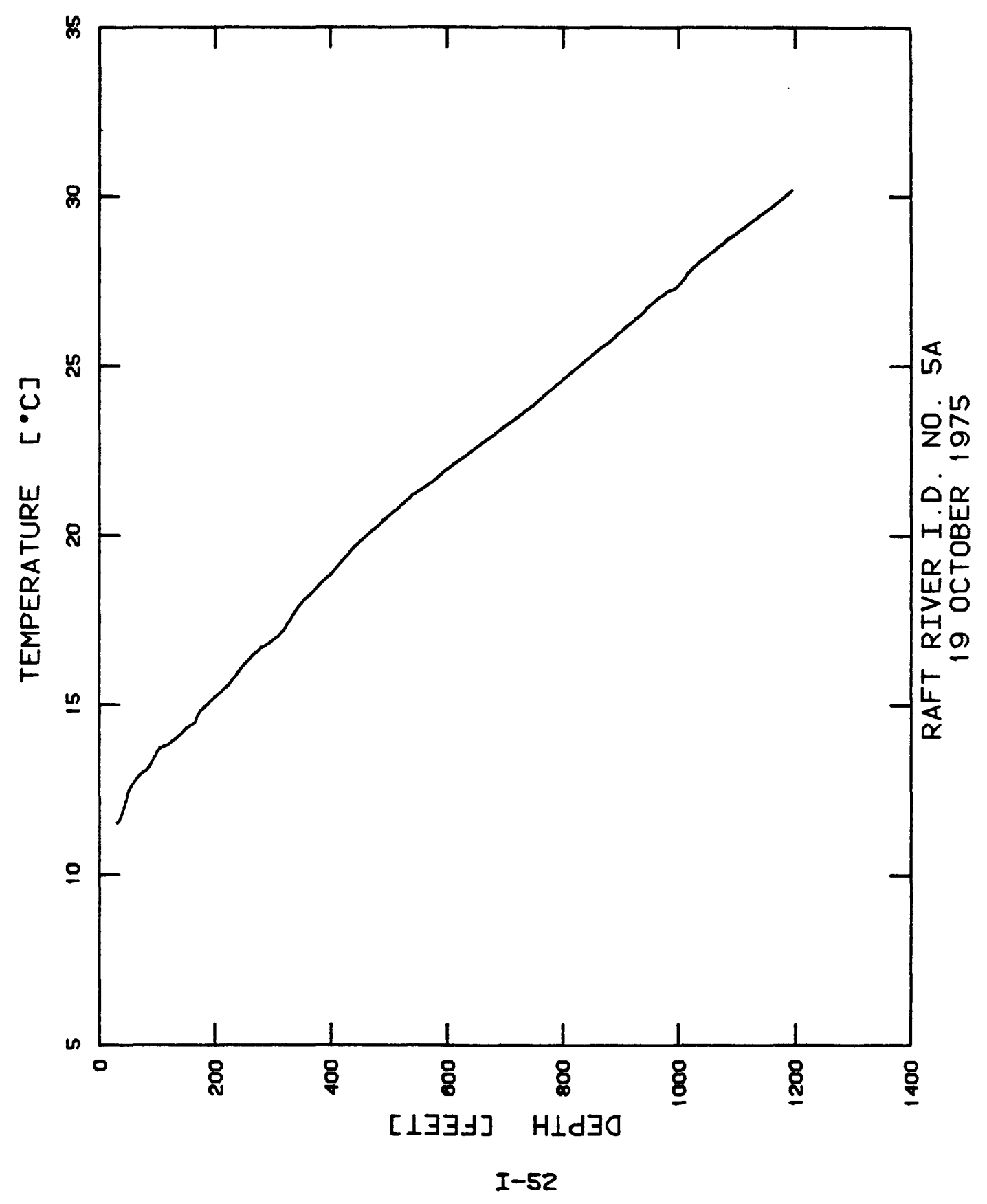




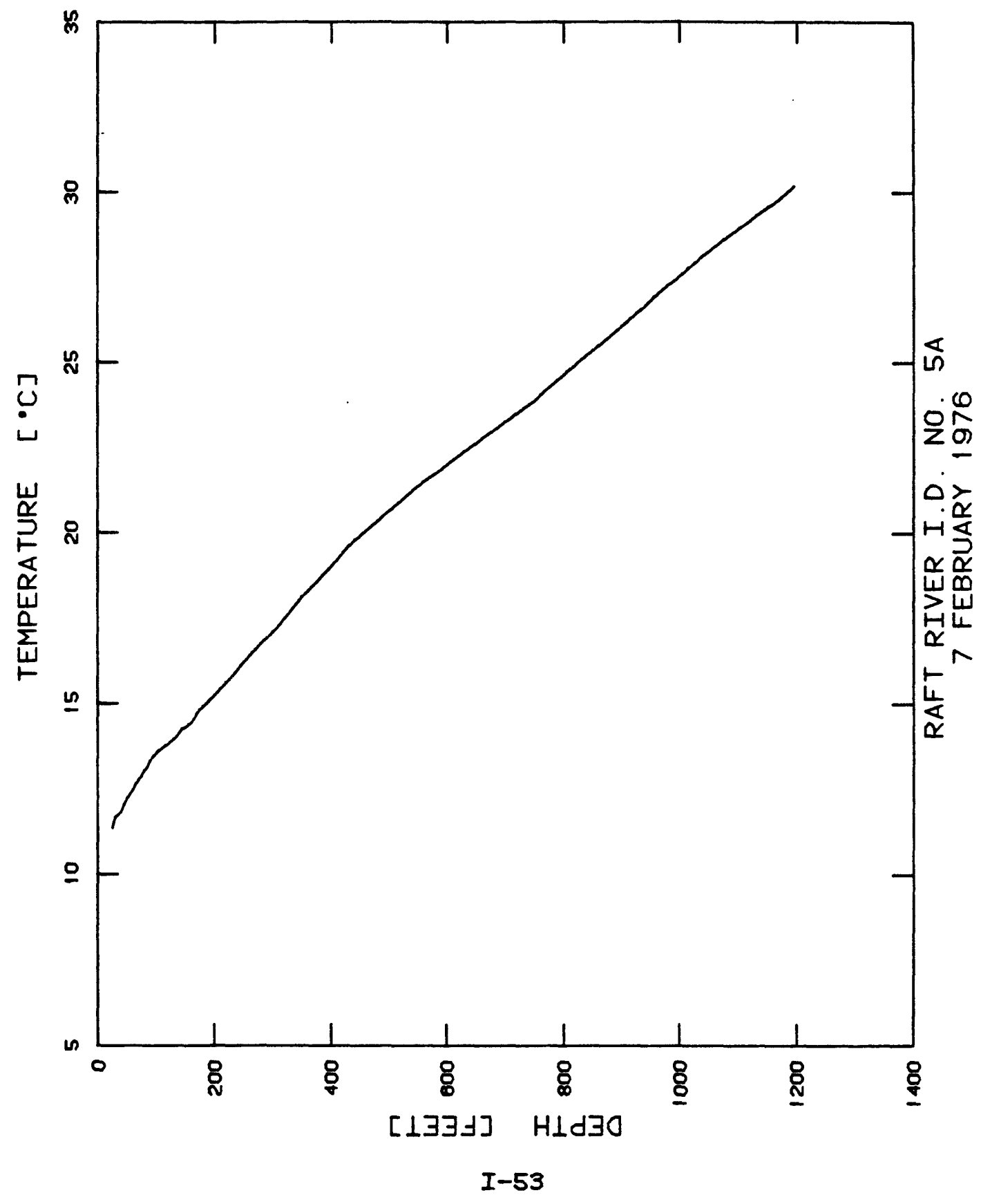




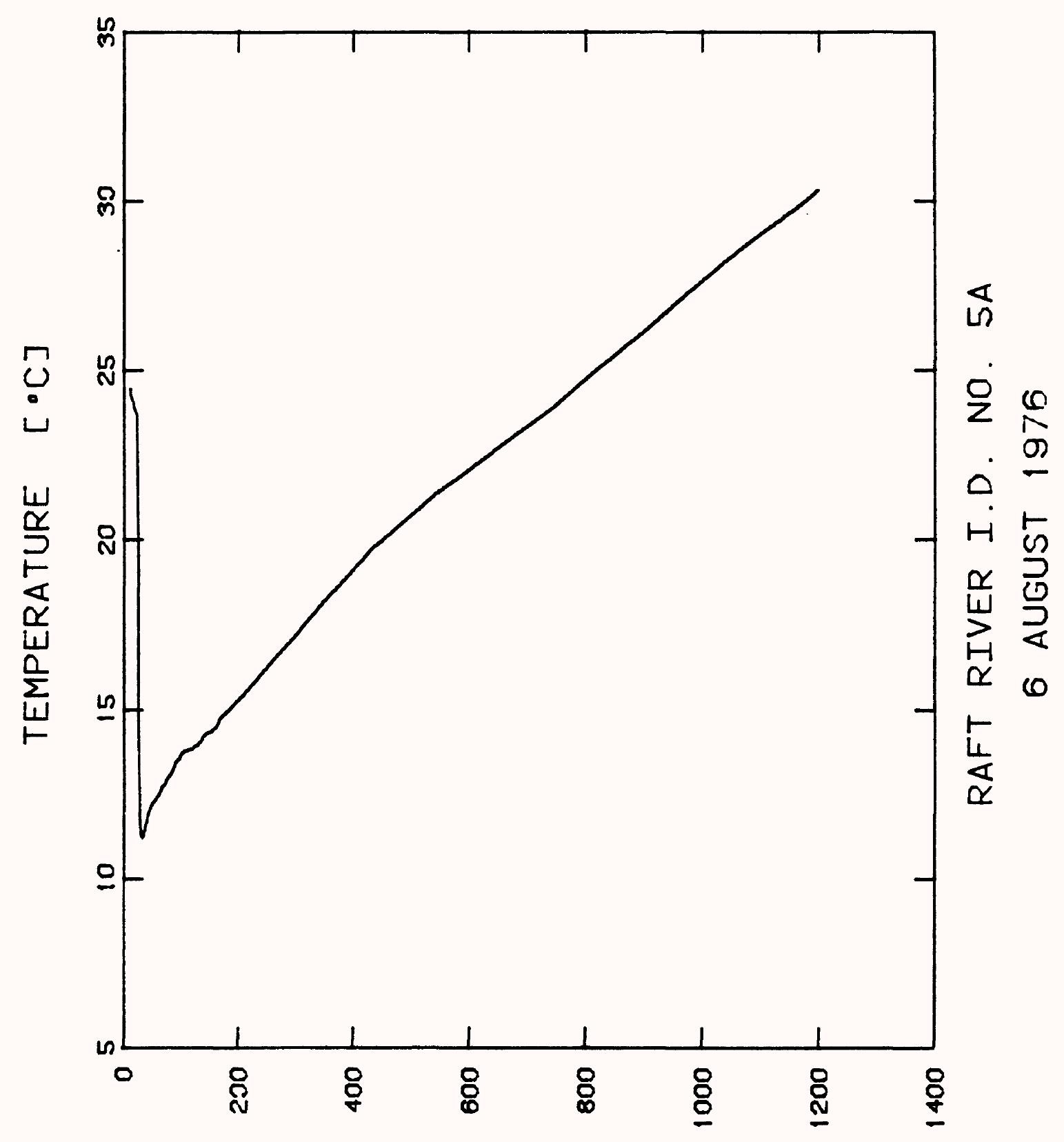

$[\perp \exists \exists\lrcorner] \quad H \perp d \exists O$ 


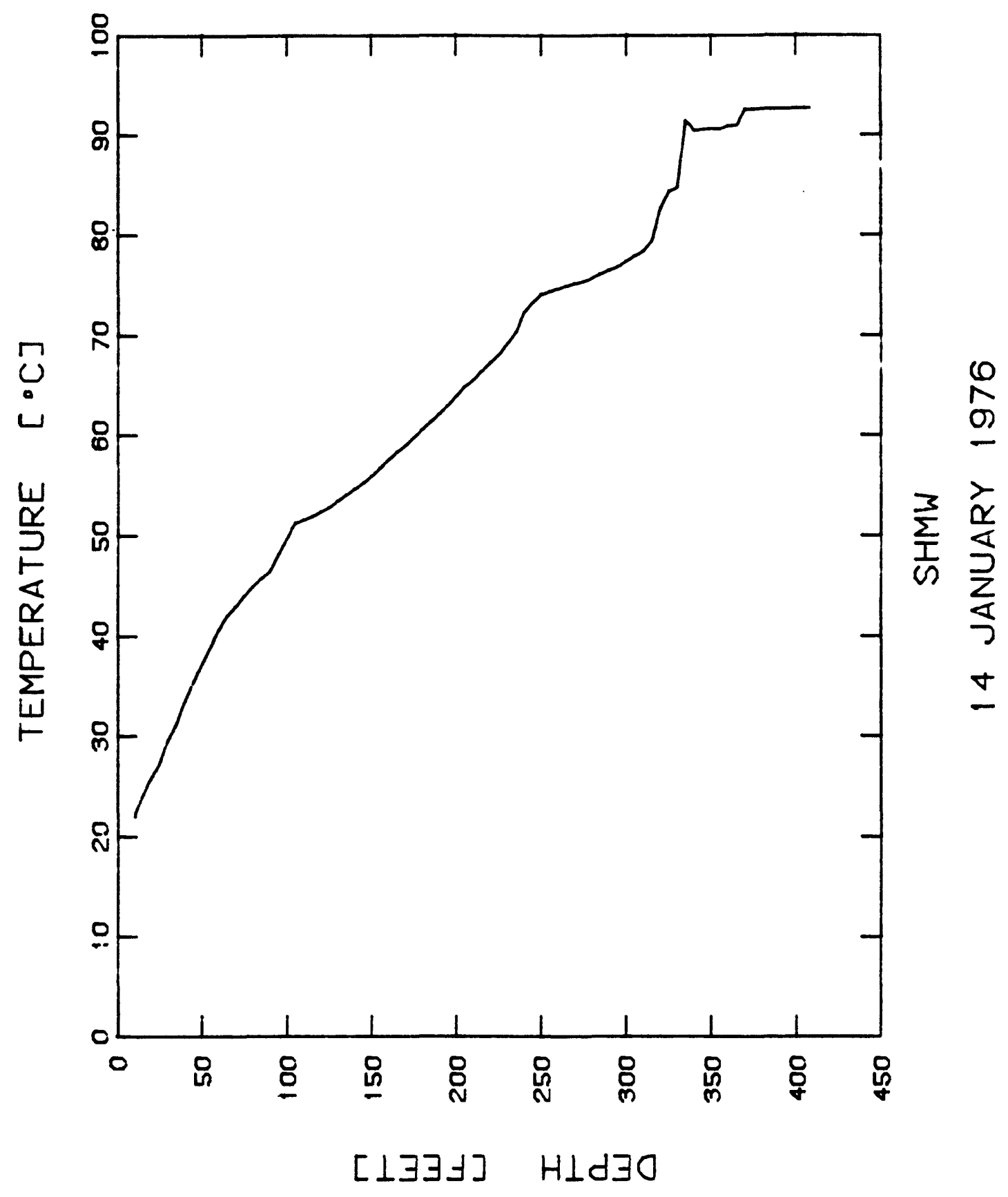

$I-55$ 


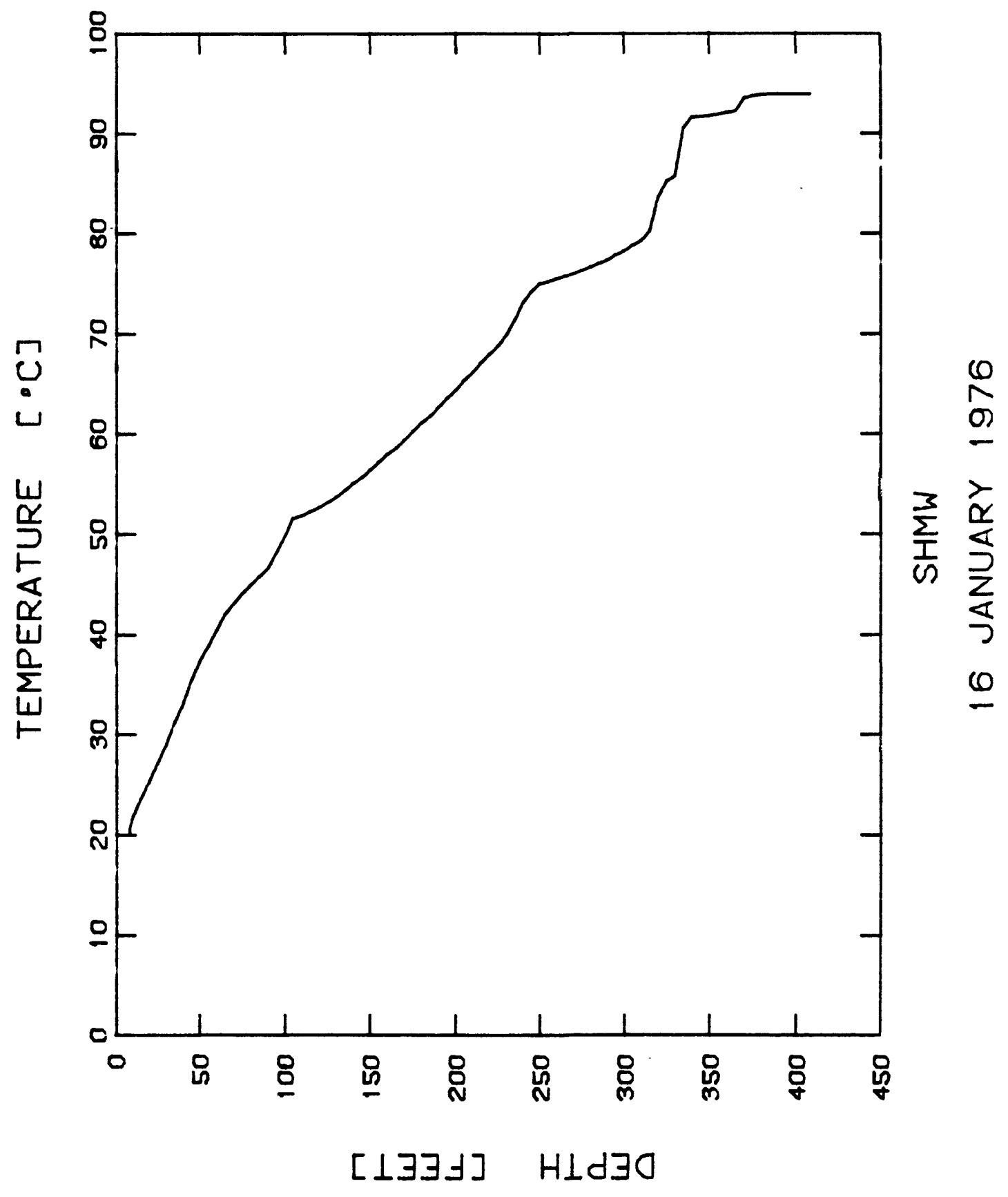




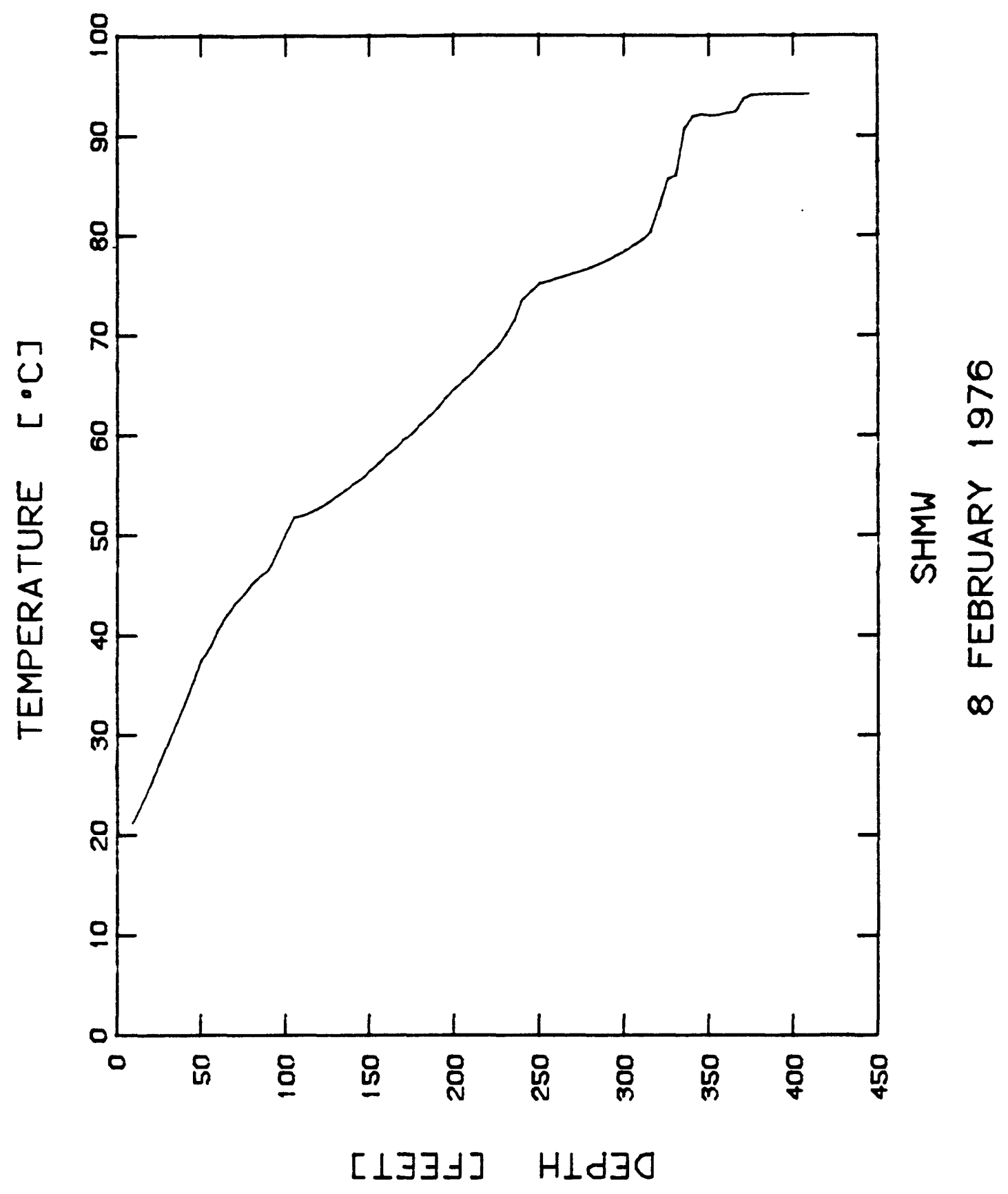

$I-57$ 


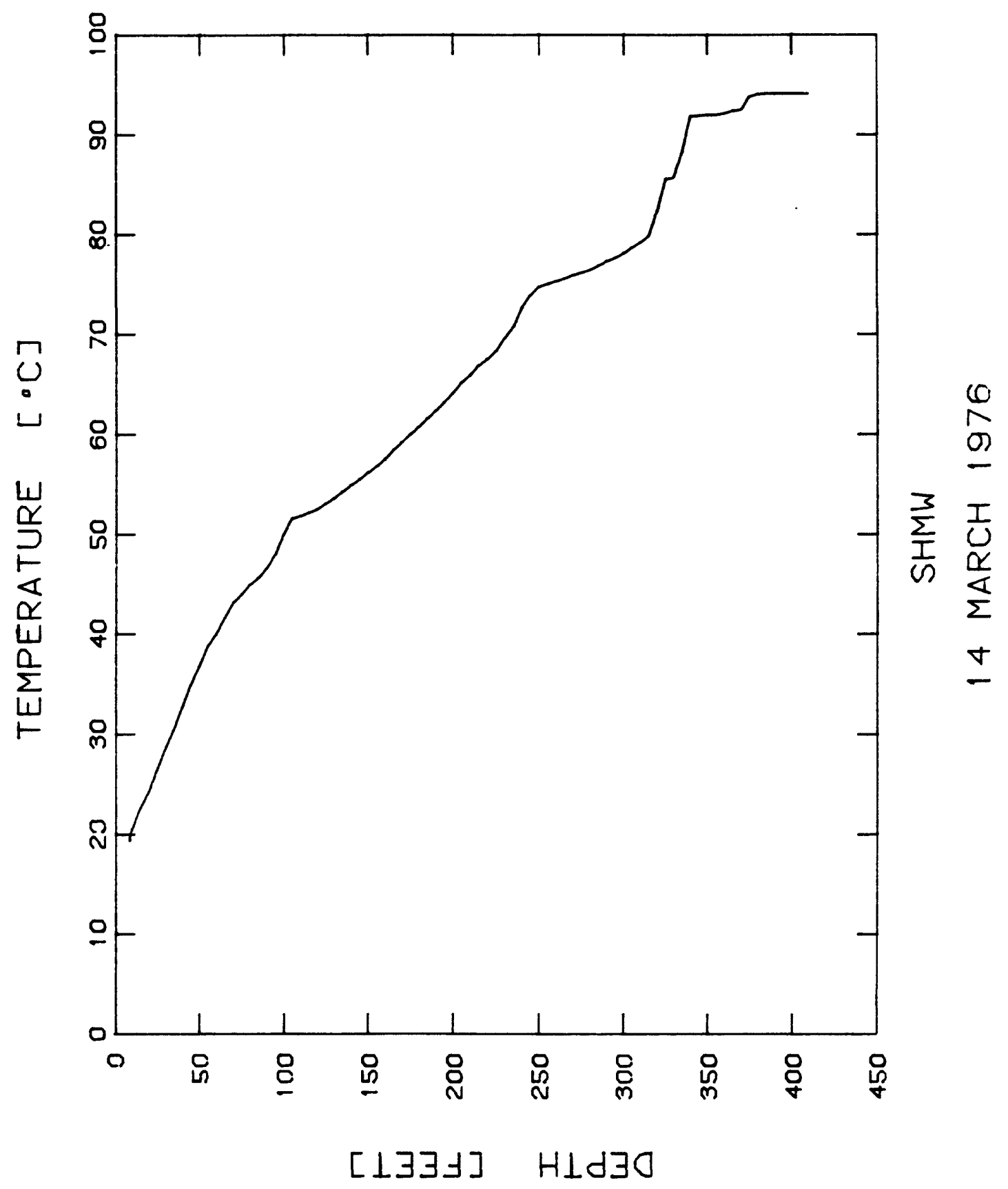

$I-58$ 


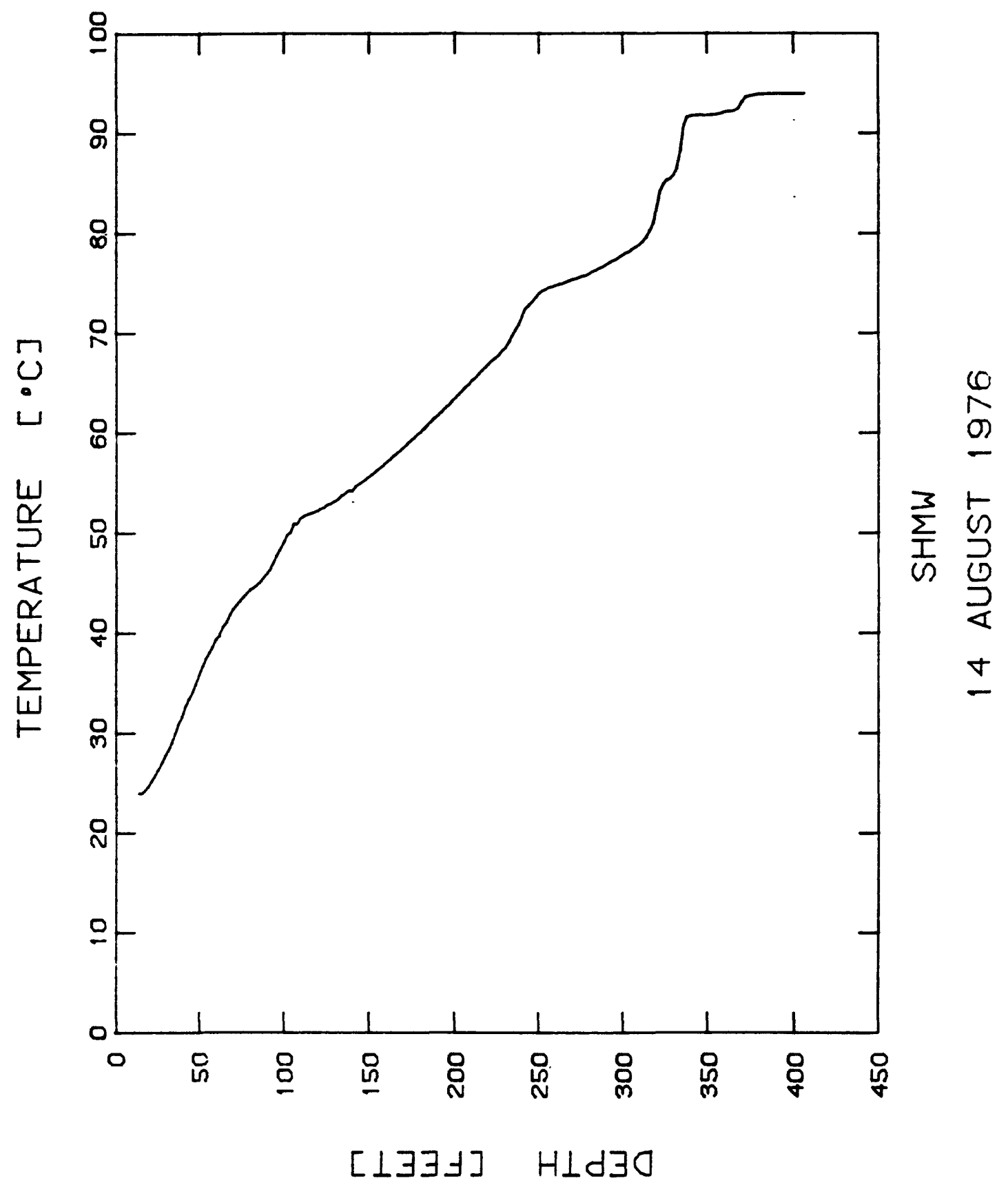

$I-59$ 


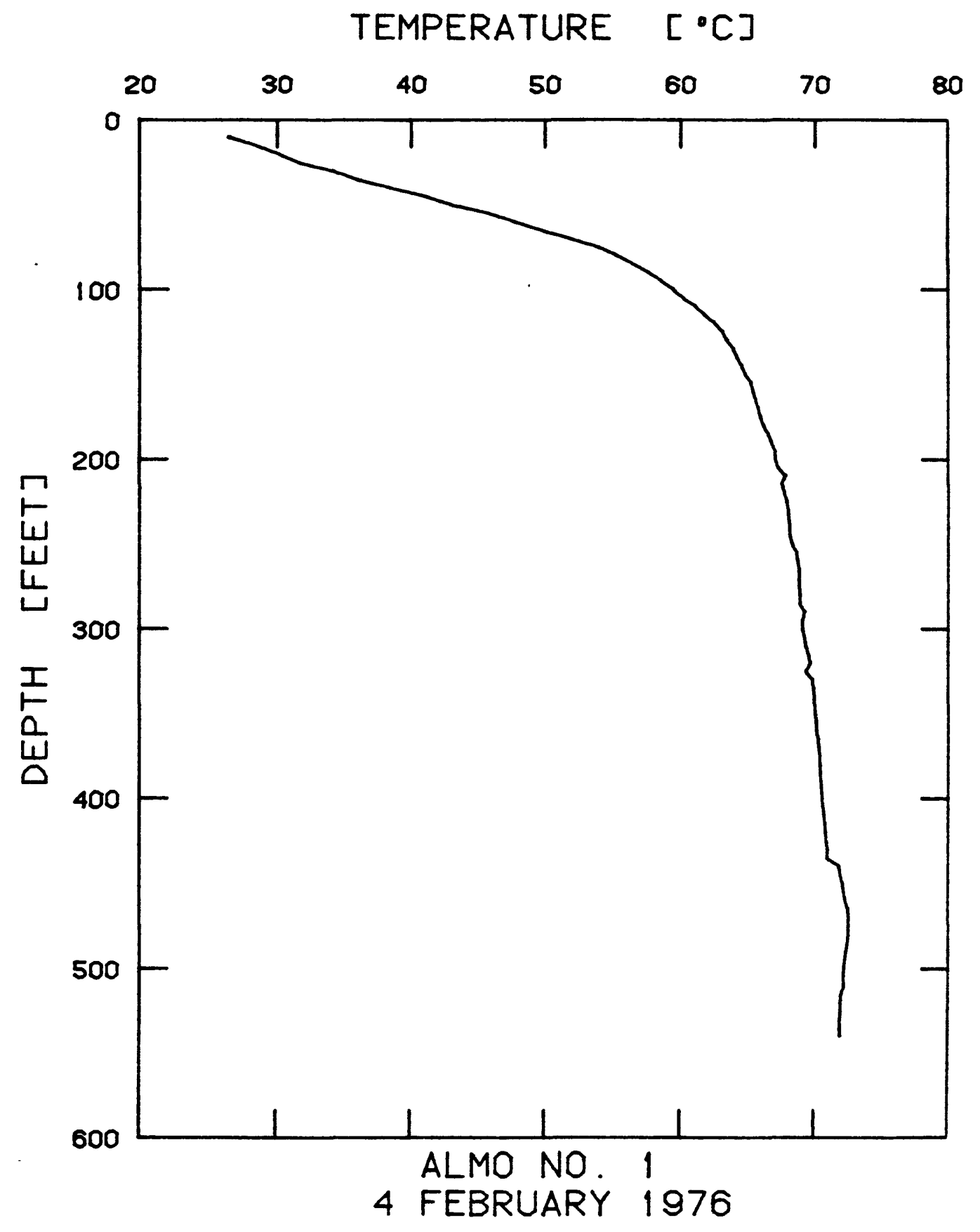

$I-60$ 


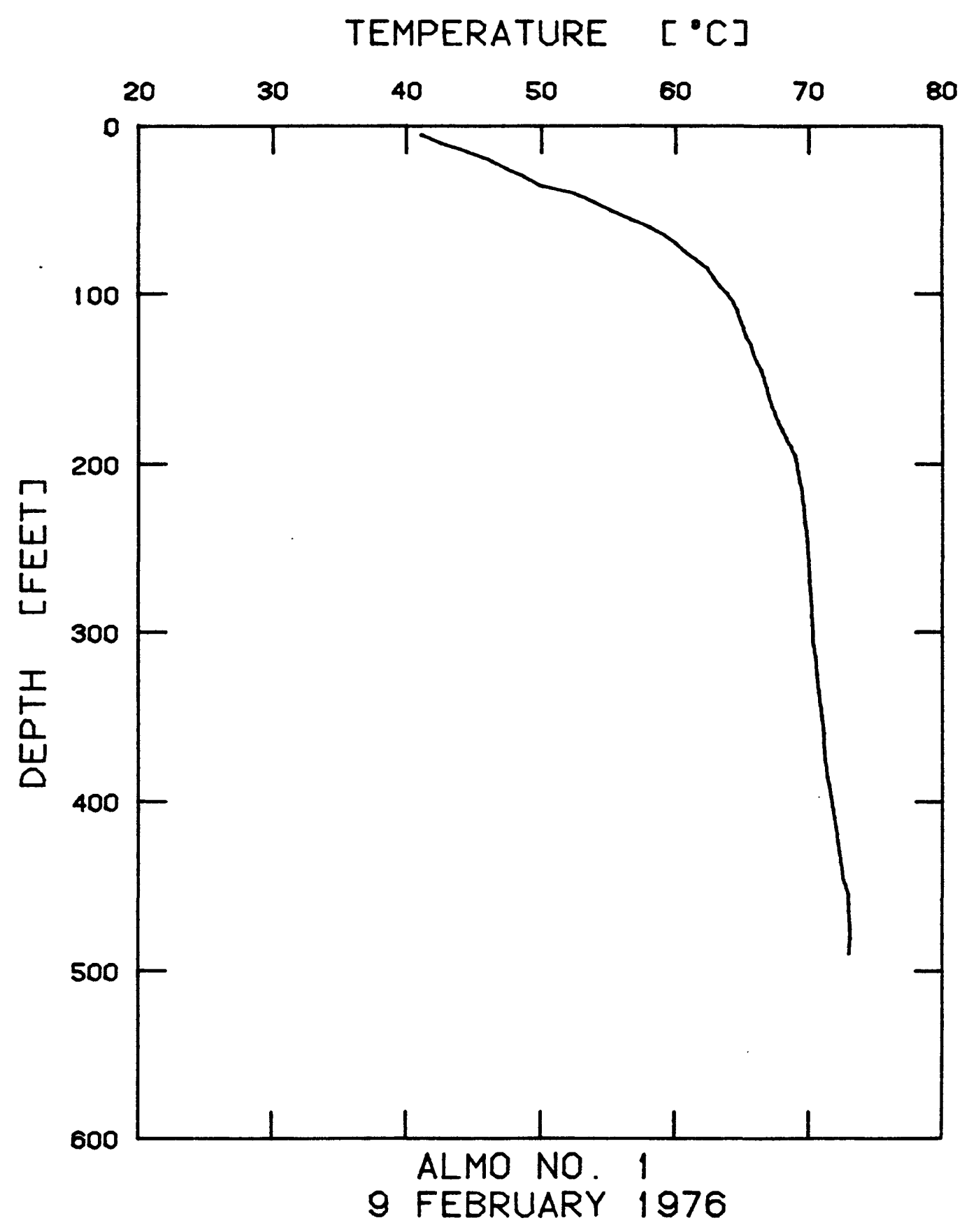

$I-61$ 


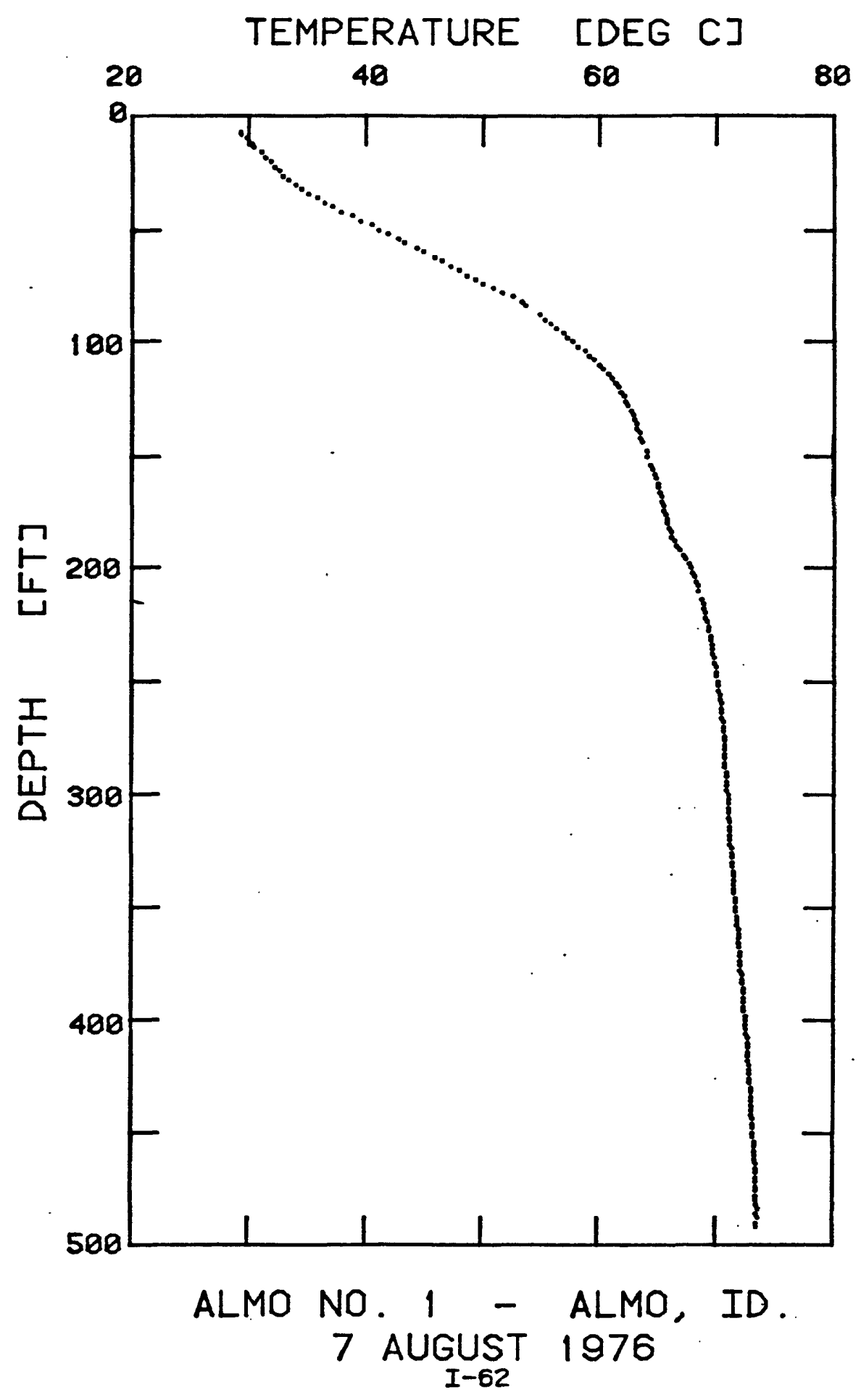




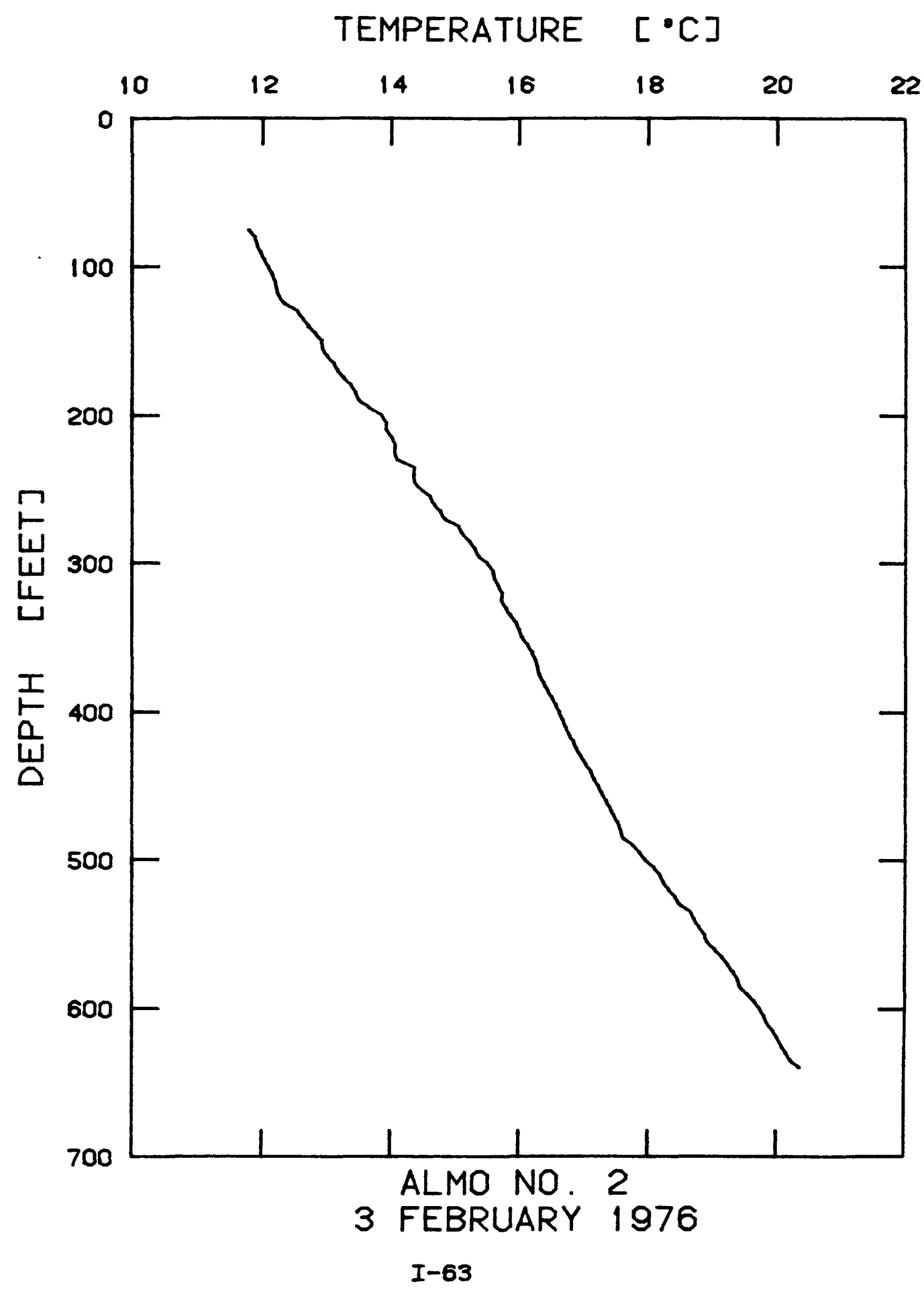




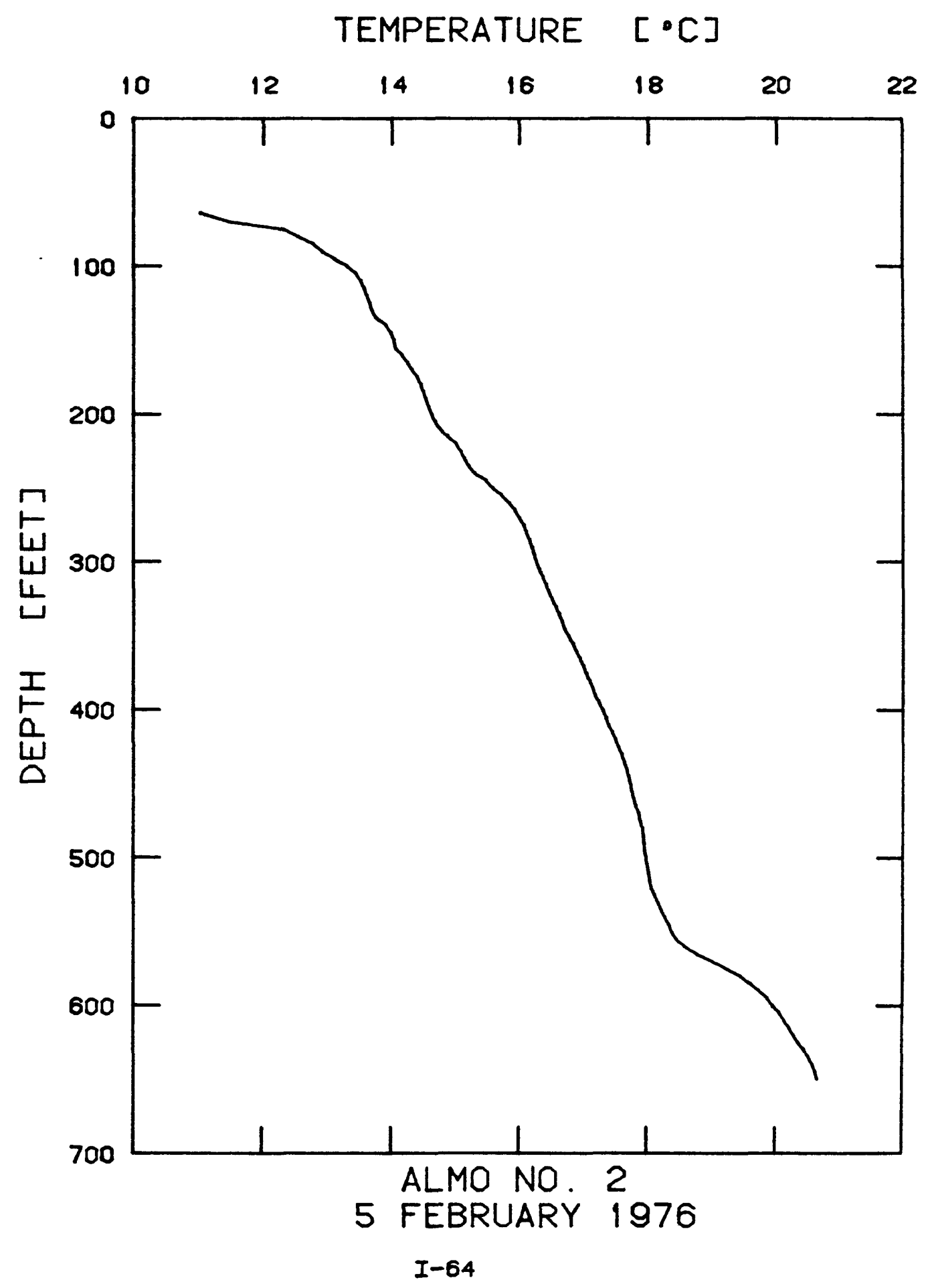




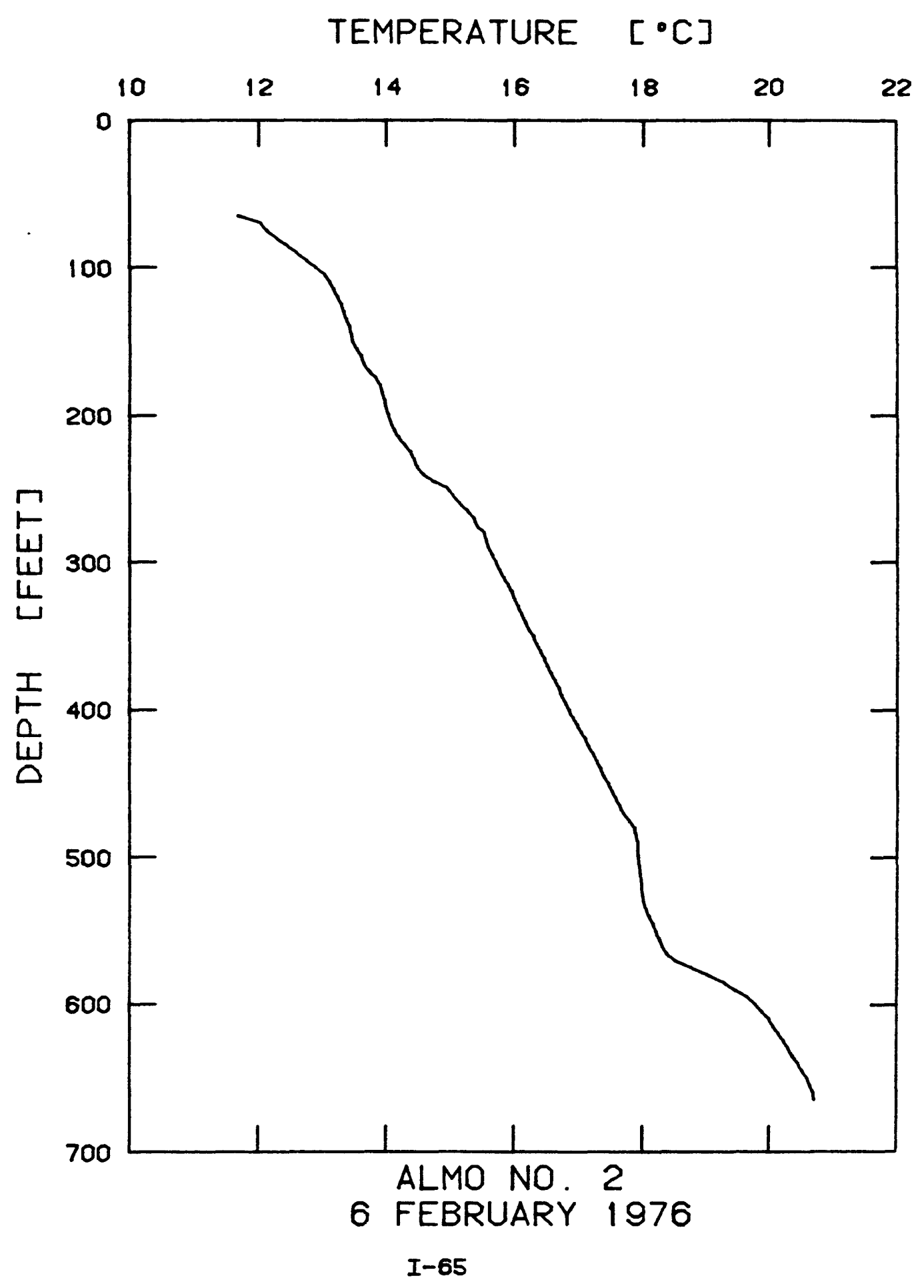




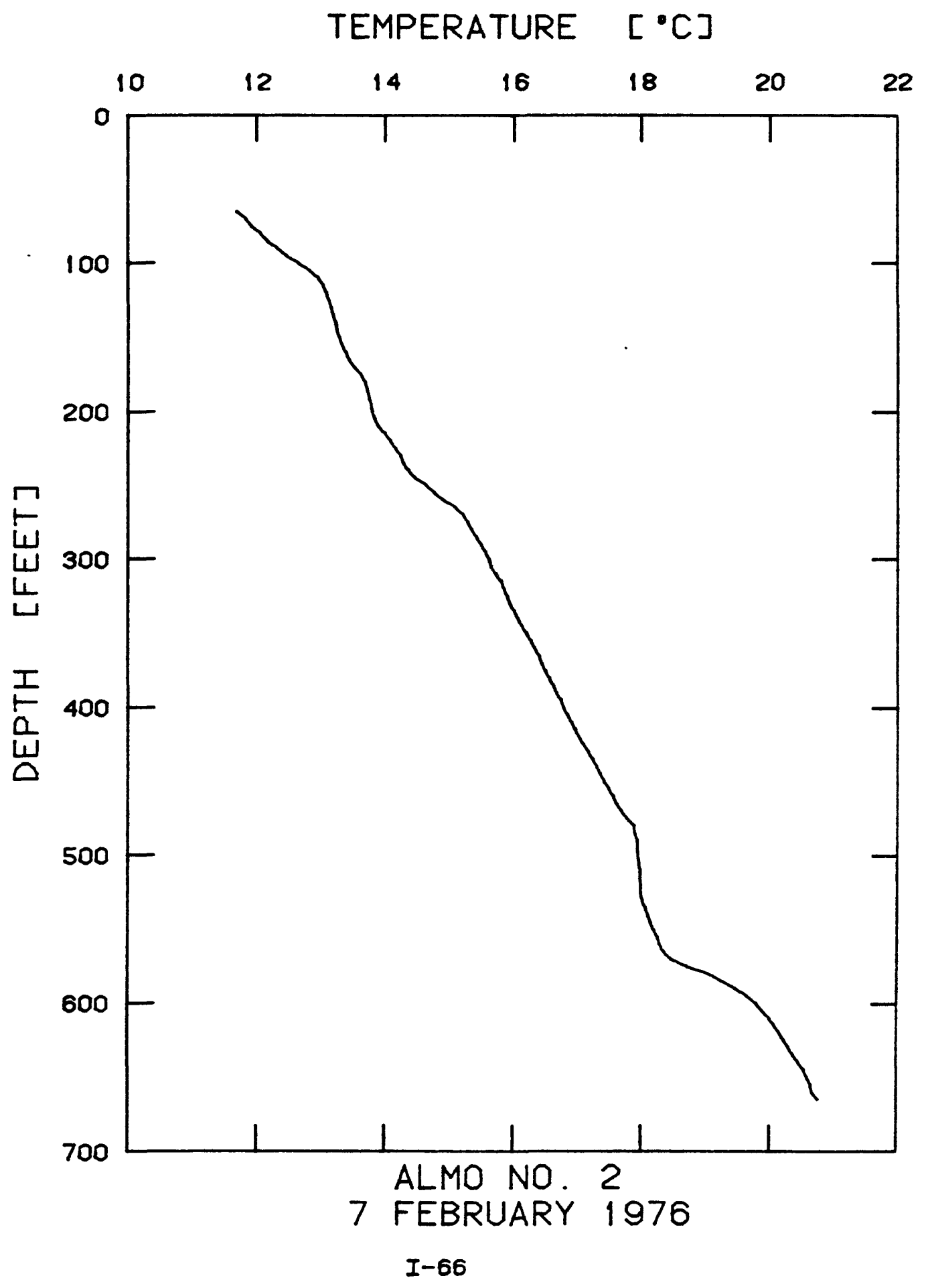




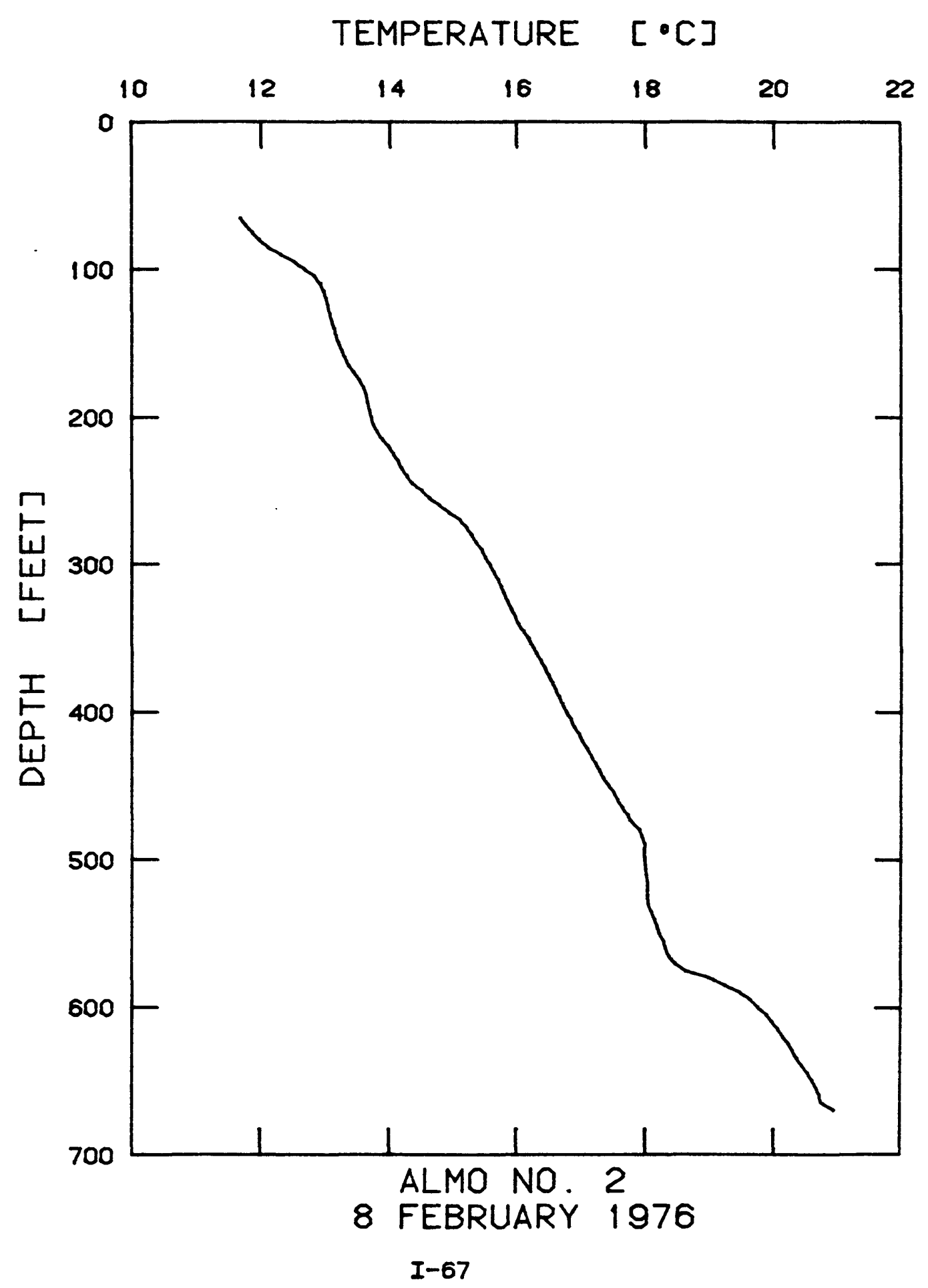




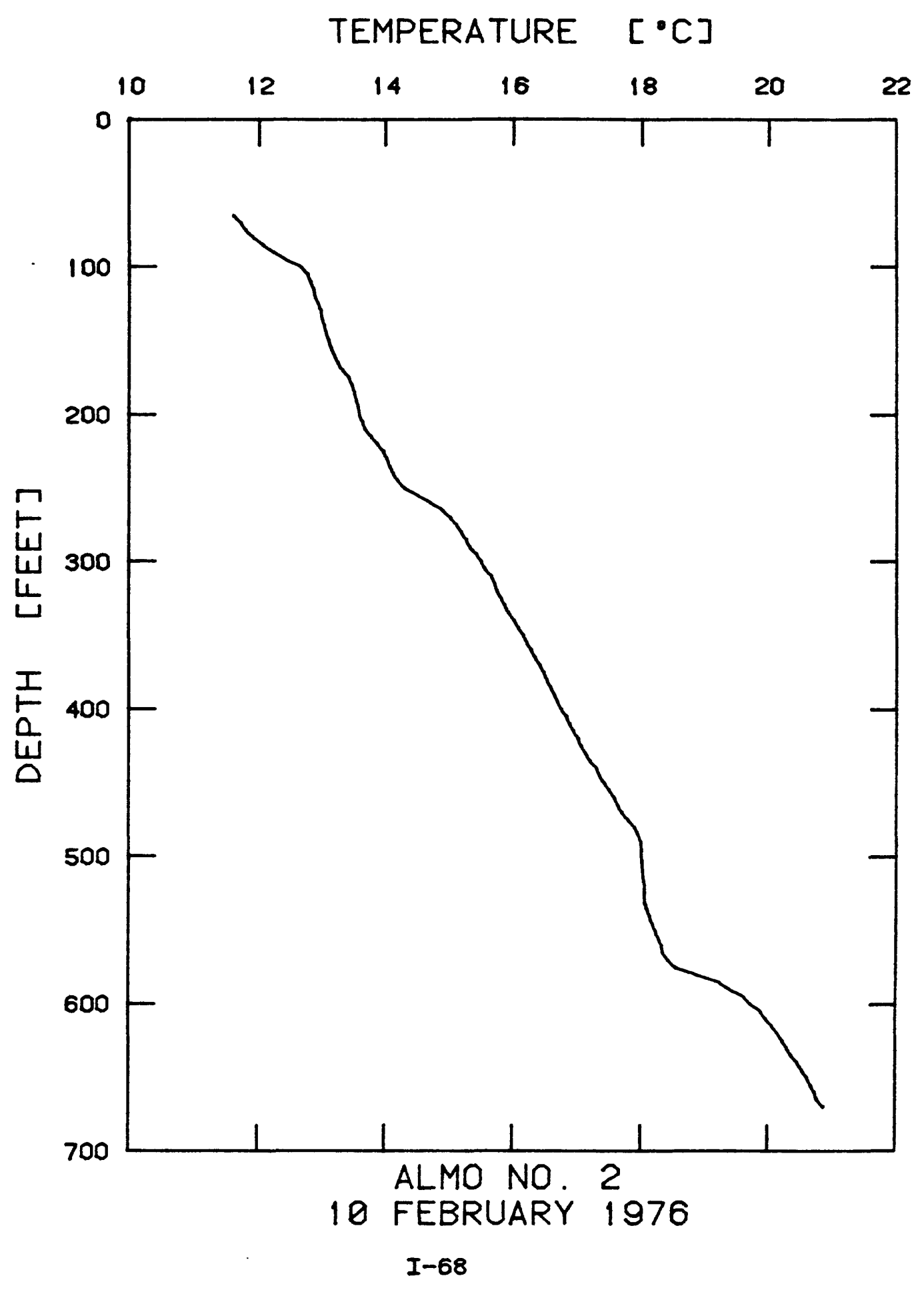




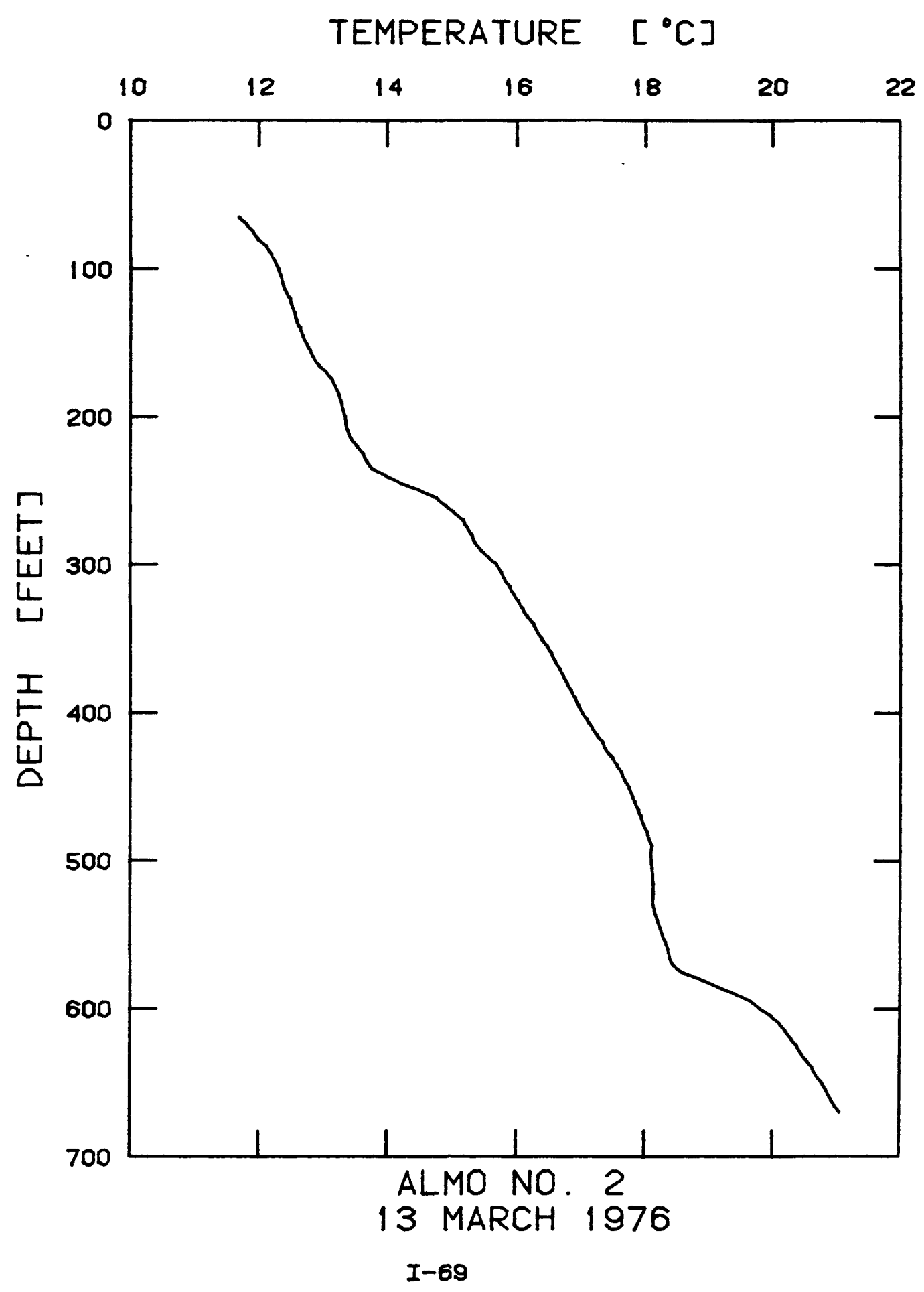




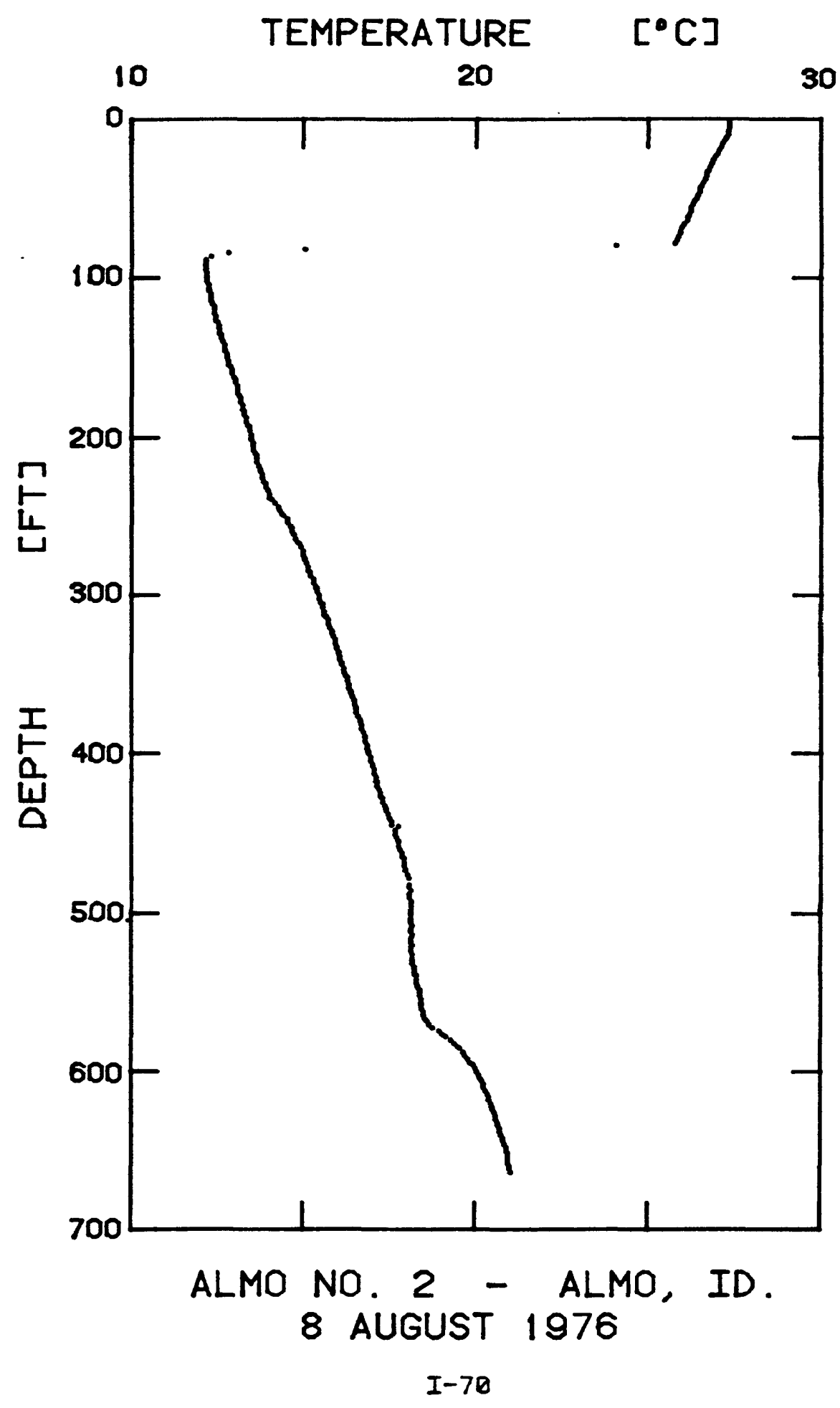




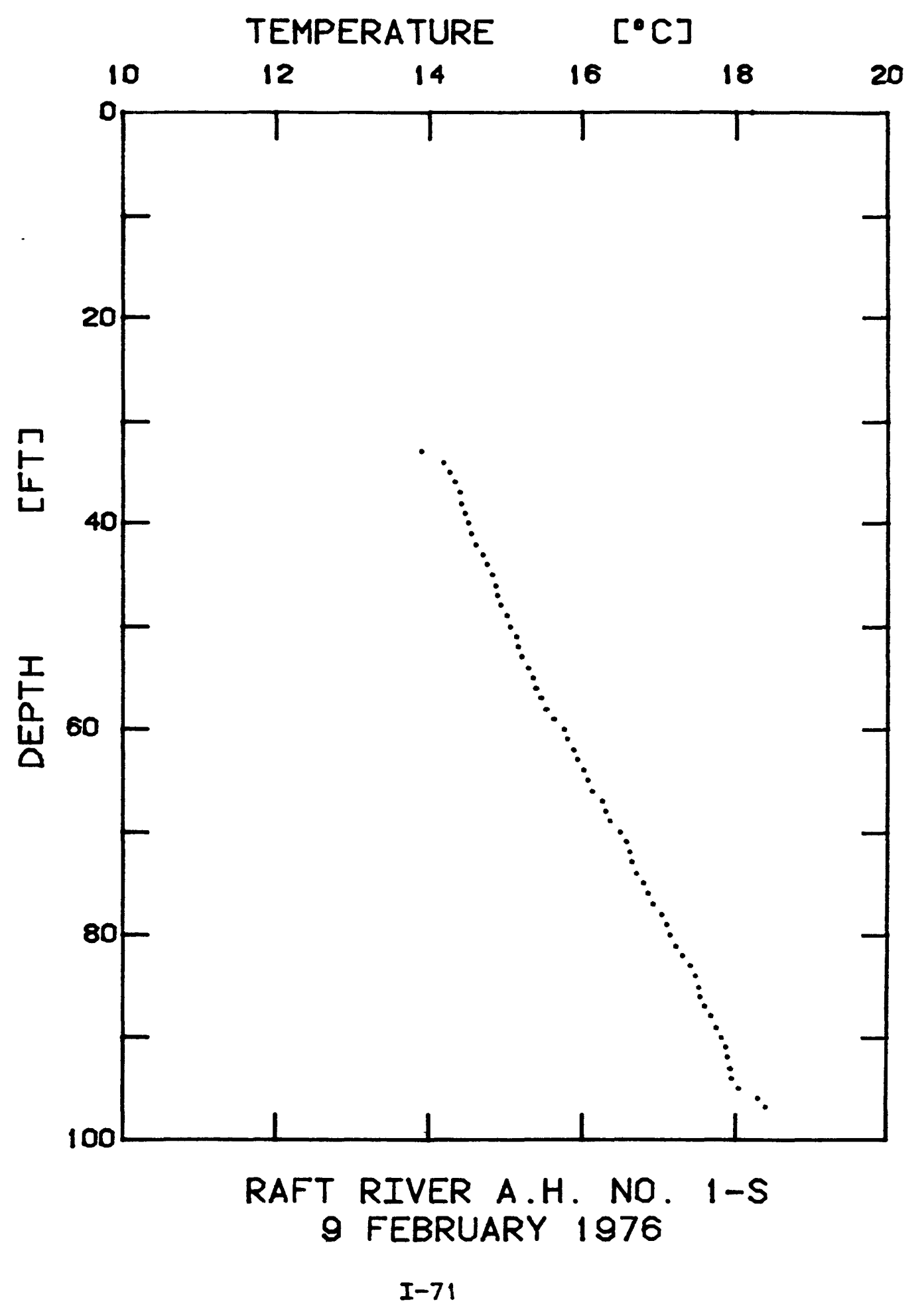




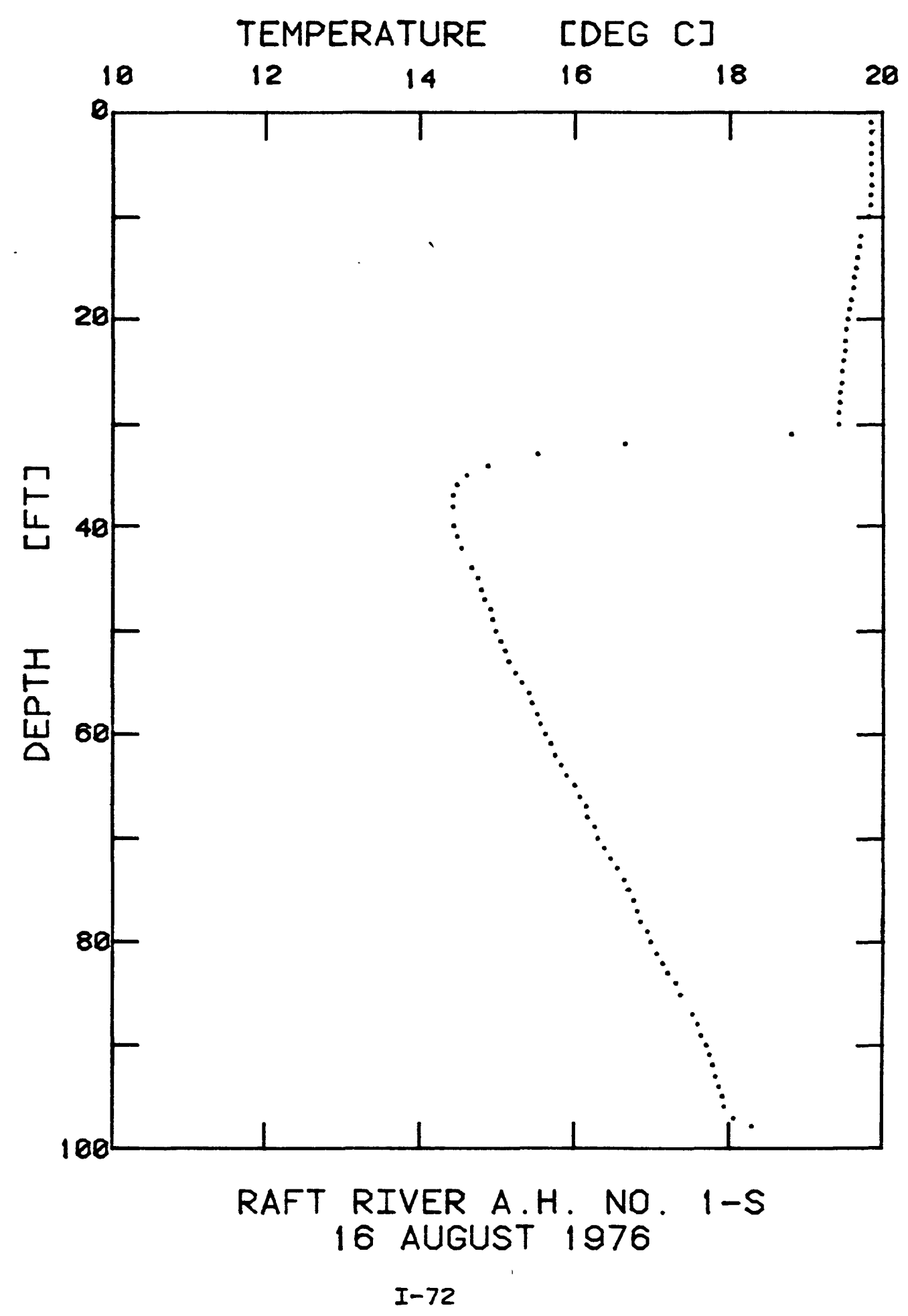




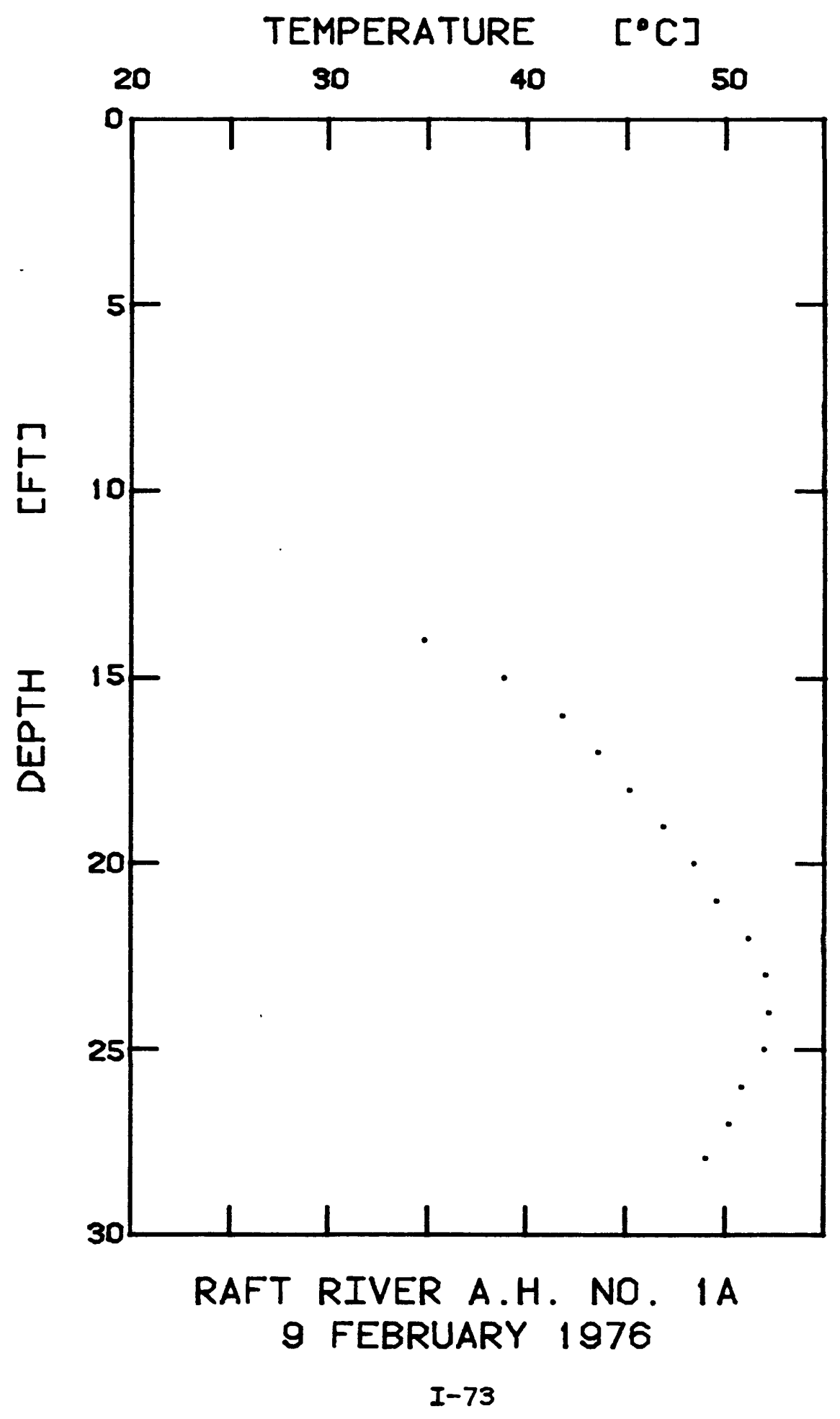




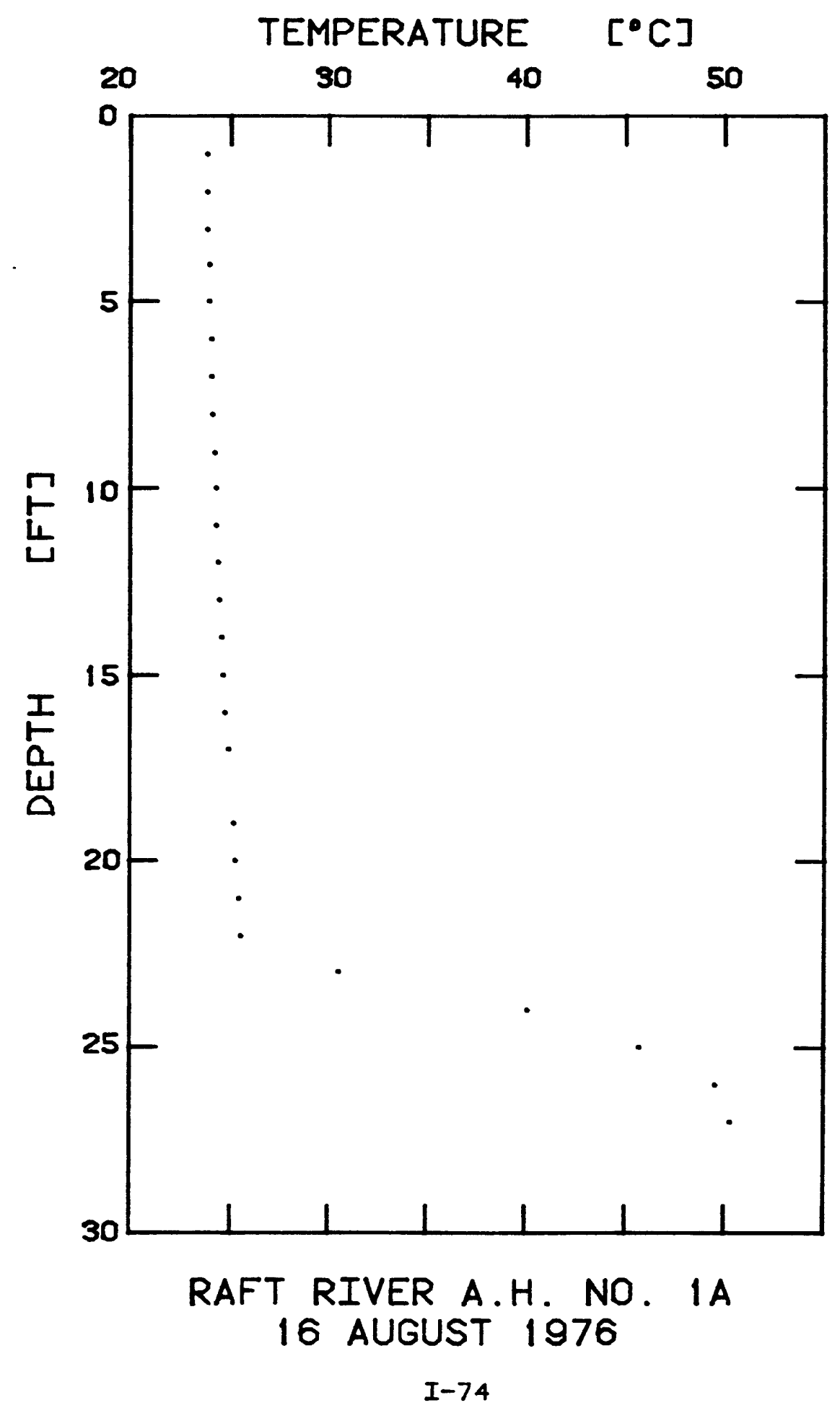




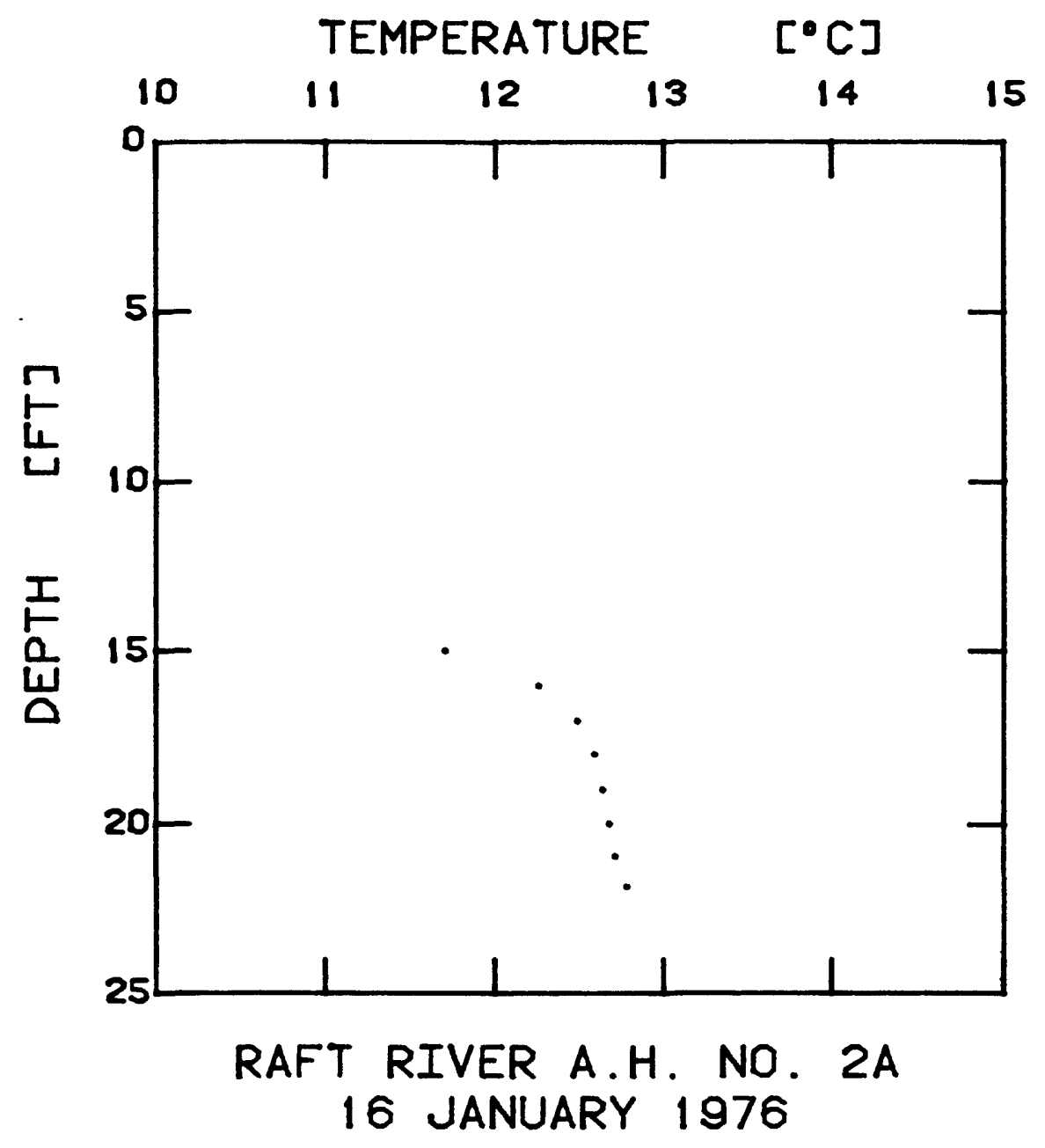

$I-75$ 


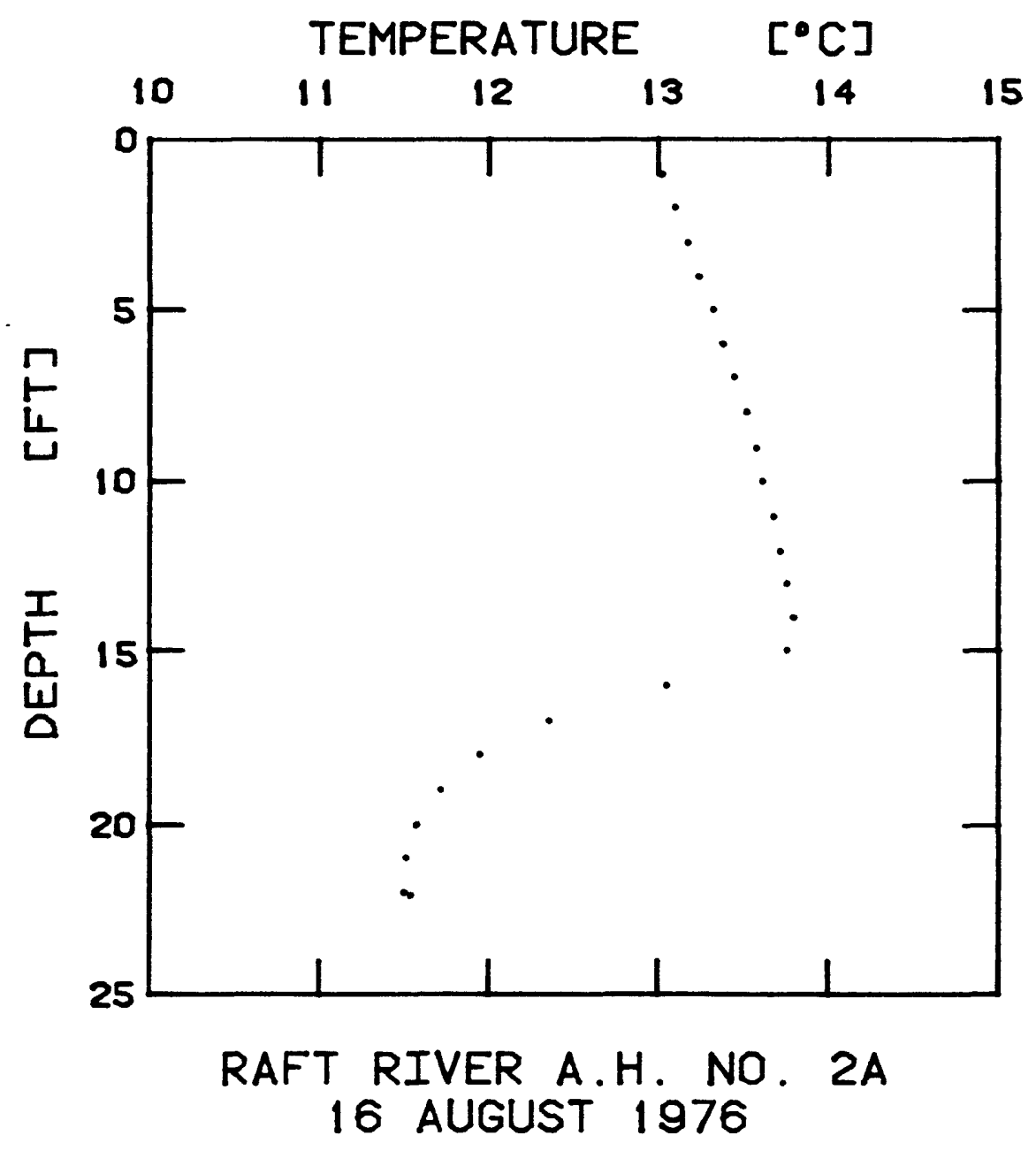




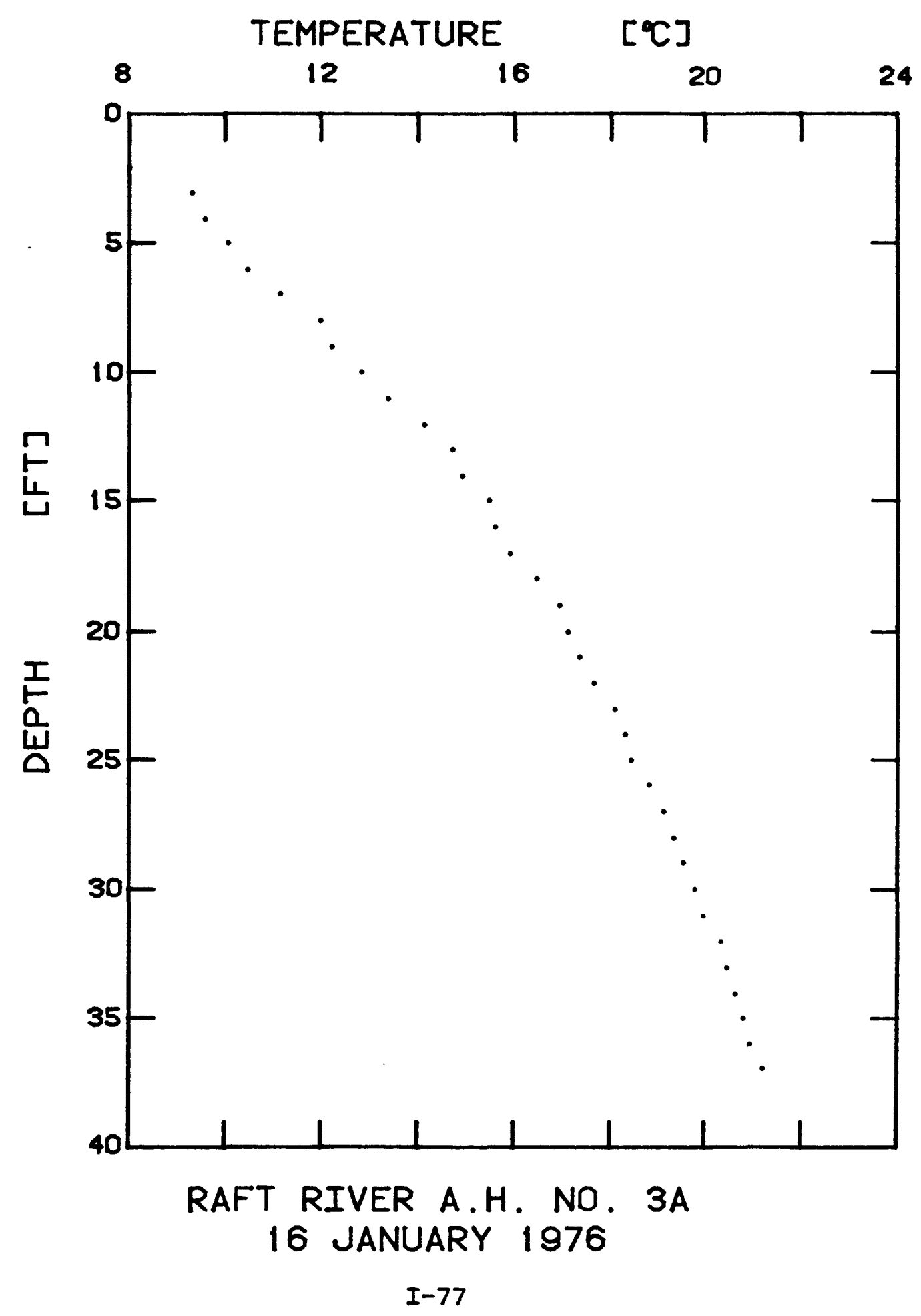




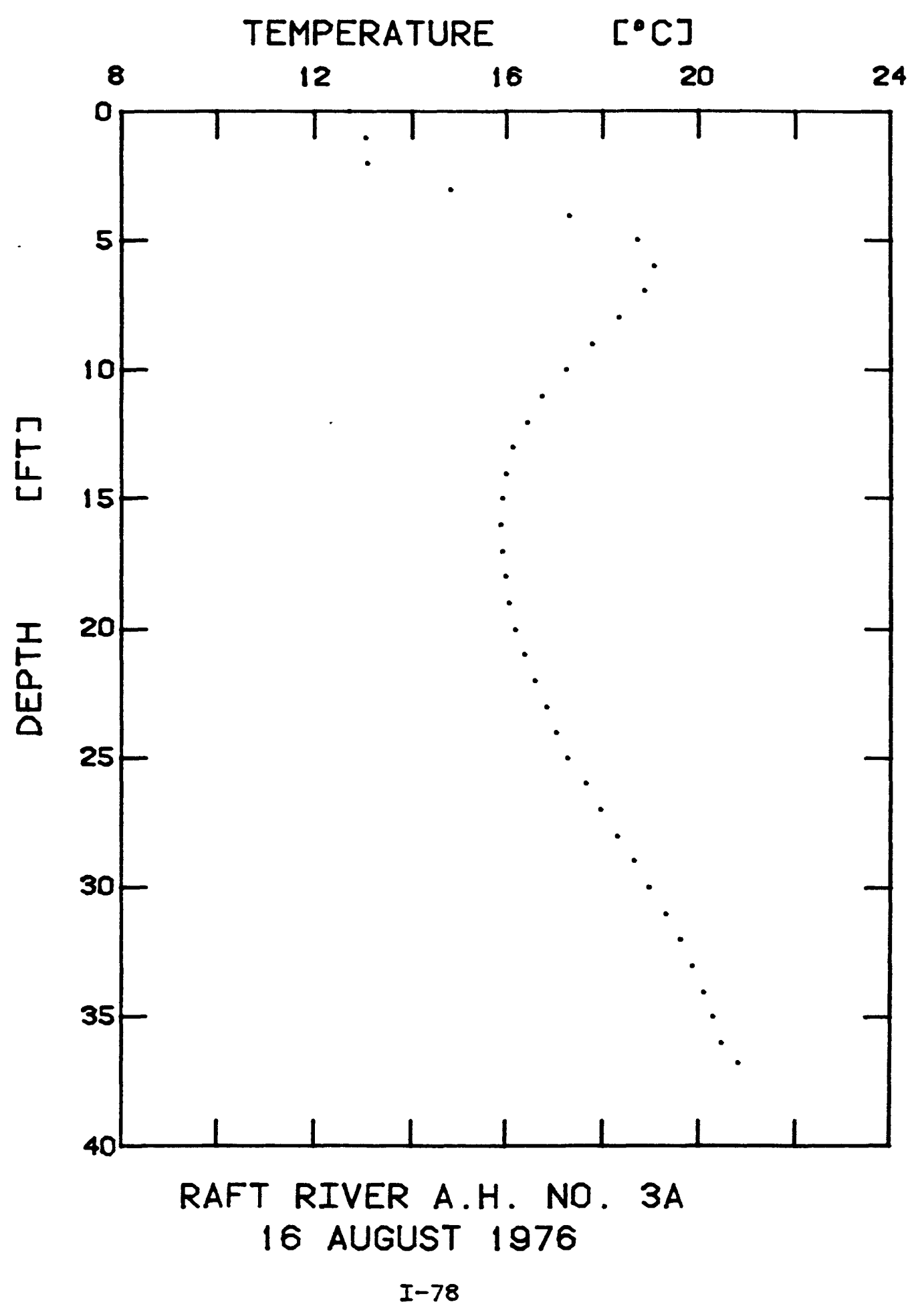




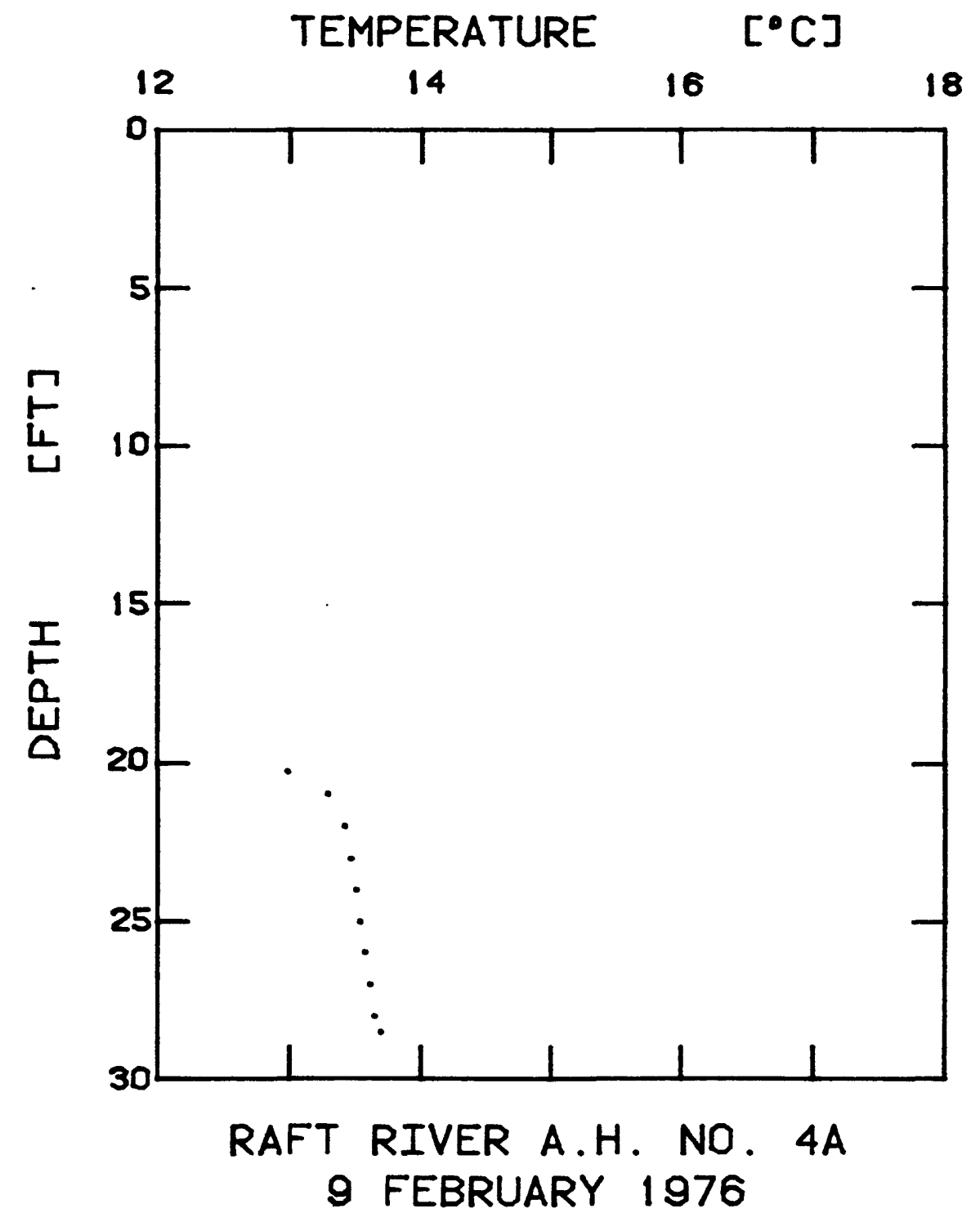




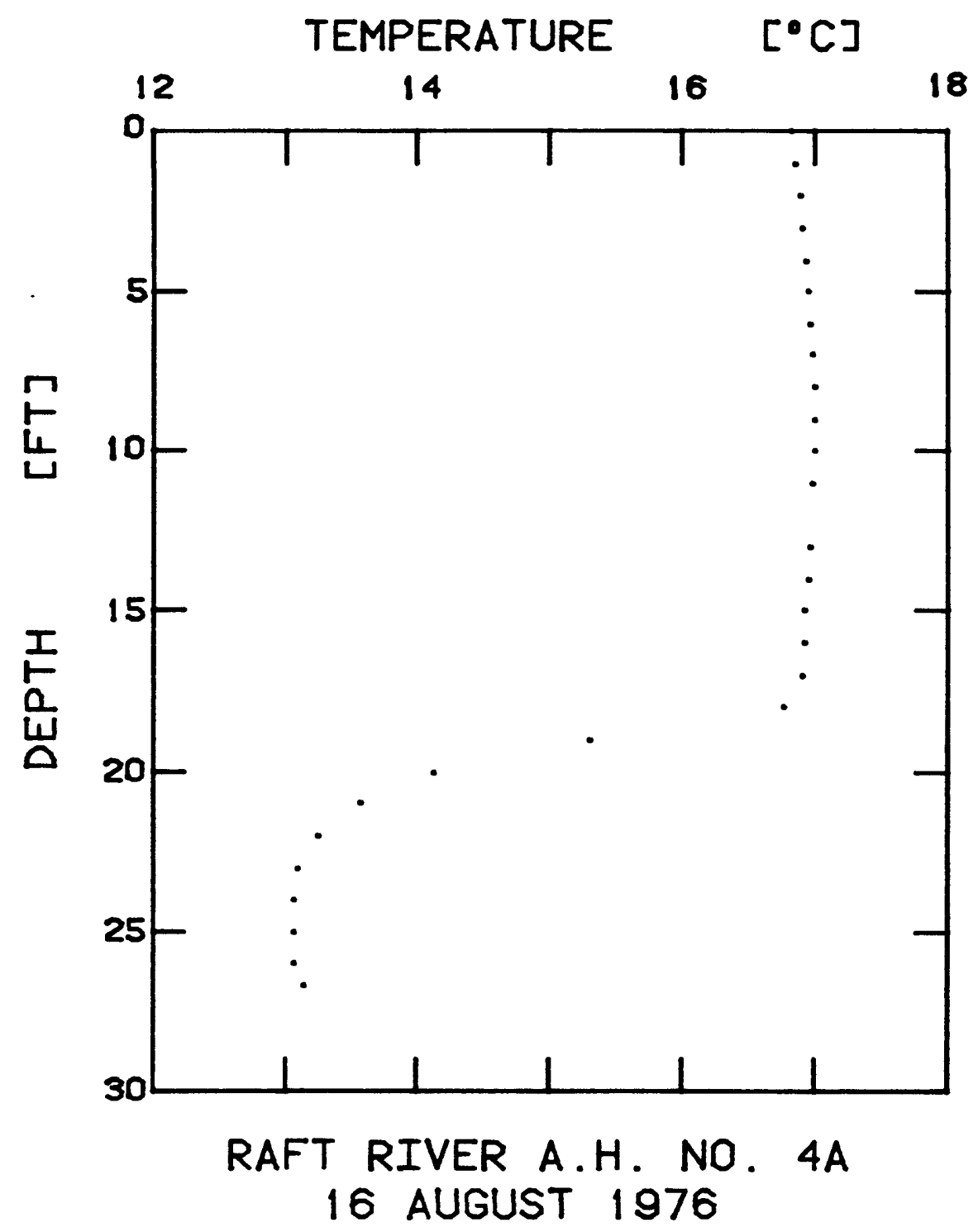




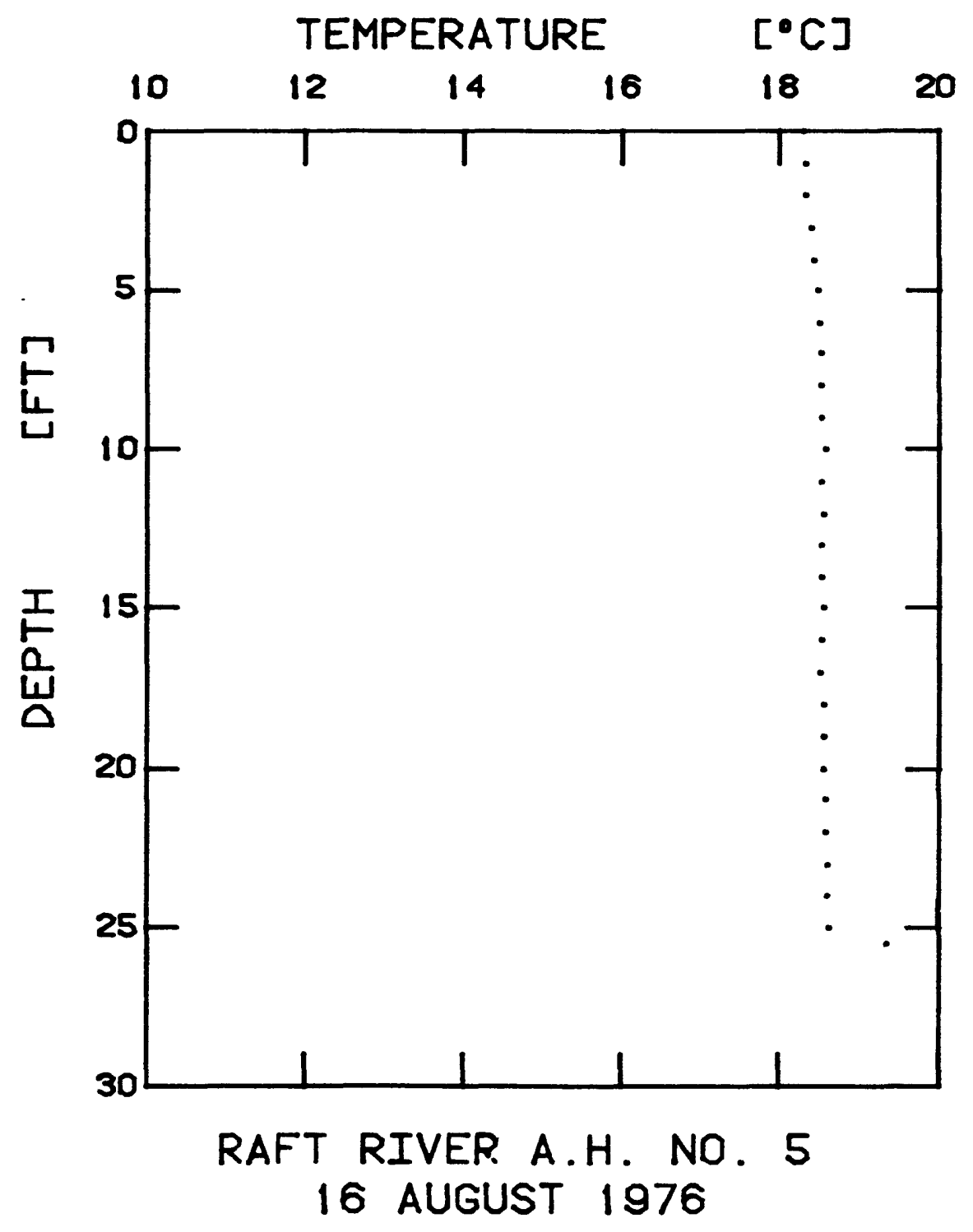

$I-81$ 


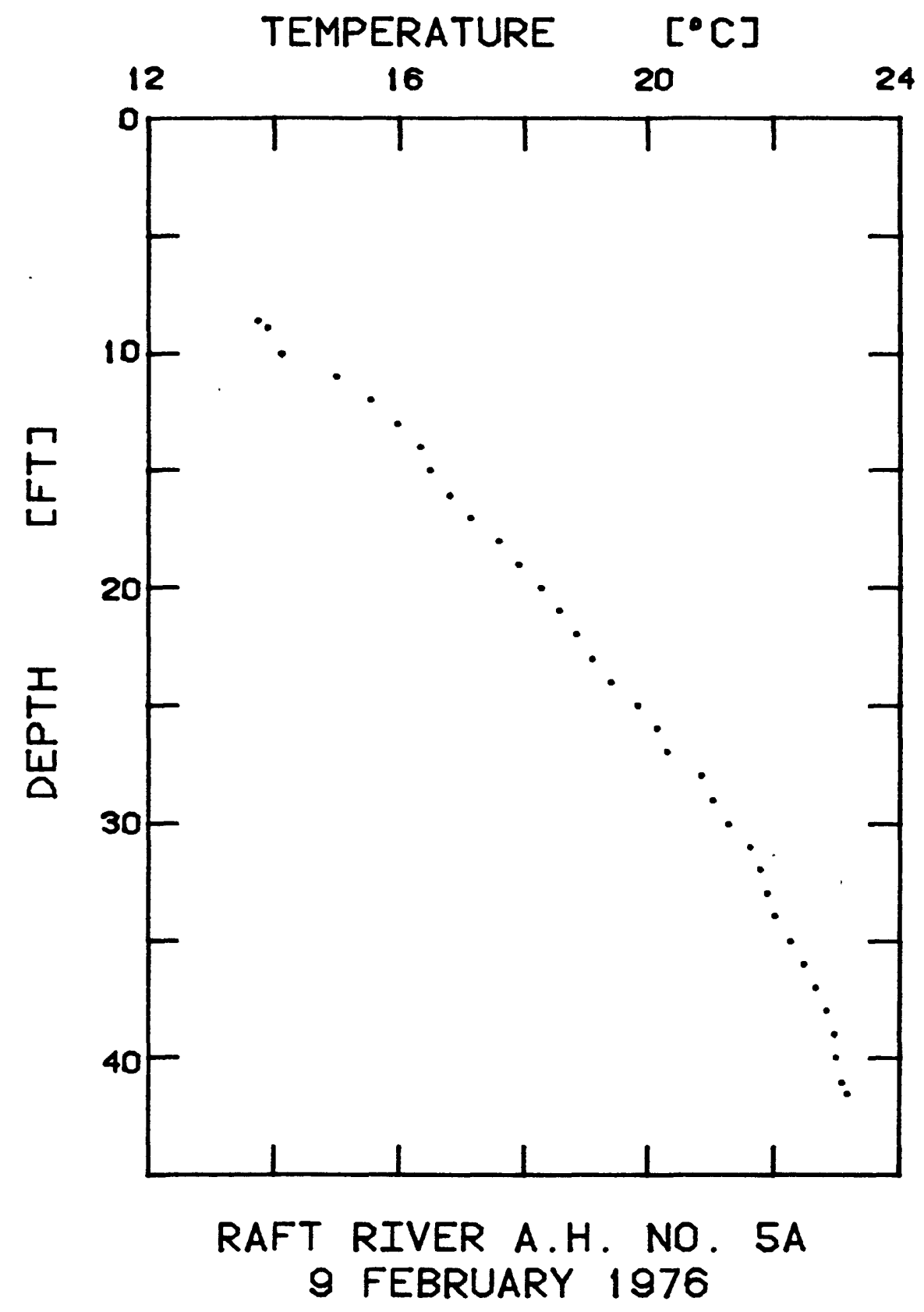

$I-82$ 


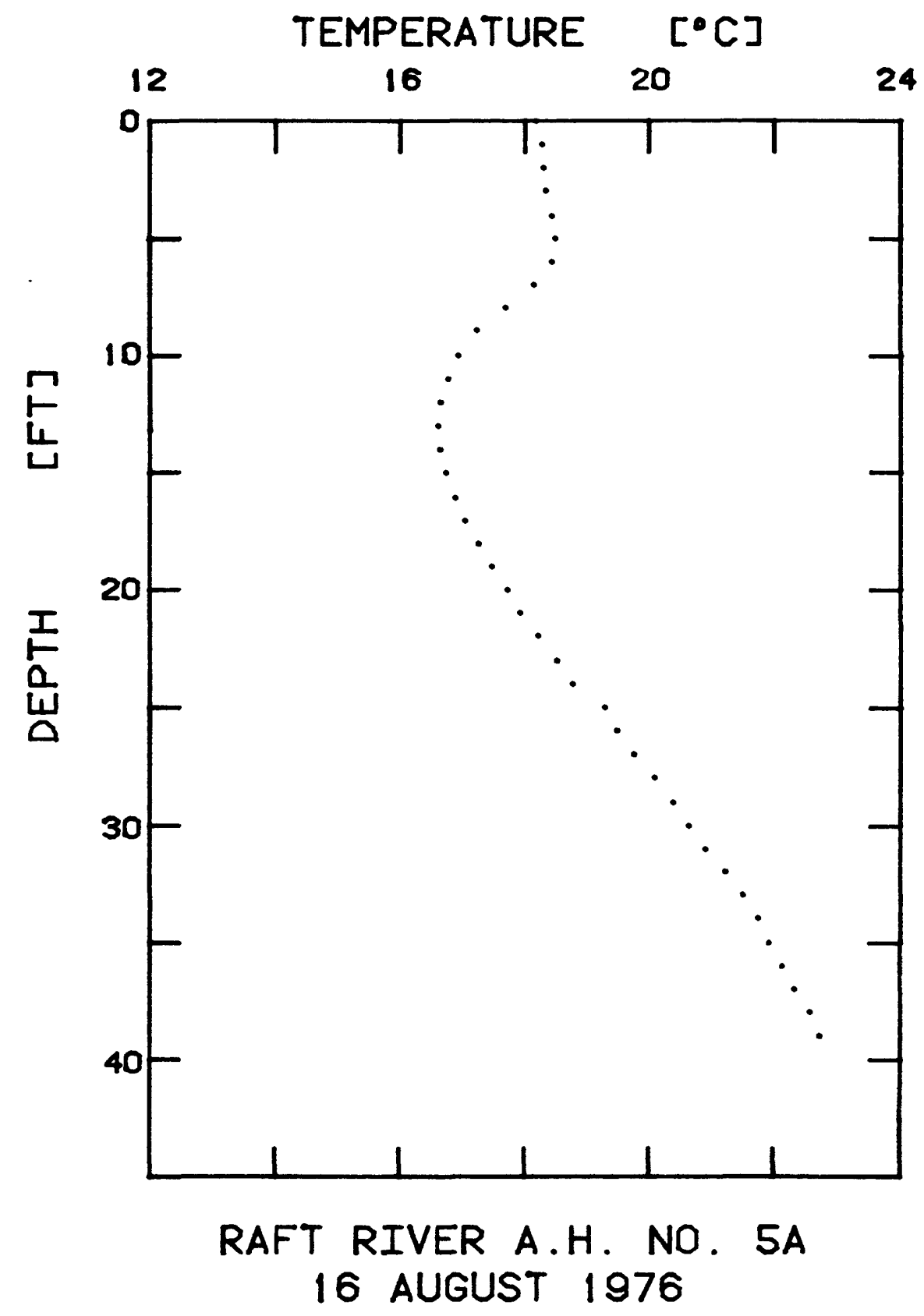

$I-83$ 


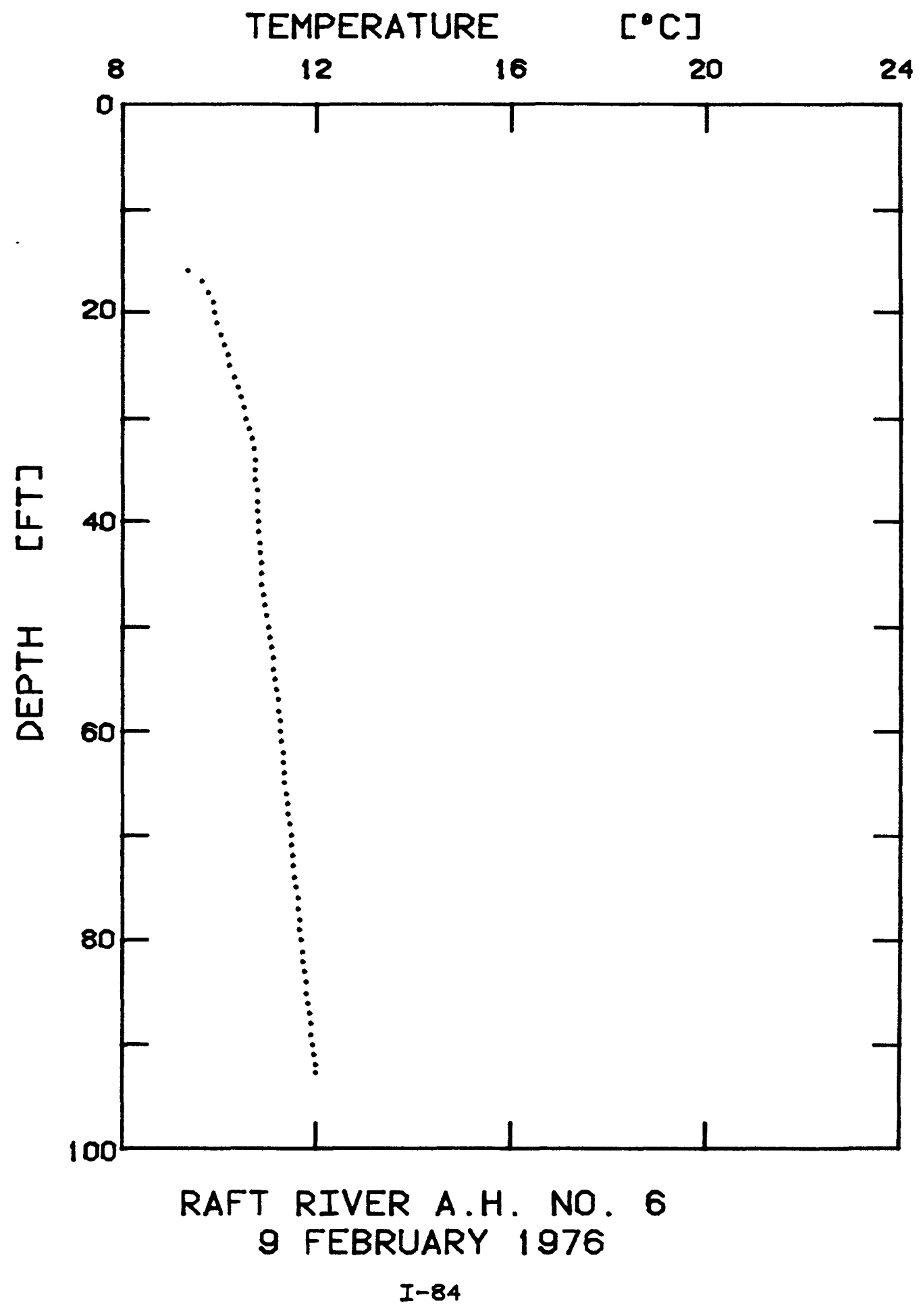




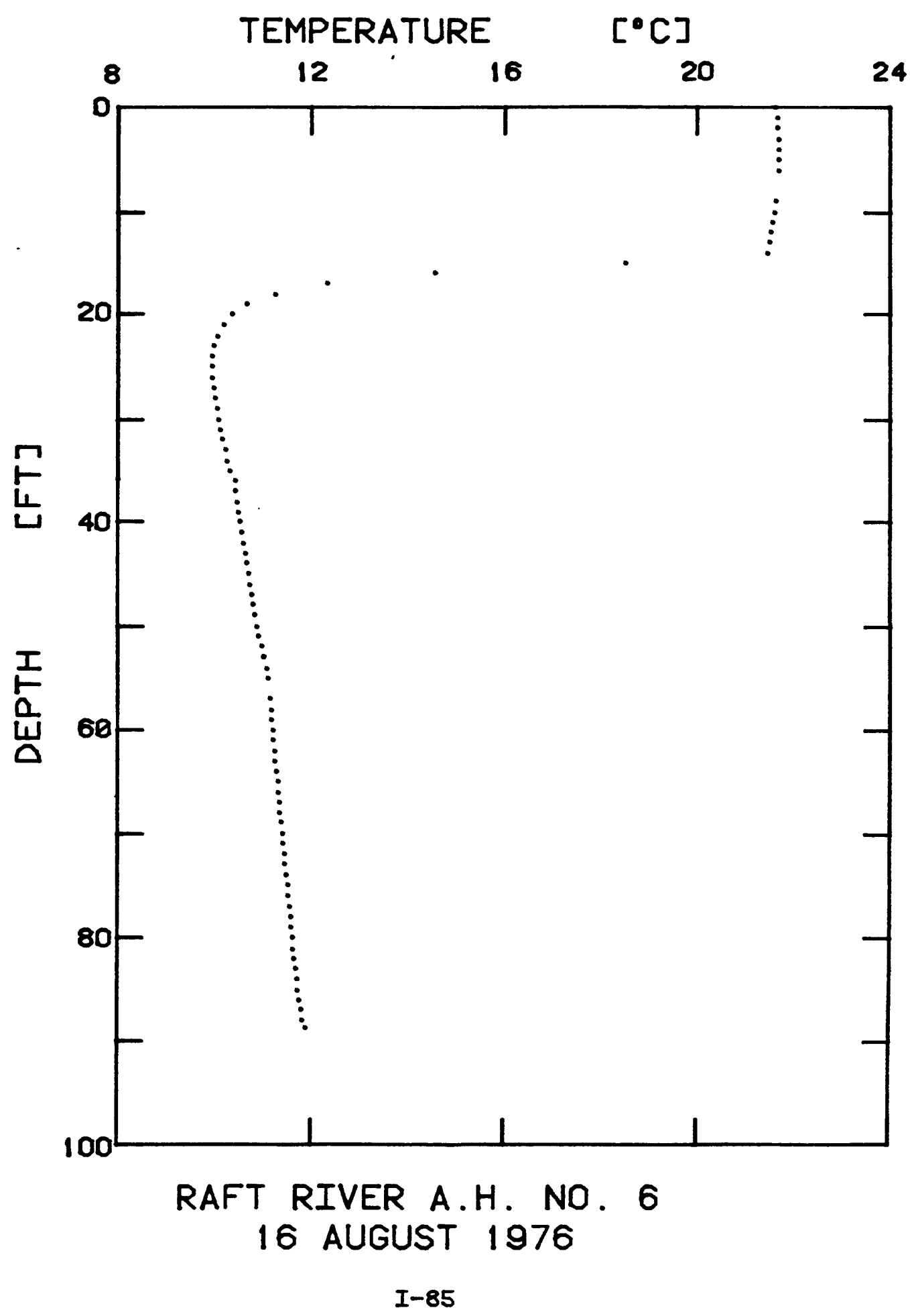




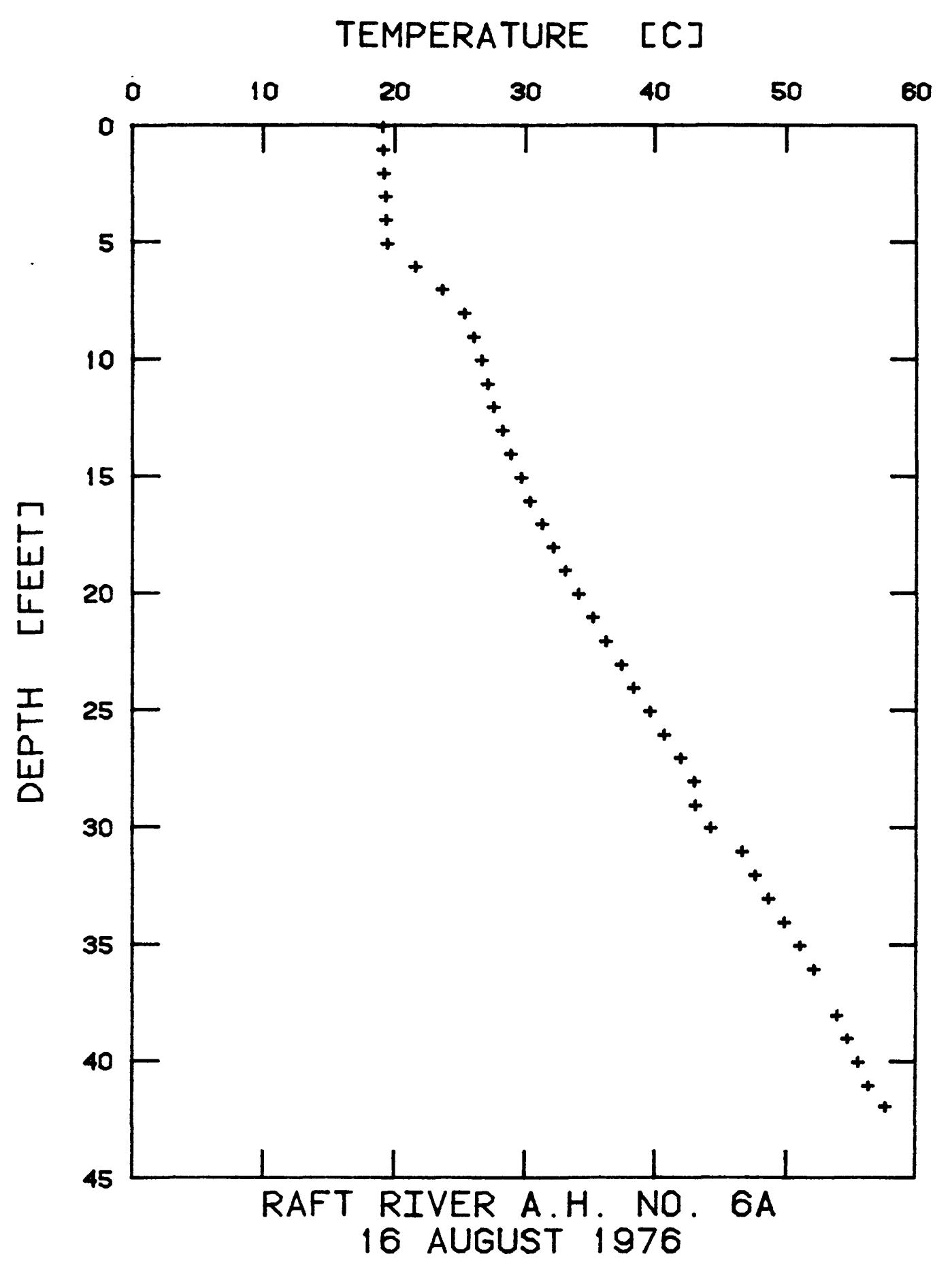

I-86 


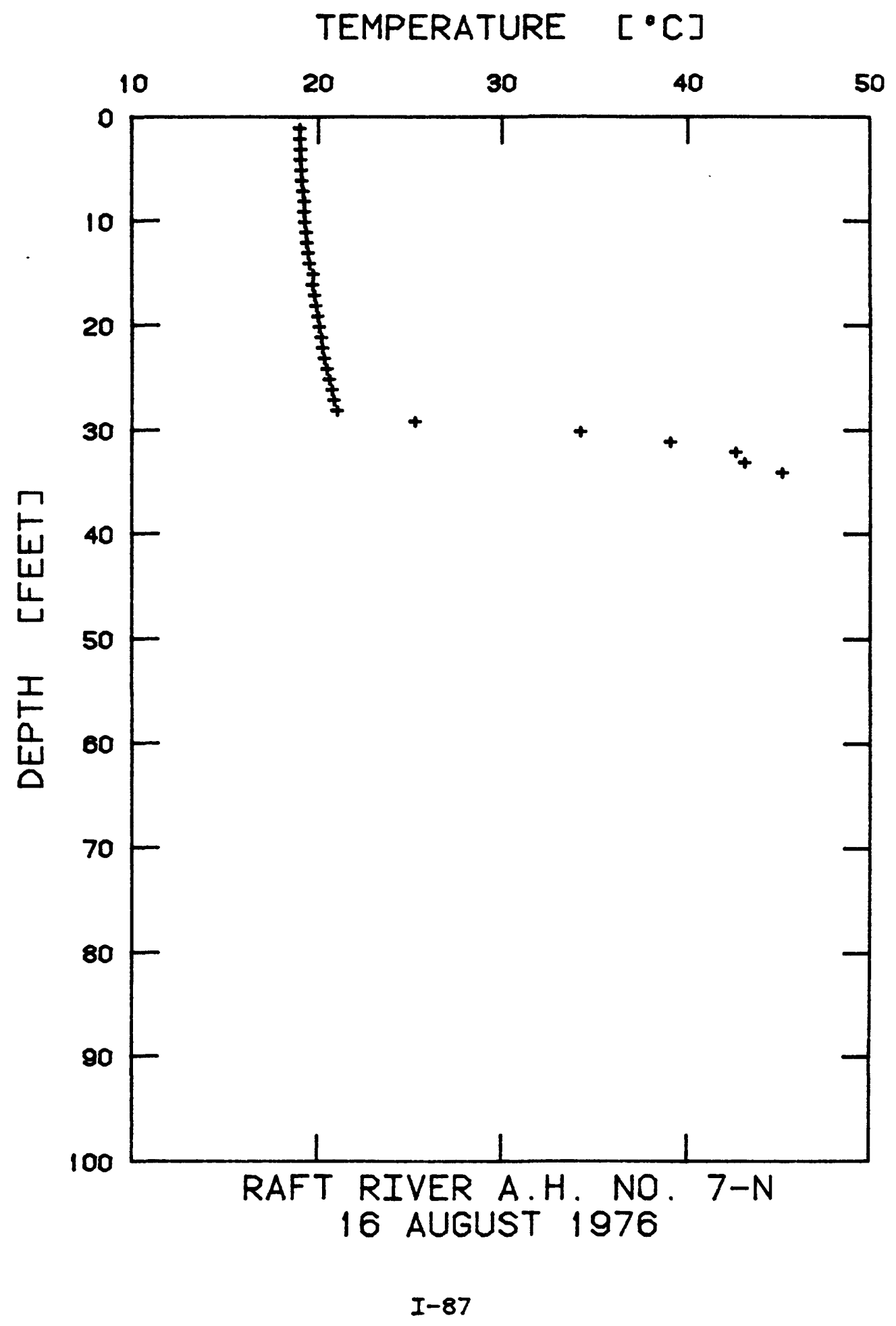




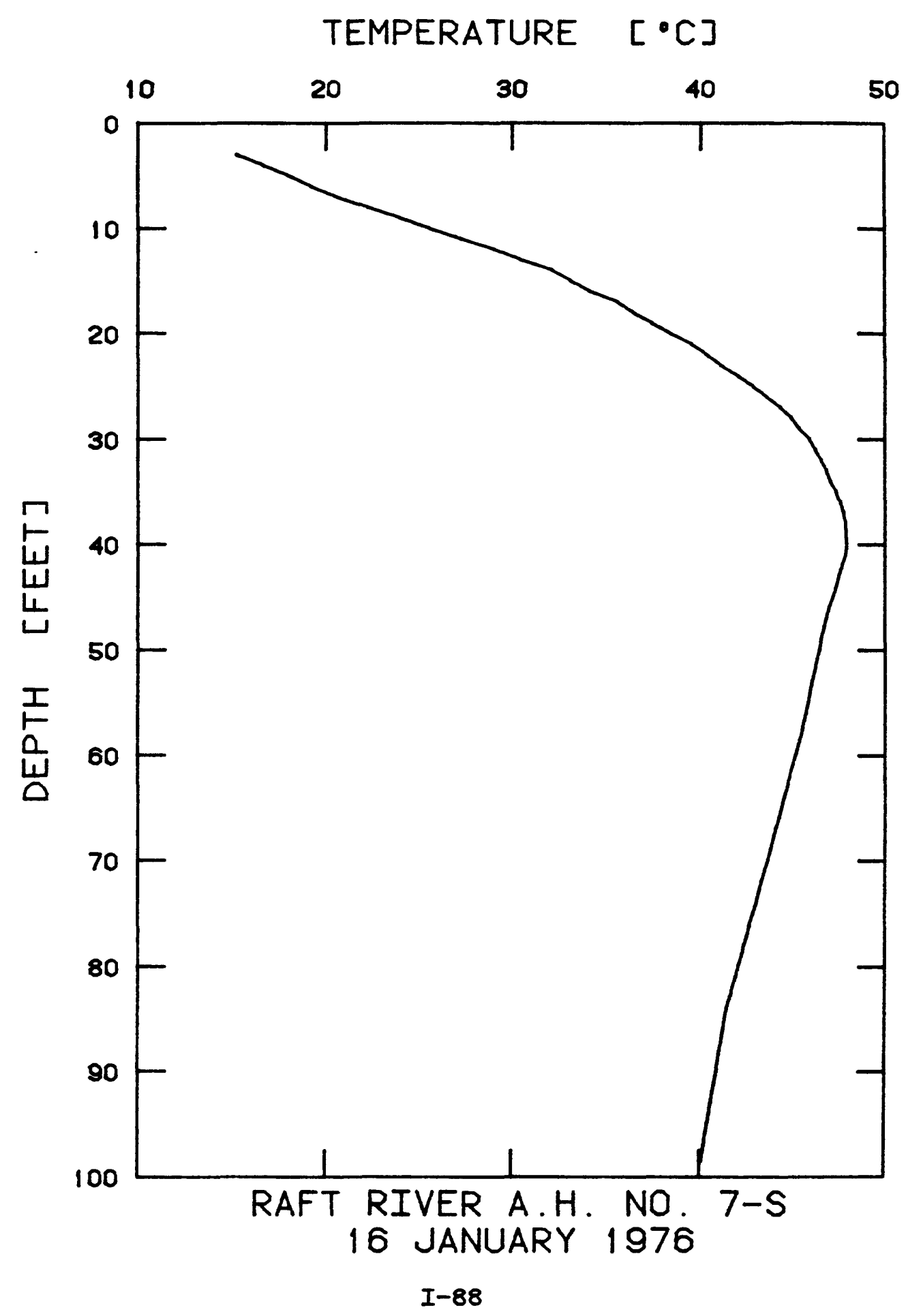




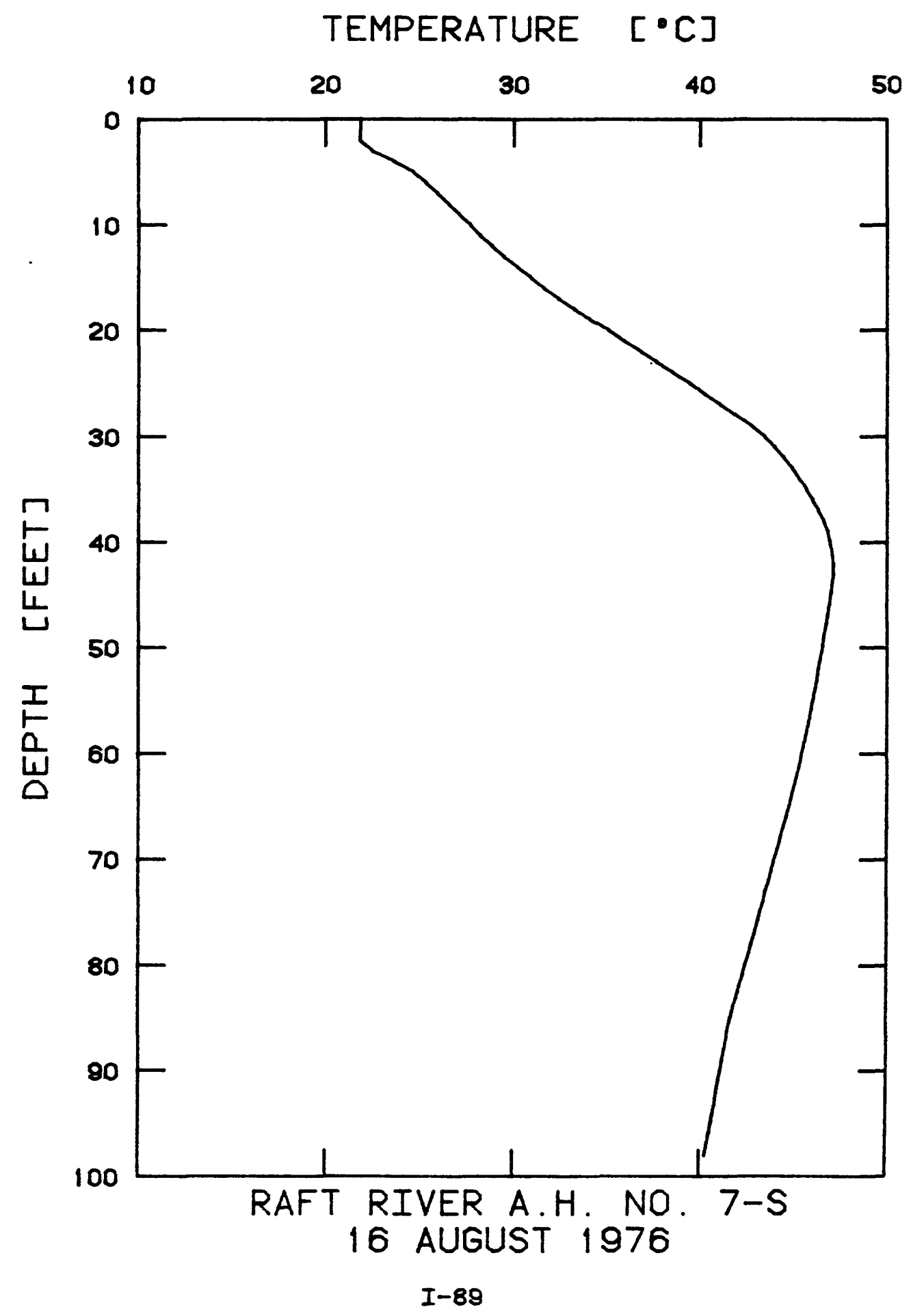




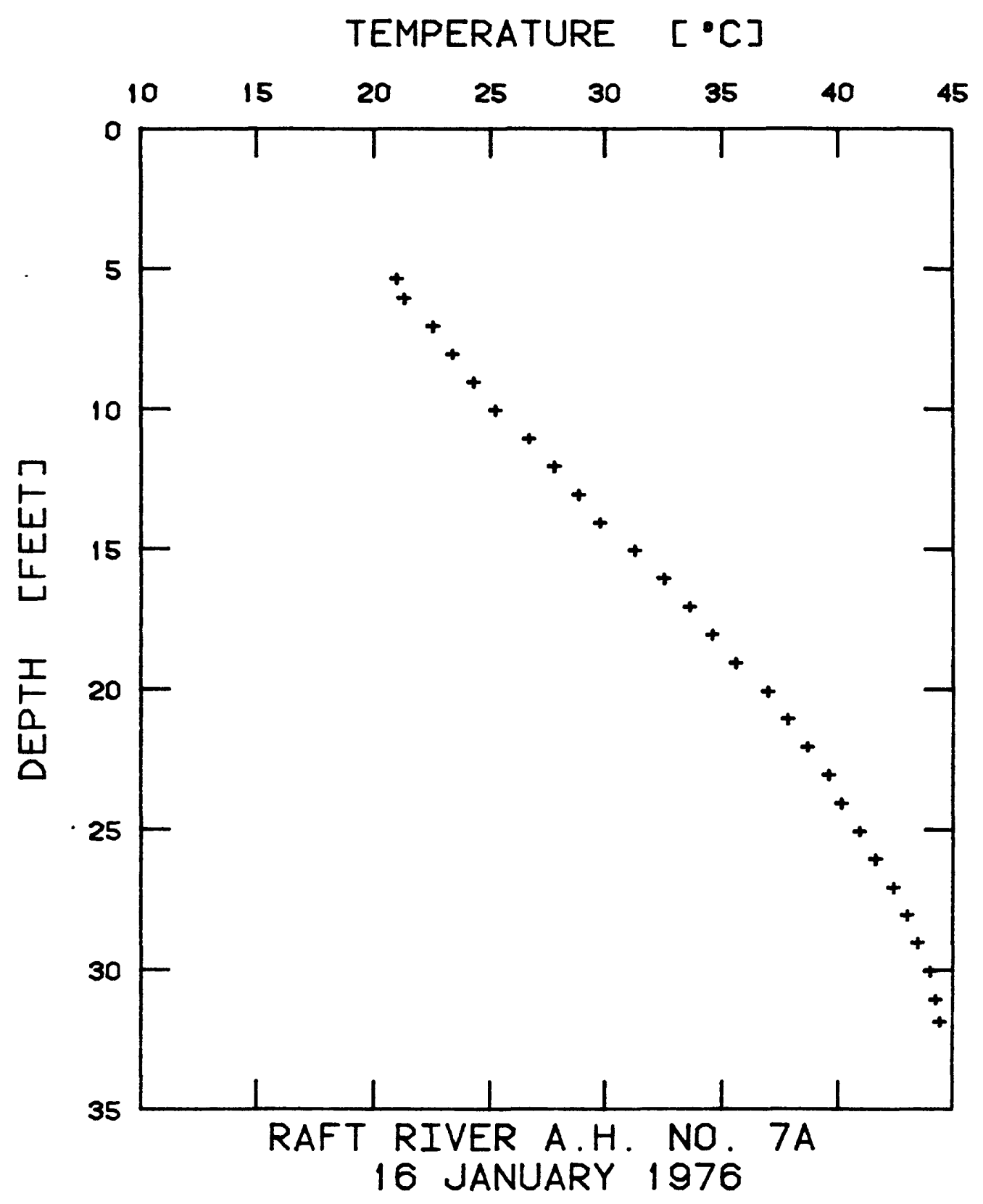

$I-90$ 


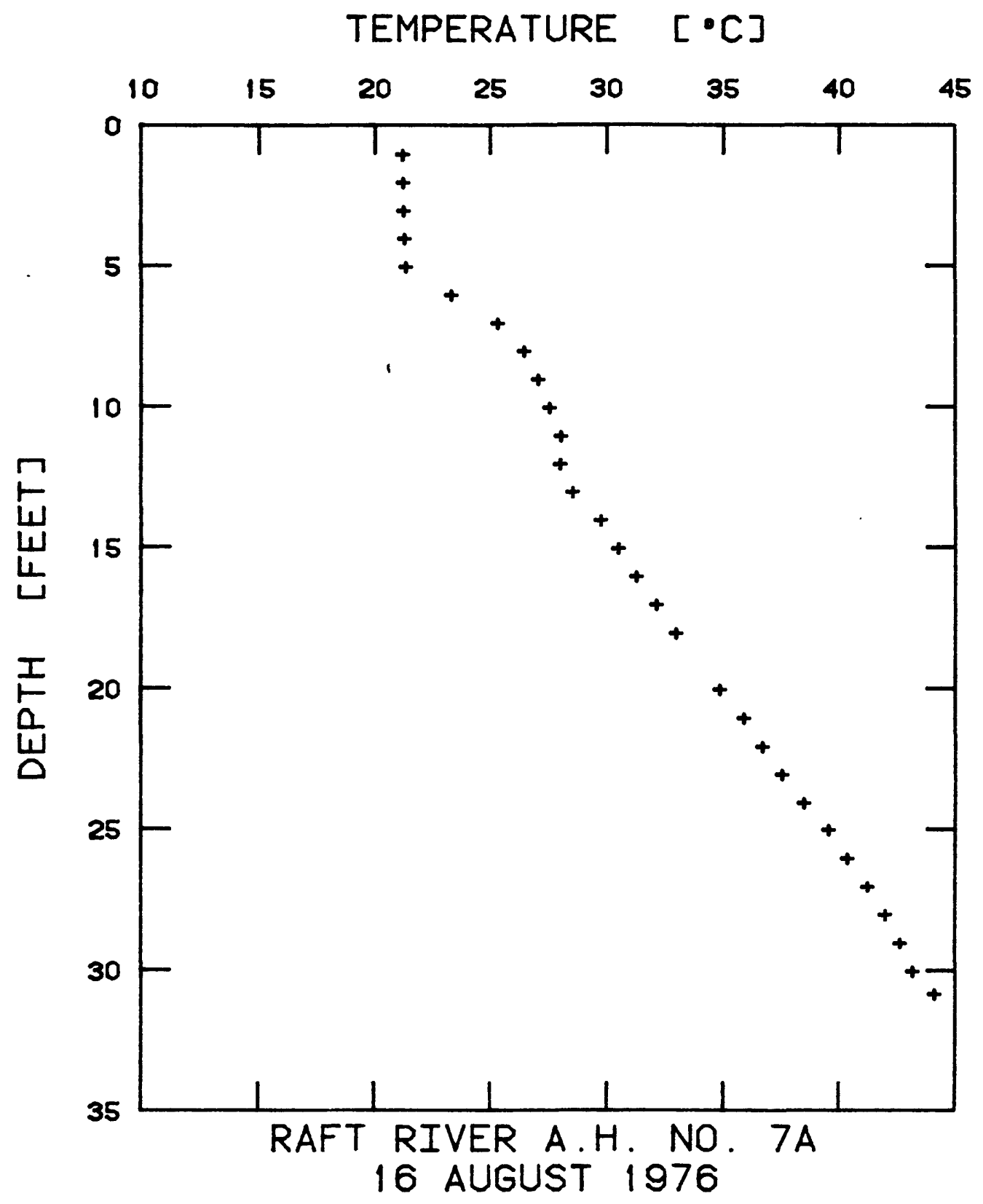

$I-91$ 


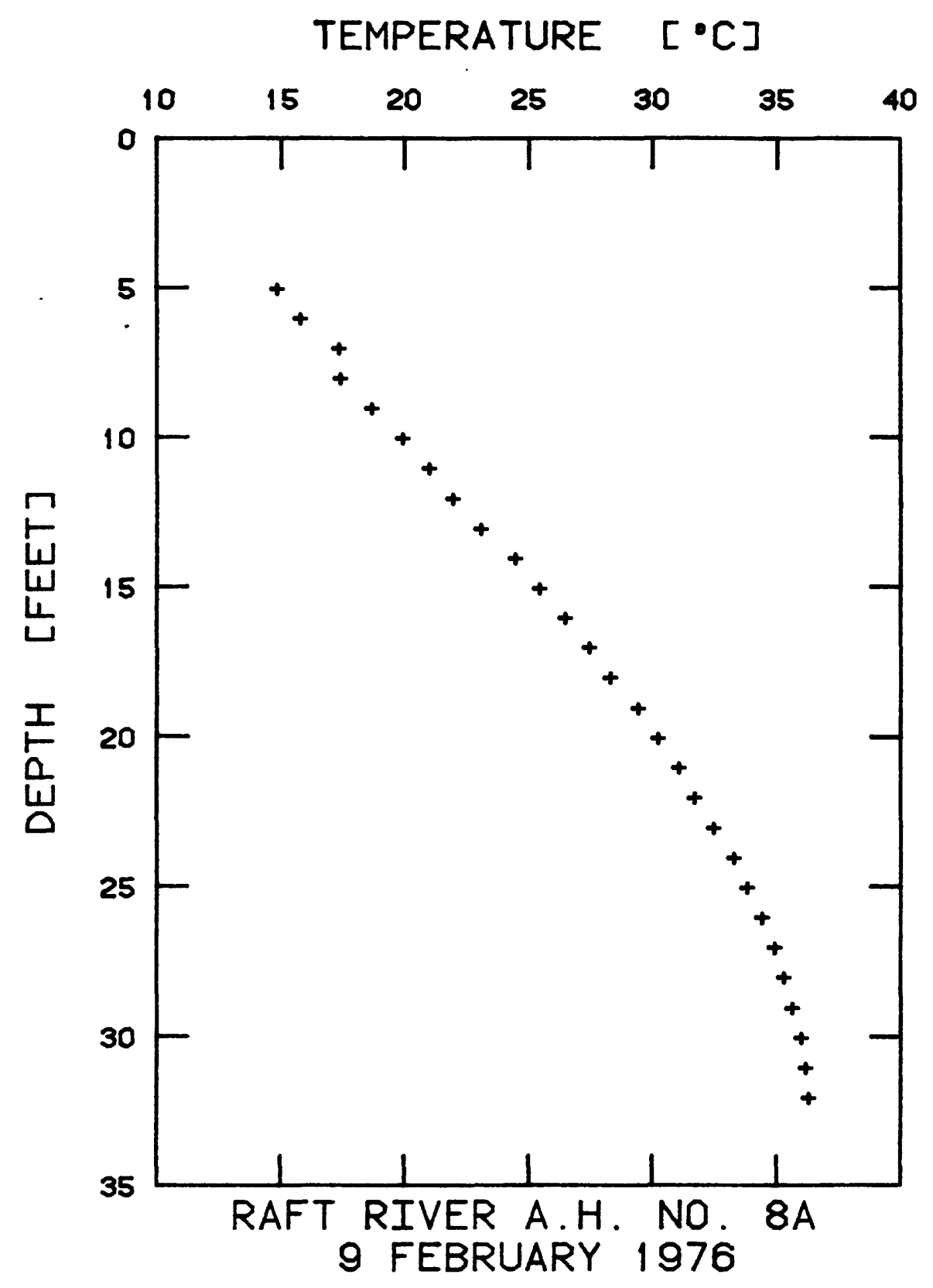

$I-92$ 


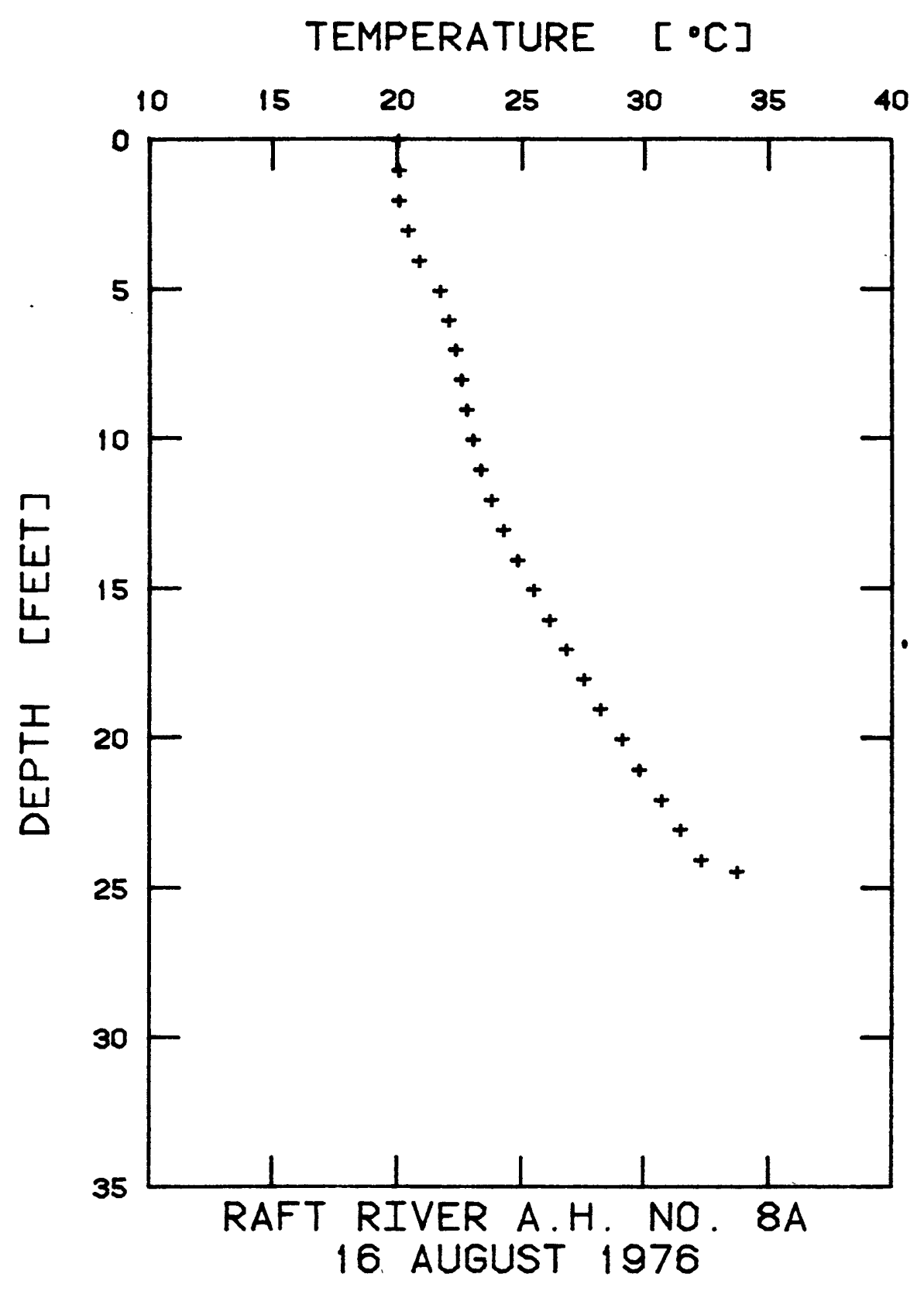

$I-93$ 


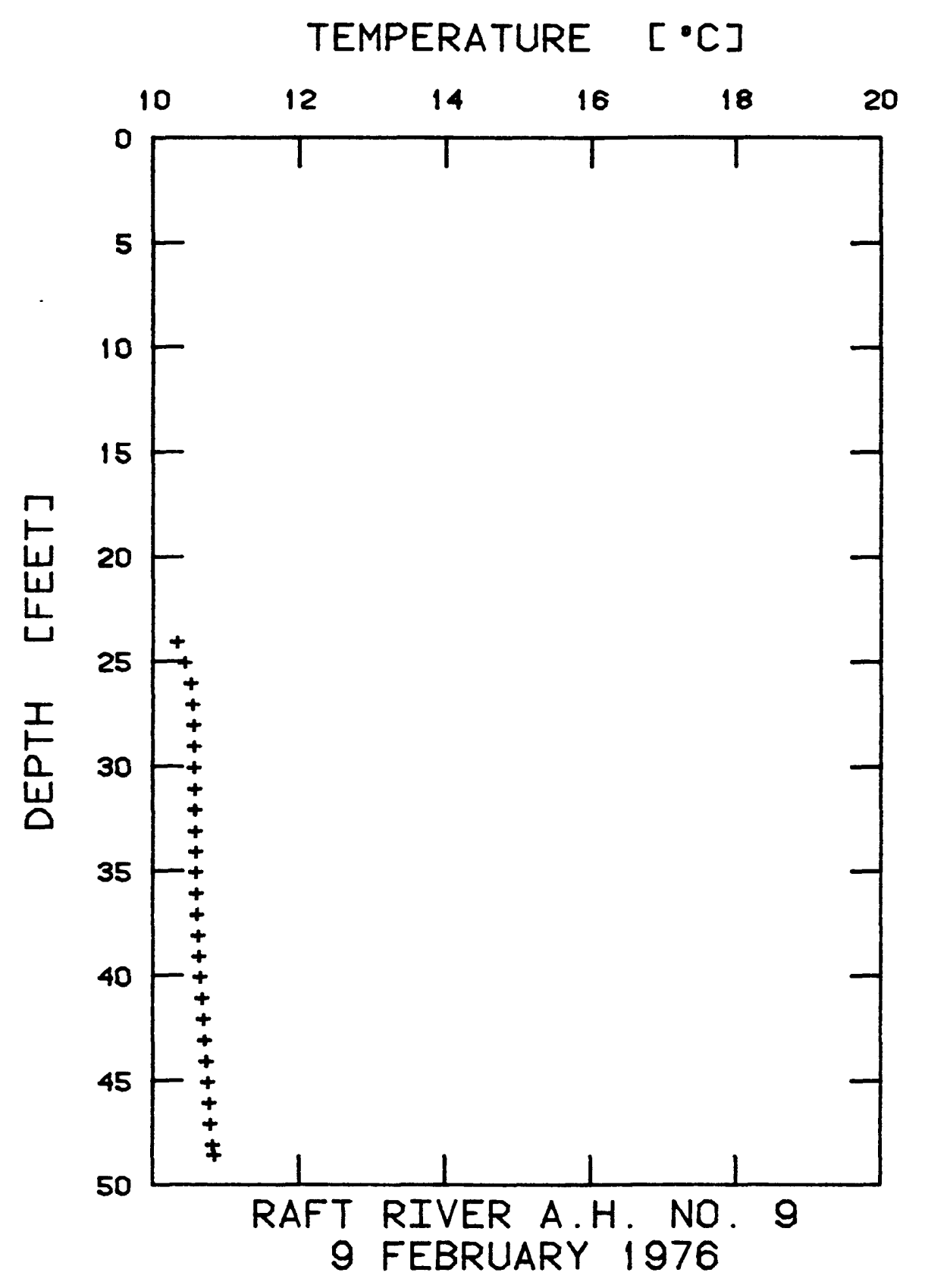

$I-94$ 


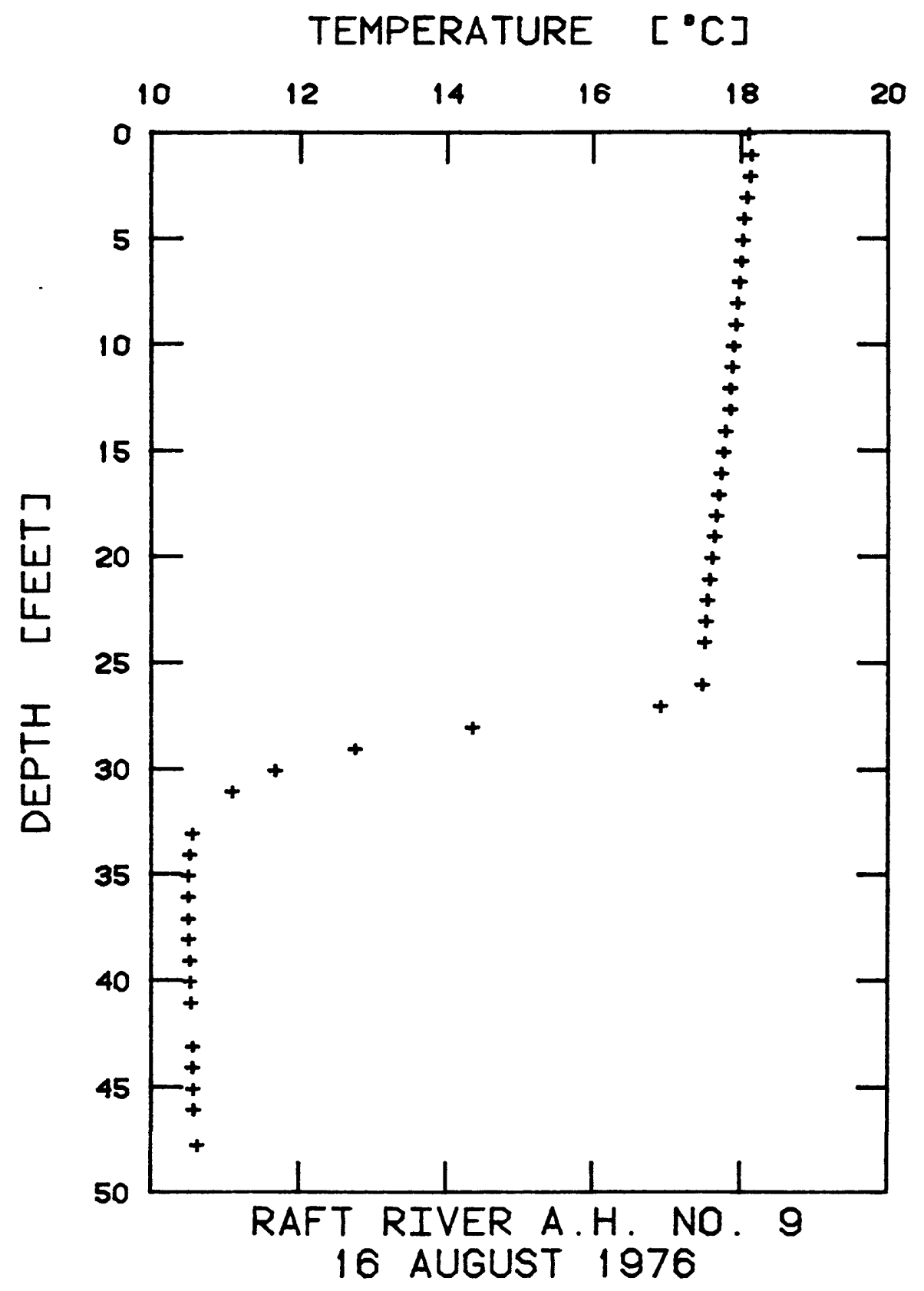

$I-85$ 


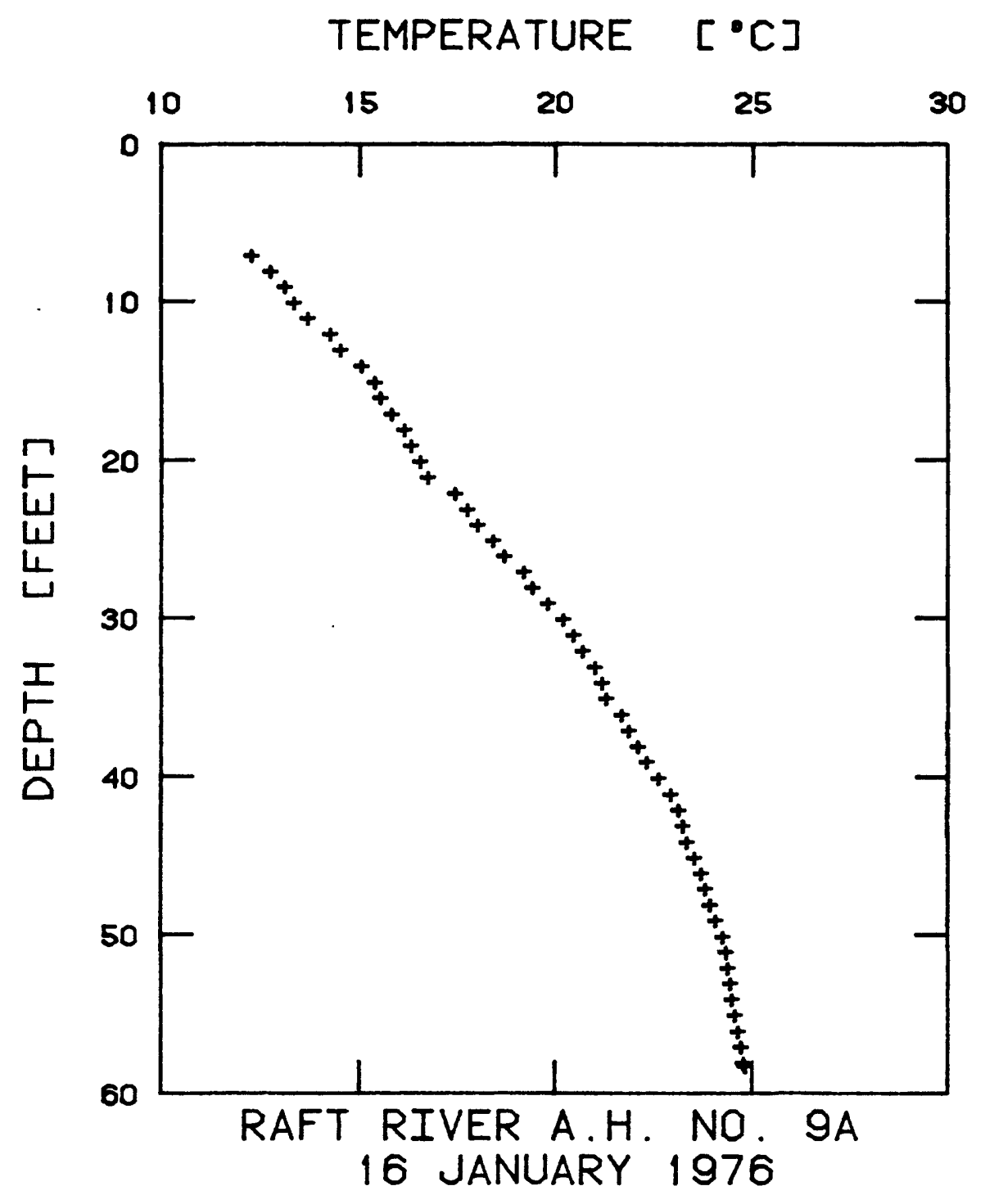

$I-86$ 


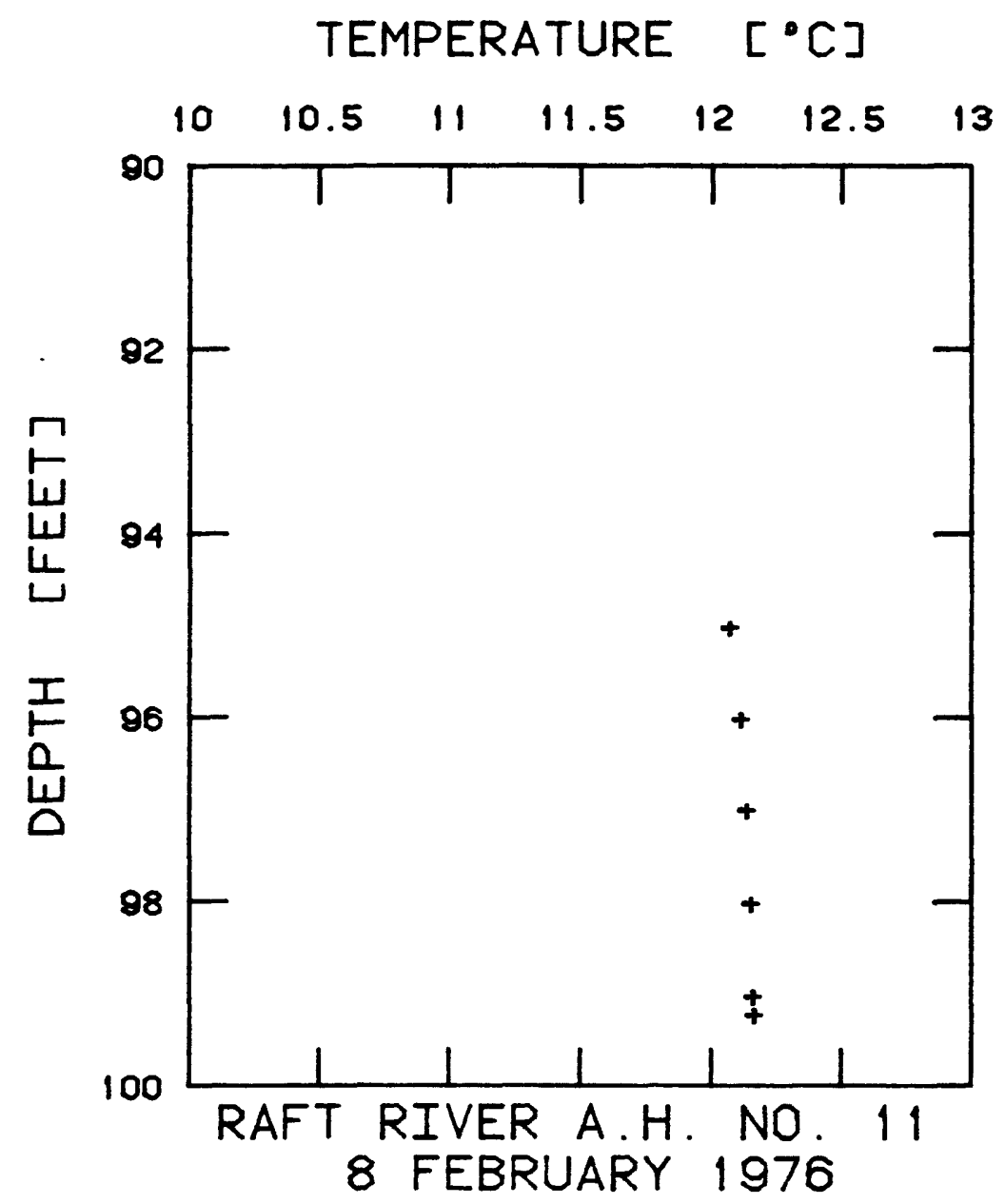




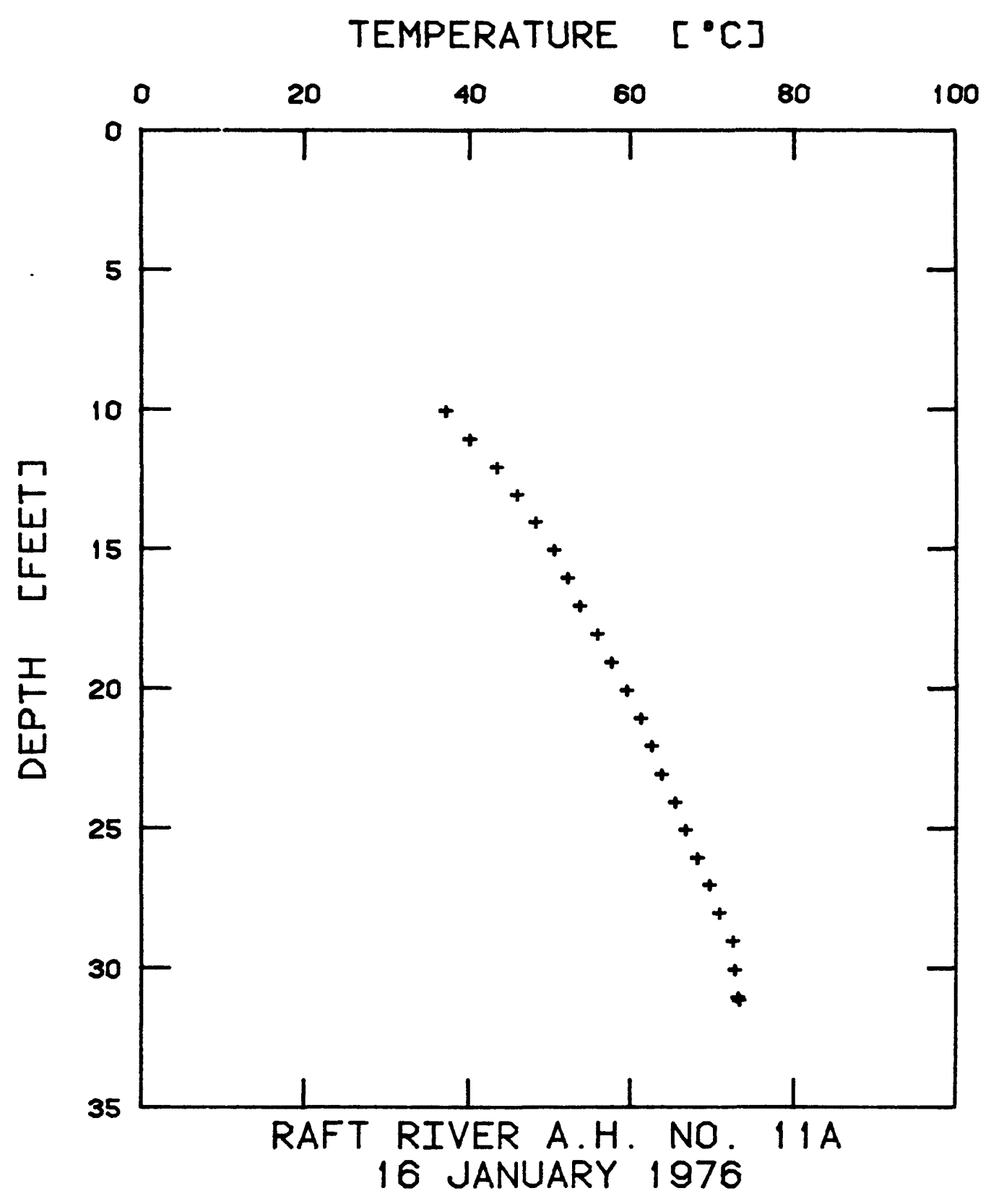

$I-88$ 


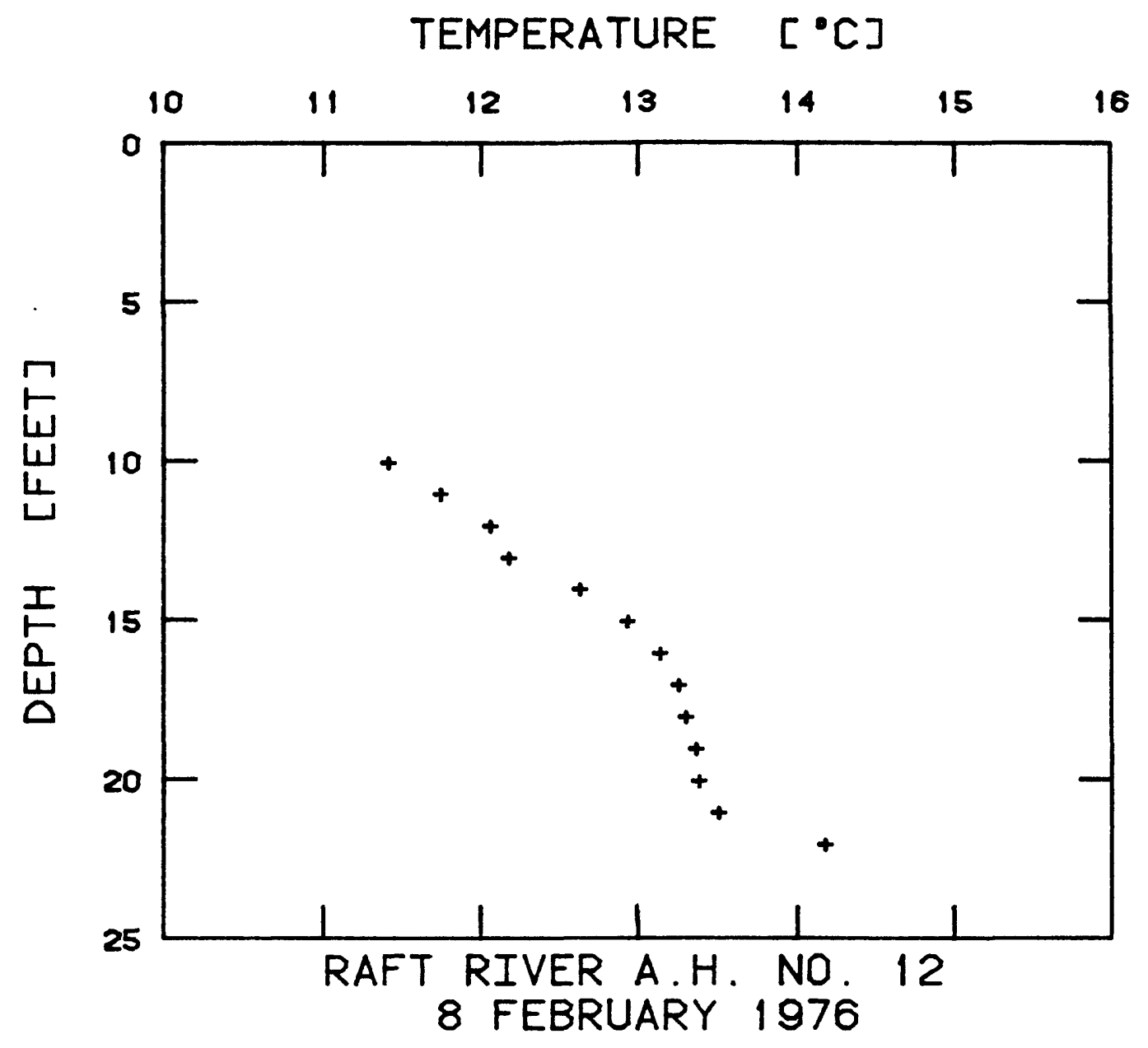

I-99 


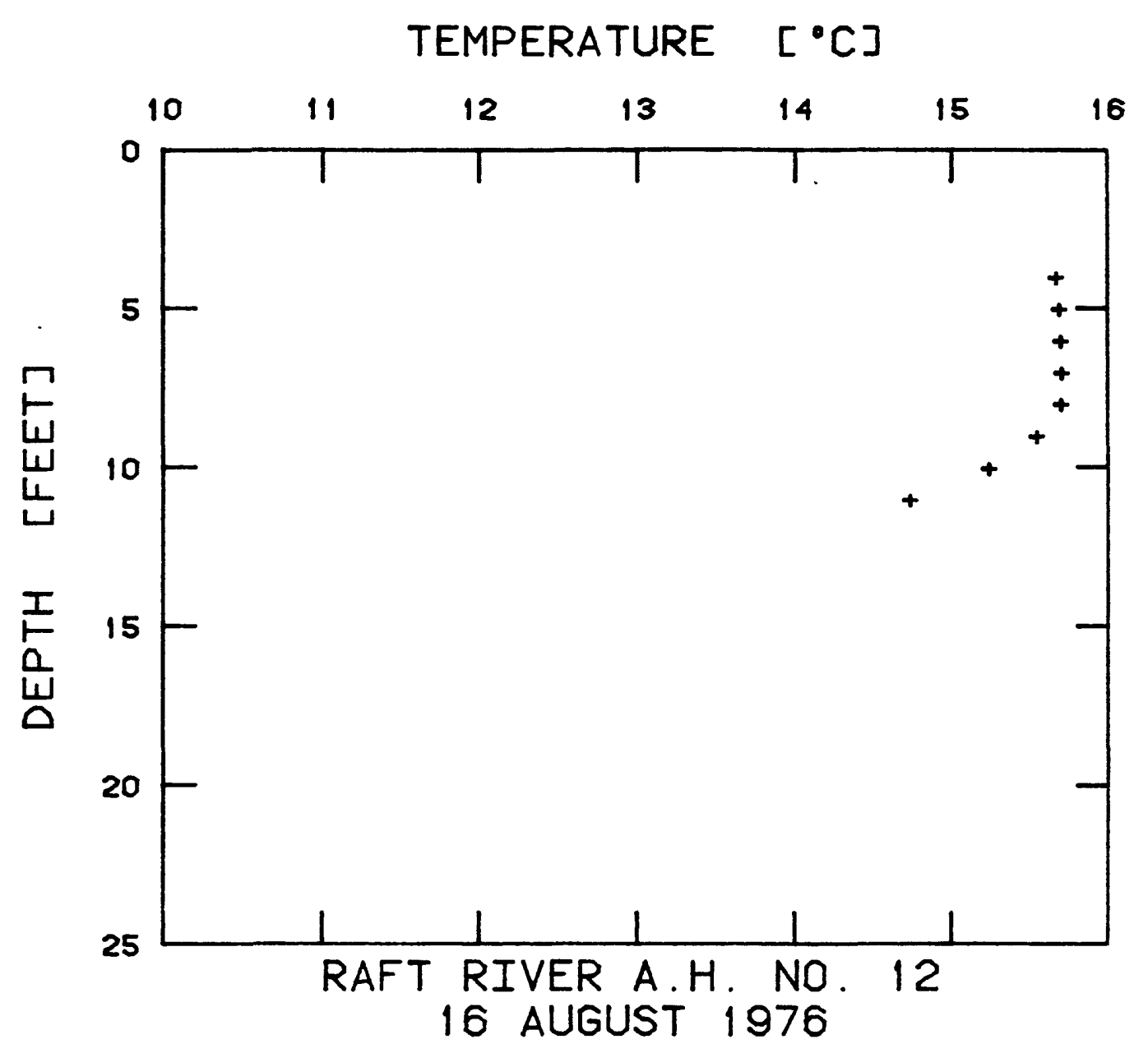




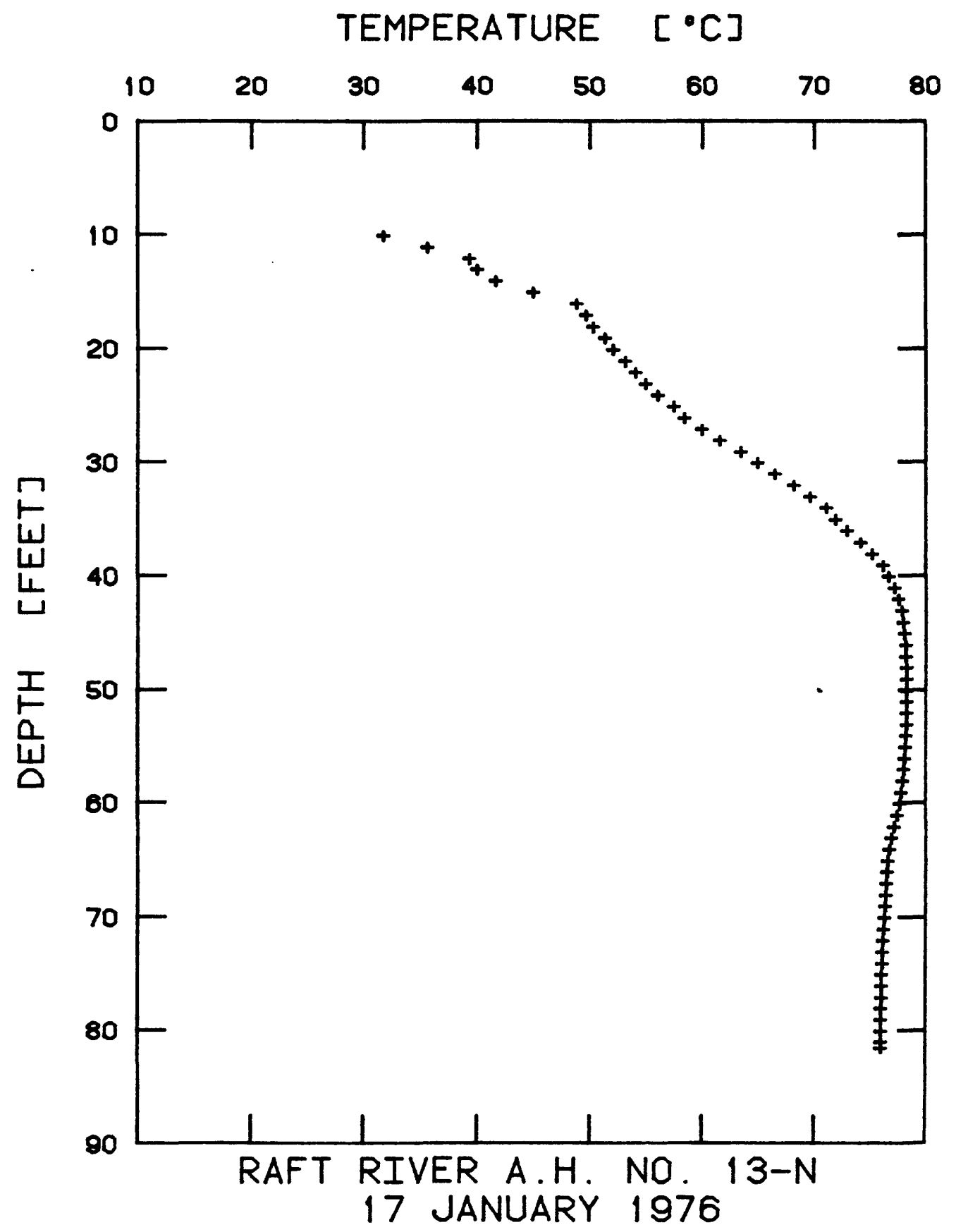

$I-101$ 


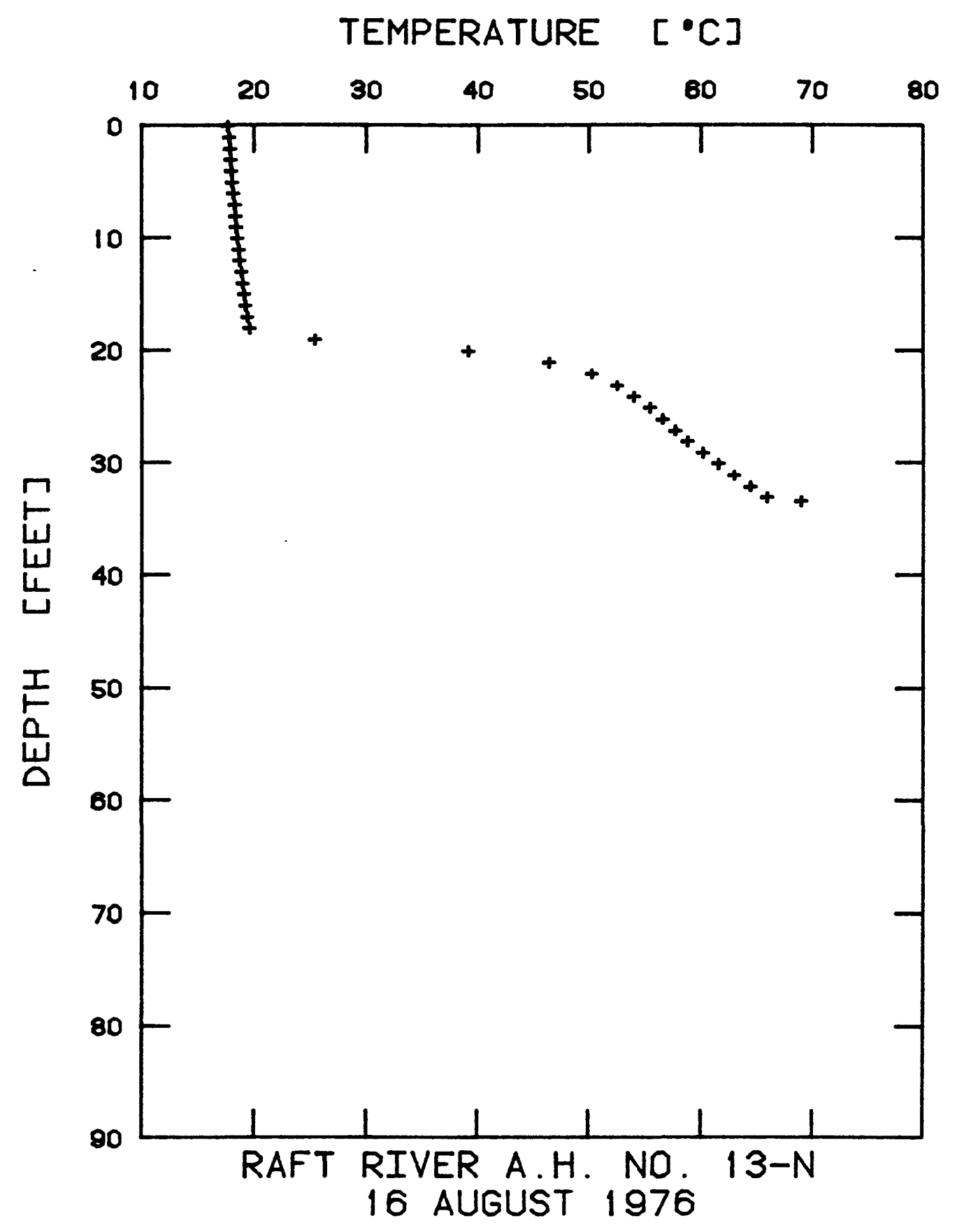

I-102 


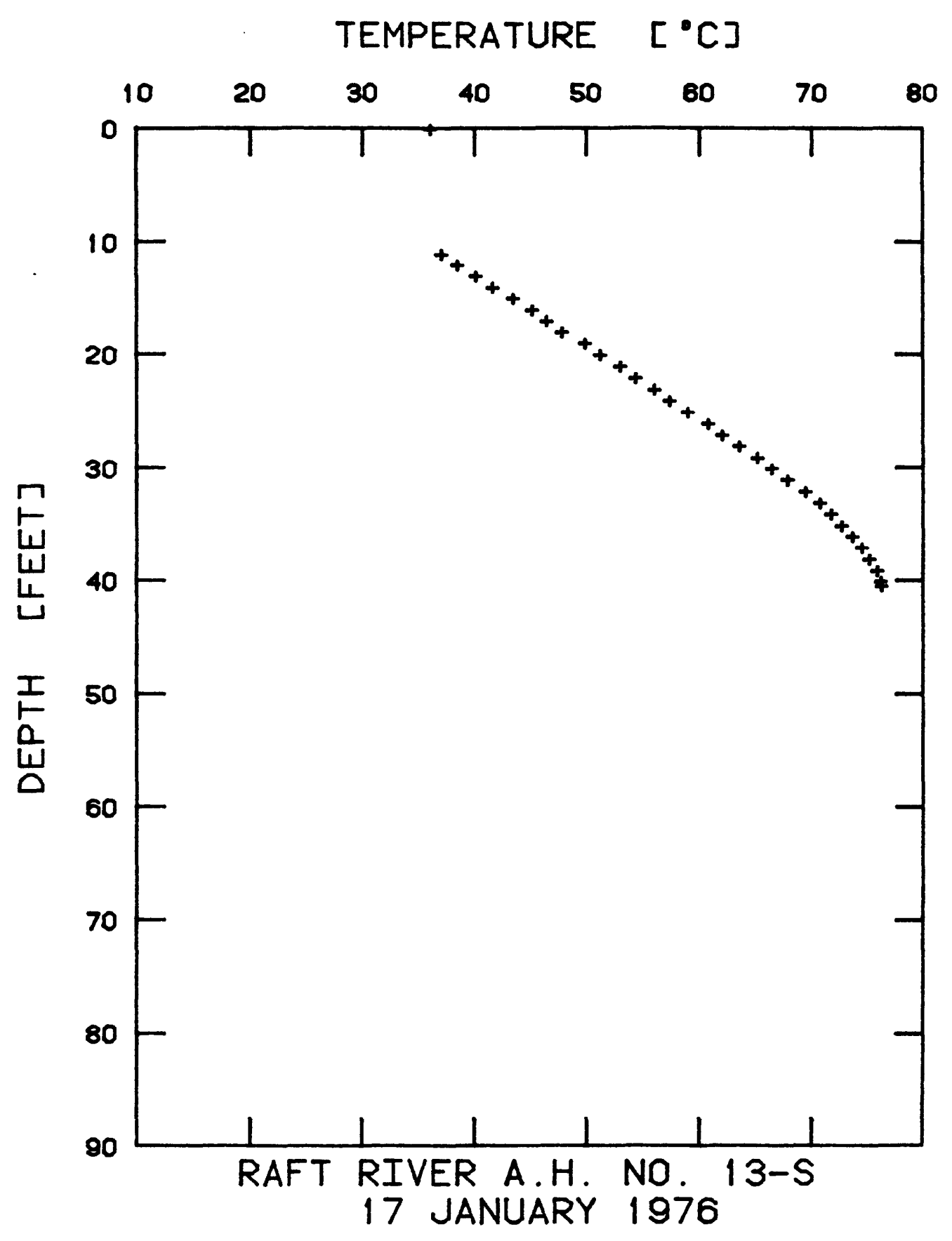

$I-109$ 


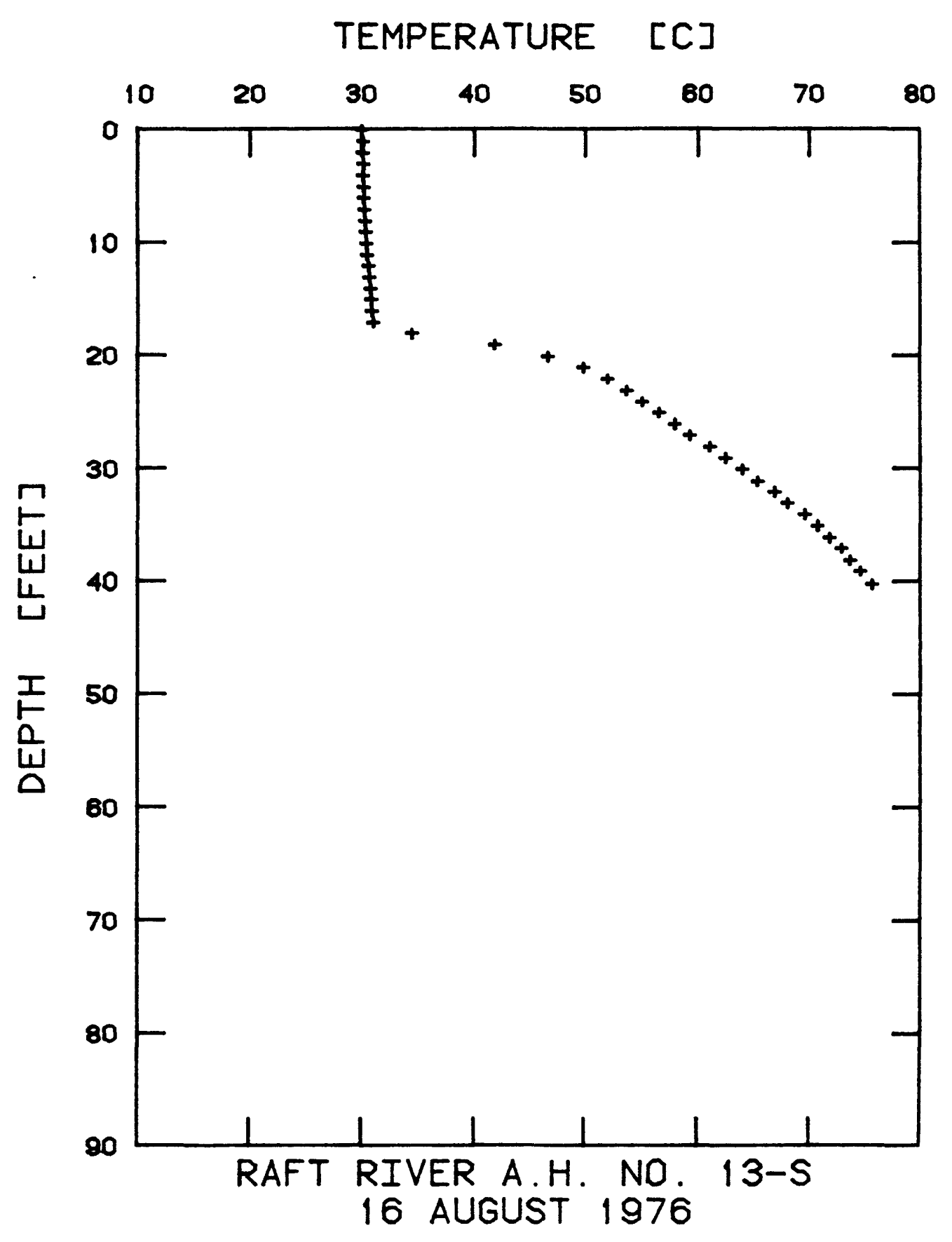




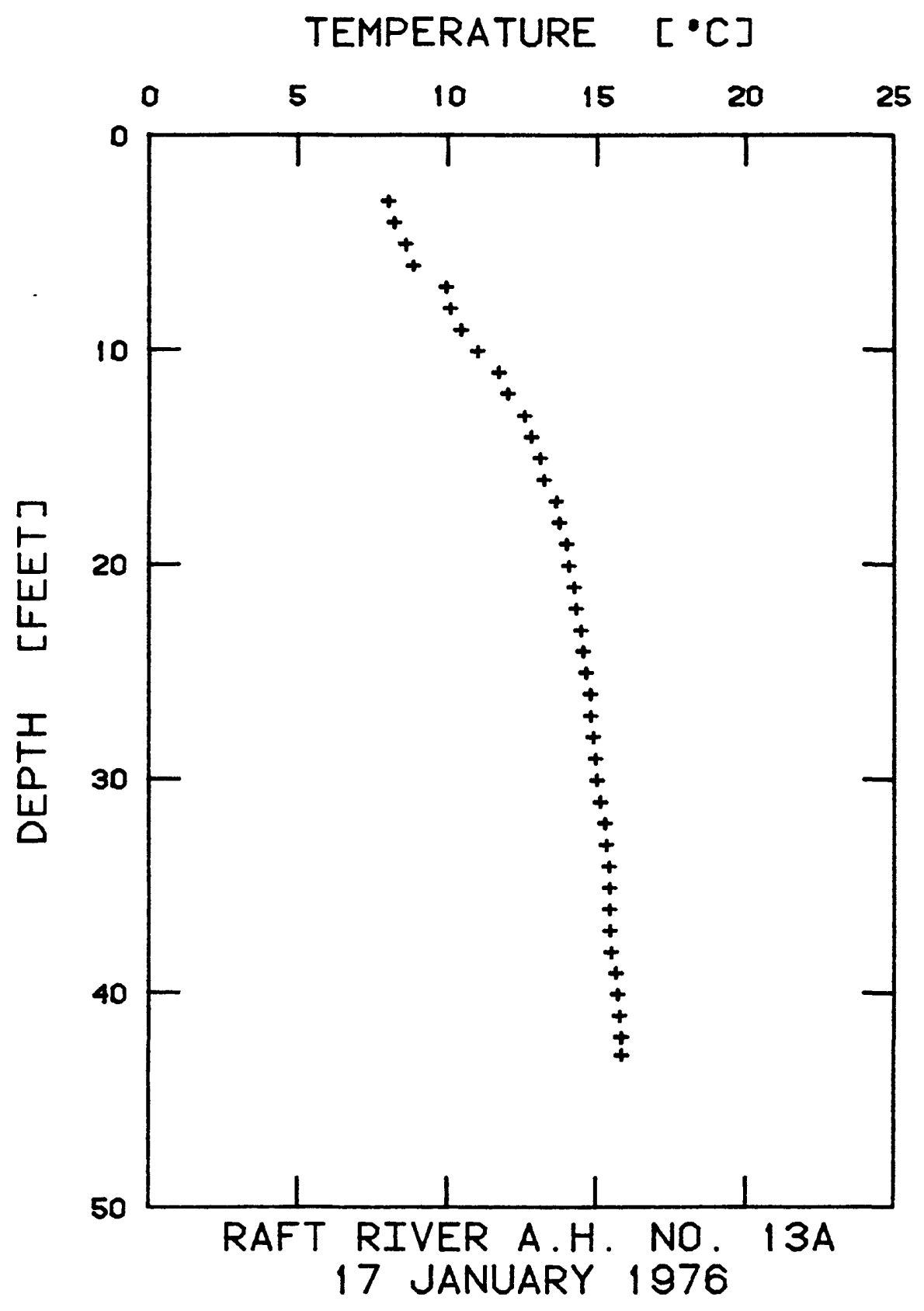

$I-105$ 


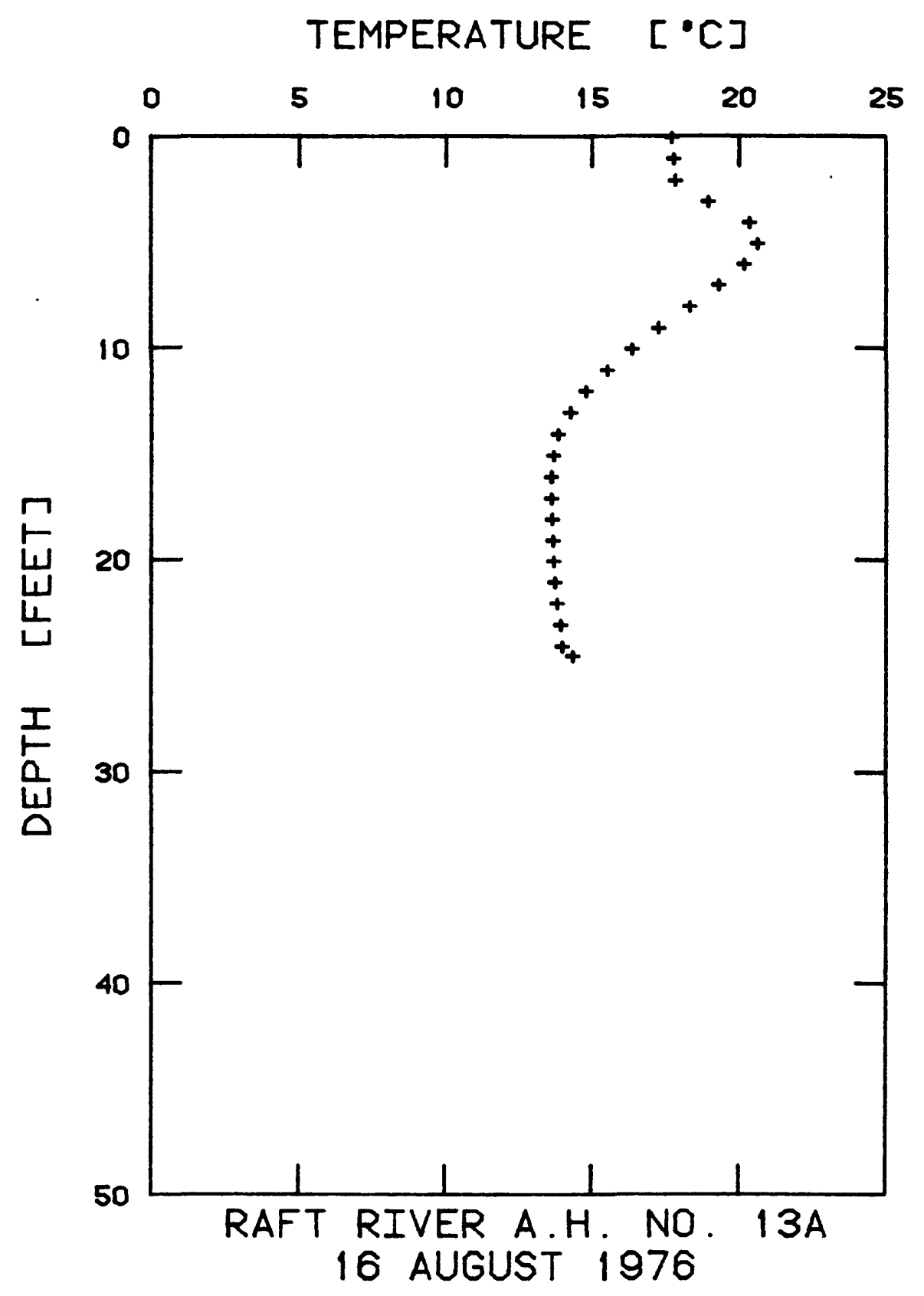

$I-106$ 


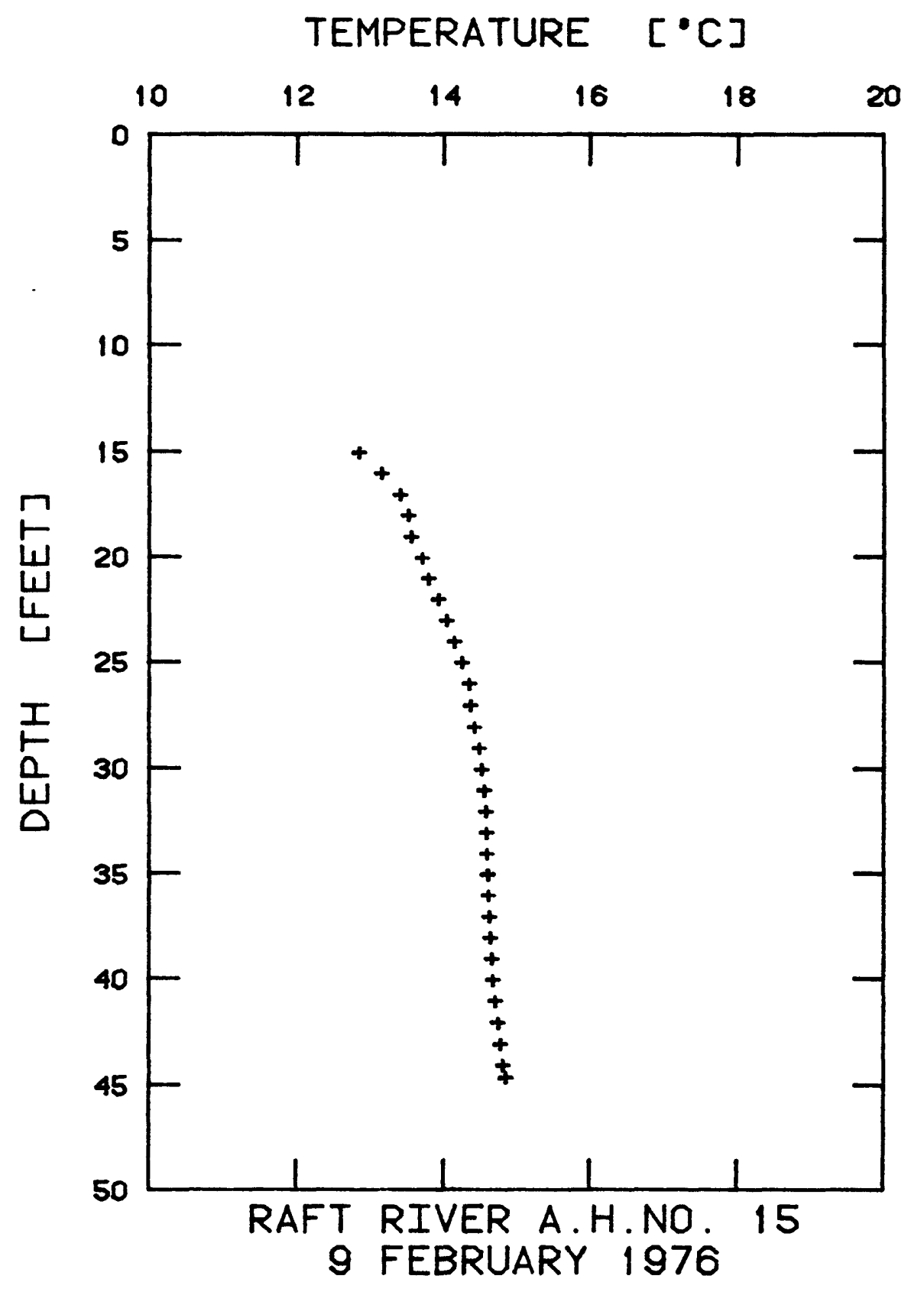

$I-107$ 


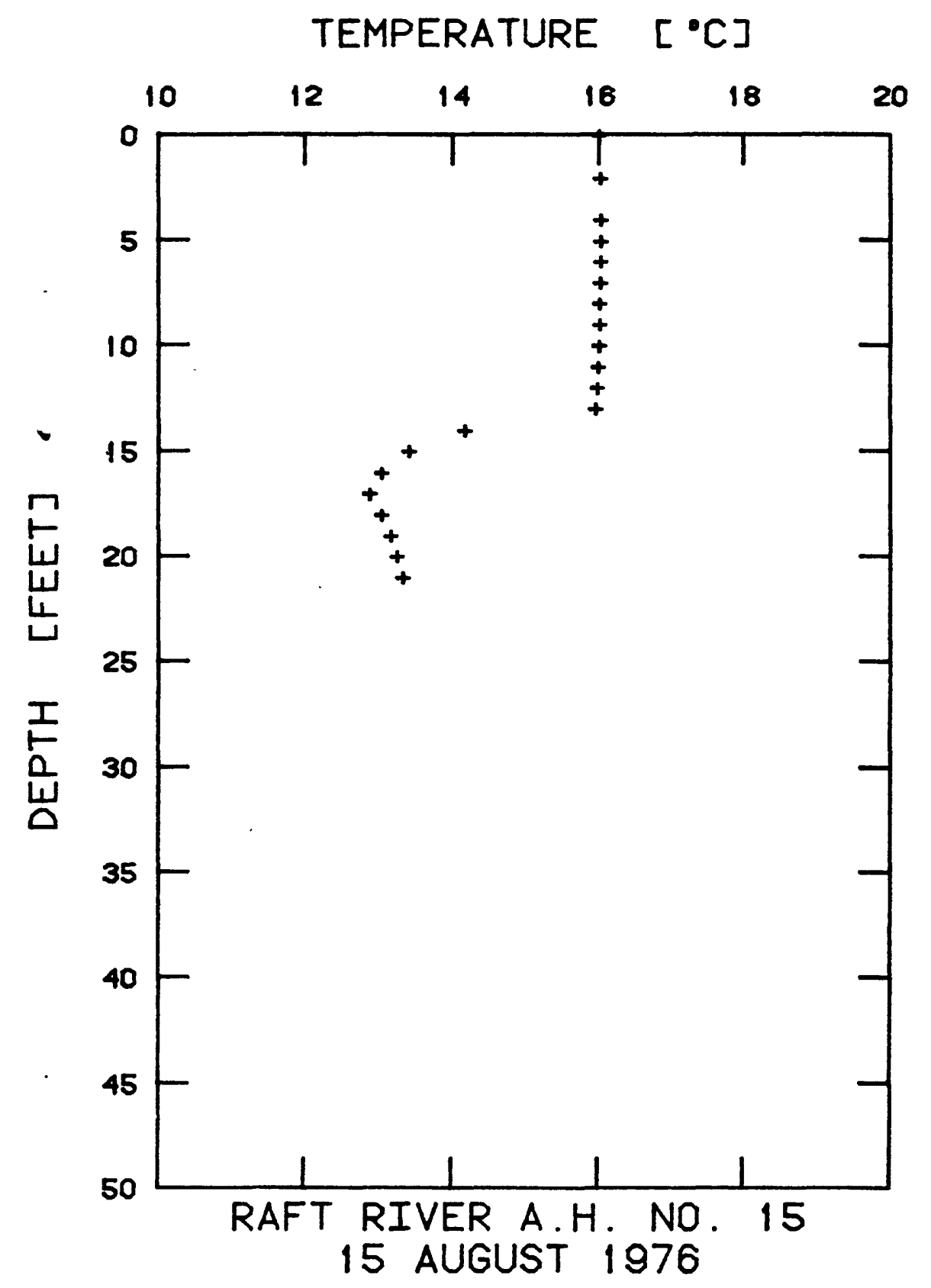

$I-108$ 


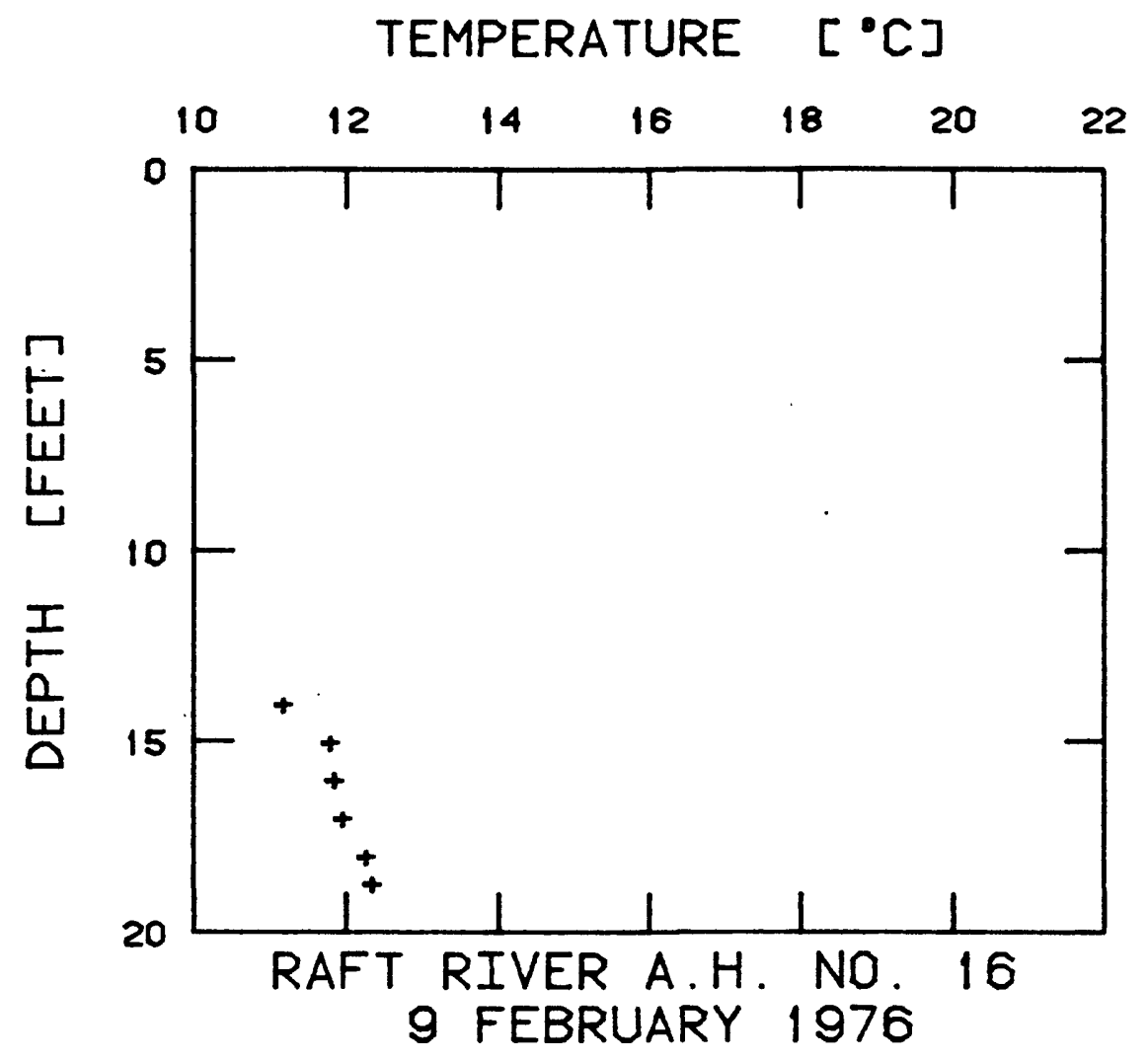




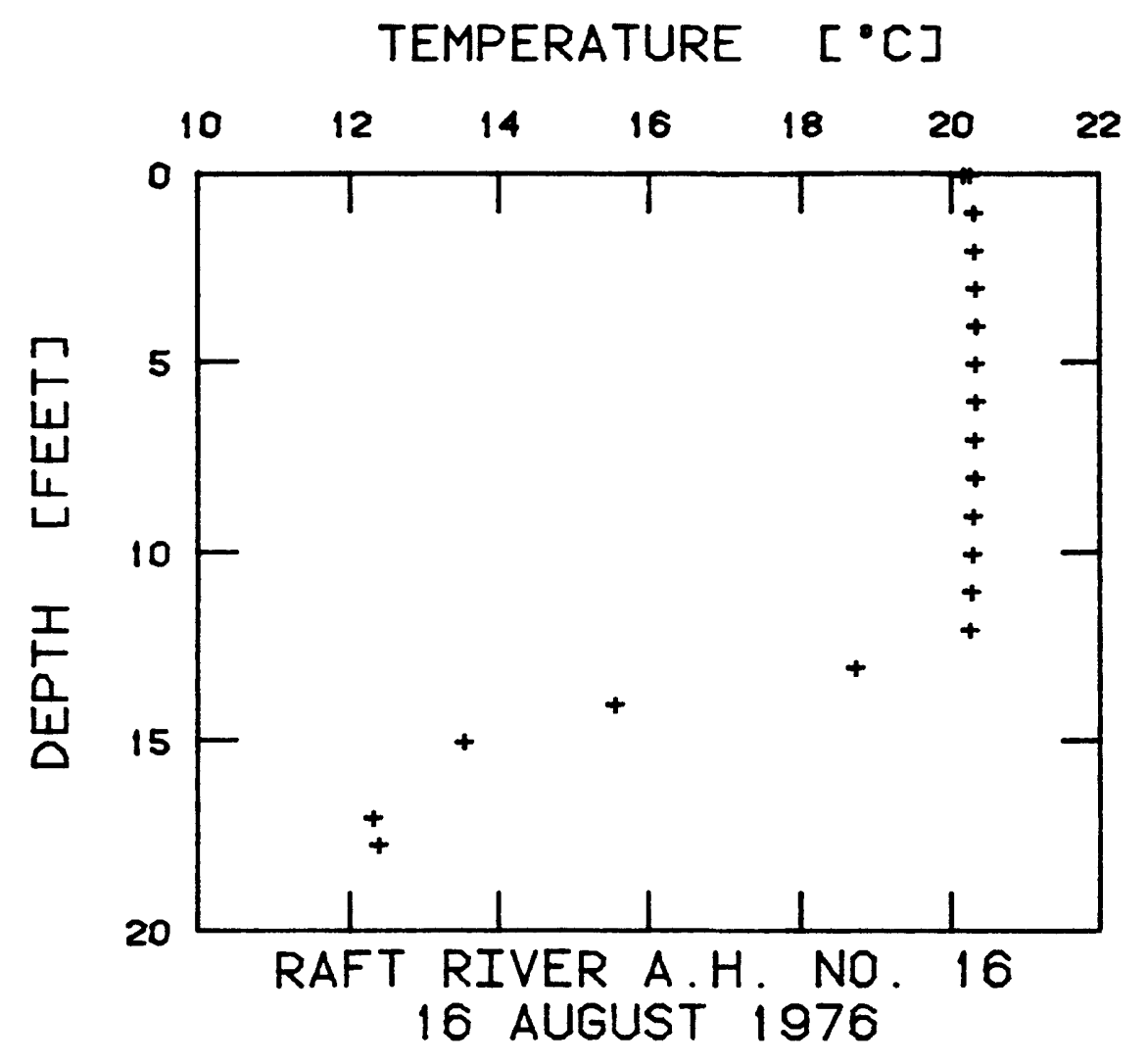




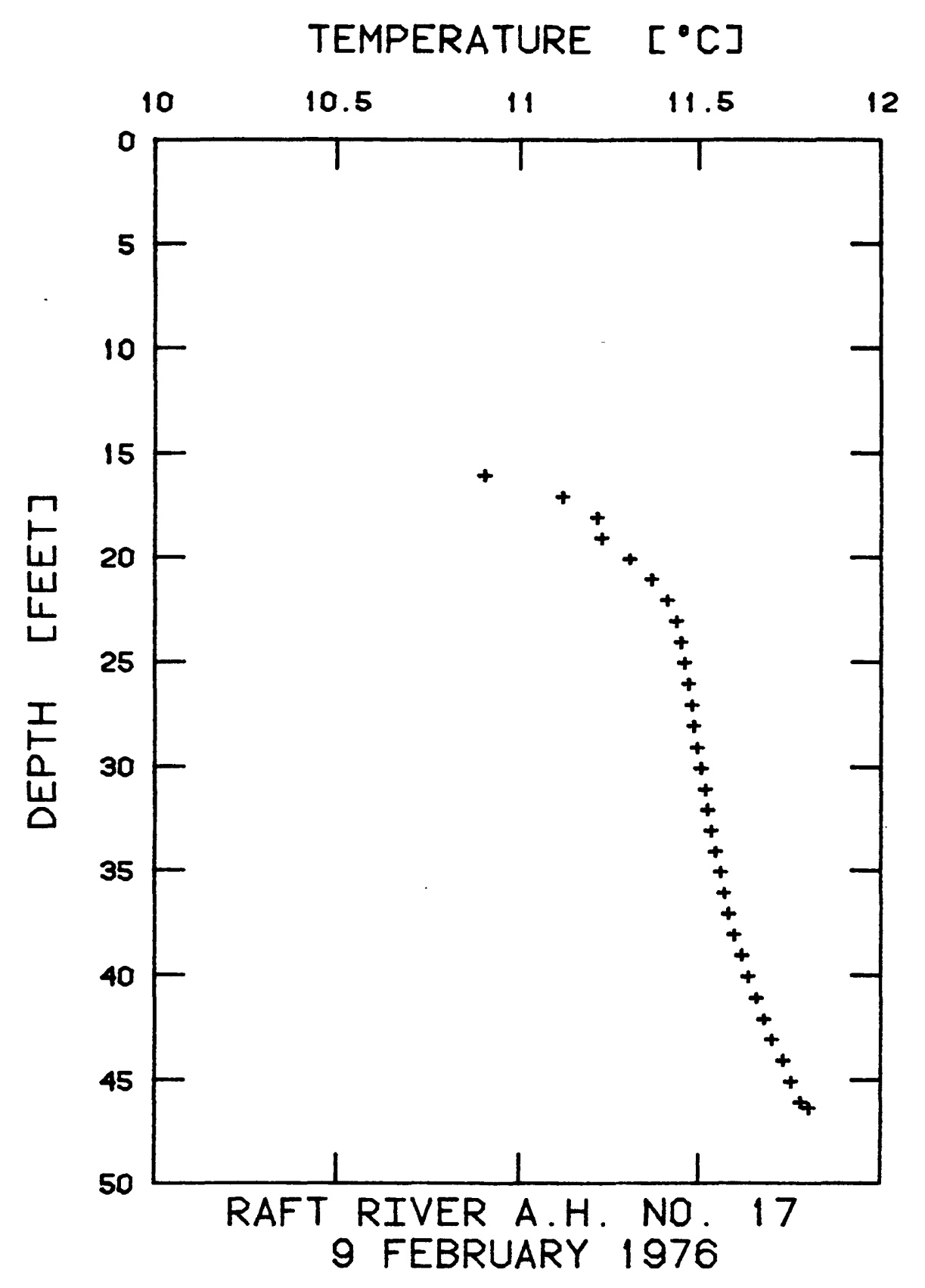

$I-111$ 


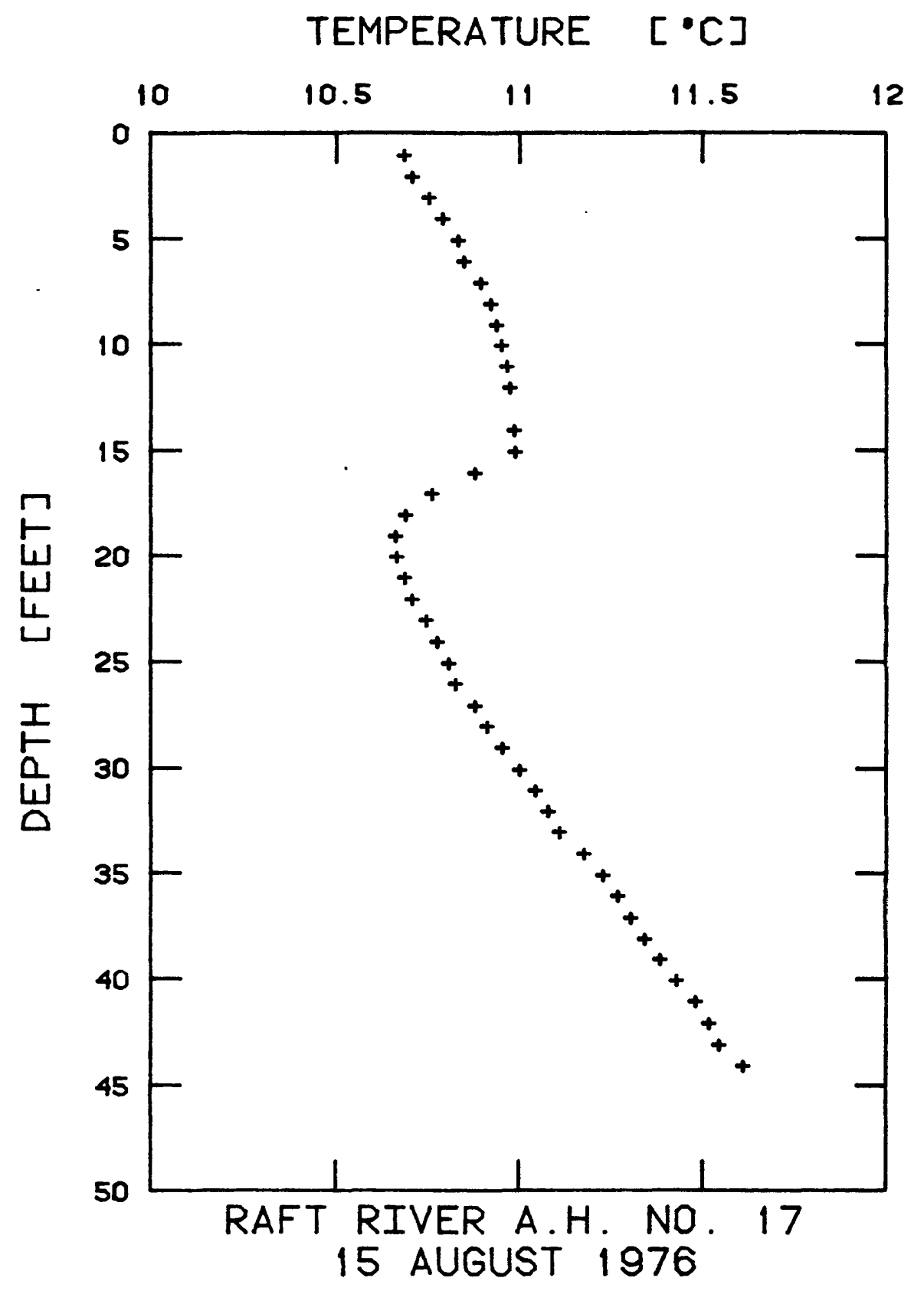

$I-112$ 


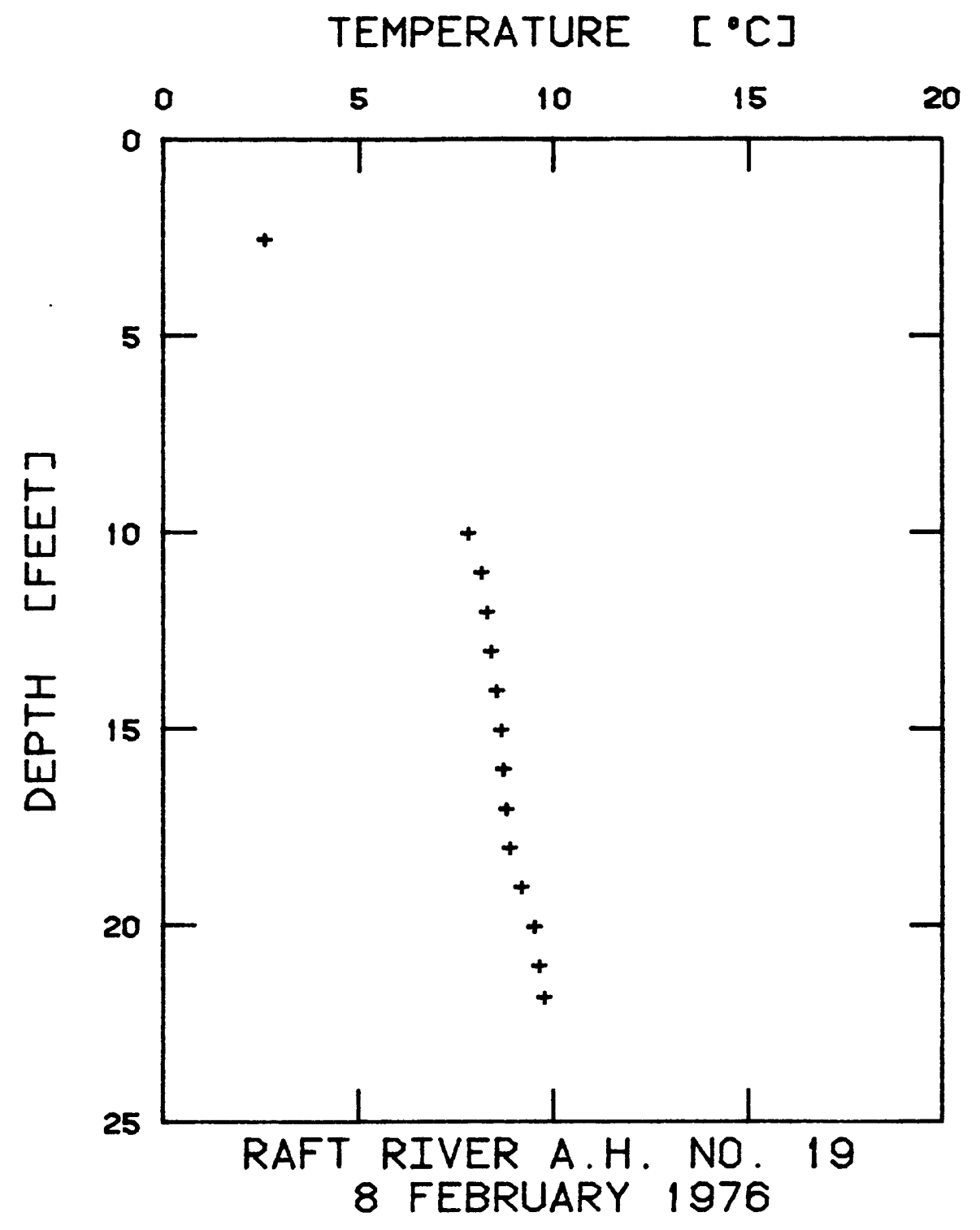

$I-113$ 


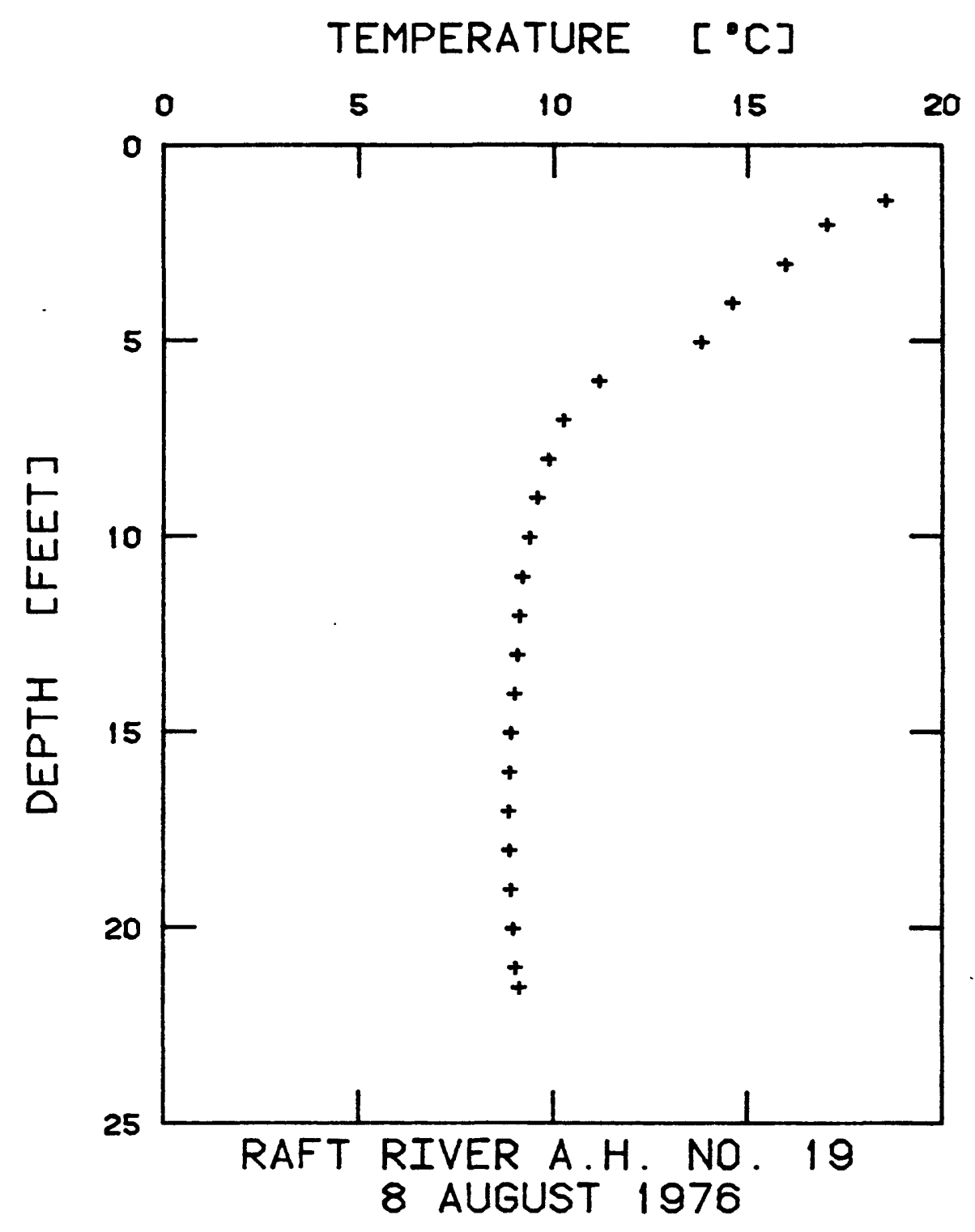

$I-114$ 


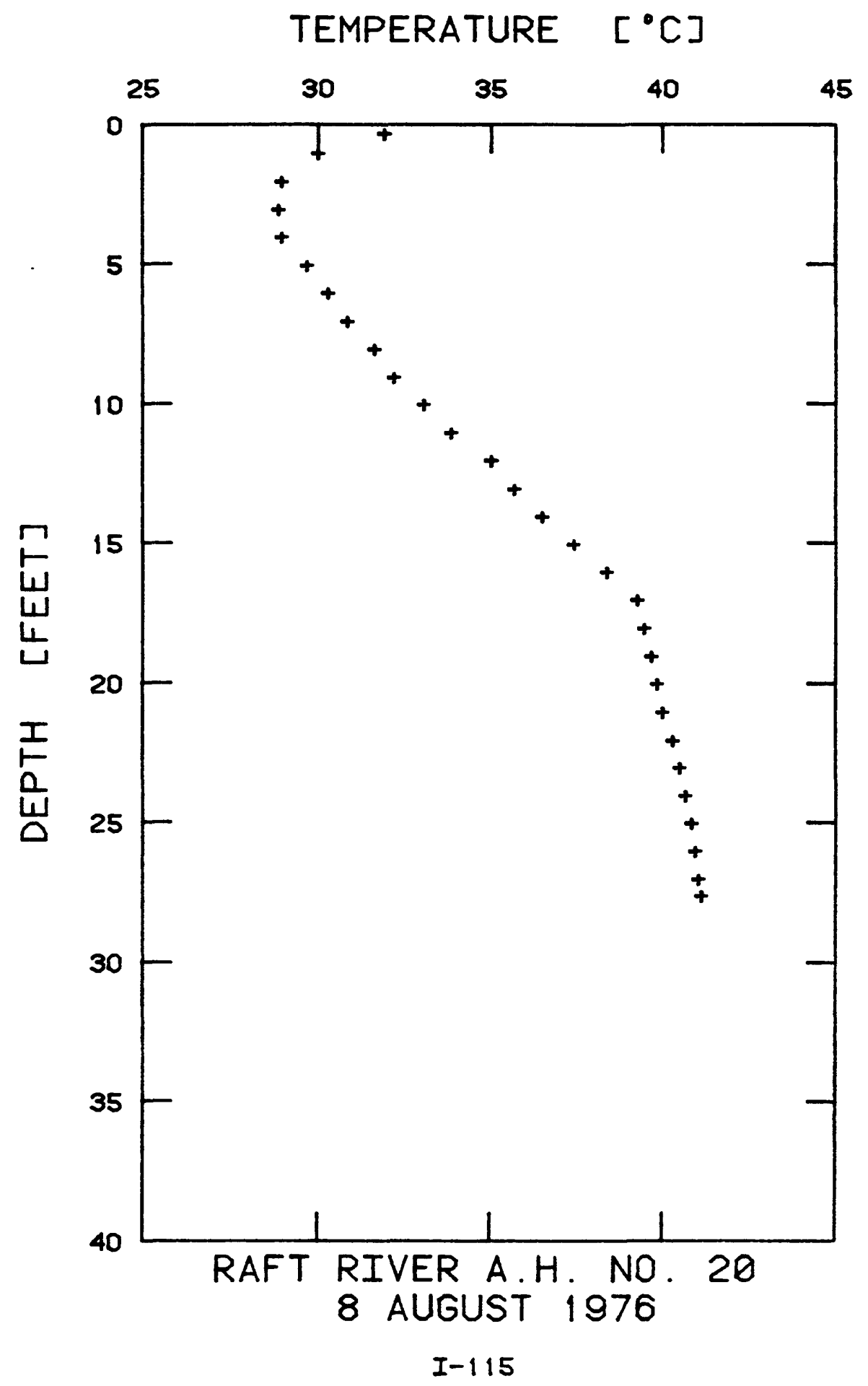




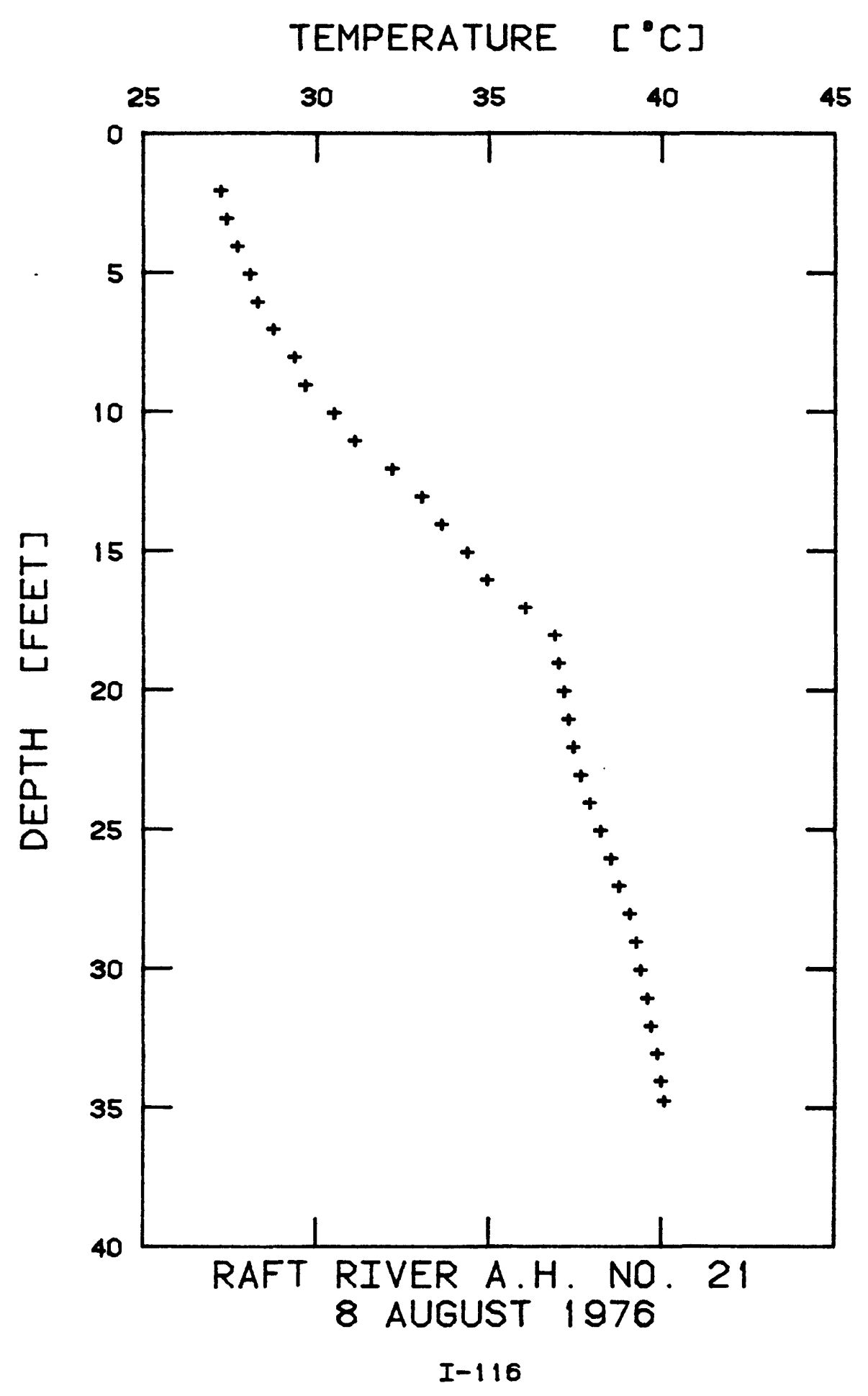




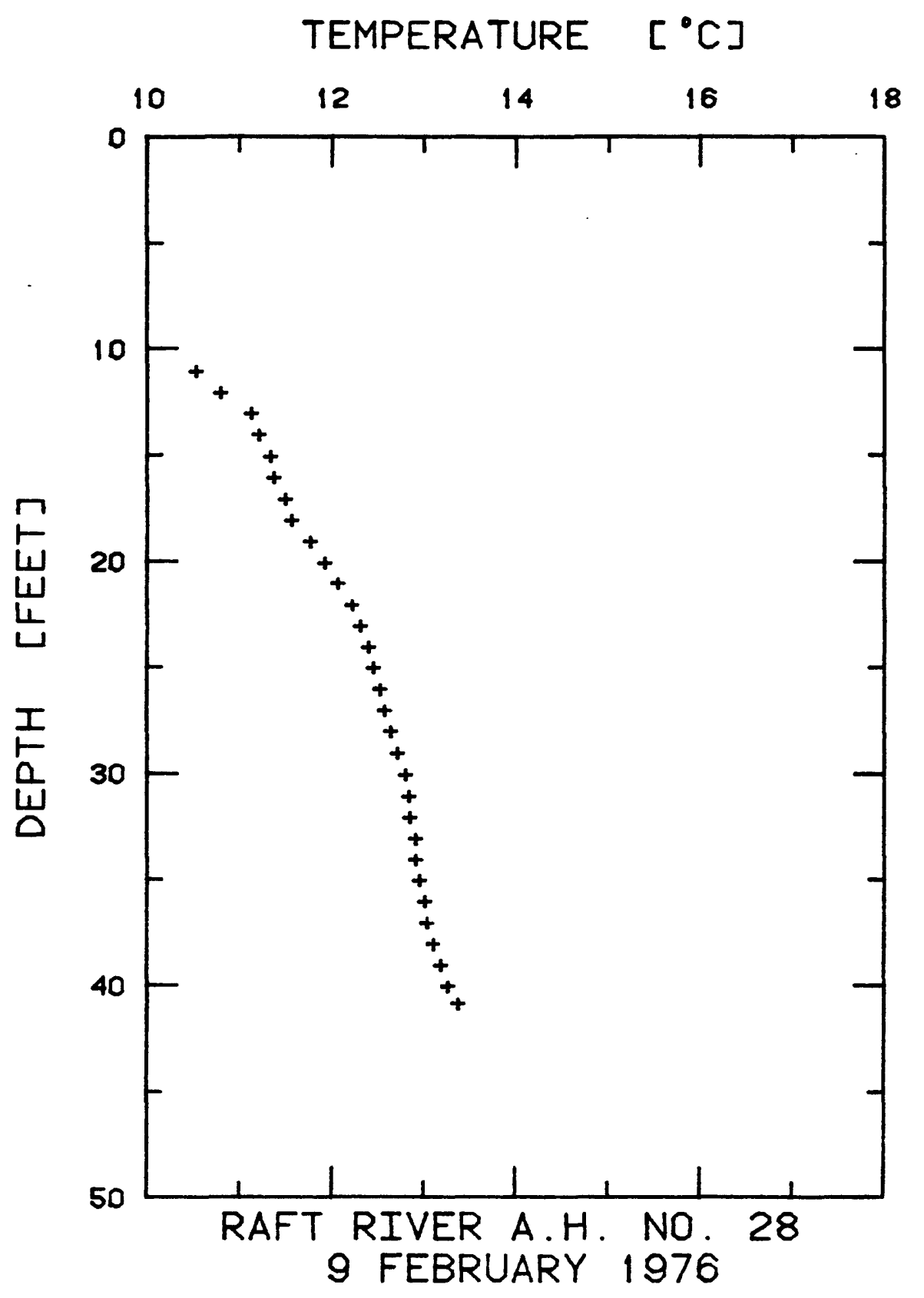

$$
I-117
$$




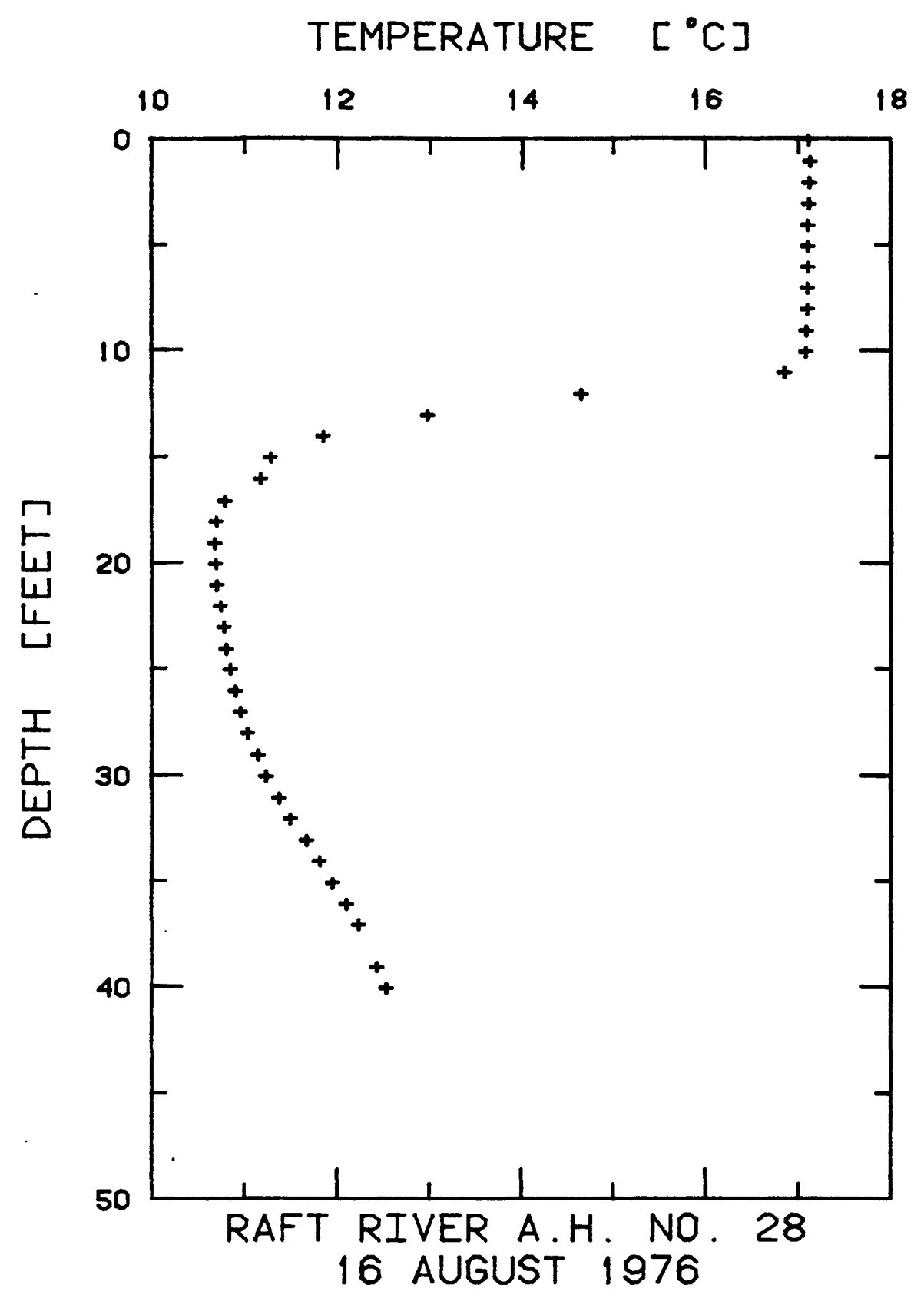

$I-118$ 


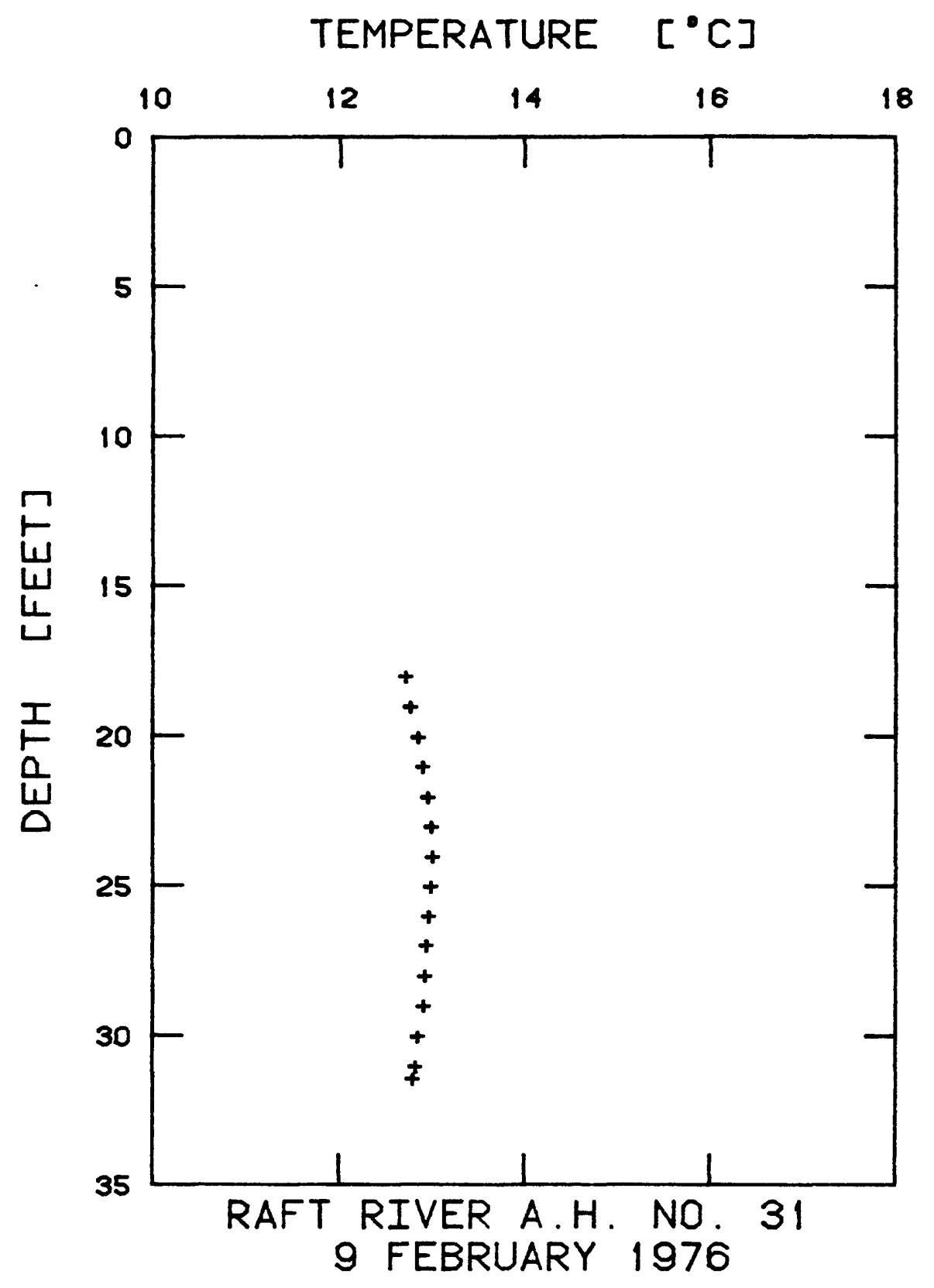

$I-119$ 


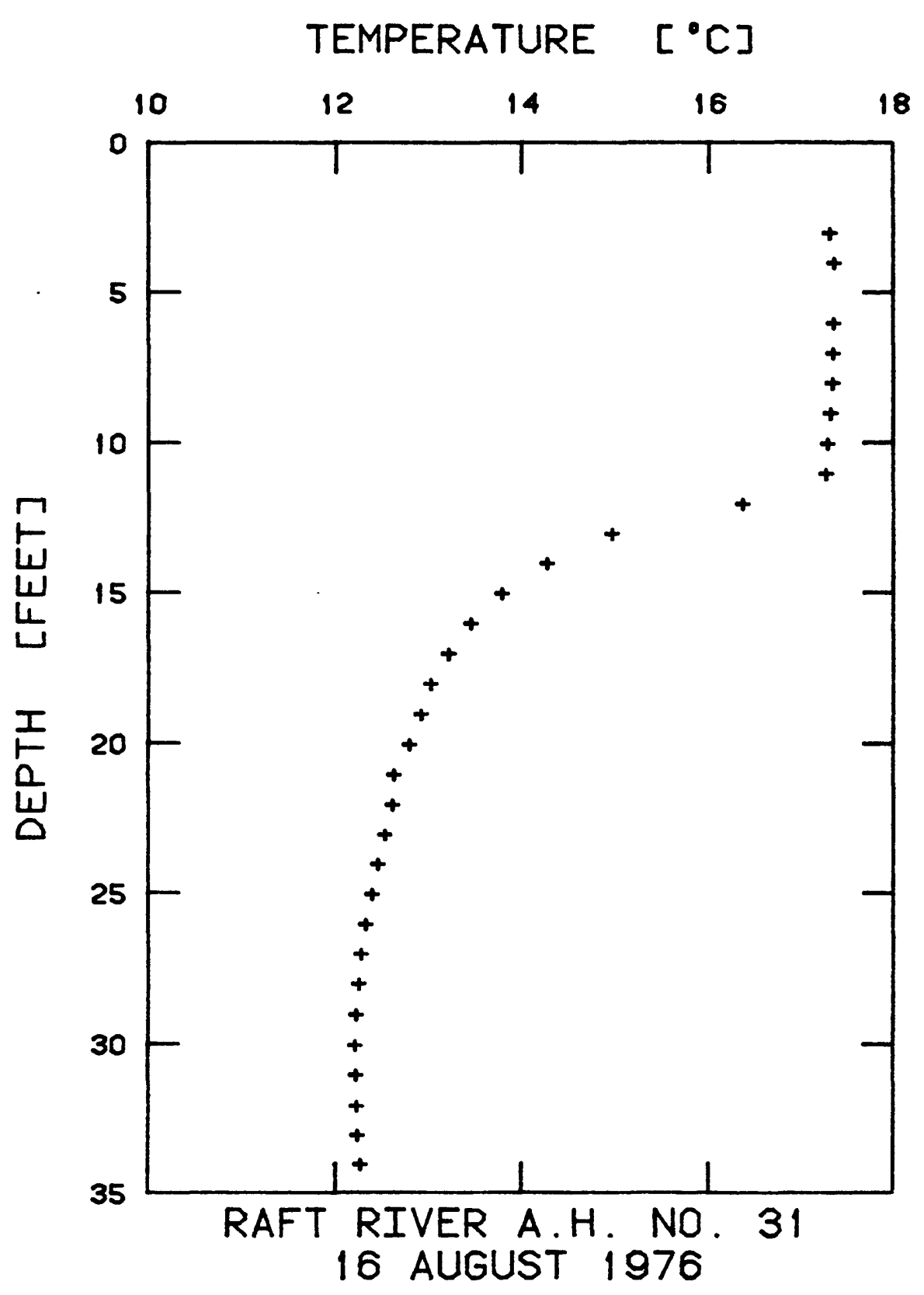

$I-120$ 


\section{APPENDIX II}

THERMAL CONDUCTIVITY PLOTTED AS A FUNCTION OF DEPTH FOR WELLS IN THE RAFT RIVER AREA, CASSIA COUNTY, IDAHO

\section{CONTENTS}

Well No.

Page

I.D. 1

II -1

I.D. 2

$I I-2$

I.D. 30.

II -3

I.D. 5

II -4 
THERMAL CONDUCTIVITY $[\mathrm{W} / \mathrm{mK}]$

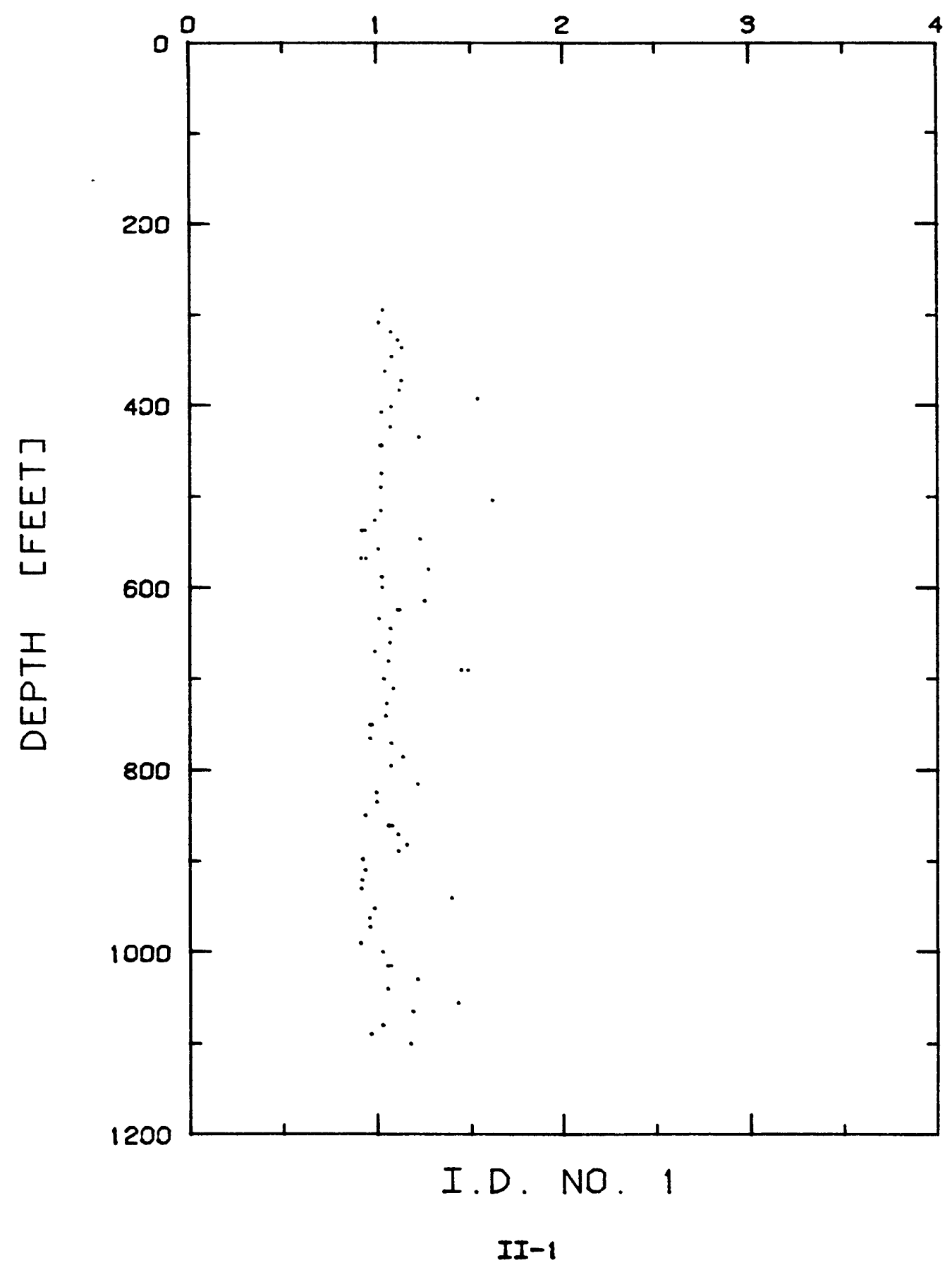


THERMAL CONDUCTIVITY [W/mK]

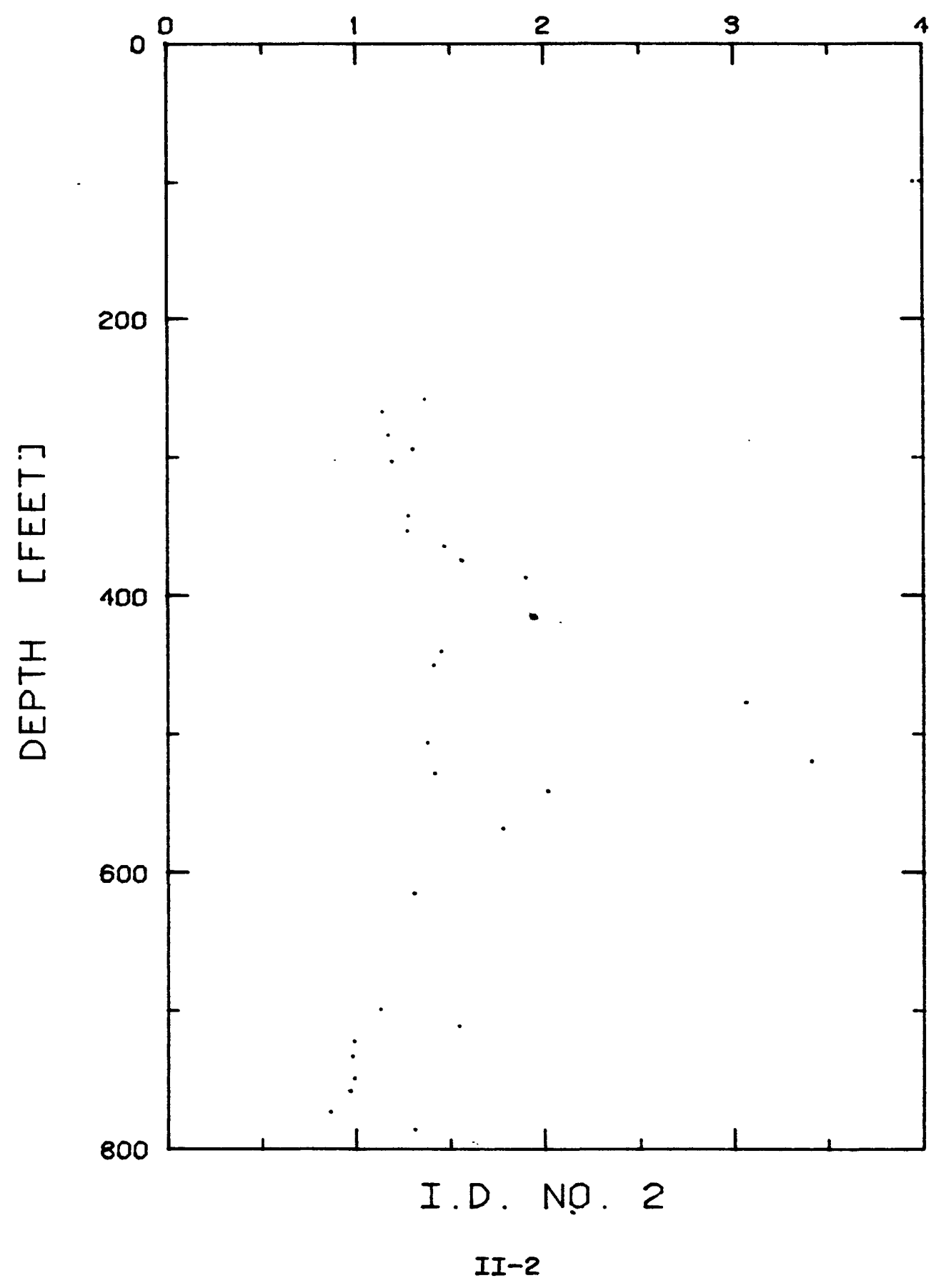


THERMAL CONDUCTIVITY [W/mK]

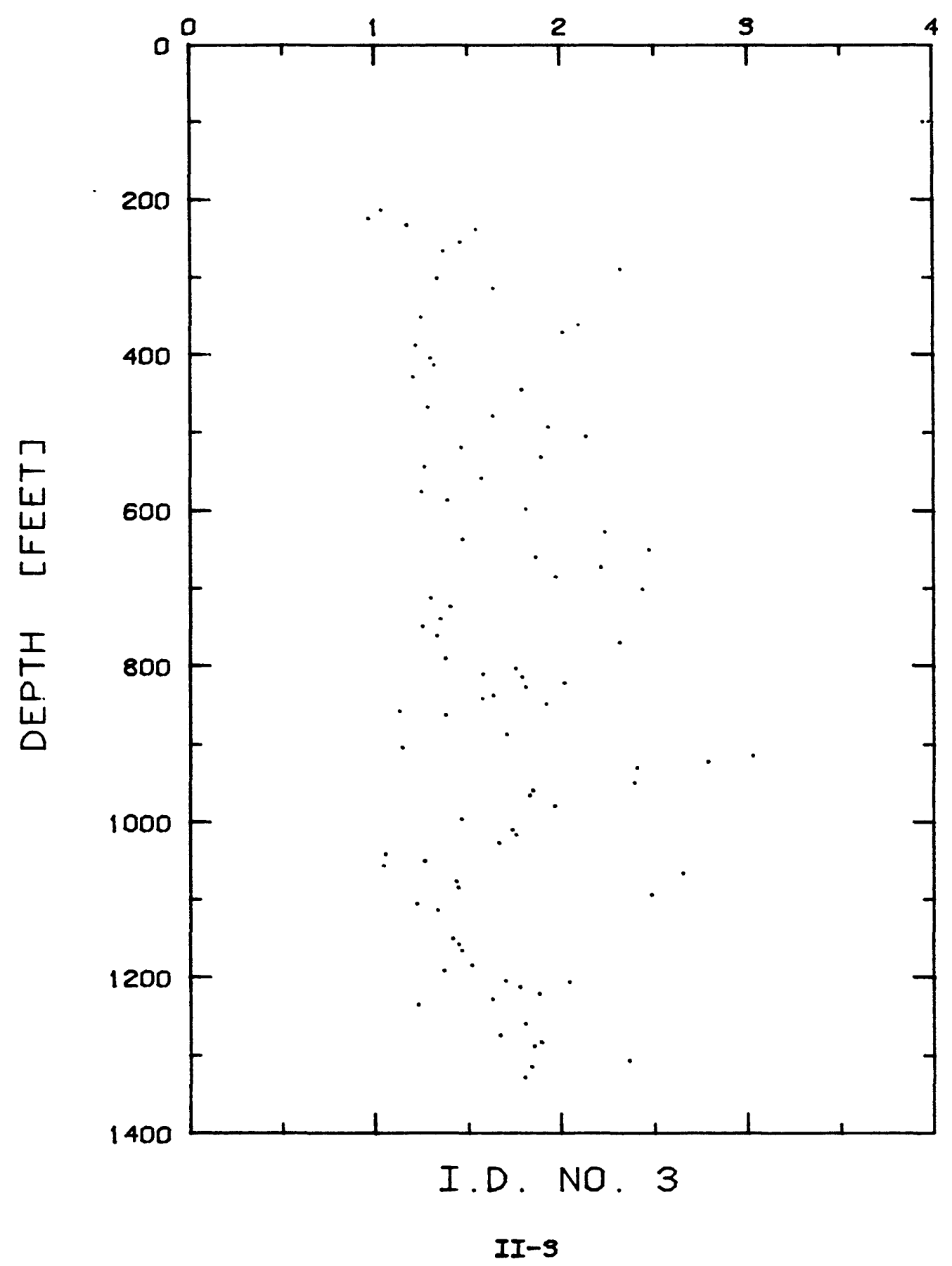


THERMAL CONDUCTIVITY [W/mK]

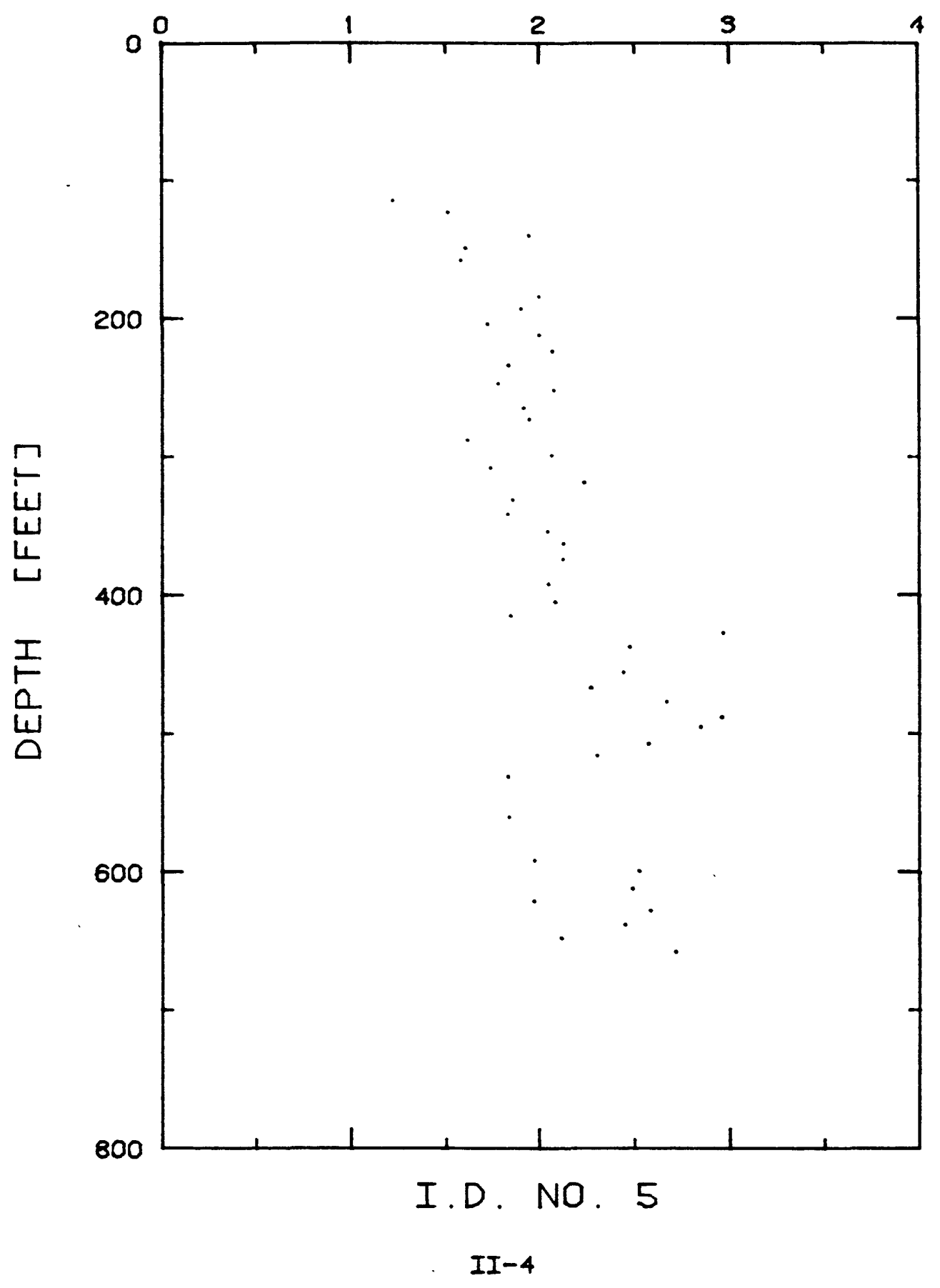


DEPTHS AND TEMPERATURES FOR WELLS LOGGED IN THE RAFT RIVER AREA, CASSIA COUNTY, IDAHO (1974-1976)

\section{CONTENTS}

Well No. $\quad$ Page

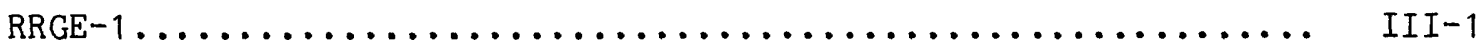

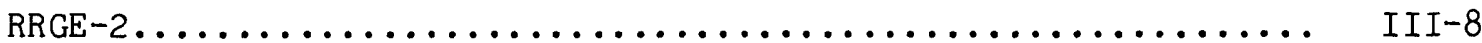

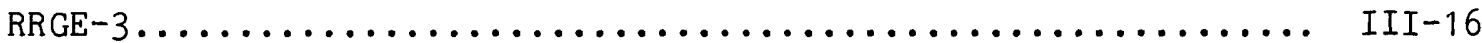

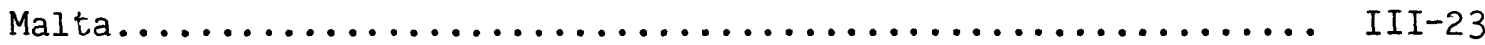

Strevell............................................ III

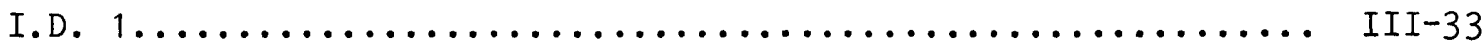

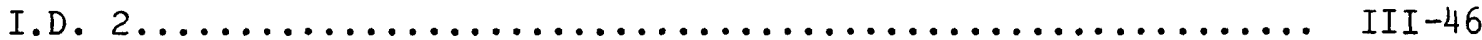

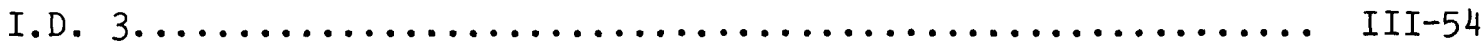

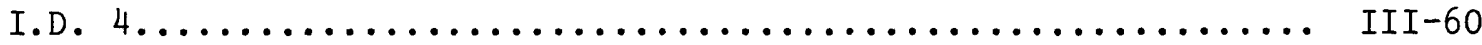

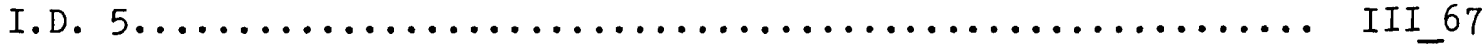

I.D. 5 A.................................... III -74

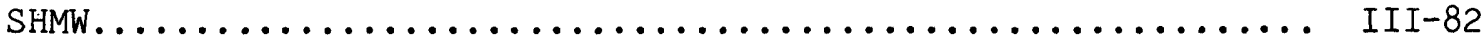

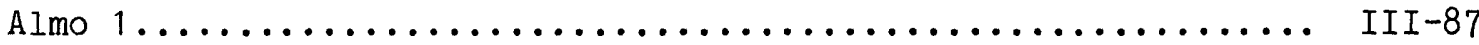

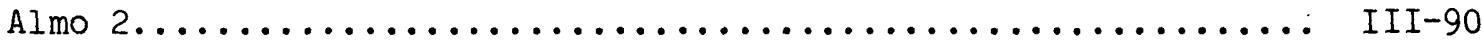

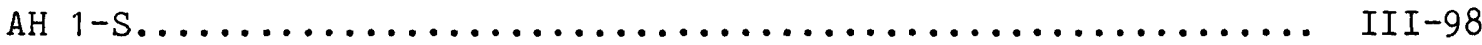

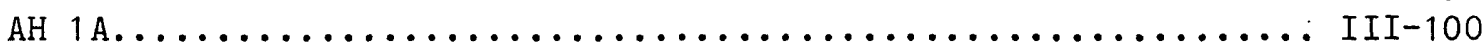

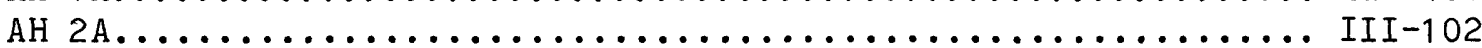

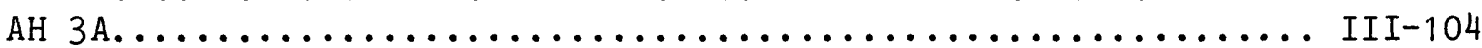

AH 4 А........................................

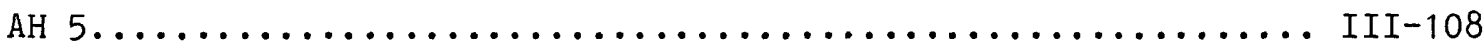

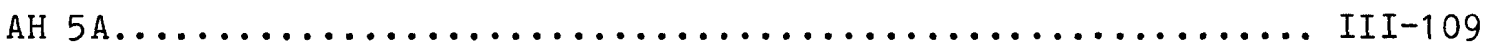

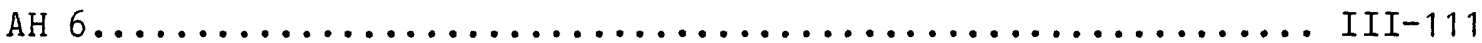

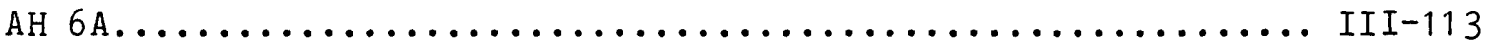

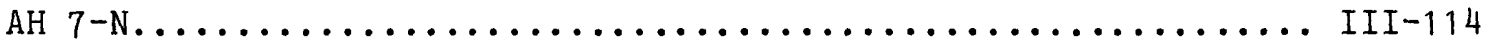

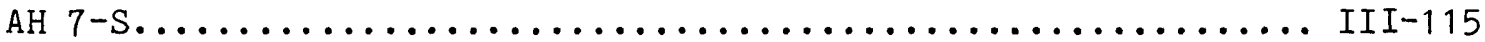

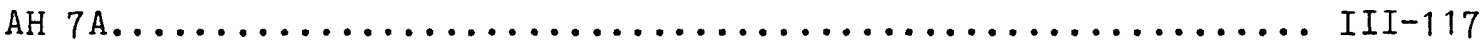

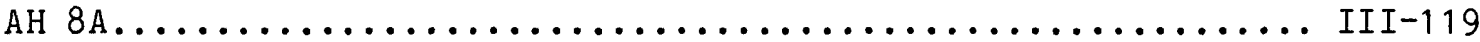

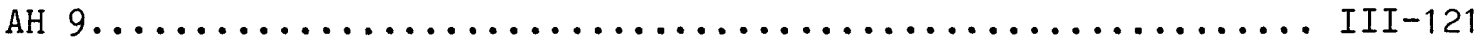

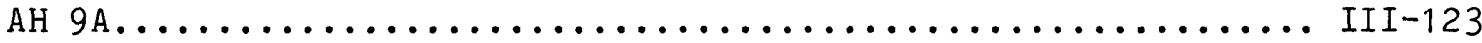

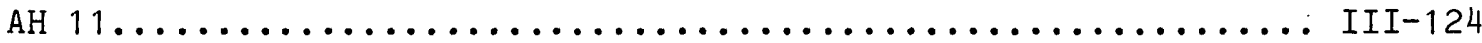

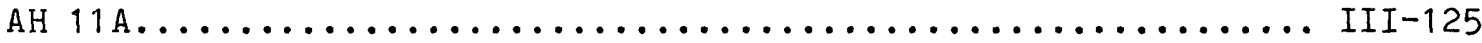

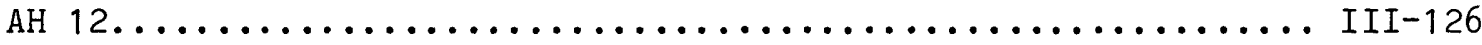

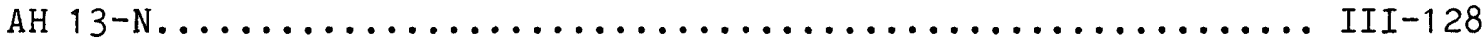

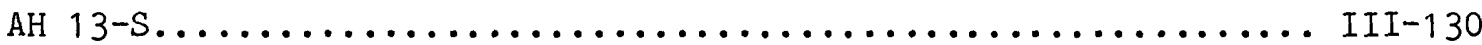

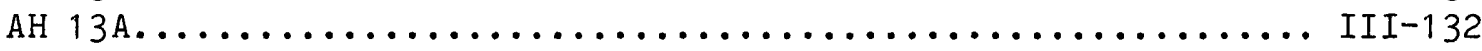

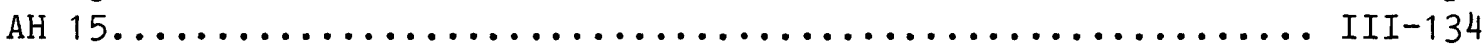

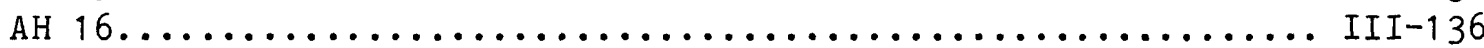

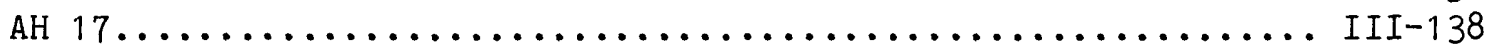

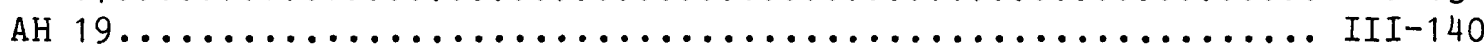

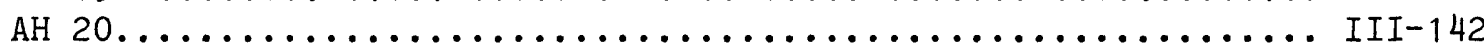

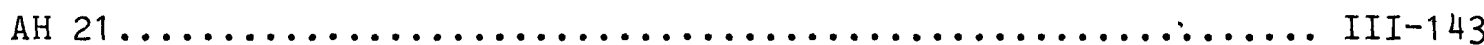

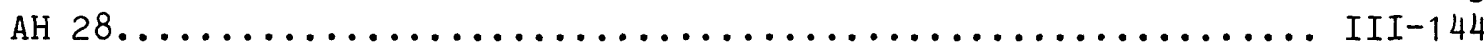

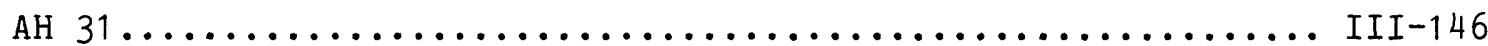


RAFT RIVER, IDAHO - RRGE-1 - 11 JAWARY 1975

\begin{tabular}{|c|c|c|c|c|c|}
\hline $\begin{array}{l}\text { DEPTH } \\
\text { (FT) }\end{array}$ & $\begin{array}{l}\text { TEXPERATURE } \\
\text { (C) }\end{array}$ & $\begin{array}{c}\text { DEPTH } \\
\text { (FT) }\end{array}$ & $\begin{array}{l}\text { TEMPERATULE } \\
\text { (C) }\end{array}$ & $\begin{array}{l}\text { DEPIH } \\
\text { (FT) }\end{array}$ & $\begin{array}{l}\text { TEMPERATU } \\
\text { (C) }\end{array}$ \\
\hline $\begin{array}{l}5 \\
10 \\
15 \\
20 \\
25 \\
30 \\
35 \\
40 \\
45 \\
50 \\
55 \\
60 \\
65 \\
70 \\
75 \\
80 \\
85 \\
90 \\
95 \\
100 \\
105 \\
110 \\
115 \\
120 \\
125 \\
130 \\
135 \\
140 \\
145 \\
150 \\
155 \\
160 \\
165 \\
170 \\
175 \\
180 \\
185 \\
190 \\
195 \\
200 \\
205 \\
210 \\
215 \\
220 \\
225 \\
230 \\
235 \\
240 \\
245 \\
250 \\
255 \\
260 \\
265 \\
270 \\
275 \\
280 \\
285 \\
290 \\
295 \\
300 \\
305 \\
310 \\
315 \\
320 \\
325 \\
330 \\
335 \\
340 \\
345 \\
350\end{array}$ & $\begin{array}{l}28.536 \\
28.352 \\
29.127 \\
29.687 \\
29.819 \\
29.963 \\
20.825 \\
30.286 \\
30.199 \\
30.529 \\
30.125 \\
30.866 \\
30.937 \\
31.086 \\
31.131 \\
31.248 \\
31.395 \\
31.474 \\
31.693 \\
31.863 \\
31.957 \\
32.109 \\
32.279 \\
32.378 \\
32.567 \\
32.761 \\
32.820 \\
33.037 \\
33.168 \\
33.292 \\
33.536 \\
33.676 \\
33.702 \\
33.905 \\
33.975 \\
34.133 \\
34.348 \\
34.423 \\
34.505 \\
31.738 \\
34.822 \\
34.936 \\
35.101 \\
35.201 \\
35.317 \\
35.418 \\
35.656 \\
35.905 \\
35.913 \\
36.037 \\
36.193 \\
36.387 \\
36.468 \\
36.542 \\
33.790 \\
36.908 \\
37.012 \\
37.133 \\
37.271 \\
37.3555 \\
37.440 \\
37.559 \\
31.906 \\
37.777 \\
37.997 \\
38.132 \\
38.197 \\
33.369 \\
38.559 \\
38\end{array}$ & $\begin{array}{l}355 \\
360 \\
365 \\
370 \\
375 \\
380 \\
385 \\
390 \\
395 \\
400 \\
405 \\
110 \\
415 \\
420 \\
425 \\
430 \\
435 \\
440 \\
445 \\
450 \\
455 \\
460 \\
465 \\
470 \\
480 \\
485 \\
490 \\
495 \\
500 \\
505 \\
510 \\
515 \\
520 \\
525 \\
530 \\
535 \\
540 \\
515 \\
550 \\
555 \\
540 \\
565 \\
570 \\
575 \\
580 \\
585 \\
590 \\
595 \\
600 \\
605 \\
610 \\
615 \\
620 \\
625 \\
630 \\
635 \\
640 \\
645 \\
650 \\
655 \\
660 \\
665 \\
670 \\
675 \\
680 \\
685 \\
690 \\
695 \\
700 \\
705\end{array}$ & $\begin{array}{l}38.795 \\
38.952 \\
38.952 \\
39.270 \\
39.307 \\
39.468 \\
39.518 \\
39.760 \\
40.013 \\
40.150 \\
40.226 \\
10.326 \\
40.445 \\
40.638 \\
40.817 \\
40.979 \\
41.043 \\
41.225 \\
41.344 \\
41.614 \\
41.690 \\
41.837 \\
41.877 \\
41.969 \\
42.241 \\
42.454 \\
42.428 \\
12.558 \\
42.652 \\
42.911 \\
12.868 \\
43.039 \\
43.033 \\
43.188 \\
43.290 \\
43.252 \\
41.187 \\
44.776 \\
44.571 \\
15.145 \\
45.087 \\
44.839 \\
44.995 \\
45.151 \\
45.337 \\
45.407 \\
45.331 \\
45.244 \\
45.343 \\
45.273 \\
45.649 \\
45.582 \\
46.260 \\
47.199 \\
49.072 \\
50.060 \\
50.060 \\
49.849 \\
49.551 \\
49.558 \\
49.447 \\
48.897 \\
48.493 \\
48.341 \\
47.998 \\
47.502 \\
46.559 \\
45.970 \\
45.601 \\
45.893\end{array}$ & $\begin{array}{l}710 \\
715 \\
720 \\
725 \\
730 \\
735 \\
740 \\
745 \\
750 \\
755 \\
760 \\
765 \\
770 \\
775 \\
780 \\
785 \\
790 \\
795 \\
800 \\
805 \\
810 \\
815 \\
820 \\
825 \\
930 \\
835 \\
840 \\
845 \\
850 \\
855 \\
860 \\
865 \\
870\end{array}$ & $\begin{array}{l}46.418 \\
48.29 \\
19.038 \\
50.500 \\
50.586 \\
47.092 \\
41.128 \\
51.419 \\
12.107 \\
51.242 \\
48.843 \\
44.799 \\
42.164 \\
41.771 \\
40.184 \\
34.892 \\
38.782 \\
46.333 \\
17.243 \\
53.218 \\
50.738 \\
48.503 \\
17.944 \\
53.289 \\
53.538 \\
54.365 \\
54.298 \\
46.911 \\
45.012 \\
52.226 \\
53.202 \\
53.417 \\
53.218\end{array}$ \\
\hline
\end{tabular}


RAFT RIVER, IDAHD - RRGE-1 - 12 JAHUARY 1975

\begin{tabular}{|c|c|c|c|c|c|}
\hline $\begin{array}{l}\text { DEPTH } \\
\text { (FT) }\end{array}$ & $\begin{array}{l}\text { TEIPERATURE } \\
\text { (C) }\end{array}$ & $\begin{array}{l}\text { DEPTH } \\
\text { (FT) }\end{array}$ & $\begin{array}{l}\text { TETPERATPE } \\
\text { (C) }\end{array}$ & $\begin{array}{r}\text { DEPTH } \\
\text { (FT) }\end{array}$ & $\begin{array}{l}\text { TEPERATR } \\
\text { (C) }\end{array}$ \\
\hline $\begin{array}{l}5 \\
10 \\
15 \\
20 \\
25 \\
30 \\
35 \\
40 \\
45 \\
50 \\
55 \\
60 \\
55 \\
70 \\
75 \\
80 \\
85 \\
90 \\
95 \\
100 \\
105 \\
110 \\
115 \\
120 \\
125 \\
130 \\
135 \\
140 \\
145 \\
150 \\
155 \\
160 \\
165 \\
170 \\
175 \\
180 \\
185 \\
190 \\
195 \\
200 \\
205 \\
210 \\
215 \\
220 \\
225 \\
230 \\
235 \\
240 \\
245 \\
250 \\
255 \\
250 \\
265 \\
270 \\
275 \\
280 \\
285 \\
290 \\
295 \\
300 \\
305 \\
310 \\
315 \\
320 \\
325 \\
330 \\
335 \\
340 \\
345 \\
350\end{array}$ & $\begin{array}{l}23.359 \\
26.805 \\
27.758 \\
29.339 \\
30.149 \\
30.280 \\
30.452 \\
30.652 \\
30.921 \\
31.209 \\
31.392 \\
31.613 \\
31.957 \\
32.180 \\
32.550 \\
32.671 \\
33.221 \\
33.207 \\
33.275 \\
33.424 \\
33.536 \\
33.774 \\
34.089 \\
34.222 \\
34.546 \\
34.693 \\
34.814 \\
34.879 \\
35.154 \\
35.527 \\
35.654 \\
35.790 \\
36.021 \\
36.326 \\
36.473 \\
36.693 \\
37.037 \\
37.288 \\
37.137 \\
37.451 \\
37.760 \\
37.824 \\
38.298 \\
38.369 \\
38.773 \\
39.120 \\
39.307 \\
39.514 \\
39.764 \\
39.639 \\
39.905 \\
40.107 \\
10.715 \\
40.808 \\
41.314 \\
41.449 \\
41.791 \\
42.025 \\
41.705 \\
42.339 \\
42.355 \\
42.495 \\
42.969 \\
43.204 \\
43.102 \\
43.231 \\
43.593 \\
43.597 \\
14.195 \\
41.550\end{array}$ & $\begin{array}{l}355 \\
360 \\
365 \\
370 \\
375 \\
380 \\
385 \\
390 \\
395 \\
400 \\
405 \\
110 \\
415 \\
420 \\
425 \\
430 \\
135 \\
440 \\
445 \\
450 \\
455 \\
460 \\
465 \\
470 \\
475 \\
480 \\
485 \\
190 \\
495 \\
500 \\
505 \\
510 \\
515 \\
520 \\
525 \\
530 \\
535 \\
540 \\
545 \\
550 \\
5555 \\
560 \\
565 \\
570 \\
575 \\
580 \\
595 \\
590 \\
595 \\
600 \\
605 \\
610 \\
315 \\
620 \\
625 \\
630 \\
335 \\
640 \\
645 \\
650 \\
6555 \\
660 \\
6655 \\
670 \\
675 \\
680 \\
6950 \\
695 \\
700\end{array}$ & $\begin{array}{l}44.458 \\
44.504 \\
14.902 \\
45.378 \\
45.525 \\
45.548 \\
45.791 \\
16.006 \\
46.546 \\
46.824 \\
45.948 \\
47.243 \\
47.325 \\
47.598 \\
47.823 \\
47.810 \\
48.095 \\
48.509 \\
48.450 \\
48.417 \\
49.157 \\
19.038 \\
49.379 \\
19.738 \\
49.814 \\
19.821 \\
50.130 \\
50.279 \\
50.236 \\
50.272 \\
50.393 \\
50.479 \\
50.912 \\
50.985 \\
52.534 \\
53.321 \\
53.162 \\
53.554 \\
53.530 \\
53.683 \\
53.822 \\
53.562 \\
53.458 \\
53.887 \\
53.911 \\
54.059 \\
53.969 \\
53.675 \\
54.825 \\
55.201 \\
55.551 \\
55.906 \\
56.029 \\
56.821 \\
57.486 \\
58.722 \\
59.077 \\
59.176 \\
59.037 \\
59.246 \\
59.265 \\
59.437 \\
59.215 \\
59.578 \\
59.125 \\
59.236 \\
59.027 \\
59.346 \\
59.165 \\
58.850\end{array}$ & $\begin{array}{l}705 \\
710 \\
715 \\
720 \\
725 \\
730 \\
735 \\
740 \\
745 \\
750 \\
755 \\
760 \\
765 \\
770 \\
775 \\
780 \\
785 \\
790 \\
795 \\
800 \\
805 \\
810 \\
815 \\
820 \\
825 \\
830 \\
835 \\
810 \\
815 \\
850 \\
855 \\
860 \\
865 \\
870\end{array}$ & $\begin{array}{l}59.116 \\
59.306 \\
59.296 \\
59.246 \\
59.527 \\
59.186 \\
59.216 \\
59.126 \\
60.441 \\
60.220 \\
61.086 \\
61.022 \\
61.140 \\
59.629 \\
57.013 \\
55.270 \\
56.377 \\
56.648 \\
62.233 \\
62.581 \\
64.573 \\
63.361 \\
60.757 \\
60.979 \\
61.086 \\
60.119 \\
58.130 \\
56.422 \\
57.328 \\
58.849 \\
62.570 \\
63.337 \\
63.747 \\
63.735\end{array}$ \\
\hline
\end{tabular}


RAFT RIVEP, IDAHD - RRGE-1 - 13 JAMUAFY 1975

\begin{tabular}{|c|c|c|c|c|c|}
\hline $\begin{array}{l}\text { DEPTH } \\
\text { (FT) }\end{array}$ & $\begin{array}{l}\text { TEPPERATLRE } \\
\text { (C) }\end{array}$ & $\begin{array}{l}\text { DEPTH } \\
\text { (FT) }\end{array}$ & $\begin{array}{l}\text { TEPERATLRE } \\
\text { (C) }\end{array}$ & $\begin{array}{c}\text { DEPTH } \\
\text { (FT) }\end{array}$ & $\begin{array}{l}\text { TEMPERATUF } \\
\text { (C) }\end{array}$ \\
\hline $\begin{array}{l}5 \\
10 \\
15 \\
20 \\
25 \\
30 \\
35 \\
40 \\
45 \\
50 \\
55 \\
60 \\
65 \\
70 \\
75 \\
80 \\
85 \\
90 \\
95 \\
100 \\
105 \\
110 \\
115 \\
120 \\
125 \\
130 \\
135 \\
140 \\
145 \\
150 \\
155 \\
160 \\
165 \\
170 \\
175 \\
180 \\
185 \\
190 \\
195 \\
200 \\
205 \\
210 \\
215 \\
220 \\
225 \\
230 \\
235 \\
240 \\
245 \\
250 \\
255 \\
260 \\
265 \\
270 \\
275 \\
280 \\
285 \\
290 \\
295 \\
300 \\
305 \\
310 \\
315 \\
320 \\
325 \\
330 \\
335 \\
340 \\
345 \\
350\end{array}$ & 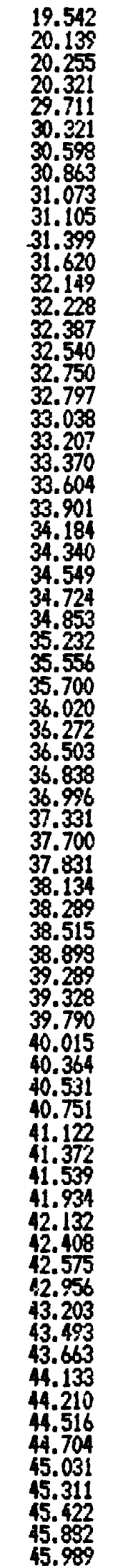 & $\begin{array}{l}355 \\
360 \\
365 \\
370 \\
375 \\
380 \\
385 \\
390 \\
395 \\
400 \\
405 \\
110 \\
415 \\
420 \\
425 \\
130 \\
435 \\
440 \\
445 \\
450 \\
455 \\
460 \\
465 \\
470 \\
475 \\
480 \\
485 \\
490 \\
495 \\
500 \\
505 \\
510 \\
515 \\
520 \\
525 \\
530 \\
538 \\
540 \\
545 \\
550 \\
555 \\
560 \\
565 \\
570 \\
575 \\
580 \\
585 \\
590 \\
575 \\
600 \\
605 \\
610 \\
615 \\
620 \\
625 \\
630 \\
635 \\
640 \\
345 \\
650 \\
655 \\
660 \\
655 \\
670 \\
675 \\
680 \\
6855 \\
690 \\
695 \\
700\end{array}$ & 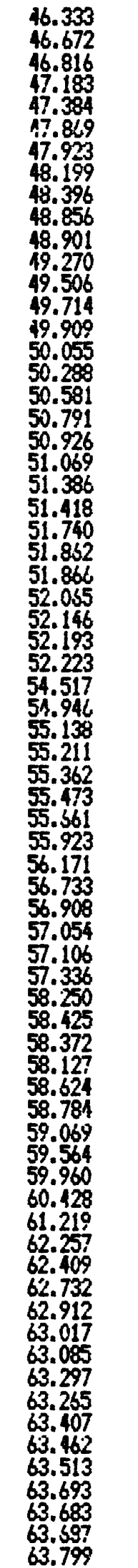 & $\begin{array}{l}705 \\
710 \\
715 \\
720 \\
725 \\
730 \\
735 \\
740 \\
745 \\
750 \\
755 \\
760 \\
765 \\
770 \\
775 \\
780 \\
785 \\
790 \\
795 \\
800\end{array}$ & $\begin{array}{l}64.009 \\
64.091 \\
64.133 \\
64.281 \\
64.698 \\
64.737 \\
65.477 \\
65.286 \\
65.318 \\
64.528 \\
64.325 \\
63.378 \\
62.747 \\
62.709 \\
62.690 \\
62.675 \\
52.665 \\
62.654 \\
62.650 \\
62.647\end{array}$ \\
\hline
\end{tabular}


RAFT RIVER, IDAHD - RRGE-1 - 15 JALARY 1975

\begin{tabular}{|c|c|c|c|c|c|}
\hline $\begin{array}{l}\text { DEPTH } \\
\text { (FT) }\end{array}$ & $\begin{array}{l}\text { TEMPERATLRE } \\
\text { (C) }\end{array}$ & $\begin{array}{l}\text { OEPTH } \\
\text { (FT) }\end{array}$ & $\begin{array}{l}\text { TERPERATLRE } \\
\text { (C) }\end{array}$ & $\begin{array}{l}\text { DEPTH } \\
\text { (FT) }\end{array}$ & $\begin{array}{l}\text { TEMPERATU } \\
\text { (C) }\end{array}$ \\
\hline $\begin{array}{l}5 \\
10 \\
15 \\
20 \\
25 \\
30 \\
35 \\
40 \\
45 \\
50 \\
55 \\
60 \\
55 \\
70 \\
75 \\
80 \\
85 \\
90 \\
95 \\
100 \\
105 \\
110 \\
115 \\
120 \\
125 \\
130 \\
135 \\
140 \\
145 \\
150 \\
155 \\
160 \\
165 \\
170 \\
175 \\
180 \\
185 \\
190 \\
195 \\
200 \\
205 \\
210 \\
215 \\
220 \\
225 \\
230 \\
235 \\
240 \\
245 \\
250 \\
255 \\
260 \\
265 \\
270 \\
275 \\
280 \\
290 \\
295 \\
300 \\
305 \\
310 \\
315 \\
320 \\
325 \\
330 \\
335 \\
340 \\
345 \\
350 \\
355\end{array}$ & $\begin{array}{l}22.108 \\
22.326 \\
22.741 \\
23.325 \\
23.848 \\
24.183 \\
24.504 \\
25.019 \\
25.449 \\
25.824 \\
.26 .298 \\
26.713 \\
27.256 \\
27.811 \\
27.981 \\
28.346 \\
28.709 \\
29.427 \\
29.823 \\
30.157 \\
30.625 \\
30.883 \\
31.370 \\
31.822 \\
34.976 \\
35.348 \\
35.976 \\
36.018 \\
36.081 \\
36.294 \\
36.414 \\
36.621 \\
36.757 \\
37.167 \\
37.214 \\
37.533 \\
37.914 \\
38.094 \\
38.374 \\
33.636 \\
38.955 \\
39.151 \\
39.383 \\
39.957 \\
30.986 \\
40.477 \\
40.790 \\
41.095 \\
41.339 \\
41.562 \\
42.023 \\
12.120 \\
42.333 \\
42.891 \\
43.227 \\
43.663 \\
14.110 \\
14.528 \\
44.865 \\
44.884 \\
45.341 \\
45.628 \\
45.985 \\
46.153 \\
46.504 \\
16.811 \\
47.106 \\
47.386 \\
47.555 \\
47.957\end{array}$ & $\begin{array}{l}350 \\
365 \\
370 \\
375 \\
380 \\
385 \\
390 \\
395 \\
400 \\
405 \\
410 \\
415 \\
420 \\
425 \\
430 \\
435 \\
440 \\
445 \\
450 \\
455 \\
460 \\
165 \\
170 \\
475 \\
480 \\
485 \\
490 \\
495 \\
500 \\
505 \\
510 \\
515 \\
520 \\
525 \\
530 \\
535 \\
540 \\
515 \\
550 \\
555 \\
560 \\
565 \\
570 \\
575 \\
590 \\
585 \\
590 \\
595 \\
600 \\
605 \\
610 \\
615 \\
320 \\
625 \\
630 \\
635 \\
640 \\
615 \\
650 \\
655 \\
660 \\
665 \\
670 \\
675 \\
680 \\
685 \\
690 \\
695 \\
700 \\
705\end{array}$ & 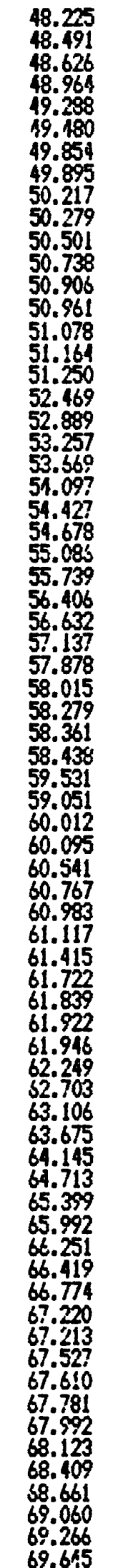 & $\begin{array}{l}710 \\
715 \\
720 \\
725 \\
730 \\
735 \\
740 \\
745 \\
750 \\
755 \\
760 \\
765 \\
770 \\
775 \\
780 \\
785 \\
790 \\
795\end{array}$ & $\begin{array}{l}69.833 \\
70.080 \\
70.381 \\
70.821 \\
70.857 \\
70.640 \\
71.054 \\
71.689 \\
71.999 \\
72.001 \\
71.993 \\
71.697 \\
71.125 \\
71.370 \\
71.953 \\
72.831 \\
72.845 \\
72.856\end{array}$ \\
\hline
\end{tabular}


RAFT RIVER, IDAHD - RRGE-1 - 13 OCTOBER 1975

\begin{tabular}{|c|c|c|c|c|c|}
\hline $\begin{array}{l}\text { DEPTH } \\
\text { (FT) }\end{array}$ & $\begin{array}{l}\text { TEMPERATURE } \\
\text { (C) }\end{array}$ & $\begin{array}{l}\text { DEPTH } \\
\text { (FT) }\end{array}$ & $\begin{array}{l}\text { TEPSERATURE } \\
\text { (C) }\end{array}$ & $\begin{array}{c}\text { DEPTH } \\
\text { (FT) }\end{array}$ & $\begin{array}{l}\text { TEMPERATLR } \\
\text { (C) }\end{array}$ \\
\hline $\begin{array}{l}5 \\
10 \\
15 \\
20 \\
25 \\
30 \\
35 \\
40 \\
45 \\
50 \\
53 \\
55 \\
58 \\
60 \\
53 \\
65 \\
38 \\
70 \\
73 \\
75 \\
78 \\
80 \\
83 \\
85 \\
88 \\
90 \\
93 \\
95 \\
99 \\
100 \\
103 \\
105 \\
108 \\
110 \\
113 \\
115 \\
118 \\
120 \\
123 \\
125 \\
128 \\
130 \\
133 \\
135 \\
138 \\
140 \\
145 \\
148 \\
150 \\
153 \\
155 \\
158 \\
160 \\
163 \\
155 \\
168 \\
170 \\
173 \\
175 \\
178 \\
180 \\
185 \\
188 \\
190 \\
193 \\
195 \\
198 \\
200 \\
203 \\
205\end{array}$ & $\begin{array}{l}18.071 \\
18.996 \\
19.580 \\
20.172 \\
20.617 \\
21.272 \\
21.939 \\
22.628 \\
23.120 \\
23.817 \\
.24 .204 \\
24.616 \\
21.876 \\
25.014 \\
25.397 \\
25.757 \\
25.952 \\
26.250 \\
26.564 \\
26.849 \\
27.246 \\
27.591 \\
27.678 \\
28.095 \\
28.380 \\
28.595 \\
28.915 \\
29.237 \\
29.483 \\
29.918 \\
30.093 \\
30.293 \\
30.697 \\
30.993 \\
31.339 \\
31.575 \\
31.735 \\
31.953 \\
32.375 \\
32.568 \\
32.836 \\
33.269 \\
33.400 \\
33.746 \\
34.011 \\
34.372 \\
34.862 \\
35.327 \\
35.489 \\
35.657 \\
35.998 \\
36.080 \\
35.438 \\
36.795 \\
37.036 \\
37.258 \\
37.578 \\
37.842 \\
38.040 \\
38.328 \\
38.5559 \\
39.126 \\
39.355 \\
39.539 \\
39.916 \\
40.143 \\
40.455 \\
40.5877 \\
40.975 \\
41.202\end{array}$ & $\begin{array}{l}208 \\
210 \\
213 \\
215 \\
218 \\
220 \\
223 \\
225 \\
228 \\
233 \\
235 \\
238 \\
240 \\
243 \\
245 \\
218 \\
250 \\
253 \\
255 \\
258 \\
260 \\
263 \\
265 \\
268 \\
270 \\
273 \\
275 \\
278 \\
280 \\
283 \\
285 \\
288 \\
290 \\
293 \\
295 \\
298 \\
300 \\
303 \\
305 \\
308 \\
310 \\
313 \\
315 \\
318 \\
320 \\
323 \\
325 \\
328 \\
330 \\
333 \\
335 \\
338 \\
340 \\
343 \\
345 \\
348 \\
350 \\
353 \\
355 \\
358 \\
360 \\
363 \\
335 \\
368 \\
370 \\
373 \\
375 \\
379 \\
330 \\
323 \\
\end{array}$ & 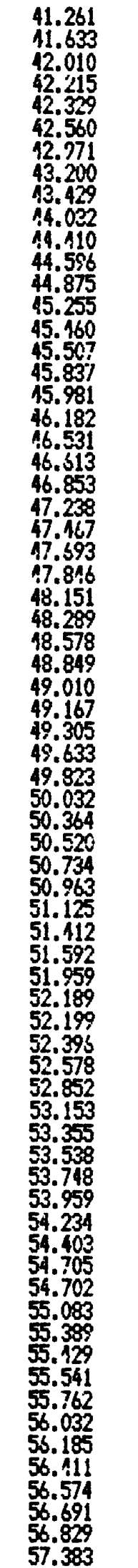 & $\begin{array}{l}385 \\
388 \\
390 \\
396 \\
400 \\
403 \\
405 \\
408 \\
110 \\
413 \\
115 \\
420 \\
423 \\
130 \\
435 \\
440 \\
445 \\
450 \\
455 \\
460 \\
465 \\
470 \\
475 \\
480 \\
485 \\
490 \\
495 \\
500 \\
505 \\
510 \\
515 \\
526 \\
525 \\
530 \\
535 \\
540 \\
545 \\
550 \\
5555 \\
560 \\
565 \\
570 \\
575 \\
580 \\
585 \\
590 \\
595 \\
600 \\
305 \\
610 \\
615 \\
620 \\
625 \\
630 \\
635 \\
640\end{array}$ & $\begin{array}{l}57.393 \\
57.593 \\
57.633 \\
58.175 \\
58.567 \\
58.788 \\
59.037 \\
59.163 \\
59.348 \\
59.086 \\
59.727 \\
60.154 \\
60.544 \\
60.876 \\
61.164 \\
61.555 \\
61.965 \\
62.335 \\
52.367 \\
63.119 \\
63.506 \\
63.850 \\
64.145 \\
61.554 \\
54.843 \\
65.152 \\
65.771 \\
65.967 \\
56.129 \\
66.763 \\
67.138 \\
67.487 \\
67.866 \\
68.236 \\
68.578 \\
68.929 \\
60.293 \\
69.622 \\
69.962 \\
70.354 \\
70.634 \\
71.153 \\
71.485 \\
71.940 \\
72.235 \\
72.597 \\
72.946 \\
73.370 \\
73.716 \\
74.423 \\
75.002 \\
75.505 \\
76.024 \\
76.532 \\
75.969 \\
77.3399\end{array}$ \\
\hline
\end{tabular}


RAFT RIVER, IDAHD - RRGE-1 - 16 OCTOEER 1975

\begin{tabular}{|c|c|c|c|c|c|c|c|c|c|c|c|}
\hline $\begin{array}{c}\text { DEPTH } \\
\text { (FT) }\end{array}$ & $\begin{array}{l}\text { TEPPER TTUPE } \\
(C)\end{array}$ & $\begin{array}{l}\text { DEPTH } \\
\text { (FT) }\end{array}$ & $\begin{array}{l}\text { TERPEKATURE } \\
\text { (C) }\end{array}$ & $\begin{array}{l}\text { DEPTH } \\
\text { (FT) }\end{array}$ & $\begin{array}{l}\text { TEMPERATURE } \\
\text { (C) }\end{array}$ & $\begin{array}{l}\text { DEPTH } \\
\text { (FT) }\end{array}$ & $\begin{array}{c}\text { TEMPERATLRE } \\
\text { (L) }\end{array}$ & $\begin{array}{l}\text { DEPTH } \\
\text { (FT) }\end{array}$ & $\begin{array}{l}\text { TEPFERATURE } \\
\text { (C) }\end{array}$ & $\begin{array}{l}\text { DEPTH } \\
\text { (FT) }\end{array}$ & $\begin{array}{l}\text { TEPPERAT } \\
\text { (C) }\end{array}$ \\
\hline $\begin{array}{l}5 \\
10 \\
15 \\
20 \\
25 \\
30 \\
35 \\
40 \\
15 \\
50 \\
55 \\
60 \\
65 \\
70 \\
75 \\
80 \\
85 \\
90 \\
95 \\
100 \\
105 \\
110 \\
115 \\
120 \\
125 \\
130 \\
135 \\
140 \\
145 \\
150 \\
155 \\
160 \\
165 \\
170 \\
175 \\
180 \\
185 \\
190 \\
195 \\
200 \\
205 \\
210 \\
215 \\
220 \\
225 \\
230 \\
235 \\
240 \\
245 \\
250 \\
255 \\
250 \\
265 \\
270 \\
275 \\
280 \\
285 \\
295 \\
300 \\
305 \\
310 \\
315 \\
320 \\
330 \\
335 \\
340 \\
345 \\
350\end{array}$ & $\begin{array}{l}19.674 \\
20.418 \\
20.569 \\
22.542 \\
22.748 \\
23.338 \\
23.870 \\
24.058 \\
24.737 \\
25.394 \\
25.944 \\
26.331 \\
27.110 \\
27.721 \\
28.231 \\
28.753 \\
29.330 \\
29.930 \\
30.533 \\
31.055 \\
31.524 \\
32.129 \\
32.741 \\
33.360 \\
33.895 \\
34.396 \\
34.934 \\
35.381 \\
36.172 \\
36.671 \\
31.110 \\
37.598 \\
38.078 \\
38.734 \\
39.304 \\
39.693 \\
40.281 \\
40.734 \\
41.298 \\
41.682 \\
42.219 \\
42.899 \\
43.305 \\
49.430 \\
43.847 \\
50.373 \\
50.820 \\
51.144 \\
51.587 \\
51.936 \\
52.376 \\
53.735 \\
45.331 \\
45.850 \\
45.839 \\
46.247 \\
46.690 \\
47.270 \\
47.536 \\
54.163 \\
54.540 \\
54.989 \\
55.351\end{array}$ & $\begin{array}{l}355 \\
360 \\
365 \\
370 \\
375 \\
380 \\
385 \\
390 \\
395 \\
400 \\
405 \\
410 \\
415 \\
420 \\
125 \\
430 \\
135 \\
440 \\
415 \\
450 \\
155 \\
130 \\
465 \\
470 \\
475 \\
480 \\
485 \\
490 \\
495 \\
500 \\
505 \\
510 \\
515 \\
520 \\
525 \\
530 \\
535 \\
540 \\
545 \\
550 \\
555 \\
560 \\
565 \\
570 \\
575 \\
590 \\
595 \\
540 \\
595 \\
600 \\
605 \\
610 \\
615 \\
620 \\
625 \\
630 \\
635 \\
640 \\
645 \\
650 \\
655 \\
660 \\
665 \\
670 \\
680 \\
685 \\
690 \\
695 \\
700\end{array}$ & 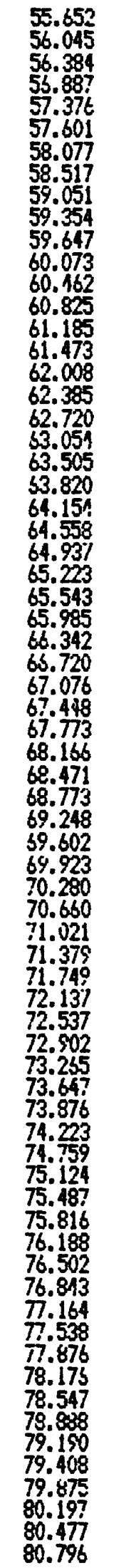 & 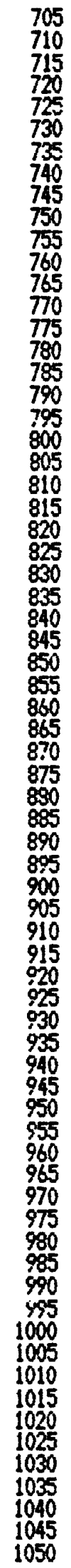 & 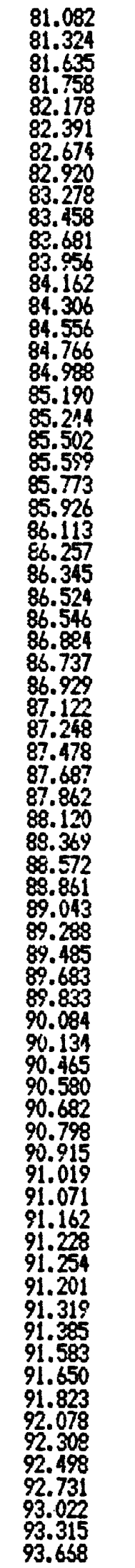 & $\begin{array}{l}1055 \\
1060 \\
1065 \\
1070 \\
1075 \\
1080 \\
1095 \\
1090 \\
1095 \\
1100 \\
1105 \\
1110 \\
1115 \\
1120 \\
1125 \\
1130 \\
1135 \\
1140 \\
1145 \\
1150 \\
1155 \\
1160 \\
1165 \\
1170 \\
1175 \\
1180 \\
1185 \\
1100 \\
1195 \\
1200 \\
1205 \\
1210 \\
1215 \\
1220 \\
1225 \\
1230 \\
1235 \\
1240 \\
1245 \\
1250 \\
1255 \\
1250 \\
1265 \\
1270 \\
1275 \\
1280 \\
1285 \\
1290 \\
1295 \\
1300 \\
1305 \\
1310 \\
1315 \\
1320 \\
1325 \\
1330 \\
1335 \\
1340 \\
1345 \\
1350 \\
1355 \\
1360 \\
1365 \\
1370 \\
1375 \\
1330 \\
1385 \\
1390 \\
1395 \\
1400\end{array}$ & 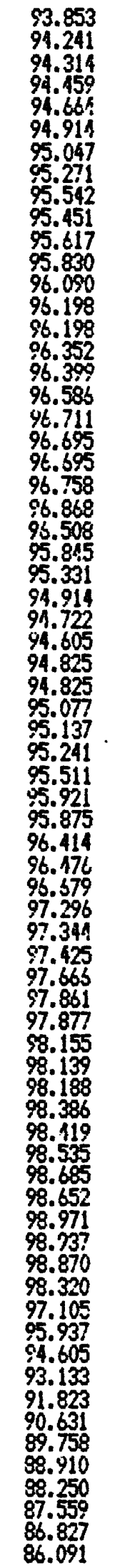 & $\begin{array}{l}1405 \\
1410 \\
1415 \\
1420 \\
1425 \\
1430 \\
1435 \\
1440 \\
1445 \\
1450 \\
1455 \\
1430 \\
1465 \\
1470 \\
1475 \\
1490 \\
1485 \\
1490 \\
1495 \\
1500 \\
1505 \\
1510 \\
1515 \\
1520 \\
1535 \\
1530 \\
1535 \\
1540 \\
1545 \\
1550 \\
1555 \\
1560 \\
1565 \\
1570 \\
1575 \\
1580 \\
1585 \\
1590 \\
1595 \\
1600 \\
1605 \\
1610 \\
1615 \\
1620 \\
1625 \\
1530 \\
1635 \\
1640 \\
1645 \\
1650 \\
1655 \\
1650 \\
1665 \\
1670 \\
1675 \\
1680 \\
1685 \\
1690 \\
1695 \\
1700 \\
1705 \\
1710 \\
1715 \\
1720 \\
1725 \\
1730 \\
1735 \\
1710 \\
1765 \\
1750\end{array}$ & $\begin{array}{l}85.126 \\
84.389 \\
83.520 \\
82.801 \\
82.073 \\
81.862 \\
81.910 \\
81.767 \\
81.767 \\
81.919 \\
82.014 \\
82.092 \\
82.372 \\
82.566 \\
82.625 \\
82.890 \\
83.238 \\
83.278 \\
83.600 \\
83.803 \\
84.151 \\
84.389 \\
84.861 \\
85.052 \\
85.655 \\
85.893 \\
86.315 \\
85.636 \\
87.054 \\
87.524 \\
87.571 \\
88.310 \\
89.116 \\
89.371 \\
89.596 \\
90.286 \\
90.631 \\
91.241 \\
91.636 \\
92.389 \\
92.911 \\
93.498 \\
94.154 \\
94.328 \\
91.488 \\
95.493 \\
95.967 \\
96.430 \\
96.994 \\
97.375 \\
97.812 \\
99.719 \\
99.106 \\
99.581 \\
100.315 \\
101.208 \\
101.353 \\
101.904 \\
102.767 \\
103.441 \\
104.069 \\
104.188 \\
104.468 \\
104.669 \\
105.240 \\
105.842 \\
106.221 \\
106.370 \\
106.929\end{array}$ & $\begin{array}{l}1755 \\
1760 \\
1765 \\
1770 \\
1775 \\
1780 \\
1785 \\
1740 \\
1795 \\
1800 \\
1805 \\
1910 \\
1815 \\
1820 \\
1825 \\
1830 \\
1835 \\
1840 \\
1845 \\
1850 \\
1855 \\
1860 \\
1865 \\
1870 \\
1875 \\
1980 \\
1805 \\
1890 \\
1895 \\
1900 \\
1905 \\
1910 \\
1915 \\
1920 \\
1925 \\
1930 \\
1935 \\
1940 \\
1945 \\
1950 \\
1955 \\
1960 \\
1945 \\
1970 \\
1975 \\
1980 \\
1985 \\
1990 \\
1995 \\
2000 \\
2005 \\
2010 \\
2015 \\
2020 \\
2025 \\
2030 \\
2035 \\
2040 \\
2045 \\
2050 \\
2055 \\
2060 \\
2065 \\
2070 \\
2075 \\
2080 \\
2085 \\
2090 \\
2095 \\
2100\end{array}$ & 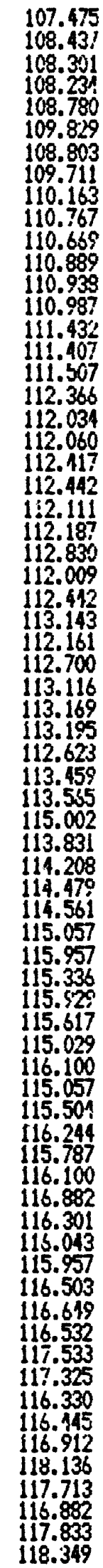 \\
\hline
\end{tabular}


RAFT RIVER, IDAHO - PRREE-1 - 16 OCTOBER 1975

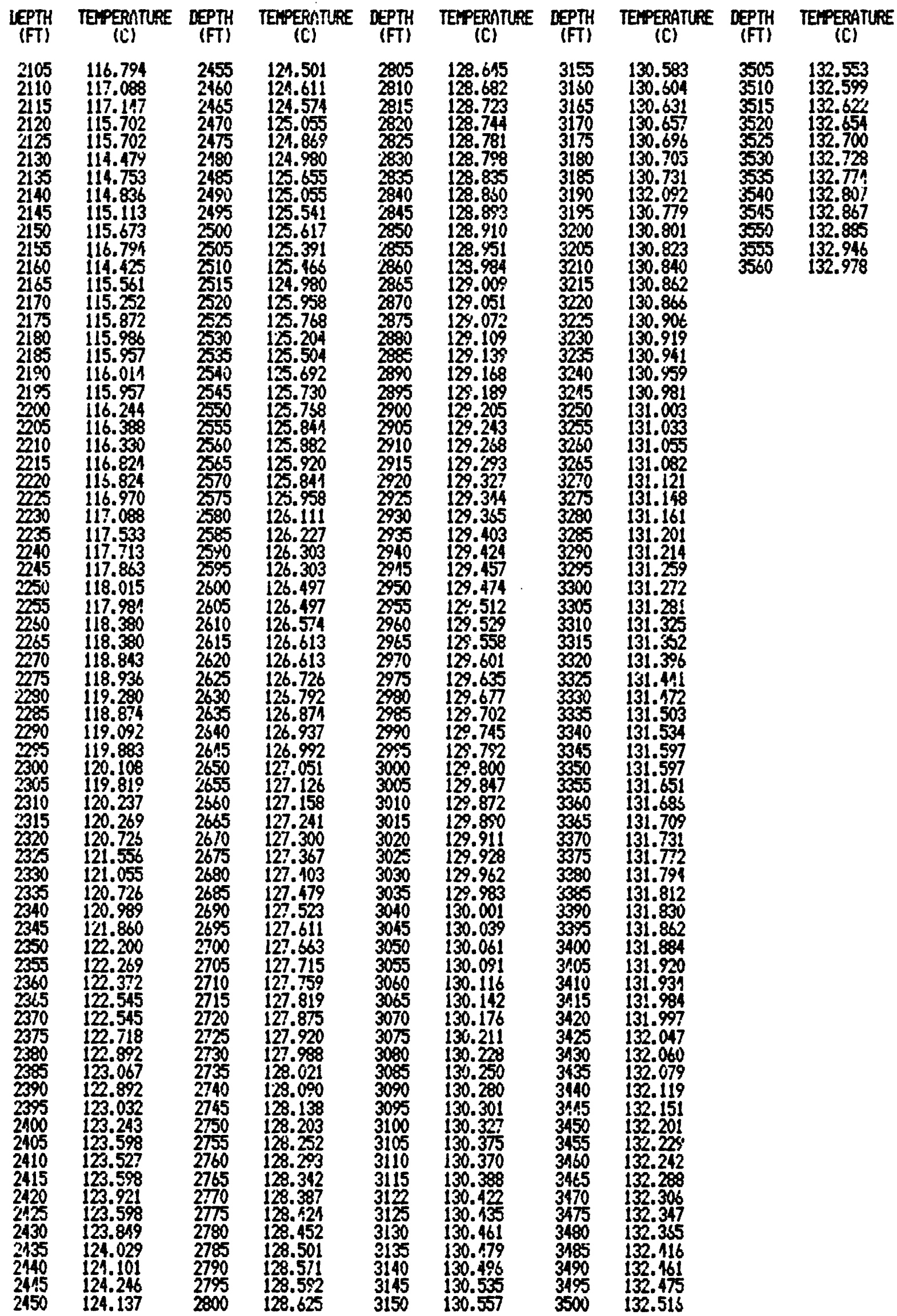


RAFT RIVER, IDAHO - RRGE-2 - 10 ALGUST 1976

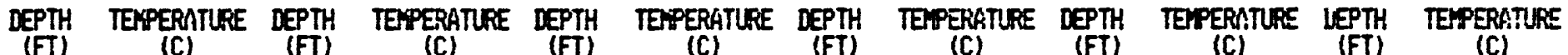

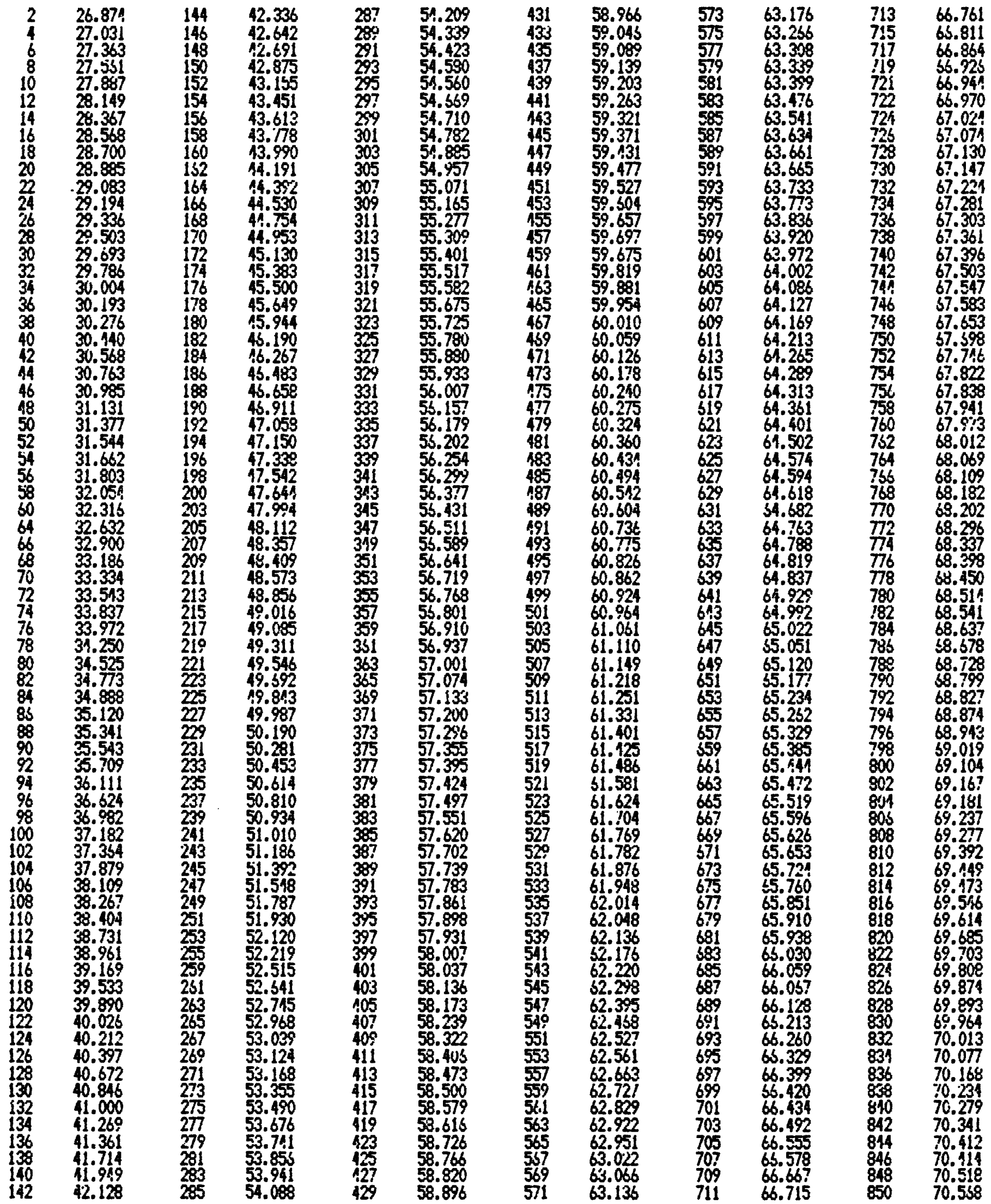


RAFT RIVER, IDAHO - RREE-2 - 10 AUCUST 1976

\begin{tabular}{|c|c|c|c|c|c|c|c|c|c|c|c|}
\hline $\begin{array}{l}\text { IEPTH } \\
\text { (FT) }\end{array}$ & $\begin{array}{l}\text { TEMPERATURE } \\
\text { (C) }\end{array}$ & $\begin{array}{c}\text { DEPTH } \\
\text { (FT) }\end{array}$ & $\begin{array}{l}\text { TEMPERATLRE } \\
\text { (C) }\end{array}$ & $\begin{array}{l}\text { DEPTH } \\
\text { (FT) }\end{array}$ & $\begin{array}{l}\text { TEPERATLRE } \\
\text { (C) }\end{array}$ & $\begin{array}{l}\text { DEPTH } \\
\text { (FT) }\end{array}$ & $\begin{array}{l}\text { TERPERATURE } \\
\text { (C) }\end{array}$ & $\begin{array}{c}\text { DEPTH } \\
\text { (FT) }\end{array}$ & $\begin{array}{l}\text { TEMPERATLHE } \\
\text { (C) }\end{array}$ & $\begin{array}{c}\text { IEPTH } \\
\text { (FT) }\end{array}$ & $\begin{array}{l}\text { TERPERPRT } \\
\text { (C) }\end{array}$ \\
\hline $\begin{array}{l}852 \\
854 \\
856 \\
958 \\
850 \\
862 \\
864 \\
866 \\
868 \\
870 \\
872 \\
874 \\
876 \\
878 \\
880 \\
882 \\
981 \\
896 \\
888 \\
890 \\
892 \\
804 \\
896 \\
898 \\
900 \\
902 \\
901 \\
906 \\
908 \\
910 \\
911 \\
913 \\
518 \\
920 \\
922 \\
924 \\
926 \\
930 \\
932 \\
934 \\
936 \\
938 \\
940 \\
942 \\
914 \\
945 \\
948 \\
950 \\
9552 \\
954 \\
956 \\
958 \\
960 \\
952 \\
964 \\
965 \\
968 \\
970 \\
972 \\
994 \\
976 \\
978 \\
990 \\
992 \\
991 \\
985 \\
998 \\
990 \\
992 \\
994\end{array}$ & 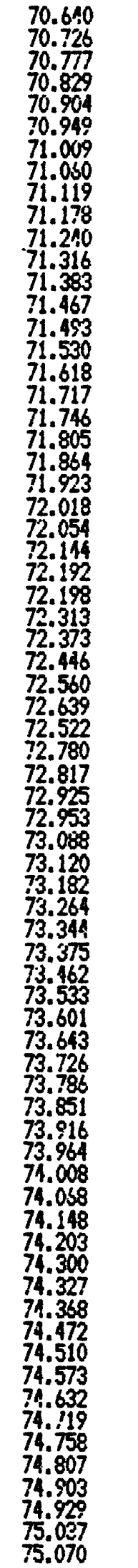 & $\begin{array}{l}998 \\
1000 \\
1002 \\
1004 \\
1006 \\
1008 \\
1010 \\
1012 \\
1014 \\
1016 \\
1018 \\
1020 \\
1024 \\
1026 \\
1028 \\
1030 \\
1032 \\
1034 \\
1036 \\
1038 \\
1040 \\
1042 \\
1044 \\
1045 \\
1048 \\
1050 \\
1052 \\
1054 \\
1056 \\
1058 \\
1060 \\
1062 \\
1064 \\
1065 \\
1068 \\
1070 \\
1072 \\
1074 \\
1076 \\
1079 \\
1080 \\
1092 \\
1094 \\
1086 \\
1088 \\
1090 \\
1092 \\
1095 \\
1098 \\
1100 \\
1102 \\
1104 \\
1106 \\
1108 \\
1110 \\
1112 \\
1114 \\
1116 \\
1118 \\
1120 \\
1122 \\
1124 \\
1126 \\
1128 \\
1130 \\
1133 \\
1134 \\
1136 \\
1138 \\
1140\end{array}$ & 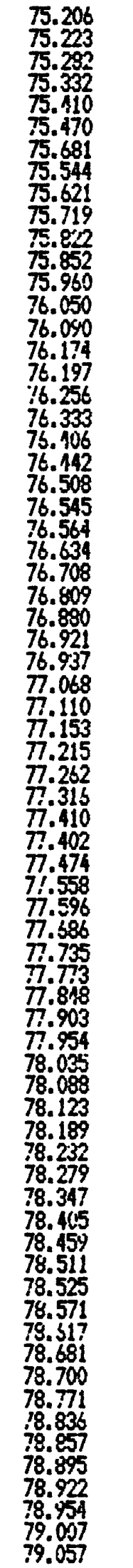 & 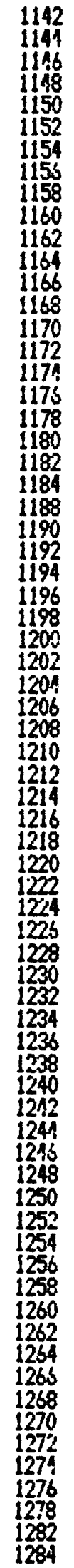 & 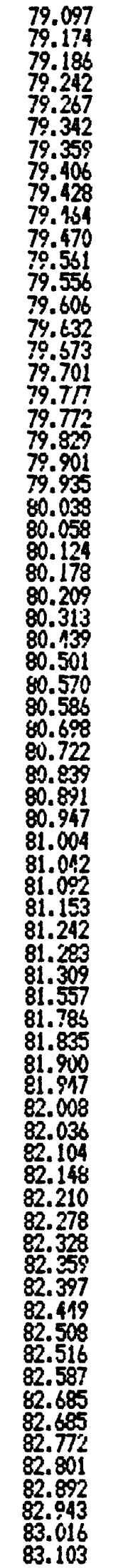 & $\begin{array}{l}1286 \\
1288 \\
1290 \\
1292 \\
1394 \\
1296 \\
1298 \\
1300 \\
1302 \\
1304 \\
1306 \\
1308 \\
1310 \\
1312 \\
1314 \\
1315 \\
1318 \\
1320 \\
1322 \\
1321 \\
1326 \\
1328 \\
1330 \\
1332 \\
1334 \\
1336 \\
1338 \\
1340 \\
1342 \\
1344 \\
1346 \\
1319 \\
1350 \\
1352 \\
1354 \\
1356 \\
1358 \\
1360 \\
1362 \\
1364 \\
1356 \\
1368 \\
1370 \\
1372 \\
1374 \\
1376 \\
1378 \\
1380 \\
13392 \\
1384 \\
1336 \\
1388 \\
1390 \\
1392 \\
1394 \\
1395 \\
1398 \\
1100 \\
1402 \\
1404 \\
1906 \\
1108 \\
1410 \\
1412 \\
1414 \\
1116 \\
1148 \\
1120 \\
1422 \\
1425\end{array}$ & 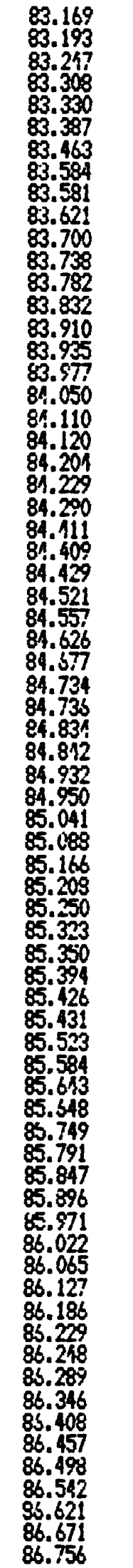 & $\begin{array}{l}1428 \\
1430 \\
1432 \\
1434 \\
1436 \\
1438 \\
1140 \\
1442 \\
1144 \\
1446 \\
1448 \\
1150 \\
1452 \\
1454 \\
1456 \\
1458 \\
1460 \\
1452 \\
1461 \\
1466 \\
1468 \\
1470 \\
1472 \\
1171 \\
1476 \\
1478 \\
1480 \\
1492 \\
1481 \\
1186 \\
1488 \\
1190 \\
1492 \\
1494 \\
1196 \\
1498 \\
1500 \\
1502 \\
1504 \\
1505 \\
1508 \\
1510 \\
1512 \\
1514 \\
1516 \\
1518 \\
1520 \\
1522 \\
1524 \\
1526 \\
1528 \\
1530 \\
1532 \\
1534 \\
1536 \\
1538 \\
1540 \\
1542 \\
1544 \\
1554 \\
1548 \\
1550 \\
1552 \\
1554 \\
1556 \\
1558 \\
1560 \\
1552 \\
1564 \\
15366\end{array}$ & 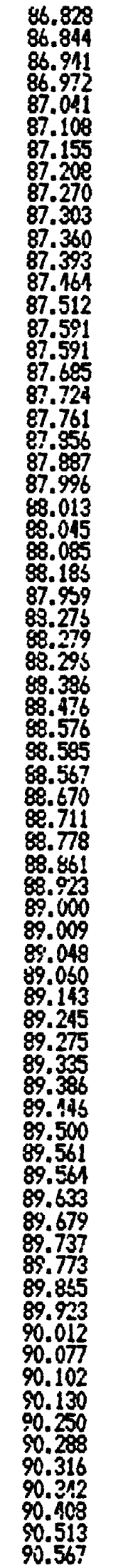 & $\begin{array}{l}1568 \\
1570 \\
1572 \\
1574 \\
1576 \\
1578 \\
1580 \\
1582 \\
1584 \\
1586 \\
1588 \\
1590 \\
1592 \\
1594 \\
1598 \\
1500 \\
1602 \\
1604 \\
1606 \\
1508 \\
1610 \\
1612 \\
1614 \\
1516 \\
1618 \\
1520 \\
1622 \\
1524 \\
1624 \\
1628 \\
1630 \\
1532 \\
1634 \\
1636 \\
1638 \\
1540 \\
1642 \\
1644 \\
1646 \\
1648 \\
1650 \\
1552 \\
1654 \\
1654 \\
1658 \\
1660 \\
1662 \\
1644 \\
1666 \\
1568 \\
1670 \\
1672 \\
1674 \\
1675 \\
1678 \\
1580 \\
1682 \\
1584 \\
1686 \\
1598 \\
1640 \\
1692 \\
1694 \\
1696 \\
1698 \\
1700 \\
1702 \\
1704 \\
1706 \\
1708\end{array}$ & 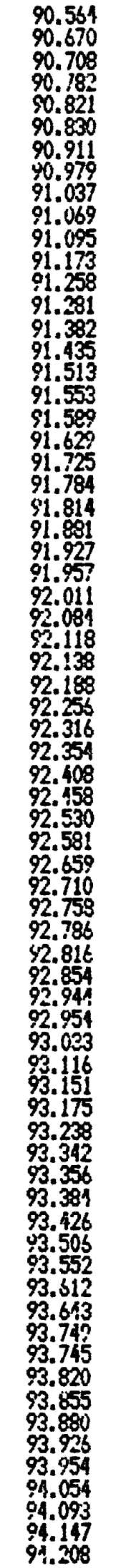 \\
\hline
\end{tabular}


RAFT RIVER, IDAHD - RRGE-2 - 10 AUGUST 1976

\begin{tabular}{|c|c|c|c|c|c|c|c|c|c|c|c|}
\hline $\begin{array}{l}\text { IEPTH } \\
\text { (FT) }\end{array}$ & $\begin{array}{l}\text { TEMPERATUPE } \\
\text { (C) }\end{array}$ & $\begin{array}{l}\text { DEPTH } \\
\text { (FT) }\end{array}$ & $\begin{array}{l}\text { TEMPERATURE } \\
\text { (C) }\end{array}$ & $\begin{array}{l}\text { IEPTH } \\
\text { (FT) }\end{array}$ & $\begin{array}{l}\text { IERFERFTLPE } \\
\text { (C) }\end{array}$ & $\begin{array}{l}\text { DEPTH } \\
\text { (FT) }\end{array}$ & $\begin{array}{l}\text { TEMPERATURE } \\
\text { (C) }\end{array}$ & $\begin{array}{l}\text { DEPTH } \\
\text { (FT) }\end{array}$ & $\begin{array}{l}\text { TEPERATLEC } \\
\text { (C) }\end{array}$ & $\begin{array}{l}\text { LEPTH } \\
\text { (FT) }\end{array}$ & $\begin{array}{l}\text { TERPERATURE } \\
\text { (C) }\end{array}$ \\
\hline $\begin{array}{l}1710 \\
1712 \\
1714 \\
1716 \\
1718 \\
1720 \\
1722 \\
1724 \\
1726 \\
1728 \\
1730 \\
1732 \\
1734 \\
1736 \\
1738 \\
1740 \\
1742 \\
1744 \\
1746 \\
1748 \\
1750 \\
1752 \\
1754 \\
1755 \\
1758 \\
1750 \\
1762 \\
1754 \\
1764 \\
1758 \\
1770 \\
1772 \\
1774 \\
1776 \\
1778 \\
1780 \\
1782 \\
1784 \\
1786 \\
1798 \\
1790 \\
1792 \\
1791 \\
1796 \\
1798 \\
1800 \\
1802 \\
1804 \\
1806 \\
1808 \\
1810 \\
1812 \\
1814 \\
1816 \\
1820 \\
1822 \\
1821 \\
1825 \\
1828 \\
1830 \\
1832 \\
1831 \\
1834 \\
1838 \\
1840 \\
1842 \\
1814 \\
1814 \\
1848 \\
1850\end{array}$ & 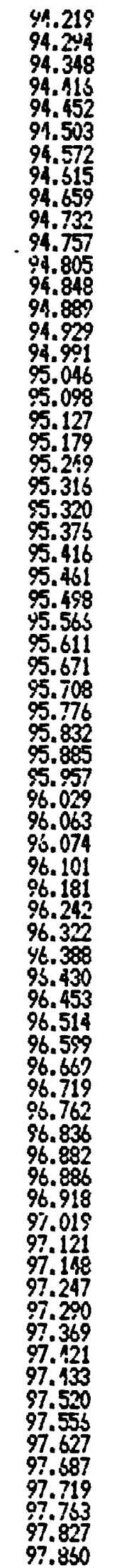 & $\begin{array}{l}1852 \\
1854 \\
1856 \\
1858 \\
1860 \\
1852 \\
1861 \\
1865 \\
1868 \\
1870 \\
1872 \\
1874 \\
1876 \\
1978 \\
1880 \\
1882 \\
1881 \\
1986 \\
1889 \\
1890 \\
1892 \\
1894 \\
1896 \\
1898 \\
1900 \\
1902 \\
1904 \\
1906 \\
1908 \\
1910 \\
1912 \\
1014 \\
1916 \\
1918 \\
1920 \\
1920 \\
1924 \\
1926 \\
1528 \\
1930 \\
1932 \\
1934 \\
1934 \\
1938 \\
1942 \\
1944 \\
1946 \\
1948 \\
1950 \\
1952 \\
1954 \\
1953 \\
1958 \\
1960 \\
1962 \\
1964 \\
1966 \\
1968 \\
1970 \\
1972 \\
1974 \\
1976 \\
1978 \\
1990 \\
1982 \\
1984 \\
1986 \\
1988 \\
1990 \\
1992 \\
\end{array}$ & 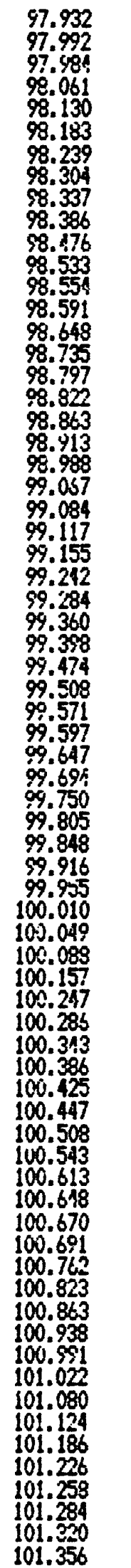 & $\begin{array}{l}1994 \\
1996 \\
1998 \\
2000 \\
2002 \\
2004 \\
2006 \\
2008 \\
2012 \\
2011 \\
2016 \\
2018 \\
2020 \\
2022 \\
2024 \\
2026 \\
2028 \\
2030 \\
2032 \\
2034 \\
2036 \\
2038 \\
2040 \\
2042 \\
2044 \\
2046 \\
2048 \\
2050 \\
2052 \\
2054 \\
2056 \\
2058 \\
2062 \\
2064 \\
2066 \\
2068 \\
2070 \\
2072 \\
2071 \\
2076 \\
2078 \\
2080 \\
2082 \\
2084 \\
2086 \\
2098 \\
2090 \\
2092 \\
2094 \\
2096 \\
2098 \\
2100 \\
2102 \\
2104 \\
2106 \\
2108 \\
2110 \\
2112 \\
2114 \\
2115 \\
2118 \\
2120 \\
2122 \\
2124 \\
2126 \\
2128 \\
2130 \\
2132 \\
2134 \\
2136\end{array}$ & $\begin{array}{l}101.387 \\
101.477 \\
101.526 \\
101.571 \\
101.558 \\
101.630 \\
101.666 \\
101.713 \\
101.820 \\
101.855 \\
101.879 \\
101.015 \\
101.966 \\
102.020 \\
102.029 \\
102.075 \\
102.114 \\
102.171 \\
102.213 \\
102.236 \\
102.314 \\
102.328 \\
102.355 \\
102.415 \\
102.438 \\
102.489 \\
102.526 \\
102.559 \\
102.531 \\
102.633 \\
102.712 \\
102.735 \\
102.815 \\
102.857 \\
102.857 \\
102.914 \\
102.942 \\
103.008 \\
103.031 \\
103.097 \\
103.116 \\
103.154 \\
103.177 \\
103.220 \\
103.202 \\
103.301 \\
103.328 \\
103.377 \\
103.420 \\
103.434 \\
103.472 \\
103.525 \\
103.582 \\
103.520 \\
103.654 \\
103.597 \\
103.731 \\
103.734 \\
103.813 \\
103.851 \\
103.504 \\
103.948 \\
103.996 \\
104.016 \\
104.040 \\
101.103 \\
104.108 \\
104.167 \\
104.211 \\
104.250\end{array}$ & 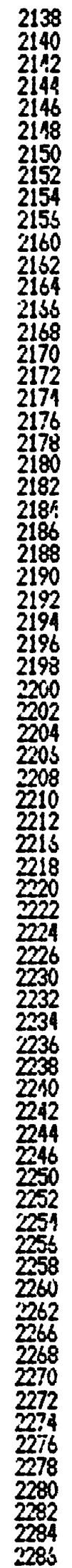 & 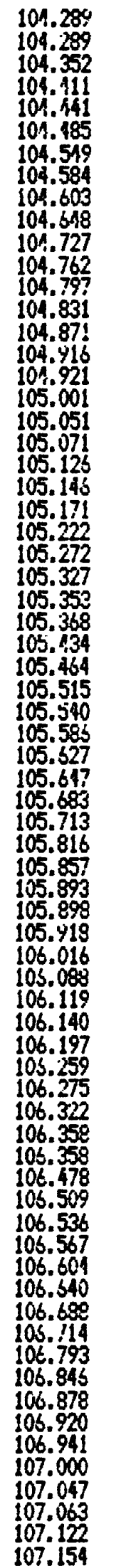 & 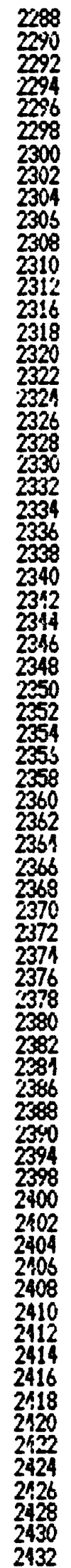 & $\begin{array}{l}107.181 \\
107.223 \\
107.239 \\
107.293 \\
107.336 \\
107.368 \\
107.127 \\
107.465 \\
107.470 \\
107.556 \\
107.567 \\
107.332 \\
107.637 \\
107.751 \\
107.800 \\
107.800 \\
107.851 \\
107.914 \\
107.931 \\
107.964 \\
108.013 \\
108.040 \\
108.051 \\
108.095 \\
108.114 \\
108.210 \\
108.238 \\
108.332 \\
108.354 \\
108.370 \\
108.420 \\
108.412 \\
108.492 \\
108.537 \\
108.548 \\
108.515 \\
108.659 \\
108.659 \\
108.732 \\
109.799 \\
108.938 \\
108.861 \\
108.945 \\
108.945 \\
108.979 \\
109.030 \\
109.109 \\
109.114 \\
108.171 \\
109.199 \\
109.256 \\
109.364 \\
109.432 \\
109.427 \\
109.501 \\
109.530 \\
109.558 \\
109.321 \\
109.673 \\
109.702 \\
109.775 \\
109.748 \\
109.829 \\
109.858 \\
109.927 \\
109.915 \\
109.950 \\
110.014 \\
110.055 \\
110.095\end{array}$ & $\begin{array}{l}2431 \\
2436 \\
2438 \\
2440 \\
2142 \\
2441 \\
2446 \\
2448 \\
2450 \\
2452 \\
2451 \\
2456 \\
2458 \\
2150 \\
2162 \\
2454 \\
2466 \\
2168 \\
2470 \\
2172 \\
2171 \\
2476 \\
2478 \\
2190 \\
2482 \\
2481 \\
2486 \\
2488 \\
2190 \\
2192 \\
2491 \\
2498 \\
2490 \\
2500 \\
2502 \\
2504 \\
2506 \\
2508 \\
2512 \\
2514 \\
2516 \\
2518 \\
2320 \\
2522 \\
2521 \\
2526 \\
2528 \\
2530 \\
2532 \\
2531 \\
2536 \\
2533 \\
2540 \\
2542 \\
2541 \\
2543 \\
2548 \\
2550 \\
2552 \\
2554 \\
2556 \\
2558 \\
2560 \\
2562 \\
2564 \\
2566 \\
2568 \\
2570 \\
2572 \\
2574\end{array}$ & $\begin{array}{l}110.148 \\
110.206 \\
110.241 \\
110.259 \\
110.300 \\
110.364 \\
110.382 \\
110.435 \\
110.476 \\
110.517 \\
110.529 \\
110.535 \\
110.612 \\
110.547 \\
110.724 \\
110.754 \\
110.778 \\
110.819 \\
110.85 \\
110.937 \\
110.950 \\
110.980 \\
111.010 \\
111.070 \\
111.082 \\
111.130 \\
111.166 \\
111.202 \\
111.244 \\
111.271 \\
111.329 \\
111.359 \\
111.707 \\
111.431 \\
111.180 \\
111.534 \\
111.541 \\
111.501 \\
111.674 \\
111.381 \\
111.723 \\
111.748 \\
111.772 \\
111.821 \\
111.864 \\
111.919 \\
111.956 \\
112.055 \\
112.036 \\
112.057 \\
112.110 \\
112.135 \\
112.185 \\
112.222 \\
112.216 \\
112.281 \\
112.315 \\
112.358 \\
112.383 \\
112.433 \\
112.961 \\
112.499 \\
112.539 \\
112.571 \\
112.627 \\
112.645 \\
112.702 \\
112.709 \\
112.759 \\
112.803\end{array}$ \\
\hline
\end{tabular}


RAFT RIVER, IDAHD - RREE-2 - 10 IUUGST 1976

\begin{tabular}{|c|c|c|c|c|c|c|c|c|c|c|c|}
\hline $\begin{array}{l}\text { IEPTH } \\
\text { (FT) }\end{array}$ & $\begin{array}{l}\text { TEXPERATLRE } \\
\text { (C) }\end{array}$ & $\begin{array}{l}\text { DEPTH } \\
\text { (FT) }\end{array}$ & $\begin{array}{l}\text { TEMPERATLRE } \\
\text { (C) }\end{array}$ & $\begin{array}{l}\text { DEPTH } \\
\text { (FT) }\end{array}$ & $\begin{array}{l}\text { TENPERATURE } \\
\text { (C) }\end{array}$ & $\begin{array}{l}\text { DEPTH } \\
\text { (FT) }\end{array}$ & $\begin{array}{l}\text { TEYPERATLRE } \\
\text { (C) }\end{array}$ & $\begin{array}{l}\text { DEPTH } \\
(F T)\end{array}$ & $\begin{array}{l}\text { TEMPERATURE } \\
\text { (C) }\end{array}$ & $\begin{array}{l}\text { EEPTH } \\
\text { (FT) }\end{array}$ & $\begin{array}{l}\text { TEPERATLR } \\
\text { (C) }\end{array}$ \\
\hline $\begin{array}{l}2576 \\
2578 \\
2580 \\
2582 \\
2584 \\
2585 \\
2588 \\
2590 \\
2592 \\
2594 \\
2596 \\
2598 \\
2600 \\
2502 \\
2604 \\
2506 \\
2608 \\
2610 \\
2612 \\
2514 \\
2616 \\
2618 \\
2520 \\
2622 \\
2621 \\
2526 \\
2628 \\
2630 \\
2632 \\
2534 \\
2638 \\
2540 \\
2612 \\
2644 \\
2646 \\
2548 \\
2650 \\
2652 \\
2654 \\
2656 \\
2658 \\
2550 \\
2662 \\
2654 \\
2666 \\
2358 \\
2670 \\
2672 \\
2674 \\
2676 \\
2678 \\
2350 \\
2682 \\
2584 \\
2686 \\
2688 \\
2650 \\
2692 \\
2694 \\
2693 \\
2658 \\
2700 \\
2702 \\
2704 \\
2706 \\
2708 \\
2710 \\
2712 \\
2714 \\
2715 \\
\end{array}$ & $\begin{array}{l}112.828 \\
112.841 \\
112.904 \\
112.911 \\
112.974 \\
112.986 \\
113.037 \\
113.075 \\
113.101 \\
113.120 \\
113.152 \\
113.203 \\
113.222 \\
113.219 \\
113.369 \\
113.330 \\
113.369 \\
113.414 \\
113.440 \\
113.459 \\
113.497 \\
113.530 \\
113.555 \\
113.600 \\
113.639 \\
113.535 \\
113.701 \\
113.743 \\
113.775 \\
113.795 \\
113.892 \\
113.896 \\
113.925 \\
113.970 \\
114.029 \\
114.049 \\
114.075 \\
111.114 \\
114.160 \\
111.213 \\
114.219 \\
114.252 \\
114.292 \\
114.338 \\
114.357 \\
114.394 \\
114.104 \\
114.457 \\
114.523 \\
114.523 \\
114.543 \\
114.556 \\
114.609 \\
111.682 \\
114.709 \\
114.722 \\
114.769 \\
114.782 \\
114.860 \\
111.876 \\
114.950 \\
111.957 \\
115.017 \\
115.010 \\
115.098 \\
115.094 \\
115.152 \\
115.203 \\
115.219 \\
115.219 \\
115\end{array}$ & $\begin{array}{l}2718 \\
2720 \\
2722 \\
2724 \\
2726 \\
2728 \\
2730 \\
2732 \\
2734 \\
2736 \\
2740 \\
2742 \\
2744 \\
2716 \\
2748 \\
2750 \\
2752 \\
2754 \\
2756 \\
2 / 58 \\
2760 \\
2762 \\
2764 \\
2756 \\
2772 \\
2774 \\
2776 \\
2778 \\
2780 \\
2782 \\
2781 \\
2786 \\
2788 \\
2700 \\
2744 \\
2795 \\
2800 \\
2802 \\
2804 \\
2808 \\
2810 \\
2812 \\
2814 \\
2816 \\
2818 \\
2820 \\
2822 \\
2924 \\
2826 \\
2828 \\
2830 \\
2832 \\
2834 \\
2836 \\
2838 \\
2840 \\
2842 \\
2844 \\
2846 \\
2848 \\
2850 \\
2852 \\
2854 \\
2858 \\
2860 \\
2852 \\
2861 \\
2865 \\
2868 \\
2870\end{array}$ & 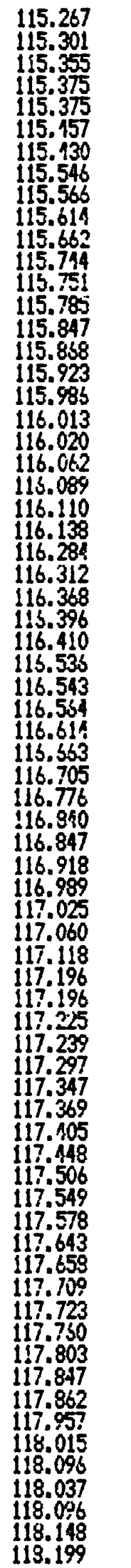 & 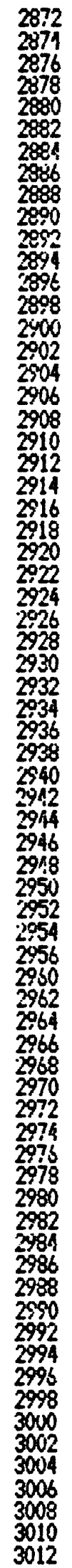 & 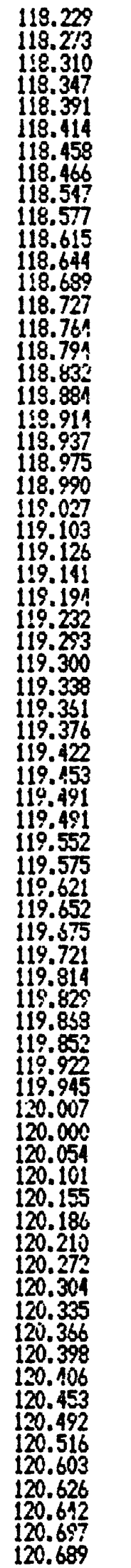 & $\begin{array}{l}3011 \\
3116 \\
3018 \\
3020 \\
302 \\
3024 \\
3026 \\
3028 \\
3030 \\
3032 \\
3031 \\
3036 \\
3038 \\
3040 \\
3012 \\
3044 \\
3046 \\
3048 \\
3050 \\
3054 \\
3056 \\
3058 \\
3050 \\
3062 \\
3064 \\
3056 \\
3068 \\
3070 \\
3072 \\
3074 \\
3076 \\
3078 \\
3080 \\
3082 \\
3084 \\
3086 \\
3008 \\
3092 \\
3094 \\
3096 \\
3098 \\
3100 \\
3102 \\
3104 \\
3106 \\
3108 \\
3110 \\
3112 \\
3114 \\
3116 \\
3118 \\
3120 \\
3122 \\
3124 \\
3126 \\
3128 \\
3130 \\
3132 \\
3134 \\
3136 \\
3138 \\
3140 \\
3142 \\
3144 \\
3146 \\
3148 \\
3150 \\
3152 \\
3154 \\
3156\end{array}$ & $\begin{array}{l}120.761 \\
120.793 \\
120.848 \\
120.864 \\
120.911 \\
120.952 \\
120.992 \\
121.049 \\
121.010 \\
121.072 \\
121.080 \\
121.144 \\
121.181 \\
121.224 \\
121.264 \\
121.312 \\
121.329 \\
121.417 \\
121.333 \\
121.434 \\
121.190 \\
121.531 \\
121.571 \\
121.601 \\
121.636 \\
121.553 \\
121.693 \\
121.731 \\
121.758 \\
121.199 \\
121.848 \\
121.840 \\
121.899 \\
121.889 \\
121.963 \\
122.037 \\
122.070 \\
122.111 \\
122.152 \\
122.185 \\
122.235 \\
122.252 \\
122.293 \\
122.318 \\
122.321 \\
122.375 \\
122.117 \\
122.150 \\
121.181 \\
122.517 \\
122.526 \\
122.601 \\
122.626 \\
122.668 \\
122.693 \\
122.710 \\
122.735 \\
122.785 \\
122.810 \\
122.852 \\
122.861 \\
122.420 \\
122.928 \\
122.951 \\
123.013 \\
123.047 \\
123.017 \\
123.097 \\
123.123 \\
123.157\end{array}$ & $\begin{array}{l}3160 \\
3152 \\
3164 \\
3166 \\
3168 \\
3170 \\
3172 \\
3174 \\
3176 \\
3178 \\
3180 \\
3182 \\
3181 \\
3186 \\
3188 \\
3190 \\
3192 \\
3194 \\
319 t \\
3198 \\
3200 \\
3202 \\
3204 \\
3206 \\
3209 \\
3210 \\
3212 \\
3214 \\
3216 \\
3218 \\
3210 \\
3222 \\
3224 \\
3226 \\
3220 \\
3230 \\
3232 \\
3234 \\
3236 \\
3238 \\
3210 \\
3242 \\
3244 \\
3246 \\
3248 \\
3250 \\
3252 \\
3254 \\
3256 \\
3258 \\
3260 \\
3252 \\
3261 \\
3266 \\
3268 \\
3270 \\
3272 \\
3274 \\
3276 \\
3278 \\
3280 \\
3282 \\
3384 \\
3286 \\
3388 \\
3290 \\
3292 \\
3294 \\
3296 \\
3298\end{array}$ & 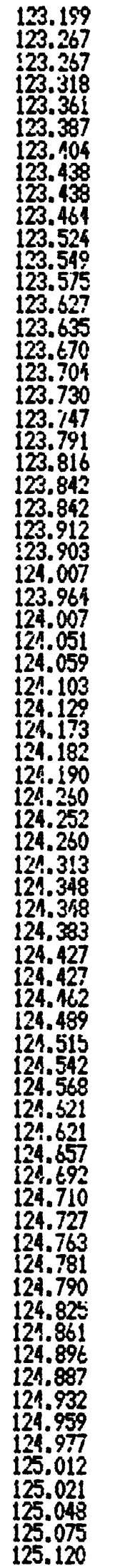 & 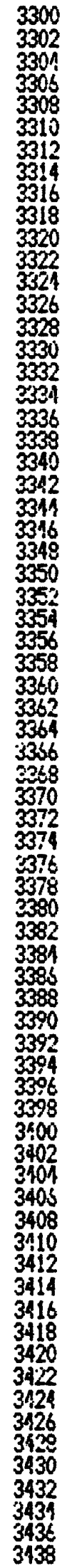 & $\begin{array}{l}125.138 \\
125.165 \\
125.210 \\
125.228 \\
125.237 \\
125.291 \\
125.318 \\
125.300 \\
125.345 \\
125.345 \\
125.390 \\
125.426 \\
125.481 \\
125.481 \\
125.508 \\
125.562 \\
125.571 \\
125.580 \\
125.635 \\
125.653 \\
125.708 \\
125.135 \\
125.714 \\
125.772 \\
125.817 \\
125.827 \\
125.863 \\
125.881 \\
125.936 \\
125.936 \\
125.973 \\
126.047 \\
126.030 \\
126.074 \\
121.094 \\
126.148 \\
126.176 \\
126.176 \\
126.213 \\
126.211 \\
126.259 \\
126.296 \\
126.333 \\
126.352 \\
126.417 \\
126.473 \\
126.529 \\
126.510 \\
126.557 \\
126.575 \\
126.603 \\
126.613 \\
126.669 \\
126.678 \\
126.706 \\
126.716 \\
126.744 \\
126.819 \\
126.828 \\
123.847 \\
126.895 \\
126.923 \\
126.551 \\
123.951 \\
126.979 \\
127.027 \\
127.674 \\
127.083 \\
127.159 \\
127.131\end{array}$ \\
\hline
\end{tabular}


PAFT RIVER, IUAHO - RRGE-2 - 10 ALULST 17\%

\begin{tabular}{|c|c|c|c|c|c|c|c|c|c|c|c|}
\hline $\begin{array}{c}\text { IEPTH } \\
\text { (FT) }\end{array}$ & $\begin{array}{l}\text { TERPERATURE } \\
\text { (C) }\end{array}$ & $\begin{array}{c}\text { OEPTH } \\
(\mathrm{FT})\end{array}$ & $\begin{array}{l}\text { TEMPERATLRE } \\
\text { (C) }\end{array}$ & $\begin{array}{l}\text { DEPTH } \\
\text { (FT) }\end{array}$ & $\begin{array}{l}\text { TEMPERATLRE } \\
\text { (C) }\end{array}$ & $\begin{array}{l}\text { DEPTH } \\
\text { (FT) }\end{array}$ & $\begin{array}{l}\text { TEPPERNTURE } \\
\text { (C) }\end{array}$ & $\begin{array}{l}\text { IEPTH } \\
\text { (FT) }\end{array}$ & $\begin{array}{l}\text { TESPERATRE } \\
\text { (C) }\end{array}$ & $\begin{array}{l}\text { DEPTH } \\
\text { (FT) }\end{array}$ & $\begin{array}{l}\text { TEPPERATLEA } \\
\text { (C) }\end{array}$ \\
\hline $\begin{array}{l}3440 \\
3442 \\
3444 \\
3446 \\
3448 \\
3450 \\
3452 \\
3454 \\
3456 \\
3458 \\
3460 \\
3462 \\
3464 \\
3456 \\
3468 \\
3470 \\
3472 \\
3474 \\
3476 \\
34.8 \\
3480 \\
3482 \\
3484 \\
3485 \\
3488 \\
3490 \\
3492 \\
3494 \\
3496 \\
3498 \\
3500 \\
3502 \\
3504 \\
3505 \\
3508 \\
3510 \\
3512 \\
3514 \\
3516 \\
3518 \\
3520 \\
3522 \\
3524 \\
3526 \\
3528 \\
3530 \\
3532 \\
3534 \\
3536 \\
3535 \\
3540 \\
3542 \\
3544 \\
3545 \\
3548 \\
3550 \\
3552 \\
3554 \\
3556 \\
3558 \\
3560 \\
3554 \\
3566 \\
3568 \\
3570 \\
3574 \\
3576 \\
3578 \\
3580\end{array}$ & 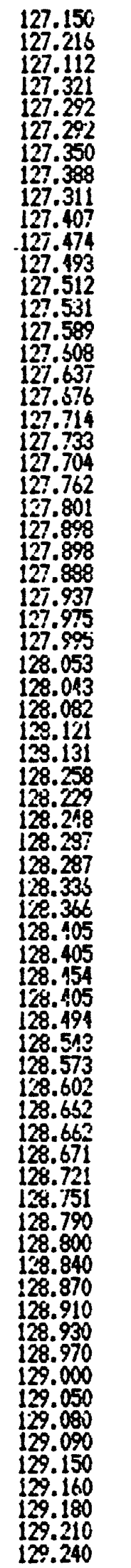 & $\begin{array}{l}3582 \\
3581 \\
3586 \\
3588 \\
3590 \\
3502 \\
3594 \\
3596 \\
3548 \\
3500 \\
3601 \\
3604 \\
3606 \\
3608 \\
3610 \\
3612 \\
3614 \\
3615 \\
3618 \\
3620 \\
3622 \\
3624 \\
3626 \\
3628 \\
3636 \\
3632 \\
3634 \\
3636 \\
3638 \\
3610 \\
3612 \\
3641 \\
3646 \\
3648 \\
3650 \\
3654 \\
3656 \\
3658 \\
3662 \\
3654 \\
3666 \\
3670 \\
3672 \\
3674 \\
3676 \\
3678 \\
3690 \\
3682 \\
3681 \\
3685 \\
3688 \\
3690 \\
3692 \\
3694 \\
3696 \\
3698 \\
3700 \\
3702 \\
3701 \\
3706 \\
3708 \\
3710 \\
3712 \\
3714 \\
3716 \\
3718 \\
3720 \\
3721 \\
3726 \\
3728\end{array}$ & 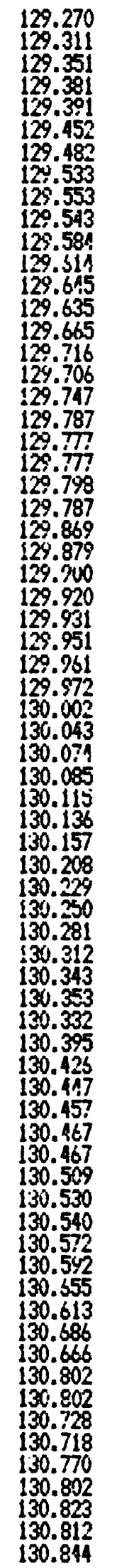 & $\begin{array}{l}3730 \\
3732 \\
3734 \\
3736 \\
3730 \\
3740 \\
3742 \\
3711 \\
3746 \\
3748 \\
3750 \\
3752 \\
3754 \\
3756 \\
3758 \\
3760 \\
3762 \\
3754 \\
3766 \\
3768 \\
3770 \\
3772 \\
3714 \\
3776 \\
3778 \\
3780 \\
3782 \\
3784 \\
3786 \\
3788 \\
3790 \\
3792 \\
3794 \\
3706 \\
3798 \\
3800 \\
3302 \\
3804 \\
3806 \\
3808 \\
3310 \\
3812 \\
3814 \\
3816 \\
3920 \\
3822 \\
3824 \\
3828 \\
3830 \\
3832 \\
3834 \\
3336 \\
3828 \\
3840 \\
3842 \\
3814 \\
3846 \\
3848 \\
3850 \\
3852 \\
3854 \\
3856 \\
3858 \\
3350 \\
3862 \\
3864 \\
3866 \\
3858 \\
3870 \\
3872\end{array}$ & $\begin{array}{l}130.875 \\
131.002 \\
130.813 \\
130.911 \\
130.938 \\
130.981 \\
130.981 \\
130.928 \\
131.023 \\
131.044 \\
131.086 \\
131.095 \\
131.107 \\
131.224 \\
131.129 \\
131.150 \\
131.192 \\
131.192 \\
131.203 \\
131.235 \\
131.288 \\
131.277 \\
131.288 \\
131.288 \\
131.351 \\
131.352 \\
131.330 \\
131.384 \\
131.394 \\
131.416 \\
131.426 \\
131.437 \\
131.458 \\
131.458 \\
131.501 \\
131.512 \\
131.541 \\
131.541 \\
131.555 \\
131.598 \\
131.598 \\
131.519 \\
131.662 \\
131.673 \\
131.759 \\
131.748 \\
131.790 \\
131.913 \\
131.813 \\
131.845 \\
131.834 \\
131.999 \\
131.888 \\
131.953 \\
131.075 \\
131.964 \\
131.975 \\
132.051 \\
131.997 \\
132.051 \\
132.094 \\
132.127 \\
132.138 \\
132.149 \\
132.160 \\
132.214 \\
132.247 \\
132.225 \\
132.260 \\
132.356\end{array}$ & 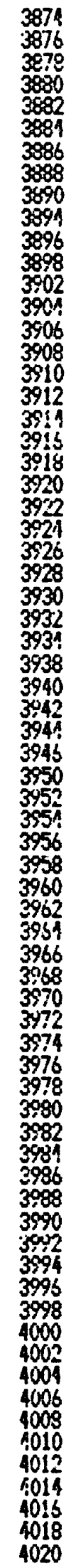 & $\begin{array}{l}132.214 \\
132.312 \\
132.356 \\
132.378 \\
132.378 \\
132.455 \\
132.455 \\
132.455 \\
132.521 \\
131.565 \\
132.587 \\
132.587 \\
132.612 \\
132.664 \\
132.675 \\
132.142 \\
132.719 \\
132.542 \\
132.775 \\
132.808 \\
132.808 \\
132.852 \\
132.863 \\
132.9836 \\
132.919 \\
132.952 \\
132.964 \\
132.995 \\
133.030 \\
133.053 \\
133.064 \\
133.097 \\
133.097 \\
133.142 \\
133.164 \\
133.220 \\
133.220 \\
133.265 \\
133.265 \\
133.277 \\
133.299 \\
133.310 \\
133.344 \\
133.355 \\
133.378 \\
133.367 \\
133.100 \\
133.145 \\
133.134 \\
133.445 \\
133.179 \\
133.191 \\
133.502 \\
133.513 \\
133.524 \\
133.513 \\
133.567 \\
133.570 \\
133.547 \\
133.581 \\
133.626 \\
133.626 \\
133.615 \\
133.528 \\
133.660 \\
133.672 \\
133.683 \\
133.549 \\
133.649 \\
133.694\end{array}$ & $\begin{array}{l}4022 \\
4024 \\
4028 \\
1030 \\
4082 \\
1034 \\
4036 \\
4038 \\
4010 \\
4042 \\
4014 \\
1046 \\
4048 \\
4050 \\
4052 \\
4054 \\
4056 \\
1058 \\
4060 \\
1062 \\
1065 \\
4056 \\
4068 \\
1070 \\
4072 \\
4074 \\
4076 \\
4078 \\
4080 \\
4082 \\
4084 \\
4086 \\
4088 \\
4090 \\
4092 \\
4094 \\
4096 \\
1098 \\
4100 \\
4102 \\
1104 \\
4106 \\
4108 \\
1110 \\
1112 \\
4114 \\
4116 \\
4118 \\
4120 \\
1122 \\
4124 \\
4126 \\
4128 \\
4130 \\
4132 \\
1134 \\
4136 \\
1138 \\
1140 \\
4112 \\
4141 \\
1146 \\
4148 \\
1150 \\
4152 \\
1154 \\
1156 \\
1158 \\
1160 \\
1152 \\
\end{array}$ & 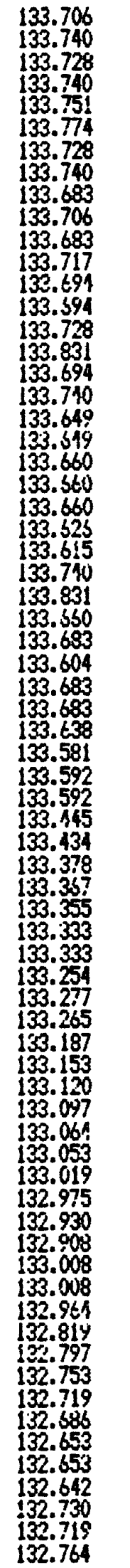 & $\begin{array}{l}4161 \\
4136 \\
4168 \\
4170 \\
4172 \\
4174 \\
4176 \\
4178 \\
4180 \\
4182 \\
4184 \\
4186 \\
4180 \\
4190 \\
1192 \\
4194 \\
1196 \\
1198 \\
1200 \\
4202 \\
1204 \\
4206 \\
4208 \\
1210 \\
4212 \\
4214 \\
4216 \\
4219 \\
4220 \\
4222 \\
4224 \\
1226 \\
4238 \\
1230 \\
4232 \\
1234 \\
4236 \\
4238 \\
4210 \\
1242 \\
1244 \\
1216 \\
4248 \\
1250 \\
4252 \\
1254 \\
4256 \\
1258 \\
1260 \\
4232 \\
4261 \\
4266 \\
4268 \\
4270 \\
4272 \\
1274 \\
4276 \\
4278 \\
4290 \\
4282 \\
4234 \\
4286 \\
4288 \\
4290 \\
4292 \\
4294 \\
4296 \\
1298 \\
4300 \\
4302\end{array}$ & 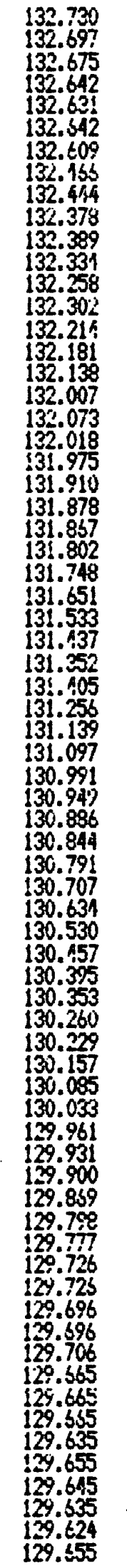 \\
\hline
\end{tabular}


RNFT RIVER, IDAHO - RROE-2 - 10 AUCUST 1976

\begin{tabular}{|c|c|c|c|c|c|c|c|c|c|c|c|}
\hline $\begin{array}{l}\text { IEPTH } \\
\text { (FT) }\end{array}$ & $\begin{array}{l}\text { TERPERATURE } \\
\text { (C) }\end{array}$ & $\begin{array}{l}\text { DEPTH } \\
(\mathrm{FT})\end{array}$ & $\begin{array}{l}\text { TEYPERANGAE } \\
\text { (C) }\end{array}$ & $\begin{array}{l}\text { DEPTH } \\
\text { (FT) }\end{array}$ & $\begin{array}{l}\text { TEMPERATLRE } \\
\text { (C) }\end{array}$ & $\begin{array}{c}\text { DEPTH } \\
\text { (FT) }\end{array}$ & $\begin{array}{l}\text { TEMPERATURE } \\
\text { (C) }\end{array}$ & $\begin{array}{l}\text { DEPTH } \\
\text { (FT) }\end{array}$ & $\begin{array}{l}\text { TEMPERATURE } \\
\text { (C) }\end{array}$ & DSPTH & $\begin{array}{l}\text { TEAPERAT } \\
\text { (C) }\end{array}$ \\
\hline 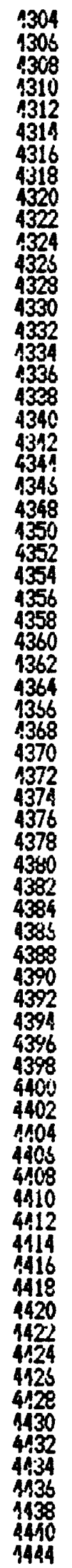 & $\begin{array}{l}129.655 \\
129.665 \\
129.717 \\
129.767 \\
129.696 \\
120.747 \\
129.757 \\
129.675 \\
129.767 \\
129.819 \\
129.869 \\
129.092 \\
129.972 \\
130.023 \\
130.126 \\
130.199 \\
130.301 \\
130.353 \\
130.331 \\
130.126 \\
130.500 \\
130.519 \\
130.519 \\
130.519 \\
130.770 \\
130.833 \\
130.896 \\
131.002 \\
131.086 \\
131.213 \\
131.245 \\
131.256 \\
131.248 \\
131.289 \\
131.330 \\
131.391 \\
131.362 \\
131.373 \\
131.362 \\
131.391 \\
131.418 \\
131.437 \\
131.426 \\
131.458 \\
131.352 \\
131.458 \\
131.480 \\
131.490 \\
131.469 \\
131.523 \\
131.480 \\
131.490 \\
131.501 \\
131.512 \\
131.533 \\
131.533 \\
131.630 \\
131.555 \\
131.576 \\
131.587 \\
131.608 \\
131.619 \\
131.640 \\
131.551 \\
131.673 \\
131.552 \\
131.727 \\
131.750 \\
131.770 \\
131.802\end{array}$ & 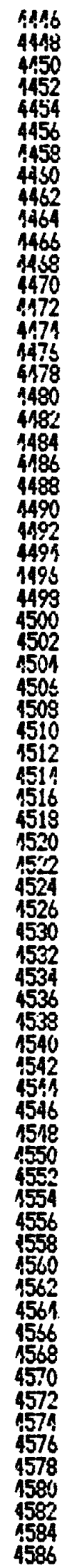 & $\begin{array}{l}131.834 \\
131.010 \\
131.921 \\
131.921 \\
131.986 \\
131.997 \\
131.997 \\
132.040 \\
132.051 \\
132.051 \\
132.116 \\
132.094 \\
132.105 \\
132.149 \\
132.149 \\
132.150 \\
132.181 \\
132.211 \\
132.225 \\
132.214 \\
132.356 \\
132.441 \\
132.466 \\
132.422 \\
132.620 \\
132.565 \\
132.598 \\
132.575 \\
132.753 \\
132.819 \\
132.808 \\
132.908 \\
132.919 \\
132.964 \\
133.042 \\
133.019 \\
133.075 \\
133.120 \\
133.254 \\
133.265 \\
133.265 \\
133.367 \\
132.400 \\
133.412 \\
133.468 \\
133.513 \\
133.502 \\
133.740 \\
135.821 \\
133.638 \\
133.706 \\
133.954 \\
133.911 \\
131.014 \\
134.151 \\
134.140 \\
134.186 \\
134.195 \\
134.278 \\
134.405 \\
134.140 \\
134.428 \\
134.405 \\
134.152 \\
134.498 \\
134.521 \\
134.514 \\
134.556 \\
134.603 \\
134.573\end{array}$ & 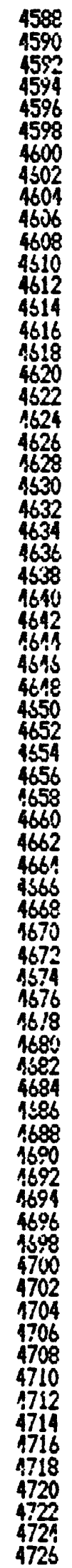 & 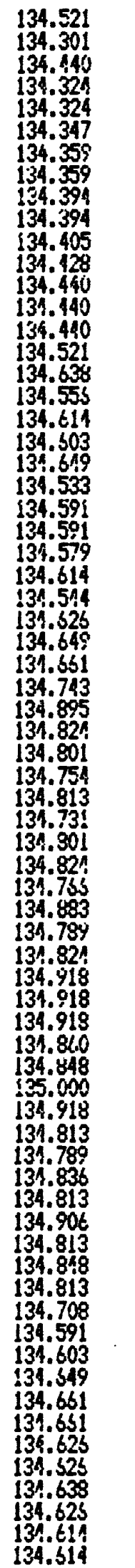 & 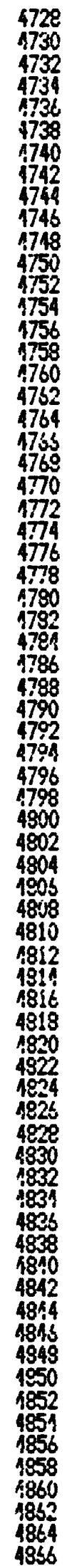 & 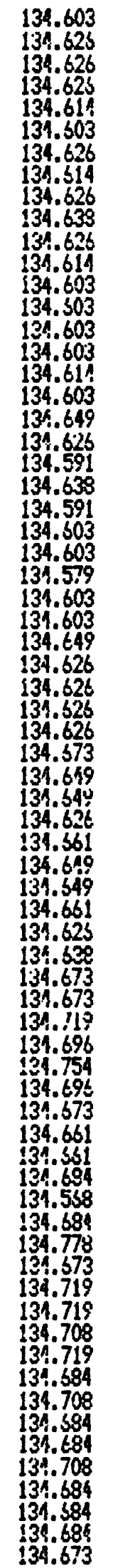 & 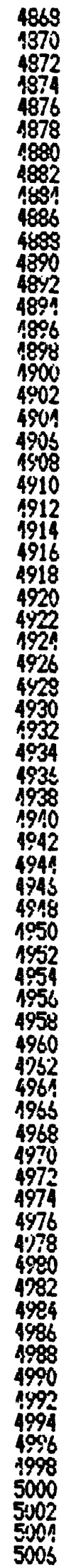 & 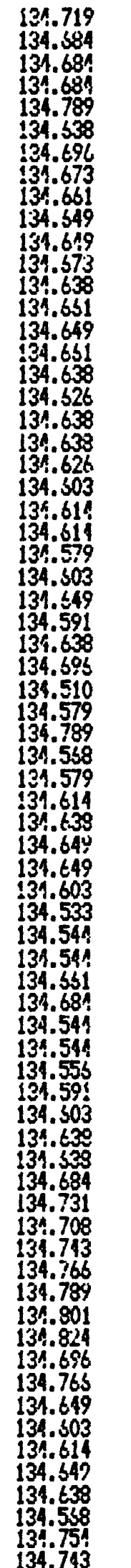 & $\begin{array}{l}5008 \\
5010 \\
5012 \\
5014 \\
5016 \\
5018 \\
5020 \\
5022 \\
5026 \\
5028 \\
5030 \\
5032 \\
5034 \\
5036 \\
5038 \\
5040 \\
5042 \\
5044 \\
5016 \\
5048 \\
5050 \\
5052 \\
5054 \\
5058 \\
5058 \\
5060 \\
5062 \\
5054 \\
5056 \\
5058 \\
5070 \\
5072 \\
5074 \\
5075 \\
5078 \\
5020 \\
5092 \\
5084 \\
5086 \\
5088 \\
5090 \\
5092 \\
5091 \\
5096 \\
5098 \\
5100 \\
5102 \\
5106 \\
5108 \\
5110 \\
5112 \\
5114 \\
5186 \\
5118 \\
5120 \\
5122 \\
5124 \\
5126 \\
5128 \\
5130 \\
5132 \\
5134 \\
5136 \\
5138 \\
5140 \\
5142 \\
5111 \\
5116 \\
5148 \\
5150\end{array}$ & 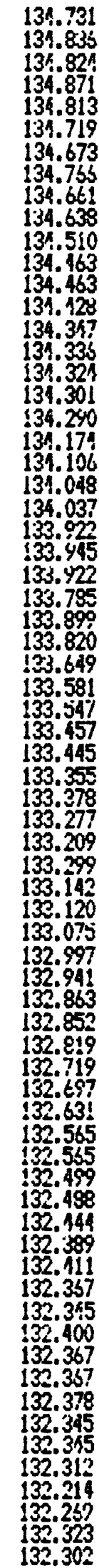 \\
\hline
\end{tabular}


PAFT RIVER, IDAHO - RREE-2 - 10 AUCUST 1976

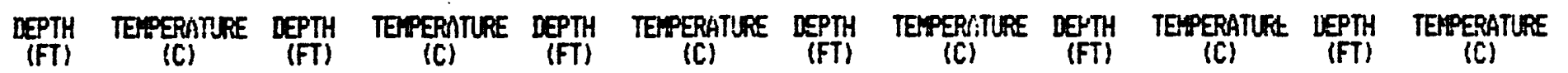

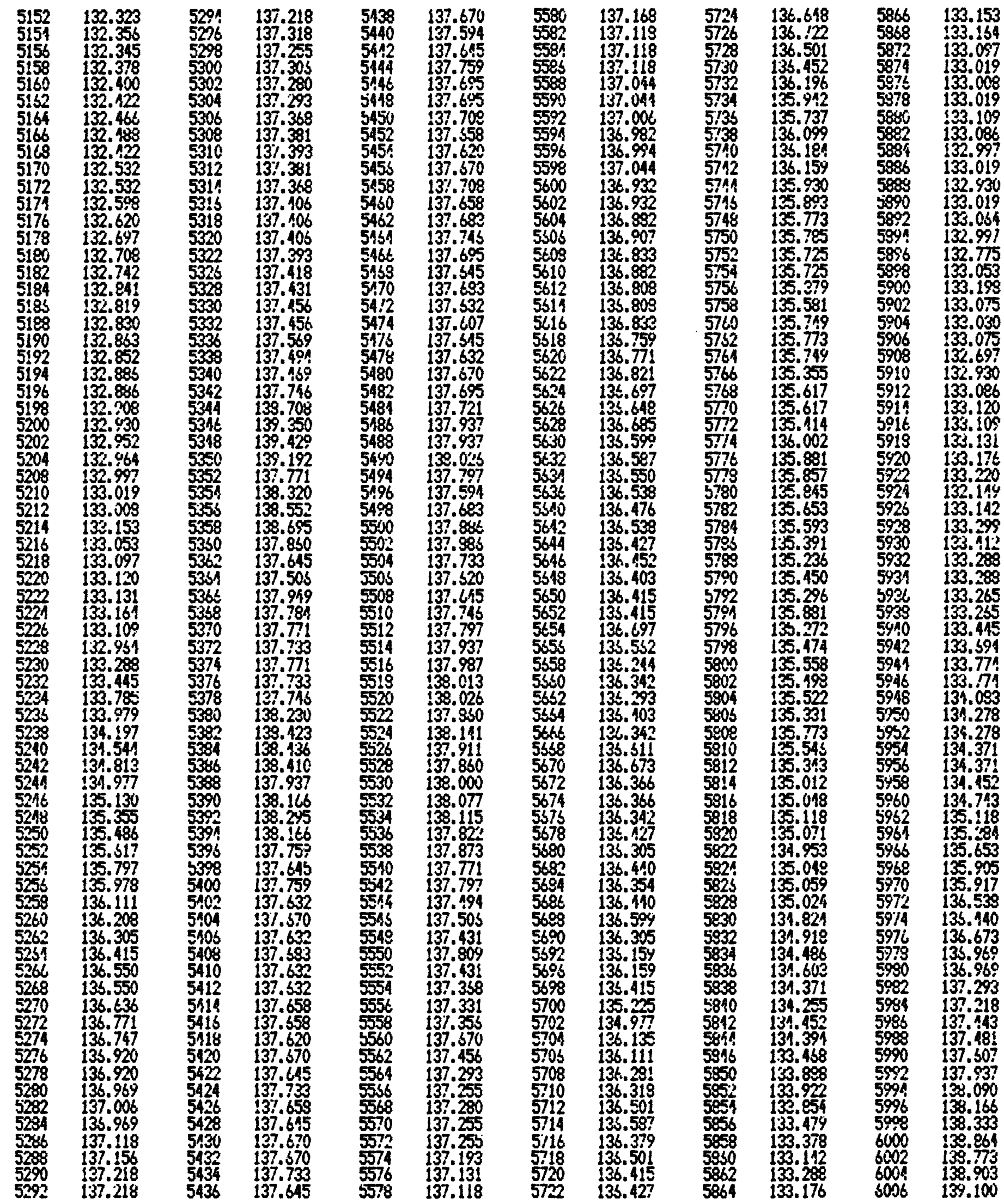


RAT RIVER, IDAHO - RRGE-2 - 10 NUSLST 1975

\begin{tabular}{|c|c|c|c|c|c|c|c|}
\hline $\begin{array}{l}\text { IEPTH } \\
\text { (FT) }\end{array}$ & $\begin{array}{l}\text { TERPERATURE } \\
\text { (C) }\end{array}$ & $\begin{array}{l}\text { DEPTH } \\
\text { (FT) }\end{array}$ & $\begin{array}{l}\text { TEPPERATURE } \\
\text { (C) }\end{array}$ & $\begin{array}{l}\text { DEMTH } \\
\text { (FT) }\end{array}$ & $\begin{array}{l}\text { TERERATLRE } \\
\text { (C) }\end{array}$ & $\begin{array}{l}\text { DEPTH } \\
\text { (FT) }\end{array}$ & $\begin{array}{l}\text { TEREERATLR } \\
\text { (C) }\end{array}$ \\
\hline $\begin{array}{l}6008 \\
5010 \\
6012 \\
5014 \\
6016 \\
5018 \\
6020 \\
5022 \\
6024 \\
6025 \\
6028 \\
6030 \\
6032 \\
5034 \\
6056 \\
5040 \\
6012 \\
5044 \\
6048 \\
6050 \\
6052 \\
6054 \\
6056 \\
5053 \\
6060 \\
5062 \\
6064 \\
6056 \\
6068 \\
6070 \\
6072 \\
5074 \\
6076 \\
6078 \\
6080 \\
6082 \\
6084 \\
6085 \\
6088 \\
6030 \\
60 \% 2 \\
6094 \\
6096 \\
6098 \\
6100 \\
6102 \\
6104 \\
6106 \\
6110 \\
6112 \\
6114 \\
5116 \\
6118 \\
6120 \\
6122 \\
6134 \\
6126 \\
6128 \\
6130 \\
5132 \\
6134 \\
6133 \\
6138 \\
6140 \\
6142 \\
6144 \\
6116 \\
6148 \\
6150 \\
6152\end{array}$ & 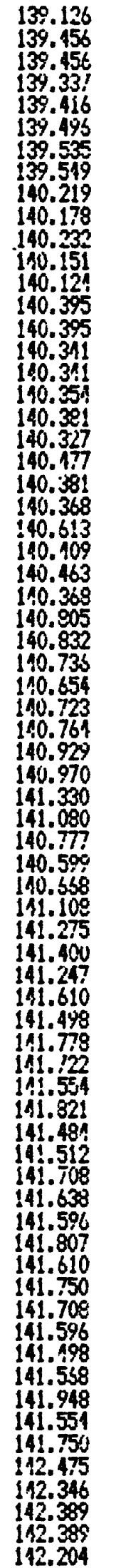 & 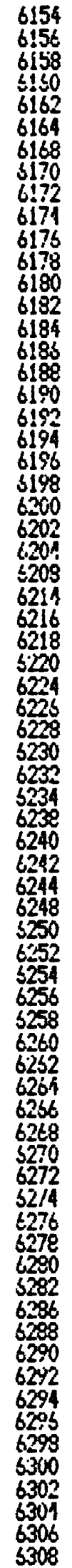 & 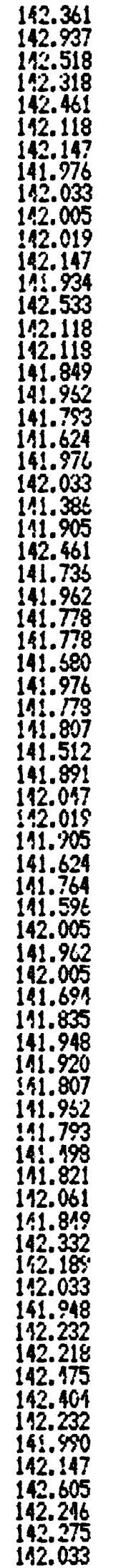 & 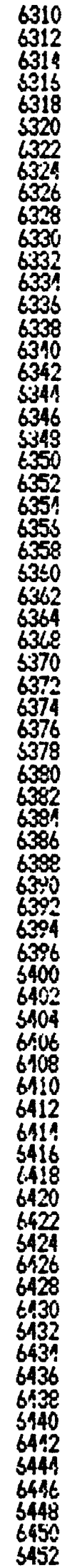 & 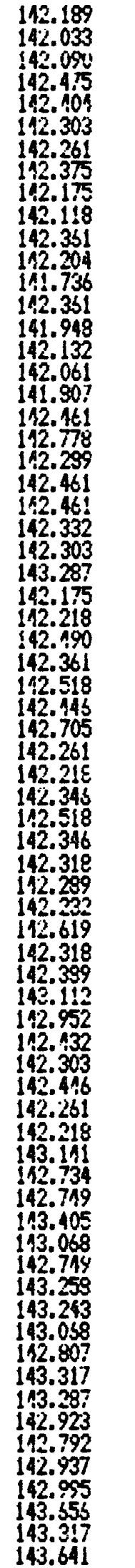 & 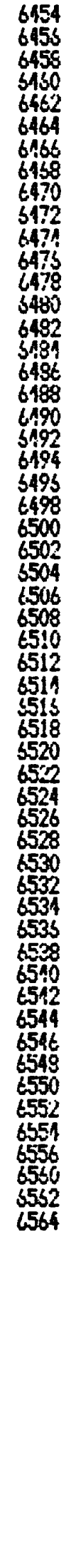 & 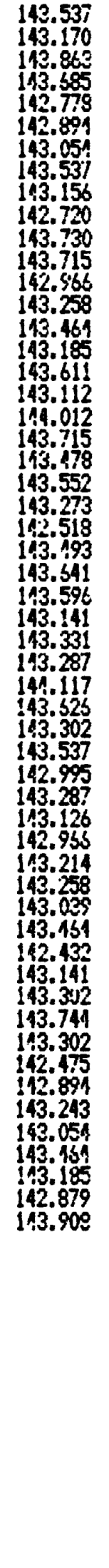 \\
\hline
\end{tabular}


RAFT RIVER, IDAHO - RRGE-3 - 12 AUEUST 1976

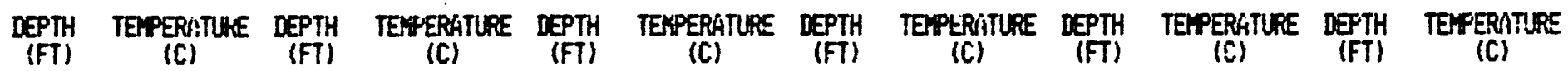

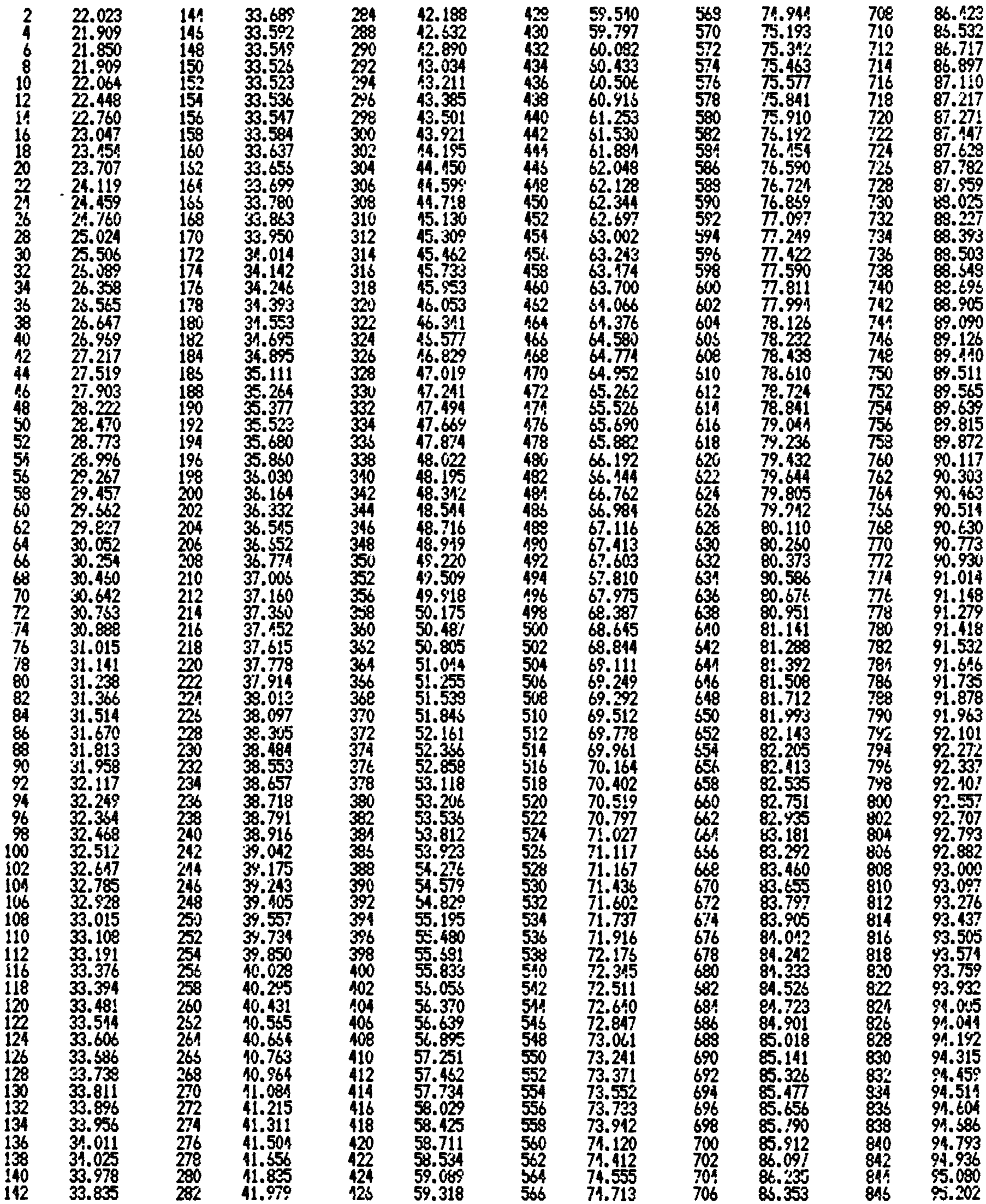


RAFT RIVER, IDAHO - PROE-3 - 12 NULUST 1976

\begin{tabular}{|c|c|c|c|c|c|c|c|c|c|c|c|}
\hline $\begin{array}{l}\text { DEPTH } \\
\text { (FT) }\end{array}$ & $\begin{array}{l}\text { TEYFERATURE } \\
\text { (C) }\end{array}$ & $\begin{array}{l}\text { DEPTH } \\
\text { (FT) }\end{array}$ & $\begin{array}{l}\text { TEYPERRITRE } \\
\text { (C) }\end{array}$ & $\begin{array}{l}\text { DEPTH } \\
\text { (FT) }\end{array}$ & $\begin{array}{l}\text { TEFEERATURE } \\
\text { (C) }\end{array}$ & $\begin{array}{l}\text { DEPTH } \\
\text { (FT) }\end{array}$ & $\begin{array}{l}\text { TEAPERATURE } \\
\text { (C) }\end{array}$ & $\begin{array}{c}\text { DEPTH } \\
\text { (FT) }\end{array}$ & $\begin{array}{l}\text { TEYPERATLRE } \\
\text { (C) }\end{array}$ & $\begin{array}{l}\text { DEPTH } \\
\text { (FT) }\end{array}$ & $\begin{array}{l}\text { TEMPERATLF } \\
\text { (C) }\end{array}$ \\
\hline $\begin{array}{l}848 \\
850 \\
952 \\
854 \\
856 \\
858 \\
860 \\
862 \\
864 \\
856 \\
868 \\
870 \\
872 \\
874 \\
876 \\
878 \\
880 \\
882 \\
834 \\
385 \\
888 \\
890 \\
892 \\
894 \\
996 \\
998 \\
900 \\
902 \\
904 \\
905 \\
908 \\
910 \\
912 \\
914 \\
916 \\
018 \\
920 \\
922 \\
926 \\
928 \\
930 \\
932 \\
931 \\
936 \\
938 \\
940 \\
942 \\
944 \\
945 \\
948 \\
950 \\
952 \\
951 \\
955 \\
958 \\
952 \\
9644 \\
966 \\
968 \\
970 \\
972 \\
974 \\
976 \\
978 \\
980 \\
982 \\
984 \\
986 \\
988 \\
990\end{array}$ & 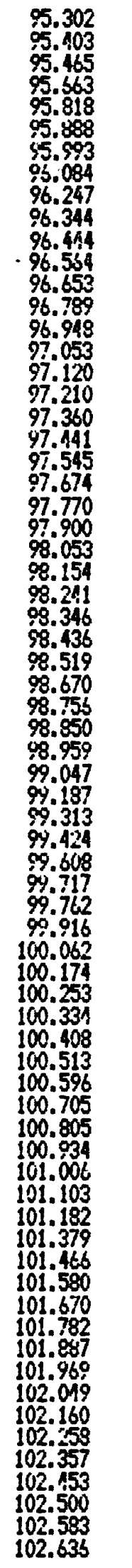 & $\begin{array}{l}992 \\
994 \\
996 \\
9000 \\
1000 \\
1002 \\
1001 \\
1006 \\
1008 \\
1010 \\
1012 \\
1011 \\
1016 \\
1019 \\
1020 \\
1022 \\
1024 \\
1023 \\
1028 \\
1030 \\
1032 \\
1036 \\
1038 \\
1040 \\
1042 \\
1041 \\
1046 \\
1018 \\
1050 \\
1052 \\
1054 \\
1056 \\
1058 \\
1050 \\
1062 \\
1061 \\
1066 \\
1068 \\
1070 \\
1072 \\
1071 \\
1075 \\
1079 \\
1080 \\
1082 \\
1084 \\
1086 \\
1088 \\
1090 \\
1092 \\
1094 \\
1096 \\
1098 \\
1100 \\
1102 \\
1104 \\
1106 \\
1108 \\
1110 \\
1112 \\
1114 \\
1115 \\
1118 \\
1120 \\
1122 \\
1124 \\
1126 \\
1128 \\
1130 \\
1132\end{array}$ & $\begin{array}{l}102.756 \\
102.853 \\
102.889 \\
103.004 \\
103.135 \\
103.220 \\
103.293 \\
103.378 \\
103.974 \\
103.573 \\
103.683 \\
103.753 \\
103.808 \\
103.881 \\
103.964 \\
104.053 \\
104.152 \\
104.214 \\
104.316 \\
104.428 \\
104.518 \\
104.671 \\
104.712 \\
104.830 \\
104.919 \\
104.972 \\
105.049 \\
105.107 \\
105.193 \\
105.206 \\
105.372 \\
105.471 \\
105.544 \\
105.619 \\
105.671 \\
105.731 \\
105.821 \\
105.911 \\
106.002 \\
105.090 \\
106.171 \\
106.232 \\
106.266 \\
105.349 \\
106.104 \\
105.513 \\
106.605 \\
105.711 \\
106.758 \\
105.870 \\
106.9914 \\
106.494 \\
107.055 \\
107.120 \\
107.221 \\
107.346 \\
107.365 \\
107.429 \\
107.506 \\
107.526 \\
107.670 \\
107.723 \\
107.801 \\
107.875 \\
107.937 \\
108.018 \\
108.059 \\
108.168 \\
108.222 \\
108.299\end{array}$ & $\begin{array}{l}1134 \\
1136 \\
1138 \\
1140 \\
1142 \\
1144 \\
1146 \\
1148 \\
1150 \\
1152 \\
1154 \\
1156 \\
1158 \\
1150 \\
1162 \\
1154 \\
1166 \\
1158 \\
1170 \\
1172 \\
1174 \\
1176 \\
1178 \\
1180 \\
1182 \\
1184 \\
1186 \\
1189 \\
1190 \\
1192 \\
1194 \\
1196 \\
1196 \\
1200 \\
1202 \\
1204 \\
1206 \\
1208 \\
1210 \\
1212 \\
1218 \\
1216 \\
1218 \\
1200 \\
1222 \\
1224 \\
1326 \\
1228 \\
1230 \\
1245 \\
1249 \\
1250 \\
1252 \\
1354 \\
1256 \\
1259 \\
1260 \\
1262 \\
1241 \\
1256 \\
1268 \\
1270 \\
1272 \\
1274 \\
1276 \\
1218 \\
1280 \\
1282 \\
1284 \\
1289\end{array}$ & 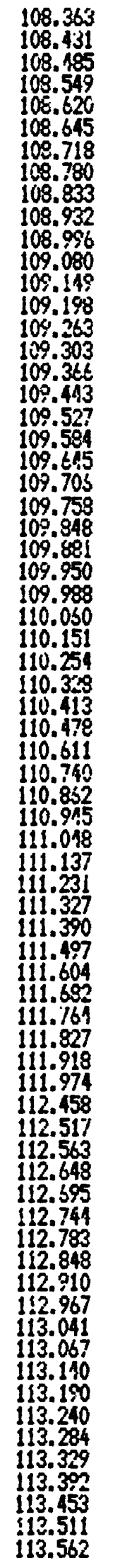 & $\begin{array}{l}1298 \\
1290 \\
1292 \\
1294 \\
1296 \\
1298 \\
1300 \\
1302 \\
1304 \\
1306 \\
1308 \\
1310 \\
1312 \\
1314 \\
1316 \\
1318 \\
1320 \\
1322 \\
1321 \\
1326 \\
1328 \\
1330 \\
1332 \\
1334 \\
1326 \\
1338 \\
1340 \\
1342 \\
1344 \\
1345 \\
1348 \\
1350 \\
1352 \\
1354 \\
1356 \\
1358 \\
1360 \\
1352 \\
1364 \\
1356 \\
1348 \\
1370 \\
1372 \\
1374 \\
1376 \\
1378 \\
1380 \\
1391 \\
1396 \\
1398 \\
1390 \\
1392 \\
1394 \\
1396 \\
1398 \\
1100 \\
1402 \\
1404 \\
1406 \\
1409 \\
1410 \\
1412 \\
1114 \\
1416 \\
1418 \\
1420 \\
1422 \\
1424 \\
1426 \\
1429 \\
\end{array}$ & 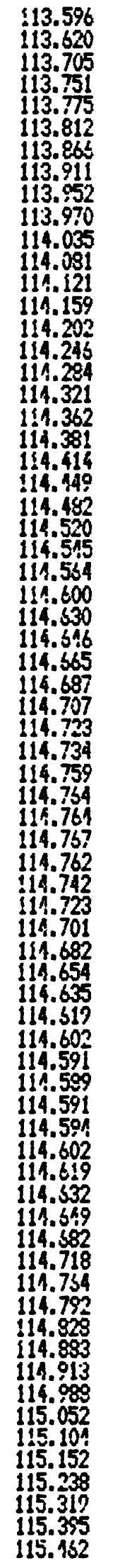 & $\begin{array}{l}1430 \\
1432 \\
1134 \\
1436 \\
1438 \\
1140 \\
1462 \\
1414 \\
1146 \\
1418 \\
1150 \\
1452 \\
1454 \\
1456 \\
1458 \\
1450 \\
1452 \\
1464 \\
1446 \\
1468 \\
1470 \\
1472 \\
1974 \\
1176 \\
1478 \\
1480 \\
1482 \\
1184 \\
1406 \\
1498 \\
1190 \\
1492 \\
1494 \\
1496 \\
1498 \\
1500 \\
1502 \\
1504 \\
1506 \\
1508 \\
1510 \\
1512 \\
1511 \\
1515 \\
1518 \\
1520 \\
1522 \\
1524 \\
1526 \\
1528 \\
1530 \\
1532 \\
1534 \\
1536 \\
1530 \\
1510 \\
1544 \\
1516 \\
1549 \\
1550 \\
1552 \\
1554 \\
1556 \\
1558 \\
1540 \\
1552 \\
1564 \\
1546 \\
1568 \\
1570\end{array}$ & 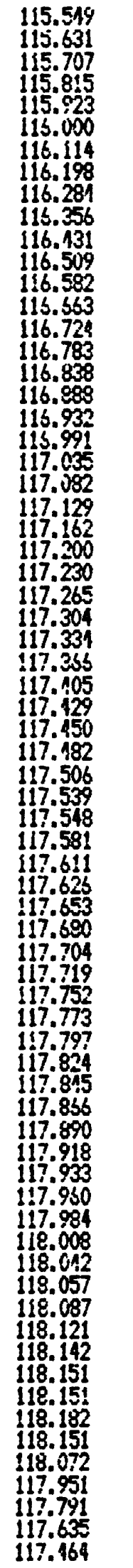 & 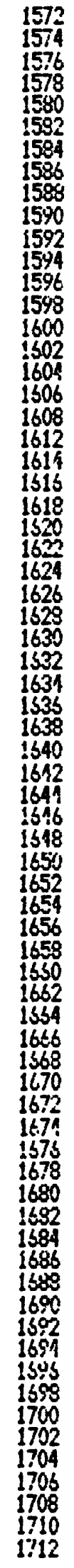 & 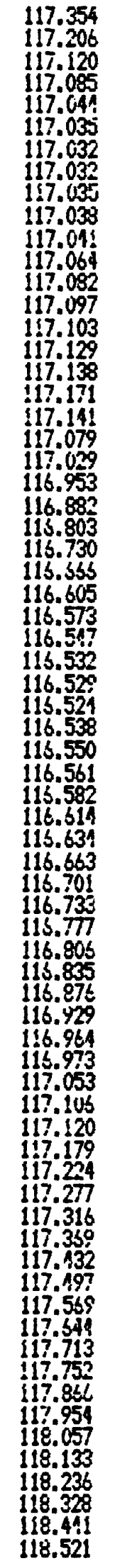 \\
\hline
\end{tabular}


RNFT RIVER, IOHHD - RPTSE-3 - 12 AULUST 19.6

\begin{tabular}{|c|c|c|c|c|c|c|c|c|c|c|c|}
\hline $\begin{array}{l}\text { IEETH } \\
\text { (FT) }\end{array}$ & $\begin{array}{l}\text { TEYPERITURE } \\
\text { (C) }\end{array}$ & $\begin{array}{l}\text { LEPTH } \\
\text { (FT) }\end{array}$ & $\begin{array}{l}\text { TEYPERATLRE } \\
\text { (C) }\end{array}$ & $\begin{array}{l}\text { DEPTH } \\
\text { (FT) }\end{array}$ & $\begin{array}{l}\text { TEPERATURE } \\
\text { (C) }\end{array}$ & $\begin{array}{l}\text { DEPTH } \\
\text { (FT) }\end{array}$ & $\begin{array}{l}\text { TERPERATURE } \\
\text { (C) }\end{array}$ & $\begin{array}{c}\text { DEPTH } \\
\text { (FT) }\end{array}$ & $\begin{array}{l}\text { TEPPERTTLFE } \\
\text { (C) }\end{array}$ & $\begin{array}{l}\text { DEPTH } \\
\text { (FT) }\end{array}$ & $\begin{array}{l}\text { TERPERATI } \\
\text { (C) }\end{array}$ \\
\hline 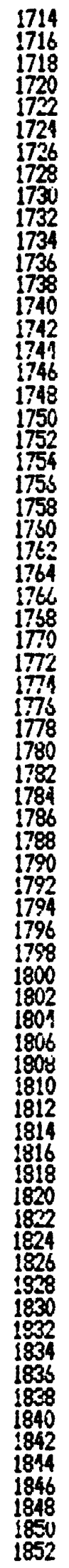 & $\begin{array}{l}118.641 \\
118.737 \\
118.815 \\
118.952 \\
119.089 \\
119.170 \\
119.286 \\
119.349 \\
119.179 \\
119.571 \\
-119.682 \\
119.771 \\
119.883 \\
119.941 \\
120.005 \\
120.079 \\
120.164 \\
120.234 \\
120.321 \\
120.396 \\
120.46 ! \\
120.493 \\
120.536 \\
120.595 \\
120.650 \\
120.699 \\
120.732 \\
120.778 \\
126.821 \\
120.867 \\
126.897 \\
120.893 \\
120.969 \\
121.002 \\
121.032 \\
121.052 \\
121.079 \\
121.095 \\
121.102 \\
121.095 \\
121.072 \\
121.035 \\
121.012 \\
120.979 \\
120.956 \\
120.943 \\
120.920 \\
120.913 \\
120.907 \\
120.907 \\
120.907 \\
120.910 \\
120.930 \\
120.943 \\
120.946 \\
120.983 \\
121.006 \\
121.030 \\
121.079 \\
121.108 \\
121.142 \\
121.189 \\
121.238 \\
121.285 \\
121.301 \\
121.331 \\
121.361 \\
121.118 \\
121.958 \\
121.502\end{array}$ & 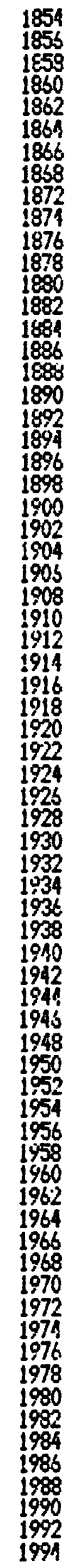 & $\begin{array}{l}121.529 \\
121.556 \\
121.586 \\
121.593 \\
121.559 \\
121.505 \\
121.438 \\
121.356 \\
121.185 \\
121.085 \\
120.963 \\
120.900 \\
120.831 \\
120.752 \\
120.676 \\
120.514 \\
120.546 \\
120.177 \\
120.419 \\
120.357 \\
120.302 \\
120.214 \\
120.150 \\
120.066 \\
119.973 \\
119.477 \\
119.771 \\
119.670 \\
119.583 \\
119.533 \\
119.419 \\
119.353 \\
119.290 \\
119.227 \\
119.177 \\
119.108 \\
119.042 \\
118.983 \\
118.930 \\
118.858 \\
118.805 \\
118.756 \\
118.697 \\
118.554 \\
118.601 \\
118.558 \\
118.506 \\
118.457 \\
118.398 \\
118.331 \\
118.267 \\
118.206 \\
118.151 \\
118.135 \\
118.060 \\
118.015 \\
117.069 \\
117.903 \\
117.845 \\
117.806 \\
117.719 \\
117.698 \\
117.647 \\
117.590 \\
117.527 \\
117.457 \\
117.393 \\
117.316 \\
117.236 \\
117.153\end{array}$ & $\begin{array}{l}1996 \\
1998 \\
2000 \\
2002 \\
2004 \\
2005 \\
2008 \\
2010 \\
2012 \\
2014 \\
2016 \\
2018 \\
2040 \\
2022 \\
2021 \\
2026 \\
2028 \\
2030 \\
2032 \\
2034 \\
2036 \\
2039 \\
2040 \\
2042 \\
2041 \\
2015 \\
2048 \\
2050 \\
2052 \\
2054 \\
2056 \\
2058 \\
2060 \\
2052 \\
2064 \\
2055 \\
2068 \\
2070 \\
2072 \\
2074 \\
2076 \\
2078 \\
2080 \\
2082 \\
2084 \\
2036 \\
2090 \\
2092 \\
2094 \\
2095 \\
2098 \\
2100 \\
2102 \\
2104 \\
2106 \\
2108 \\
2110 \\
2112 \\
2114 \\
2115 \\
2158 \\
2120 \\
2122 \\
2124 \\
2126 \\
2128 \\
2130 \\
2132 \\
2134 \\
2136\end{array}$ & 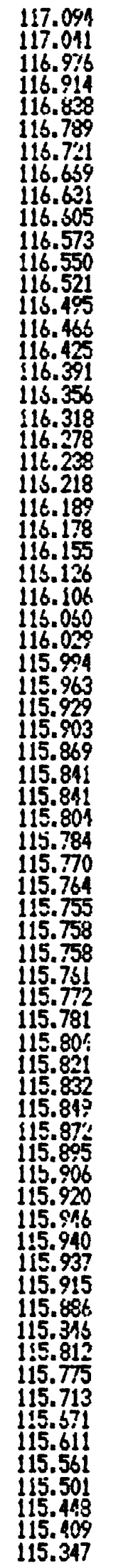 & 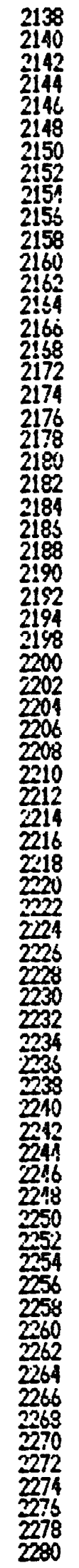 & $\begin{array}{l}115.302 \\
115.255 \\
115.188 \\
115.157 \\
115.102 \\
115.054 \\
115.010 \\
114.968 \\
114.924 \\
111.974 \\
114.830 \\
114.792 \\
111.745 \\
11.701 \\
111.646 \\
114.602 \\
111.501 \\
114.855 \\
114.399 \\
111.341 \\
111.284 \\
111.232 \\
114.189 \\
114.167 \\
114.137 \\
111.102 \\
111.116 \\
114.118 \\
114.118 \\
111.118 \\
114.121 \\
114.135 \\
114.135 \\
114.137 \\
114.154 \\
114.151 \\
114.124 \\
114.099 \\
111.048 \\
113.989 \\
113.035 \\
113.877 \\
113.820 \\
113.753 \\
113.700 \\
113.542 \\
113.591 \\
113.530 \\
113.474 \\
113.114 \\
113.337 \\
113.279 \\
113.195 \\
113.129 \\
113.064 \\
112.989 \\
112.921 \\
112.848 \\
112.793 \\
112.741 \\
112.692 \\
112.566 \\
112.597 \\
112.571 \\
112.535 \\
112.191 \\
112.468 \\
112.440 \\
112.417 \\
112.399\end{array}$ & 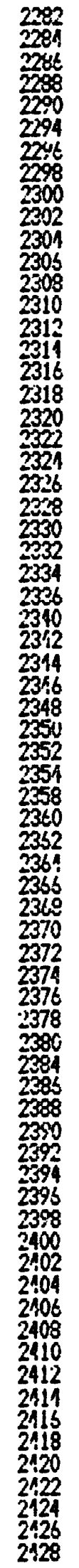 & $\begin{array}{l}112.389 \\
112.336 \\
112.376 \\
112.371 \\
112.376 \\
112.396 \\
112.353 \\
112.340 \\
112.317 \\
112.312 \\
112.286 \\
112.230 \\
112.200 \\
112.156 \\
112.113 \\
112.080 \\
112.032 \\
112.004 \\
111.971 \\
111.946 \\
111.928 \\
111.918 \\
111.916 \\
111.921 \\
111.913 \\
111.018 \\
111.923 \\
111.935 \\
111.959 \\
111.971 \\
111.976 \\
111.996 \\
112.014 \\
112.037 \\
112.017 \\
112.079 \\
112.093 \\
112.106 \\
112.116 \\
112.128 \\
112.131 \\
112.111 \\
112.134 \\
112.141 \\
113.136 \\
112.136 \\
112.139 \\
112.136 \\
112.139 \\
112.13 ! \\
112.126 \\
112.126 \\
112.116 \\
112.111 \\
112.093 \\
112.095 \\
112.073 \\
112.042 \\
112.024 \\
111.099 \\
111.969 \\
111.931 \\
111.890 \\
111.847 \\
111.797 \\
111.747 \\
111.694 \\
111.641 \\
111.584 \\
111.544\end{array}$ & 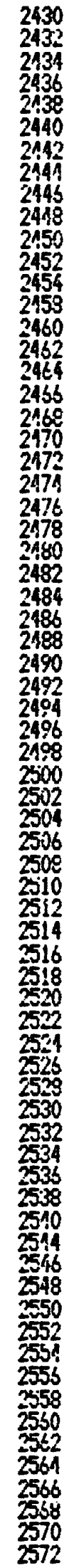 & $\begin{array}{l}111.492 \\
111.452 \\
111.409 \\
11.375 \\
111.390 \\
111.320 \\
111.395 \\
11.280 \\
111.275 \\
111.258 \\
111.261 \\
111.265 \\
11.270 \\
111.275 \\
11.290 \\
111.308 \\
111.315 \\
111.320 \\
111.335 \\
11.337 \\
111.315 \\
111.352 \\
111.350 \\
111.340 \\
111.335\end{array}$ \\
\hline
\end{tabular}


RAFT RIVER, INAHO - PRIE-3 - 12 NLCUST 1976

\begin{tabular}{|c|c|c|c|c|c|c|c|c|c|c|c|}
\hline $\begin{array}{l}\text { IEPTH } \\
\text { (FT) }\end{array}$ & $\begin{array}{l}\text { TEHEERTTURE- } \\
\text { (C) }\end{array}$ & $\begin{array}{l}\text { DEPIH } \\
\text { (FT) }\end{array}$ & $\begin{array}{l}\text { TEMPSRATURE } \\
\text { (C) }\end{array}$ & $\begin{array}{l}\text { DSPTH } \\
\text { (FT) }\end{array}$ & $\begin{array}{l}\text { TEMPERATUFE } \\
\text { (C) }\end{array}$ & $\begin{array}{c}\text { LEPTH } \\
\text { (FT) }\end{array}$ & $\begin{array}{l}\text { TEPPERSTIRE } \\
\text { (C) }\end{array}$ & $\begin{array}{l}\text { DEPTH } \\
\text { (FT) }\end{array}$ & $\begin{array}{l}\text { TEAPERGTTRE } \\
\text { (C) }\end{array}$ & $\begin{array}{l}\text { DEPTH } \\
\text { (FT) }\end{array}$ & $\begin{array}{l}\text { TERPERATISTE } \\
\text { (C) }\end{array}$ \\
\hline 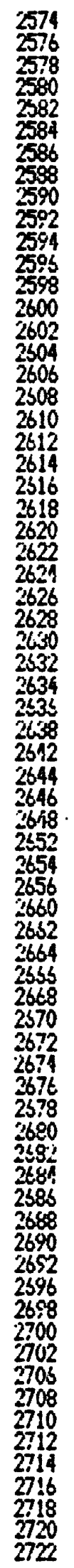 & 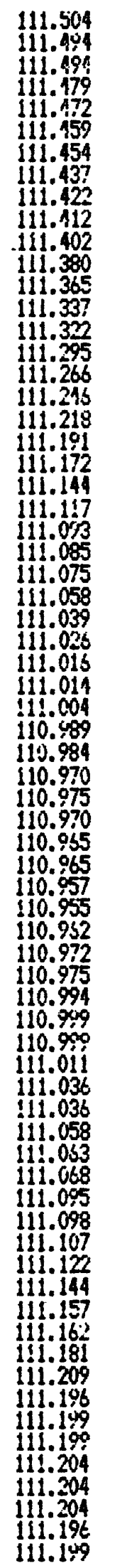 & 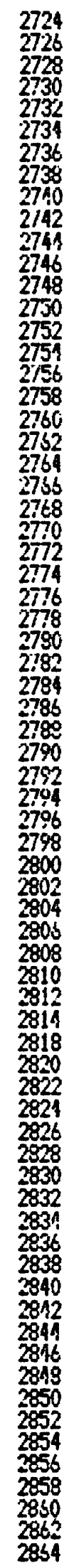 & $\begin{array}{l}111.194 \\
111.196 \\
111.199 \\
111.211 \\
11.211 \\
111.201 \\
111.211 \\
111.223 \\
111.233 \\
111.251 \\
11.275 \\
111.283 \\
111.285 \\
111.305 \\
111.322 \\
111.342 \\
111.362 \\
111.362 \\
111.395 \\
111.429 \\
111.412 \\
111.457 \\
111.482 \\
111.502 \\
111.509 \\
111.527 \\
111.536 \\
111.544 \\
111.554 \\
111.554 \\
111.551 \\
111.556 \\
111.559 \\
111.551 \\
111.561 \\
111.554 \\
111.554 \\
111.559 \\
111.561 \\
111.559 \\
111.544 \\
111.571 \\
111.571 \\
111.576 \\
111.571 \\
111.576 \\
111.581 \\
111.584 \\
111.599 \\
111.593 \\
111.501 \\
111.614 \\
111.626 \\
111.644 \\
111.652 \\
111.367 \\
111.692 \\
111.38 ! \\
111.702 \\
111.714 \\
111.739 \\
111.754 \\
111.774 \\
111.792 \\
111.817 \\
111.830 \\
111.835 \\
111.875 \\
111.898 \\
111.931\end{array}$ & 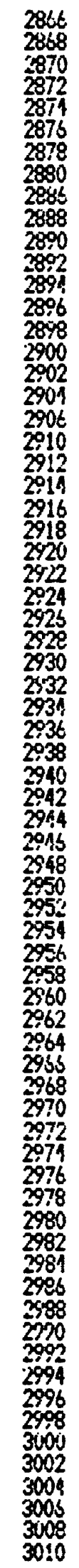 & $\begin{array}{l}111.996 \\
111.959 \\
111.976 \\
111.049 \\
112.032 \\
112.050 \\
112.060 \\
112.073 \\
112.111 \\
112.115 \\
112.123 \\
112.139 \\
112.149 \\
112.149 \\
112.161 \\
112.169 \\
112.172 \\
112.177 \\
112.184 \\
112.189 \\
112.187 \\
112.184 \\
112.164 \\
112.187 \\
112.179 \\
112.179 \\
112.177 \\
112.177 \\
112.169 \\
112.167 \\
112.159 \\
112.159 \\
112.159 \\
112.161 \\
112.151 \\
112.154 \\
112.151 \\
112.159 \\
112.154 \\
112.156 \\
112.161 \\
112.159 \\
112.154 \\
112.161 \\
112.167 \\
112.187 \\
112.172 \\
112.167 \\
112.179 \\
112.172 \\
112.182 \\
112.174 \\
112.169 \\
112.174 \\
112.164 \\
112.154 \\
112.156 \\
112.151 \\
112.136 \\
112.118 \\
112.108 \\
112.093 \\
112.073 \\
112.055 \\
112.012 \\
112.012 \\
111.994 \\
111.979 \\
111.953 \\
111.913\end{array}$ & $\begin{array}{l}3012 \\
3014 \\
30 ! 6 \\
3018 \\
3020 \\
3022 \\
3024 \\
3026 \\
3028 \\
3030 \\
3032 \\
3034 \\
3036 \\
3038 \\
3040 \\
3042 \\
3044 \\
3045 \\
3048 \\
3050 \\
3052 \\
3054 \\
3056 \\
3058 \\
3060 \\
3052 \\
3054 \\
3055 \\
3068 \\
31570 \\
3072 \\
3074 \\
3074 \\
3078 \\
3080 \\
3082 \\
3084 \\
3085 \\
3088 \\
3090 \\
3002 \\
3094 \\
3096 \\
3098 \\
3100 \\
3102 \\
3104 \\
3106 \\
3108 \\
3110 \\
3112 \\
3114 \\
3116 \\
3118 \\
3120 \\
3122 \\
3124 \\
3126 \\
3123 \\
3130 \\
3132 \\
3134 \\
3136 \\
3138 \\
3140 \\
3112 \\
3144 \\
3145 \\
3110 \\
3150\end{array}$ & 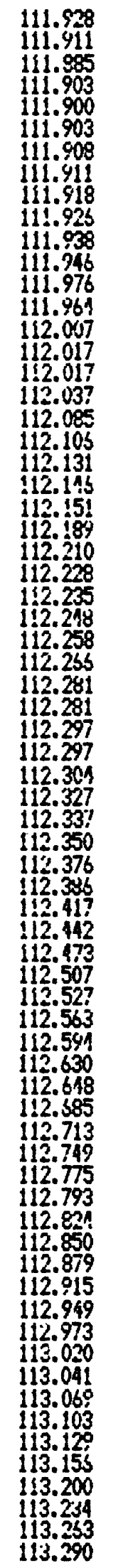 & 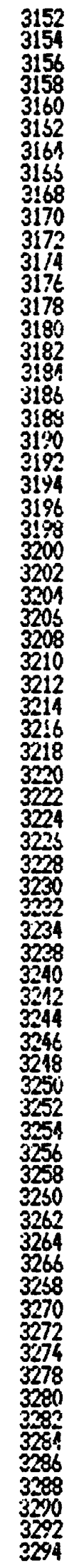 & 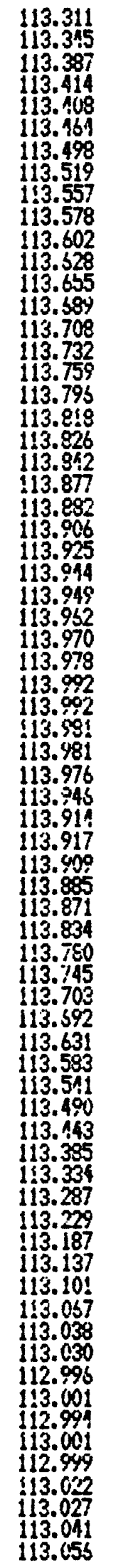 & 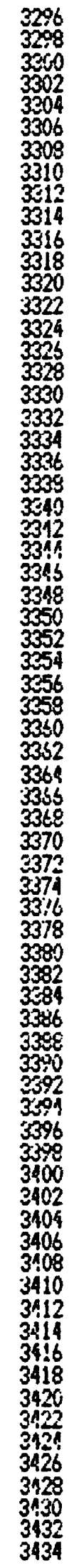 & 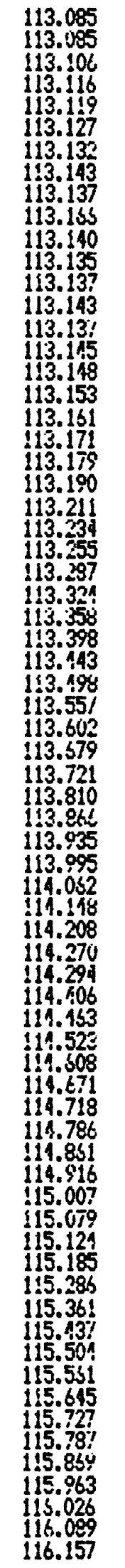 \\
\hline
\end{tabular}


RHFT RIVER, IOAHO - RRGE-3 - 12 AUEUST $19 \% 6$

\begin{tabular}{|c|c|c|c|c|c|c|c|c|c|c|c|}
\hline $\begin{array}{l}\text { IEPTH } \\
\text { (FT) }\end{array}$ & $\begin{array}{l}\text { TEPPERATLGE } \\
\text { (C) }\end{array}$ & $\begin{array}{l}\text { DEPTH } \\
\text { (FT) }\end{array}$ & $\begin{array}{l}\text { TERPERATURE } \\
\text { (C) }\end{array}$ & $\begin{array}{l}\text { DEPTH } \\
\text { (FT) }\end{array}$ & $\begin{array}{l}\text { TEPPEPATURE } \\
\text { (C) }\end{array}$ & $\begin{array}{l}\text { DEPTH } \\
\text { (FT) }\end{array}$ & $\begin{array}{l}\text { TERPEFATLPL } \\
\text { (C) }\end{array}$ & $\begin{array}{l}\text { DEPTH } \\
\text { (FT) }\end{array}$ & $\begin{array}{l}\text { TERERATLRE } \\
\text { (C) }\end{array}$ & $\begin{array}{l}\text { LEPTH } \\
\text { (FT) }\end{array}$ & $\begin{array}{l}\text { TEAFERATIRE } \\
\text { (C) }\end{array}$ \\
\hline 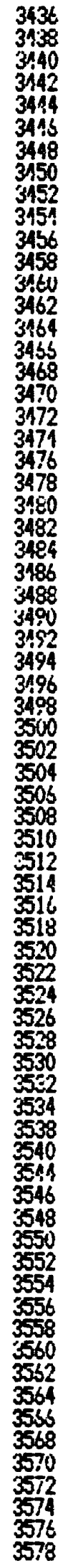 & 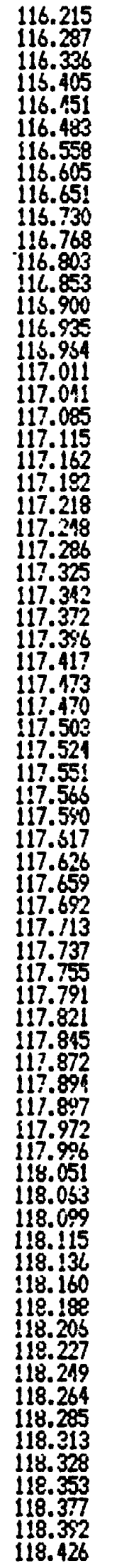 & 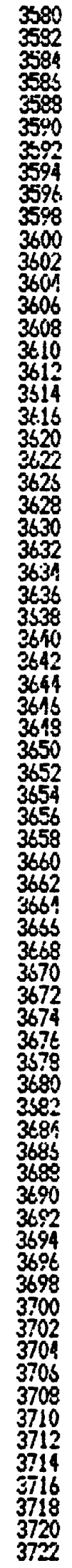 & 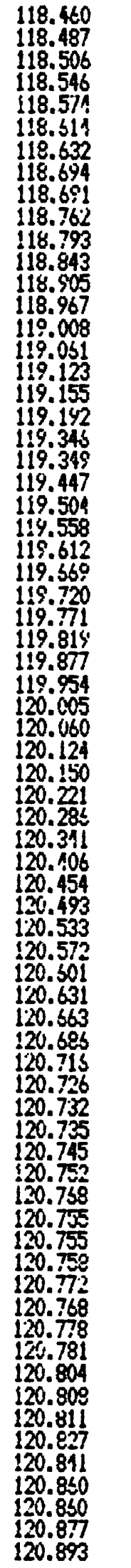 & 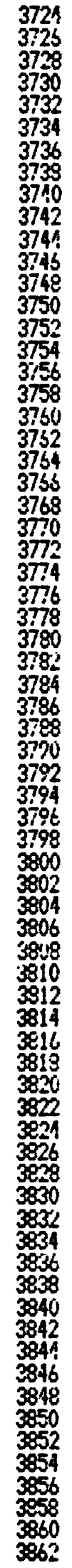 & 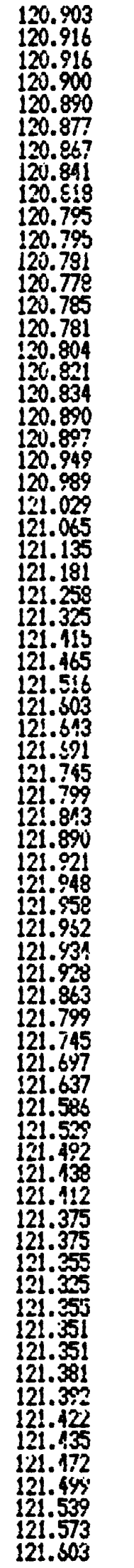 & 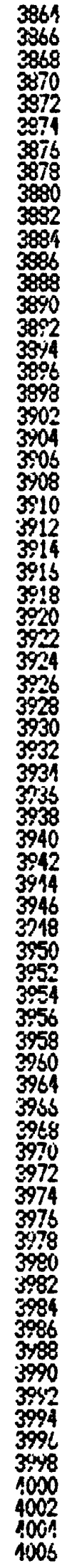 & 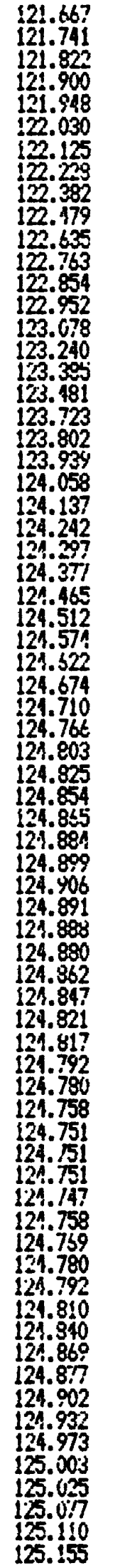 & 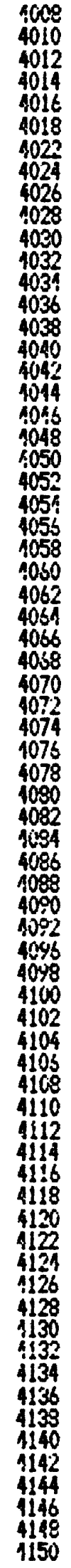 & 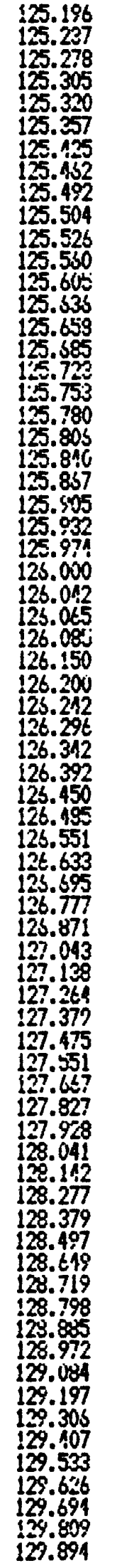 & 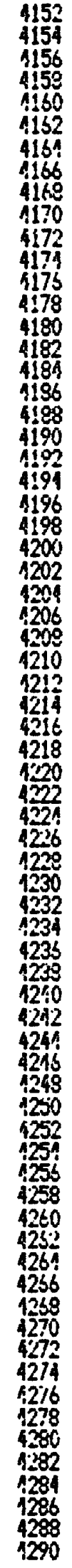 & 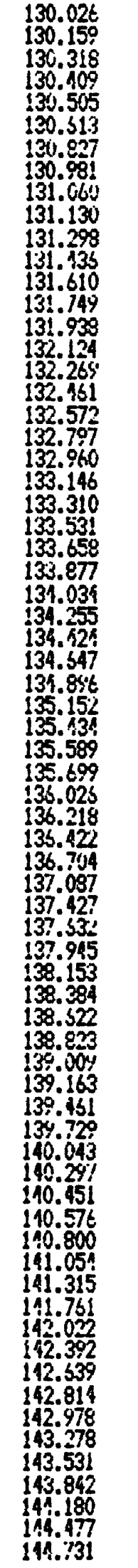 \\
\hline
\end{tabular}


PAFT RIVER, IOAHD - PACE-3 - 12 AUGUST 1975

\begin{tabular}{|c|c|c|c|c|c|c|c|c|c|c|c|}
\hline $\begin{array}{l}\text { IEPTH } \\
\text { (FT) }\end{array}$ & $\begin{array}{l}\text { TEXPEPATURE } \\
\text { (C) }\end{array}$ & $\begin{array}{l}\text { DEPTH } \\
\text { (FT) }\end{array}$ & $\begin{array}{l}\text { TEYPERAILPE } \\
\text { (C) }\end{array}$ & $\begin{array}{l}\text { DETH } \\
\text { (FT) }\end{array}$ & $\begin{array}{l}\text { TEAPERATLRE } \\
\text { (C) }\end{array}$ & $\begin{array}{l}\text { DEFTH } \\
\text { (FT) }\end{array}$ & $\begin{array}{l}\text { TEMPERATURE } \\
\text { (C) }\end{array}$ & $\begin{array}{l}\text { DEPTH } \\
\text { (FT) }\end{array}$ & $\begin{array}{l}\text { TEMPERATLPE } \\
\text { (C) }\end{array}$ & $\begin{array}{l}\text { DEPTH } \\
\text { (FT) }\end{array}$ & $\begin{array}{l}\text { TENHERATLHE } \\
\text { (C) }\end{array}$ \\
\hline 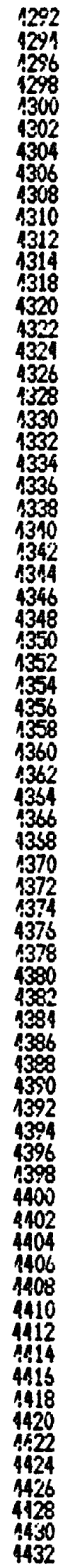 & 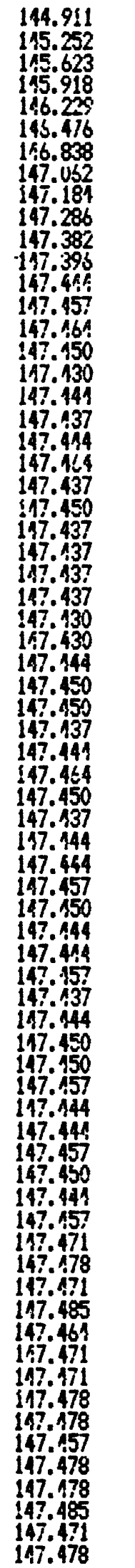 & 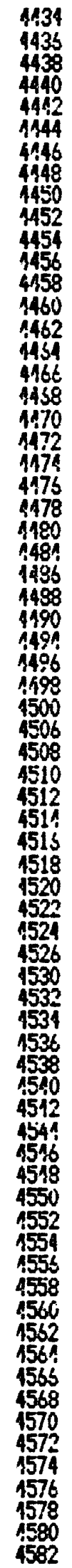 & 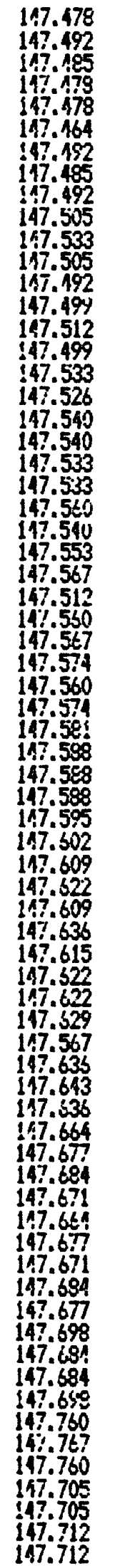 & 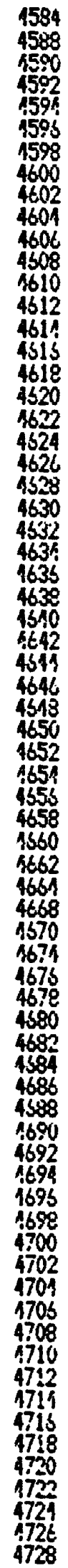 & 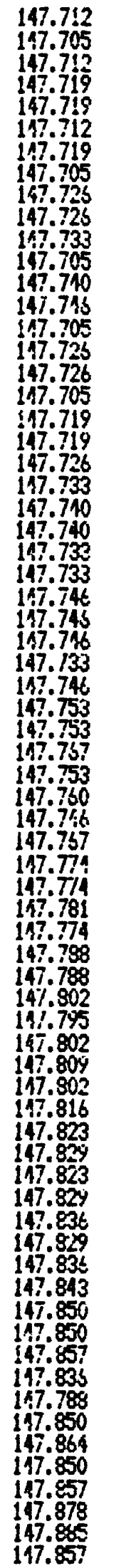 & 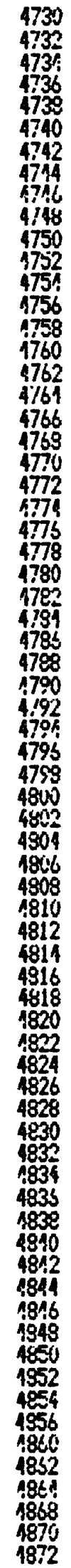 & 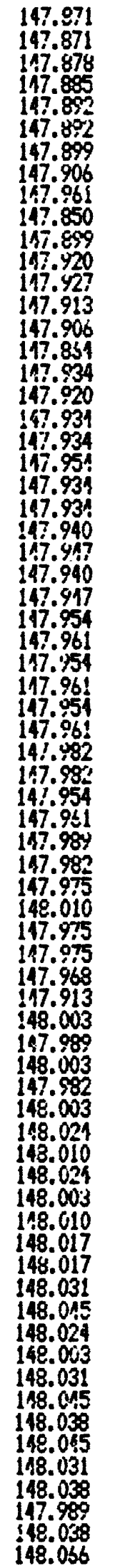 & 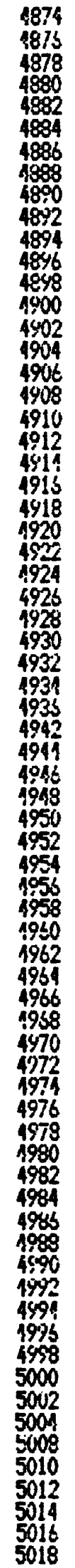 & 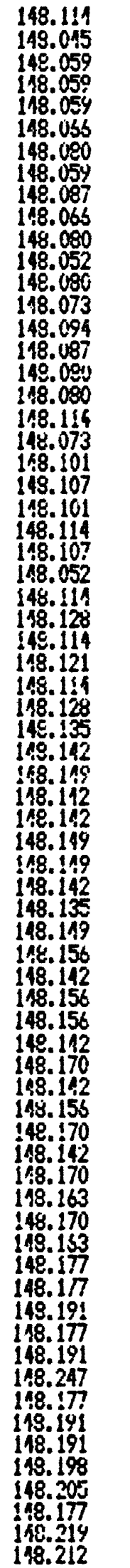 & $\begin{array}{l}5020 \\
5022 \\
5024 \\
5026 \\
5028 \\
5030 \\
5032 \\
5034 \\
5036 \\
5038 \\
5010 \\
5042 \\
5044 \\
5046 \\
5048 \\
5050 \\
5052 \\
5054 \\
5056 \\
5058 \\
5060 \\
5052 \\
5064 \\
5056 \\
5069 \\
5070 \\
51072 \\
5074 \\
5076 \\
5078 \\
5090 \\
5082 \\
5084 \\
5086 \\
5080 \\
5090 \\
5002 \\
5044 \\
5096 \\
5093 \\
5100 \\
5102 \\
5101 \\
5106 \\
5108 \\
5110 \\
5112 \\
5114 \\
5116 \\
5118 \\
5120 \\
5122 \\
5124 \\
5123 \\
5128 \\
5130 \\
5132 \\
5134 \\
5136 \\
5138 \\
5140 \\
5182 \\
5114 \\
5146 \\
5148 \\
5150 \\
5152 \\
5154 \\
5156 \\
5158\end{array}$ & 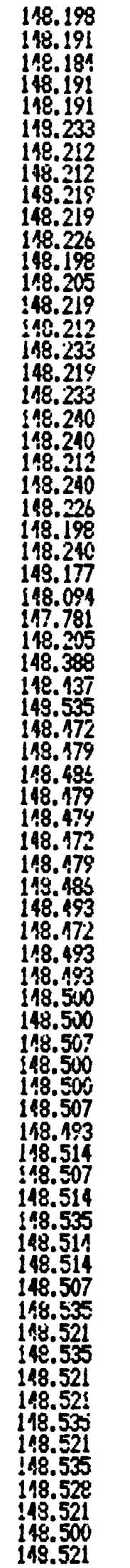 \\
\hline
\end{tabular}


RAFT RIVER, ILAHO - PRGE-3 - 12 NUGAST 1976

\begin{tabular}{|c|c|c|c|c|c|c|c|c|c|c|c|}
\hline $\begin{array}{l}\text { IEPTH } \\
\text { (FT) }\end{array}$ & $\begin{array}{c}\text { TEREERATIRE } \\
\text { (C) }\end{array}$ & $\begin{array}{l}\text { DEPTH } \\
\text { (FT) }\end{array}$ & $\begin{array}{l}\text { TEPPERATRE } \\
\text { (C) }\end{array}$ & $\begin{array}{l}\text { DEPTH } \\
\text { (FT) }\end{array}$ & $\begin{array}{l}\text { TEFERATLRE } \\
\text { (C) }\end{array}$ & $\begin{array}{c}\text { DEPTH } \\
\text { (FT) }\end{array}$ & $\begin{array}{l}\text { TEMPERSTURE } \\
\text { (C) }\end{array}$ & $\begin{array}{l}\text { LEPTH } \\
\text { (FT) }\end{array}$ & $\begin{array}{l}\text { TEMPERATLRE } \\
\text { (C) }\end{array}$ & $\begin{array}{l}\text { DEPTH } \\
\text { (FT) }\end{array}$ & $\begin{array}{l}\text { TEMPERATURE } \\
\text { (C) }\end{array}$ \\
\hline 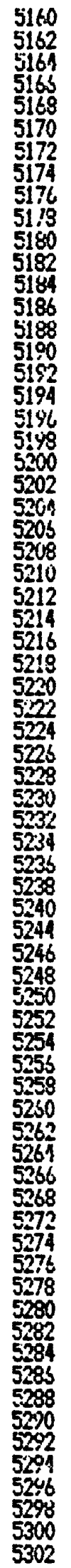 & 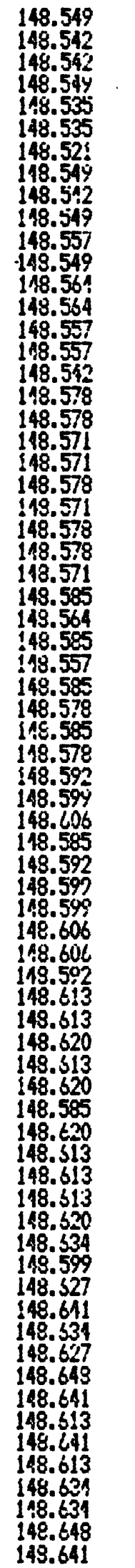 & 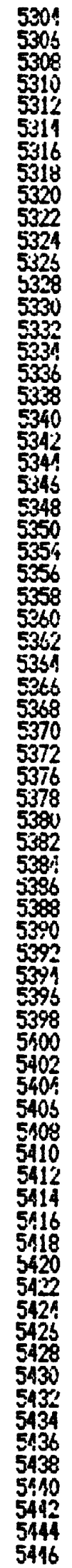 & 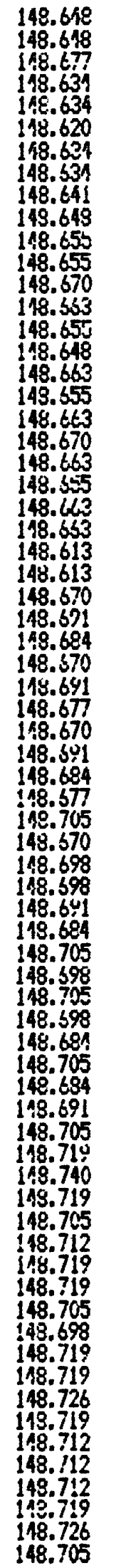 & 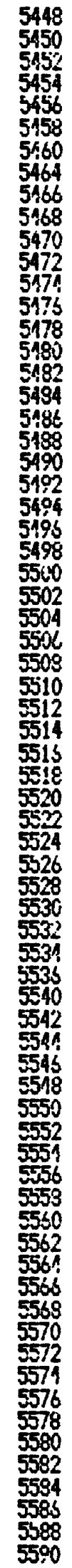 & 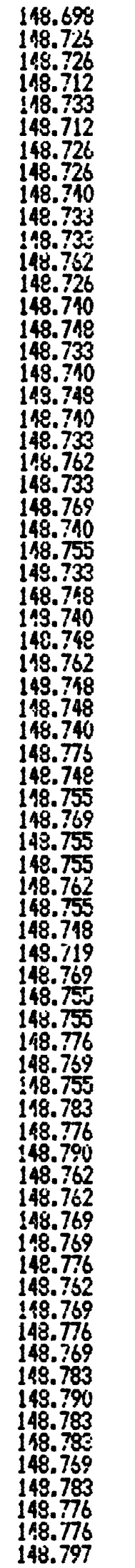 & 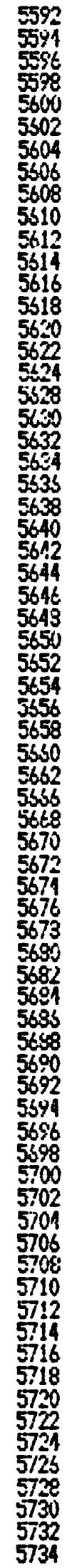 & 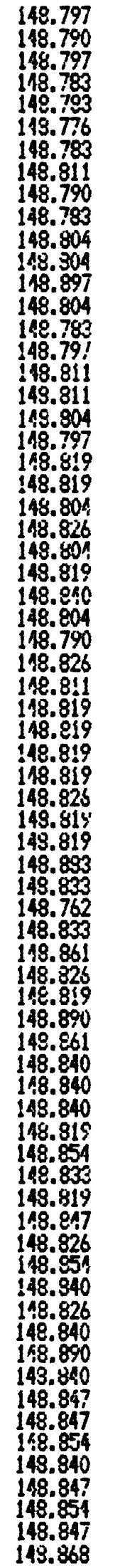 & 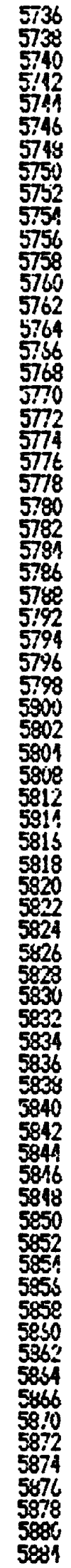 & 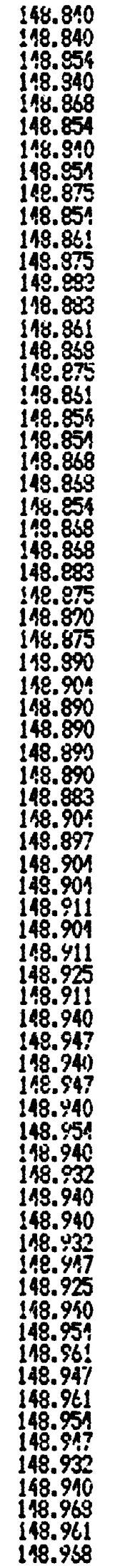 & $\begin{array}{l}5986 \\
5808 \\
5890 \\
5902 \\
5996 \\
5900 \\
5902 \\
5904 \\
5906 \\
5908 \\
5910 \\
5012 \\
5914 \\
5913 \\
5918 \\
5920\end{array}$ & $\begin{array}{l}118.997 \\
118.975 \\
148.997 \\
148.407 \\
148.975 \\
149.999 \\
149.001 \\
148.432 \\
148.911 \\
148.911 \\
148.925 \\
148.040 \\
148.422 \\
148.025 \\
118.925 \\
148.932\end{array}$ \\
\hline
\end{tabular}


RAFT RIVER, IONHO - HLTI WEL - 20 OCTOBER 5975

\begin{tabular}{|c|c|c|c|c|c|c|c|}
\hline $\begin{array}{l}\text { IEPTH } \\
\text { (FT) }\end{array}$ & $\begin{array}{l}\text { TEMPERATURE } \\
\text { (C) }\end{array}$ & $\begin{array}{c}\text { DEPTH } \\
\text { (FT) }\end{array}$ & $\begin{array}{l}\text { IEYPSRATLRE } \\
\text { (C) }\end{array}$ & $\begin{array}{l}\text { DEPTH } \\
\text { (FT! }\end{array}$ & $\begin{array}{l}\text { TEMPERATURE } \\
\text { (C) }\end{array}$ & $\underset{(F T)}{\text { DEPTH }}$ & $\begin{array}{l}\text { TEPERATRR } \\
\text { (C) }\end{array}$ \\
\hline $\begin{array}{l}5 \\
10 \\
15 \\
20 \\
25 \\
30 \\
35 \\
40 \\
15 \\
50 \\
55 \\
60 \\
65 \\
70 \\
75 \\
80 \\
85 \\
90 \\
95 \\
100 \\
105 \\
110 \\
115 \\
120 \\
125 \\
130 \\
135 \\
140 \\
145 \\
150 \\
155 \\
160 \\
165 \\
170 \\
175 \\
180 \\
185 \\
190 \\
195 \\
200 \\
205 \\
210 \\
215 \\
220 \\
225 \\
230 \\
235 \\
240 \\
245 \\
250 \\
255 \\
260 \\
265 \\
270 \\
280 \\
285 \\
290 \\
295 \\
300 \\
305 \\
310 \\
315 \\
325 \\
330 \\
355 \\
310 \\
345 \\
350 \\
355 \\
360\end{array}$ & $\begin{array}{l}13.438 \\
12.601 \\
12.711 \\
12.061 \\
13.280 \\
13.608 \\
13.915 \\
11.183 \\
11.501 \\
14.759 \\
11.985 \\
15.242 \\
15.121 \\
15.661 \\
15.845 \\
16.010 \\
16.186 \\
16.296 \\
16.456 \\
16.594 \\
16.760 \\
16.943 \\
17.093 \\
17.306 \\
11.441 \\
17.664 \\
17.871 \\
18.060 \\
18.305 \\
18.539 \\
18.760 \\
18.969 \\
19.216 \\
19.405 \\
19.630 \\
19.871 \\
20.066 \\
20.327 \\
20.520 \\
20.749 \\
20.924 \\
21.082 \\
21.271 \\
21.453 \\
21.607 \\
21.820 \\
21.970 \\
22.155 \\
22.209 \\
22.479 \\
22.597 \\
22.736 \\
22.933 \\
23.107 \\
23.396 \\
23.609 \\
23.754 \\
23.914 \\
24.040 \\
24.213 \\
24.434 \\
24.512 \\
24.858 \\
25.016 \\
25.181 \\
25.304 \\
25.491 \\
25.633 \\
25.712 \\
25.891\end{array}$ & $\begin{array}{l}345 \\
370 \\
375 \\
380 \\
355 \\
390 \\
395 \\
100 \\
405 \\
410 \\
415 \\
420 \\
425 \\
430 \\
135 \\
440 \\
145 \\
150 \\
455 \\
460 \\
465 \\
470 \\
475 \\
480 \\
495 \\
490 \\
495 \\
500 \\
505 \\
510 \\
515 \\
520 \\
525 \\
530 \\
535 \\
540 \\
545 \\
550 \\
555 \\
560 \\
545 \\
570 \\
575 \\
560 \\
585 \\
590 \\
595 \\
600 \\
505 \\
610 \\
615 \\
620 \\
625 \\
630 \\
635 \\
640 \\
345 \\
650 \\
655 \\
660 \\
655 \\
670 \\
675 \\
680 \\
695 \\
690 \\
695 \\
700 \\
705 \\
710\end{array}$ & 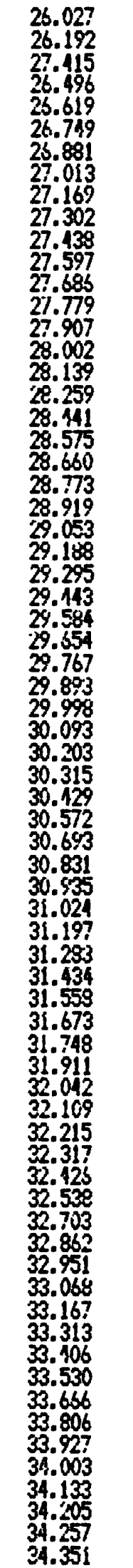 & $\begin{array}{l}715 \\
720 \\
725 \\
730 \\
735 \\
740 \\
715 \\
750 \\
755 \\
760 \\
770 \\
775 \\
780 \\
785 \\
790 \\
795 \\
800 \\
805 \\
810 \\
815 \\
820 \\
825 \\
830 \\
835 \\
840 \\
845 \\
850 \\
855 \\
860 \\
865 \\
870 \\
875 \\
890 \\
885 \\
890 \\
895 \\
900 \\
905 \\
110 \\
915 \\
920 \\
925 \\
730 \\
935 \\
940 \\
945 \\
950 \\
955 \\
980 \\
965 \\
970 \\
975 \\
980 \\
985 \\
990 \\
995 \\
1000 \\
1005 \\
1010 \\
1015 \\
1020 \\
1025 \\
1030 \\
1035 \\
1010 \\
1015 \\
1050 \\
1055 \\
1060 \\
1065\end{array}$ & 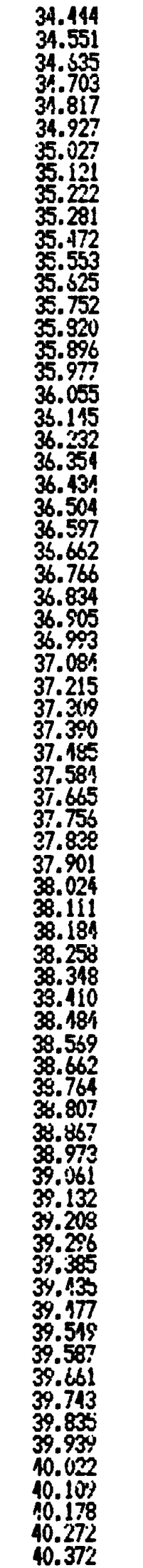 & $\begin{array}{l}1070 \\
1075 \\
1080 \\
1085 \\
1090 \\
1095 \\
1100 \\
1105 \\
1110 \\
1115 \\
1120 \\
1125 \\
1130 \\
1135 \\
1140 \\
1145 \\
1150 \\
1155 \\
1160 \\
1165 \\
1170 \\
1175 \\
1180 \\
1185 \\
1190 \\
1195 \\
1200 \\
1205 \\
1210 \\
1215 \\
1220 \\
1225 \\
1230 \\
1235 \\
1240 \\
1265 \\
1250 \\
1255 \\
1250 \\
1265 \\
1270 \\
1275 \\
1280 \\
1285\end{array}$ & $\begin{array}{l}40.412 \\
40.520 \\
40.586 \\
40.678 \\
40.732 \\
40.826 \\
40.885 \\
40.978 \\
41.029 \\
41.111 \\
41.192 \\
41.316 \\
11.391 \\
41.431 \\
41.487 \\
11.560 \\
41.598 \\
41.700 \\
11.755 \\
11.831 \\
41.913 \\
11.993 \\
12.049 \\
12.142 \\
12.277 \\
12.343 \\
12.417 \\
12.535 \\
42.583 \\
32.664 \\
42.815 \\
12.942 \\
43.032 \\
13.108 \\
43.191 \\
43.281 \\
43.281 \\
43.318 \\
43.333 \\
13.364 \\
43.533 \\
43.656 \\
43.775 \\
43.862\end{array}$ \\
\hline
\end{tabular}




\begin{tabular}{|c|c|c|}
\hline $\begin{array}{c}\text { IEPTH } \\
\text { (FT) }\end{array}$ & $\begin{array}{l}\text { TEPERATLR } \\
\text { (C) }\end{array}$ & DEP \\
\hline
\end{tabular}

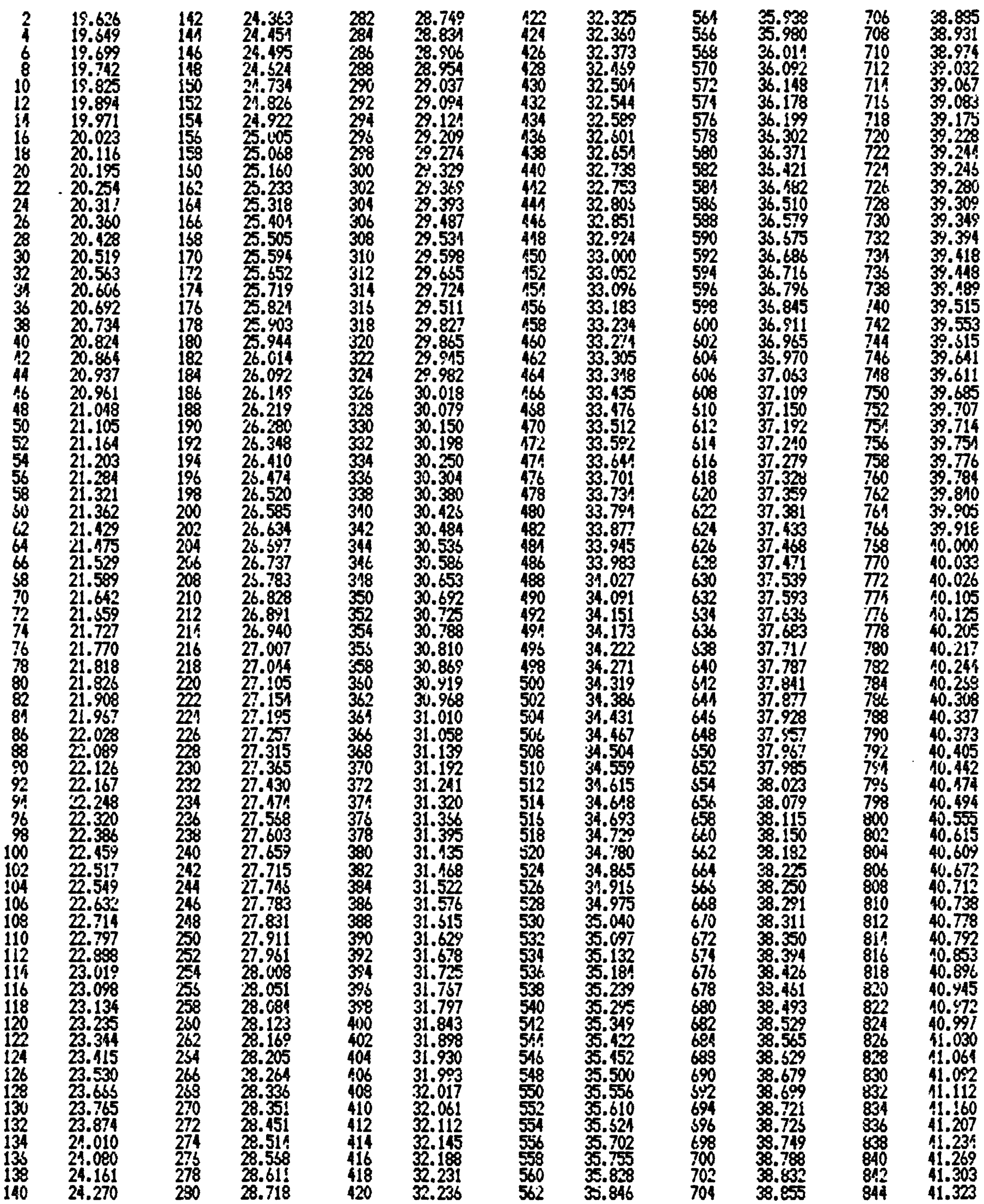


RAFT RIVER, IOHO - MLTA HEUL - 9 NLLUST 1975

\begin{tabular}{|c|c|c|c|c|c|c|c|}
\hline $\begin{array}{l}\text { DEPTH } \\
\text { (FT) }\end{array}$ & $\begin{array}{l}\text { TEMPERATLRE } \\
\text { (C) }\end{array}$ & $\begin{array}{l}\text { DEFTH } \\
\text { (FT) }\end{array}$ & $\begin{array}{l}\text { TEVPERATURE } \\
\text { (C) }\end{array}$ & $\begin{array}{l}\text { LEPTH } \\
\text { (FT) }\end{array}$ & $\begin{array}{l}\text { EEPPERINURE } \\
\text { (C) }\end{array}$ & $\begin{array}{l}\text { DEFTH } \\
\text { (FT) }\end{array}$ & $\begin{array}{l}\text { IEPERATI } \\
\text { (C) }\end{array}$ \\
\hline $\begin{array}{l}846 \\
848 \\
850 \\
852 \\
854 \\
856 \\
850 \\
860 \\
862 \\
854 \\
866 \\
858 \\
870 \\
872 \\
874 \\
876 \\
878 \\
880 \\
882 \\
884 \\
886 \\
898 \\
840 \\
892 \\
894 \\
895 \\
898 \\
900 \\
902 \\
904 \\
906 \\
908 \\
910 \\
912 \\
914 \\
916 \\
918 \\
920 \\
922 \\
924 \\
926 \\
428 \\
930 \\
932 \\
931 \\
936 \\
938 \\
940 \\
912 \\
944 \\
946 \\
948 \\
950 \\
952 \\
951 \\
953 \\
958 \\
950 \\
462 \\
964 \\
966 \\
989 \\
970 \\
972 \\
974 \\
976 \\
978 \\
980 \\
982 \\
984\end{array}$ & $\begin{array}{l}41.353 \\
41.374 \\
41.411 \\
11.144 \\
41.475 \\
11.494 \\
41.510 \\
11.535 \\
41.568 \\
41.306 \\
41.617 \\
41.657 \\
41.687 \\
41.722 \\
41.749 \\
11.763 \\
11.736 \\
41.805 \\
41.842 \\
11.970 \\
41.897 \\
41.043 \\
41.979 \\
42.000 \\
42.025 \\
42.055 \\
42.112 \\
42.1132 \\
42.210 \\
12.277 \\
42.303 \\
42.313 \\
42.335 \\
42.369 \\
42.338 \\
42.430 \\
42.753 \\
12.182 \\
42.525 \\
42.559 \\
42.580 \\
42.615 \\
42.692 \\
42.678 \\
42.707 \\
42.755 \\
42.766 \\
42.794 \\
42.824 \\
12.849 \\
42.840 \\
42.908 \\
42.934 \\
42.965 \\
42.985 \\
43.028 \\
43.059 \\
43.104 \\
43.124 \\
13.151 \\
43.194 \\
43.234 \\
43.237 \\
13.278 \\
43.296 \\
43.311 \\
43.350 \\
13.383 \\
43.815 \\
43.445\end{array}$ & $\begin{array}{l}986 \\
988 \\
900 \\
992 \\
994 \\
996 \\
998 \\
1000 \\
1002 \\
1004 \\
1006 \\
1008 \\
1010 \\
1012 \\
1011 \\
1016 \\
1018 \\
1020 \\
1024 \\
1025 \\
1028 \\
1030 \\
1032 \\
1034 \\
1036 \\
1038 \\
1040 \\
1042 \\
1044 \\
1046 \\
1048 \\
1050 \\
1052 \\
1054 \\
1056 \\
1058 \\
1060 \\
1062 \\
1061 \\
1035 \\
1068 \\
1070 \\
1072 \\
1074 \\
1076 \\
1078 \\
1080 \\
1082 \\
1094 \\
1086 \\
1088 \\
1090 \\
1092 \\
1096 \\
1098 \\
1100 \\
1102 \\
1104 \\
1106 \\
1108 \\
1110 \\
1112 \\
1114 \\
1116 \\
1118 \\
1120 \\
1122 \\
1124 \\
1126 \\
1128\end{array}$ & 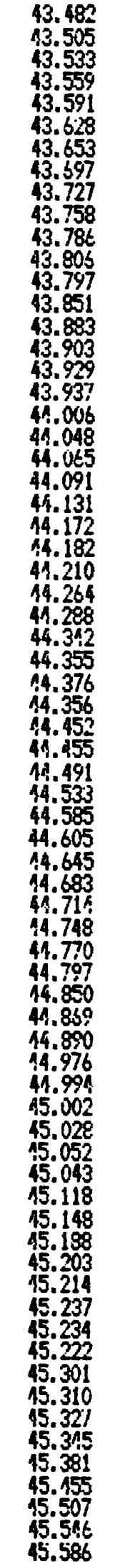 & $\begin{array}{l}1130 \\
1132 \\
1134 \\
1135 \\
1138 \\
1190 \\
1142 \\
1144 \\
1146 \\
1148 \\
1150 \\
1152 \\
1154 \\
1156 \\
1158 \\
1160 \\
1162 \\
1151 \\
1166 \\
1168 \\
1170 \\
1172 \\
1174 \\
1116 \\
1178 \\
1180 \\
1182 \\
1184 \\
1186 \\
1188 \\
1190 \\
1192 \\
1194 \\
1195 \\
1198 \\
1200 \\
1202 \\
1204 \\
1206 \\
1208 \\
1212 \\
1214 \\
1216 \\
1218 \\
1220 \\
1222 \\
1224 \\
1226 \\
1228 \\
1230 \\
1232 \\
1234 \\
1236 \\
1238 \\
1240 \\
1242 \\
1244 \\
1246 \\
1248 \\
1250 \\
1252 \\
1256 \\
1258 \\
1260 \\
1262 \\
1264 \\
1266 \\
1238 \\
1270 \\
1272\end{array}$ & 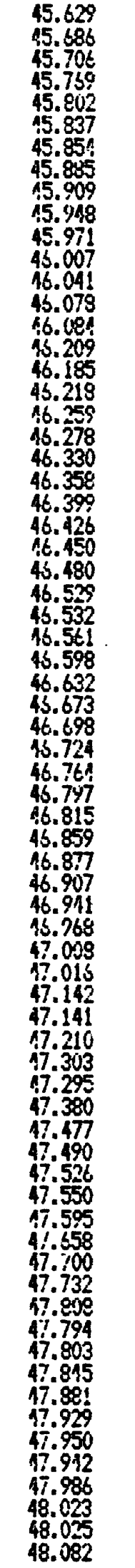 & $\begin{array}{l}1274 \\
1276 \\
1278 \\
1280 \\
1282 \\
1284 \\
1386 \\
1288 \\
1290 \\
1292 \\
1294 \\
1295 \\
1298 \\
1300 \\
1302 \\
1304 \\
1306 \\
1308 \\
1310 \\
1312 \\
1314 \\
1316 \\
1318 \\
1322 \\
1321 \\
1326 \\
1328 \\
1330 \\
1332 \\
1334 \\
1336 \\
1339 \\
1340 \\
1342 \\
1341 \\
1346 \\
1348 \\
1350 \\
1352 \\
1354 \\
1356 \\
1358 \\
1360 \\
1362 \\
1364 \\
1366 \\
1360 \\
1370 \\
1372 \\
1374 \\
1376 \\
1378 \\
1380 \\
1382 \\
1384 \\
1384 \\
1398 \\
1390 \\
1392 \\
1394 \\
1396 \\
1399 \\
1400 \\
1402 \\
1404 \\
1406 \\
1408 \\
1110 \\
1412 \\
1416\end{array}$ & 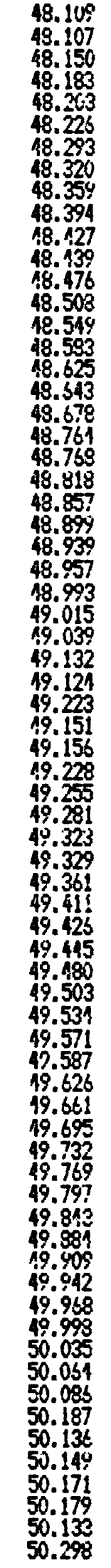 \\
\hline
\end{tabular}


RAFT RIVER, IDAHD - MALTA HELL - 18 DELERBER I976

\begin{tabular}{|c|c|c|c|c|c|c|c|c|c|c|c|}
\hline $\begin{array}{l}\text { DEFTH } \\
\text { (FT) }\end{array}$ & $\begin{array}{l}\text { TEMPEEATLRE } \\
\text { (C) }\end{array}$ & $\begin{array}{l}\text { DEPTH } \\
\text { (FT) }\end{array}$ & $\begin{array}{l}\text { TEMPERATLRE } \\
\text { (C) }\end{array}$ & $\begin{array}{l}\text { DEPTH } \\
\text { (FT) }\end{array}$ & $\begin{array}{l}\text { TEMPERATLRE } \\
\text { (C) }\end{array}$ & $\begin{array}{c}\text { DEPTH } \\
\text { (FT) }\end{array}$ & $\begin{array}{l}\text { TEMPEFATLRE } \\
\text { (C) }\end{array}$ & $\begin{array}{l}\text { DEPTH } \\
\text { (FT) }\end{array}$ & $\begin{array}{l}\text { TEFPERATURE } \\
\text { (C) }\end{array}$ & $\begin{array}{l}\text { DEPTH } \\
\text { (FT) }\end{array}$ & $\begin{array}{l}\text { IEMPERATURE } \\
\text { (C) }\end{array}$ \\
\hline $\begin{array}{r}1 \\
3 \\
4 \\
6 \\
8 \\
10 \\
12 \\
14 \\
16 \\
19 \\
21 \\
23 \\
75 \\
27 \\
38 \\
30 \\
32 \\
34 \\
36 \\
38 \\
10 \\
12 \\
14 \\
46 \\
48 \\
50 \\
52 \\
54 \\
56 \\
58 \\
60 \\
32 \\
64 \\
35 \\
68 \\
70 \\
72 \\
74 \\
76 \\
78 \\
80 \\
82 \\
84 \\
86 \\
88 \\
90 \\
92 \\
91 \\
96 \\
48 \\
100 \\
102 \\
104 \\
105 \\
108 \\
110 \\
112 \\
114 \\
116 \\
118 \\
120 \\
122 \\
124 \\
126 \\
128 \\
130 \\
132 \\
134 \\
136 \\
138\end{array}$ & $\begin{array}{l}51.014 \\
51.250 \\
51.449 \\
52.507 \\
53.489 \\
53.849 \\
54.119 \\
54.993 \\
55.723 \\
56.135 \\
56.839 \\
.57 .131 \\
57.731 \\
57.801 \\
58.000 \\
58.157 \\
58.315 \\
58.500 \\
58.626 \\
58.739 \\
58.831 \\
58.021 \\
58.962 \\
59.026 \\
59.078 \\
59.135 \\
59.163 \\
59.200 \\
59.223 \\
50.258 \\
59.278 \\
59.289 \\
59.295 \\
59.304 \\
59.317 \\
59.326 \\
59.378 \\
59.335 \\
59.332 \\
59.326 \\
59.319 \\
59.321 \\
59.302 \\
59.304 \\
59.302 \\
59.297 \\
59.302 \\
59.293 \\
59.274 \\
59.241 \\
59.195 \\
59.180 \\
59.167 \\
50.156 \\
59.180 \\
59.204 \\
59.269 \\
59.324 \\
59.365 \\
59.380 \\
59.359 \\
59.351 \\
59.311 \\
50.375 \\
59.421 \\
59.475 \\
59.595 \\
59.375 \\
59.733 \\
59.780\end{array}$ & $\begin{array}{l}140 \\
112 \\
144 \\
146 \\
148 \\
150 \\
152 \\
154 \\
155 \\
158 \\
1150 \\
162 \\
164 \\
165 \\
167 \\
167 \\
169 \\
171 \\
173 \\
175 \\
177 \\
179 \\
181 \\
182 \\
184 \\
186 \\
189 \\
190 \\
192 \\
199 \\
198 \\
200 \\
202 \\
204 \\
206 \\
204 \\
210 \\
214 \\
216 \\
218 \\
220 \\
222 \\
221 \\
226 \\
228 \\
230 \\
232 \\
234 \\
236 \\
238 \\
240 \\
242 \\
244 \\
246 \\
248 \\
250 \\
252 \\
254 \\
256 \\
258 \\
260 \\
262 \\
264 \\
256 \\
268 \\
270 \\
272 \\
274 \\
276 \\
278\end{array}$ & $\begin{array}{l}59.847 \\
59.899 \\
59.934 \\
50.979 \\
60.019 \\
60.091 \\
60.166 \\
60.236 \\
60.293 \\
60.335 \\
60.250 \\
30.419 \\
60.144 \\
60.475 \\
60.602 \\
30.499 \\
60.517 \\
50.538 \\
60.568 \\
50.500 \\
60.632 \\
60.381 \\
60.713 \\
50.718 \\
60.785 \\
30.839 \\
60.860 \\
30.934 \\
60.953 \\
60.997 \\
61.068 \\
51.087 \\
61.070 \\
51.059 \\
61.047 \\
61.033 \\
61.016 \\
60.958 \\
60.937 \\
60.941 \\
60.911 \\
50.955 \\
60.972 \\
50.957 \\
60.997 \\
61.054 \\
61.136 \\
51.155 \\
61.217 \\
51.278 \\
61.302 \\
61.330 \\
61.385 \\
51.419 \\
61.438 \\
51.412 \\
61.616 \\
61.336 \\
61.316 \\
61.245 \\
61.191 \\
61.146 \\
61.137 \\
51.171 \\
51.219 \\
51.243 \\
51.264 \\
61.278 \\
61.283 \\
61.316 \\
\end{array}$ & $\begin{array}{l}280 \\
282 \\
284 \\
296 \\
228 \\
270 \\
292 \\
204 \\
296 \\
298 \\
300 \\
302 \\
304 \\
306 \\
308 \\
310 \\
312 \\
314 \\
316 \\
318 \\
320 \\
322 \\
324 \\
326 \\
328 \\
330 \\
332 \\
334 \\
336 \\
338 \\
340 \\
342 \\
344 \\
346 \\
349 \\
350 \\
352 \\
356 \\
358 \\
360 \\
-362 \\
354 \\
366 \\
368 \\
370 \\
372 \\
374 \\
376 \\
378 \\
380 \\
382 \\
381 \\
386 \\
338 \\
390 \\
392 \\
394 \\
395 \\
398 \\
400 \\
40 \% \\
404 \\
406 \\
709 \\
410 \\
412 \\
414 \\
415 \\
418 \\
420\end{array}$ & 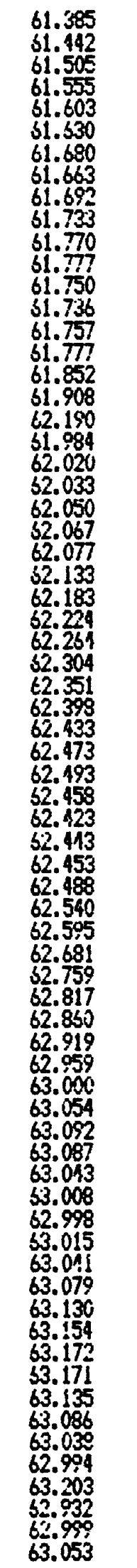 & 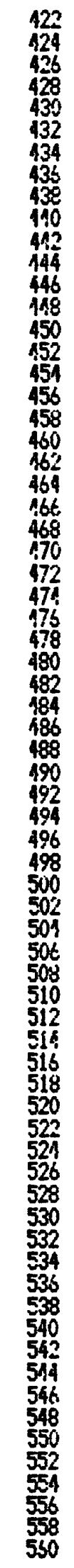 & 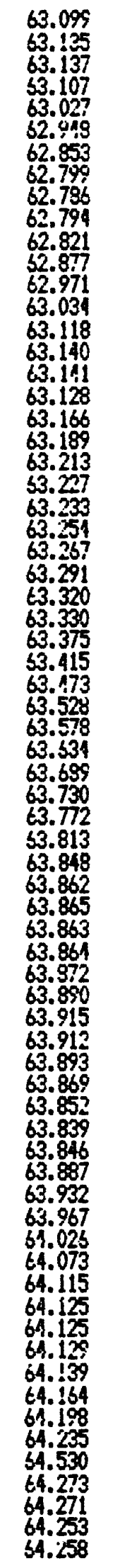 & 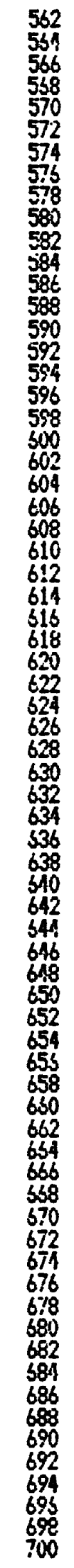 & 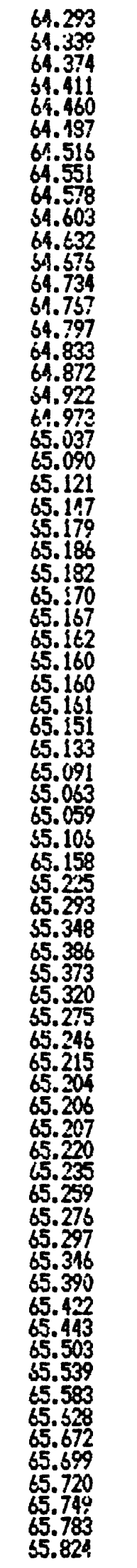 & $\begin{array}{l}702 \\
704 \\
706 \\
708 \\
710 \\
712 \\
714 \\
715 \\
718 \\
720 \\
722 \\
724 \\
726 \\
728 \\
730 \\
732 \\
734 \\
735 \\
736 \\
740 \\
742 \\
744 \\
746 \\
748 \\
750 \\
752 \\
751 \\
754 \\
758 \\
750 \\
751 \\
764 \\
768 \\
770 \\
772 \\
774 \\
776 \\
778 \\
780 \\
782 \\
781 \\
786 \\
790 \\
792 \\
794 \\
796 \\
798 \\
900 \\
804 \\
806 \\
808 \\
810 \\
812 \\
814 \\
816 \\
818 \\
820 \\
922 \\
824 \\
826 \\
828 \\
820 \\
832 \\
834 \\
834 \\
839 \\
890 \\
842 \\
894 \\
816\end{array}$ & 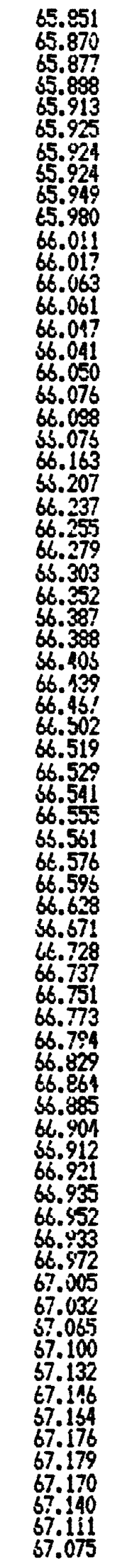 \\
\hline
\end{tabular}


PAFT RIVER, IDAHD - MALTA HELL - 19 DECEMBER 1976

\begin{tabular}{|c|c|c|c|c|c|c|c|c|c|c|c|}
\hline $\begin{array}{l}\text { DEPTH } \\
\text { (FT) }\end{array}$ & $\begin{array}{l}\text { TEPERATLRE } \\
\text { (C) }\end{array}$ & $\begin{array}{l}\text { DEPTH } \\
\text { (FT) }\end{array}$ & $\begin{array}{l}\text { TEMPERATURE } \\
\text { (C) }\end{array}$ & $\begin{array}{c}\text { DEPTH } \\
\text { (FT) }\end{array}$ & $\begin{array}{l}\text { TEMPERATLRE } \\
\text { (C) }\end{array}$ & $\begin{array}{c}\text { DEPTH } \\
\text { IFTI }\end{array}$ & $\begin{array}{l}\text { TEMPERSITLRE } \\
\text { (C) }\end{array}$ & $\begin{array}{c}\text { DEPTH } \\
\text { (FT) }\end{array}$ & $\begin{array}{l}\text { TEAPERATURE } \\
\text { (C) }\end{array}$ & $\begin{array}{c}\text { DEFTH } \\
\text { (FT) }\end{array}$ & $\begin{array}{c}\text { TEYPERATU } \\
\text { (C) }\end{array}$ \\
\hline 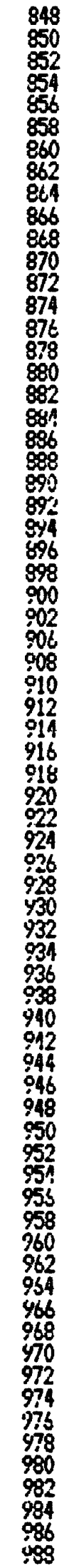 & 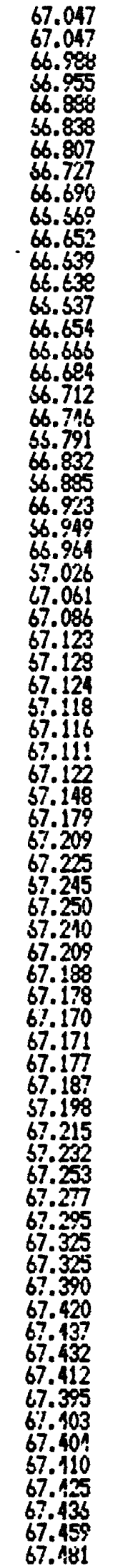 & $\begin{array}{l}990 \\
992 \\
994 \\
995 \\
998 \\
1000 \\
1002 \\
1004 \\
1006 \\
1008 \\
1010 \\
1012 \\
1014 \\
1016 \\
1018 \\
1020 \\
1022 \\
1024 \\
1026 \\
1028 \\
1030 \\
1032 \\
1034 \\
1036 \\
1038 \\
1040 \\
1042 \\
1044 \\
1016 \\
1048 \\
1050 \\
1052 \\
1054 \\
1056 \\
1058 \\
1060 \\
1062 \\
1054 \\
1066 \\
1058 \\
1070 \\
1072 \\
1071 \\
1079 \\
1078 \\
1080 \\
1089 \\
1094 \\
1086 \\
1088 \\
1050 \\
1092 \\
1094 \\
1095 \\
1098 \\
100 \\
1102 \\
1104 \\
1106 \\
1108 \\
110 \\
1112 \\
1114 \\
1115 \\
1118 \\
1120 \\
1122 \\
1124 \\
1126 \\
1128\end{array}$ & 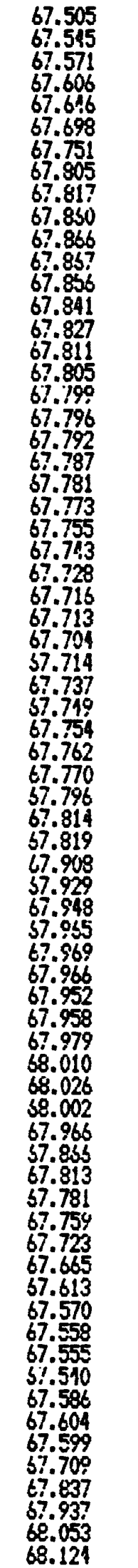 & $\begin{array}{l}1130 \\
1132 \\
1134 \\
1136 \\
1138 \\
1140 \\
1142 \\
1144 \\
1146 \\
1118 \\
1150 \\
1152 \\
1154 \\
1155 \\
1158 \\
1160 \\
1162 \\
1144 \\
1166 \\
1169 \\
1170 \\
1172 \\
1174 \\
1176 \\
1178 \\
1180 \\
1182 \\
1184 \\
1186 \\
1188 \\
1190 \\
1192 \\
1194 \\
1196 \\
1198 \\
1200 \\
1202 \\
1204 \\
1206 \\
1209 \\
1210 \\
1212 \\
1214 \\
1188 \\
1220 \\
1222 \\
1224 \\
1226 \\
1230 \\
134 \\
1236 \\
1238 \\
1210 \\
1299 \\
1292 \\
1993 \\
1295 \\
1297 \\
1298 \\
1300 \\
1304 \\
1308 \\
1308 \\
1310 \\
1312 \\
1314 \\
1316 \\
1318 \\
1320 \\
1322\end{array}$ & 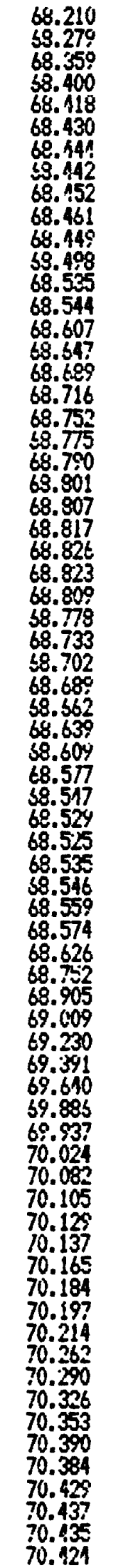 & 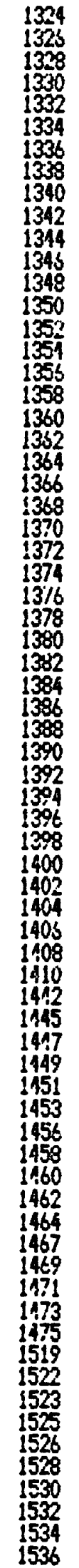 & 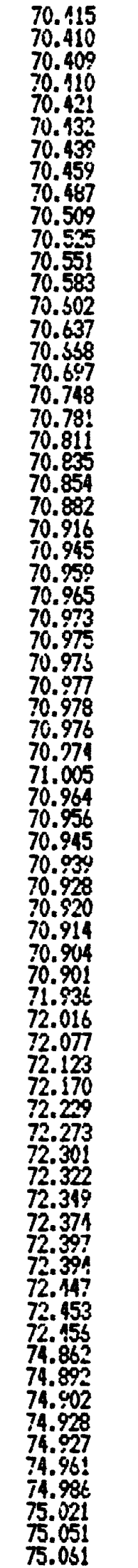 & 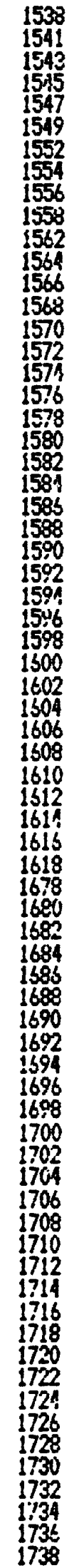 & 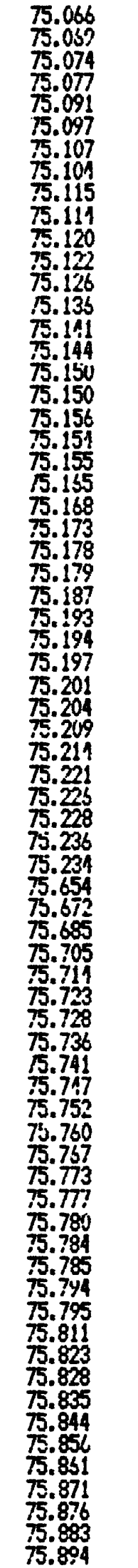 & 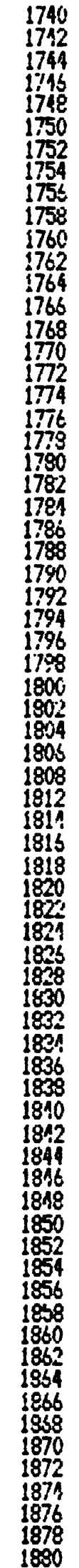 & 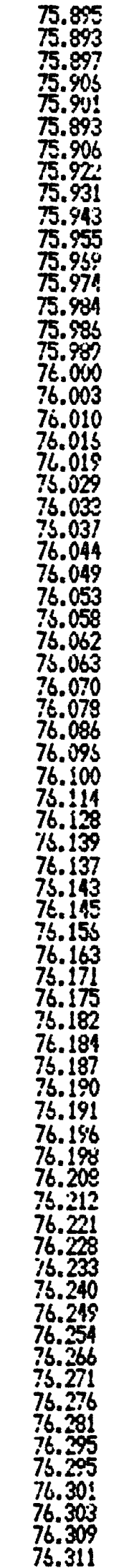 \\
\hline
\end{tabular}


RAFT RIVER, IOA40 - MALT WEL - 18 DECEMEER 1973

\begin{tabular}{|c|c|c|c|c|c|c|c|c|c|c|c|}
\hline $\begin{array}{l}\text { IIEPTH } \\
\text { (FT) }\end{array}$ & $\begin{array}{l}\text { TEMERATURE } \\
\text { (C) }\end{array}$ & $\begin{array}{l}\text { DEPTH } \\
\text { (FT) }\end{array}$ & $\begin{array}{l}\text { TEMPERATLRE } \\
\text { (C) }\end{array}$ & $\begin{array}{l}\text { DEPTH } \\
\text { (FT) }\end{array}$ & $\begin{array}{l}\text { TERPERATLRE } \\
\text { (C) }\end{array}$ & $\begin{array}{l}\text { DEFTH } \\
\text { (FT) }\end{array}$ & $\begin{array}{l}\text { TENPERATLRE } \\
\text { (C) }\end{array}$ & $\begin{array}{l}\text { DEPTH } \\
\text { (FT) }\end{array}$ & $\begin{array}{l}\text { TERPERATURE } \\
(C)\end{array}$ & $\begin{array}{c}\text { DEPTH } \\
\text { (FT) }\end{array}$ & $\begin{array}{l}\text { TEYPERSTL } \\
\text { (C) }\end{array}$ \\
\hline $\begin{array}{l}1882 \\
1884 \\
1986 \\
1988 \\
1890 \\
1992 \\
1894 \\
1895 \\
1898 \\
1900 \\
1902 \\
1904 \\
1906 \\
1908 \\
1910 \\
1012 \\
1914 \\
1016 \\
1918 \\
1920 \\
1992 \\
1924 \\
1926 \\
1928 \\
1930 \\
1932 \\
1934 \\
1935 \\
1910 \\
1042 \\
1944 \\
1946 \\
1948 \\
1950 \\
1952 \\
1951 \\
1956 \\
1958 \\
1960 \\
1962 \\
1964 \\
1956 \\
1968 \\
1970 \\
1972 \\
1974 \\
1976 \\
19979 \\
1980 \\
1982 \\
1984 \\
1986 \\
1998 \\
1990 \\
1992 \\
1994 \\
1996 \\
1908 \\
2000 \\
2002 \\
2004 \\
2005 \\
2008 \\
2010 \\
2012 \\
2014 \\
2016 \\
2018 \\
2020 \\
2022 \\
\end{array}$ & 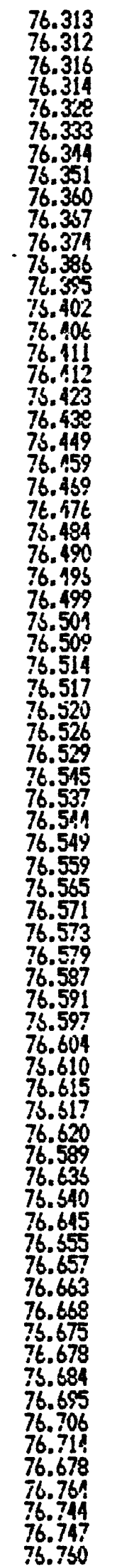 & $\begin{array}{l}2024 \\
2026 \\
2028 \\
2030 \\
2032 \\
2034 \\
2036 \\
2038 \\
2040 \\
2042 \\
2044 \\
2046 \\
2018 \\
2050 \\
2052 \\
2054 \\
2056 \\
2058 \\
2060 \\
2052 \\
2064 \\
2046 \\
2048 \\
2070 \\
2072 \\
2074 \\
2076 \\
2078 \\
2090 \\
2082 \\
2081 \\
2083 \\
2088 \\
2090 \\
2092 \\
2094 \\
2096 \\
2098 \\
2100 \\
2104 \\
2106 \\
2108 \\
2110 \\
2112 \\
2114 \\
2116 \\
2118 \\
2120 \\
2132 \\
2124 \\
2126 \\
2128 \\
2130 \\
2132 \\
2134 \\
2135 \\
2138 \\
2140 \\
2142 \\
2144 \\
2146 \\
2150 \\
2154 \\
2158 \\
2160 \\
2162 \\
2164\end{array}$ & 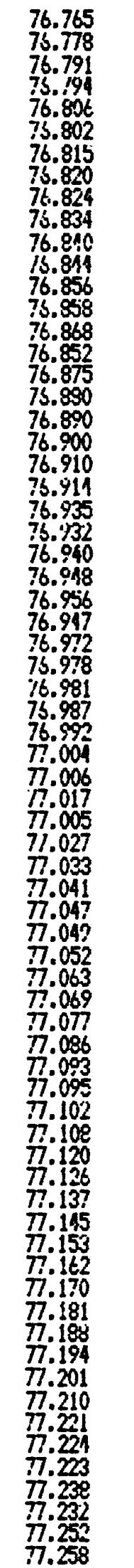 & 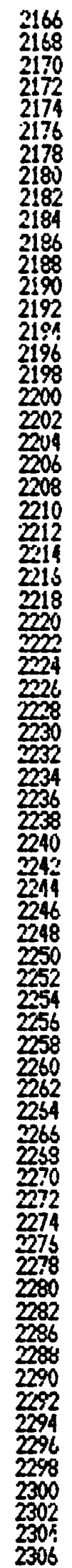 & 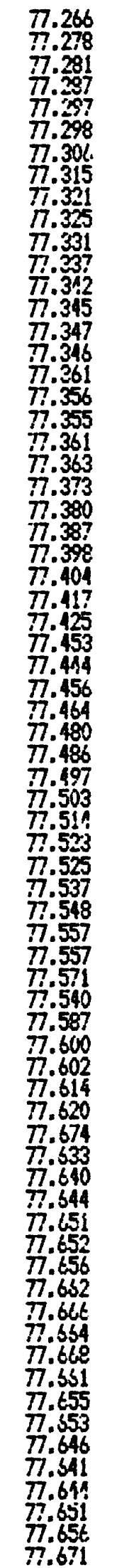 & 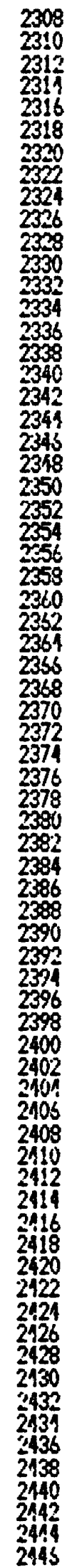 & 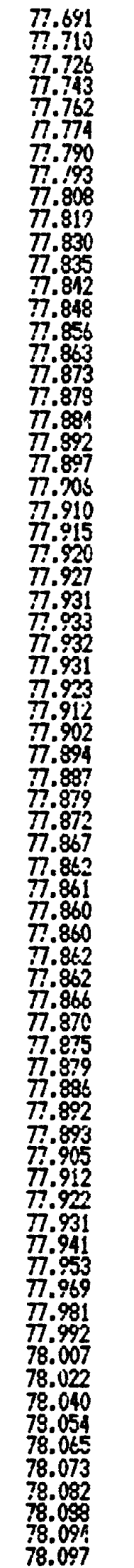 & 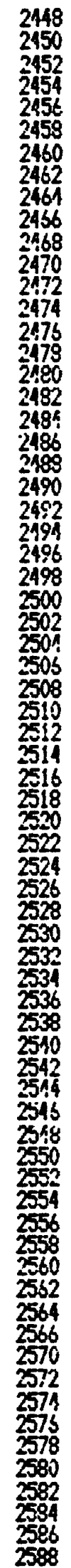 & 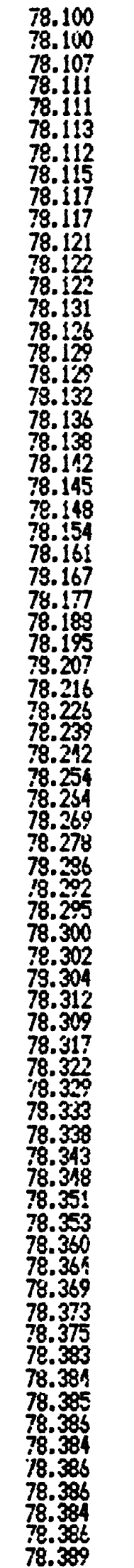 & 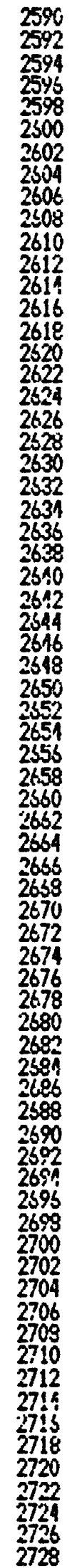 & 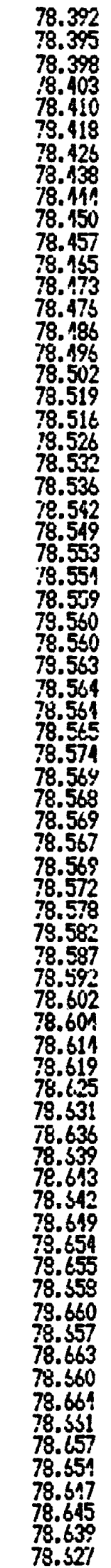 \\
\hline
\end{tabular}




\begin{tabular}{|c|c|c|c|c|c|c|c|c|c|c|c|}
\hline $\begin{array}{l}\text { IEPTH } \\
\text { (FT) }\end{array}$ & $\begin{array}{l}\text { TEMPERATURE } \\
\text { (C) }\end{array}$ & $\begin{array}{l}\text { DEPTH } \\
\text { (FT) }\end{array}$ & $\begin{array}{l}\text { TERPERGTLRE } \\
\text { (C) }\end{array}$ & $\begin{array}{l}\text { DEPTH } \\
\text { (FT) }\end{array}$ & $\begin{array}{l}\text { TEYPERATURE } \\
\text { (C) }\end{array}$ & $\begin{array}{l}\text { DEFTH } \\
\text { (FT) }\end{array}$ & $\begin{array}{l}\text { TEMPERATURE } \\
\text { (C) }\end{array}$ & $\begin{array}{c}\text { DEPTH } \\
\text { (FT) }\end{array}$ & $\begin{array}{l}\text { TEMPERATLRE } \\
\text { (C) }\end{array}$ & $\begin{array}{l}\text { DEPTH } \\
\text { (FT) }\end{array}$ & $\begin{array}{l}\text { TEMPERAT } \\
(C)\end{array}$ \\
\hline 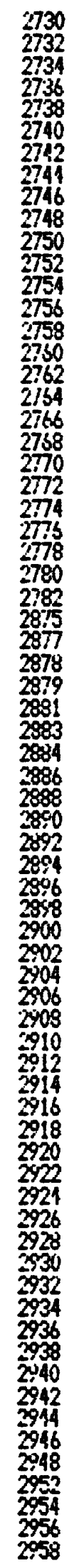 & 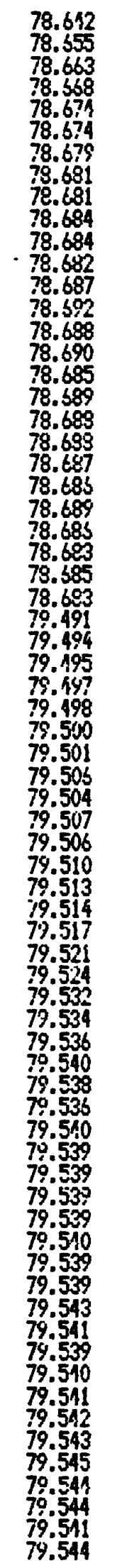 & $\begin{array}{l}3960 \\
2962 \\
2961 \\
2966 \\
3968 \\
2970 \\
2972 \\
2074 \\
2976 \\
2978 \\
2980 \\
2982 \\
2984 \\
2986 \\
2988 \\
2990 \\
2592 \\
2994 \\
2996 \\
2908 \\
3000 \\
3002 \\
3004 \\
3006 \\
3008 \\
3010 \\
3012 \\
3014 \\
3016 \\
3019 \\
3020 \\
3022 \\
3024 \\
3026 \\
3028 \\
3030 \\
3032 \\
3034 \\
3036 \\
3038 \\
3040 \\
3042 \\
3041 \\
3046 \\
3048 \\
3050 \\
3052 \\
3054 \\
3054 \\
3058 \\
3060 \\
3062 \\
3064 \\
3055 \\
3070 \\
3072 \\
3074 \\
3076 \\
3078 \\
3090 \\
3092 \\
3084 \\
3086 \\
3088 \\
3090 \\
3092 \\
3091 \\
3096 \\
3098 \\
3100\end{array}$ & 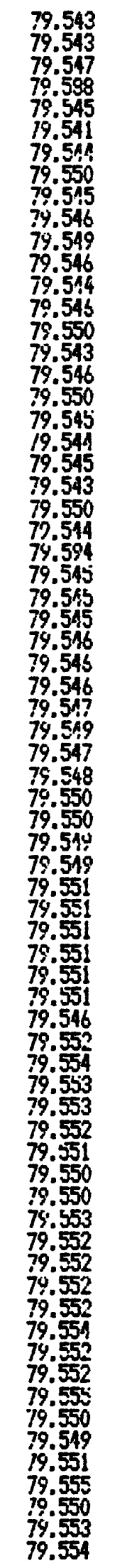 & $\begin{array}{l}3102 \\
3104 \\
3106 \\
3108 \\
3110 \\
3112 \\
3114 \\
3116 \\
3118 \\
3120 \\
3122 \\
3124 \\
3126 \\
3128 \\
3130 \\
3132 \\
3134 \\
3135 \\
3134 \\
3110 \\
3142 \\
3144 \\
3116 \\
3149 \\
3150 \\
3152 \\
3151 \\
3154 \\
2158 \\
3150 \\
3162 \\
3164 \\
3164 \\
3168 \\
3170 \\
3172 \\
3174 \\
3176 \\
3178 \\
3180 \\
3182 \\
3184 \\
3186 \\
3198 \\
3190 \\
3192 \\
3194 \\
3196 \\
3198 \\
3200 \\
3202 \\
3204 \\
3206 \\
3208 \\
3210 \\
3212 \\
3214 \\
3216 \\
3218 \\
3220 \\
3222 \\
3224 \\
3226 \\
3228 \\
3235 \\
3232 \\
3234 \\
3234 \\
3238 \\
3240\end{array}$ & 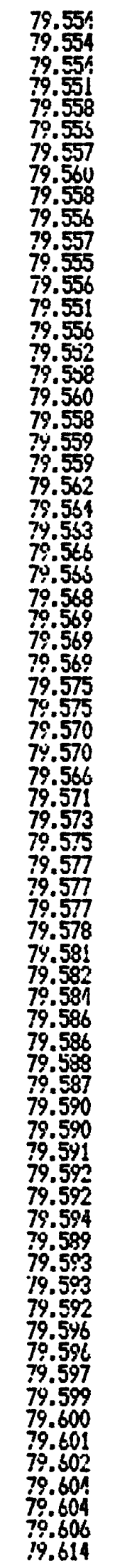 & 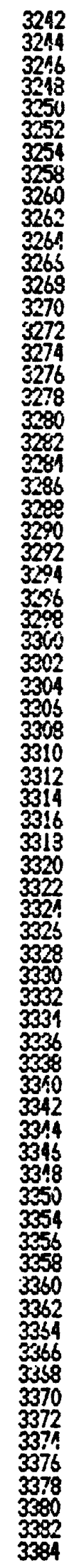 & 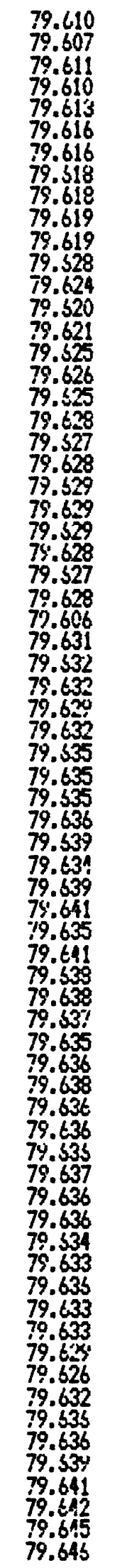 & 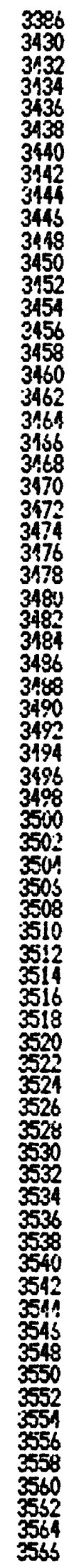 & 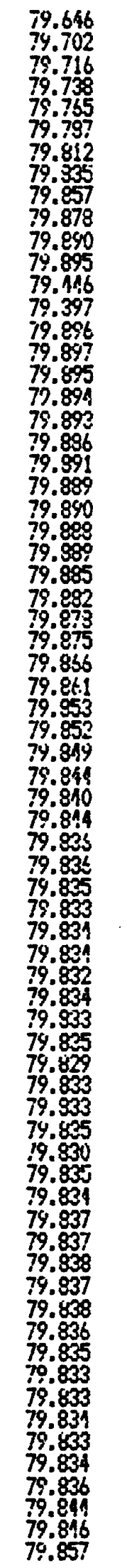 & 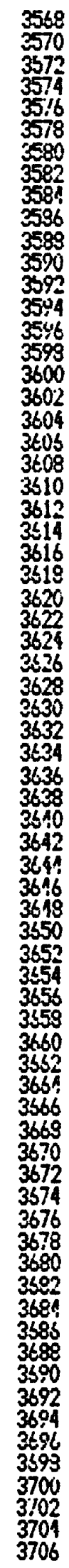 & 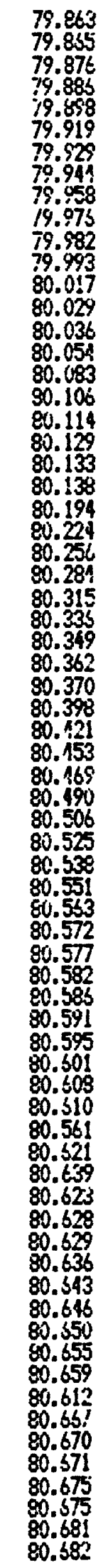 \\
\hline
\end{tabular}


RAFT RIVER, IDAHO - MLTA HELL - 18 DECEMEER 1976

\begin{tabular}{|c|c|c|c|c|c|c|c|c|c|c|c|}
\hline $\begin{array}{l}\text { DEPTH } \\
\text { (FT) }\end{array}$ & $\begin{array}{l}\text { TEPPERATURE } \\
\text { (C) }\end{array}$ & $\begin{array}{c}\text { DEPTH } \\
\text { (FT) }\end{array}$ & $\begin{array}{l}\text { TEMPERATURE } \\
\text { (C) }\end{array}$ & $\begin{array}{l}\text { DEPTH } \\
\text { (FT) }\end{array}$ & $\begin{array}{l}\text { TEPEERATURE } \\
\text { (C) }\end{array}$ & $\begin{array}{l}\text { DEFTH } \\
\text { (FT) }\end{array}$ & $\begin{array}{l}\text { TEMFERATURE } \\
\text { (C) }\end{array}$ & $\begin{array}{l}\text { LEPTH } \\
\text { (FT) }\end{array}$ & $\begin{array}{l}\text { TEPPERATLRE } \\
\text { (C) }\end{array}$ & $\begin{array}{l}\text { DEPTH } \\
\text { (FT) }\end{array}$ & $\begin{array}{l}\text { TEMPERATL } \\
\text { (C) }\end{array}$ \\
\hline 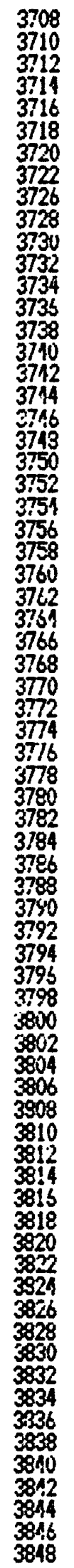 & 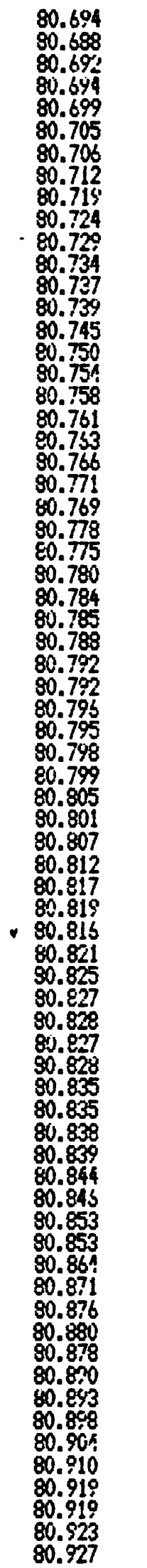 & 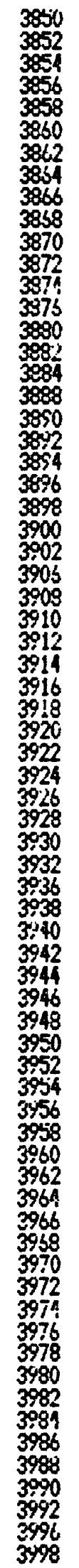 & $\begin{array}{l}80.932 \\
80.935 \\
80.941 \\
80.947 \\
80.951 \\
80.957 \\
80.962 \\
80.974 \\
80.981 \\
80.990 \\
80.999 \\
81.012 \\
81.022 \\
81.031 \\
81.054 \\
81.052 \\
81.070 \\
81.086 \\
81.055 \\
81.101 \\
81.110 \\
81.115 \\
81.124 \\
81.128 \\
81.137 \\
81.141 \\
81.145 \\
81.150 \\
81.153 \\
81.155 \\
81.161 \\
81.165 \\
81.168 \\
81.772 \\
81.177 \\
81.180 \\
81.174 \\
81.185 \\
81.190 \\
81.196 \\
81.200 \\
81.206 \\
81.208 \\
81.213 \\
81.215 \\
81.215 \\
81.215 \\
81.225 \\
81.227 \\
81.235 \\
81.211 \\
81.242 \\
81.253 \\
81.253 \\
81.700 \\
81.776 \\
81.287 \\
81.295 \\
81.306 \\
81.312 \\
81.321 \\
81.331 \\
81.310 \\
81.3552 \\
81.311 \\
81.3355 \\
81.403 \\
81.422 \\
81.467 \\
81.497\end{array}$ & $\begin{array}{l}4002 \\
4004 \\
4006 \\
4008 \\
4010 \\
4012 \\
4016 \\
4018 \\
4020 \\
4022 \\
4024 \\
4026 \\
4028 \\
4030 \\
4031 \\
1031 \\
4036 \\
4038 \\
4040 \\
4042 \\
4044 \\
4046 \\
4048 \\
1050 \\
4052 \\
4054 \\
4056 \\
1054 \\
4060 \\
4062 \\
4064 \\
4056 \\
4068 \\
4070 \\
4072 \\
4071 \\
4076 \\
4078 \\
4080 \\
4082 \\
4084 \\
4088 \\
4090 \\
1092 \\
400 \% 4 \\
4096 \\
4098 \\
1100 \\
4102 \\
4104 \\
4108 \\
4110 \\
1112 \\
4114 \\
4 ! 16 \\
4118 \\
4120 \\
4122 \\
4124 \\
4126 \\
4128 \\
4130 \\
4132 \\
4134 \\
1136 \\
1138 \\
1140 \\
4142 \\
1144 \\
1116 \\
\end{array}$ & 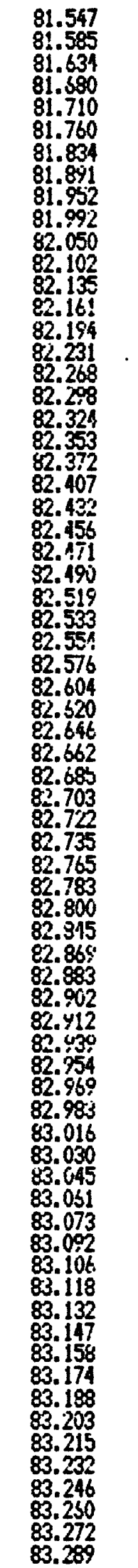 & 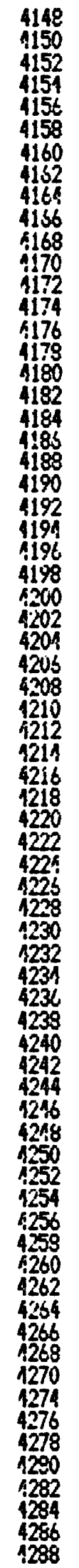 & 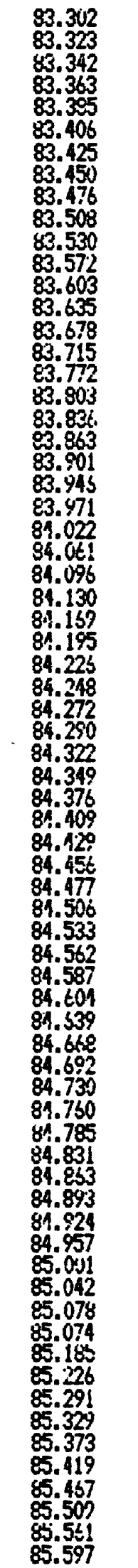 & $\begin{array}{l}4290 \\
4292 \\
4296 \\
1298 \\
1300 \\
1302 \\
4304 \\
4306 \\
4308 \\
1310 \\
4312 \\
4314 \\
4316 \\
4318 \\
4320 \\
4322 \\
4324 \\
4326 \\
4329 \\
1330 \\
4332 \\
4334 \\
4335 \\
4338 \\
4340 \\
4342 \\
4341 \\
4345 \\
4348 \\
1350 \\
4352 \\
1354 \\
4356 \\
4358 \\
4360 \\
4362 \\
4364 \\
4366 \\
4368 \\
4370 \\
4372 \\
4371 \\
4376 \\
4378 \\
4380 \\
4332 \\
4394 \\
1386 \\
4398 \\
4390 \\
4392 \\
1394 \\
4396 \\
4398 \\
4400 \\
1402 \\
4404 \\
4106 \\
4408 \\
1410 \\
4112 \\
4414 \\
4116 \\
4118 \\
4420 \\
4422 \\
4121 \\
1125 \\
4128 \\
4430\end{array}$ & 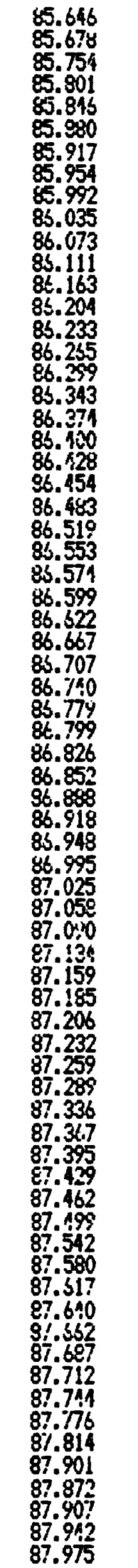 & 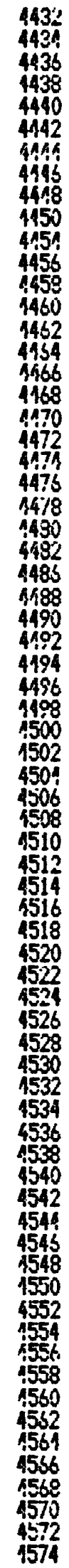 & 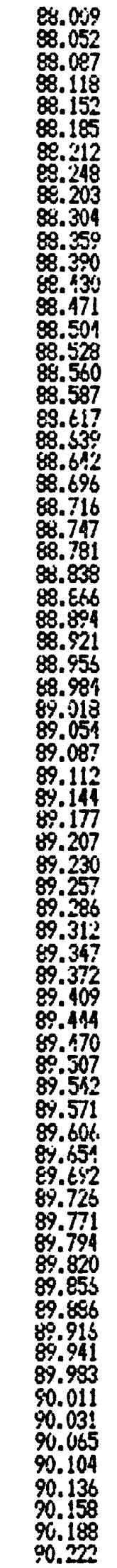 \\
\hline
\end{tabular}


PAFT RIVER, IDAHO - MATA IEUL - 18 DECENAEER 1976

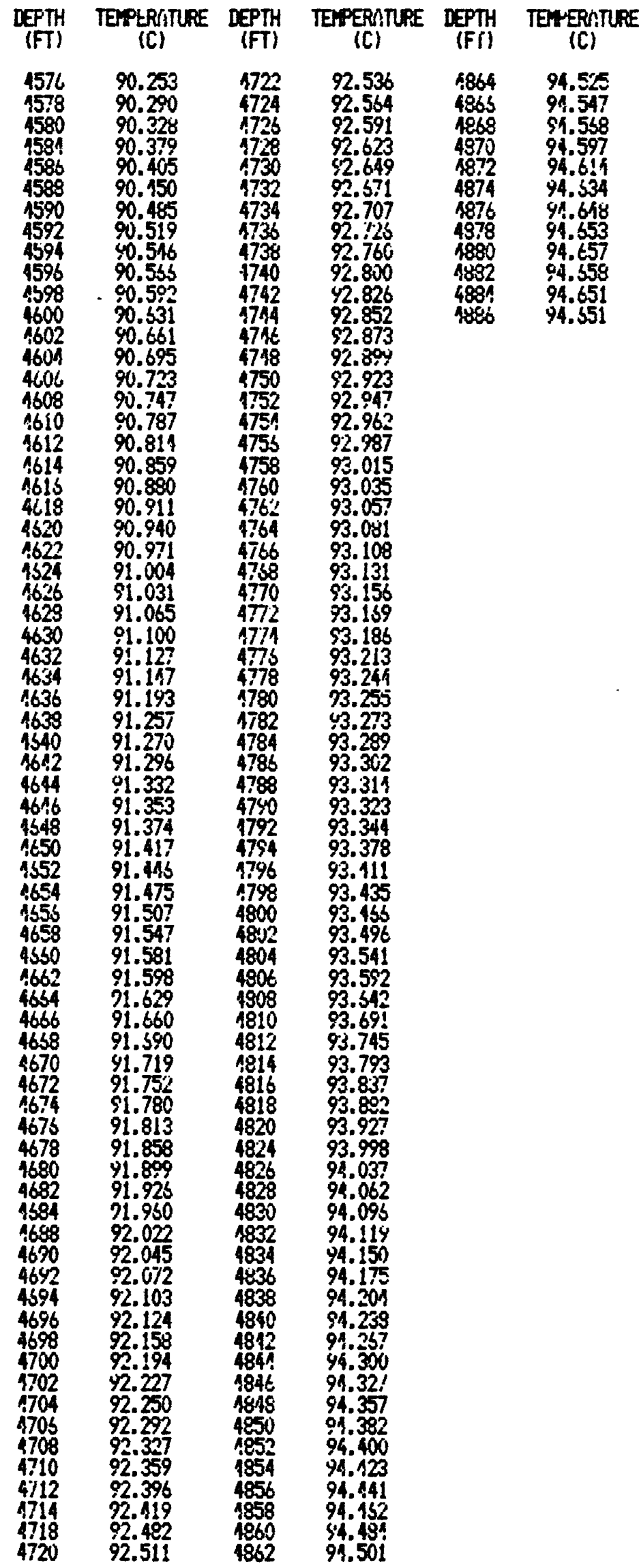


RAFT RIVER, IDAHO - STREVELL IELL - 17 OCTOBER 1975

DEPTH TEPPRATURE DEPTH TEMPERATLRE DEPTH TEPERATURE
(FT)
(C)

$\begin{array}{llllll}10 & 15.600 & 370 & 15.860 & 720 & 21.759 \\ 15 & 15.513 & 375 & 15.950 & 725 & 21.789 \\ 20 & 15.134 & 390 & 16.045 & 730 & 22.057\end{array}$

$25 \quad 15.327 \quad 3$

$15.199 \quad 390$

15.100 395

$15.019 \quad 400$

$14.935 \quad 105$

$14.858 \quad 410$

$14.793 \quad 415$

- 11.139

$\begin{array}{ll}14.689 & 425 \\ 14.639 & 430\end{array}$

$14.603 \quad 435$

$14.564 \quad 440$

$\begin{array}{ll}14.531 & 445 \\ 14.498 & 950\end{array}$

$14.477 \quad 955$

$14.449 \quad 450$

$14.433 \quad 465$

$14.417 \quad 170$

$14.396 \quad 475$

11.3864430

$14.377 \quad 495$

$14.350 \quad 495$

$14.351 \quad 500$

$\begin{array}{ll}14.338 & 505 \\ 14.341 & 510\end{array}$

14.311 515

$14.334 \quad 520$

14.332

14.332

11.348

13.378

$12.8 \% 5$

12.918

13. 089

16.127

16.304

16.419

16.526

16.631

16.720

16.802

16.892

16.966

17.074

17.181

17.237

17.348

17.115

17.509

17.600

17.810

17.879

17.970

18.055

18.128

18.232

18.255

18.372

18.458

18.513

18.578

18.543

18.720

18.765

18.818

18.884

18.961

13.147

19.011

13.218

19.079

19.138

$13.306 \quad 575 \quad 19.220$

13.380

13.458

19.285

12.996

13.005

13.736

13.837

13.915

13.978

11.083

14.167
14.256

14. 361

14.149
14.522

14.595

14.700

14.786

14.916

14.997

15.075

15.161

15.243

15.323

15.427

15.509

15.603

15.690

15.761

19.362

10.158

19.537

19.638

19.736

19.813

19.911

20.035

20.102

20.185

20.293

20.401

20.493

20.773

20.855

20.953

21.065

21.148

21.242

21.318

21.503

21.571

360

365

680

69

695

700

710
715

21.7504 
RAFT RIVER, IDAHO - I.D. AO. 1 - Z1 SEPTEMBER 1971

\begin{tabular}{|c|c|c|c|c|c|}
\hline $\begin{array}{l}\text { DEPTH } \\
\text { (FT) }\end{array}$ & $\begin{array}{l}\text { TERPERATLRE } \\
\text { (C) }\end{array}$ & $\begin{array}{l}\text { DEPTH } \\
\text { (FT) }\end{array}$ & $\begin{array}{l}\text { TEMPERATLKE } \\
\text { (C) }\end{array}$ & $\begin{array}{l}\text { DEPTH } \\
\text { (FT) }\end{array}$ & $\begin{array}{l}\text { TERPERITU } \\
\text { (C) }\end{array}$ \\
\hline $\begin{array}{l}10 \\
25 \\
50 \\
75 \\
100 \\
125 \\
135 \\
140 \\
145 \\
150 \\
155 \\
160 \\
165 \\
170 \\
175 \\
180 \\
185 \\
190 \\
195 \\
200 \\
205 \\
210 \\
215 \\
220 \\
225 \\
230 \\
235 \\
240 \\
245 \\
250 \\
255 \\
260 \\
265 \\
270 \\
275 \\
280 \\
285 \\
290 \\
295 \\
300 \\
305 \\
310 \\
315 \\
320 \\
325 \\
330 \\
335 \\
340 \\
345 \\
350 \\
3555 \\
360 \\
365 \\
370 \\
375 \\
3300 \\
3355 \\
350 \\
395 \\
400 \\
405 \\
410 \\
415 \\
420 \\
425 \\
430 \\
435 \\
440 \\
445 \\
450\end{array}$ & 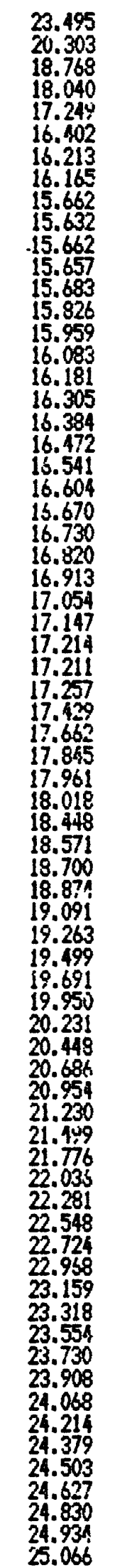 & $\begin{array}{l}455 \\
460 \\
465 \\
770 \\
475 \\
480 \\
485 \\
490 \\
495 \\
500 \\
505 \\
510 \\
515 \\
520 \\
525 \\
530 \\
535 \\
540 \\
545 \\
550 \\
555 \\
560 \\
565 \\
570 \\
575 \\
580 \\
585 \\
590 \\
595 \\
600 \\
605 \\
610 \\
615 \\
620 \\
625 \\
630 \\
535 \\
640 \\
545 \\
650 \\
655 \\
660 \\
655 \\
670 \\
675 \\
680 \\
385 \\
690 \\
695 \\
700 \\
705 \\
710 \\
715 \\
720 \\
725 \\
730 \\
735 \\
740 \\
745 \\
750 \\
755 \\
760 \\
765 \\
770 \\
775 \\
780 \\
785 \\
790 \\
795 \\
800\end{array}$ & 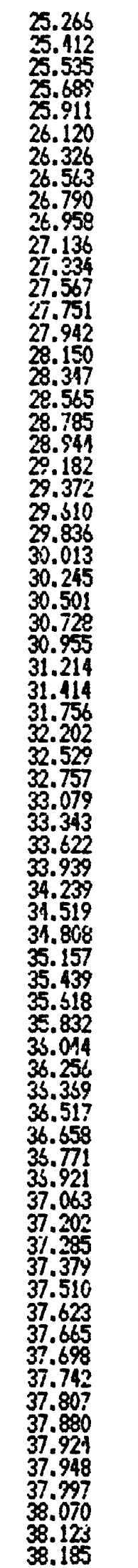 & $\begin{array}{l}805 \\
810 \\
815 \\
820 \\
825 \\
830 \\
835 \\
840 \\
845 \\
850 \\
955 \\
860 \\
865 \\
870 \\
975 \\
880 \\
885 \\
890 \\
895 \\
900 \\
905 \\
910 \\
915 \\
920 \\
925 \\
930 \\
935 \\
910 \\
945 \\
950 \\
955 \\
960 \\
965 \\
970 \\
975 \\
980 \\
495 \\
990 \\
995 \\
1006 \\
1005 \\
1010 \\
1015 \\
1020 \\
1025 \\
1030 \\
1035 \\
1040 \\
1045 \\
1050 \\
1051\end{array}$ & $\begin{array}{l}38.219 \\
38.232 \\
33.255 \\
38.301 \\
38.352 \\
38.367 \\
38.387 \\
38.415 \\
38.414 \\
38.478 \\
38.533 \\
38.654 \\
38.718 \\
38.775 \\
38.815 \\
33.846 \\
38.872 \\
39.012 \\
39.187 \\
39.237 \\
39.275 \\
39.375 \\
39.510 \\
39.631 \\
39.750 \\
39.848 \\
34.907 \\
40.022 \\
40.148 \\
40.307 \\
40.420 \\
40.532 \\
40.527 \\
40.721 \\
40.850 \\
40.950 \\
41.051 \\
41.171 \\
41.287 \\
41.390 \\
41.484 \\
41.576 \\
41.654 \\
41.712 \\
41.751 \\
41.805 \\
41.920 \\
13.068 \\
42.199 \\
42.332 \\
42.364\end{array}$ \\
\hline
\end{tabular}


RAFT RIVER, IDAHO - I.D. W. 1 - 12 OCTOBER 1971

\begin{tabular}{|c|c|c|c|c|c|}
\hline $\begin{array}{l}\text { DEPTH } \\
\text { (FT) }\end{array}$ & $\begin{array}{l}\text { TEPEERATURE } \\
\text { (C) }\end{array}$ & $\begin{array}{l}\text { DEPTH } \\
\text { (FT) }\end{array}$ & $\begin{array}{l}\text { TENPERATLPE } \\
\text { (C) }\end{array}$ & $\begin{array}{l}\text { LEPTH } \\
\text { (FT) }\end{array}$ & $\begin{array}{l}\text { TEMPERATUF } \\
\text { (C) }\end{array}$ \\
\hline $\begin{array}{l}10 \\
25 \\
50 \\
75 \\
100 \\
125 \\
135 \\
140 \\
145 \\
150 \\
155 \\
160 \\
165 \\
170 \\
175 \\
180 \\
185 \\
190 \\
195 \\
200 \\
205 \\
210 \\
215 \\
220 \\
225 \\
230 \\
235 \\
240 \\
245 \\
250 \\
255 \\
260 \\
265 \\
270 \\
275 \\
280 \\
285 \\
290 \\
295 \\
300 \\
305 \\
310 \\
315 \\
320 \\
325 \\
330 \\
335 \\
340 \\
345 \\
350 \\
3555 \\
360 \\
365 \\
370 \\
375 \\
330 \\
395 \\
330 \\
395 \\
400 \\
105 \\
410 \\
415 \\
120 \\
425 \\
130 \\
135 \\
140 \\
145 \\
450\end{array}$ & $\begin{array}{l}17.311 \\
14.123 \\
13.921 \\
13.820 \\
13.882 \\
14.140 \\
14.250 \\
14.401 \\
14.968 \\
15.026 \\
15.065 \\
15.076 \\
15.141 \\
15.283 \\
15.406 \\
15.522 \\
15.541 \\
15.723 \\
15.826 \\
15.904 \\
15.487 \\
16.055 \\
16.125 \\
16.199 \\
13.298 \\
16.412 \\
16.526 \\
16.670 \\
15.754 \\
16.818 \\
16.896 \\
17.029 \\
17.178 \\
17.345 \\
17.472 \\
17.607 \\
18.018 \\
18.151 \\
19.253 \\
18.426 \\
18.650 \\
18.839 \\
19.053 \\
19.349 \\
19.543 \\
19.823 \\
20.125 \\
20.331 \\
20.605 \\
20.858 \\
21.189 \\
21.439 \\
21.717 \\
22.014 \\
22.250 \\
22.178 \\
22.683 \\
22.909 \\
23.077 \\
23.273 \\
23.527 \\
23.710 \\
23.852 \\
24.009 \\
24.144 \\
24.307 \\
24.411 \\
24.593 \\
24.769 \\
24.907\end{array}$ & $\begin{array}{l}455 \\
460 \\
465 \\
470 \\
475 \\
480 \\
185 \\
490 \\
495 \\
506 \\
505 \\
510 \\
515 \\
520 \\
525 \\
530 \\
535 \\
540 \\
545 \\
550 \\
555 \\
560 \\
565 \\
570 \\
575 \\
580 \\
585 \\
590 \\
595 \\
600 \\
605 \\
610 \\
615 \\
620 \\
625 \\
630 \\
635 \\
510 \\
645 \\
650 \\
655 \\
660 \\
665 \\
670 \\
675 \\
680 \\
685 \\
690 \\
695 \\
700 \\
705 \\
710 \\
715 \\
720 \\
725 \\
730 \\
735 \\
740 \\
745 \\
750 \\
755 \\
760 \\
765 \\
770 \\
775 \\
780 \\
785 \\
790 \\
795 \\
800\end{array}$ & 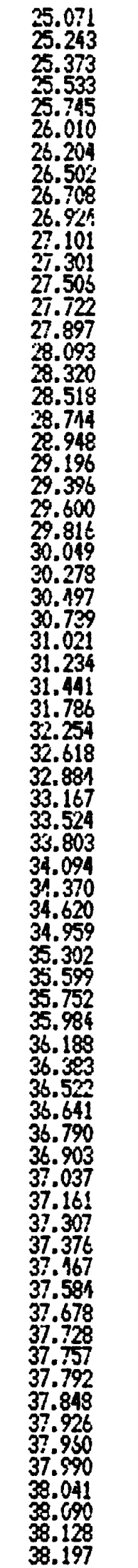 & $\begin{array}{l}805 \\
810 \\
815 \\
820 \\
825 \\
820 \\
835 \\
846 \\
845 \\
850 \\
855 \\
860 \\
855 \\
870 \\
875 \\
880 \\
885 \\
890 \\
895 \\
900 \\
905 \\
910 \\
915 \\
920 \\
925 \\
930 \\
935 \\
940 \\
945 \\
950 \\
955 \\
960 \\
965 \\
970 \\
975 \\
980 \\
985 \\
990 \\
995 \\
1000 \\
1005 \\
1010 \\
1015 \\
1020 \\
1025 \\
1030 \\
1035 \\
1040 \\
1042\end{array}$ & $\begin{array}{l}38.237 \\
38.254 \\
38.271 \\
38.316 \\
38.350 \\
38.373 \\
38.384 \\
38.409 \\
38.432 \\
38.465 \\
38.525 \\
38.635 \\
38.698 \\
38.760 \\
38.791 \\
38.823 \\
38.847 \\
38.981 \\
39.180 \\
39.234 \\
39.268 \\
39.351 \\
39.512 \\
30.641 \\
39.741 \\
39.854 \\
30.914 \\
40.020 \\
40.154 \\
40.340 \\
40.447 \\
40.541 \\
40.629 \\
40.724 \\
40.932 \\
40.947 \\
11.036 \\
41.142 \\
11.253 \\
41.350 \\
41.436 \\
11.510 \\
41.577 \\
41.624 \\
41.572 \\
11.741 \\
41.822 \\
41.9096 \\
42.068\end{array}$ \\
\hline
\end{tabular}


RAST RIVER, IDAHO - I.D. N. 1 - 25 VCTOEER 1974

\begin{tabular}{|c|c|c|c|c|c|}
\hline $\begin{array}{l}\text { DEPTH } \\
\text { (FT) }\end{array}$ & $\begin{array}{l}\text { TEHPERATLRE } \\
\text { (C) }\end{array}$ & $\begin{array}{l}\text { DEPTH } \\
\text { (FT) }\end{array}$ & $\begin{array}{l}\text { TERPERATURE } \\
\text { (C) }\end{array}$ & $\begin{array}{l}\text { DEPTH } \\
\text { (FT) }\end{array}$ & $\begin{array}{l}\text { TEREERATH } \\
\text { (C) }\end{array}$ \\
\hline $\begin{array}{l}140 \\
145 \\
150 \\
155 \\
160 \\
165 \\
170 \\
175 \\
180 \\
185 \\
190 \\
195 \\
200 \\
205 \\
210 \\
215 \\
220 \\
225 \\
230 \\
235 \\
240 \\
245 \\
250 \\
255 \\
260 \\
265 \\
270 \\
275 \\
280 \\
285 \\
290 \\
295 \\
300 \\
305 \\
310 \\
315 \\
320 \\
325 \\
330 \\
335 \\
340 \\
345 \\
350 \\
355 \\
360 \\
365 \\
370 \\
375 \\
380 \\
385 \\
300 \\
395 \\
400 \\
405 \\
110 \\
115 \\
420 \\
425 \\
430 \\
435 \\
140 \\
445 \\
450 \\
455 \\
460 \\
465 \\
170 \\
475 \\
485 \\
\end{array}$ & 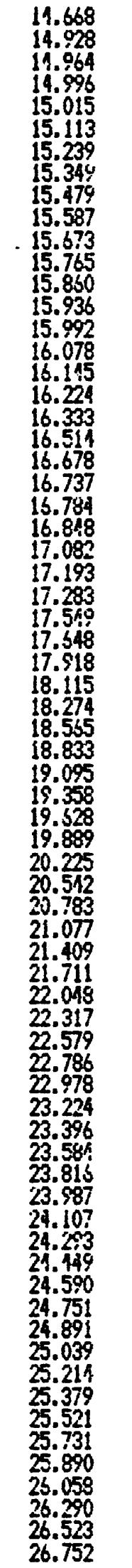 & $\begin{array}{l}490 \\
495 \\
500 \\
505 \\
510 \\
515 \\
520 \\
525 \\
530 \\
535 \\
540 \\
545 \\
550 \\
5555 \\
560 \\
565 \\
570 \\
575 \\
580 \\
585 \\
590 \\
595 \\
600 \\
605 \\
510 \\
615 \\
620 \\
625 \\
530 \\
635 \\
640 \\
645 \\
650 \\
655 \\
650 \\
665 \\
670 \\
675 \\
680 \\
685 \\
690 \\
695 \\
700 \\
705 \\
710 \\
715 \\
720 \\
725 \\
730 \\
735 \\
740 \\
745 \\
750 \\
755 \\
760 \\
765 \\
770 \\
775 \\
780 \\
785 \\
790 \\
795 \\
800 \\
805 \\
810 \\
815 \\
820 \\
825 \\
820 \\
835\end{array}$ & 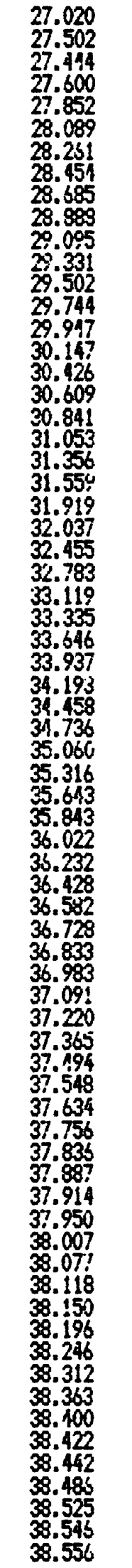 & $\begin{array}{l}840 \\
845 \\
850 \\
855 \\
850 \\
865 \\
870 \\
875 \\
880 \\
895 \\
890 \\
895 \\
900 \\
905 \\
910 \\
915 \\
920 \\
925 \\
930 \\
935 \\
940 \\
945 \\
950 \\
955 \\
960 \\
965 \\
970 \\
975 \\
980 \\
985 \\
990 \\
995 \\
1000 \\
1005 \\
1010 \\
1015 \\
1020 \\
1025 \\
1030 \\
1035 \\
1040 \\
1040\end{array}$ & $\begin{array}{l}38.571 \\
38.605 \\
38.527 \\
38.696 \\
38.801 \\
38.865 \\
38.920 \\
38.956 \\
38.985 \\
39.002 \\
39.126 \\
39.345 \\
39.417 \\
30.414 \\
39.523 \\
39.684 \\
39.832 \\
39.939 \\
40.039 \\
40.106 \\
40.212 \\
40.352 \\
40.545 \\
40.640 \\
40.743 \\
40.820 \\
40.919 \\
11.031 \\
41.134 \\
41.229 \\
41.339 \\
41.447 \\
41.532 \\
41.619 \\
11.699 \\
41.758 \\
41.705 \\
41.811 \\
41.918 \\
42.027 \\
12.193 \\
42.206\end{array}$ \\
\hline
\end{tabular}


RSFT RIVER, ILFHO - I.D. MO. 1 - 3 DECEYBER 1974

\begin{tabular}{|c|c|c|c|c|c|}
\hline $\begin{array}{l}\text { IEPTH } \\
\text { (FT) }\end{array}$ & $\begin{array}{l}\text { TEAPERATURE } \\
(\mathrm{C})\end{array}$ & $\begin{array}{l}\text { DEPTH } \\
\text { (FT) }\end{array}$ & $\begin{array}{l}\text { TELERATLRE } \\
\text { (C) }\end{array}$ & $\begin{array}{c}\text { DEPTH } \\
\text { (FT) }\end{array}$ & $\begin{array}{l}\text { TEPERATU } \\
\text { (C) }\end{array}$ \\
\hline $\begin{array}{l}140 \\
145 \\
150 \\
155 \\
150 \\
165 \\
170 \\
175 \\
180 \\
185 \\
190 \\
195 \\
200 \\
205 \\
210 \\
215 \\
220 \\
225 \\
230 \\
235 \\
240 \\
245 \\
250 \\
255 \\
250 \\
265 \\
270 \\
275 \\
280 \\
285 \\
290 \\
295 \\
300 \\
305 \\
310 \\
315 \\
320 \\
325 \\
330 \\
335 \\
340 \\
345 \\
350 \\
355 \\
360 \\
365 \\
370 \\
375 \\
330 \\
385 \\
3390 \\
395 \\
400 \\
405 \\
410 \\
115 \\
420 \\
4255 \\
430 \\
135 \\
4140 \\
445 \\
4550 \\
455 \\
460 \\
465 \\
470 \\
475 \\
480 \\
485\end{array}$ & $\begin{array}{l}13.105 \\
14.645 \\
14.713 \\
14.763 \\
14.829 \\
14.907 \\
15.017 \\
15.115 \\
15.234 \\
15.331 \\
15.123 \\
15.517 \\
15.582 \\
15.730 \\
15.791 \\
15.825 \\
16.003 \\
16.072 \\
15.160 \\
16.268 \\
16.336 \\
16.592 \\
16.645 \\
16.735 \\
16.826 \\
16.595 \\
11.128 \\
17.301 \\
17.467 \\
17.577 \\
17.979 \\
18.117 \\
18.368 \\
18.591 \\
18.891 \\
19.142 \\
19.140 \\
19.751 \\
20.012 \\
20.323 \\
20.6007 \\
20.919 \\
21.234 \\
21.548 \\
21.844 \\
22.174 \\
22.386 \\
22.631 \\
22.864 \\
23.049 \\
23.268 \\
23.444 \\
23.329 \\
23.847 \\
24.001 \\
24.250 \\
24.3777 \\
21.529 \\
21.5951 \\
21.917 \\
25.057 \\
25.127 \\
25.282 \\
25.466 \\
25.641 \\
25.813 \\
26.0399 \\
26.213 \\
26.452\end{array}$ & $\begin{array}{l}490 \\
495 \\
500 \\
505 \\
510 \\
515 \\
520 \\
525 \\
530 \\
535 \\
540 \\
545 \\
550 \\
555 \\
550 \\
565 \\
570 \\
575 \\
580 \\
585 \\
590 \\
595 \\
600 \\
605 \\
610 \\
615 \\
620 \\
625 \\
630 \\
635 \\
640 \\
645 \\
650 \\
655 \\
360 \\
665 \\
670 \\
675 \\
380 \\
685 \\
390 \\
695 \\
700 \\
705 \\
710 \\
715 \\
720 \\
725 \\
730 \\
735 \\
740 \\
745 \\
750 \\
755 \\
730 \\
765 \\
770 \\
775 \\
780 \\
785 \\
790 \\
755 \\
800 \\
805 \\
810 \\
815 \\
820 \\
8250 \\
835\end{array}$ & 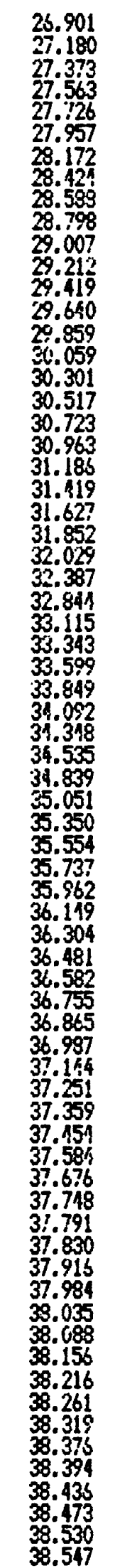 & $\begin{array}{l}840 \\
845 \\
850 \\
855 \\
860 \\
865 \\
870 \\
875 \\
880 \\
895 \\
890 \\
895 \\
900 \\
905 \\
910 \\
915 \\
920 \\
925 \\
930 \\
935 \\
910 \\
945 \\
950 \\
955 \\
960 \\
965 \\
970 \\
975 \\
980 \\
985 \\
990 \\
995 \\
1000 \\
1005 \\
1010 \\
1015 \\
1020 \\
1025 \\
1030 \\
1035 \\
1040 \\
1045 \\
1049\end{array}$ & $\begin{array}{l}38.557 \\
38.579 \\
38.502 \\
38.634 \\
38.653 \\
38.785 \\
33.849 \\
33.910 \\
38.956 \\
38.991 \\
39.009 \\
39.106 \\
39.348 \\
39.429 \\
30.461 \\
39.526 \\
39.565 \\
39.798 \\
39.940 \\
40.039 \\
40.132 \\
40.213 \\
40.322 \\
40.188 \\
40.632 \\
10.730 \\
40.828 \\
40.907 \\
41.013 \\
41.127 \\
41.231 \\
41.321 \\
41.421 \\
41.515 \\
41.503 \\
41.680 \\
41.743 \\
41.793 \\
41.831 \\
61.886 \\
42.005 \\
42.114 \\
42.253\end{array}$ \\
\hline
\end{tabular}


RAFT RIYER, IDAHD - I.D. NU. 1 - 8 DECENBER 1974

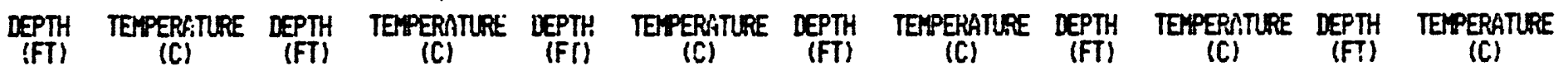

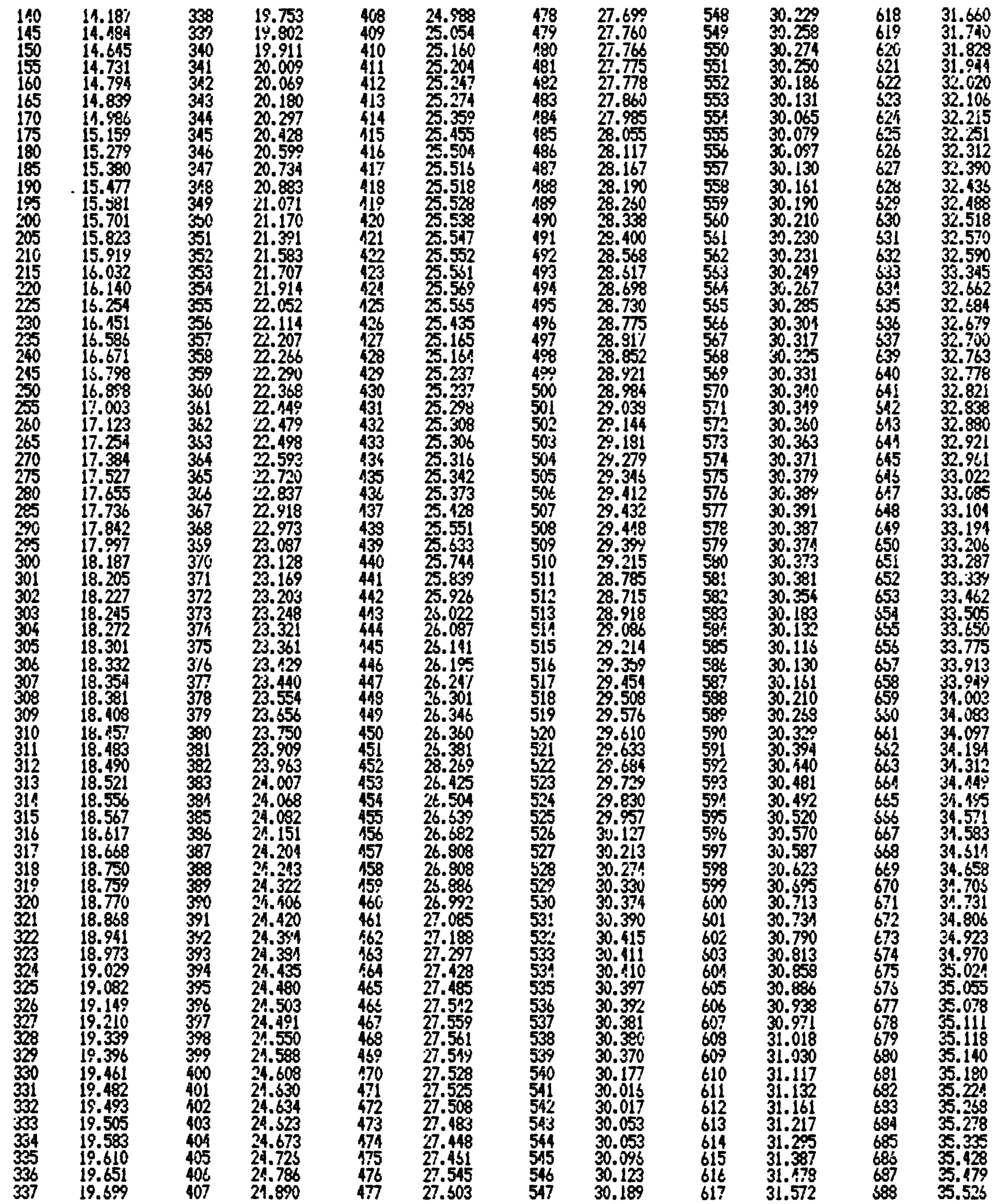


RAFT RIVER, IDAHO - 1.D. MO. 1 - 8 DECEYRER 1974

DEPTH TEMPERTIRE DEPTH TEMPERATURE DEPTH TEMPERATURE
(FT) (C) (C)

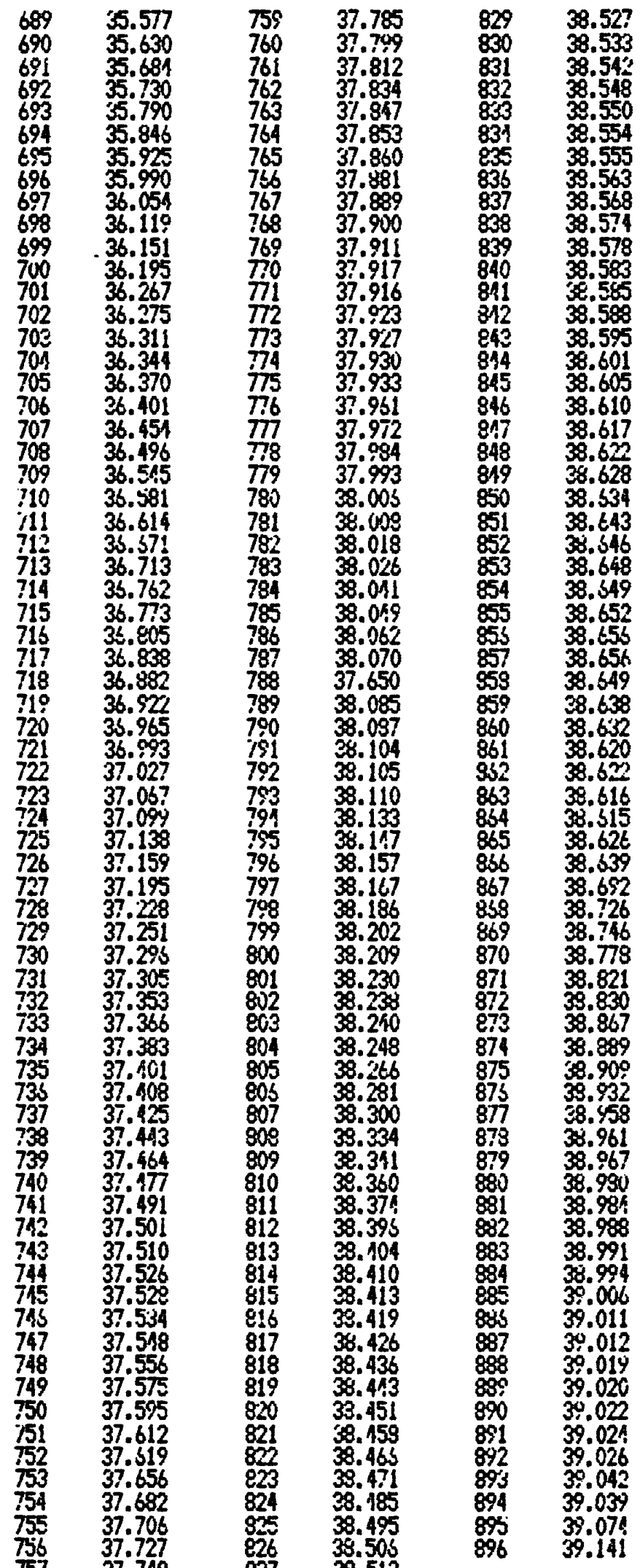


RAFT RIVER, IOAHD - I.D. NO. 1 - 11 JALUFAY 1975

\begin{tabular}{|c|c|c|c|c|c|}
\hline $\begin{array}{l}\text { DEPTH } \\
\text { (FT) }\end{array}$ & $\begin{array}{l}\text { TERPEPATURE } \\
\text { (C) }\end{array}$ & $\begin{array}{l}\text { DEPTH } \\
\text { (FT) }\end{array}$ & $\begin{array}{l}\text { TEMPERATUPE } \\
\text { (C) }\end{array}$ & $\begin{array}{l}\text { DEPTH } \\
\text { (FT) }\end{array}$ & $\begin{array}{l}\text { TEPPERATU } \\
\text { (C) }\end{array}$ \\
\hline $\begin{array}{l}5 \\
10 \\
20 \\
25 \\
30 \\
35 \\
10 \\
15 \\
50 \\
55 \\
50 \\
65 \\
70 \\
75 \\
80 \\
90 \\
65 \\
100 \\
105 \\
110 \\
115 \\
120 \\
125 \\
130 \\
135 \\
140 \\
115 \\
150 \\
155 \\
160 \\
165 \\
170 \\
175 \\
180 \\
185 \\
190 \\
195 \\
200 \\
205 \\
210 \\
215 \\
220 \\
225 \\
230 \\
235 \\
240 \\
245 \\
250 \\
255 \\
260 \\
265 \\
270 \\
275 \\
280 \\
285 \\
290 \\
295 \\
300 \\
305 \\
310 \\
315 \\
320 \\
325 \\
330 \\
335 \\
340 \\
315 \\
350 \\
355 \\
360\end{array}$ & 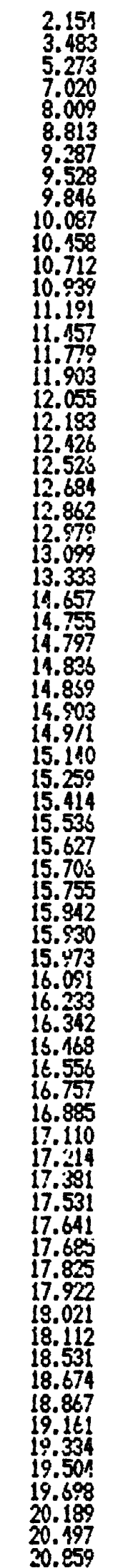 & $\begin{array}{l}365 \\
370 \\
375 \\
380 \\
395 \\
390 \\
395 \\
400 \\
405 \\
110 \\
415 \\
420 \\
425 \\
430 \\
435 \\
140 \\
415 \\
450 \\
455 \\
460 \\
465 \\
470 \\
475 \\
480 \\
495 \\
490 \\
495 \\
500 \\
505 \\
510 \\
515 \\
540 \\
525 \\
530 \\
535 \\
540 \\
545 \\
550 \\
555 \\
560 \\
565 \\
570 \\
575 \\
580 \\
585 \\
590 \\
595 \\
600 \\
315 \\
620 \\
625 \\
630 \\
635 \\
640 \\
645 \\
650 \\
5555 \\
660 \\
645 \\
670 \\
675 \\
680 \\
385 \\
690 \\
595 \\
700 \\
705 \\
710 \\
715 \\
720\end{array}$ & 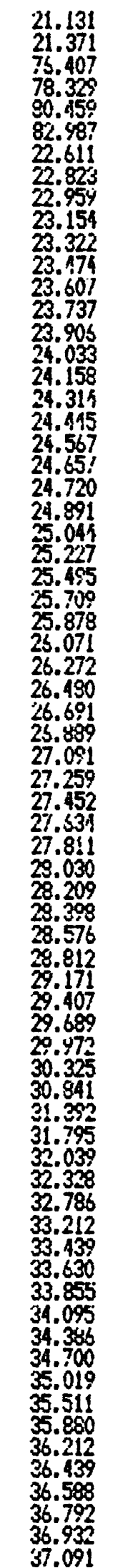 & $\begin{array}{l}725 \\
730 \\
735 \\
710 \\
745 \\
750 \\
755 \\
760 \\
765 \\
770 \\
775 \\
780 \\
785 \\
790 \\
795 \\
800 \\
805 \\
810 \\
815 \\
820 \\
825 \\
830 \\
835 \\
810 \\
845 \\
850 \\
855 \\
860 \\
855 \\
870 \\
875 \\
980 \\
895 \\
890 \\
892\end{array}$ & $\begin{array}{l}37.251 \\
37.285 \\
37.467 \\
37.510 \\
37.524 \\
37.616 \\
37.727 \\
37.811 \\
37.855 \\
37.859 \\
37.927 \\
37.979 \\
33.049 \\
38.091 \\
38.138 \\
38.179 \\
38.243 \\
38.357 \\
38.417 \\
38.454 \\
38.496 \\
38.532 \\
38.559 \\
39.597 \\
38.614 \\
38.649 \\
38.652 \\
33.600 \\
38.707 \\
38.838 \\
38.450 \\
38.997 \\
39.029 \\
39.019 \\
39.110\end{array}$ \\
\hline
\end{tabular}


RAFT RIVER, IDAHO - I.D. NO. 1 - 29 JAHUAYY IS75

\begin{tabular}{|c|c|c|c|c|c|}
\hline $\begin{array}{l}\text { DEPTH } \\
\text { (FT) }\end{array}$ & $\begin{array}{l}\text { TEAPERATURE } \\
\text { (C) }\end{array}$ & $\begin{array}{c}\text { DEPTH } \\
\text { (FT) }\end{array}$ & $\begin{array}{l}\text { TEMPERATLRE } \\
\text { (C) }\end{array}$ & $\begin{array}{l}\text { IEPTH } \\
\text { (FT) }\end{array}$ & $\begin{array}{l}\text { TEEPERSTR } \\
(C)\end{array}$ \\
\hline $\begin{array}{l}5 \\
10 \\
15 \\
20 \\
25 \\
30 \\
35 \\
10 \\
45 \\
50 \\
55 \\
60 \\
655 \\
70 \\
80 \\
90 \\
100 \\
110 \\
120 \\
130 \\
140 \\
145 \\
150 \\
155 \\
160 \\
165 \\
170 \\
175 \\
180 \\
195 \\
190 \\
195 \\
200 \\
205 \\
210 \\
215 \\
220 \\
225 \\
230 \\
235 \\
240 \\
245 \\
250 \\
255 \\
250 \\
265 \\
270 \\
275 \\
230 \\
285 \\
290 \\
2095 \\
300 \\
305 \\
310 \\
315 \\
3320 \\
325 \\
330 \\
335 \\
340 \\
345 \\
3500 \\
355 \\
360 \\
365 \\
370 \\
3375 \\
330 \\
385\end{array}$ & 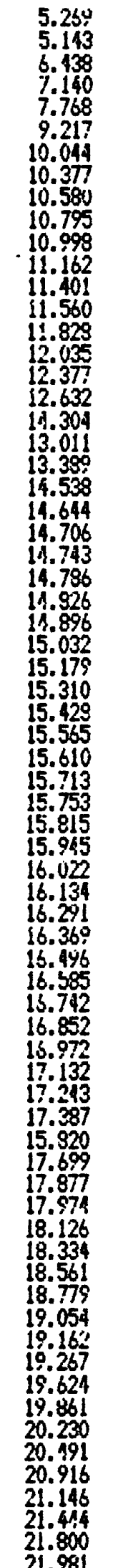 & 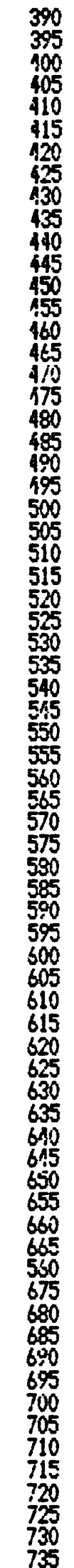 & 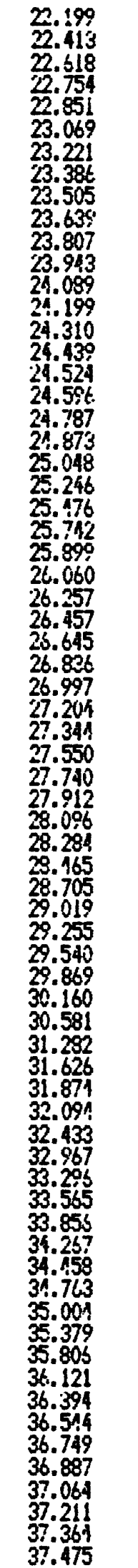 & $\begin{array}{l}710 \\
745 \\
750 \\
7555 \\
750 \\
765 \\
770 \\
775 \\
780 \\
785 \\
790 \\
795 \\
800 \\
805 \\
810 \\
815 \\
820 \\
825 \\
830 \\
835 \\
890 \\
845 \\
850 \\
855 \\
860 \\
865 \\
870 \\
875 \\
820 \\
895 \\
890 \\
865 \\
897\end{array}$ & $\begin{array}{l}37.545 \\
37.560 \\
37.543 \\
37.732 \\
37.965 \\
37.892 \\
37.932 \\
37.955 \\
38.010 \\
38.055 \\
38.116 \\
38.170 \\
38.255 \\
38.267 \\
39.370 \\
38.156 \\
33.494 \\
38.542 \\
38.578 \\
38.610 \\
38.663 \\
38.687 \\
38.708 \\
38.733 \\
38.736 \\
38.706 \\
38.816 \\
38.962 \\
39.050 \\
39.099 \\
34.124 \\
39.137 \\
39.243\end{array}$ \\
\hline
\end{tabular}


RAFT RIVER, IDAHO - I.D. MO. I - 31 MARH 1975

\begin{tabular}{|c|c|c|c|c|c|}
\hline $\begin{array}{l}\text { IEPTH } \\
\text { (ITT) }\end{array}$ & $\begin{array}{l}\text { TEAPERATURE } \\
\text { (C) }\end{array}$ & $\begin{array}{l}\text { IEPTH } \\
\text { (FT) }\end{array}$ & $\begin{array}{l}\text { TEPEERATLRE } \\
\text { (C) }\end{array}$ & $\begin{array}{l}\text { DEPTH } \\
\text { (FT) }\end{array}$ & $\begin{array}{l}\text { TERERATUR } \\
\text { (C) }\end{array}$ \\
\hline $\begin{array}{l}142 \\
145 \\
150 \\
155 \\
160 \\
165 \\
170 \\
175 \\
180 \\
185 \\
190 \\
195 \\
200 \\
205 \\
210 \\
215 \\
220 \\
225 \\
230 \\
235 \\
240 \\
245 \\
250 \\
255 \\
260 \\
265 \\
270 \\
275 \\
280 \\
285 \\
290 \\
295 \\
300 \\
305 \\
310 \\
315 \\
320 \\
325 \\
330 \\
335 \\
340 \\
345 \\
350 \\
355 \\
350 \\
365 \\
370 \\
375 \\
390 \\
385 \\
390 \\
395 \\
100 \\
405 \\
410 \\
415 \\
420 \\
425 \\
430 \\
435 \\
440 \\
445 \\
450 \\
455 \\
460 \\
465 \\
470 \\
175 \\
480 \\
485\end{array}$ & $\begin{array}{l}14.440 \\
11.489 \\
19.557 \\
14.615 \\
11.674 \\
11.115 \\
11.790 \\
14.871 \\
14.909 \\
15.147 \\
15.292 \\
15.390 \\
15.475 \\
15.575 \\
15.637 \\
15.736 \\
15.800 \\
15.935 \\
16.008 \\
16.153 \\
16.257 \\
16.364 \\
16.498 \\
16.601 \\
16.735 \\
16.907 \\
16.990 \\
17.130 \\
17.263 \\
17.120 \\
17.511 \\
17.723 \\
17.903 \\
18.050 \\
18.123 \\
18.128 \\
18.559 \\
18.824 \\
19.041 \\
19.214 \\
19.432 \\
19.615 \\
19.920 \\
20.235 \\
20.527 \\
20.856 \\
21.113 \\
21.368 \\
21.521 \\
21.848 \\
22.085 \\
22.292 \\
22.170 \\
22.622 \\
22.781 \\
22.950 \\
23.128 \\
23.302 \\
23.100 \\
23.591 \\
23.680 \\
23.822 \\
23.940 \\
24.073 \\
24.199 \\
24.293 \\
24.3904 \\
24.472 \\
24.607 \\
24.757\end{array}$ & $\begin{array}{l}490 \\
495 \\
500 \\
505 \\
510 \\
515 \\
520 \\
525 \\
530 \\
535 \\
540 \\
515 \\
550 \\
555 \\
550 \\
565 \\
570 \\
575 \\
580 \\
585 \\
590 \\
595 \\
600 \\
605 \\
610 \\
615 \\
620 \\
625 \\
630 \\
635 \\
540 \\
645 \\
650 \\
655 \\
560 \\
655 \\
670 \\
675 \\
690 \\
695 \\
690 \\
695 \\
700 \\
705 \\
710 \\
715 \\
720 \\
725 \\
730 \\
735 \\
710 \\
745 \\
750 \\
755 \\
760 \\
765 \\
770 \\
775 \\
790 \\
785 \\
790 \\
7995 \\
800 \\
805 \\
810 \\
815 \\
820 \\
8250 \\
835\end{array}$ & 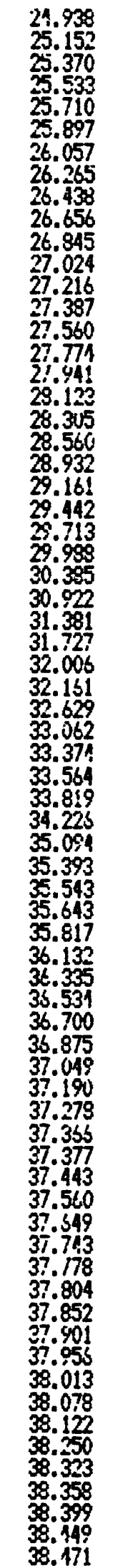 & $\begin{array}{l}840 \\
845 \\
850 \\
855 \\
950 \\
865 \\
870 \\
875 \\
880 \\
825 \\
890 \\
895 \\
896\end{array}$ & $\begin{array}{l}38.500 \\
38.528 \\
38.555 \\
38.578 \\
38.578 \\
38.554 \\
38.693 \\
38.938 \\
38.898 \\
38.912 \\
38.973 \\
38.990 \\
39.112\end{array}$ \\
\hline
\end{tabular}


RAFT RIVER, IDAHO - I.D. MO. 1 - 29 NUGUST 1975

\begin{tabular}{|c|c|c|c|c|c|}
\hline $\begin{array}{l}\text { DEPTH } \\
\text { (FT) }\end{array}$ & $\begin{array}{l}\text { TEPEERATLAE } \\
\text { (C) }\end{array}$ & $\begin{array}{l}\text { DEPTH } \\
\text { (FT) }\end{array}$ & $\begin{array}{l}\text { TEPERATLRE } \\
\text { (C) }\end{array}$ & $\begin{array}{c}\text { DEPTH } \\
\text { (FT) }\end{array}$ & $\begin{array}{l}\text { TENPERATU } \\
\text { (C) }\end{array}$ \\
\hline $\begin{array}{l}5 \\
10 \\
15 \\
20 \\
25 \\
30 \\
35 \\
10 \\
15 \\
50 \\
60 \\
65 \\
70 \\
75 \\
80 \\
50 \\
95 \\
100 \\
105 \\
101 \\
115 \\
1100 \\
125 \\
130 \\
135 \\
140 \\
145 \\
150 \\
155 \\
160 \\
155 \\
170 \\
115 \\
180 \\
185 \\
190 \\
195 \\
200 \\
205 \\
210 \\
215 \\
220 \\
225 \\
230 \\
235 \\
240 \\
245 \\
250 \\
255 \\
260 \\
265 \\
270 \\
275 \\
280 \\
235 \\
290 \\
2455 \\
300 \\
305 \\
310 \\
315 \\
330 \\
325 \\
330 \\
355 \\
340 \\
345 \\
350 \\
355 \\
360\end{array}$ & 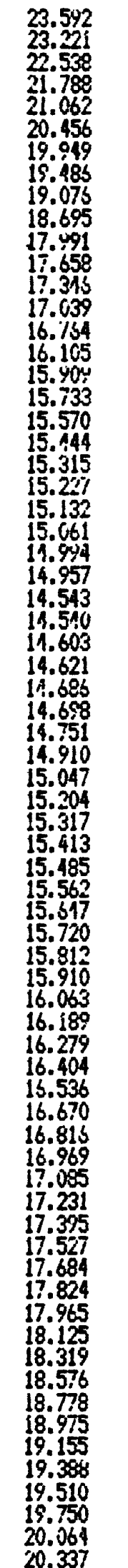 & 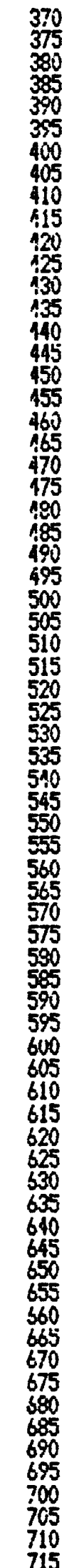 & 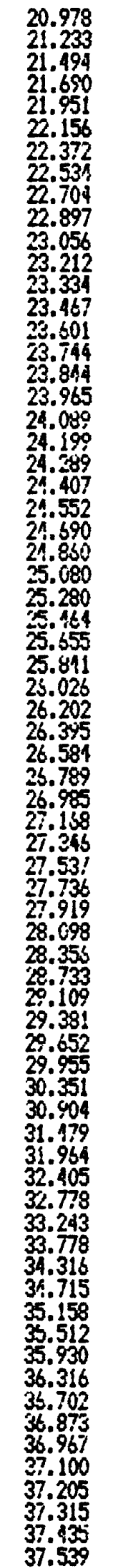 & $\begin{array}{l}720 \\
725 \\
730 \\
735 \\
7100 \\
715 \\
750 \\
755 \\
760 \\
765 \\
770 \\
775 \\
780 \\
785 \\
790 \\
795 \\
800 \\
805 \\
810 \\
815 \\
920 \\
825 \\
830 \\
835 \\
840 \\
845 \\
850 \\
855 \\
860 \\
855 \\
870 \\
875 \\
890 \\
885 \\
887\end{array}$ & $\begin{array}{l}37.652 \\
37.748 \\
37.824 \\
37.875 \\
37.891 \\
37.944 \\
38.018 \\
38.094 \\
33.135 \\
38.156 \\
38.171 \\
38.219 \\
38.261 \\
38.294 \\
38.335 \\
38.377 \\
38.416 \\
38.499 \\
38.545 \\
38.568 \\
38.599 \\
39.621 \\
38.647 \\
38.652 \\
38.487 \\
35.720 \\
38.724 \\
38.704 \\
38.730 \\
38.825 \\
38.926 \\
39.002 \\
39.041 \\
34.063 \\
39.171\end{array}$ \\
\hline
\end{tabular}


RAFT RIVER, IDAHO - 1.D. NO. 1 - 16 SEPTEMPER 1975

\begin{tabular}{|c|c|c|c|c|c|}
\hline $\begin{array}{l}\text { DEPTH } \\
\text { (FT) }\end{array}$ & $\begin{array}{l}\text { TEYPERATLRE } \\
\text { (C) }\end{array}$ & $\begin{array}{l}\text { DEPTH } \\
\text { (FT) }\end{array}$ & $\begin{array}{l}\text { TEPPERATURE } \\
\text { (C) }\end{array}$ & $\begin{array}{c}\text { DEPTH } \\
\text { (FT) }\end{array}$ & $\begin{array}{l}\text { TERPERATURE } \\
\text { (C) }\end{array}$ \\
\hline $\begin{array}{l}5 \\
10 \\
15 \\
20 \\
25 \\
30 \\
35 \\
40 \\
15 \\
50 \\
55 \\
60 \\
55 \\
70 \\
75 \\
80 \\
85 \\
90 \\
95 \\
100 \\
105 \\
110 \\
115 \\
120 \\
125 \\
130 \\
135 \\
140 \\
145 \\
150 \\
155 \\
160 \\
155 \\
170 \\
175 \\
180 \\
185 \\
190 \\
195 \\
200 \\
205 \\
210 \\
215 \\
200 \\
225 \\
230 \\
235 \\
240 \\
215 \\
250 \\
255 \\
260 \\
265 \\
270 \\
275 \\
290 \\
285 \\
290 \\
205 \\
300 \\
305 \\
310 \\
315 \\
320 \\
325 \\
330 \\
335 \\
340 \\
345 \\
350\end{array}$ & $\begin{array}{l}21.091 \\
20.951 \\
10.305 \\
18.562 \\
18.080 \\
17.591 \\
17.007 \\
16.291 \\
15.901 \\
15.787 \\
15.39 \\
15.050 \\
14.279 \\
14.135 \\
11.038 \\
11.185 \\
13.973 \\
13.920 \\
13.871 \\
13.812 \\
13.823 \\
13.817 \\
13.819 \\
13.825 \\
13.835 \\
13.841 \\
13.945 \\
14.005 \\
11.341 \\
11.551 \\
11.628 \\
14.661 \\
14.593 \\
14.746 \\
14.821 \\
11.941 \\
15.082 \\
15.233 \\
15.320 \\
15.415 \\
15.503 \\
15.558 \\
15.331 \\
15.711 \\
15.821 \\
15.930 \\
16.031 \\
16.175 \\
16.317 \\
16.457 \\
16.591 \\
16.702 \\
16.783 \\
16.919 \\
17.097 \\
17.207 \\
17.324 \\
17.497 \\
17.628 \\
17.754 \\
17.861 \\
18.035 \\
18.224 \\
18.190 \\
18.728 \\
18.899 \\
10.080 \\
19.354 \\
19.510 \\
10.721\end{array}$ & $\begin{array}{l}355 \\
365 \\
370 \\
375 \\
380 \\
385 \\
390 \\
395 \\
100 \\
405 \\
410 \\
415 \\
420 \\
125 \\
430 \\
435 \\
140 \\
145 \\
450 \\
455 \\
450 \\
465 \\
170 \\
975 \\
480 \\
485 \\
490 \\
495 \\
500 \\
505 \\
510 \\
515 \\
520 \\
525 \\
530 \\
535 \\
540 \\
545 \\
550 \\
555 \\
560 \\
555 \\
570 \\
575 \\
580 \\
585 \\
590 \\
595 \\
300 \\
605 \\
510 \\
615 \\
620 \\
625 \\
330 \\
635 \\
640 \\
645 \\
550 \\
655 \\
350 \\
665 \\
670 \\
675 \\
680 \\
685 \\
690 \\
695 \\
700 \\
705\end{array}$ & $\begin{array}{l}20.338 \\
20.564 \\
20.989 \\
21.153 \\
21.166 \\
21.691 \\
21.891 \\
22.101 \\
22.319 \\
22.511 \\
22.691 \\
22.771 \\
22.935 \\
23.106 \\
23.219 \\
23.372 \\
23.557 \\
23.702 \\
23.832 \\
23.975 \\
24.108 \\
24.210 \\
24.305 \\
24.398 \\
24.535 \\
24.686 \\
21.833 \\
25.010 \\
25.182 \\
25.427 \\
25.578 \\
25.781 \\
25.915 \\
26.158 \\
26.309 \\
26.477 \\
26.587 \\
26.913 \\
27.091 \\
27.276 \\
27.143 \\
27.660 \\
27.840 \\
28.043 \\
28.225 \\
28.163 \\
28.941 \\
39.233 \\
29.489 \\
29.738 \\
30.068 \\
30.401 \\
31.007 \\
31.629 \\
32.042 \\
32.485 \\
32.182 \\
33.342 \\
33.898 \\
34.398 \\
31.73 ! \\
35.211 \\
35.600 \\
36.027 \\
33.371 \\
36.675 \\
35.900 \\
37.000 \\
37.076 \\
37.175\end{array}$ & $\begin{array}{l}710 \\
715 \\
720 \\
725 \\
730 \\
735 \\
740 \\
745 \\
750 \\
755 \\
760 \\
765 \\
770 \\
775 \\
780 \\
785 \\
790 \\
795 \\
800 \\
805 \\
810 \\
815 \\
920 \\
825 \\
830 \\
835 \\
840 \\
845 \\
850 \\
855 \\
960 \\
865 \\
870 \\
875 \\
880 \\
895 \\
890 \\
895 \\
897\end{array}$ & 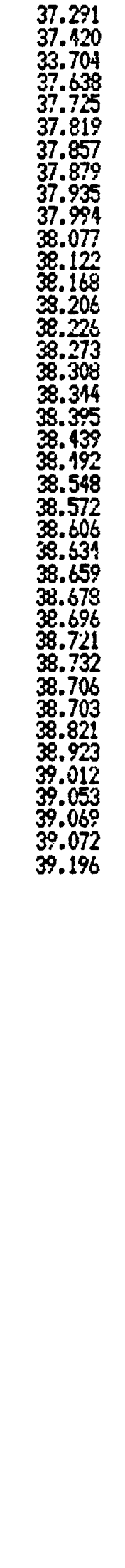 \\
\hline
\end{tabular}


HAFT RIVER, IOHHO - I.D. NO. I - 21 OCTOEER 1975

\begin{tabular}{|c|c|c|c|c|c|}
\hline $\begin{array}{l}\text { DEPTH } \\
\text { (FT) }\end{array}$ & $\begin{array}{l}\text { TEMERATURE } \\
\text { (C) }\end{array}$ & $\begin{array}{l}\text { DEPTH } \\
\text { (FT) }\end{array}$ & $\begin{array}{l}\text { TEPPEATURE } \\
(C)\end{array}$ & $\begin{array}{l}\text { DEPTH } \\
\text { (FT) }\end{array}$ & $\begin{array}{l}\text { TEEFERATU } \\
\text { (C) }\end{array}$ \\
\hline $\begin{array}{l}5 \\
10 \\
15 \\
20 \\
25 \\
30 \\
35 \\
10 \\
45 \\
50 \\
55 \\
60 \\
65 \\
70 \\
75 \\
80 \\
90 \\
45 \\
100 \\
105 \\
110 \\
115 \\
120 \\
125 \\
130 \\
135 \\
140 \\
145 \\
150 \\
155 \\
150 \\
165 \\
170 \\
175 \\
180 \\
185 \\
190 \\
195 \\
200 \\
205 \\
210 \\
215 \\
220 \\
225 \\
230 \\
235 \\
240 \\
245 \\
250 \\
255 \\
250 \\
265 \\
270 \\
275 \\
280 \\
285 \\
290 \\
295 \\
300 \\
305 \\
310 \\
315 \\
320 \\
325 \\
330 \\
335 \\
340 \\
3355 \\
350 \\
355\end{array}$ & 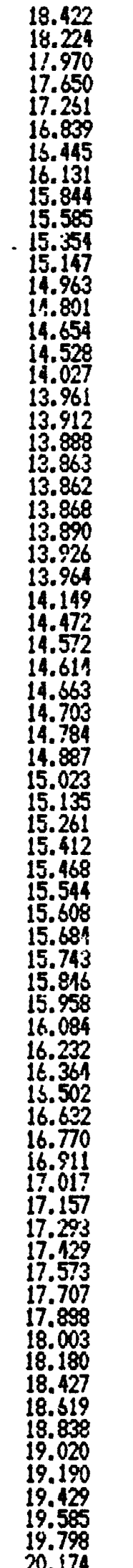 & 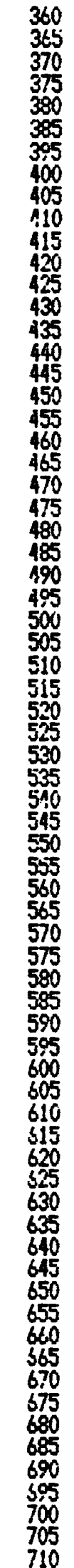 & 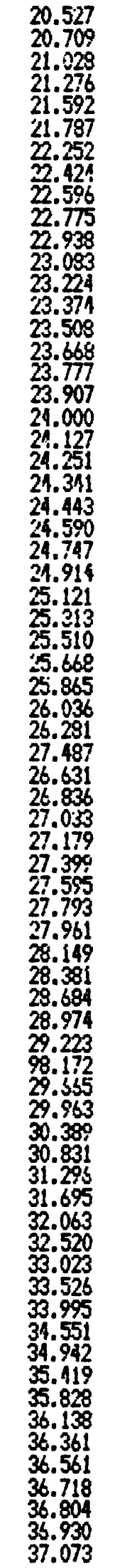 & $\begin{array}{l}715 \\
720 \\
725 \\
730 \\
735 \\
740 \\
745 \\
750 \\
755 \\
760 \\
765 \\
770 \\
775 \\
780 \\
785 \\
790 \\
795 \\
800 \\
805 \\
810 \\
815 \\
820 \\
825 \\
830 \\
835 \\
840 \\
815 \\
850 \\
855 \\
860 \\
865 \\
870 \\
875 \\
880 \\
885 \\
889\end{array}$ & 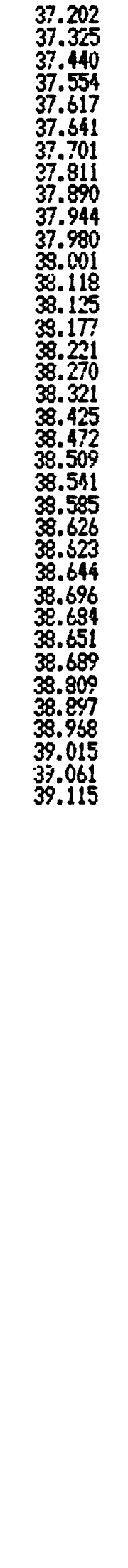 \\
\hline
\end{tabular}


RATT RIVER, IDAHO - I.D. NO. I - 15 JWAUARY 1976

IEPTH TEPPEPATLRE DEPTH TEPPERATUPE DEPTH

(FT)

(C)

14.727

(FT)

(C)

(FT)

Tupe

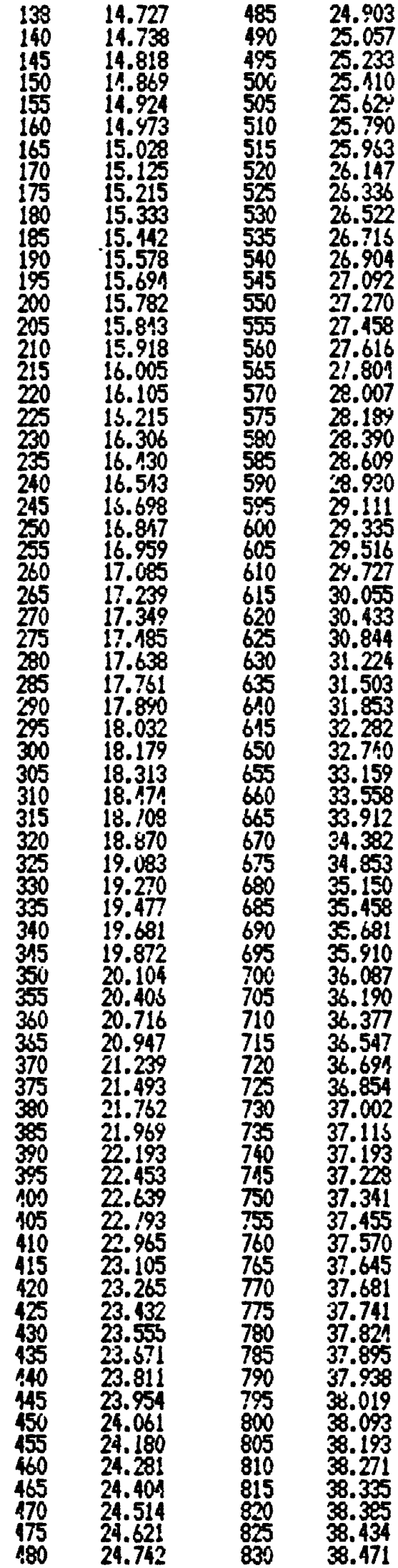

\author{
(C)
}

38.497

$\begin{array}{ll}835 & 38.497 \\ 810 & 38.524 \\ 945 & 38.548 \\ 850 & 38.575 \\ 855 & 39.569 \\ 860 & 38.566 \\ 845 & 33.619 \\ 870 & 38.732 \\ 875 & 33.838 \\ 880 & 38.902 \\ 985 & 38.940 \\ 890 & 38.959 \\ 893 & 39.025\end{array}$ 
RAFT RIVER, IDAH - I.D. NO. 2 - 3 LECEMBER 1974

\begin{tabular}{|c|c|c|c|}
\hline $\begin{array}{l}\text { DEPTH } \\
\text { (FT) }\end{array}$ & $\begin{array}{l}\text { TEYPERATURE } \\
\text { (C) }\end{array}$ & $\begin{array}{l}\text { DEPTH } \\
\text { (FT) }\end{array}$ & $\begin{array}{l}\text { TEMEERAT } \\
\text { (C) }\end{array}$ \\
\hline $\begin{array}{l}60 \\
65 \\
70 \\
75 \\
80 \\
85 \\
90 \\
95 \\
100 \\
105 \\
110 \\
115 \\
120 \\
125 \\
130 \\
135 \\
140 \\
145 \\
150 \\
155 \\
160 \\
165 \\
170 \\
175 \\
180 \\
185 \\
190 \\
195 \\
200 \\
205 \\
210 \\
215 \\
220 \\
225 \\
230 \\
235 \\
240 \\
245 \\
250 \\
255 \\
260 \\
265 \\
270 \\
275 \\
280 \\
285 \\
290 \\
255 \\
300 \\
305 \\
310 \\
315 \\
320 \\
325 \\
330 \\
335 \\
340 \\
345 \\
350 \\
355 \\
360 \\
365 \\
370 \\
375 \\
380 \\
385 \\
390 \\
395 \\
100 \\
405\end{array}$ & 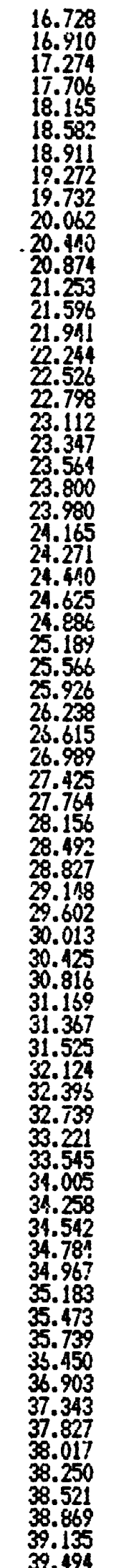 & $\begin{array}{l}410 \\
415 \\
420 \\
425 \\
130 \\
135 \\
410 \\
445 \\
450 \\
455 \\
460 \\
465 \\
470 \\
475 \\
180 \\
485 \\
490 \\
495 \\
500 \\
505 \\
510 \\
515 \\
520 \\
525 \\
530 \\
535 \\
540 \\
545 \\
550 \\
555 \\
560 \\
565 \\
570 \\
575 \\
580 \\
585 \\
590 \\
595 \\
600 \\
605 \\
610 \\
615 \\
620 \\
625 \\
330 \\
635 \\
540\end{array}$ & $\begin{array}{l}39.858 \\
40.129 \\
40.453 \\
40.672 \\
40.976 \\
11.252 \\
41.501 \\
41.721 \\
41.997 \\
42.283 \\
42.495 \\
42.741 \\
43.000 \\
43.215 \\
43.164 \\
13.702 \\
43.942 \\
44.141 \\
14.416 \\
44.782 \\
45.082 \\
45.356 \\
45.864 \\
46.076 \\
16.336 \\
46.698 \\
47.160 \\
47.522 \\
47.936 \\
48.255 \\
48.493 \\
48.843 \\
99.130 \\
49.523 \\
49.846 \\
50.276 \\
50.380 \\
51.015 \\
51.309 \\
51.579 \\
51.811 \\
52.048 \\
52.414 \\
52.701 \\
52.930 \\
53.230 \\
53.498\end{array}$ \\
\hline
\end{tabular}


RAFT RIVER, IDAHO - I.D. MO. 2 - 7 DECEHEER 1974

\begin{tabular}{|c|c|c|}
\hline $\begin{array}{l}\text { DEPTH } \\
\text { (FT) }\end{array}$ & $\begin{array}{l}\text { TEPFERITLRE } \\
\text { (C) }\end{array}$ & $\begin{array}{l}\text { DEPTH } \\
\text { (FT) }\end{array}$ \\
\hline $\begin{array}{l}55 \\
56 \\
60 \\
65 \\
70 \\
75 \\
80 \\
85 \\
90 \\
45 \\
100 \\
105 \\
110 \\
115 \\
120 \\
125 \\
130 \\
135 \\
140 \\
145 \\
150 \\
155 \\
160 \\
165 \\
170 \\
175 \\
190 \\
185 \\
190 \\
195 \\
200 \\
205 \\
210 \\
215 \\
220 \\
225 \\
230 \\
235 \\
240 \\
245 \\
250 \\
255 \\
260 \\
265 \\
270 \\
275 \\
280 \\
285 \\
200 \\
295 \\
300 \\
305 \\
310 \\
315 \\
320 \\
325 \\
330 \\
335 \\
340 \\
345 \\
350 \\
355 \\
350 \\
365 \\
370 \\
375 \\
390 \\
335 \\
390 \\
395\end{array}$ & $\begin{array}{l}15.164 \\
16.512 \\
15.323 \\
16.855 \\
17.183 \\
17.572 \\
19.090 \\
18.529 \\
18.976 \\
19.229 \\
19.572 \\
20.003 \\
20.368 \\
20.789 \\
21.187 \\
21.545 \\
21.829 \\
22.155 \\
22.452 \\
22.792 \\
23.064 \\
23.275 \\
23.532 \\
23.760 \\
23.914 \\
24.098 \\
24.264 \\
24.391 \\
24.315 \\
24.856 \\
25.156 \\
25.537 \\
25.875 \\
26.223 \\
26.566 \\
26.976 \\
27.400 \\
27.755 \\
28.137 \\
28.501 \\
28.848 \\
29.188 \\
29.580 \\
30.018 \\
30.376 \\
30.805 \\
31.155 \\
31.396 \\
31.611 \\
32.127 \\
32.475 \\
32.700 \\
33.243 \\
33.565 \\
34.009 \\
34.256 \\
34.515 \\
34.902 \\
35.036 \\
35.311 \\
35.307 \\
35.835 \\
35.435 \\
37.003 \\
37.440 \\
37.849 \\
38.065 \\
38.314 \\
39.610 \\
38.910\end{array}$ & $\begin{array}{l}100 \\
105 \\
110 \\
415 \\
420 \\
425 \\
430 \\
435 \\
440 \\
445 \\
450 \\
155 \\
460 \\
465 \\
470 \\
475 \\
480 \\
485 \\
190 \\
495 \\
500 \\
505 \\
510 \\
515 \\
520 \\
525 \\
530 \\
535 \\
540 \\
545 \\
550 \\
555 \\
540 \\
565 \\
570 \\
575 \\
580 \\
585 \\
540 \\
595 \\
600 \\
605 \\
610 \\
615 \\
620 \\
625 \\
630 \\
635 \\
640\end{array}$ \\
\hline
\end{tabular}


RAFT RIVER, IDAHO - I.D. MO. 2 - 11 AMUARY 1975

IEPTH TEPERATLRE IEPTH TEPERATLRE
(FT)

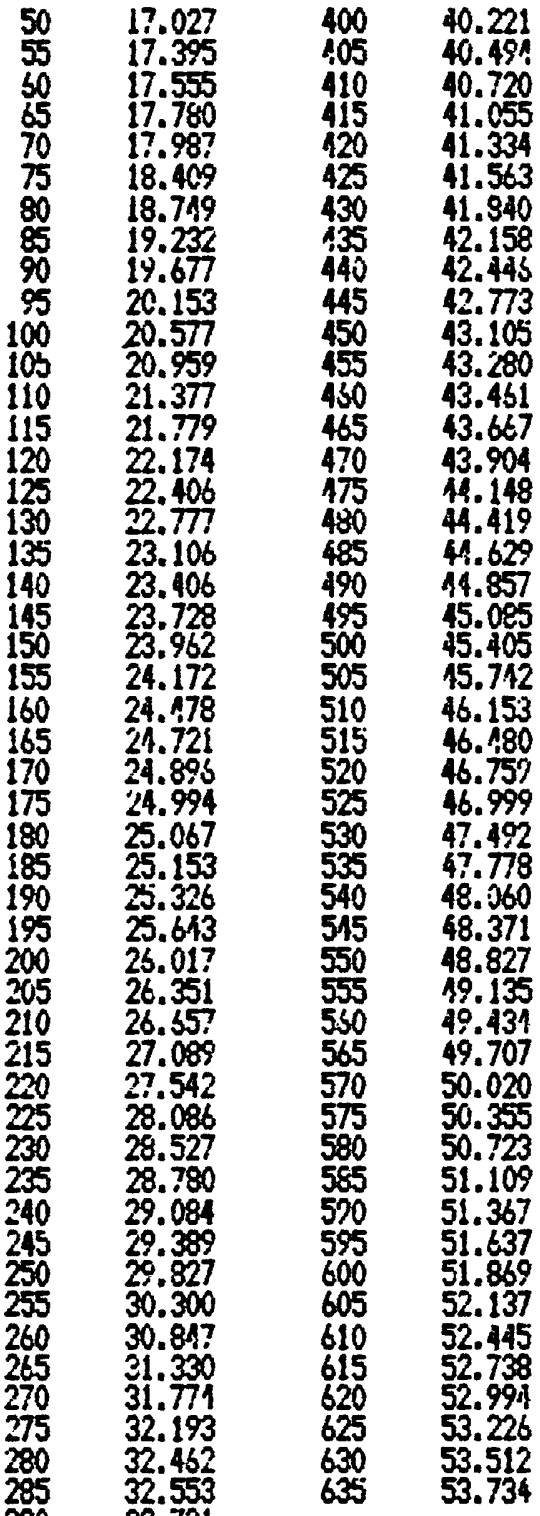

$\quad 33.099$

$300 \quad 33.528$

$305 \quad 33.834$

$310 \quad 34.076$

$315 \quad 34.459$

$320 \quad 34.867$

$325 \quad 35.232$

$330 \quad 35.596$

$335 \quad 35.979$

$340 \quad 36.272$

$350 \quad 37.047$

$355 \quad 37.366$

$360 \quad 37.678$

$365 \quad 38.012$

$370 \quad 38.339$

$375 \quad 38.669$

$390 \quad 39.004$

$390 \quad 39.308$

$395 \quad 39.923$ 
RAFT RIVER, IOALD - I.D. ND. 2 - 30 JAUUARY 1975

\begin{tabular}{|c|c|c|c|}
\hline $\begin{array}{l}\text { DEPTH } \\
\text { (FT) }\end{array}$ & $\begin{array}{l}\text { TEYPERATURE } \\
\text { (C) }\end{array}$ & $\begin{array}{c}\text { DEPTH } \\
\text { (FT) }\end{array}$ & $\begin{array}{l}\text { TEMERATL } \\
\text { (C) }\end{array}$ \\
\hline $\begin{array}{l}55 \\
60 \\
65 \\
70 \\
75 \\
80 \\
85 \\
90 \\
95 \\
100 \\
105 \\
110 \\
115 \\
120 \\
125 \\
130 \\
135 \\
140 \\
145 \\
150 \\
155 \\
160 \\
165 \\
170 \\
175 \\
180 \\
185 \\
190 \\
195 \\
200 \\
205 \\
210 \\
215 \\
250 \\
225 \\
230 \\
235 \\
240 \\
245 \\
250 \\
255 \\
260 \\
265 \\
270 \\
275 \\
280 \\
285 \\
290 \\
295 \\
300 \\
305 \\
310 \\
315 \\
320 \\
325 \\
330 \\
335 \\
340 \\
345 \\
350 \\
355 \\
360 \\
365 \\
370 \\
375 \\
380 \\
335 \\
390 \\
305 \\
400\end{array}$ & $\begin{array}{l}16.384 \\
16.665 \\
15.503 \\
17.248 \\
17.510 \\
18.055 \\
19.374 \\
18.822 \\
19.285 \\
19.600 \\
20.058 \\
20.393 \\
20.769 \\
21.149 \\
21.519 \\
21.804 \\
22.152 \\
22.158 \\
22.779 \\
23.066 \\
23.315 \\
23.527 \\
23.761 \\
23.921 \\
24.138 \\
24.291 \\
24.481 \\
24.681 \\
24.931 \\
25.295 \\
25.617 \\
25.976 \\
26.286 \\
26.669 \\
27.121 \\
27.555 \\
27.990 \\
28.282 \\
29.587 \\
29.026 \\
29.363 \\
29.799 \\
30.246 \\
30.738 \\
31.001 \\
31.381 \\
31.723 \\
31.857 \\
32.558 \\
32.727 \\
33.275 \\
33.524 \\
33.862 \\
31.152 \\
31.460 \\
34.726 \\
35.055 \\
35.267 \\
35.150 \\
35.869 \\
36.150 \\
36.917 \\
37.350 \\
37.689 \\
33.110 \\
38.352 \\
38.583 \\
339.932 \\
39.220 \\
39.530\end{array}$ & $\begin{array}{l}405 \\
410 \\
415 \\
420 \\
425 \\
430 \\
135 \\
140 \\
415 \\
450 \\
455 \\
460 \\
155 \\
470 \\
115 \\
180 \\
485 \\
490 \\
495 \\
500 \\
505 \\
510 \\
515 \\
520 \\
525 \\
530 \\
535 \\
540 \\
545 \\
550 \\
5555 \\
560 \\
565 \\
570 \\
575 \\
580 \\
585 \\
590 \\
595 \\
600 \\
605 \\
610 \\
515 \\
620 \\
625 \\
630 \\
635 \\
640\end{array}$ & $\begin{array}{l}30.816 \\
40.173 \\
40.353 \\
40.554 \\
40.843 \\
41.159 \\
11.191 \\
11.795 \\
12.040 \\
12.378 \\
12.578 \\
12.861 \\
43.110 \\
42.293 \\
43.645 \\
43.843 \\
44.074 \\
44.269 \\
44.509 \\
44.802 \\
45.158 \\
45.458 \\
45.821 \\
16.173 \\
45.480 \\
46.758 \\
47.126 \\
77.454 \\
17.859 \\
48.207 \\
48.514 \\
48.742 \\
49.221 \\
49.574 \\
49.945 \\
50.274 \\
50.439 \\
51.032 \\
51.322 \\
51.587 \\
51.826 \\
52.082 \\
55.404 \\
52.740 \\
52.939 \\
53.244 \\
53.516 \\
53.762\end{array}$ \\
\hline
\end{tabular}


RAFT RIVER, IDAHO - 1.D. NO. 2 - 31 MAPCH 1975

\begin{tabular}{|c|c|c|}
\hline $\begin{array}{l}\text { DEPTH } \\
\text { (FT) }\end{array}$ & $\begin{array}{l}\text { TERPERATURE } \\
\text { (C) }\end{array}$ & $\begin{array}{l}\text { DEPTH } \\
\text { (FT) }\end{array}$ \\
\hline $\begin{array}{l}50 \\
55 \\
50 \\
65 \\
70 \\
75 \\
80 \\
85 \\
90 \\
45 \\
100 \\
105 \\
110 \\
115 \\
120 \\
125 \\
130 \\
135 \\
140 \\
145 \\
150 \\
155 \\
160 \\
165 \\
170 \\
175 \\
180 \\
185 \\
190 \\
195 \\
200 \\
205 \\
210 \\
215 \\
220 \\
225 \\
230 \\
235 \\
240 \\
245 \\
255 \\
260 \\
245 \\
270 \\
275 \\
280 \\
255 \\
290\end{array}$ & $\begin{array}{l}16.031 \\
16.355 \\
15.637 \\
16.941 \\
17.240 \\
17.573 \\
17.957 \\
18.311 \\
18.683 \\
19.138 \\
19.534 \\
19.882 \\
20.274 \\
20.634 \\
20.996 \\
21.351 \\
21.727 \\
21.990 \\
22.302 \\
22.636 \\
22.909 \\
23.166 \\
23.370 \\
23.635 \\
23.830 \\
24.076 \\
24.249 \\
24.135 \\
24.665 \\
21.916 \\
25.221 \\
25.546 \\
25.962 \\
26.182 \\
26.608 \\
27.009 \\
27.462 \\
27.801 \\
28.193 \\
28.540 \\
29.267 \\
29.704 \\
30.125 \\
30.496 \\
30.867 \\
31.159 \\
31.764 \\
31.935\end{array}$ & $\begin{array}{l}405 \\
410 \\
415 \\
120 \\
125 \\
430 \\
435 \\
440 \\
415 \\
450 \\
455 \\
460 \\
465 \\
470 \\
475 \\
480 \\
485 \\
490 \\
495 \\
500 \\
505 \\
510 \\
515 \\
520 \\
525 \\
530 \\
535 \\
540 \\
545 \\
550 \\
555 \\
560 \\
555 \\
570 \\
575 \\
580 \\
585 \\
590 \\
575 \\
600 \\
605 \\
610 \\
615 \\
620 \\
525 \\
630 \\
635 \\
640\end{array}$ \\
\hline
\end{tabular}

32.244

32.681

33.310

33.621

33.863

34.058

34.343

34.643

31.893

35.137

35.276

35.891

$36.3 \% 3$

36.851

37.322

37.694

38.079

38.311

38.560

38.868

39.159 
RFFT RIVER, IDWHO - I.D. MO. 2 - 30 MULLET 1975

\begin{tabular}{|c|c|c|c|}
\hline $\begin{array}{l}\text { DEPTH } \\
\text { (FT) }\end{array}$ & $\begin{array}{l}\text { TEXPERATLRE } \\
(C)\end{array}$ & $\begin{array}{l}\text { DEPTH } \\
\text { (FT) }\end{array}$ & $\begin{array}{l}\text { TEPPERATU } \\
\text { (C) }\end{array}$ \\
\hline $\begin{array}{l}55 \\
60 \\
65 \\
70 \\
75 \\
80 \\
85 \\
90 \\
95 \\
100 \\
105 \\
110 \\
115 \\
120 \\
125 \\
130 \\
135 \\
140 \\
115 \\
150 \\
155 \\
160 \\
165 \\
170 \\
175 \\
180 \\
185 \\
190 \\
195 \\
200 \\
205 \\
210 \\
215 \\
220 \\
225 \\
230 \\
235 \\
240 \\
245 \\
250 \\
255 \\
260 \\
265 \\
270 \\
275 \\
280 \\
285 \\
290 \\
295 \\
300 \\
305 \\
310 \\
315 \\
320 \\
325 \\
330 \\
335 \\
340 \\
345 \\
350 \\
355 \\
360 \\
355 \\
370 \\
375 \\
380 \\
385 \\
390 \\
395 \\
400\end{array}$ & 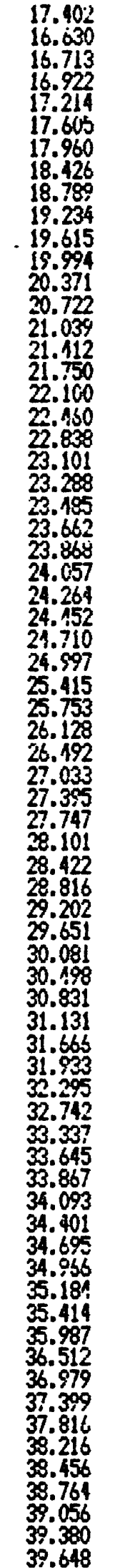 & $\begin{array}{l}405 \\
410 \\
415 \\
420 \\
425 \\
430 \\
435 \\
440 \\
445 \\
450 \\
455 \\
160 \\
470 \\
475 \\
480 \\
185 \\
490 \\
495 \\
500 \\
505 \\
510 \\
515 \\
520 \\
525 \\
530 \\
535 \\
540 \\
515 \\
550 \\
555 \\
560 \\
565 \\
570 \\
575 \\
580 \\
585 \\
590 \\
595 \\
600 \\
605 \\
610 \\
615 \\
620 \\
625 \\
630 \\
635\end{array}$ & $\begin{array}{l}40.035 \\
40.276 \\
40.540 \\
40.740 \\
11.050 \\
41.335 \\
41.567 \\
41.825 \\
42.263 \\
42.626 \\
42.876 \\
43.023 \\
43.459 \\
43.699 \\
43.930 \\
44.153 \\
44.38 ! \\
44.653 \\
44.973 \\
45.267 \\
45.677 \\
46.016 \\
46.294 \\
46.551 \\
46.957 \\
17.382 \\
47.734 \\
48.070 \\
48.392 \\
48.620 \\
49.042 \\
49.433 \\
49.798 \\
50.150 \\
50.505 \\
50.885 \\
51.213 \\
51.528 \\
51.802 \\
52.030 \\
52.355 \\
52.661 \\
52.058 \\
53.190 \\
53.699\end{array}$ \\
\hline
\end{tabular}


RRFT RIVER, IDAHO - 1.D. NO. 2 - 18 OCTOBER 1975

DEPTH TEPERATURE IEPTH TEPERATURE
(FT) (C)

\begin{tabular}{|c|c|}
\hline 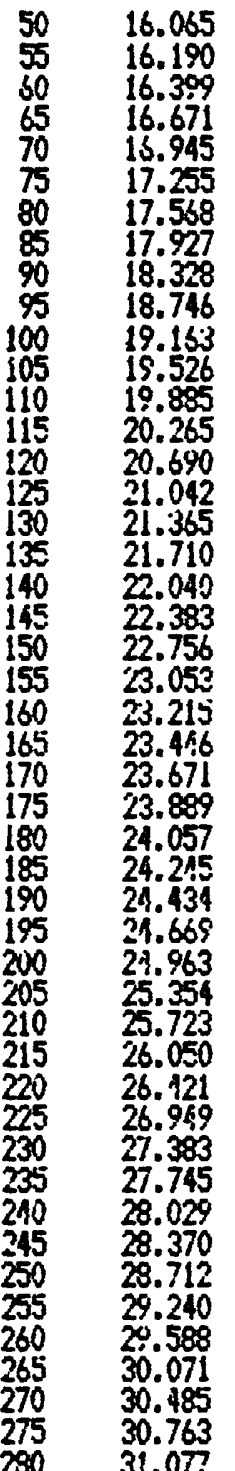 & $\begin{array}{l}400 \\
405 \\
410 \\
415 \\
420 \\
425 \\
430 \\
435 \\
440 \\
445 \\
450 \\
455 \\
460 \\
465 \\
770 \\
475 \\
430 \\
485 \\
490 \\
495 \\
500 \\
505 \\
510 \\
515 \\
520 \\
525 \\
530 \\
534 \\
540 \\
515 \\
550 \\
5555 \\
560 \\
565 \\
570 \\
575 \\
590 \\
585 \\
590 \\
595 \\
600 \\
605 \\
610 \\
615 \\
620 \\
625 \\
630\end{array}$ \\
\hline
\end{tabular}

$285 \quad 31.59$

$\% 00$

$300 \quad 32.732$

$305 \quad 33.253$

$310 \quad 33.597$

$315 \quad 33.834$

$320 \quad 34.083$

$325 \quad 34.374$

$330 \quad 34.665$

$335 \quad 34.936$

310

$350 \quad 35.806$

$355 \quad 36.387$

$360 \quad 36.918$

$365 \quad 37.311$

$370 \quad 37.720$

$375 \quad 38.107$

$380 \quad 38.379$

$385 \quad 38.659$

$390 \quad 38.935$

$395 \quad 39.234$ 
KAFT RIVER, IDAHD - 1.ע. KO. 2 - 15 JAWUARY 1976

\begin{tabular}{|c|c|c|}
\hline $\begin{array}{l}\text { DEPTH } \\
\text { (FT) }\end{array}$ & $\begin{array}{l}\text { TEAPERATUKE } \\
\text { (C) }\end{array}$ & $\begin{array}{c}\text { DEPTH } \\
\text { (FT) }\end{array}$ \\
\hline $\begin{array}{l}15 \\
50 \\
55 \\
60 \\
65 \\
70 \\
75 \\
80 \\
85 \\
90 \\
95 \\
100 \\
105 \\
110 \\
115 \\
120 \\
125 \\
130 \\
135 \\
140 \\
145 \\
150 \\
155 \\
160 \\
165 \\
170 \\
175 \\
180 \\
105 \\
190 \\
195 \\
200 \\
205 \\
210 \\
215 \\
220 \\
225 \\
230 \\
235 \\
210 \\
245 \\
250 \\
255 \\
260 \\
255 \\
270 \\
275 \\
280 \\
285 \\
290 \\
295 \\
300 \\
305 \\
310 \\
315 \\
320 \\
325 \\
330 \\
335 \\
340 \\
345 \\
350 \\
3555 \\
360 \\
365 \\
370 \\
375 \\
380 \\
335 \\
390\end{array}$ & $\begin{array}{l}15.818 \\
16.018 \\
16.305 \\
16.620 \\
13.921 \\
17.172 \\
17.181 \\
17.846 \\
18.185 \\
18.587 \\
18.098 \\
19.406 \\
19.760 \\
20.135 \\
20.485 \\
20.819 \\
21.211 \\
21.602 \\
21.934 \\
22.251 \\
22.559 \\
22.893 \\
23.124 \\
23.360 \\
23.582 \\
23.788 \\
21.029 \\
24.220 \\
24.407 \\
24.628 \\
21.907 \\
25.219 \\
25.577 \\
25.855 \\
26.226 \\
26.628 \\
27.044 \\
27.447 \\
27.857 \\
28.205 \\
28.560 \\
28.966 \\
29.373 \\
29.801 \\
30.209 \\
30.523 \\
30.879 \\
31.269 \\
31.840 \\
32.013 \\
32.428 \\
32.867 \\
33.503 \\
33.711 \\
33.937 \\
34.175 \\
34.995 \\
34.772 \\
35.086 \\
35.268 \\
35.421 \\
36.107 \\
36.607 \\
37.071 \\
37.499 \\
37.932 \\
38.263 \\
38.468 \\
38.751 \\
38.945\end{array}$ & $\begin{array}{l}395 \\
400 \\
405 \\
410 \\
415 \\
420 \\
425 \\
130 \\
135 \\
140 \\
445 \\
450 \\
455 \\
460 \\
465 \\
470 \\
475 \\
180 \\
485 \\
490 \\
495 \\
500 \\
505 \\
510 \\
515 \\
520 \\
525 \\
530 \\
535 \\
540 \\
545 \\
550 \\
550 \\
560 \\
545 \\
570 \\
575 \\
580 \\
585 \\
590 \\
595 \\
600 \\
605 \\
610 \\
615 \\
620 \\
625 \\
630 \\
635 \\
640\end{array}$ \\
\hline
\end{tabular}


PAFT RIVER, IDNHO - I.D. NO. 3 - 8 DECEMBER 1STA

\begin{tabular}{|c|c|c|c|c|c|}
\hline $\begin{array}{l}\text { DEPTH } \\
\text { (FT) }\end{array}$ & $\begin{array}{l}\text { TEPERATURE } \\
\text { (C) }\end{array}$ & $\begin{array}{l}\text { DEPTH } \\
(F T)\end{array}$ & $\begin{array}{l}\text { TEPERITLRE } \\
\text { (C) }\end{array}$ & $\begin{array}{l}\text { DEPFH } \\
\text { (FT) }\end{array}$ & $\begin{array}{c}\text { TEXPERMTU } \\
\text { (C) }\end{array}$ \\
\hline $\begin{array}{l}5 \\
10 \\
15 \\
20 \\
25 \\
30 \\
35 \\
10 \\
45 \\
50 \\
55 \\
60 \\
65 \\
70 \\
75 \\
160 \\
165 \\
170 \\
175 \\
180 \\
185 \\
190 \\
195 \\
200 \\
205 \\
210 \\
215 \\
220 \\
225 \\
230 \\
235 \\
240 \\
245 \\
250 \\
255 \\
260 \\
265 \\
270 \\
275 \\
280 \\
295 \\
290 \\
295 \\
300 \\
305 \\
310 \\
315 \\
320 \\
325 \\
330 \\
335 \\
340 \\
350 \\
360 \\
370 \\
386 \\
390 \\
400 \\
410 \\
420 \\
330 \\
440 \\
450 \\
460 \\
170 \\
480 \\
490 \\
500 \\
510 \\
520\end{array}$ & 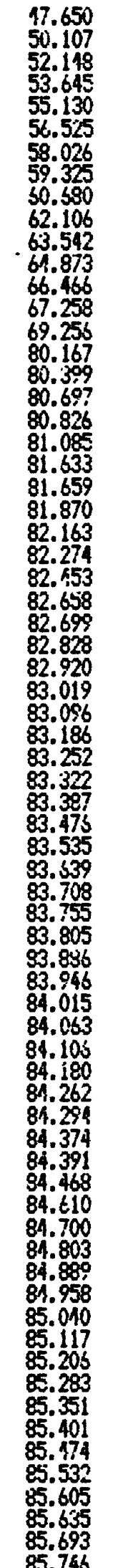 & $\begin{array}{l}530 \\
540 \\
550 \\
560 \\
570 \\
580 \\
590 \\
600 \\
610 \\
620 \\
630 \\
640 \\
650 \\
660 \\
670 \\
680 \\
690 \\
700 \\
710 \\
720 \\
730 \\
740 \\
750 \\
760 \\
770 \\
780 \\
790 \\
800 \\
810 \\
820 \\
830 \\
810 \\
850 \\
860 \\
870 \\
880 \\
890 \\
900 \\
910 \\
920 \\
930 \\
940 \\
950 \\
960 \\
990 \\
980 \\
990 \\
1000 \\
1010 \\
1020 \\
1030 \\
1040 \\
1050 \\
1060 \\
1070 \\
1000 \\
1090 \\
1100 \\
1110 \\
1120 \\
1130 \\
1140 \\
1115 \\
1150 \\
1155 \\
1160 \\
1155 \\
1170 \\
1175 \\
1180\end{array}$ & 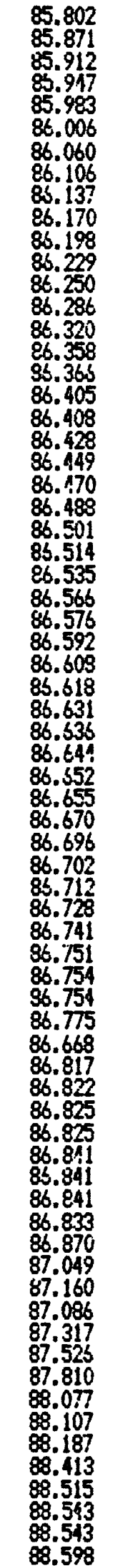 & $\begin{array}{l}1185 \\
1190 \\
1195 \\
1200 \\
1205 \\
1210 \\
1215 \\
1220 \\
1225 \\
1230 \\
1235 \\
1240 \\
1245 \\
1250 \\
1255 \\
1260 \\
1265 \\
1270\end{array}$ & $\begin{array}{l}88.601 \\
88.598 \\
89.507 \\
88.794 \\
88.953 \\
88.996 \\
89.033 \\
89.150 \\
89.044 \\
89.070 \\
89.104 \\
89.106 \\
89.118 \\
89.123 \\
89.126 \\
89.132 \\
89.135 \\
89.135\end{array}$ \\
\hline
\end{tabular}


RATT RIVER, IDAHO - I.D. HO. 3 - 12 JANUARY 1975

\begin{tabular}{|c|c|}
\hline $\begin{array}{l}\text { IEPTH } \\
\text { (FT) }\end{array}$ & $\begin{array}{l}\text { TERPERATU } \\
\text { (C) }\end{array}$ \\
\hline $\begin{array}{l}5 \\
50 \\
100 \\
150 \\
200 \\
250 \\
300 \\
350 \\
400 \\
450 \\
500 \\
450 \\
600 \\
650 \\
700 \\
7150 \\
800 \\
250 \\
900 \\
950 \\
1000 \\
1010 \\
1020 \\
1030 \\
1040 \\
1050 \\
1050 \\
1070 \\
1080 \\
1090 \\
1100 \\
1110 \\
1120 \\
1130 \\
1140 \\
1150 \\
1100 \\
1170 \\
1180 \\
1190 \\
1200 \\
1210 \\
1220 \\
1230 \\
1240 \\
1250 \\
1260 \\
1270 \\
1280 \\
1290 \\
1300 \\
1310 \\
1320 \\
1330 \\
1340 \\
1350 \\
1360 \\
1370 \\
1380 \\
1390 \\
1400 \\
1410 \\
1420 \\
1426\end{array}$ & 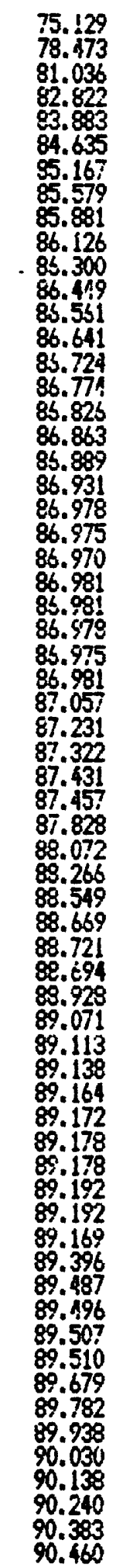 \\
\hline
\end{tabular}


RAFT RIVER, IDAHO - I.D. KO. 3 - 31 MAPCH 1975

\begin{tabular}{|c|c|c|c|}
\hline $\begin{array}{l}\text { DEPTH } \\
\text { (FT) }\end{array}$ & $\begin{array}{l}\text { TEPPERAIUPE } \\
\text { (C) }\end{array}$ & $\begin{array}{l}\text { DEPTH } \\
\text { (FT) }\end{array}$ & $\begin{array}{l}\text { TEPPERATU } \\
\text { (C) }\end{array}$ \\
\hline $\begin{array}{l}35 \\
10 \\
15 \\
50 \\
555 \\
60 \\
65 \\
70 \\
75 \\
80 \\
895 \\
90 \\
95 \\
100 \\
105 \\
110 \\
115 \\
120 \\
125 \\
130 \\
135 \\
140 \\
145 \\
150 \\
155 \\
160 \\
155 \\
170 \\
175 \\
180 \\
185 \\
190 \\
195 \\
200 \\
205 \\
210 \\
215 \\
220 \\
225 \\
230 \\
235 \\
240 \\
245 \\
250 \\
255 \\
260 \\
265 \\
270 \\
275 \\
200 \\
285 \\
290 \\
295 \\
300 \\
305 \\
310 \\
315 \\
320 \\
325 \\
3300 \\
335 \\
340 \\
345 \\
350 \\
3555 \\
360 \\
365 \\
370 \\
375 \\
200\end{array}$ & 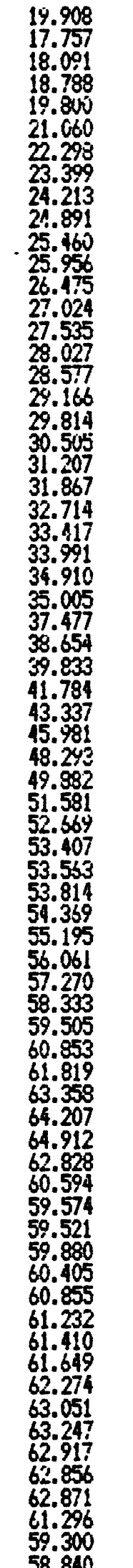 & $\begin{array}{l}385 \\
390 \\
305 \\
398\end{array}$ & $\begin{array}{l}58.900 \\
59.161 \\
59.433 \\
60.136\end{array}$ \\
\hline
\end{tabular}


RIFT RIVER, IDAHO - I.D. NO. 3 - 30 NLGLST 1975

\begin{tabular}{|c|c|c|c|c|c|c|c|}
\hline $\begin{array}{l}\text { DEPTH } \\
\text { (FT) }\end{array}$ & $\begin{array}{l}\text { TEPPERATURE } \\
\text { (C) }\end{array}$ & $\begin{array}{l}\text { DEPTH } \\
\text { (FT) }\end{array}$ & $\begin{array}{l}\text { TEMPERATURE } \\
\text { (C) }\end{array}$ & $\begin{array}{l}\text { DEPTH } \\
\text { (FT) }\end{array}$ & $\begin{array}{l}\text { TEYPERATURE } \\
\text { (C) }\end{array}$ & $\begin{array}{l}\text { DEPTH } \\
\text { (FT) }\end{array}$ & $\begin{array}{l}\text { TERERATLRE } \\
\text { (C) }\end{array}$ \\
\hline $\begin{array}{l}5 \\
10 \\
15 \\
22 \\
25 \\
30 \\
35 \\
10 \\
45 \\
50 \\
555 \\
60 \\
65 \\
70 \\
75 \\
80 \\
85 \\
90 \\
95 \\
100 \\
105 \\
110 \\
115 \\
120 \\
125 \\
130 \\
135 \\
140 \\
145 \\
150 \\
155 \\
160 \\
165 \\
170 \\
175 \\
180 \\
185 \\
190 \\
195 \\
200 \\
205 \\
210 \\
215 \\
220 \\
225 \\
220 \\
235 \\
240 \\
245 \\
250 \\
255 \\
260 \\
265 \\
270 \\
275 \\
280 \\
265 \\
290 \\
295 \\
300 \\
305 \\
310 \\
315 \\
320 \\
325 \\
330 \\
335 \\
340 \\
345 \\
350\end{array}$ & 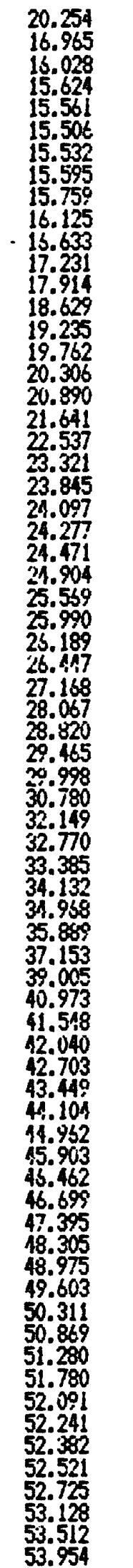 & $\begin{array}{l}355 \\
360 \\
365 \\
370 \\
375 \\
390 \\
385 \\
390 \\
395 \\
400 \\
405 \\
410 \\
415 \\
420 \\
425 \\
430 \\
435 \\
440 \\
455 \\
150 \\
155 \\
460 \\
465 \\
470 \\
175 \\
480 \\
485 \\
490 \\
495 \\
500 \\
505 \\
510 \\
515 \\
520 \\
575 \\
530 \\
555 \\
540 \\
545 \\
5550 \\
555 \\
560 \\
565 \\
570 \\
555 \\
580 \\
595 \\
590 \\
595 \\
600 \\
610 \\
615 \\
620 \\
625 \\
630 \\
635 \\
540 \\
645 \\
650 \\
655 \\
650 \\
665 \\
670 \\
675 \\
680 \\
655 \\
390 \\
695 \\
700 \\
705\end{array}$ & 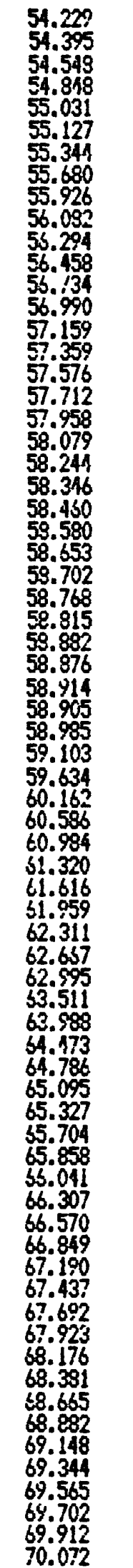 & $\begin{array}{l}710 \\
715 \\
720 \\
725 \\
730 \\
735 \\
740 \\
745 \\
750 \\
755 \\
760 \\
765 \\
770 \\
775 \\
780 \\
785 \\
790 \\
795 \\
800 \\
805 \\
810 \\
815 \\
820 \\
9255 \\
830 \\
835 \\
840 \\
815 \\
850 \\
855 \\
960 \\
865 \\
870 \\
875 \\
880 \\
885 \\
890 \\
895 \\
900 \\
905 \\
910 \\
915 \\
920 \\
925 \\
930 \\
935 \\
940 \\
915 \\
950 \\
955 \\
960 \\
965 \\
970 \\
975 \\
980 \\
9855 \\
990 \\
995 \\
1000 \\
1005 \\
1010 \\
1015 \\
1020 \\
1025 \\
1030 \\
1005 \\
1040 \\
1045 \\
1050 \\
1055\end{array}$ & $\begin{array}{l}70.289 \\
70.579 \\
70.831 \\
71.099 \\
71.375 \\
71.729 \\
72.038 \\
72.481 \\
72.954 \\
73.524 \\
74.134 \\
74.708 \\
75.130 \\
75.160 \\
75.731 \\
76.017 \\
75.374 \\
76.732 \\
76.927 \\
77.082 \\
77.173 \\
77.212 \\
77.197 \\
77.133 \\
77.052 \\
77.026 \\
77.048 \\
77.096 \\
77.191 \\
77.342 \\
77.521 \\
77.706 \\
77.914 \\
78.123 \\
78.307 \\
78.768 \\
78.488 \\
78.493 \\
79.357 \\
80.051 \\
81.845 \\
82.502 \\
82.715 \\
82.904 \\
83.181 \\
83.772 \\
83.837 \\
84.124 \\
94.445 \\
84.746 \\
85.241 \\
85.16 t \\
85.763 \\
85.948 \\
85.084 \\
86.186 \\
85.309 \\
86.380 \\
85.453 \\
85.527 \\
86.7355 \\
86.967 \\
87.203 \\
87.368 \\
87.549 \\
87.727 \\
87.895 \\
88.078 \\
89.252 \\
83.741\end{array}$ & $\begin{array}{l}1050 \\
1065 \\
1070 \\
1075\end{array}$ & $\begin{array}{l}88.760 \\
88.882 \\
88.900 \\
88.954\end{array}$ \\
\hline
\end{tabular}


RAFT RIVER, IDAHD - 1.D. NO. 3 - 11 AUEUST 1976

DEPTH TEPERGTURE DEPTH TEMPERGTLRE DEPTH TEMERATLRE DEPTH TEPPERATLFE DEPTH TEFPERATURE DEPTH TEYPERATLRE
(FT) (C)

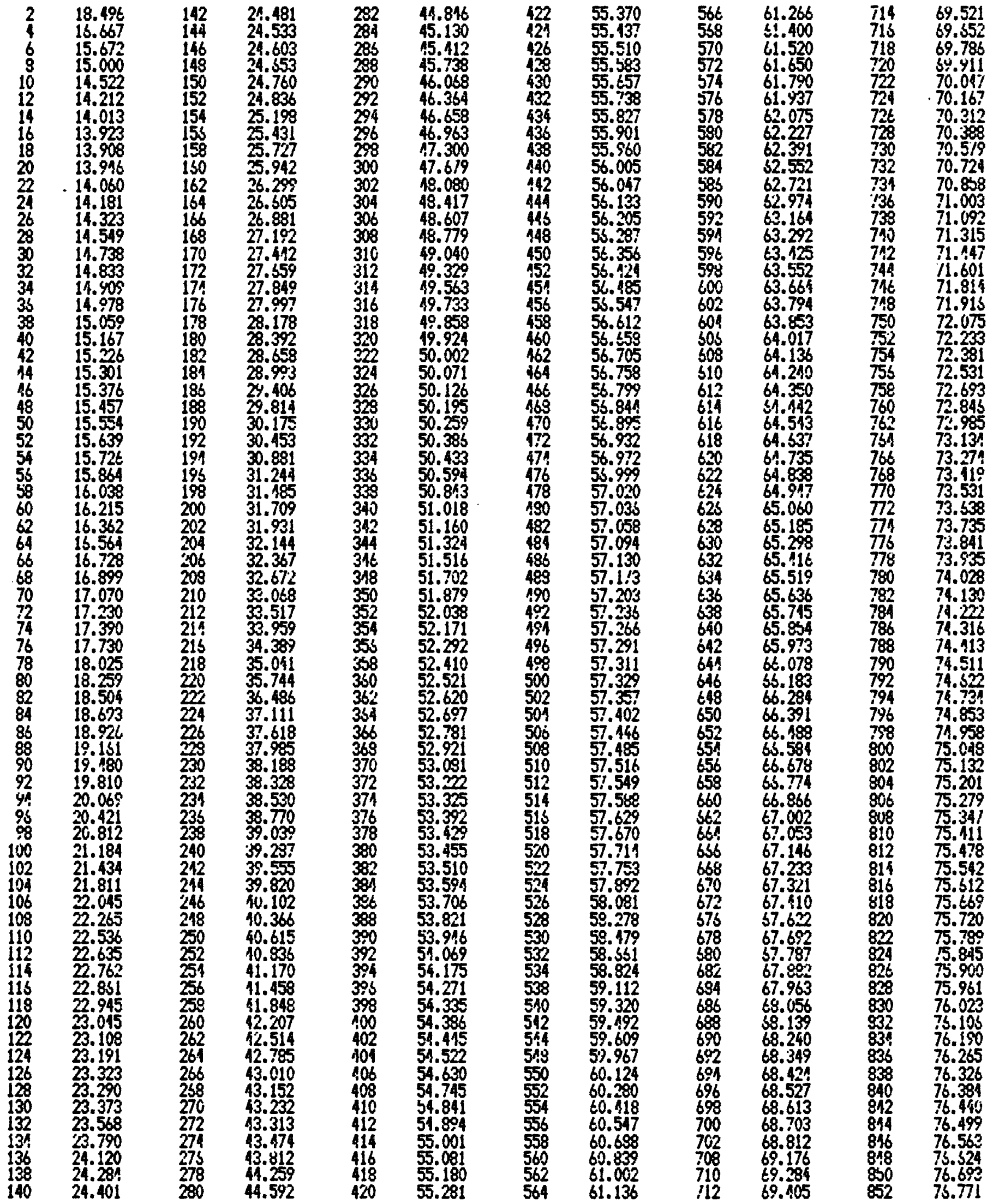


RAFT RIVER, IDAHO - I.D. HO. 3 - 11 NUGUST 1975

\begin{tabular}{|c|c|c|}
\hline $\begin{array}{l}\text { DEPTH } \\
\text { (FT) }\end{array}$ & $\begin{array}{l}\text { TEPERATLRE } \\
\text { (C) }\end{array}$ & $\begin{array}{l}\text { DEPTH } \\
\text { (FT) }\end{array}$ \\
\hline 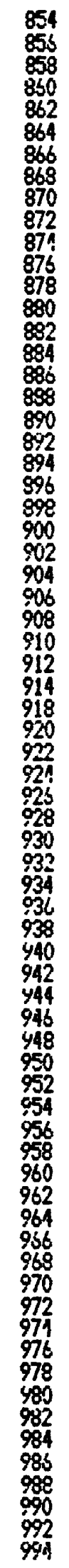 & 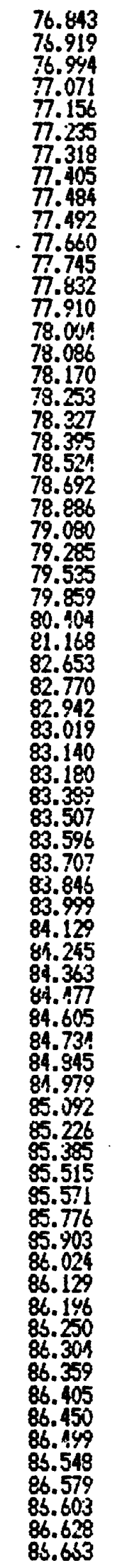 & $\begin{array}{c}996 \\
908 \\
1000 \\
1002 \\
1004 \\
1006 \\
1008 \\
1010 \\
1012 \\
1014 \\
1016 \\
1018 \\
1020 \\
1022 \\
1024 \\
1026 \\
1028 \\
1030 \\
1032 \\
1034 \\
1024 \\
1039 \\
1040 \\
1012 \\
1044 \\
1046 \\
1048 \\
1050 \\
1052 \\
1054 \\
1056 \\
1058 \\
1060 \\
1052 \\
1061 \\
1056 \\
1068 \\
1070 \\
1072\end{array}$ \\
\hline
\end{tabular}


RAFT RIVER, IDAHO - 1.D. M. 4 - 12 JAHULEY 1975

$\begin{array}{cc}\begin{array}{c}\text { DEPTH } \\ \text { (FT) }\end{array} & \begin{array}{c}\text { TEFERTITU } \\ \text { (C) }\end{array} \\ 20 & 35.141 \\ 25 & 36.545 \\ 30 & 37.589 \\ 35 & 38.605 \\ 40 & 39.478 \\ 45 & 40.311 \\ 50 & 40.712 \\ 55 & 41.111 \\ 60 & 41.110 \\ 65 & 41.657 \\ 70 & 11.768 \\ 75 & 41.753 \\ 80 & 11.723 \\ 85 & 41.844 \\ 90 & 12.046 \\ 95 & 42.235 \\ 100 & 12.260 \\ 105 & 42.216 \\ 105 & 42.172\end{array}$


RAT RIVER, IDAHD - I.D. NO. A - 31 MARCH 1975

\begin{tabular}{|c|c|}
\hline $\begin{array}{l}\text { DEPTH } \\
\text { (FT) }\end{array}$ & $\begin{array}{l}\text { TEMPERATLRE } \\
\text { (C) }\end{array}$ \\
\hline $\begin{array}{l}20 \\
25 \\
30 \\
35 \\
40 \\
45 \\
50 \\
55 \\
60 \\
65 \\
70 \\
75 \\
80 \\
85 \\
90 \\
95 \\
100 \\
105 \\
110 \\
115 \\
120 \\
125 \\
130 \\
135 \\
140 \\
145 \\
150 \\
155 \\
150 \\
165 \\
170 \\
175 \\
180 \\
185 \\
190 \\
195 \\
200 \\
205 \\
210 \\
215 \\
220 \\
221\end{array}$ & $\begin{array}{l}35.256 \\
36.315 \\
37.403 \\
38.359 \\
39.695 \\
40.431 \\
11.092 \\
41.407 \\
41.601 \\
41.710 \\
41.746 \\
41.731 \\
41.683 \\
41.657 \\
41.506 \\
41.125 \\
40.995 \\
40.131 \\
39.677 \\
39.219 \\
38.811 \\
38.446 \\
39.090 \\
37.700 \\
37.440 \\
37.238 \\
35.987 \\
36.658 \\
36.362 \\
36.112 \\
35.922 \\
35.531 \\
35.333 \\
35.240 \\
34.997 \\
34.553 \\
34.413 \\
34.209 \\
33.985 \\
33.718 \\
33.443 \\
33.400\end{array}$ \\
\hline
\end{tabular}

III-6I 
RGFT RIVER, IDAHO - I.D. MO. 4 - 18 OCTOBER 1975

\section{DEPTH TEMPERATURE}

(FT) (C)

$\begin{array}{cc}20 & 30.503 \\ 25 & 35.917 \\ 30 & 37.106 \\ 35 & 33.235 \\ 10 & 3 \% .326 \\ 15 & 40.211 \\ 50 & 40.964 \\ 55 & 41.143 \\ 50 & 11.747 \\ 65 & 41.937 \\ 70 & 42.063 \\ 75 & 42.117 \\ 80 & 42.123 \\ 85 & 42.090 \\ 90 & 42.028 \\ 95 & 41.952 \\ 100 & 41.986 \\ 105 & 41.714 \\ 110 & 41.412 \\ 115 & 11.027 \\ 120 & 40.579 \\ 125 & 40.079 \\ 130 & 39.657 \\ 135 & 39.262 \\ 140 & 38.771 \\ 145 & 38.263 \\ 150 & 37.835 \\ 155 & 37.418 \\ 150 & 37.084 \\ 165 & 36.736 \\ 170 & 36.413 \\ 175 & 33.110 \\ 180 & 35.793 \\ 185 & 35.506 \\ 190 & 35.208\end{array}$


RAFT RIVER, IDAHO - !.D. NO. A - 14 JAHUAYY 1976

DEPTH TEMPERATUPE

(FT) (C)

$\begin{array}{ll}20 & 35.453 \\ 25 & 36.608 \\ 30 & 37.498 \\ 35 & 33.636 \\ 50 & 33.749 \\ 55 & 40.665 \\ 50 & 41.326 \\ 55 & 41.662 \\ 60 & 41.960 \\ 65 & 52.134 \\ 70 & 42.237 \\ 75 & 42.288 \\ 80 & 42.309 \\ 85 & 42.298 \\ 90 & 42.247 \\ 95 & 42.130 \\ 100 & 42.015 \\ 105 & 41.801 \\ 110 & 41.496 \\ 115 & 41.107 \\ 120 & 40.635 \\ 125 & 40.185 \\ 130 & 39.775 \\ 135 & 39.384 \\ 140 & 33.862 \\ 145 & 33.358 \\ 150 & 37.938 \\ 155 & 37.519 \\ 160 & 37.107 \\ 165 & 36.725 \\ 170 & 36.406 \\ 175 & 36.053 \\ 180 & 35.750 \\ 181 & 35.458\end{array}$


RAFT RIVER, IOAHD - I.D. NO. 4 - 8 FEBRUAARY 1976

BEPTH TETERITURE

(FT) (C)

$\begin{array}{ll}20 & 35.299 \\ 25 & 36.497 \\ 30 & 37.613 \\ 35 & 33.758 \\ 40 & 39.940 \\ 15 & 40.764 \\ 50 & 41.323 \\ 55 & 41.826 \\ 30 & 12.120 \\ 65 & 42.283 \\ 70 & 42.396 \\ 75 & 42.410 \\ 80 & 12.470 \\ 85 & 42.159 \\ 90 & 42.391 \\ 95 & 42.282 \\ 100 & 42.153 \\ 105 & 41.917 \\ 110 & 41.621 \\ 115 & 11.226 \\ 120 & 40.775 \\ 125 & 40.296 \\ 130 & 39.996 \\ 135 & 39.512 \\ 140 & 38.455 \\ 145 & 38.435 \\ 150 & 38.003 \\ 155 & 37.591 \\ 150 & 37.188 \\ 165 & 36.773 \\ 170 & 36.427 \\ 175 & 36.079 \\ 180 & 35.733 \\ 185 & 35.426 \\ 126 & 35.403 \\ 15 & \end{array}$


PAFT RIVER, IDAHO - I.D. NO. 4 - 14 MARCH 1976

DEPTH TEMPERATLRE

(FT) (C)

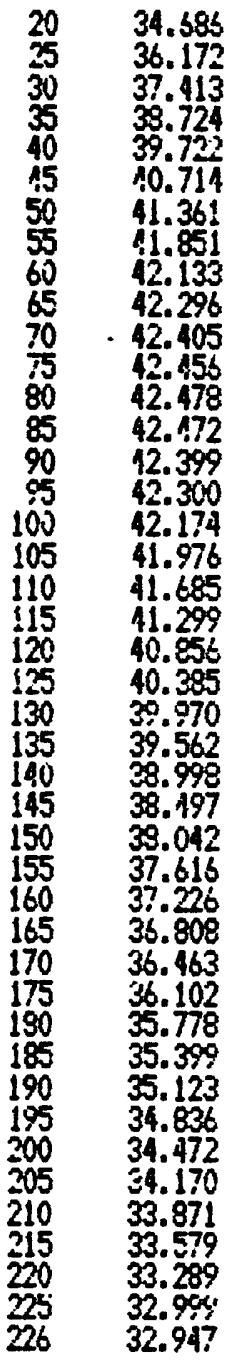


RAFT RIVER, IDAHO - I.U. N. 4 - 8 N MUGST 1976

\begin{tabular}{|c|c|c|c|}
\hline $\begin{array}{l}\text { DEPTH } \\
\text { (FT) }\end{array}$ & $\begin{array}{l}\text { TEMPERATURE } \\
\text { (C) }\end{array}$ & $\begin{array}{l}\text { DEPTH } \\
\text { (FT) }\end{array}$ & $\begin{array}{l}\text { TENPERATL } \\
\text { (C) }\end{array}$ \\
\hline 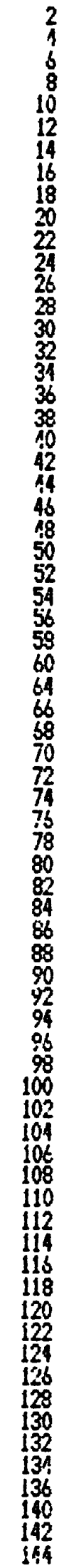 & $\begin{array}{l}27.332 \\
27.354 \\
27.376 \\
27.391 \\
27.422 \\
27.419 \\
27.484 \\
27.533 \\
27.728 \\
33.762 \\
35.085 \\
35.618 \\
36.043 \\
36.517 \\
36.951 \\
37.440 \\
31.944 \\
38.401 \\
38.953 \\
39.375 \\
39.857 \\
40.271 \\
40.621 \\
40.929 \\
41.183 \\
41.405 \\
41.584 \\
41.805 \\
41.951 \\
42.090 \\
42.249 \\
42.366 \\
42.355 \\
42.414 \\
42.140 \\
42.469 \\
42.484 \\
42.195 \\
42.502 \\
42.507 \\
42.505 \\
12.494 \\
42.470 \\
42.444 \\
42.115 \\
42.375 \\
42.324 \\
12.265 \\
42.212 \\
42.148 \\
42.085 \\
42.000 \\
41.889 \\
41.764 \\
41.615 \\
41.976 \\
41.322 \\
41.149 \\
41.024 \\
40.810 \\
10.507 \\
40.426 \\
40.295 \\
40.103 \\
39.940 \\
39.797 \\
39.313 \\
38.182 \\
38.710\end{array}$ & $\begin{array}{l}118 \\
150 \\
152 \\
151 \\
156 \\
158 \\
150 \\
162 \\
134 \\
166 \\
168 \\
170 \\
172 \\
174 \\
176 \\
178 \\
180 \\
182 \\
184 \\
186 \\
128 \\
190 \\
192 \\
194 \\
196 \\
198 \\
200 \\
202 \\
204 \\
206 \\
209 \\
210 \\
212 \\
214 \\
216 \\
218 \\
220 \\
222 \\
224 \\
226 \\
228 \\
230 \\
232 \\
236 \\
238\end{array}$ & $\begin{array}{l}38.329 \\
38.155 \\
37.964 \\
37.786 \\
37.519 \\
37.454 \\
37.339 \\
37.123 \\
37.003 \\
36.921 \\
33.841 \\
36.710 \\
36.600 \\
36.445 \\
35.292 \\
35.220 \\
36.190 \\
36.102 \\
35.755 \\
35.570 \\
35.438 \\
35.225 \\
35.135 \\
35.010 \\
34.875 \\
34.842 \\
34.840 \\
34.779 \\
34.673 \\
34.511 \\
31.129 \\
34.347 \\
34.226 \\
34.061 \\
33.909 \\
33.804 \\
33.645 \\
33.503 \\
33.391 \\
33.291 \\
33.174 \\
33.075 \\
33.012 \\
32.785 \\
32.557\end{array}$ \\
\hline
\end{tabular}


RAFT RIVER, IDA40 - I.D. NO. 5 - 1 APRIL 1975

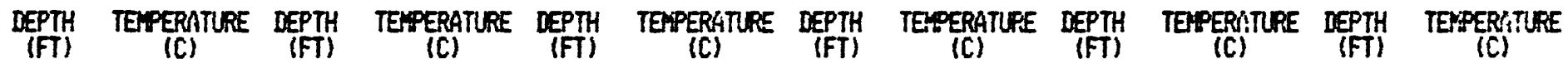

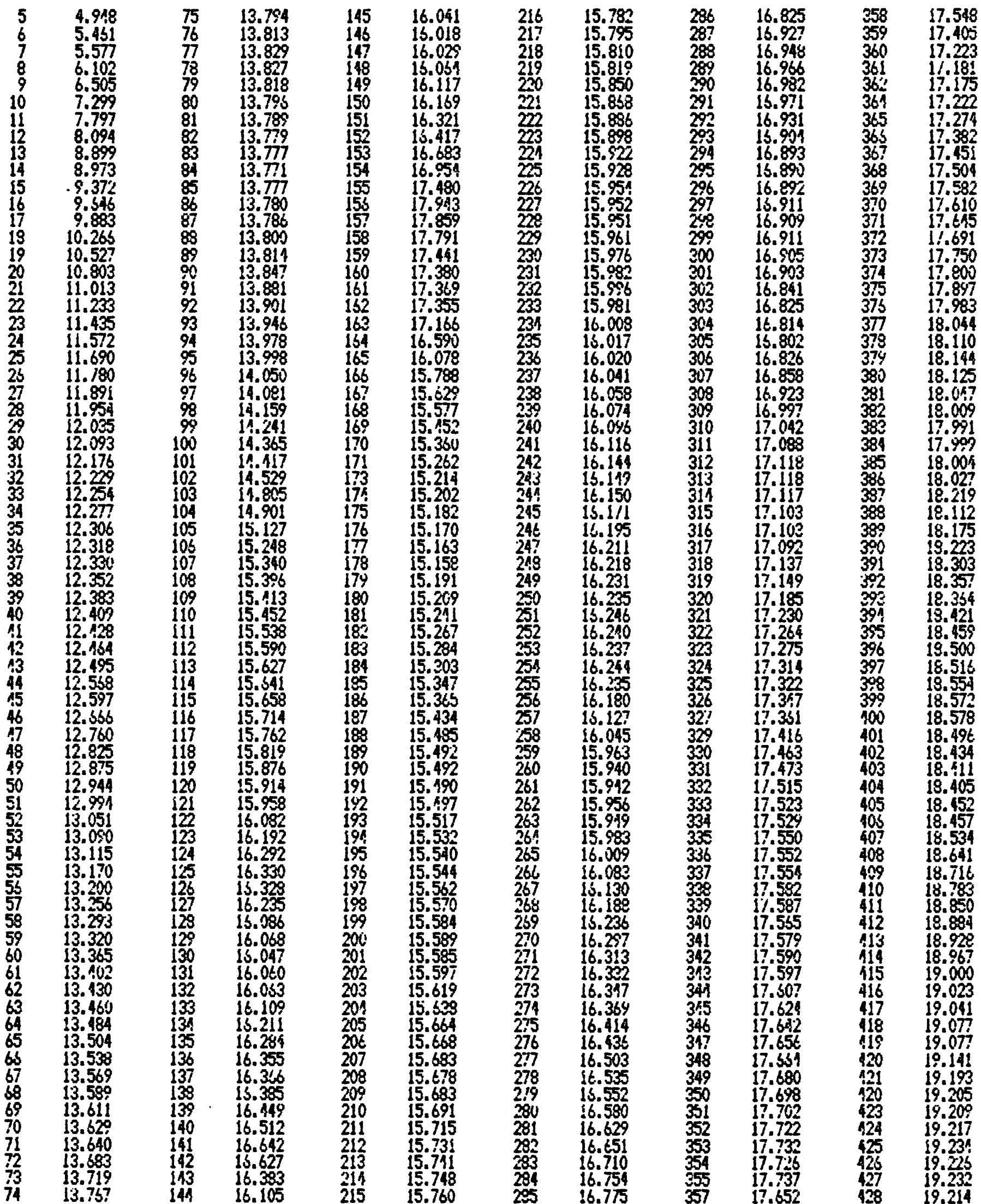


RAFT RIVER, IDSHO - I.D. NO. 5 - 1 APRIL 1975

\begin{tabular}{|c|c|c|c|c|c|c|c|}
\hline $\begin{array}{l}\text { DEPTH } \\
\text { (FT) }\end{array}$ & $\begin{array}{l}\text { TEWPERATURE } \\
\text { (C) }\end{array}$ & $\begin{array}{l}\text { DEPTH } \\
\text { (FT) }\end{array}$ & $\begin{array}{l}\text { TERPERATURE } \\
\text { (C) }\end{array}$ & $\begin{array}{l}\text { DEPTH } \\
\text { (FT) }\end{array}$ & $\begin{array}{l}\text { TEMPERATLRE } \\
\text { (C) }\end{array}$ & $\begin{array}{l}\text { DEFTH } \\
\text { (FT) }\end{array}$ & $\begin{array}{l}\text { TEMPERATUR } \\
\text { (C) }\end{array}$ \\
\hline 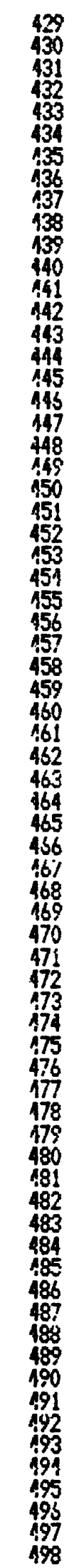 & $\begin{array}{l}19.252 \\
19.300 \\
10.307 \\
10.329 \\
19.261 \\
19.384 \\
19.107 \\
19.415 \\
19.457 \\
19.164 \\
19.495 \\
19.514 \\
19.520 \\
19.533 \\
19.570 \\
19.599 \\
19.609 \\
19.623 \\
19.617 \\
19.521 \\
19.659 \\
19.631 \\
19.692 \\
19.670 \\
19.720 \\
19.746 \\
19.761 \\
10.783 \\
19.791 \\
19.805 \\
19.819 \\
19.860 \\
19.858 \\
10.903 \\
19.911 \\
19.934 \\
19.941 \\
10.995 \\
19.979 \\
19.998 \\
10.990 \\
20.038 \\
20.038 \\
20.087 \\
20.094 \\
20.078 \\
20.199 \\
20.159 \\
20.185 \\
20.166 \\
20.206 \\
20.217 \\
20.237 \\
20.272 \\
20.256 \\
20.303 \\
20.238 \\
20.301 \\
20.316 \\
20.365 \\
20.350 \\
20.397 \\
20.410 \\
20.436 \\
20.451 \\
20.455 \\
20.428 \\
20.473 \\
20.482 \\
20.509\end{array}$ & $\begin{array}{l}499 \\
500 \\
501 \\
502 \\
503 \\
504 \\
505 \\
505 \\
507 \\
508 \\
509 \\
510 \\
511 \\
512 \\
513 \\
514 \\
515 \\
516 \\
517 \\
519 \\
519 \\
520 \\
521 \\
522 \\
523 \\
524 \\
525 \\
526 \\
527 \\
528 \\
529 \\
530 \\
521 \\
532 \\
533 \\
534 \\
535 \\
535 \\
537 \\
538 \\
539 \\
540 \\
541 \\
542 \\
513 \\
514 \\
545 \\
515 \\
547 \\
548 \\
549 \\
550 \\
551 \\
552 \\
553 \\
554 \\
555 \\
556 \\
557 \\
558 \\
559 \\
560 \\
561 \\
552 \\
563 \\
554 \\
565 \\
556 \\
567 \\
548\end{array}$ & 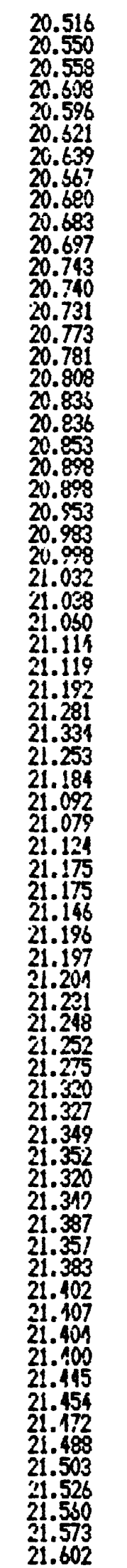 & 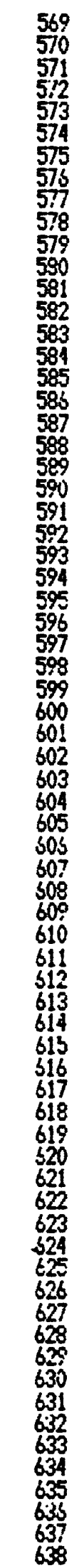 & 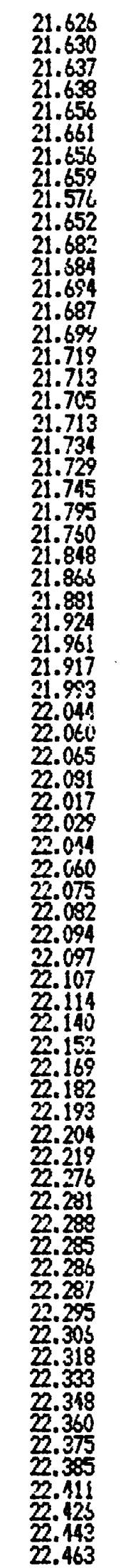 & $\begin{array}{l}639 \\
540 \\
641 \\
642 \\
643 \\
314 \\
645 \\
545 \\
647 \\
548 \\
649 \\
650 \\
651 \\
352 \\
653 \\
651 \\
655 \\
656 \\
657 \\
358 \\
659 \\
650 \\
661 \\
662 \\
663 \\
654 \\
665 \\
666 \\
667 \\
668 \\
669 \\
670 \\
671 \\
312 \\
673 \\
674 \\
675 \\
676 \\
677 \\
678 \\
679 \\
380 \\
681 \\
682 \\
693 \\
584 \\
685 \\
595 \\
687 \\
698 \\
699 \\
690 \\
691 \\
692 \\
693 \\
691 \\
695 \\
696 \\
697 \\
698 \\
699 \\
700 \\
701 \\
702\end{array}$ & $\begin{array}{l}22.465 \\
22.497 \\
22.195 \\
22.509 \\
22.531 \\
22.551 \\
22.568 \\
22.576 \\
22.596 \\
22.509 \\
22.617 \\
22.527 \\
22.639 \\
22.566 \\
22.690 \\
22.587 \\
22.701 \\
22.704 \\
22.705 \\
22.711 \\
22.710 \\
22.724 \\
22.726 \\
22.731 \\
22.757 \\
22.772 \\
22.798 \\
22.839 \\
22.865 \\
22.029 \\
22.948 \\
23.009 \\
23.022 \\
23.021 \\
23.017 \\
23.011 \\
22.005 \\
23.005 \\
23.015 \\
23.016 \\
23.027 \\
23.040 \\
23.050 \\
23.051 \\
23.065 \\
23.068 \\
23.073 \\
23.083 \\
23.085 \\
23.093 \\
22.096 \\
23.099 \\
23.104 \\
23.104 \\
23.101 \\
23.102 \\
23.104 \\
23.104 \\
23.104 \\
23.103 \\
23.105 \\
23.099 \\
23.101 \\
23.139\end{array}$ \\
\hline
\end{tabular}


RAFT RIVER, IDAHO - I.D. NO. 5 - 25 APRIL 1975

\begin{tabular}{|c|c|c|c|}
\hline $\begin{array}{l}\text { DEPTH } \\
\text { (FT) }\end{array}$ & $\begin{array}{l}\text { TERPERATURE } \\
\text { (C) }\end{array}$ & $\begin{array}{l}\text { DEPTH } \\
\text { (FT) }\end{array}$ & $\begin{array}{l}\text { TEPEERATU } \\
\text { (C) }\end{array}$ \\
\hline $\begin{array}{l}10 \\
25 \\
30 \\
35 \\
40 \\
45 \\
50 \\
55 \\
60 \\
65 \\
70 \\
75 \\
80 \\
85 \\
90 \\
95 \\
100 \\
105 \\
110 \\
115 \\
120 \\
125 \\
130 \\
135 \\
140 \\
145 \\
150 \\
155 \\
160 \\
165 \\
170 \\
175 \\
180 \\
185 \\
190 \\
195 \\
200 \\
205 \\
210 \\
215 \\
220 \\
225 \\
230 \\
235 \\
240 \\
245 \\
250 \\
255 \\
260 \\
265 \\
270 \\
275 \\
280 \\
225 \\
290 \\
205 \\
300 \\
305 \\
310 \\
315 \\
320 \\
325 \\
330 \\
335 \\
340 \\
315 \\
350 \\
355 \\
360 \\
365\end{array}$ & $\begin{array}{l}10.462 \\
11.221 \\
11.708 \\
12.006 \\
12.136 \\
12.242 \\
12.367 \\
12.512 \\
12.820 \\
12.951 \\
13.057 \\
13.071 \\
13.159 \\
13.268 \\
13.393 \\
13.526 \\
13.545 \\
13.814 \\
13.950 \\
11.012 \\
11.105 \\
14.204 \\
14.273 \\
14.370 \\
14.472 \\
14.551 \\
11.659 \\
14.797 \\
14.915 \\
14.871 \\
14.853 \\
14.896 \\
14.933 \\
15.021 \\
15.099 \\
15.173 \\
15.254 \\
15.341 \\
15.416 \\
15.520 \\
15.599 \\
15.683 \\
15.755 \\
15.864 \\
15.956 \\
16.020 \\
13.063 \\
16.055 \\
16.015 \\
16.096 \\
15.246 \\
16.407 \\
16.577 \\
16.730 \\
16.850 \\
16.924 \\
17.019 \\
17.102 \\
17.205 \\
17.293 \\
17.388 \\
17.479 \\
17.793 \\
17.449 \\
17.719 \\
17.790 \\
17.842 \\
17.866 \\
17.972 \\
17.937\end{array}$ & $\begin{array}{l}370 \\
375 \\
390 \\
385 \\
390 \\
395 \\
400 \\
405 \\
110 \\
115 \\
420 \\
4255 \\
430 \\
435 \\
440 \\
455 \\
450 \\
455 \\
450 \\
465 \\
470 \\
475 \\
490 \\
485 \\
490 \\
495 \\
500 \\
505 \\
510 \\
515 \\
520 \\
525 \\
530 \\
5355 \\
510 \\
515 \\
550 \\
555 \\
560 \\
565 \\
570 \\
575 \\
580 \\
585 \\
590 \\
595 \\
600 \\
605 \\
610 \\
615 \\
620 \\
625 \\
630 \\
635 \\
610 \\
645 \\
650 \\
655 \\
650 \\
665 \\
670 \\
675 \\
680 \\
685 \\
690 \\
695 \\
700\end{array}$ & $\begin{array}{l}18.040 \\
18.248 \\
18.424 \\
18.534 \\
18.675 \\
18.774 \\
18.857 \\
18.949 \\
19.065 \\
19.196 \\
10.310 \\
19.403 \\
10.502 \\
19.568 \\
19.535 \\
19.714 \\
19.791 \\
10.860 \\
19.941 \\
20.007 \\
20.090 \\
20.167 \\
20.245 \\
20.316 \\
20.391 \\
20.165 \\
20.540 \\
20.624 \\
20.692 \\
20.765 \\
20.863 \\
20.938 \\
21.012 \\
21.054 \\
21.121 \\
21.179 \\
21.250 \\
21.291 \\
21.356 \\
21.416 \\
21.183 \\
21.533 \\
21.575 \\
21.626 \\
21.675 \\
21.745 \\
21.811 \\
21.904 \\
21.993 \\
22.067 \\
22.135 \\
22.229 \\
22.294 \\
22.366 \\
22.141 \\
22.511 \\
22.576 \\
22.658 \\
22.728 \\
22.794 \\
22.869 \\
22.934 \\
22.987 \\
23.045 \\
23.087 \\
23.134 \\
23.178\end{array}$ \\
\hline
\end{tabular}


HGFT RIVER, IDAHO - I.D. NO. 5 - 29 NUOUST 1975

\begin{tabular}{|c|c|c|c|}
\hline $\begin{array}{l}\text { DEPTH } \\
\text { (FT) }\end{array}$ & $\begin{array}{l}\text { TEPPERATURE } \\
\text { (i) }\end{array}$ & $\begin{array}{l}\text { DEPTH } \\
\text { (FT) }\end{array}$ & $\begin{array}{l}\text { TERPEPATU } \\
\text { (C) }\end{array}$ \\
\hline $\begin{array}{l}155 \\
160 \\
165 \\
170 \\
175 \\
180 \\
185 \\
190 \\
195 \\
200 \\
210 \\
200 \\
2255 \\
230 \\
2355 \\
240 \\
245 \\
250 \\
255 \\
260 \\
265 \\
270 \\
275 \\
280 \\
285 \\
290 \\
295 \\
300 \\
305 \\
310 \\
315 \\
320 \\
325 \\
330 \\
335 \\
340 \\
345 \\
350 \\
360 \\
365 \\
370 \\
375 \\
380 \\
385 \\
390 \\
395 \\
400 \\
405 \\
410 \\
115 \\
420 \\
225 \\
430 \\
135 \\
140 \\
445 \\
450 \\
455 \\
450 \\
365 \\
470 \\
475 \\
480 \\
485 \\
490 \\
495 \\
500 \\
505 \\
510 \\
\end{array}$ & 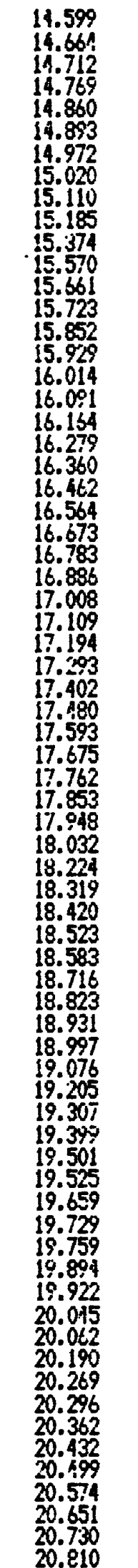 & $\begin{array}{l}520 \\
525 \\
530 \\
535 \\
540 \\
545 \\
550 \\
555 \\
560 \\
565 \\
570 \\
575 \\
580 \\
585 \\
590 \\
575 \\
500 \\
605 \\
610 \\
615 \\
620 \\
625 \\
530 \\
635 \\
610 \\
615 \\
650 \\
655 \\
660 \\
665 \\
670 \\
675 \\
680 \\
685 \\
690 \\
695 \\
700 \\
705 \\
710 \\
715\end{array}$ & $\begin{array}{l}20.894 \\
20.961 \\
21.031 \\
21.095 \\
21.159 \\
21.221 \\
21.278 \\
21.335 \\
21.395 \\
21.158 \\
21.518 \\
21.582 \\
21.643 \\
21.703 \\
21.760 \\
21.825 \\
21.895 \\
21.972 \\
22.046 \\
22.121 \\
22.197 \\
22.269 \\
22.341 \\
22.131 \\
22.197 \\
22.562 \\
22.631 \\
22.708 \\
22.770 \\
22.842 \\
22.912 \\
22.980 \\
23.041 \\
23.100 \\
23.159 \\
23.221 \\
23.275 \\
23.359 \\
23.434 \\
23.477\end{array}$ \\
\hline
\end{tabular}


RAFT RIVER, IDAHO - I.D. ND. 5 - 19 CCTOBER 1975

DEPTH TEPERATURE DEPTH TEPERATLRE
(FT) (C)

\begin{tabular}{|c|c|}
\hline $\begin{array}{l}15.698 \\
14.702 \\
14.692 \\
14.759 \\
14.835 \\
11.901 \\
14.962 \\
15.019 \\
15.108 \\
15.184 \\
15.213 \\
15.370 \\
15.457 \\
15.531 \\
15.326 \\
15.717 \\
15.804 \\
15.908 \\
15.991 \\
16.080 \\
16.173 \\
16.248 \\
16.351 \\
16.425 \\
15.528 \\
16.626 \\
16.753 \\
16.831 \\
16.941 \\
17.040 \\
17.144 \\
17.244 \\
17.356 \\
17.448 \\
17.537 \\
17.636 \\
17.244 \\
17.824 \\
17.912 \\
18.003 \\
18.488 \\
18.179 \\
18.281\end{array}$ & $\begin{array}{l}500 \\
510 \\
515 \\
520 \\
525 \\
530 \\
535 \\
540 \\
545 \\
550 \\
555 \\
560 \\
565 \\
570 \\
575 \\
580 \\
585 \\
590 \\
595 \\
600 \\
605 \\
610 \\
615 \\
620 \\
625 \\
630 \\
635 \\
640 \\
645 \\
650 \\
6555 \\
660 \\
665 \\
670 \\
675 \\
680 \\
695 \\
690 \\
695 \\
700 \\
705 \\
710 \\
715\end{array}$ \\
\hline
\end{tabular}

18.468

18.563

18.658

18.749

18.841

18.951

19.041

10.169

19.24)

19.333

19.441

19.516

19.504

19.661

19.736

19.84 !

19.879

19.957

20.030

20.105

20.185

20.257

20.319

20.104

20.16 .

20.555 
RAFT RIVER, IDAHD - I.D. HO. 5 - 6 AUGUST 1976

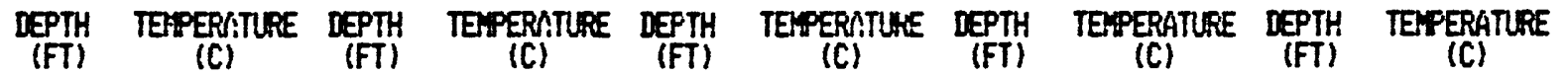

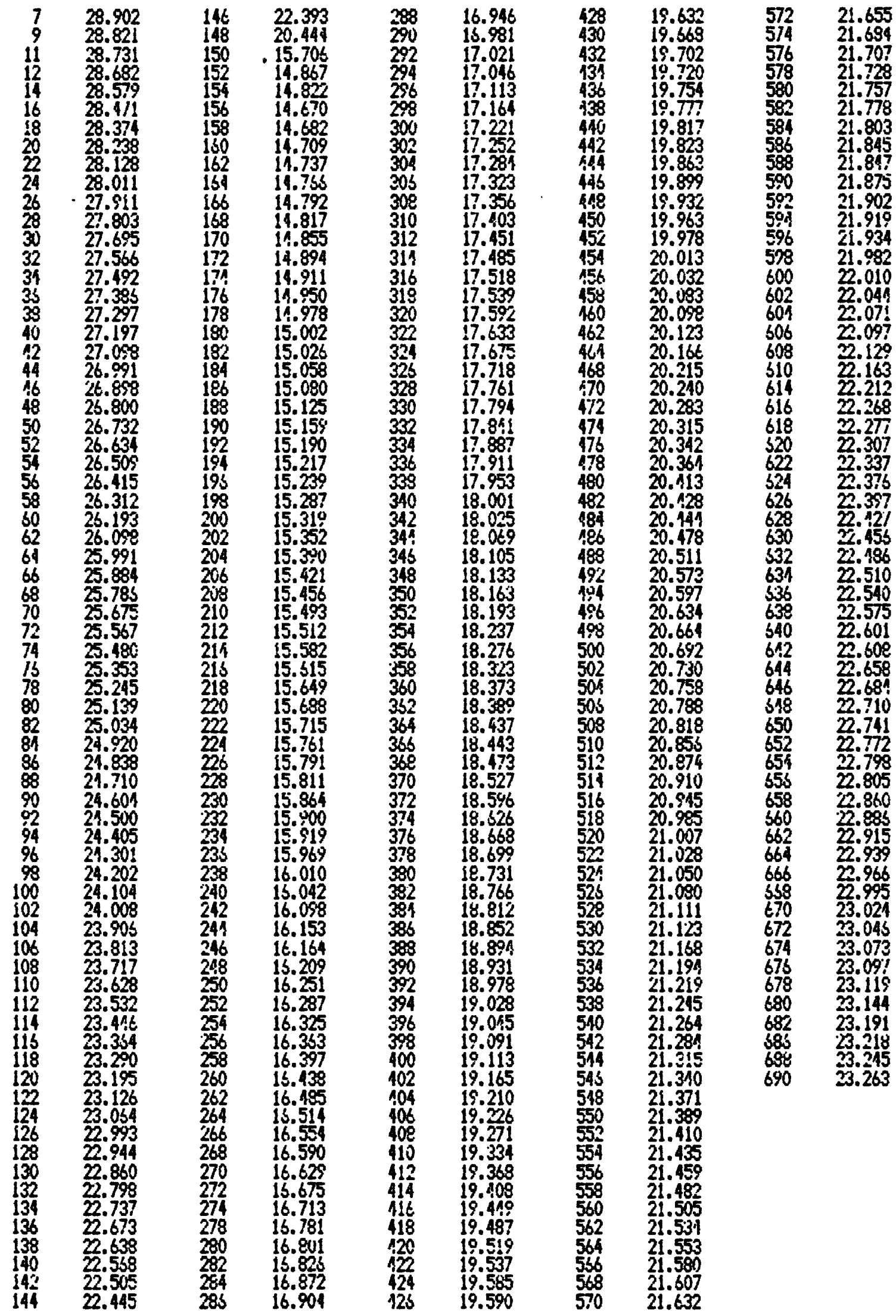


RAFT RIVER, IDHD - I.D. HO. 5A - 17 SEPTEMER 1975

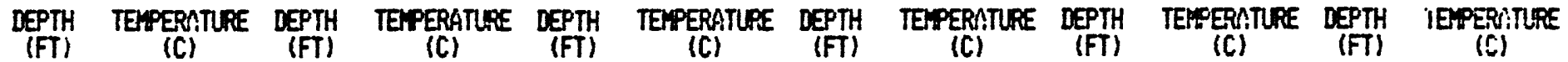

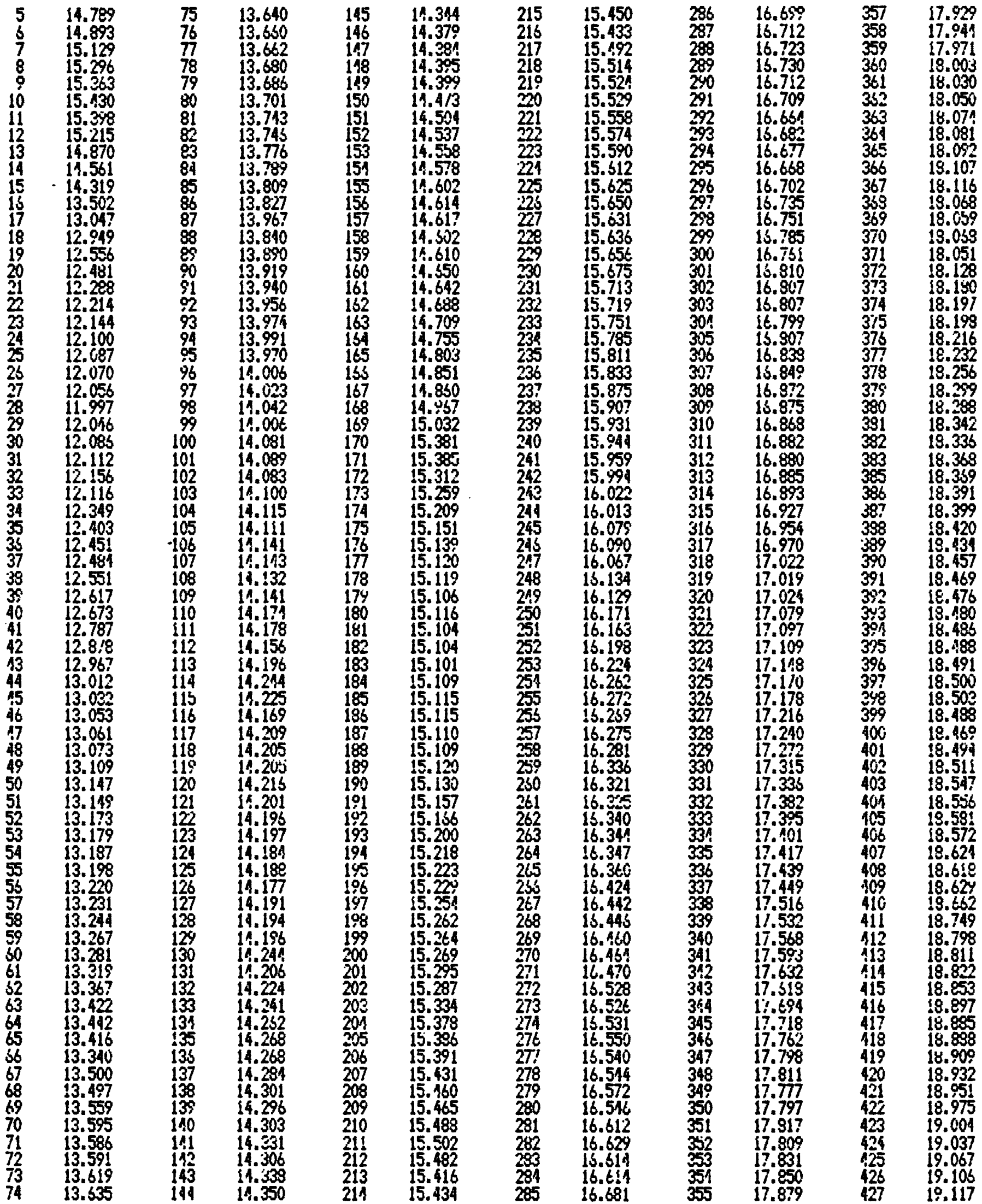


RAFT RIVEP, ILAHO - I.D. M. 5月 - 17 SEPTEMEER 1475

\begin{tabular}{|c|c|c|c|c|c|c|c|c|c|c|c|}
\hline $\begin{array}{l}\text { DEPTH } \\
\text { (FT) }\end{array}$ & $\begin{array}{l}\text { TEMPERATLRE } \\
\text { (C) }\end{array}$ & $\begin{array}{l}\text { DEPTH } \\
\text { (FT) }\end{array}$ & $\begin{array}{l}\text { TEMPERGTLPE } \\
\text { (C) }\end{array}$ & $\begin{array}{l}\text { DEPTH } \\
\text { (FT) }\end{array}$ & $\begin{array}{l}\text { TEMPERATURE } \\
\text { (C) }\end{array}$ & $\begin{array}{l}\text { DEPTH } \\
\text { (FT) }\end{array}$ & $\begin{array}{l}\text { TERPERATLUE } \\
\text { (C) }\end{array}$ & $\begin{array}{l}\text { DEFTH } \\
\text { (FT) }\end{array}$ & $\begin{array}{l}\text { TEIPERMTLRE } \\
\text { (C) }\end{array}$ & $\begin{array}{l}\text { DEPTH } \\
\text { (FT) }\end{array}$ & $\begin{array}{l}\text { TEYPERATU } \\
\text { (C) }\end{array}$ \\
\hline $\begin{array}{l}128 \\
429 \\
430 \\
131 \\
132 \\
433 \\
134 \\
435 \\
436 \\
437 \\
430 \\
430 \\
440 \\
441 \\
442 \\
443 \\
441 \\
445 \\
446 \\
447 \\
448 \\
149 \\
450 \\
451 \\
452 \\
453 \\
451 \\
155 \\
156 \\
157 \\
458 \\
459 \\
460 \\
451 \\
462 \\
463 \\
464 \\
465 \\
466 \\
467 \\
468 \\
139 \\
470 \\
471 \\
472 \\
473 \\
171 \\
475 \\
476 \\
477 \\
478 \\
179 \\
480 \\
481 \\
482 \\
483 \\
484 \\
485 \\
486 \\
187 \\
488 \\
489 \\
490 \\
191 \\
492 \\
493 \\
494 \\
495 \\
496 \\
497\end{array}$ & $\begin{array}{l}19.123 \\
19.131 \\
19.152 \\
19.184 \\
19.239 \\
19.249 \\
19.266 \\
19.321 \\
19.341 \\
19.365 \\
19.396 \\
19.404 \\
19.117 \\
19.453 \\
19.440 \\
19.471 \\
19.495 \\
19.505 \\
19.523 \\
19.544 \\
19.566 \\
19.572 \\
19.544 \\
19.636 \\
19.658 \\
10.674 \\
19.689 \\
19.705 \\
19.721 \\
19.754 \\
19.798 \\
10.813 \\
19.833 \\
19.837 \\
19.855 \\
19.882 \\
19.862 \\
19.932 \\
19.9914 \\
10.947 \\
10.999 \\
20.019 \\
20.009 \\
20.044 \\
20.054 \\
20.070 \\
20.097 \\
20.103 \\
20.129 \\
20.139 \\
20.152 \\
20.185 \\
20.193 \\
20.225 \\
20.268 \\
20.244 \\
20.259 \\
20.277 \\
20.319 \\
20.333 \\
20.353 \\
20.339 \\
20.398 \\
20.424 \\
20.126 \\
20.457 \\
20.472 \\
20.493 \\
20.510 \\
20.531\end{array}$ & 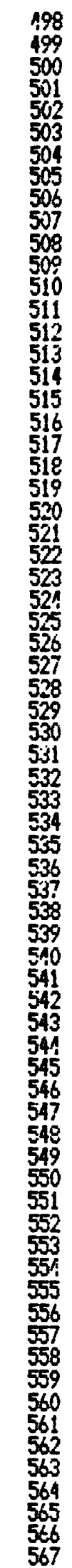 & $\begin{array}{l}20.550 \\
20.555 \\
20.591 \\
20.514 \\
20.633 \\
20.602 \\
20.631 \\
20.671 \\
20.689 \\
20.731 \\
20.718 \\
20.773 \\
20.762 \\
20.815 \\
20.818 \\
20.790 \\
20.829 \\
20.848 \\
20.843 \\
20.868 \\
20.882 \\
20.738 \\
20.919 \\
20.919 \\
20.940 \\
20.959 \\
20.990 \\
21.023 \\
21.025 \\
21.059 \\
21.075 \\
21.099 \\
21.105 \\
21.109 \\
21.121 \\
21.137 \\
21.139 \\
21.145 \\
21.168 \\
21.180 \\
21.189 \\
21.200 \\
21.238 \\
21.236 \\
21.234 \\
21 . .265 \\
21.274 \\
21.310 \\
21.3137 \\
21.353 \\
21.364 \\
21.318 \\
21.411 \\
21.435 \\
21.141 \\
21.399 \\
21.356 \\
21.375 \\
21.359 \\
21.335 \\
21.398 \\
21.415 \\
21.435 \\
21.442 \\
21.452 \\
21.468 \\
21.466 \\
21.475 \\
21.178\end{array}$ & $\begin{array}{l}568 \\
569 \\
570 \\
571 \\
572 \\
573 \\
571 \\
575 \\
516 \\
577 \\
578 \\
579 \\
580 \\
581 \\
582 \\
583 \\
584 \\
585 \\
586 \\
587 \\
589 \\
589 \\
540 \\
591 \\
592 \\
593 \\
591 \\
595 \\
596 \\
547 \\
598 \\
549 \\
600 \\
601 \\
602 \\
503 \\
604 \\
505 \\
606 \\
507 \\
608 \\
609 \\
610 \\
511 \\
612 \\
613 \\
614 \\
615 \\
616 \\
517 \\
618 \\
519 \\
620 \\
621 \\
622 \\
623 \\
624 \\
625 \\
626 \\
627 \\
628 \\
529 \\
630 \\
531 \\
632 \\
633 \\
634 \\
635 \\
636 \\
637\end{array}$ & 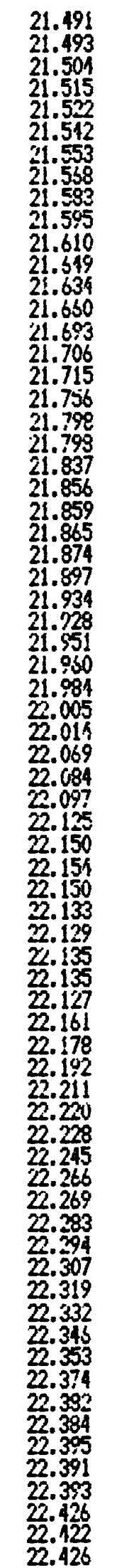 & $\begin{array}{l}638 \\
639 \\
640 \\
611 \\
692 \\
641 \\
645 \\
346 \\
647 \\
649 \\
649 \\
650 \\
651 \\
652 \\
653 \\
654 \\
655 \\
655 \\
657 \\
358 \\
659 \\
650 \\
661 \\
652 \\
663 \\
654 \\
665 \\
656 \\
667 \\
668 \\
664 \\
670 \\
671 \\
672 \\
673 \\
674 \\
675 \\
375 \\
677 \\
678 \\
679 \\
630 \\
681 \\
682 \\
683 \\
684 \\
695 \\
696 \\
687 \\
688 \\
699 \\
390 \\
691 \\
692 \\
693 \\
694 \\
655 \\
696 \\
697 \\
698 \\
699 \\
700 \\
701 \\
702 \\
103 \\
704 \\
705 \\
705 \\
707 \\
708\end{array}$ & 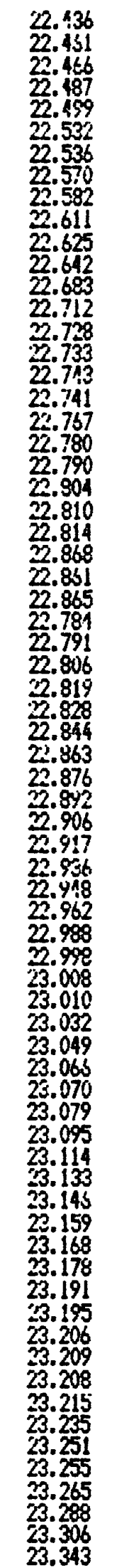 & $\begin{array}{l}709 \\
710 \\
711 \\
712 \\
713 \\
714 \\
715 \\
715 \\
717 \\
718 \\
719 \\
720 \\
72 ! \\
722 \\
723 \\
724 \\
725 \\
725 \\
727 \\
729 \\
729 \\
730 \\
731 \\
732 \\
733 \\
731 \\
735 \\
736 \\
737 \\
138 \\
739 \\
740 \\
741 \\
712 \\
713 \\
744 \\
745 \\
746 \\
747 \\
748 \\
749 \\
750 \\
751 \\
752 \\
753 \\
754 \\
755 \\
736 \\
757 \\
758 \\
754 \\
730 \\
761 \\
732 \\
763 \\
764 \\
765 \\
765 \\
767 \\
769 \\
765 \\
770 \\
771 \\
772 \\
773 \\
774 \\
775 \\
776 \\
777 \\
778\end{array}$ & 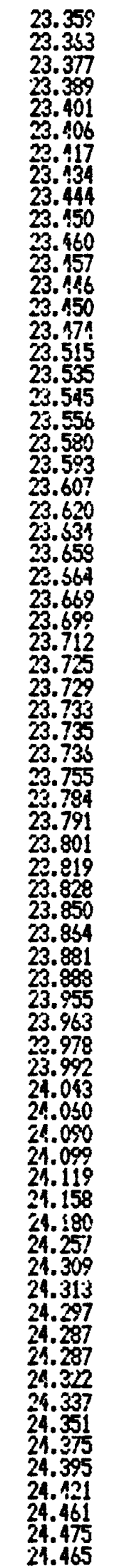 & $\begin{array}{l}779 \\
780 \\
781 \\
742 \\
783 \\
784 \\
795 \\
785 \\
787 \\
788 \\
799 \\
790 \\
791 \\
792 \\
793 \\
794 \\
795 \\
796 \\
797 \\
790 \\
790 \\
800 \\
801 \\
802 \\
803 \\
804 \\
805 \\
805 \\
807 \\
808 \\
809 \\
810 \\
811 \\
812 \\
813 \\
814 \\
815 \\
815 \\
817 \\
818 \\
810 \\
820 \\
821 \\
822 \\
823 \\
824 \\
825 \\
855 \\
827 \\
828 \\
829 \\
830 \\
831 \\
832 \\
833 \\
831 \\
835 \\
836 \\
837 \\
839 \\
839 \\
840 \\
811 \\
842 \\
843 \\
844 \\
845 \\
845 \\
817 \\
848\end{array}$ & 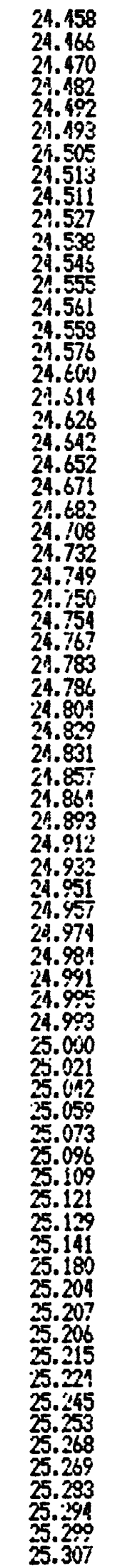 \\
\hline
\end{tabular}


RAFT RIUER, IDAHO - I.D. M. 5A - 17 SEPTEMBEP. 1975

\begin{tabular}{|c|c|c|c|c|c|c|c|c|c|c|c|}
\hline $\begin{array}{l}\text { DEPTH } \\
\text { (FT) }\end{array}$ & $\begin{array}{l}\text { TEMPERATURE } \\
\text { (C) }\end{array}$ & $\begin{array}{c}\text { DEPTH } \\
\text { (FT) }\end{array}$ & $\begin{array}{l}\text { TERPERGTURE } \\
\text { (C) }\end{array}$ & $\begin{array}{l}\text { DEPTH } \\
\text { (FT) }\end{array}$ & $\begin{array}{l}\text { TEAFERATURE } \\
\text { (C) }\end{array}$ & $\begin{array}{l}\text { DEPTH } \\
\text { (FT) }\end{array}$ & $\begin{array}{l}\text { TEMPERATLRE } \\
\text { (C) }\end{array}$ & $\begin{array}{l}\text { IEPTH } \\
\text { (FT) }\end{array}$ & $\begin{array}{l}\text { TEMPEEATTRE } \\
\text { (C) }\end{array}$ & $\begin{array}{l}\text { DEPTH } \\
\text { (FT) }\end{array}$ & $\begin{array}{l}\text { TEPPERATL } \\
\text { (C) }\end{array}$ \\
\hline $\begin{array}{l}844 \\
850 \\
851 \\
852 \\
853 \\
854 \\
855 \\
856 \\
857 \\
859 \\
859 \\
850 \\
861 \\
862 \\
863 \\
864 \\
865 \\
856 \\
867 \\
868 \\
865 \\
970 \\
871 \\
872 \\
873 \\
874 \\
875 \\
875 \\
871 \\
878 \\
879 \\
880 \\
881 \\
882 \\
883 \\
894 \\
985 \\
885 \\
897 \\
888 \\
899 \\
890 \\
991 \\
892 \\
893 \\
994 \\
895 \\
896 \\
897 \\
889 \\
8999 \\
900 \\
901 \\
902 \\
993 \\
904 \\
905 \\
905 \\
907 \\
908 \\
909 \\
910 \\
911 \\
912 \\
913 \\
9111 \\
915 \\
916 \\
917 \\
918\end{array}$ & 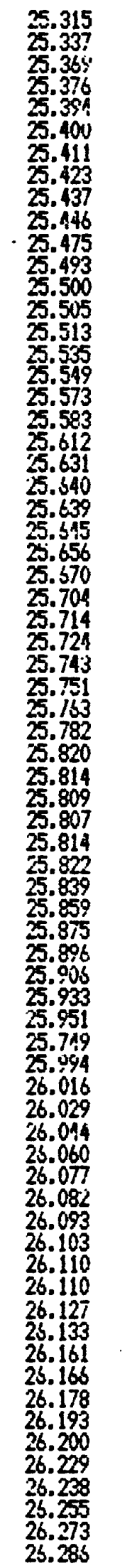 & $\begin{array}{l}919 \\
920 \\
921 \\
922 \\
923 \\
921 \\
925 \\
926 \\
927 \\
928 \\
929 \\
930 \\
931 \\
932 \\
933 \\
931 \\
935 \\
936 \\
937 \\
038 \\
939 \\
940 \\
941 \\
942 \\
943 \\
944 \\
945 \\
946 \\
947 \\
948 \\
940 \\
950 \\
951 \\
952 \\
953 \\
954 \\
955 \\
456 \\
951 \\
958 \\
955 \\
960 \\
961 \\
962 \\
964 \\
955 \\
960 \\
967 \\
968 \\
969 \\
970 \\
971 \\
972 \\
973 \\
970 \\
975 \\
976 \\
977 \\
978 \\
970 \\
480 \\
981 \\
982 \\
983 \\
981 \\
485 \\
986 \\
987 \\
998 \\
989\end{array}$ & 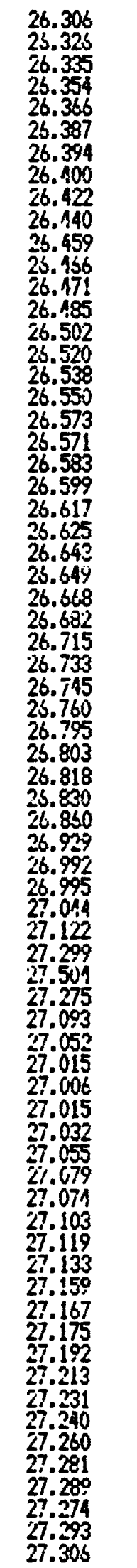 & $\begin{array}{l}990 \\
991 \\
992 \\
993 \\
994 \\
995 \\
996 \\
997 \\
998 \\
999 \\
1000 \\
1001 \\
1002 \\
1003 \\
1004 \\
1005 \\
1006 \\
1007 \\
1008 \\
1009 \\
1010 \\
1011 \\
1012 \\
1013 \\
1014 \\
1015 \\
1016 \\
1017 \\
1019 \\
1020 \\
1021 \\
1022 \\
1023 \\
1024 \\
1025 \\
1026 \\
1027 \\
1028 \\
1029 \\
1030 \\
1031 \\
1032 \\
1633 \\
1034 \\
1035 \\
1036 \\
1037 \\
1038 \\
1039 \\
1040 \\
1041 \\
1042 \\
1043 \\
1044 \\
1045 \\
1016 \\
1017 \\
1049 \\
1049 \\
1050 \\
1051 \\
1052 \\
1053 \\
1054 \\
1055 \\
1055 \\
1057 \\
1058 \\
1059 \\
1050\end{array}$ & 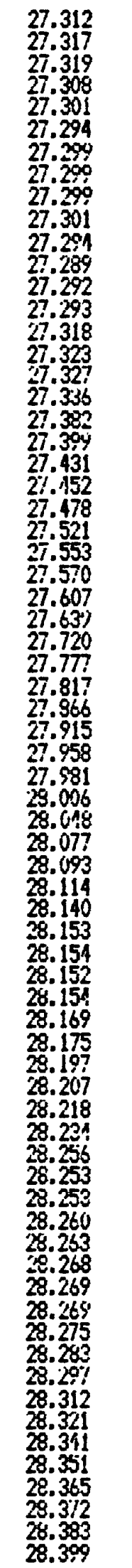 & $\begin{array}{l}1061 \\
1062 \\
1063 \\
1061 \\
1065 \\
1066 \\
1067 \\
1068 \\
1069 \\
1070 \\
1071 \\
1072 \\
1073 \\
1074 \\
1075 \\
1076 \\
1077 \\
1078 \\
1079 \\
1080 \\
1081 \\
1082 \\
1093 \\
1084 \\
1085 \\
1086 \\
1087 \\
1089 \\
1089 \\
1090 \\
1091 \\
1092 \\
1093 \\
1094 \\
1095 \\
1096 \\
1097 \\
1098 \\
1099 \\
1100 \\
1101 \\
1102 \\
1103 \\
1104 \\
1105 \\
1106 \\
1107 \\
1108 \\
1109 \\
1110 \\
1111 \\
1112 \\
1113 \\
1114 \\
1115 \\
1116 \\
1117 \\
1119 \\
1119 \\
1120 \\
1121 \\
1122 \\
1123 \\
1121 \\
1125 \\
1126 \\
1127 \\
1128 \\
1139 \\
1130\end{array}$ & 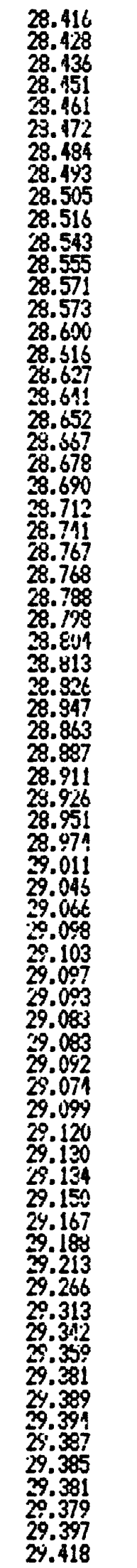 & $\begin{array}{l}1131 \\
1132 \\
1133 \\
1134 \\
1135 \\
1136 \\
1137 \\
1138 \\
1134 \\
1141 \\
1142 \\
1143 \\
1144 \\
1145 \\
1146 \\
1147 \\
1148 \\
1149 \\
1150 \\
1151 \\
1152 \\
1153 \\
1151 \\
1155 \\
1156 \\
1157 \\
1158 \\
1159 \\
1160 \\
1161 \\
1162 \\
1153 \\
1164 \\
1165 \\
1166 \\
1167 \\
1168 \\
1169 \\
1170 \\
1171 \\
1172 \\
1173 \\
1171 \\
1175 \\
1176 \\
1177 \\
1178 \\
1110 \\
1180 \\
1181 \\
1182 \\
1183 \\
1184 \\
1185 \\
1186 \\
1187 \\
1189 \\
1189 \\
1190 \\
1191 \\
1192 \\
1193 \\
1194 \\
1195 \\
1196 \\
1197 \\
1198 \\
1199 \\
1200 \\
1201\end{array}$ & 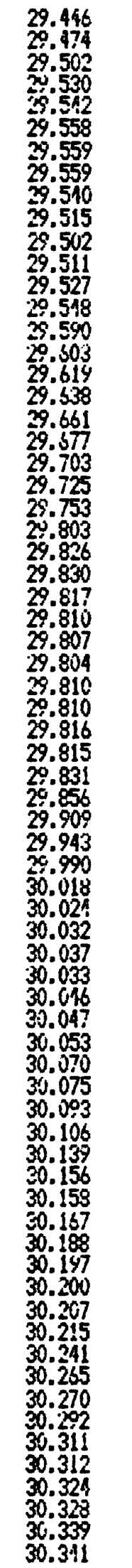 & $\begin{array}{l}1202 \\
1203 \\
1204 \\
1205 \\
1206 \\
1207 \\
1208 \\
1209 \\
1210 \\
1211 \\
1212 \\
1213 \\
1214 \\
1215 \\
1216 \\
1217 \\
1218 \\
1219 \\
1220 \\
1221 \\
1222 \\
1223 \\
1224 \\
1255 \\
1226 \\
1227 \\
1228 \\
1229 \\
1220 \\
1231 \\
1232 \\
1233 \\
1234 \\
1235 \\
1236 \\
1237 \\
1238 \\
1230 \\
1240 \\
1241 \\
1262 \\
1243 \\
1211 \\
1245 \\
1246 \\
1247 \\
1248 \\
1249 \\
1250 \\
1251 \\
1252 \\
1253 \\
1251 \\
1255 \\
1256 \\
1257 \\
1250 \\
1259 \\
1260 \\
1251 \\
1252 \\
1253 \\
1264 \\
1265 \\
1266 \\
1267 \\
1268 \\
1259 \\
1270 \\
1271\end{array}$ & 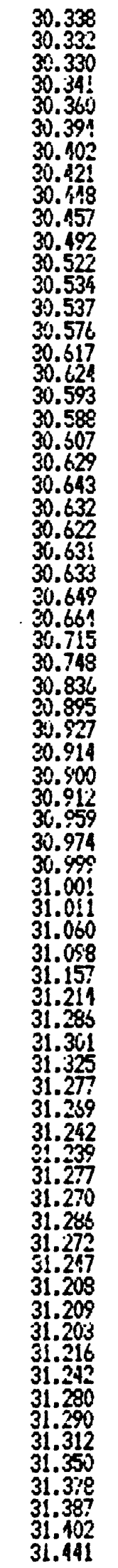 \\
\hline
\end{tabular}


RAFT RIVER, IDHHO - I.D. MO. 5A - 17 SEPTEULER 1975

DEPTH TEMPERATURE

(FT) (C)

$1272 \quad 31.181$

$1273 \quad 31.540$

127431.716

$127531.86 !$

$1276 \quad 31.953$

$1277 \quad 31.981$

$1278 \quad 31.956$

$1279 \quad 31.816$

$1280 \quad 31.673$

$1281 \quad 31.602$

$1282 \cdot 31.592$

$1283-31.535$

128431.529

129531.543

$1386 \quad 31.511$

$1287 \quad 31.534$

$1260 \quad 31.592$

129931.585

$1250 \quad 31.641$

$1201 \quad 31.652$

$1202 \quad 31.692$

$1293 \quad 31.722$

120431.88 ?

129531.893

$1296-31.98$

$1297 \quad 32.019$

$1298 \quad 32.010$

$1299 \quad 32.086$

$1300 \quad 32.138$ 
RAFT RIVER, IDAHD - I.D. NO. 5A - 19 C.TOBER 1975

\begin{tabular}{|c|c|c|c|c|c|c|c|}
\hline $\begin{array}{l}\text { DEPTH } \\
\text { (FT) }\end{array}$ & $\begin{array}{l}\text { TERESKATUPE } \\
\text { (C) }\end{array}$ & $\begin{array}{c}\text { UEPTH } \\
\text { (FT) }\end{array}$ & $\begin{array}{l}\text { TEYPERATLYE } \\
\text { (C) }\end{array}$ & $\begin{array}{l}\text { BEPTH } \\
\text { (FT) }\end{array}$ & $\begin{array}{l}\text { TEHPERATURE } \\
\text { (C) }\end{array}$ & $\begin{array}{l}\text { LEPTH } \\
\text { (FT) }\end{array}$ & $\begin{array}{l}\text { TEROERATLEE } \\
\text { (C) }\end{array}$ \\
\hline $\begin{array}{l}30 \\
35 \\
40 \\
45 \\
50 \\
55 \\
30 \\
655 \\
70 \\
75 \\
80 \\
85 \\
90 \\
95 \\
100 \\
105 \\
110 \\
115 \\
120 \\
125 \\
130 \\
135 \\
100 \\
145 \\
150 \\
155 \\
150 \\
155 \\
170 \\
175 \\
190 \\
185 \\
190 \\
195 \\
200 \\
205 \\
210 \\
215 \\
220 \\
2255 \\
230 \\
235 \\
240 \\
245 \\
250 \\
255 \\
250 \\
265 \\
270 \\
275 \\
290 \\
285 \\
290 \\
295 \\
300 \\
305 \\
310 \\
315 \\
320 \\
3255 \\
330 \\
335 \\
340 \\
3155 \\
350 \\
3555 \\
350 \\
365 \\
370 \\
375\end{array}$ & 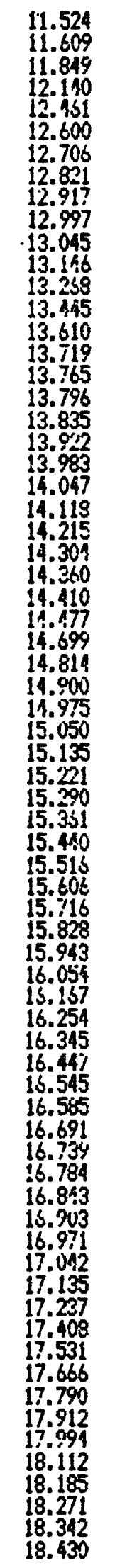 & $\begin{array}{l}390 \\
385 \\
390 \\
395 \\
100 \\
405 \\
110 \\
120 \\
425 \\
430 \\
435 \\
440 \\
445 \\
450 \\
455 \\
460 \\
465 \\
470 \\
475 \\
480 \\
485 \\
490 \\
495 \\
500 \\
505 \\
510 \\
515 \\
520 \\
525 \\
530 \\
535 \\
540 \\
545 \\
550 \\
555 \\
560 \\
565 \\
570 \\
575 \\
580 \\
555 \\
590 \\
595 \\
600 \\
505 \\
610 \\
615 \\
620 \\
325 \\
630 \\
3355 \\
640 \\
645 \\
650 \\
355 \\
660 \\
5655 \\
670 \\
575 \\
680 \\
6655 \\
690 \\
395 \\
700 \\
775 \\
770 \\
715 \\
720 \\
725 \\
730\end{array}$ & 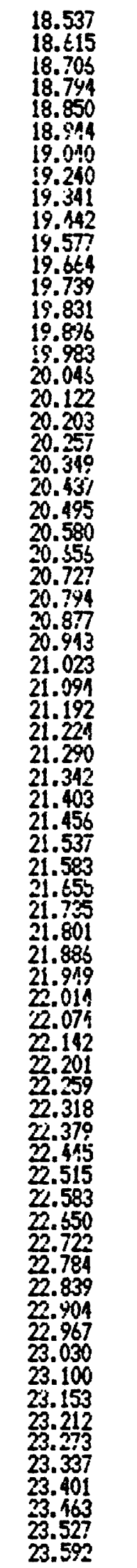 & $\begin{array}{l}735 \\
740 \\
745 \\
750 \\
755 \\
760 \\
755 \\
770 \\
775 \\
780 \\
785 \\
790 \\
795 \\
800 \\
805 \\
810 \\
815 \\
820 \\
825 \\
830 \\
835 \\
840 \\
895 \\
850 \\
855 \\
860 \\
855 \\
870 \\
875 \\
880 \\
885 \\
896 \\
895 \\
900 \\
905 \\
910 \\
915 \\
920 \\
925 \\
930 \\
935 \\
910 \\
945 \\
950 \\
955 \\
960 \\
965 \\
970 \\
975 \\
990 \\
9855 \\
990 \\
995 \\
1000 \\
1010 \\
1105 \\
1020 \\
1025 \\
1030 \\
1055 \\
1040 \\
1045 \\
1050 \\
1055 \\
1060 \\
1065 \\
1070 \\
1075 \\
1080 \\
1085\end{array}$ & 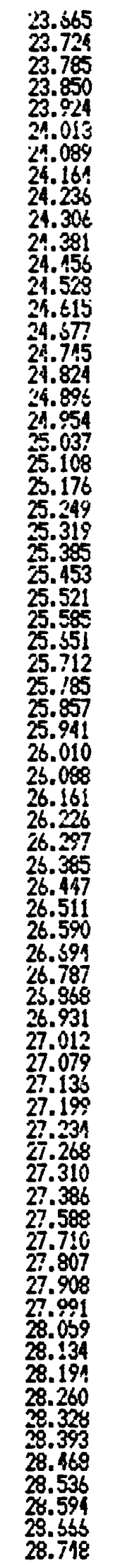 & $\begin{array}{l}1090 \\
1095 \\
1100 \\
1105 \\
1110 \\
1115 \\
1120 \\
1125 \\
1130 \\
1135 \\
1140 \\
1145 \\
1150 \\
1155 \\
1150 \\
1165 \\
1170 \\
1175 \\
1190 \\
1185 \\
1190 \\
1105\end{array}$ & 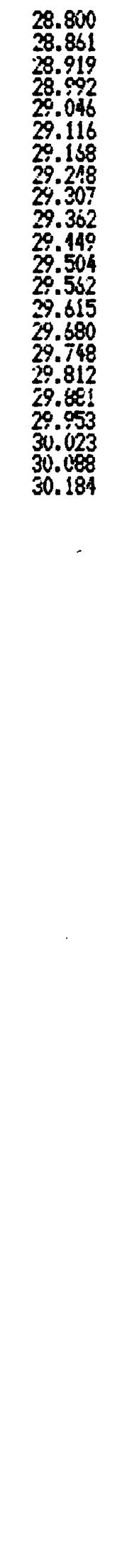 \\
\hline
\end{tabular}


RAFT RIVER, IDAHO - I.D. W. 5A - ? FEBRLARY 1976

\begin{tabular}{|c|c|c|c|c|c|c|c|}
\hline $\begin{array}{l}\text { DEPTH } \\
\text { (FT) }\end{array}$ & $\begin{array}{l}\text { TERERATURE } \\
\text { (C) }\end{array}$ & $\begin{array}{l}\text { DEPTH } \\
\text { (FT) }\end{array}$ & $\begin{array}{l}\text { IEYERATURE } \\
\text { (C) }\end{array}$ & $\begin{array}{l}\text { DEPTH } \\
\text { (FT) }\end{array}$ & $\begin{array}{l}\text { TEMPERATLRE } \\
\text { (C) }\end{array}$ & $\begin{array}{c}\text { DEPTH } \\
\text { (FT) }\end{array}$ & $\begin{array}{l}\text { TEMPERATLRE } \\
\text { (C) }\end{array}$ \\
\hline $\begin{array}{l}25 \\
30 \\
355 \\
10 \\
15 \\
50 \\
555 \\
60 \\
65 \\
70 \\
75 \\
80 \\
855 \\
90 \\
95 \\
100 \\
105 \\
110 \\
115 \\
120 \\
125 \\
130 \\
135 \\
110 \\
115 \\
150 \\
155 \\
160 \\
165 \\
170 \\
175 \\
180 \\
185 \\
190 \\
105 \\
200 \\
205 \\
210 \\
215 \\
250 \\
225 \\
230 \\
235 \\
240 \\
245 \\
250 \\
255 \\
260 \\
255 \\
270 \\
275 \\
280 \\
285 \\
290 \\
295 \\
300 \\
305 \\
310 \\
315 \\
320 \\
335 \\
330 \\
335 \\
340 \\
315 \\
350 \\
3355 \\
360 \\
365 \\
370\end{array}$ & 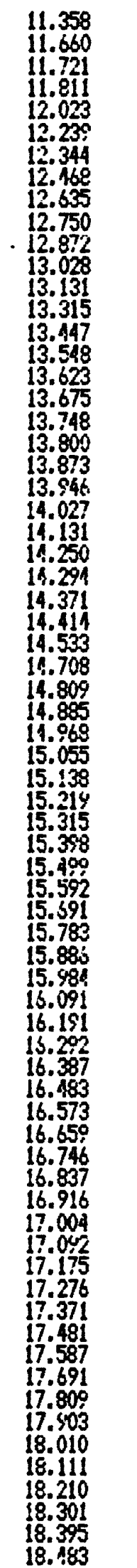 & $\begin{array}{l}375 \\
380 \\
385 \\
390 \\
395 \\
100 \\
405 \\
110 \\
415 \\
420 \\
425 \\
430 \\
435 \\
410 \\
415 \\
450 \\
455 \\
460 \\
465 \\
170 \\
475 \\
480 \\
495 \\
490 \\
495 \\
500 \\
505 \\
510 \\
515 \\
520 \\
525 \\
530 \\
535 \\
510 \\
545 \\
550 \\
555 \\
560 \\
565 \\
570 \\
575 \\
580 \\
595 \\
590 \\
5695 \\
600 \\
605 \\
610 \\
615 \\
620 \\
625 \\
630 \\
335 \\
640 \\
615 \\
650 \\
6555 \\
660 \\
365 \\
670 \\
5755 \\
680 \\
6955 \\
690 \\
695 \\
700 \\
7055 \\
710 \\
715 \\
720\end{array}$ & 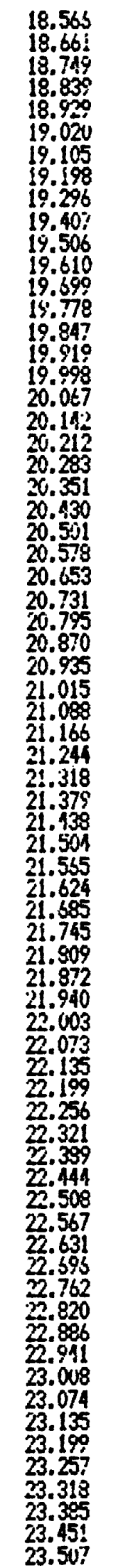 & 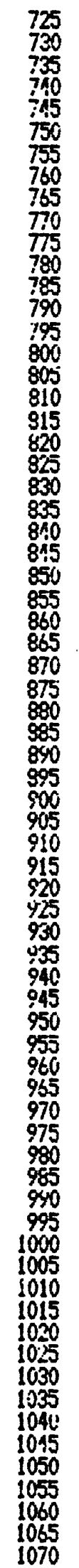 & 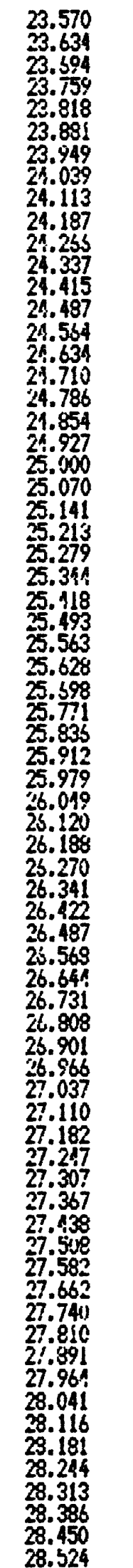 & $\begin{array}{l}1075 \\
1080 \\
1085 \\
1090 \\
1095 \\
1100 \\
1105 \\
1110 \\
1115 \\
1120 \\
1125 \\
1130 \\
1135 \\
1140 \\
1145 \\
1150 \\
1155 \\
1160 \\
1155 \\
1170 \\
1175 \\
1180 \\
1185 \\
119 \\
1195\end{array}$ & $\begin{array}{l}28.595 \\
28.653 \\
28.724 \\
28.795 \\
28.847 \\
28.913 \\
28.970 \\
29.012 \\
20.103 \\
29.163 \\
20.226 \\
39.30 ! \\
29.352 \\
29.131 \\
29.189 \\
29.555 \\
29.112 \\
29.659 \\
29.723 \\
29.787 \\
29.859 \\
29.932 \\
30.015 \\
30.077 \\
30.151\end{array}$ \\
\hline
\end{tabular}


RAFT RIVER, IDANO - 1.D. KO. SF - 6 AUEUST 1976

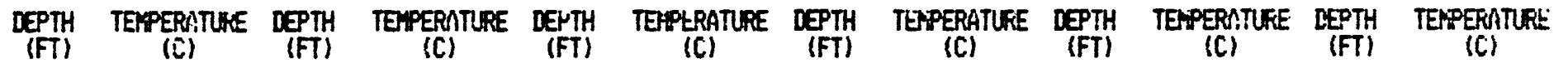

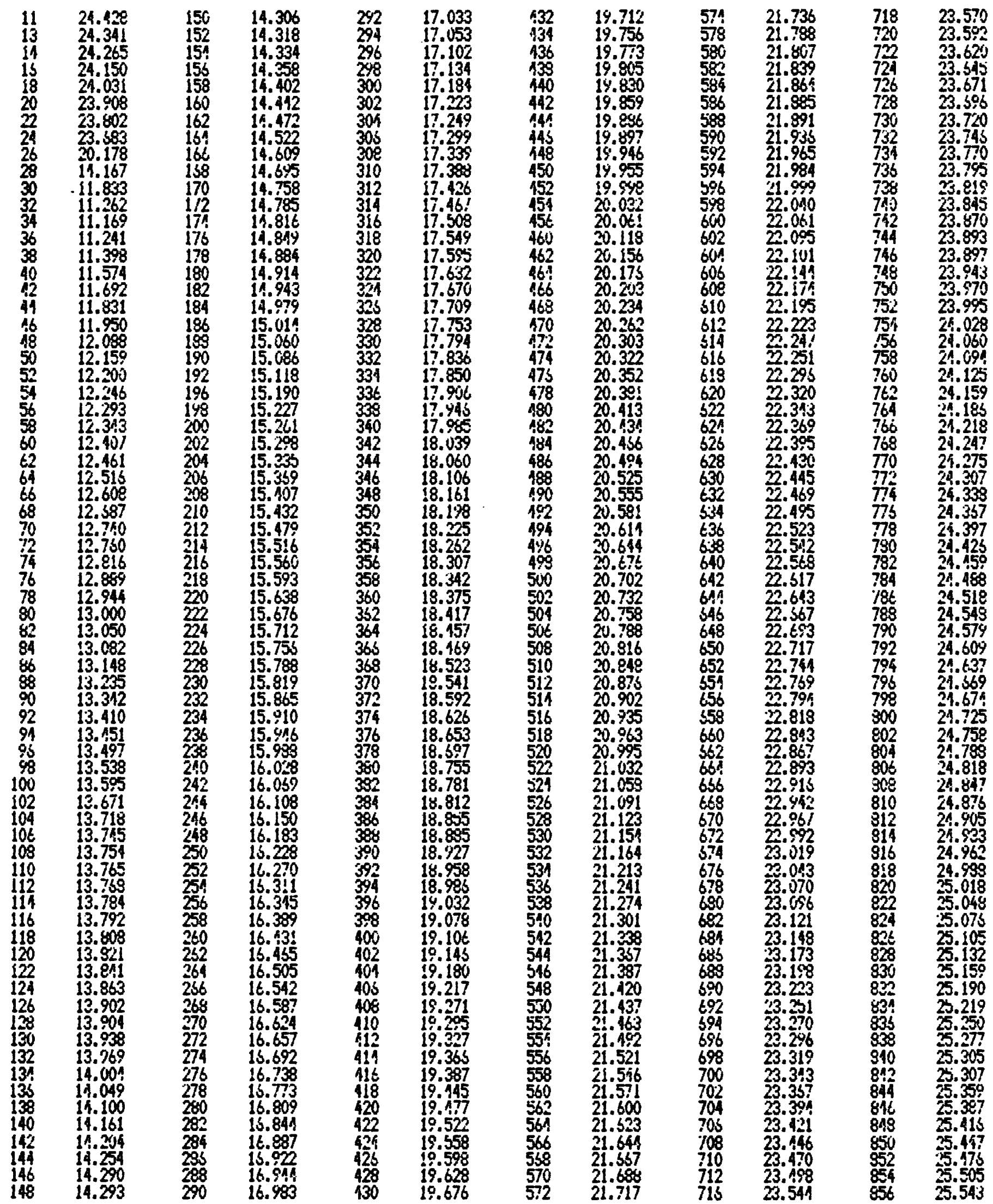


PAFT RIVER, IDHO - I.D. NO. 5 - 6 AUCUST 197S

\begin{tabular}{|c|c|c|c|c|c|}
\hline $\begin{array}{l}\text { IEPTH } \\
\text { (FT) }\end{array}$ & $\begin{array}{l}\text { TEMPERATURE } \\
\text { (C) }\end{array}$ & $\begin{array}{l}\text { DEPTH } \\
\text { (FT) }\end{array}$ & $\begin{array}{l}\text { TEPERATLRE } \\
\text { (C) }\end{array}$ & $\begin{array}{l}\text { DEPTH } \\
\text { (FT) }\end{array}$ & $\begin{array}{l}\text { TERPERATLRE } \\
\text { (C) }\end{array}$ \\
\hline 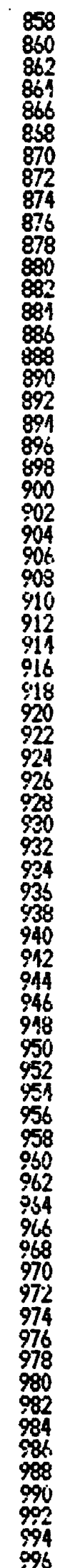 & 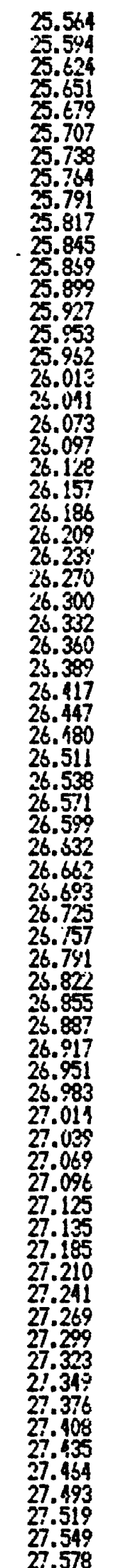 & $\begin{array}{l}998 \\
1000 \\
1002 \\
1004 \\
1006 \\
1008 \\
1010 \\
1012 \\
1014 \\
1016 \\
1018 \\
1020 \\
1022 \\
1024 \\
1026 \\
1028 \\
1030 \\
1032 \\
1031 \\
1036 \\
1039 \\
1010 \\
1042 \\
1041 \\
1046 \\
1048 \\
1050 \\
1052 \\
1054 \\
1055 \\
1059 \\
1060 \\
1062 \\
1054 \\
1066 \\
1068 \\
1070 \\
1072 \\
1074 \\
1076 \\
1078 \\
1080 \\
1082 \\
1084 \\
1086 \\
1098 \\
1090 \\
1092 \\
1094 \\
1006 \\
1098 \\
1100 \\
1102 \\
1104 \\
1106 \\
1108 \\
1110 \\
1112 \\
1111 \\
1115 \\
1118 \\
1120 \\
1122 \\
1124 \\
1126 \\
1128 \\
1130 \\
1132 \\
1134 \\
1136\end{array}$ & 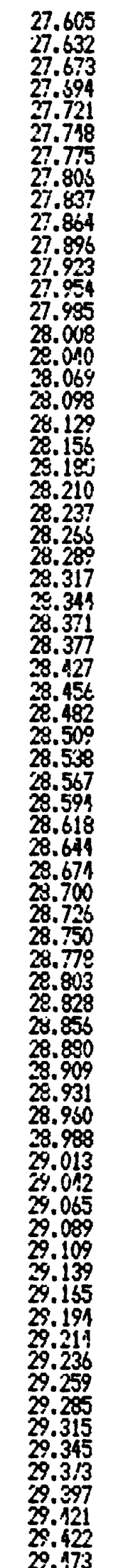 & $\begin{array}{l}1138 \\
1140 \\
1142 \\
1144 \\
1116 \\
1148 \\
1150 \\
1152 \\
1154 \\
1156 \\
1158 \\
1160 \\
1162 \\
1164 \\
1166 \\
1168 \\
1170 \\
1172 \\
1174 \\
1175 \\
1178 \\
1180 \\
1182 \\
1184 \\
1186 \\
1188 \\
1190 \\
1102 \\
1994 \\
1196 \\
1198\end{array}$ & $\begin{array}{l}29.501 \\
20.525 \\
28.551 \\
29.577 \\
29.600 \\
29.528 \\
29.651 \\
29.571 \\
29.491 \\
29.710 \\
29.732 \\
29.759 \\
29.786 \\
29.821 \\
29.841 \\
29.858 \\
29.896 \\
29.927 \\
20.957 \\
29.934 \\
30.011 \\
30.042 \\
20.073 \\
30.103 \\
30.133 \\
30.162 \\
30.190 \\
30.227 \\
30.255 \\
30.293 \\
30.313\end{array}$ \\
\hline
\end{tabular}


RAFT RIVER, IDAHO - SWH HELL - II MUWHAY 1976

\begin{tabular}{|c|c|c|c|}
\hline $\begin{array}{l}\text { DEPTH } \\
\text { (FT) }\end{array}$ & $\begin{array}{l}\text { TEMPERATLRE } \\
\text { (C) }\end{array}$ & $\begin{array}{l}\text { DEPTH } \\
\text { (FT) }\end{array}$ & $\begin{array}{l}\text { TERPERATL } \\
\text { (C) }\end{array}$ \\
\hline $\begin{array}{l}10 \\
15 \\
20 \\
25 \\
30 \\
35 \\
10 \\
15 \\
50 \\
55 \\
60 \\
655 \\
70 \\
75 \\
80 \\
85 \\
90 \\
95 \\
100 \\
105 \\
110 \\
115 \\
120 \\
125 \\
130 \\
135 \\
140 \\
145 \\
150 \\
155 \\
160 \\
165 \\
170 \\
175 \\
180 \\
185 \\
190 \\
195 \\
200 \\
205 \\
210 \\
215 \\
220 \\
225 \\
230 \\
235 \\
240 \\
245 \\
250 \\
255 \\
260 \\
2655 \\
270 \\
275 \\
280 \\
285 \\
290 \\
295 \\
300 \\
305 \\
310 \\
315 \\
320 \\
325 \\
330 \\
335 \\
340 \\
345 \\
350 \\
355\end{array}$ & $\begin{array}{l}21.916 \\
23.829 \\
25.602 \\
27.053 \\
29.446 \\
30.976 \\
33.346 \\
35.336 \\
37.116 \\
38.929 \\
.40 .507 \\
11.984 \\
42.956 \\
44.015 \\
44.854 \\
45.704 \\
46.382 \\
42.050 \\
49.650 \\
51.205 \\
51.559 \\
51.997 \\
52.315 \\
52.753 \\
53.384 \\
53.990 \\
54.568 \\
55.171 \\
55.887 \\
56.635 \\
57.469 \\
58.247 \\
58.864 \\
59.682 \\
60.496 \\
61.270 \\
62.065 \\
62.891 \\
63.787 \\
64.769 \\
65.484 \\
66.310 \\
67.213 \\
68.009 \\
69.143 \\
70.283 \\
72.288 \\
73.249 \\
74.088 \\
74.341 \\
74.592 \\
74.838 \\
75.115 \\
75.363 \\
75.688 \\
76.058 \\
76.413 \\
76.809 \\
77.335 \\
77.843 \\
78.303 \\
79.336 \\
82.667 \\
84.301 \\
84.541 \\
91.423 \\
90.422 \\
90.553 \\
90.619 \\
90.541\end{array}$ & $\begin{array}{l}360 \\
365 \\
370 \\
375 \\
390 \\
385 \\
390 \\
395 \\
400 \\
405 \\
408\end{array}$ & $\begin{array}{l}90.858 \\
90.922 \\
92.561 \\
92.521 \\
92.584 \\
92.611 \\
92.611 \\
92.624 \\
92.641 \\
92.617 \\
92.647\end{array}$ \\
\hline
\end{tabular}


RAFT RIVER, IDAHO - SHA WELL - 16 JANUAY 1976

DEPTH TEYPERATUPE DEPTH TEPPERATURE
(FT) (C)

$\begin{array}{rrrr}8 & 20.192 & 355 & 91.844 \\ 10 & 21.740 & 360 & 92.097 \\ 15 & 23.550 & 365 & 92.169 \\ 20 & 25.226 & 370 & 92.559 \\ 25 & 27.042 & 375 & 93.755 \\ 30 & 28.951 & 330 & 93.892 \\ 35 & 31.085 & 395 & 93.901 \\ 30 & 32.918 & 390 & 93.911 \\ 15 & 35.375 & 395 & 93.917 \\ 50 & 37.288 & 400 & 93.920 \\ 55 & -38.810 & 405 & 93.927 \\ 60 & 40.311 & 408 & 93.927\end{array}$

6542.027

$70 \quad 13.038$

$75 \quad 44.044$

$85 \quad 45.737$

$90 \quad 16.551$

$95 \quad 18.139$

$100-49.743$

$105 \quad 51.506$

$115 \quad 52.173$

$120 \quad 52.604$

$125 \quad 53.041$

$130 \quad 53.619$

$135 \quad 54.267$

$140 \quad 55.033$

$150 \quad 56.341$

$155 \quad 57.110$

$160 \quad 57.932$

$165 \quad 58.537$

$170 \quad 59.348$

$175 \quad 60.171$

$180 \quad 61.090$

$185 \quad 61.712$

$190 \quad 62.672$

$195 \quad 63.517$

$200 \quad 64.313$

$205 \quad 65.365$

$210 \quad 66.139$

$215 \quad 67.054$

$220 \quad 67.95$

225068.722

$235 \quad 71.400$

$240 \quad 73.044$

$245 \quad 74.176$

$350 \quad 75.001$

$255 \quad 75.22$

$260 \quad 75.46$

$265 \quad 75.727$

$270 \quad 75.95 t$

$275 \quad 76.275$

$280 \quad 76.579$

$285 \quad 75.953$

$290 \quad 77.279$

$295 \quad 77.773$

$300 \quad 78.215$

$305 \quad 78.733$

$310 \quad 79.211$

$315 \quad 80.160$

$320 \quad 83.577$

325

85.232

85.618

90.547

91.620

91.352

$350 \quad 91.745$ 
RAFT RIVER, IOFHO - SHA WELL - 8 FEBRUARY 1976

DEPTH TEPERATURE DEPTH TERERATLRE
(FT) (C)

$\begin{array}{rrrr}9 & 21.076 & 355 & 91.921 \\ 10 & 21.417 & 360 & 92.158 \\ 15 & 23.112 & 365 & 92.275 \\ 20 & 24.872 & 370 & 93.608 \\ 25 & 26.972 & 375 & 93.921 \\ 30 & 28.997 & 330 & 94.006 \\ 35 & 30.949 & 395 & 94.069 \\ 40 & 32.962 & 390 & 94.078 \\ 45 & 35.125 & 395 & 94.095 \\ 50 & 37.112 & 400 & 94.075 \\ 55 & .38 .720 & 105 & 94.098 \\ 50 & 40.195 & 109 & 91.078\end{array}$

$\begin{array}{llll}65 & 40.195 & 408 \quad 94.078\end{array}$

$70 \quad 43.142$

$75 \quad 43.991$

$80 \quad 45.006$

$85 \quad 45.799$

$90 \quad 46.492$

$95 \quad 48.300$

$105 \quad 51.684$

$110 \quad 51.876$

$115 \quad 52.219$

$120 \quad 52.651$

$125 \quad 53.158$

$130 \quad 53.763$

$135 \quad 54.347$

$140 \quad 54.999$

$145 \quad 55.564$

$150 \quad 56.351$

$155 \quad 57.084$

$160 \quad 57.901$

$170 \quad 59.107$

$175 \quad 60.033$

$180 \quad 60.912$

$185 \quad 61.712$

$190 \quad 62.561$

$195 \quad 53.523$

$200 \quad 64.446$

$205 \quad 65.241$

$210 \quad 66.031$

$215 \quad 66.950$

$200 \quad 67.784$

$225 \quad 68.632$

$230 \quad 69.853$

$235 \quad 71.240$

$240 \quad 73.413$

$245 \quad 74.204$

$250 \quad 75.025$

$2555 \quad 75.227$

$260 \quad 75.473$

$265 \quad 75.741$

$270 \quad 75.992$

$275 \quad 76.275$

$280 \quad 76.613$

$285 \quad 76.920$

$290 \quad 77.36$

$295 \quad 77.792$

$300 \quad 78.264$

$305 \quad 78.769$

$310 \quad 79.322$

$315 \quad 80.140$

$320 \quad 82.734$

$325 \quad 85.530$

$330 \quad 85.870$

$335 \quad 90.542$

$340 \quad 91.793$

$345 \quad 91.981$ 
RAFT RIVER, IDAHO - SWW WELL - 14 MRCH 1976

DEPTH TEMPERATURE DEPTH TEPERATUPE
(FT) (C)

\begin{tabular}{|c|c|c|}
\hline $\begin{array}{l}8 \\
10 \\
15 \\
20 \\
25 \\
30 \\
35 \\
10 \\
45 \\
50 \\
55 \\
60 \\
65 \\
70 \\
75 \\
80 \\
85 \\
90 \\
95 \\
100 \\
105 \\
110 \\
15 \\
120 \\
25 \\
130 \\
135 \\
140 \\
145 \\
150 \\
155 \\
160 \\
65 \\
70 \\
75 \\
180 \\
85\end{array}$ & $\begin{array}{l}19.321 \\
20.484 \\
22.434 \\
24.158 \\
26.398 \\
28.593 \\
30.367 \\
32.674 \\
34.922 \\
36.818 \\
33.793 \\
39.963 \\
41.511 \\
43.112 \\
44.018 \\
44.931 \\
45.627 \\
46.568 \\
47.900 \\
19.995 \\
51.548 \\
51.819 \\
52.104 \\
55.499 \\
52.976 \\
53.532 \\
54.202 \\
54.818 \\
55.440 \\
56.128 \\
56.741 \\
57.464 \\
58.393 \\
59.165 \\
59.922 \\
60.681 \\
61.532 \\
62.312 \\
63.146 \\
64.070 \\
65.109 \\
65.847 \\
65.806 \\
67.197 \\
68.268 \\
69.588 \\
70.717 \\
72.583 \\
73.893 \\
74.725 \\
75.005 \\
75.275 \\
75.551 \\
75.860 \\
73.114 \\
76.121 \\
76.823 \\
77.248 \\
77.578 \\
78.045 \\
78.619 \\
79.140 \\
79.807 \\
82.341 \\
85.520 \\
85.679 \\
8.148 \\
91.815 \\
91.844 \\
91.937\end{array}$ & $\begin{array}{l}355 \\
360 \\
355 \\
370 \\
375 \\
390 \\
385 \\
390 \\
395 \\
100 \\
405 \\
110\end{array}$ \\
\hline
\end{tabular}


RAFT RIVER, IDAHD - SHW HELL - 14 ALEUST 1976

\begin{tabular}{|c|c|c|c|c|c|}
\hline $\begin{array}{l}\text { DEPTH } \\
\text { (FT) }\end{array}$ & $\begin{array}{l}\text { TEMPERATURE } \\
\text { (C) }\end{array}$ & $\begin{array}{l}\text { DEPTH } \\
\text { (FT) }\end{array}$ & $\begin{array}{l}\text { TEMPERATLRE } \\
\text { (C) }\end{array}$ & $\begin{array}{c}\text { DEPTH } \\
\text { (FT) }\end{array}$ & $\begin{array}{l}\text { TEMPERAT } \\
\text { (C) }\end{array}$ \\
\hline $\begin{array}{l}14 \\
16 \\
18 \\
20 \\
22 \\
24 \\
26 \\
28 \\
30 \\
32 \\
34 \\
36 \\
39 \\
40 \\
42 \\
44 \\
46 \\
50 \\
52 \\
54 \\
56 \\
58 \\
60 \\
62 \\
64 \\
66 \\
68 \\
70 \\
72 \\
74 \\
76 \\
78 \\
80 \\
82 \\
84 \\
86 \\
88 \\
90 \\
92 \\
91 \\
96 \\
98 \\
100 \\
102 \\
104 \\
106 \\
108 \\
110 \\
112 \\
114 \\
116 \\
118 \\
120 \\
122 \\
124 \\
126 \\
128 \\
130 \\
132 \\
134 \\
136 \\
138 \\
140 \\
142 \\
144 \\
146 \\
148 \\
150 \\
152 \\
154\end{array}$ & $\begin{array}{l}23.933 \\
23.946 \\
24.194 \\
24.615 \\
25.151 \\
25.693 \\
26.285 \\
27.020 \\
27.732 \\
28.305 \\
.29 .075 \\
30.022 \\
30.934 \\
31.580 \\
32.616 \\
33.359 \\
34.058 \\
35.768 \\
36.630 \\
37.306 \\
37.011 \\
38.541 \\
39.276 \\
39.669 \\
40.576 \\
41.021 \\
41.729 \\
42.300 \\
42.745 \\
13.156 \\
43.507 \\
43.852 \\
44.245 \\
41.525 \\
44.832 \\
45.135 \\
45.500 \\
45.910 \\
46.356 \\
46.532 \\
47.654 \\
48.280 \\
48.998 \\
49.721 \\
50.010 \\
50.859 \\
50.892 \\
51.404 \\
51.642 \\
51.790 \\
51.923 \\
52.067 \\
52.229 \\
52.106 \\
52.556 \\
52.767 \\
52.951 \\
53.144 \\
53.362 \\
53.648 \\
53.909 \\
54.179 \\
54.109 \\
54.628 \\
54.849 \\
55.052 \\
55.332 \\
55.586 \\
55.890 \\
56.170\end{array}$ & $\begin{array}{l}156 \\
158 \\
160 \\
162 \\
164 \\
166 \\
168 \\
170 \\
172 \\
174 \\
176 \\
178 \\
180 \\
182 \\
184 \\
186 \\
188 \\
190 \\
192 \\
194 \\
196 \\
198 \\
200 \\
202 \\
204 \\
206 \\
208 \\
210 \\
212 \\
214 \\
216 \\
218 \\
220 \\
222 \\
224 \\
226 \\
228 \\
230 \\
232 \\
234 \\
236 \\
238 \\
242 \\
244 \\
246 \\
248 \\
250 \\
252 \\
254 \\
256 \\
258 \\
260 \\
262 \\
264 \\
266 \\
268 \\
270 \\
272 \\
274 \\
276 \\
278 \\
280 \\
282 \\
284 \\
286 \\
288 \\
290 \\
292 \\
294 \\
296\end{array}$ & $\begin{array}{l}55.453 \\
56.728 \\
57.037 \\
57.322 \\
57.640 \\
57.925 \\
58.225 \\
58.547 \\
58.895 \\
59.186 \\
59.452 \\
59.772 \\
50.047 \\
60.370 \\
60.742 \\
61.087 \\
61.376 \\
61.709 \\
62.066 \\
62.384 \\
52.684 \\
63.061 \\
63.390 \\
63.718 \\
34.090 \\
64.456 \\
64.919 \\
65.178 \\
55.446 \\
65.869 \\
65.226 \\
66.544 \\
66.866 \\
67.191 \\
67.456 \\
67.916 \\
69.206 \\
68.566 \\
39.061 \\
69.636 \\
70.246 \\
70.772 \\
72.391 \\
72.789 \\
73.147 \\
73.553 \\
73.920 \\
74.181 \\
74.363 \\
74.507 \\
74.627 \\
74.738 \\
74.838 \\
74.944 \\
75.048 \\
75.196 \\
75.315 \\
75.431 \\
75.555 \\
75.679 \\
75.751 \\
75.902 \\
76.133 \\
76.281 \\
76.462 \\
76.624 \\
76.8002 \\
76.991 \\
77.180 \\
77.358\end{array}$ & $\begin{array}{l}298 \\
300 \\
302 \\
301 \\
305 \\
308 \\
310 \\
312 \\
314 \\
316 \\
318 \\
320 \\
322 \\
324 \\
326 \\
328 \\
330 \\
332 \\
334 \\
336 \\
338 \\
340 \\
342 \\
344 \\
346 \\
348 \\
350 \\
352 \\
354 \\
356 \\
358 \\
360 \\
352 \\
364 \\
356 \\
368 \\
370 \\
372 \\
374 \\
376 \\
378 \\
380 \\
382 \\
384 \\
386 \\
388 \\
390 \\
392 \\
394 \\
396 \\
398 \\
400 \\
402 \\
404 \\
406\end{array}$ & $\begin{array}{l}77.571 \\
77.787 \\
77.993 \\
78.166 \\
79.392 \\
78.614 \\
78.820 \\
79.120 \\
74.526 \\
80.276 \\
81.003 \\
82.552 \\
84.128 \\
84.876 \\
85.249 \\
85.120 \\
85.704 \\
86.366 \\
88.241 \\
90.742 \\
91.500 \\
91.652 \\
91.733 \\
91.757 \\
91.789 \\
91.797 \\
91.749 \\
91.789 \\
91.833 \\
91.860 \\
91.914 \\
92.036 \\
92.114 \\
92.159 \\
92.187 \\
92.129 \\
93.040 \\
93.508 \\
93.659 \\
93.756 \\
93.800 \\
93.857 \\
93.893 \\
93.894 \\
93.905 \\
93.899 \\
93.910 \\
93.920 \\
93.926 \\
93.933 \\
93.933 \\
93.943 \\
93.949 \\
93.946 \\
93.947\end{array}$ \\
\hline
\end{tabular}


RAFT RIVER, IONHO - NUNO NO. 1 - 4 FEBRUAFY 1976

\begin{tabular}{|c|c|c|}
\hline $\begin{array}{l}\text { OEPTH } \\
\text { (FT) }\end{array}$ & $\begin{array}{l}\text { TEPPERATURE } \\
\text { (C) }\end{array}$ & $\begin{array}{c}\text { DEPTH } \\
\text { (FT) }\end{array}$ \\
\hline $\begin{array}{l}10 \\
15 \\
20 \\
25 \\
30 \\
35 \\
40 \\
15 \\
50 \\
55 \\
80 \\
65 \\
70 \\
75 \\
80 \\
85 \\
90 \\
95 \\
100 \\
105 \\
110 \\
115 \\
120 \\
125 \\
130 \\
135 \\
140 \\
145 \\
150 \\
155 \\
160 \\
165 \\
170 \\
175 \\
180 \\
185 \\
190 \\
195 \\
200 \\
205 \\
210 \\
215 \\
220 \\
225 \\
230 \\
235 \\
240 \\
245 \\
250 \\
255 \\
250 \\
265 \\
270 \\
275 \\
280 \\
285 \\
290 \\
295 \\
300 \\
305 \\
310 \\
315 \\
320 \\
325 \\
330 \\
335 \\
340 \\
345 \\
350 \\
355\end{array}$ & 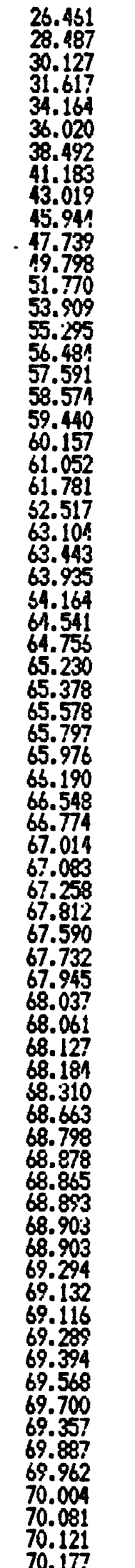 & $\begin{array}{l}350 \\
365 \\
370 \\
375 \\
380 \\
335 \\
390 \\
395 \\
400 \\
405 \\
410 \\
415 \\
420 \\
425 \\
430 \\
435 \\
440 \\
445 \\
450 \\
455 \\
460 \\
465 \\
470 \\
475 \\
480 \\
485 \\
490 \\
495 \\
500 \\
505 \\
510 \\
515 \\
520 \\
525 \\
530 \\
535 \\
540\end{array}$ \\
\hline
\end{tabular}


RAFT RIVER, IDAHO - NLMO NO. 1 - 9 FEERUYARY 1976

\begin{tabular}{|c|c|c|c|}
\hline $\begin{array}{l}\text { DEPTH } \\
\text { (FT) }\end{array}$ & $\begin{array}{l}\text { TEPPERATURE } \\
\text { (C) }\end{array}$ & $\begin{array}{l}\text { DEPTH } \\
\text { (FT) }\end{array}$ & $\begin{array}{l}\text { TEPEPATURE } \\
\text { (C) }\end{array}$ \\
\hline $\begin{array}{l}5 \\
10 \\
15 \\
20 \\
25 \\
30 \\
355 \\
10 \\
45 \\
50 \\
55 \\
60 \\
65 \\
70 \\
75 \\
80 \\
855 \\
90 \\
95 \\
100 \\
105 \\
110 \\
115 \\
120 \\
125 \\
130 \\
135 \\
140 \\
145 \\
150 \\
155 \\
160 \\
165 \\
170 \\
175 \\
180 \\
185 \\
190 \\
195 \\
200 \\
205 \\
210 \\
215 \\
220 \\
225 \\
230 \\
235 \\
240 \\
245 \\
250 \\
255 \\
260 \\
265 \\
270 \\
275 \\
280 \\
285 \\
290 \\
295 \\
300 \\
305 \\
310 \\
315 \\
320 \\
325 \\
330 \\
335 \\
340 \\
345 \\
350\end{array}$ & $\begin{array}{l}41.108 \\
42.459 \\
41.485 \\
46.136 \\
47.370 \\
48.739 \\
49.897 \\
52.493 \\
53.729 \\
55.112 \\
56.577 \\
58.002 \\
59.203 \\
60.093 \\
60.771 \\
61.647 \\
62.151 \\
62.857 \\
63.344 \\
63.931 \\
64.374 \\
64.673 \\
64.868 \\
65.129 \\
65.340 \\
65.693 \\
65.890 \\
66.112 \\
55.470 \\
66.677 \\
65.990 \\
67.049 \\
67.225 \\
67.469 \\
67.722 \\
68.015 \\
68.327 \\
68.691 \\
68.962 \\
69.110 \\
69.212 \\
69.340 \\
69.455 \\
69.543 \\
69.608 \\
69.684 \\
59.7477 \\
69.807 \\
69.879 \\
69.930 \\
69.971 \\
70.026 \\
70.060 \\
70.088 \\
70.112 \\
70.1159 \\
70.205 \\
70.241 \\
70.292 \\
70.324 \\
70.338 \\
70.430 \\
70.550 \\
70.554 \\
70.305 \\
70.684 \\
70.733 \\
70.843 \\
70.735 \\
70.998\end{array}$ & $\begin{array}{l}355 \\
360 \\
355 \\
370 \\
375 \\
380 \\
395 \\
390 \\
395 \\
400 \\
405 \\
410 \\
415 \\
420 \\
425 \\
430 \\
435 \\
140 \\
445 \\
450 \\
455 \\
160 \\
465 \\
170 \\
475 \\
480 \\
485 \\
190\end{array}$ & $\begin{array}{l}71.054 \\
71.107 \\
71.147 \\
71.190 \\
71.244 \\
71.302 \\
71.360 \\
71.525 \\
71.630 \\
71.723 \\
71.820 \\
71.923 \\
72.010 \\
72.119 \\
72.192 \\
72.255 \\
72.358 \\
72.455 \\
72.547 \\
72.731 \\
72.915 \\
72.960 \\
72.995 \\
73.011 \\
73.088 \\
73.093 \\
73.006 \\
72.972\end{array}$ \\
\hline
\end{tabular}


RAT RIVER, IDAHO - ALMO MO. 1 - 7 ALULST 1976

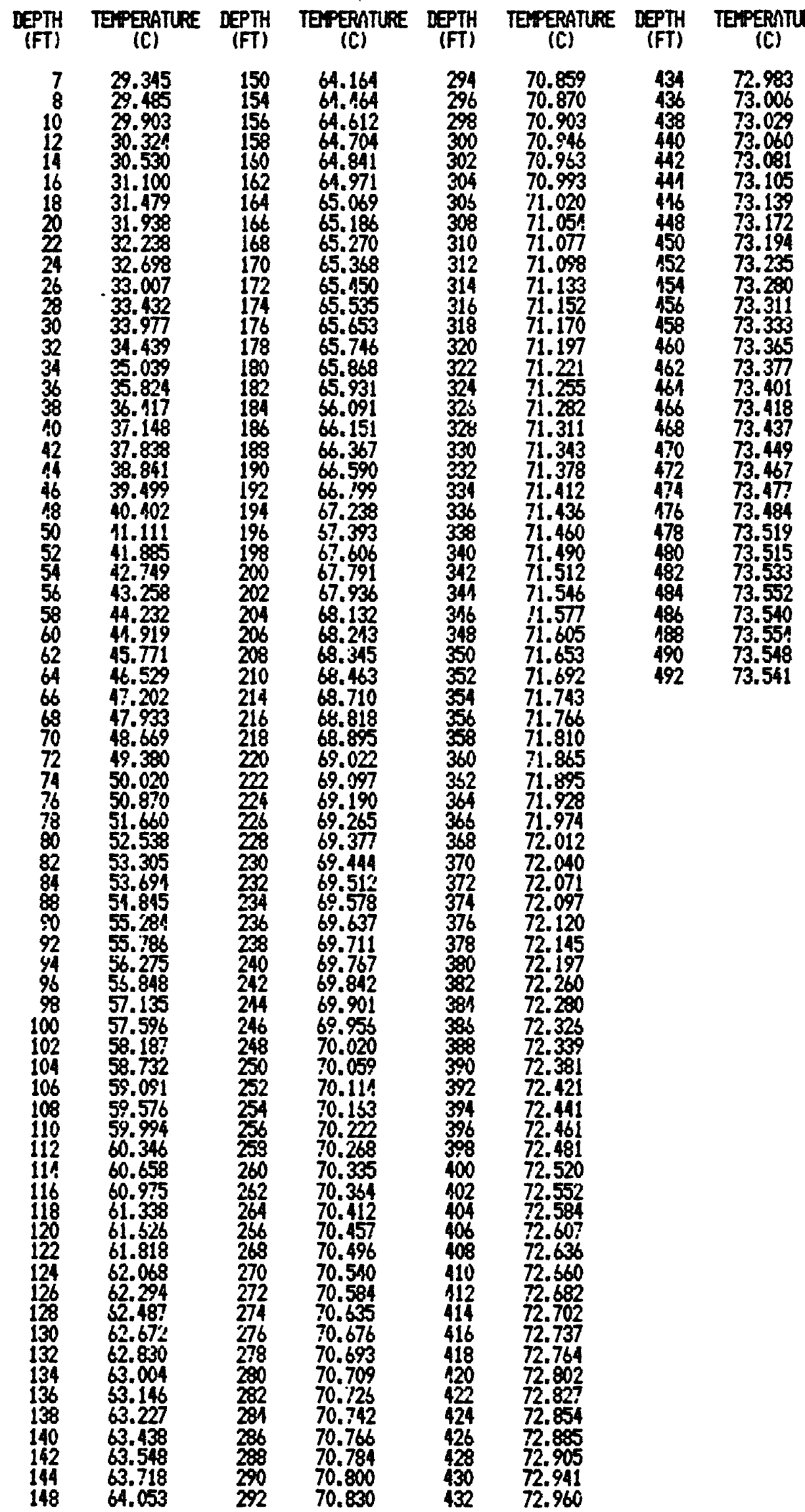


RAFT RIVER, IDAHO - ALMO NO. 2 - 3 FEBRIAAYY 1976

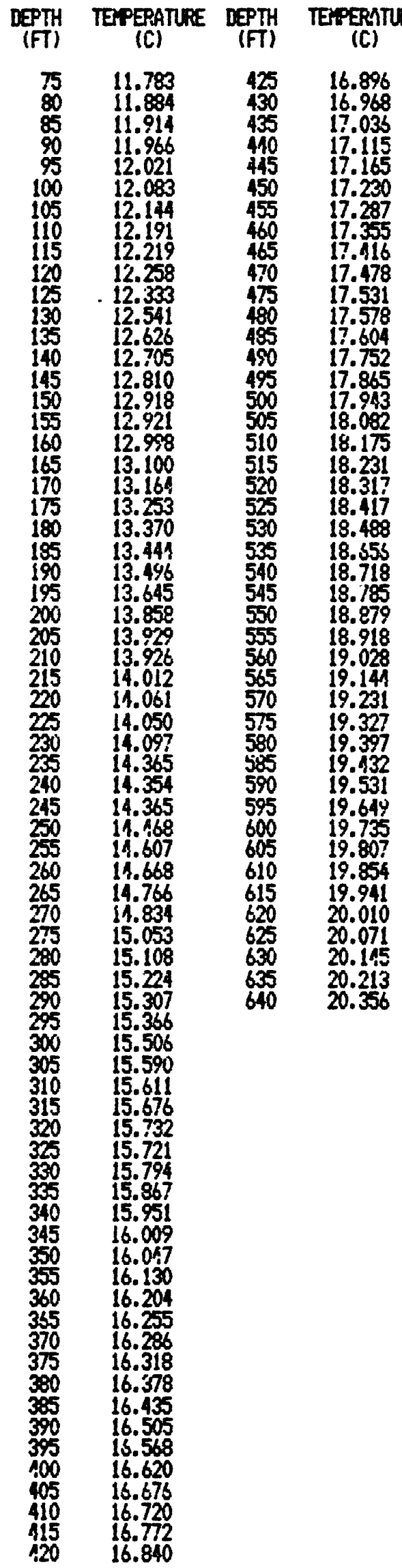


RAFT RIVER, IDAHO - ALMO MO. 2 - 5 FEBRLARY 1976

\begin{tabular}{|c|c|c|}
\hline $\begin{array}{l}\text { DEPTH } \\
\text { (FT) }\end{array}$ & $\begin{array}{l}\text { TERERATLRE } \\
\text { (C) }\end{array}$ & $\begin{array}{c}\text { DEPTH } \\
\text { (FT) }\end{array}$ \\
\hline $\begin{array}{l}64 \\
65 \\
70 \\
75 \\
80 \\
85 \\
90 \\
95 \\
100 \\
105 \\
110 \\
115 \\
120 \\
125 \\
130 \\
135 \\
140 \\
145 \\
150 \\
155 \\
160 \\
165 \\
170 \\
175 \\
180 \\
185 \\
190 \\
195 \\
200 \\
205 \\
210 \\
215 \\
220 \\
225 \\
230 \\
235 \\
240 \\
245 \\
250 \\
255 \\
250 \\
265 \\
270 \\
275 \\
280 \\
285 \\
290 \\
295 \\
300 \\
305 \\
310 \\
315 \\
320 \\
325 \\
330 \\
335 \\
340 \\
345 \\
3500 \\
355 \\
360 \\
355 \\
330 \\
375 \\
380 \\
385 \\
390 \\
395 \\
400 \\
105\end{array}$ & $\begin{array}{l}11.026 \\
11.105 \\
11.179 \\
12.321 \\
12.543 \\
12.785 \\
12.919 \\
13.112 \\
13.307 \\
13.446 \\
113.516 \\
13.567 \\
13.612 \\
13.650 \\
13.697 \\
13.757 \\
13.904 \\
13.980 \\
11.039 \\
11.053 \\
14.151 \\
11.219 \\
11.311 \\
14.396 \\
14.450 \\
14.494 \\
14.535 \\
14.571 \\
14.620 \\
14.674 \\
14.718 \\
14.869 \\
14.999 \\
15.076 \\
15.130 \\
15.209 \\
15.297 \\
15.472 \\
15.589 \\
15.727 \\
15.844 \\
15.933 \\
16.009 \\
16.072 \\
16.110 \\
16.167 \\
16.202 \\
16.243 \\
16.273 \\
16.325 \\
16.378 \\
16.426 \\
15.469 \\
16.518 \\
13.572 \\
16.624 \\
16.671 \\
16.711 \\
16.772 \\
16.840 \\
16.993 \\
17.001 \\
17.050 \\
17.104 \\
17.150 \\
17.196 \\
17.252 \\
17.312 \\
17.368\end{array}$ & $\begin{array}{l}410 \\
415 \\
420 \\
425 \\
430 \\
435 \\
440 \\
445 \\
450 \\
455 \\
460 \\
465 \\
170 \\
475 \\
490 \\
485 \\
490 \\
495 \\
500 \\
505 \\
510 \\
515 \\
520 \\
525 \\
530 \\
535 \\
540 \\
545 \\
550 \\
555 \\
560 \\
565 \\
570 \\
575 \\
580 \\
585 \\
590 \\
595 \\
600 \\
605 \\
510 \\
615 \\
620 \\
625 \\
630 \\
635 \\
640 \\
645 \\
650\end{array}$ \\
\hline
\end{tabular}


PAFT RIVER, IDAHD - ALMO ND. 2 - 6 FEBRUARY 1976

\begin{tabular}{|c|c|c|c|}
\hline $\begin{array}{l}\text { DEPTH } \\
\text { (FT) }\end{array}$ & $\begin{array}{l}\text { TEXPERATLRE } \\
\text { (C) }\end{array}$ & $\begin{array}{c}\text { DEPTH } \\
\text { (FT) }\end{array}$ & $\begin{array}{l}\text { TEPERATL } \\
\text { (C) }\end{array}$ \\
\hline $\begin{array}{l}65 \\
70 \\
75 \\
80 \\
85 \\
90 \\
95 \\
100 \\
105 \\
110 \\
115 \\
120 \\
125 \\
130 \\
135 \\
140 \\
145 \\
150 \\
155 \\
160 \\
165 \\
170 \\
175 \\
180 \\
185 \\
190 \\
195 \\
200 \\
205 \\
210 \\
215 \\
220 \\
225 \\
230 \\
235 \\
240 \\
245 \\
250 \\
255 \\
260 \\
265 \\
270 \\
275 \\
280 \\
285 \\
290 \\
295 \\
300 \\
305 \\
310 \\
315 \\
320 \\
325 \\
330 \\
335 \\
340 \\
345 \\
350 \\
355 \\
360 \\
365 \\
370 \\
375 \\
330 \\
385 \\
390 \\
395 \\
400 \\
405 \\
410\end{array}$ & $\begin{array}{l}11.686 \\
12.038 \\
12.131 \\
12.273 \\
12.442 \\
12.593 \\
12.753 \\
12.907 \\
13.043 \\
13.123 \\
13.188 \\
13.241 \\
13.305 \\
13.341 \\
13.399 \\
13.433 \\
13.463 \\
13.485 \\
13.541 \\
13.611 \\
13.658 \\
13.735 \\
13.841 \\
13.910 \\
13.947 \\
13.983 \\
14.007 \\
14.047 \\
14.076 \\
14.122 \\
14.190 \\
14.285 \\
14.385 \\
14.139 \\
14.489 \\
14.579 \\
14.728 \\
14.961 \\
15.044 \\
15.145 \\
15.260 \\
15.377 \\
15.411 \\
15.539 \\
15.561 \\
15.608 \\
15.666 \\
15.725 \\
15.777 \\
15.839 \\
15.900 \\
15.965 \\
16.009 \\
16.066 \\
16.110 \\
16.177 \\
16.229 \\
16.300 \\
16.342 \\
16.407 \\
16.464 \\
16.514 \\
16.5773 \\
16.631 \\
16.691 \\
16.732 \\
16.796 \\
16.951 \\
16.909 \\
16.977\end{array}$ & $\begin{array}{l}415 \\
420 \\
425 \\
430 \\
435 \\
440 \\
445 \\
450 \\
455 \\
460 \\
465 \\
470 \\
475 \\
480 \\
485 \\
490 \\
495 \\
500 \\
505 \\
510 \\
515 \\
520 \\
525 \\
530 \\
535 \\
540 \\
545 \\
550 \\
555 \\
560 \\
565 \\
570 \\
575 \\
580 \\
585 \\
590 \\
595 \\
600 \\
605 \\
610 \\
615 \\
620 \\
625 \\
630 \\
635 \\
640 \\
645 \\
650 \\
655 \\
660 \\
6655\end{array}$ & $\begin{array}{l}17.039 \\
17.106 \\
17.159 \\
17.228 \\
17.286 \\
17.345 \\
17.396 \\
17.461 \\
17.519 \\
17.578 \\
17.638 \\
17.699 \\
17.795 \\
17.871 \\
17.895 \\
17.924 \\
17.928 \\
17.936 \\
17.945 \\
17.957 \\
17.976 \\
17.986 \\
17.991 \\
18.018 \\
18.059 \\
18.109 \\
18.160 \\
18.212 \\
18.268 \\
18.307 \\
18.378 \\
18.506 \\
18.787 \\
19.030 \\
19.299 \\
19.457 \\
19.655 \\
19.786 \\
19.898 \\
19.996 \\
20.057 \\
20.111 \\
20.222 \\
20.291 \\
20.368 \\
20.454 \\
20.522 \\
20.593 \\
20.646 \\
20.685 \\
20.707\end{array}$ \\
\hline
\end{tabular}


RAFT RIVER, IDAHO - ALHO NO. 2 - 7 FEERUARY 1976

\begin{tabular}{|c|c|c|c|}
\hline $\begin{array}{l}\text { DEPTH } \\
\text { (FT) }\end{array}$ & $\begin{array}{l}\text { TERPERATLRE } \\
\text { (C) }\end{array}$ & $\begin{array}{l}\text { DEPTH } \\
\text { (FT) }\end{array}$ & $\begin{array}{l}\text { TEPERAT } \\
\text { (C) }\end{array}$ \\
\hline $\begin{array}{l}65 \\
70 \\
75 \\
80 \\
85 \\
90 \\
995 \\
100 \\
105 \\
110 \\
115 \\
120 \\
125 \\
130 \\
135 \\
140 \\
145 \\
150 \\
155 \\
160 \\
165 \\
170 \\
175 \\
180 \\
185 \\
190 \\
195 \\
200 \\
205 \\
210 \\
215 \\
220 \\
225 \\
230 \\
2355 \\
240 \\
245 \\
250 \\
255 \\
2200 \\
265 \\
270 \\
275 \\
280 \\
2850 \\
290 \\
295 \\
300 \\
305 \\
310 \\
315 \\
320 \\
325 \\
330 \\
3355 \\
340 \\
345 \\
350 \\
335 \\
360 \\
365 \\
370 \\
375 \\
330 \\
335 \\
390 \\
395 \\
400 \\
405 \\
910\end{array}$ & $\begin{array}{l}11.696 \\
11.833 \\
11.926 \\
12.061 \\
12.175 \\
12.329 \\
12.476 \\
12.655 \\
12.825 \\
12.960 \\
13.043 \\
13.091 \\
13.130 \\
13.162 \\
13.199 \\
13.231 \\
13.251 \\
13.290 \\
13.330 \\
13.390 \\
13.441 \\
13.522 \\
13.627 \\
13.696 \\
13.721 \\
13.751 \\
13.785 \\
13.804 \\
13.847 \\
13.897 \\
14.009 \\
14.089 \\
14.162 \\
14.241 \\
14.289 \\
14.365 \\
11.450 \\
14.633 \\
14.752 \\
14.994 \\
15.084 \\
15.211 \\
15.287 \\
15.349 \\
15.21 \\
15.493 \\
15.568 \\
15.623 \\
15.663 \\
15.737 \\
15.813 \\
15.853 \\
15.900 \\
15.947 \\
16.019 \\
16.074 \\
16.135 \\
16.204 \\
16.270 \\
16.332 \\
16.403 \\
16.418 \\
16.509 \\
16.566 \\
16.620 \\
16.676 \\
16.740 \\
16.785 \\
16.845 \\
16.913\end{array}$ & $\begin{array}{l}415 \\
420 \\
425 \\
430 \\
435 \\
440 \\
445 \\
450 \\
455 \\
460 \\
465 \\
170 \\
475 \\
480 \\
485 \\
490 \\
495 \\
500 \\
505 \\
510 \\
515 \\
520 \\
525 \\
530 \\
535 \\
540 \\
545 \\
550 \\
555 \\
560 \\
565 \\
570 \\
575 \\
580 \\
590 \\
595 \\
600 \\
605 \\
610 \\
615 \\
620 \\
625 \\
630 \\
635 \\
640 \\
645 \\
650 \\
655 \\
660 \\
665\end{array}$ & $\begin{array}{l}16.977 \\
17.039 \\
17.100 \\
17.176 \\
17.243 \\
17.302 \\
17.364 \\
17.426 \\
17.497 \\
17.561 \\
17.614 \\
17.655 \\
17.771 \\
17.890 \\
17.907 \\
17.942 \\
17.942 \\
17.955 \\
17.968 \\
17.986 \\
17.995 \\
17.993 \\
17.993 \\
18.015 \\
18.063 \\
18.105 \\
18.140 \\
18.193 \\
18.251 \\
18.280 \\
18.350 \\
18.466 \\
18.724 \\
19.061 \\
19.477 \\
19.656 \\
19.793 \\
19.884 \\
19.995 \\
20.075 \\
20.152 \\
20.222 \\
20.295 \\
20.368 \\
20.441 \\
20.524 \\
20.579 \\
20.632 \\
20.659 \\
20.744\end{array}$ \\
\hline
\end{tabular}


RAFT RIVER, IDAHO - ALIO NO. 2 - 8 FEBRLARY 1976

\begin{tabular}{|c|c|c|c|}
\hline $\begin{array}{l}\text { DEPTH } \\
\text { (FT) }\end{array}$ & $\begin{array}{l}\text { TEPPERATLRE } \\
\text { (C) }\end{array}$ & $\begin{array}{l}\text { DEPTH } \\
\text { (FT) }\end{array}$ & $\begin{array}{l}\text { TEMPERATU } \\
\text { (C) }\end{array}$ \\
\hline $\begin{array}{l}65 \\
70 \\
75 \\
80 \\
85 \\
90 \\
95 \\
100 \\
105 \\
110 \\
115 \\
120 \\
125 \\
130 \\
135 \\
140 \\
145 \\
150 \\
155 \\
160 \\
165 \\
170 \\
175 \\
180 \\
185 \\
190 \\
195 \\
200 \\
205 \\
210 \\
215 \\
220 \\
225 \\
230 \\
235 \\
240 \\
245 \\
250 \\
255 \\
260 \\
265 \\
270 \\
275 \\
280 \\
205 \\
200 \\
295 \\
300 \\
305 \\
310 \\
315 \\
320 \\
325 \\
330 \\
335 \\
340 \\
345 \\
350 \\
355 \\
360 \\
365 \\
370 \\
375 \\
330 \\
385 \\
390 \\
395 \\
400 \\
405 \\
410\end{array}$ & $\begin{array}{l}11.585 \\
11.767 \\
11.872 \\
11.971 \\
12.112 \\
12.303 \\
12.523 \\
12.676 \\
12.834 \\
12.920 \\
12.974 \\
13.011 \\
13.040 \\
13.068 \\
13.095 \\
13.134 \\
13.169 \\
13.209 \\
13.254 \\
13.301 \\
13.360 \\
13.449 \\
13.528 \\
13.595 \\
13.632 \\
13.654 \\
13.685 \\
13.719 \\
13.742 \\
13.805 \\
13.884 \\
13.984 \\
14.061 \\
14.136 \\
14.195 \\
14.271 \\
14.342 \\
14.502 \\
14.619 \\
14.780 \\
14.937 \\
15.114 \\
15.216 \\
15.293 \\
15.364 \\
15.456 \\
15.513 \\
15.584 \\
15.641 \\
15.717 \\
15.763 \\
15.818 \\
15.865 \\
15.920 \\
15.980 \\
16.038 \\
16.119 \\
16.197 \\
16.251 \\
16.315 \\
15.3300 \\
16.445 \\
16.508 \\
16.566 \\
16.612 \\
16.670 \\
16.24 \\
16.788 \\
16.8555 \\
16.906\end{array}$ & $\begin{array}{l}415 \\
420 \\
425 \\
430 \\
435 \\
440 \\
415 \\
450 \\
455 \\
460 \\
465 \\
170 \\
475 \\
180 \\
485 \\
490 \\
495 \\
500 \\
505 \\
510 \\
515 \\
520 \\
525 \\
530 \\
535 \\
540 \\
545 \\
550 \\
555 \\
560 \\
565 \\
570 \\
575 \\
580 \\
585 \\
590 \\
595 \\
600 \\
605 \\
610 \\
615 \\
620 \\
625 \\
630 \\
635 \\
640 \\
645 \\
650 \\
655 \\
660 \\
665 \\
670\end{array}$ & $\begin{array}{l}16.983 \\
17.035 \\
17.116 \\
17.176 \\
17.249 \\
17.303 \\
17.365 \\
17.142 \\
17.525 \\
17.581 \\
17.653 \\
17.733 \\
17.796 \\
17.918 \\
17.951 \\
17.995 \\
17.985 \\
17.991 \\
18.000 \\
18.013 \\
18.030 \\
18.038 \\
18.036 \\
18.017 \\
18.095 \\
18.142 \\
18.180 \\
18.229 \\
18.283 \\
18.319 \\
18.361 \\
18.451 \\
18.525 \\
18.993 \\
19.248 \\
19.171 \\
19.633 \\
19.751 \\
19.882 \\
19.977 \\
20.065 \\
20.114 \\
20.230 \\
20.296 \\
20.363 \\
20.148 \\
20.536 \\
20.602 \\
20.670 \\
20.720 \\
20.749 \\
20.956\end{array}$ \\
\hline
\end{tabular}


RAFT RIVER, IDAHO - NLM MO. 2 - 10 FEBRIARY 1976

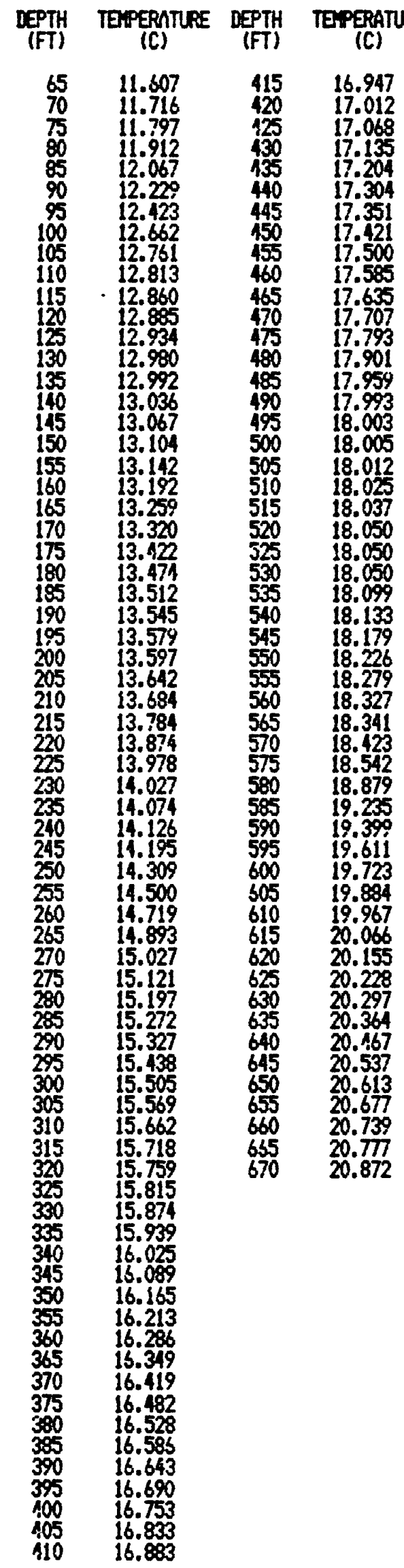


RAFT RIVER, IDAHO - ALMO NO. 2 - 13 MARCH 1976

\begin{tabular}{|c|c|c|c|}
\hline $\begin{array}{l}\text { DEPTH } \\
\text { (FT) }\end{array}$ & $\begin{array}{l}\text { TEPFERATURE } \\
\text { (C) }\end{array}$ & $\begin{array}{c}\text { DEPTH } \\
\text { (FT) }\end{array}$ & $\begin{array}{l}\text { TEPERATU } \\
\text { (C) }\end{array}$ \\
\hline $\begin{array}{l}65 \\
70 \\
75 \\
80 \\
85 \\
90 \\
95 \\
100 \\
105 \\
110 \\
115 \\
120 \\
125 \\
130 \\
135 \\
140 \\
145 \\
150 \\
155 \\
160 \\
165 \\
170 \\
175 \\
180 \\
185 \\
190 \\
195 \\
200 \\
205 \\
210 \\
215 \\
220 \\
225 \\
230 \\
235 \\
240 \\
245 \\
250 \\
255 \\
260 \\
265 \\
270 \\
275 \\
280 \\
285 \\
290 \\
295 \\
300 \\
305 \\
310 \\
315 \\
320 \\
325 \\
330 \\
335 \\
340 \\
345 \\
350 \\
355 \\
360 \\
365 \\
370 \\
3755 \\
3300 \\
335 \\
390 \\
395 \\
000 \\
405 \\
410\end{array}$ & 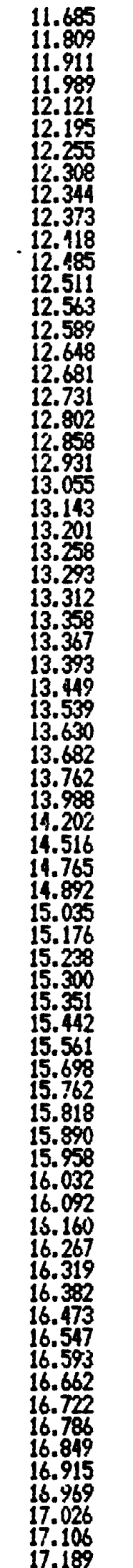 & $\begin{array}{l}415 \\
420 \\
125 \\
430 \\
435 \\
440 \\
445 \\
450 \\
155 \\
160 \\
465 \\
170 \\
475 \\
480 \\
495 \\
190 \\
495 \\
500 \\
505 \\
510 \\
515 \\
520 \\
525 \\
530 \\
535 \\
540 \\
545 \\
550 \\
555 \\
560 \\
565 \\
570 \\
575 \\
580 \\
585 \\
590 \\
595 \\
600 \\
605 \\
610 \\
615 \\
620 \\
625 \\
630 \\
635 \\
640 \\
645 \\
650 \\
655 \\
660 \\
665 \\
670\end{array}$ & $\begin{array}{l}17.251 \\
17.346 \\
17.394 \\
17.492 \\
17.564 \\
17.636 \\
17.687 \\
17.757 \\
17.795 \\
17.849 \\
17.897 \\
17.912 \\
17.974 \\
18.033 \\
18.064 \\
18.110 \\
18.097 \\
18.106 \\
18.114 \\
18.124 \\
18.133 \\
18.137 \\
18.133 \\
18.137 \\
18.169 \\
18.205 \\
18.241 \\
18.285 \\
18.337 \\
18.371 \\
18.383 \\
18.438 \\
18.579 \\
18.883 \\
19.153 \\
19.425 \\
19.660 \\
19.800 \\
19.975 \\
20.101 \\
20.190 \\
20.280 \\
20.372 \\
20.431 \\
20.524 \\
20.612 \\
20.678 \\
20.761 \\
20.830 \\
20.895 \\
20.950 \\
21.041\end{array}$ \\
\hline
\end{tabular}


RGFT RIVER, IDAHO - ALMO MO. 2 - 8 NUGUST 1976

\begin{tabular}{|c|c|c|c|c|c|c|c|c|c|}
\hline $\begin{array}{l}\text { IEPTH } \\
\text { (FT) }\end{array}$ & $\begin{array}{l}\text { TERPERATURE } \\
\text { (C) }\end{array}$ & $\begin{array}{c}\text { DEPTH } \\
\text { (FT) }\end{array}$ & $\begin{array}{l}\text { TEMPERATLE } \\
\text { (C) }\end{array}$ & $\begin{array}{c}\text { IEPTH } \\
\text { (FT) }\end{array}$ & $\begin{array}{l}\text { TEPEERATURE } \\
\text { (C) }\end{array}$ & $\begin{array}{l}\text { DEPTH } \\
\text { (FT) }\end{array}$ & $\begin{array}{l}\text { TERPERATLRE } \\
\text { (C) }\end{array}$ & $\begin{array}{c}\text { DEPTH } \\
\text { (FT) }\end{array}$ & $\begin{array}{l}\text { TEPERATURE } \\
\text { (C) }\end{array}$ \\
\hline $\begin{array}{r}2 \\
4 \\
6 \\
8 \\
10 \\
12 \\
14 \\
16 \\
18 \\
20 \\
22 \\
24 \\
26 \\
28 \\
30 \\
32 \\
34 \\
36 \\
39 \\
40 \\
42 \\
44 \\
46 \\
48 \\
50 \\
52 \\
54 \\
56 \\
58 \\
60 \\
62 \\
64 \\
66 \\
68 \\
70 \\
72 \\
74 \\
76 \\
78 \\
80 \\
82 \\
84 \\
86 \\
88 \\
90 \\
92 \\
94 \\
98 \\
98 \\
100 \\
102 \\
104 \\
106 \\
108 \\
110 \\
112 \\
114 \\
116 \\
118 \\
120 \\
122 \\
124 \\
126 \\
128 \\
130 \\
132 \\
134 \\
136 \\
138 \\
140\end{array}$ & $\begin{array}{l}27.373 \\
27.381 \\
27.357 \\
27.330 \\
27.300 \\
27.257 \\
27.208 \\
27.152 \\
27.103 \\
27.042 \\
26.996 \\
26.943 \\
26.917 \\
26.842 \\
26.813 \\
26.766 \\
26.724 \\
26.682 \\
26.645 \\
26.595 \\
26.558 \\
26.517 \\
26.475 \\
26.418 \\
26.381 \\
26.343 \\
26.304 \\
26.262 \\
26.221 \\
26.172 \\
26.131 \\
26.111 \\
26.014 \\
26.001 \\
25.953 \\
25.907 \\
25.866 \\
25.818 \\
25.773 \\
24.104 \\
15.016 \\
12.841 \\
12.297 \\
12.170 \\
12.163 \\
12.170 \\
12.184 \\
12.192 \\
12.203 \\
12.218 \\
12.226 \\
12.217 \\
12.264 \\
12.272 \\
12.308 \\
12.323 \\
12.340 \\
12.375 \\
12.399 \\
12.426 \\
12.446 \\
12.474 \\
12.196 \\
12.519 \\
12.555 \\
12.544 \\
12.590 \\
12.642 \\
12.639 \\
12.682\end{array}$ & $\begin{array}{l}142 \\
144 \\
146 \\
118 \\
150 \\
152 \\
154 \\
156 \\
158 \\
150 \\
162 \\
164 \\
166 \\
168 \\
170 \\
172 \\
174 \\
176 \\
178 \\
180 \\
182 \\
184 \\
186 \\
188 \\
190 \\
192 \\
194 \\
196 \\
198 \\
200 \\
202 \\
204 \\
206 \\
208 \\
210 \\
212 \\
214 \\
216 \\
218 \\
220 \\
222 \\
224 \\
226 \\
228 \\
230 \\
232 \\
234 \\
233 \\
238 \\
240 \\
242 \\
244 \\
246 \\
248 \\
250 \\
252 \\
254 \\
256 \\
258 \\
260 \\
262 \\
264 \\
266 \\
258 \\
270 \\
272 \\
274 \\
276 \\
278 \\
280\end{array}$ & $\begin{array}{l}12.710 \\
12.733 \\
12.714 \\
12.781 \\
12.805 \\
12.828 \\
12.853 \\
12.896 \\
12.921 \\
12.951 \\
12.978 \\
13.011 \\
13.039 \\
13.062 \\
13.097 \\
13.111 \\
13.147 \\
13.171 \\
13.208 \\
13.235 \\
13.260 \\
13.289 \\
13.314 \\
13.338 \\
13.365 \\
13.393 \\
13.423 \\
13.447 \\
13.476 \\
13.483 \\
13.521 \\
13.530 \\
13.548 \\
13.554 \\
13.602 \\
13.628 \\
13.662 \\
13.662 \\
13.704 \\
13.735 \\
13.752 \\
13.794 \\
13.818 \\
13.851 \\
13.897 \\
13.907 \\
13.942 \\
13.985 \\
11.027 \\
14.074 \\
14.167 \\
14.242 \\
14.318 \\
14.390 \\
14.433 \\
14.503 \\
14.543 \\
14.598 \\
14.661 \\
14.676 \\
14.736 \\
14.785 \\
14.829 \\
14.887 \\
11.938 \\
14.961 \\
14.997 \\
15.022 \\
15.043 \\
15.089\end{array}$ & $\begin{array}{l}282 \\
284 \\
286 \\
288 \\
290 \\
292 \\
294 \\
296 \\
298 \\
300 \\
302 \\
304 \\
306 \\
308 \\
310 \\
312 \\
314 \\
316 \\
318 \\
320 \\
322 \\
324 \\
326 \\
328 \\
330 \\
332 \\
334 \\
336 \\
338 \\
340 \\
342 \\
344 \\
316 \\
348 \\
350 \\
352 \\
354 \\
356 \\
358 \\
350 \\
362 \\
354 \\
366 \\
368 \\
370 \\
372 \\
374 \\
376 \\
378 \\
390 \\
382 \\
384 \\
3360 \\
388 \\
390 \\
392 \\
394 \\
396 \\
398 \\
400 \\
102 \\
404 \\
406 \\
408 \\
410 \\
412 \\
414 \\
416 \\
118 \\
420\end{array}$ & $\begin{array}{l}15.112 \\
15.153 \\
15.174 \\
15.204 \\
15.273 \\
15.304 \\
15.339 \\
15.391 \\
15.414 \\
15.432 \\
15.159 \\
15.494 \\
15.527 \\
15.560 \\
15.599 \\
15.610 \\
15.638 \\
15.679 \\
15.707 \\
15.756 \\
15.788 \\
15.813 \\
15.846 \\
15.883 \\
15.903 \\
15.942 \\
15.965 \\
16.010 \\
16.042 \\
16.065 \\
16.098 \\
16.119 \\
16.154 \\
16.180 \\
16.219 \\
16.210 \\
16.266 \\
16.287 \\
16.329 \\
16.361 \\
16.376 \\
16.412 \\
16.449 \\
16.477 \\
16.503 \\
16.532 \\
16.541 \\
16.565 \\
16.606 \\
16.642 \\
16.655 \\
16.677 \\
16.722 \\
16.750 \\
16.776 \\
16.786 \\
16.821 \\
16.849 \\
16.867 \\
16.896 \\
16.9907 \\
16.942 \\
16.966 \\
16.993 \\
17.014 \\
17.029 \\
17.073 \\
17.078 \\
17.125 \\
17.154\end{array}$ & $\begin{array}{l}422 \\
424 \\
426 \\
428 \\
430 \\
432 \\
434 \\
436 \\
438 \\
440 \\
442 \\
444 \\
446 \\
448 \\
450 \\
452 \\
454 \\
456 \\
458 \\
460 \\
162 \\
464 \\
466 \\
468 \\
470 \\
472 \\
474 \\
176 \\
478 \\
482 \\
484 \\
496 \\
498 \\
490 \\
492 \\
194 \\
496 \\
498 \\
500 \\
502 \\
504 \\
506 \\
508 \\
510 \\
512 \\
514 \\
516 \\
518 \\
520 \\
522 \\
524 \\
526 \\
528 \\
530 \\
532 \\
534 \\
536 \\
538 \\
540 \\
542 \\
541 \\
546 \\
548 \\
550 \\
552 \\
554 \\
5566 \\
558 \\
560 \\
\end{array}$ & $\begin{array}{l}17.181 \\
17.210 \\
17.238 \\
17.271 \\
17.305 \\
17.344 \\
17.374 \\
17.402 \\
17.446 \\
17.479 \\
17.515 \\
17.554 \\
17.735 \\
17.621 \\
17.652 \\
17.677 \\
17.723 \\
17.753 \\
17.767 \\
17.800 \\
17.831 \\
17.864 \\
17.876 \\
17.890 \\
17.920 \\
17.945 \\
17.969 \\
17.992 \\
18.034 \\
18.013 \\
18.053 \\
18.078 \\
18.075 \\
18.073 \\
18.082 \\
18.087 \\
18.092 \\
18.094 \\
18.099 \\
18.105 \\
18.105 \\
18.113 \\
18.136 \\
18.120 \\
18.127 \\
18.133 \\
18.127 \\
18.127 \\
18.131 \\
18.127 \\
18.124 \\
18.131 \\
18.140 \\
18.161 \\
18.172 \\
18.196 \\
18.209 \\
18.227 \\
18.255 \\
18.257 \\
18.280 \\
18.305 \\
18.335 \\
18.348 \\
18.355 \\
18.355 \\
18.403 \\
18.401 \\
18.111 \\
18.439\end{array}$ & $\begin{array}{l}564 \\
566 \\
568 \\
570 \\
572 \\
574 \\
576 \\
578 \\
580 \\
592 \\
584 \\
596 \\
568 \\
590 \\
592 \\
594 \\
596 \\
598 \\
600 \\
602 \\
604 \\
606 \\
608 \\
610 \\
612 \\
614 \\
616 \\
618 \\
620 \\
622 \\
624 \\
626 \\
628 \\
630 \\
632 \\
634 \\
636 \\
638 \\
640 \\
542 \\
644 \\
646 \\
648 \\
650 \\
652 \\
654 \\
656 \\
558 \\
660 \\
662 \\
664\end{array}$ & $\begin{array}{l}18.453 \\
18.509 \\
18.572 \\
18.612 \\
18.741 \\
18.914 \\
19.039 \\
19.146 \\
19.267 \\
19.381 \\
19.460 \\
19.555 \\
19.645 \\
19.712 \\
19.788 \\
19.855 \\
19.932 \\
19.998 \\
20.050 \\
20.104 \\
20.139 \\
20.203 \\
20.232 \\
20.266 \\
20.311 \\
20.350 \\
20.399 \\
20.409 \\
20.450 \\
20.511 \\
20.501 \\
20.551 \\
20.595 \\
20.606 \\
20.646 \\
20.674 \\
20.722 \\
20.726 \\
20.776 \\
20.816 \\
20.828 \\
20.870 \\
20.906 \\
20.939 \\
20.951 \\
20.979 \\
20.965 \\
20.999 \\
21.012 \\
21.032 \\
21.080\end{array}$ \\
\hline
\end{tabular}


RAFT RIVER, IDAHO - AH MO. I-S - 9 FEERIARY 1976

$\begin{array}{cc}\text { DEPTH } & \text { TEPERATURE } \\ \text { (FT) } & \text { (C) } \\ 33 & 13.893 \\ 33 & 14.181 \\ 34 & 11.282 \\ 35 & 14.344 \\ 36 & 14.392 \\ 37 & 11.429 \\ 38 & 11.465 \\ 39 & 11.45 \\ 10 & 11.512 \\ 41 & 14.558 \\ 12 & 14.619 \\ 43 & 14.706 \\ 4 & 14.770 \\ 45 & 14.820 \\ 46 & 14.875 \\ 47 & 14.882 \\ 48 & 11.923 \\ 49 & 15.019 \\ 50 & 15.067 \\ 51 & 15.147 \\ 52 & 15.166 \\ 53 & 15.212 \\ 54 & 15.285 \\ 55 & 15.356 \\ 56 & 15.397 \\ 57 & 15.170 \\ 58 & 15.519 \\ 59 & 15.627 \\ 60 & 15.757 \\ 61 & 15.811 \\ 62 & 15.897 \\ 63 & 15.943 \\ 64 & 16.010 \\ 65 & 16.072 \\ 66 & 16.137 \\ 67 & 16.250 \\ 68 & 16.297 \\ 69 & 16.360 \\ 70 & 16.479 \\ 71 & 16.574 \\ 72 & 16.611 \\ 73 & 16.637 \\ 74 & 16.692 \\ 75 & 16.779 \\ 76 & 16.860 \\ 77 & 16.920 \\ 78 & 17.016 \\ 70 & 17.091 \\ 80 & 17.121 \\ 81 & 17.205 \\ 82 & 17.304 \\ 83 & 17.408 \\ 84 & 17.459 \\ 85 & 17.509 \\ 86 & 17.540 \\ 87 & 17.596 \\ 88 & 17.681 \\ 84 & 17.755 \\ 90 & 17.802 \\ 91 & 17.882 \\ 92 & 17.903 \\ 93 & 17.933 \\ 94 & 17.963 \\ 95 & 18.048 \\ 96 & 18.306 \\ 97 & 18.413 \\ & \end{array}$


RAFT RIVER, IDAHO - AH NO. 1-S - 16 AUGUST 1976

DEPTH TEPERATLRE DEPTH TEPERATLRE
(FT)
(C)

\begin{tabular}{|c|c|}
\hline $\begin{array}{l}19.857 \\
19.858 \\
19.956 \\
19.857 \\
19.861 \\
19.862 \\
19.856 \\
19.811 \\
19.826 \\
19.807 \\
19.708 \\
19.691 \\
19.663 \\
19.638 \\
19.620 \\
19.595 \\
19.573 \\
19.554 \\
19.531 \\
19.515 \\
19.449 \\
19.485 \\
19.473 \\
19.457 \\
19.450 \\
10.135 \\
10.122 \\
19.412 \\
19.400 \\
18.798 \\
16.637 \\
15.501 \\
14.875 \\
14.590 \\
14.464 \\
14.911 \\
19.417 \\
14.426 \\
14.461 \\
11.527 \\
14.665 \\
14.732 \\
14.790 \\
14.830 \\
14.901 \\
14.940 \\
19.984 \\
15.043 \\
15.105 \\
15.153 \\
15.236 \\
15.311 \\
15.394 \\
15.451 \\
15.505 \\
15.558 \\
15.620 \\
15.673 \\
15.739 \\
15.823 \\
15.899 \\
15.991 \\
16.069 \\
16.142 \\
16.175 \\
16.256 \\
13.307 \\
16.398 \\
16.471 \\
16.551\end{array}$ & $\begin{array}{l}74 \\
75 \\
76 \\
77 \\
78 \\
79 \\
80 \\
81 \\
82 \\
83 \\
84 \\
85 \\
87 \\
88 \\
89 \\
90 \\
91 \\
92 \\
93 \\
94 \\
95 \\
96 \\
97 \\
98\end{array}$ \\
\hline
\end{tabular}


RAFT RIVER, IDAHO - AH NO. IA - 9 FEERLARY 1976

DEPTH TERERATRE

(FT) (C)

$14 \quad 34.798$

$15 \quad 38.875$

$16 \quad 41.833$

$17 \quad 13.559$

$19 \quad 46.896$

$20 \quad 48.462$

$21 \quad 49.589$

$22 \quad 51.164$

2352.106

$24 \quad 52.287$

$25 \quad 52.015$

$26 \quad 50.845$

50.172

$28 \quad 49.015$ 
RAFT RIVER, IDAHD - AH NO. IA - 16 AUGUST 1976

DEPTH TEPERATURE
(FT)

$\begin{array}{cc}1 & 23.870 \\ 2 & 23.886 \\ 3 & 23.918 \\ 4 & 23.951 \\ 5 & 23.998 \\ 6 & 24.057 \\ 1 & 24.103 \\ 8 & 24.174 \\ 9 & 24.232 \\ 10 & 24.291 \\ 11 & 24.357 \\ 12 & 24.123 \\ 13 & 21.510 \\ 14 & 24.568 \\ 15 & 24.718 \\ 16 & 24.805 \\ 17 & 24.949 \\ 19 & 25.181 \\ 20 & 25.317 \\ 21 & 25.450 \\ 22 & 25.542 \\ 23 & 30.498 \\ 24 & 40.092 \\ 25 & 45.738 \\ 26 & 40.554 \\ 27 & 50.318\end{array}$


RAFT RIVER, IDAHO - AH MD. 2A - 16 JANUARY 1976

DEPTH TEYPERATURE
(FT) (C)

$\begin{array}{ll}15 & 11.710 \\ 16 & 12.260 \\ 17 & 12.491 \\ 18 & 12.593 \\ 10 & 12.536 \\ 20 & 12.672 \\ 21 & 12.712 \\ 22 & 12.774\end{array}$


RAFT RIVER, IDAHO - AH NO. 2A - 16 NUGUST 1976

\section{DEPTH TEUPERITURE}

(FT) (C)

$\begin{array}{ll}1 & 13.021 \\ 2 & 13.094 \\ 3 & 13.174 \\ 1 & 13.24 \\ 5 & 13.322 \\ 5 & 13.386 \\ 6 & 13.450 \\ 7 & 13.450 \\ 8 & 13.516 \\ 9 & 13.773 \\ 10 & 13.607 \\ 11 & 13.676 \\ 12 & -13.718 \\ 13 & 13.754 \\ 14 & 13.789 \\ 15 & 13.752 \\ 16 & 13.045 \\ 17 & 12.359 \\ 18 & 11.952 \\ 19 & 11.720 \\ 20 & 11.577 \\ 21 & 11.515 \\ 22 & 11.505 \\ 22 & 11.537\end{array}$


RAFT RIVER, IDAHO - AH ND. 3A - 16 JAMUAPY 1976

DEPTH TEPPERTURE

(FT) (C)

$\begin{array}{cc}3 & 9.317 \\ 4 & 9.586 \\ 5 & 10.051 \\ 6 & 10.476 \\ 7 & 11.143 \\ 8 & 11.994 \\ 9 & 12.228 \\ 10 & 12.860 \\ 11 & 13.395 \\ 12 & 14.167 \\ 13 & 14.734 \\ 14 & 14.947 \\ 15 & 15.483 \\ 16 & 15.626 \\ 17 & 15.915 \\ 18 & 16.455 \\ 19 & 16.952 \\ 20 & 17.124 \\ 21 & 17.369 \\ 25 & 17.667 \\ 23 & 18.112 \\ 24 & 18.318 \\ 25 & 18.451 \\ 26 & 18.815 \\ 27 & 19.134 \\ 28 & 19.323 \\ 29 & 19.553 \\ 30 & 19.794 \\ 31 & 19.956 \\ 32 & 20.319 \\ 33 & 20.475 \\ 34 & 20.648 \\ 35 & 20.795 \\ 36 & 20.952 \\ 37 & 21.205\end{array}$


RATT RIVER, IINHD - NH MO. 3A - 16 AUCUST 1976

DEPTH TERPERATURE

(FT) (C)

$\begin{array}{ll}1 & 13.072 \\ 2 & 13.108 \\ 3 & 14.840 \\ 4 & 17.330 \\ 5 & 18.734 \\ 6 & 19.077 \\ 7 & 18.848 \\ 8 & 18.345 \\ 9 & 17.818 \\ 10 & 17.553 \\ 11 & 16.761 \\ 12 & 16.427 \\ 13 & 16.147 \\ 14 & 15.992 \\ 15 & 15.924 \\ 16 & 15.908 \\ 17 & 15.931 \\ 18 & 15.993 \\ 19 & 16.075 \\ 20 & 16.200 \\ 21 & 16.338 \\ 22 & 16.599 \\ 23 & 16.845 \\ 24 & 17.067 \\ 25 & 17.307 \\ 26 & 17.675 \\ 27 & 17.975 \\ 28 & 18.327 \\ 29 & 18.642 \\ 30 & 18.972 \\ 31 & 19.316 \\ 32 & 19.600 \\ 33 & 19.850 \\ 34 & 20.102 \\ 35 & 20.297 \\ 36 & 20.168 \\ 37 & 20.815\end{array}$

III -105 
PAFT RIVER, IDAHO - AH NO. 4 - 9 FEERUARY 1976

DEPTH TEPERATLRE

(FT) (C)

$\begin{array}{ll}20 & 12.985 \\ 21 & 13.299 \\ 22 & 13.414 \\ 23 & 13.166 \\ 21 & 13.513 \\ 25 & 13.544 \\ 26 & 13.570 \\ 27 & 13.621 \\ 28 & 13.644 \\ 29 & 13.697\end{array}$

$111-106$ 
RAFT RIVER, IOAHO - AH MO. IA - It AUCUST 1976

DEPTH TEPPERATURE

$\begin{array}{rc}\text { (FT) } & \text { (C) } \\ 1 & 16.856 \\ 2 & 16.863 \\ 3 & 16.909 \\ 1 & 16.931 \\ 5 & 16.950 \\ 6 & 16.968 \\ 7 & 16.994 \\ 8 & 16.995 \\ 9 & 16.994 \\ 10 & 16.989 \\ 11 & 16.981 \\ 13 & 16.959 \\ 14 & 16.942 \\ 15 & 16.924 \\ 16 & 16.917 \\ 17 & 16.903 \\ 18 & 13.764 \\ 19 & 15.310 \\ 20 & 14.122 \\ 21 & 13.566 \\ 22 & 13.242 \\ 23 & 13.101 \\ 24 & 13.062 \\ 25 & 13.061 \\ 26 & 13.065 \\ 27 & 13.137\end{array}$


RAFT RIVER, IDAHO - AH NO. 5 - 16 MUCUST 1976

$\begin{array}{cc}\begin{array}{c}\text { OEPTH } \\ \text { (FT) }\end{array} & \begin{array}{c}\text { TEPEERATUP } \\ \text { (C) }\end{array} \\ 1 & 18.320 \\ 2 & 18.317 \\ 3 & 18.390 \\ 4 & 18.428 \\ 5 & 18.457 \\ 6 & 18.494 \\ 7 & 18.520 \\ 8 & 18.530 \\ 9 & 18.541 \\ 10 & 18.577 \\ 11 & 18.541 \\ 12 & 18.542 \\ 13 & 18.539 \\ 14 & 18.539 \\ 15 & 18.542 \\ 16 & 18.539 \\ 17 & 18.513 \\ 18 & 18.549 \\ 19 & 18.553 \\ 20 & 18.566 \\ 21 & 18.578 \\ 22 & 18.587 \\ 23 & 18.601 \\ 24 & 18.616 \\ 25 & 18.633 \\ 26 & 19.343\end{array}$


RAFT RIVER, IDAHO - NH NO. 5 A - 9 FEBRUMAY 1976

$\begin{array}{cc}\text { DEPTH } & \text { TEPERATRR } \\ \text { (FT) } & \begin{array}{c}\text { (C) } \\ 9\end{array} \\ 4 & 13.745 \\ y & 13.908 \\ 10 & 14.143 \\ 11 & 14.978 \\ 12 & 15.540 \\ 13 & 15.956 \\ 14 & 16.340 \\ 15 & 16.487 \\ 16 & 16.811 \\ 17 & 17.140 \\ 18 & .17 .599 \\ 19 & 17.919 \\ 20 & 18.264 \\ 21 & 18.584 \\ 22 & 18.843 \\ 23 & 19.089 \\ 24 & 19.105 \\ 25 & 19.845 \\ 26 & 20.142 \\ 27 & 20.313 \\ 28 & 20.844 \\ 29 & 21.049 \\ 30 & 21.281 \\ 31 & 21.625 \\ 32 & 21.755 \\ 33 & 21.898 \\ 34 & 22.031 \\ 35 & 21.268 \\ 35 & 22.490 \\ 37 & 22.658 \\ 38 & 22.858 \\ 39 & 22.970 \\ 40 & 23.011 \\ 41 & 23.085 \\ 42 & 23.177\end{array}$


RAFT RIVER, IDAHO - AH KD. 5A - 16 ALCUST 1976

$\begin{array}{cc}\begin{array}{c}\text { DEPTH } \\ \text { (FT) }\end{array} & \begin{array}{c}\text { TEPERATURE } \\ (C)\end{array} \\ 1 & 18.263 \\ 2 & 18.319 \\ 3 & 18.349 \\ 4 & 18.132 \\ 5 & 18.478 \\ 6 & 18.424 \\ 7 & 18.139 \\ 8 & 17.703 \\ 9 & 17.237 \\ 10 & 16.915 \\ 11 & 16.755 \\ 12 & 16.448 \\ 13 & 16.621 \\ 11 & 16.645 \\ 15 & 16.739 \\ 16 & 16.897 \\ 17 & 17.044 \\ 18 & 17.253 \\ 19 & 17.495 \\ 20 & 17.717 \\ 21 & 17.936 \\ 22 & 18.217 \\ 23 & 18.558 \\ 24 & 18.780 \\ 25 & 19.274 \\ 26 & 19.471 \\ 27 & 19.747 \\ 28 & 20.076 \\ 29 & 20.405 \\ 30 & 20.652 \\ 31 & 20.917 \\ 32 & 21.220 \\ 33 & 21.483 \\ 34 & 21.758 \\ 35 & 21.933 \\ 36 & 22.144 \\ 37 & 22.335 \\ 38 & 22.564 \\ 39 & 22.714\end{array}$


RAFT RIVER, IDFH - AH NO. 6 - 9 FEERLARY 1976

\begin{tabular}{|c|c|c|c|}
\hline $\begin{array}{l}\text { DEPTH } \\
\text { (FT) }\end{array}$ & $\begin{array}{l}\text { TERERATRE } \\
\text { (C) }\end{array}$ & $\begin{array}{c}\text { DEPTH } \\
\text { (FT) }\end{array}$ & $\begin{array}{l}\text { TEPEERATL } \\
\text { (C) }\end{array}$ \\
\hline $\begin{array}{l}16 \\
17 \\
18 \\
10 \\
20 \\
21 \\
22 \\
23 \\
24 \\
25 \\
26 \\
27 \\
28 \\
29 \\
30 \\
31 \\
32 \\
33 \\
34 \\
35 \\
36 \\
37 \\
38 \\
39 \\
40 \\
41 \\
42 \\
43 \\
41 \\
15 \\
46 \\
77 \\
48 \\
19 \\
50 \\
51 \\
52 \\
53 \\
51 \\
55 \\
56 \\
57 \\
58 \\
50 \\
61 \\
62 \\
63 \\
64 \\
65 \\
66 \\
67 \\
68 \\
69 \\
70 \\
71 \\
72 \\
73 \\
74 \\
75 \\
76 \\
77 \\
78 \\
79 \\
80 \\
81 \\
82 \\
83 \\
84 \\
85\end{array}$ & $\begin{array}{l}9.330 \\
9.624 \\
9.764 \\
9.838 \\
9.895 \\
9.937 \\
10.029 \\
10.085 \\
10.159 \\
10.197 \\
10.294 \\
10.379 \\
10.439 \\
10.196 \\
10.550 \\
10.606 \\
10.674 \\
10.710 \\
10.730 \\
10.745 \\
10.754 \\
10.766 \\
10.778 \\
10.786 \\
10.800 \\
10.816 \\
10.832 \\
10.847 \\
10.663 \\
10.876 \\
10.890 \\
10.913 \\
10.947 \\
10.977 \\
11.016 \\
11.058 \\
11.088 \\
11.107 \\
11.132 \\
11.162 \\
11.186 \\
11.206 \\
11.227 \\
11.247 \\
111.267 \\
11.285 \\
11.313 \\
11.331 \\
11.354 \\
11.369 \\
11.397 \\
11.415 \\
11.436 \\
11.455 \\
11.177 \\
11.497 \\
11.520 \\
11.540 \\
11.566 \\
11.582 \\
11.611 \\
11.637 \\
11.655 \\
11.678 \\
11.698 \\
11.720 \\
11.741 \\
11.767 \\
11.799 \\
11.813\end{array}$ & $\begin{array}{l}86 \\
87 \\
88 \\
89 \\
90 \\
91 \\
92 \\
93\end{array}$ & $\begin{array}{l}11.836 \\
11.862 \\
11.988 \\
11.911 \\
11.930 \\
11.957 \\
11.987 \\
12.013\end{array}$ \\
\hline
\end{tabular}


FAFT RIVER, IDAHD - AH NO. 6 - 16 ALGUT 1976

\begin{tabular}{|c|c|c|c|}
\hline $\begin{array}{l}\text { DEPTH } \\
\text { (FT) }\end{array}$ & $\begin{array}{l}\text { TEPPERATLRE } \\
\text { (C) }\end{array}$ & $\begin{array}{c}\text { DEPTH } \\
\text { (FT) }\end{array}$ & $\begin{array}{l}\text { TEMPERATUR } \\
\text { (C) }\end{array}$ \\
\hline $\begin{array}{l}1 \\
2 \\
3 \\
1 \\
5 \\
6 \\
9 \\
10 \\
11 \\
12 \\
13 \\
14 \\
15 \\
16 \\
17 \\
18 \\
19 \\
20 \\
21 \\
22 \\
23 \\
24 \\
25 \\
26 \\
27 \\
28 \\
29 \\
30 \\
31 \\
32 \\
33 \\
34 \\
35 \\
36 \\
37 \\
38 \\
39 \\
40 \\
41 \\
42 \\
43 \\
44 \\
45 \\
46 \\
47 \\
48 \\
49 \\
50 \\
51 \\
52 \\
53 \\
54 \\
55 \\
58 \\
59 \\
60 \\
61 \\
62 \\
63 \\
64 \\
65 \\
66 \\
67 \\
68 \\
69 \\
70 \\
71 \\
72 \\
73\end{array}$ & 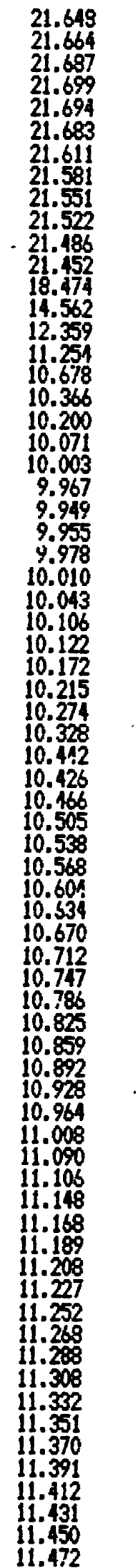 & $\begin{array}{l}74 \\
75 \\
76 \\
77 \\
78 \\
79 \\
80 \\
81 \\
82 \\
83 \\
84 \\
85 \\
86 \\
87 \\
88 \\
89\end{array}$ & $\begin{array}{l}11.492 \\
11.515 \\
11.537 \\
11.559 \\
11.578 \\
11.606 \\
11.626 \\
11.622 \\
11.671 \\
11.693 \\
11.720 \\
11.744 \\
11.771 \\
11.800 \\
11.829 \\
11.886\end{array}$ \\
\hline
\end{tabular}


RATT RIYER, IDAHO - AH MD. 6A - 16 AUEUET 1976

$\begin{array}{cc}\text { DEPTH } & \text { TEPERATLF } \\ \text { (FT) } & \text { (C) } \\ 1 & 19.192 \\ 2 & 19.239 \\ 2 & 19.300 \\ 3 & 19.378 \\ 4 & 10.487 \\ 5 & 21.596 \\ 6 & 25 \\ 7 & 23.673 \\ 8 & 25.367 \\ 9 & 26.080 \\ 10 & 26.697 \\ 11 & .27 .163 \\ 12 & 27.635 \\ 13 & 28.303 \\ 14 & 28.914 \\ 15 & 29.734 \\ 16 & 30.387 \\ 17 & 31.349 \\ 18 & 32.210 \\ 10 & 33.160 \\ 20 & 34.175 \\ 21 & 35.256 \\ 22 & 36.331 \\ 23 & 37.463 \\ 24 & 38.434 \\ 25 & 39.673 \\ 26 & 40.745 \\ 27 & 41.949 \\ 28 & 43.035 \\ 29 & 43.105 \\ 30 & 44.274 \\ 31 & 46.566 \\ 32 & 47.596 \\ 33 & 48.349 \\ 34 & 49.871 \\ 35 & 51.094 \\ 36 & 52.099 \\ 33 & 53.965 \\ 39 & 54.686 \\ 40 & 55.530 \\ 41 & 56.307 \\ 12 & 57.641\end{array}$


RAFT RIVER, IDAHO - AH NO. 7-N - 16 NUGUST 1976

DEPTH TEPERITLRE

(FT) (C)

$\begin{array}{cc}1 & 18.995 \\ 2 & 19.008 \\ 3 & 19.029 \\ 1 & 19.055 \\ 5 & 19.097 \\ 6 & 19.128 \\ 7 & 19.177 \\ 8 & 19.219 \\ 9 & 19.258 \\ 10 & 19.301 \\ 11 & 19.357 \\ 12 & 19.109 \\ 13 & 19.468 \\ 14 & 19.548 \\ 15 & 19.741 \\ 16 & 19.704 \\ 17 & 19.814 \\ 18 & 19.897 \\ 19 & 10.971 \\ 20 & 20.058 \\ 21 & 20.151 \\ 22 & 20.237 \\ 23 & 20.357 \\ 24 & 20.475 \\ 25 & 20.609 \\ 26 & 20.760 \\ 27 & 20.882 \\ 23 & 21.036 \\ 29 & 25.250 \\ 30 & 34.272 \\ 31 & 39.111 \\ 32 & 42.632 \\ 33 & 43.135 \\ 34 & 45.171\end{array}$


WAFT RIVER, IDAHO - AH NO. 7-5 - 16 JANLARY 1976

\begin{tabular}{|c|c|c|c|}
\hline $\begin{array}{l}\text { DEPTH } \\
\text { (FT) }\end{array}$ & $\begin{array}{l}\text { TERPERATLRE } \\
\text { (C) }\end{array}$ & $\begin{array}{c}\text { DEPTH } \\
\text { (FT) }\end{array}$ & $\begin{array}{l}\text { TEPERAT } \\
\text { (C) }\end{array}$ \\
\hline $\begin{array}{l}3 \\
4 \\
5 \\
6 \\
7 \\
8 \\
9 \\
10 \\
11 \\
12 \\
13 \\
14 \\
15 \\
16 \\
17 \\
18 \\
10 \\
20 \\
21 \\
22 \\
23 \\
24 \\
25 \\
26 \\
27 \\
28 \\
29 \\
30 \\
31 \\
32 \\
33 \\
34 \\
35 \\
36 \\
37 \\
38 \\
39 \\
40 \\
41 \\
12 \\
43 \\
44 \\
45 \\
46 \\
47 \\
18 \\
49 \\
50 \\
51 \\
52 \\
53 \\
54 \\
55 \\
36 \\
57 \\
58 \\
59 \\
60 \\
61 \\
62 \\
63 \\
64 \\
65 \\
66 \\
67 \\
68 \\
69 \\
70 \\
78\end{array}$ & $\begin{array}{l}15.211 \\
16.582 \\
17.997 \\
19.186 \\
20.595 \\
22.314 \\
23.975 \\
25.557 \\
27.283 \\
29.054 \\
30.427 \\
32.091 \\
33.079 \\
34.083 \\
35.563 \\
36.407 \\
37.405 \\
38.452 \\
30.555 \\
40.347 \\
41.097 \\
42.046 \\
12.871 \\
43.610 \\
44.341 \\
41.910 \\
45.379 \\
45.925 \\
46.244 \\
46.565 \\
46.851 \\
47.051 \\
47.358 \\
47.577 \\
47.739 \\
47.842 \\
47.895 \\
47.916 \\
47.844 \\
47.681 \\
47.502 \\
47.352 \\
47.175 \\
46.990 \\
46.853 \\
46.713 \\
46.573 \\
46.444 \\
46.318 \\
46.206 \\
46.065 \\
45 \\
45.956 \\
45.862 \\
45.748 \\
45.622 \\
45.491 \\
45.324 \\
44.181 \\
44.4130 \\
44.274 \\
43.124 \\
43.821 \\
43.672 \\
43.511 \\
43.333\end{array}$ & $\begin{array}{l}73 \\
74 \\
75 \\
76 \\
77 \\
78 \\
79 \\
80 \\
81 \\
82 \\
83 \\
84 \\
85 \\
86 \\
87 \\
88 \\
89 \\
90 \\
91 \\
92 \\
93 \\
94 \\
95 \\
96 \\
97 \\
98 \\
99\end{array}$ & $\begin{array}{l}13.190 \\
43.041 \\
12.892 \\
12.720 \\
42.569 \\
42.404 \\
42.265 \\
42.094 \\
41.938 \\
11.779 \\
41.611 \\
41.454 \\
41.342 \\
41.255 \\
41.177 \\
11.077 \\
40.988 \\
40.871 \\
40.773 \\
40.676 \\
40.596 \\
40.485 \\
40.110 \\
40.296 \\
40.210 \\
10.108 \\
40.066\end{array}$ \\
\hline
\end{tabular}


RAFT RIVER, IOAHO - AH NO. 7-S - 16 AUGUST 1976

\begin{tabular}{|c|c|c|c|}
\hline $\begin{array}{l}\text { DEPTH } \\
\text { (FT) }\end{array}$ & $\begin{array}{l}\text { TEAPERATLRE } \\
\text { (C) }\end{array}$ & $\begin{array}{l}\text { DEPTH } \\
\text { (FT) }\end{array}$ & $\begin{array}{l}\text { TEPERATRE } \\
\text { (C) }\end{array}$ \\
\hline $\begin{array}{l}1 \\
2 \\
3 \\
1 \\
5 \\
6 \\
7 \\
8 \\
9 \\
10 \\
11 \\
12 \\
13 \\
11 \\
15 \\
16 \\
17 \\
18 \\
19 \\
20 \\
21 \\
22 \\
23 \\
24 \\
25 \\
26 \\
27 \\
29 \\
30 \\
31 \\
32 \\
33 \\
35 \\
36 \\
37 \\
38 \\
39 \\
10 \\
41 \\
42 \\
43 \\
14 \\
15 \\
146 \\
47 \\
48 \\
49 \\
50 \\
51 \\
52 \\
53 \\
54 \\
55 \\
56 \\
57 \\
58 \\
59 \\
61 \\
62 \\
63 \\
64 \\
65 \\
66 \\
67 \\
68 \\
69 \\
70 \\
71 \\
72 \\
73\end{array}$ & $\begin{array}{l}21.836 \\
21.811 \\
22.474 \\
23.633 \\
24.638 \\
25.300 \\
25.917 \\
26.516 \\
27.052 \\
27.670 \\
28.176 \\
28.800 \\
29.433 \\
30.160 \\
30.907 \\
31.628 \\
32.417 \\
33.245 \\
31.140 \\
35.140 \\
35.954 \\
36.826 \\
37.682 \\
38.554 \\
39.437 \\
40.211 \\
41.110 \\
42.711 \\
43.439 \\
43.951 \\
44.456 \\
44.897 \\
45.563 \\
45.979 \\
46.283 \\
46.547 \\
46.766 \\
46.920 \\
47.024 \\
47.084 \\
47.087 \\
47.025 \\
46.942 \\
46.861 \\
46.774 \\
46.666 \\
46.567 \\
46.471 \\
46.384 \\
46.288 \\
46.192 \\
46.095 \\
46.002 \\
45.902 \\
45.800 \\
45.679 \\
45.559 \\
45.302 \\
45.165 \\
45.026 \\
44.894 \\
44.726 \\
44.589 \\
44.429 \\
44.289 \\
44.132 \\
43.975 \\
43.815 \\
43.674 \\
43.523\end{array}$ & $\begin{array}{l}71 \\
75 \\
76 \\
77 \\
78 \\
79 \\
80 \\
81 \\
82 \\
83 \\
84 \\
85 \\
86 \\
87 \\
88 \\
89 \\
90 \\
91 \\
92 \\
93 \\
94 \\
95 \\
93 \\
97 \\
98\end{array}$ & $\begin{array}{l}13.365 \\
43.201 \\
43.074 \\
42.906 \\
42.744 \\
42.591 \\
42.432 \\
42.286 \\
12.124 \\
11.961 \\
41.814 \\
41.648 \\
41.527 \\
41.407 \\
41.314 \\
41.207 \\
41.088 \\
41.002 \\
40.896 \\
40.801 \\
40.695 \\
40.595 \\
40.493 \\
40.007 \\
40.304\end{array}$ \\
\hline
\end{tabular}


RAFT RIVER, IDAHO - AH NO. 7A - 16 JAHUAY 1976

DEPTH TEPERATLRE

(FT) (C)

$5 \quad 20.979$

$6 \quad 21.297$

722.535

$8 \quad 23.353$

9 24.289

$10 \quad 25.259$

$11 \quad 26.673$

$13 \quad 28.858$

$14 \quad 29.791$

15.31 .305

$16 \quad 32.539$

$18 \quad 34.614$

$19 \quad 35.625$

$20 \quad 36.970$

$21 \quad 37.862$

38.707

3.625

40.158

40.934

41.643

42.404

43.005

43.449

43.997

44.254 
AAFT RIVER, IDAH - AH NO. 7A - 16 ALCUST 1976

LEPTH TEPEERTUPE

(FT) (C)

$\begin{array}{cc}1 & 21.203 \\ 2 & 21.224 \\ 3 & 21.249 \\ 1 & 21.299 \\ 5 & 21.347 \\ 6 & 23.332 \\ 7 & 25.338 \\ 8 & 26.472 \\ 9 & 27.096 \\ 10 & 27.557 \\ 11 & 28.034 \\ 12 & 28.066 \\ 13 & 28.566 \\ 14 & 29.720 \\ 15 & 30.47 \\ 16 & 31.253 \\ 17 & 32.132 \\ 18 & 32.965 \\ 20 & 34.842 \\ 21 & 35.911 \\ 22 & 36.677 \\ 23 & 37.540 \\ 24 & 38.476 \\ 25 & 39.569 \\ 26 & 40.384 \\ 27 & 41.256 \\ 28 & 41.979 \\ 29 & 42.629 \\ 30 & 43.164 \\ 31 & 41.101\end{array}$


RAFT RIVER, IDAHO - NH NO. 8A - 9 FEBRULARY 1976

$\begin{array}{cc}\begin{array}{c}\text { IEPTH } \\ \text { (FT) }\end{array} & \begin{array}{c}\text { TEIPERATR } \\ \text { (C) }\end{array} \\ 5 & 14.830 \\ 5 & 15.764 \\ 7 & 17.341 \\ 8 & 17.397 \\ 0 & 18.697 \\ 10 & 19.934 \\ 11 & 21.031 \\ 12 & 21.963 \\ 13 & 23.100 \\ 14 & 24.487 \\ 15 & 25.458 \\ 16 & 26.503 \\ 17 & 27.473 \\ 18 & 28.314 \\ 19 & 29.408 \\ 20 & 30.245 \\ 21 & 31.066 \\ 22 & 31.688 \\ 23 & 32.452 \\ 24 & 33.260 \\ 25 & 33.802 \\ 26 & 34.411 \\ 27 & 34.907 \\ 28 & 35514 \\ 29 & 35.625 \\ 30 & 35.995 \\ 31 & 36.160 \\ 32 & 36.287\end{array}$


RAFT RIVER, IDAHO - AH ND. 8A - 16 ALGUST 1976

$\begin{array}{cc}\begin{array}{c}\text { IEPTH } \\ \text { (FT) }\end{array} & \begin{array}{c}\text { TEMPERATU } \\ \text { (C) }\end{array} \\ 1 & 20.091 \\ 2 & 20.105 \\ 3 & 20.447 \\ 4 & 20.904 \\ 5 & 21.756 \\ 6 & 22.110 \\ 7 & 22.372 \\ 8 & 22.610 \\ 8 & 22.825 \\ 10 & 23.067 \\ 11 & 23.390 \\ 12 & 23.816 \\ 13 & 24.296 \\ 14 & 24.855 \\ 15 & 25.528 \\ 16 & 26.154 \\ 17 & 26.848 \\ 18 & 27.568 \\ 19 & 28.233 \\ 20 & 29.137 \\ 21 & 29.801 \\ 22 & 30.714 \\ 23 & 31.465 \\ 24 & 32.295 \\ 24 & 33.734\end{array}$


RAT RIVER, IDAH - AH NO. 9 - 9 FEERLARY 1976

$\begin{array}{cc}\text { DEPTH } & \text { TEPERATUR } \\ \text { (FT) } & \text { (C) } \\ 24 & 10.335 \\ 25 & 10.447 \\ 26 & 10.529 \\ 27 & 10.549 \\ 28 & 10.559 \\ 29 & 10.565 \\ 30 & 10.569 \\ 31 & 10.573 \\ 32 & 10.575 \\ 33 & 10.579 \\ 34 & 10.591 \\ 35 & 10.587 \\ 36 & 10.595 \\ 37 & 10.604 \\ 38 & 10.617 \\ 39 & 10.633 \\ 40 & 10.556 \\ 11 & 10.675 \\ 42 & 10.695 \\ 43 & 10.716 \\ 44 & 10.731 \\ 15 & 10.754 \\ 45 & 10.770 \\ 47 & 10.790 \\ 48 & 10.824 \\ 19 & 10.844\end{array}$


PAFT RIVER, IDAHO - NH NO. 9 - 16 AUGUST 1976 DEPTH TERPERATLPE

\begin{tabular}{rc} 
(FT) & (C) \\
1 & 18.145 \\
2 & 18.132 \\
2 & 18.091 \\
3 & 18.059 \\
1 & 18.032 \\
5 & 18.011 \\
6 & 17.990 \\
7 & 17.990 \\
8 & 17.966 \\
9 & 17.949 \\
10 & 17.917 \\
11 & 17.893 \\
12 & 17.866 \\
13 & 17.861 \\
14 & 17.809 \\
15 & 17.779 \\
16 & 17.746 \\
17 & 17.719 \\
18 & 17.686 \\
19 & 17.558 \\
20 & 17.628 \\
21 & 17.597 \\
27 & 17.569 \\
23 & 17.543 \\
24 & 17.524 \\
26 & 17.483 \\
27 & 16.923 \\
28 & 14.369 \\
29 & 12.762 \\
30 & 11.672 \\
31 & 11.094 \\
33 & 10.551 \\
34 & 10.514 \\
35 & 10.492 \\
36 & 10.492 \\
37 & 10.497 \\
38 & 10.509 \\
39 & 10.516 \\
10 & 10.529 \\
41 & 10.538 \\
13 & 10.554 \\
14 & 10.557 \\
45 & 10.568 \\
46 & 10.576 \\
48 & 10.527 \\
& \\
\hline
\end{tabular}


RAFT RIVER, IDAHO - AH NO. 9A - 16 AMUARY 1976

$\begin{array}{rc}\text { DEPTH } & \text { TEPERAIU } \\ \text { (FT) } & \text { (C) } \\ 7 & 12.261 \\ 8 & 12.711 \\ 9 & 13.116 \\ 10 & 13.357 \\ 11 & 13.691 \\ 12 & 14.268 \\ 13 & 14.534 \\ 14 & 15.064 \\ 15 & 15.409 \\ 16 & 15.556 \\ 17 & 15.831 \\ 18 & 16.167 \\ 19 & 16.330 \\ 20 & 16.569 \\ 21 & 16.774 \\ 22 & 17.411 \\ 23 & 17.711 \\ 24 & 18.029 \\ 25 & 18.438 \\ 26 & 18.713 \\ 27 & 19.201 \\ 28 & 19.126 \\ 29 & 19.833 \\ 30 & 20.21 \\ 31 & 20.479 \\ 32 & 20.727 \\ 33 & 21.031 \\ 34 & 21.203 \\ 35 & 21.337 \\ 36 & 21.713 \\ 37 & 21.891 \\ 38 & 22.138 \\ 39 & 22.355 \\ 10 & 22.646 \\ 41 & 22.946 \\ 12 & 23.146 \\ 43 & 23.263 \\ 45 & 23.364 \\ 15 & 23.557 \\ 46 & 23.728 \\ 77 & 23.830 \\ 18 & 23.943 \\ 49 & 24.085 \\ 50 & 24.263 \\ 51 & 24.315 \\ 52 & 24.386 \\ 53 & 24.444 \\ 54 & 24.187 \\ 55 & 24.563 \\ 56 & 24.642 \\ 57 & 24.720 \\ 58 & 24.788 \\ 58 & 24.824\end{array}$


RAFT RIVER, IDAHO - AH NO. 11 - 8 FEERLARY 1976

DEPTH TEMPERATLRE

(FT) (C)

$95 \quad 12.071$

$96 \quad 12.111$

$97 \quad 12.138$

$98 \quad 12.151$

$99 \quad 12.165$ 
RAFT RIVER, IDAHO - NH HO. 11A - 16 JAMUARY 1976

$\begin{array}{cc}\begin{array}{c}\text { DEPTH } \\ \text { (FT) }\end{array} & \begin{array}{c}\text { TEPERATUF } \\ \text { (C) }\end{array} \\ 10 & 37.185 \\ 11 & 40.072 \\ 12 & 43.384 \\ 13 & 45.977 \\ 14 & 49.270 \\ 15 & 50.522 \\ 16 & 52.200 \\ 17 & 53.745 \\ 18 & 55.857 \\ 19 & 57.700 \\ 20 & -59.560 \\ 21 & 61.246 \\ 22 & 62.584 \\ 23 & 63.843 \\ 21 & 65.513 \\ 25 & 66.724 \\ 26 & 68.181 \\ 27 & 69.611 \\ 28 & 70.824 \\ 29 & 72.577 \\ 30 & 72.743 \\ 31 & 73.094 \\ 31 & 73.319\end{array}$


RAFT RIVER, IDAHD - AH NO. 12 - 8 FEBRUARY 1976

\begin{tabular}{|c|c|}
\hline $\begin{array}{l}\text { DEPTH } \\
\text { (FT) }\end{array}$ & $\begin{array}{l}\text { TEPERATL } \\
\text { (C) }\end{array}$ \\
\hline $\begin{array}{l}10 \\
11 \\
12 \\
13 \\
14 \\
15 \\
16 \\
17 \\
18 \\
19 \\
20 \\
21 \\
22\end{array}$ & $\begin{array}{l}11.410 \\
11.746 \\
12.061 \\
12.181 \\
12.635 \\
12.940 \\
13.140 \\
13.256 \\
13.303 \\
13.343 \\
13.383 \\
13.505 \\
14.179\end{array}$ \\
\hline
\end{tabular}


RAFT RIVER, ILAHO - AH NO. 12 - 16 ALCUST 1976

$\begin{array}{rc}\text { DEPTH } & \text { TEPERATRE } \\ \text { (FT) } & \begin{array}{c}\text { (C) } \\ 4\end{array} \\ 4 & 15.668 \\ 5 & 15.686 \\ 6 & 15.691 \\ 7 & 15.703 \\ 8 & 15.696 \\ 9 & 15.543 \\ 10 & 15.239 \\ 11 & 14.739\end{array}$


RAFT RIVER, IDHO - AH MO. 13-N - 17 JWUARY 1976

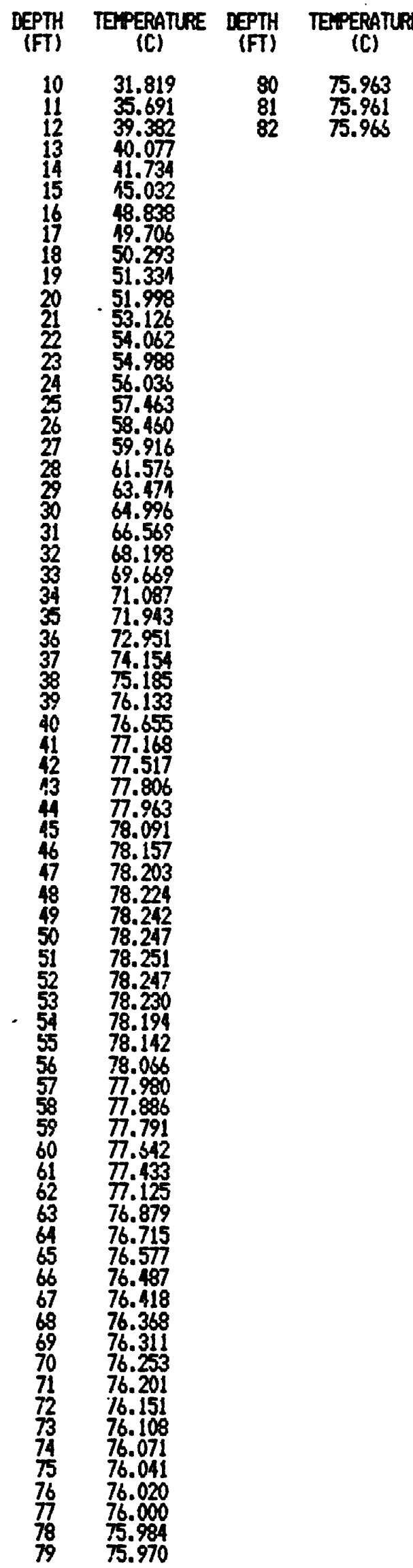


RAFT RIVER, IOAHO - AH NO. 13-N - 16 AUCUST 1976

$\begin{array}{cc}\begin{array}{c}\text { DEPTH } \\ \text { (FT) }\end{array} & \begin{array}{c}\text { TEPERATU } \\ \text { (C) }\end{array} \\ 1 & 17.811 \\ 2 & 17.863 \\ 3 & 17.945 \\ 1 & 18.025 \\ 5 & 18.093 \\ 6 & 18.215 \\ 7 & 18.296 \\ 8 & 18.376 \\ 9 & 18.454 \\ 10 & 18.515 \\ 11 & 18.636 \\ 12 & 18.750 \\ 13 & 18.912 \\ 14 & 19.030 \\ 15 & 19.150 \\ 16 & 19.261 \\ 17 & 19.446 \\ 18 & 19.630 \\ 19 & 25.466 \\ 20 & 39.184 \\ 21 & 46.447 \\ 22 & 50.293 \\ 23 & 52.543 \\ 24 & 54.032 \\ 25 & 55.452 \\ 26 & 56.609 \\ 27 & 57.743 \\ 28 & 58.786 \\ 29 & 60.198 \\ 30 & 61.593 \\ 31 & 62.946 \\ 32 & 64.470 \\ 33 & 65.916 \\ 33 & 68.976\end{array}$


RAFT RIVER, IDAHO - AH NO. 13-S - 17 JAIURY 1976

DEPTH TERERATLRE

(FT) (C)

$10 \quad 36.086$

$11 \quad 37.100$

$12 \quad 38.540$

$13 \quad 40.175$

$\begin{array}{ll}14 & 41.648 \\ 15 & 43.463\end{array}$

$16 \quad 45.130$

$17 \quad 46.125$

$19 \quad \mathbf{4 9 . 8 4 2}$

20.51 .264

$21 \quad 52.986$

$23 \quad 56.050$

$24 \quad 57.370$

$25 \quad 59.014$

$26 \quad 60.766$

$27 \quad 62.018$

$28 \quad 63.517$

$29 \quad 65.164$

$30 \quad 66.480$

$31 \quad 67.845$

$32 \quad 69.473$

$33 \quad 70.817$

$34 \quad 11.762$

$35 \quad 72.72$

$36 \quad 73.639$

$37 \quad 74.462$

$38 \quad 75.188$

$39 \quad 75.823$

$\begin{array}{ll}40 & 76.192 \\ 40 & 76.276\end{array}$ 
RAFT RIVER, IDAHO - AH NO. 13-S - 16 NULUST 1976

$\begin{array}{rc}\begin{array}{r}\text { DEPTH } \\ \text { (FT) }\end{array} & \begin{array}{c}\text { TEPERATU } \\ \text { (C) }\end{array} \\ 1 & 30.205 \\ 2 & 30.197 \\ 3 & 30.167 \\ 4 & 30.189 \\ 5 & 30.220 \\ 6 & 30.257 \\ 7 & 30.307 \\ 8 & 30.352 \\ 9 & 30.407 \\ 10 & 30.472 \\ 11 & .30 .549 \\ 12 & 30.651 \\ 13 & 30.745 \\ 14 & 30.823 \\ 15 & 30.905 \\ 16 & 30.939 \\ 17 & 31.094 \\ 18 & 34.525 \\ 19 & 41.873 \\ 20 & 16.666 \\ 21 & 49.965 \\ 22 & 51.999 \\ 23 & 53.723 \\ 24 & 55.079 \\ 25 & 56.603 \\ 26 & 57.971 \\ 27 & 59.293 \\ 28 & 61.081 \\ 29 & 62.499 \\ 30 & 63.912 \\ 31 & 65.350 \\ 32 & 66.955 \\ 33 & 68.042 \\ 34 & 69.607 \\ 35 & 70.811 \\ 36 & 71.825 \\ 37 & 72.879 \\ 38 & 73.694 \\ 39 & 74.558 \\ 10 & 75.678\end{array}$


RAFT RIVER, IOAHO - AH MO. 13A - 17 JAWARY 1976

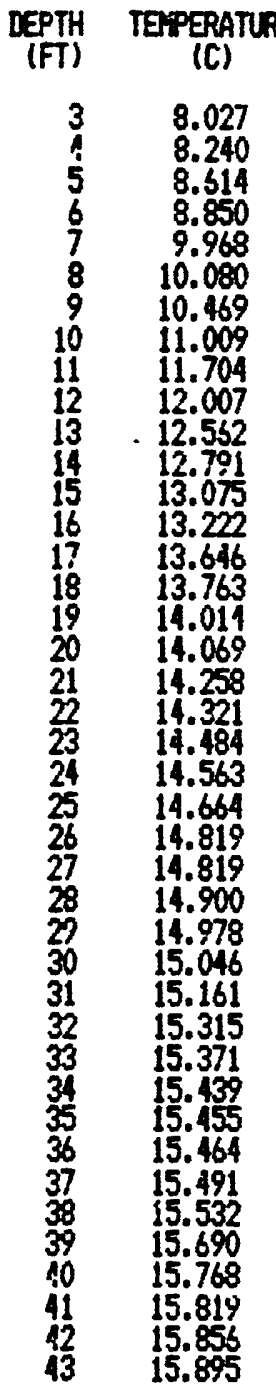


RAFT RIVER, IDAHO - NH NO. 134 - 16 NUCUST 1976

DEPTH TEPERATIRE

(FT) (C)

$\begin{array}{ll}1 & 17.803 \\ 2 & 17.857 \\ 3 & 18.992 \\ 1 & 20.369 \\ 5 & 20.624 \\ 6 & 20.185 \\ 7 & 19.331 \\ 8 & 18.364 \\ 9 & 17.235 \\ 10 & 16.331 \\ 11 & .15 .529 \\ 12 & 14.821 \\ 13 & 14.279 \\ 14 & 13.857 \\ 15 & 13.703 \\ 16 & 13.630 \\ 17 & 13.533 \\ 18 & 13.648 \\ 19 & 13.673 \\ 20 & 13.709 \\ 21 & 13.764 \\ 22 & 13.847 \\ 23 & 13.953 \\ 24 & 14.020 \\ 25 & 14.392\end{array}$


RAFT RIVER, IDAHO - AH MO. 15 - 9 FEERUHAY 1976

DEPTH TEYPERATURE

$\begin{array}{ll}15 & 12.853 \\ 15 & 13.167 \\ 17 & 13.414 \\ 18 & 13.526 \\ 10 & 13.565 \\ 20 & 13.702 \\ 21 & 13.793 \\ 22 & 13.937 \\ 23 & 14.041 \\ 24 & 14.141 \\ 25 & 14.250 \\ 26 & 14.347 \\ 27 & 14.363 \\ 28 & 14.426 \\ 29 & 14.482 \\ 30 & 14.521 \\ 31 & 14.563 \\ 32 & 14.575 \\ 33 & 14.583 \\ 34 & 14.590 \\ 35 & 14.601 \\ 36 & 14.616 \\ 37 & 14.630 \\ 38 & 14.645 \\ 39 & 14.659 \\ 40 & 14.678 \\ 41 & 14.706 \\ 42 & 14.743 \\ 43 & 14.782 \\ 44 & 14.816 \\ 45 & 14.850\end{array}$


RAFT RIVER, IDAHD - AH NO. 15 - 15 ALCLST 1976

DEPTH TEMPERATLRE

(FT) (C)

$\begin{array}{ll}2 & 16.031 \\ 4 & 16.032 \\ 5 & 16.032 \\ 6 & 16.032 \\ 7 & 16.030 \\ 8 & 16.029 \\ 9 & 16.023 \\ 10 & 16.016 \\ 11 & 16.003 \\ 12 & 15.993 \\ 13 & 15.978 \\ 14 & 14.178 \\ 15 & 13.422 \\ 16 & 13.051 \\ 17 & 12.892 \\ 18 & 13.050 \\ 19 & 13.177 \\ 20 & 13.268 \\ 21 & 13.342\end{array}$


RAFT RIVER, IDNHO - AH NO. 16 - 9 FEERIKRY 1976

DEPTH TIPPERATLRE

(FT) (C)

$14 \quad 11.181$

$15 \quad 11.806$

$16 \quad 11.851$

$17 \quad 11.962$

$18 \quad 12.26$

$19 \quad 12.342$ 
RAFT RIVER, IDAHD - AH M. 16 - 16 AUEUST 1976

$\begin{array}{rc}\begin{array}{c}\text { IEPTH } \\ \text { (FT) }\end{array} & \begin{array}{c}\text { TEPERATURE } \\ (C)\end{array} \\ 1 & 20.303 \\ 2 & 20.322 \\ 2 & 20.331 \\ 3 & 20.330 \\ 4 & 20.331 \\ 5 & 20.327 \\ 6 & 20 \\ 7 & 20.323 \\ 8 & 20.321 \\ 9 & 20.311 \\ 10 & 20.258 \\ 11 & 20.281 \\ 12 & 20.260 \\ 13 & 18.727 \\ 14 & 15.565 \\ 15 & 13.539 \\ 17 & 12.317 \\ 18 & 12.396\end{array}$


RAFT RIVER, IDAHD - NH NO. 17 - 9 FEERUARY 1976

\section{DEPTH TEPERATLRE}

(FT) (C)

$\begin{array}{ll}16 & 10.904 \\ 17 & 11.120 \\ 18 & 11.216 \\ 19 & 11.230 \\ 20 & 11.309 \\ 21 & 11.371 \\ 22 & 11.412 \\ 23 & 11.138 \\ 24 & 11.450 \\ 25 & 11.461 \\ 26 & 11.473 \\ 27 & 11.480 \\ 28 & 11.436 \\ 29 & 11.496 \\ 30 & 11.507 \\ 31 & 11.518 \\ 32 & 11.524 \\ 33 & 11.534 \\ 34 & 11.546 \\ 35 & 11.560 \\ 36 & 11.570 \\ 37 & 11.582 \\ 38 & 11.598 \\ 39 & 11.619 \\ 40 & 11.637 \\ 11 & 11.658 \\ 42 & 11.678 \\ 43 & 11.700 \\ 44 & 11.730 \\ 45 & 11.753 \\ 46 & 11.779 \\ 46 & 11.801\end{array}$


RAFT RIVER, IDHO - AH MO. 17 - 15 AUEUST 1976

DEPTH TEPPERATUPE

(FT) (C)

$\begin{array}{ll}1 & 10.687 \\ 2 & 10.709 \\ 3 & 10.755 \\ 1 & 10.790 \\ 5 & 10.832 \\ 6 & 10.848 \\ 7 & 10.895 \\ 8 & 10.923 \\ 9 & 10.939 \\ 10 & 10.953 \\ 11 & 10.966 \\ 12 & 10.974 \\ 14 & 10.996 \\ 15 & 10.99 \\ 16 & 10.979 \\ 17 & 10.762 \\ 18 & 10.690 \\ 19 & 10.662 \\ 20 & 10.666 \\ 21 & 10.689 \\ 22 & 10.709 \\ 23 & 10.746 \\ 24 & 10.777 \\ 25 & 10.808 \\ 26 & 10.826 \\ 27 & 10.890 \\ 28 & 10.913 \\ 29 & 10.954 \\ 30 & 11.002 \\ 31 & 11.046 \\ 32 & 11.082 \\ 33 & 11.113 \\ 34 & 11.181 \\ 35 & 11.230 \\ 36 & 11.271 \\ 37 & 11.307 \\ 38 & 11.344 \\ 39 & 11.386 \\ 40 & 11.428 \\ 11 & 11.481 \\ 42 & 11.515 \\ 43 & 11.544 \\ 44 & 11.609\end{array}$


RAFT RIVER, IDFHO - AH NO. 19 - 8 FEBRUARY 1976

DEPTH TERERTTLRE

(FT) (C)

$\begin{array}{rr}3 & 2.580 \\ 10 & 7.845 \\ 11 & 8.187 \\ 12 & 8.335 \\ 13 & 8.123 \\ 14 & 8.561 \\ 15 & 8.593 \\ 16 & 8.748 \\ 17 & 8.831 \\ 18 & 8.918 \\ 19 & 9.212 \\ 20 & 9.538 \\ 21 & 9.653 \\ 22 & 9.787\end{array}$


RAFT RIVER, IDAHO - AH NO. 19 - 8 IUGUST 1976

DEPTH TEPERATUPE
(FT)

$\begin{array}{cc}1 & 18.576 \\ 2 & 17.049 \\ 3 & 15.972 \\ 4 & 14.617 \\ 5 & 13.812 \\ 6 & 11.189 \\ 7 & 10.263 \\ 8 & 9.886 \\ 9 & 9.595 \\ 10 & 9.388 \\ 11 & 9.204 \\ 12 & 9.124 \\ 13 & 9.050 \\ 14 & 8.994 \\ 15 & 8.900 \\ 16 & 8.859 \\ 17 & 8.841 \\ 18 & 8.859 \\ 19 & 8.912 \\ 20 & 8.978 \\ 21 & 9.030 \\ 22 & 9.119\end{array}$


RAFT RIVER, IDAHO - AH MO. 20-8 AUCUST 1976

$\begin{array}{rc}\text { DEPTH } & \text { TERPERTT } \\ \text { (FT) } & \begin{array}{c}\text { (C) } \\ 0\end{array} \\ 31.925 \\ 1 & 30.003 \\ 2 & 28.965 \\ 3 & 28.871 \\ 4 & 28.975 \\ 5 & 29.680 \\ 6 & 30.317 \\ 7 & 30.840 \\ 8 & 31.653 \\ 9 & 32.204 \\ 10 & 33.078 \\ 11 & 33.845 \\ 12 & 35.035 \\ 13 & 35.699 \\ 14 & 36.502 \\ 15 & 37.400 \\ 16 & 38.363 \\ 17 & 39.272 \\ 18 & 39.470 \\ 19 & 39.655 \\ 20 & 39.851 \\ 21 & 40.018 \\ 27 & 40.304 \\ 23 & 40.516 \\ 24 & 40.678 \\ 25 & 40.841 \\ 26 & 40.941 \\ 27 & 41.041 \\ 28 & 41.153\end{array}$

$111-142$ 
RAFT RIVER, IDAHO - AH MO. 21 - 8 NUGUST 1976

IEPTH TEPERATURE

$\begin{array}{rc}\text { (FT) } & \text { (C) } \\ 2 & 27.237 \\ 3 & 27.399 \\ 4 & 27.715 \\ 5 & 28.078 \\ 6 & 28.310 \\ 7 & 28.774 \\ 8 & 29.365 \\ 9 & 29.690 \\ 10 & 30.536 \\ 11 & 31.135 \\ 12 & -32.208 \\ 13 & 33.075 \\ 11 & 33.646 \\ 15 & 34.364 \\ 16 & 34.55 \\ 17 & 36.076 \\ 18 & 36.918 \\ 19 & 37.034 \\ 20 & 37.165 \\ 21 & 37.314 \\ 22 & 37.151 \\ 23 & 37.666 \\ 24 & 37.935 \\ 25 & 38.215 \\ 26 & 33.530 \\ 27 & 38.765 \\ 28 & 39.091 \\ 29 & 39.263 \\ 30 & 39.416 \\ 31 & 39.605 \\ 32 & 39.700 \\ 33 & 39.882 \\ 34 & 39.985 \\ 35 & 40.085\end{array}$


RAFT RIVER, IDAHO - AH NO. 28 - 4 FEBRLAAY 1976

\section{EEPTH TEPERATLRE}

(FT) (C)

$11 \quad 10.532$

$12 \quad 10.802$

$13 \quad 11.138$

$14 \quad 11.219$

$16 \quad 11.380$

$17 \quad 11.500$

$18 \quad 11.574$

$19 \quad 11.749$

$20 \quad 11.933$

$21 \cdot 12.078$

$22 \quad 12.22$

$23 \quad 12.316$

24 12.11

2512.461

$26 \quad 12.531$

2712.581

$28 \quad 12.648$

$29 \quad 12.723$

$30 \quad 12.811$

$31 \quad 12.853$

$32 \quad 12.858$

$33 \quad 12.920$

$34 \quad 12.922$

$35 \quad 12.960$

$36 \quad 13.016$

$37 \quad 13.046$

$38 \quad 13.116$

$39 \quad 13.197$

10 13.265

$41 \quad 13.380$ 
RAFT RIVER, IDAHD - AH NO. 28 - 16 AUGUST 1976

\section{DEPTH TEPPEPATURE}

(FT) (C)

\begin{tabular}{ll}
1 & 17.131 \\
2 & 17.124 \\
3 & 17.113 \\
4 & 17.107 \\
5 & 17.111 \\
6 & 17.108 \\
7 & 17.102 \\
8 & 17.101 \\
9 & 17.092 \\
10 & 17.085 \\
11 & 11.850 \\
12 & 14.658 \\
13 & 12.978 \\
14 & 11.853 \\
15 & 11.286 \\
16 & 11.180 \\
17 & 10.799 \\
18 & 10.702 \\
19 & 10.681 \\
20 & 10.688 \\
21 & 10.707 \\
22 & 10.745 \\
23 & 10.784 \\
24 & 10.815 \\
25 & 10.854 \\
26 & 10.906 \\
27 & 10.967 \\
28 & 11.043 \\
29 & 11.155 \\
30 & 11.247 \\
31 & 11.37 \\
32 & 11.48 \\
33 & 11.673 \\
34 & 11.815 \\
35 & 11.956 \\
36 & 12.111 \\
37 & 12.237 \\
39 & 12.135 \\
40 & 12.543 \\
& \\
\hline
\end{tabular}


RYT RIVER, IDAHO - AH NO. 31 - 9 FEBRUAAY 1976

\section{DEPTH TEPEPATURE}

$\begin{array}{cc}\text { (FT) } & \text { (C) } \\ 18 & 12.718 \\ 19 & 12.763 \\ 20 & 12.852 \\ 21 & 12.902 \\ 22 & 12.954 \\ 23 & 12.998 \\ 24 & 13.010 \\ 25 & 12.996 \\ 26 & 12.974 \\ 27 & 12.550 \\ 28 & 12.923 \\ 29 & 12.905 \\ 30 & 12.851 \\ 31 & 12.821 \\ 31 & 12.793\end{array}$


RAFT RIVER, IDAHO - AH NO. 31 - 16 ALGUST 1976

DEPTH TEMPERATURE

$\begin{array}{cc}3 & 17.310 \\ 4 & 17.352 \\ 6 & 17.349 \\ 7 & 17.340 \\ 8 & 17.337 \\ 9 & 17.319 \\ 10 & 17.280 \\ 11 & 17.271 \\ 12 & 16.365 \\ 13 & 14.964 \\ 14 & 114.276 \\ 15 & 13.792 \\ 16 & 13.171 \\ 17 & 13.229 \\ 18 & 13.037 \\ 19 & 12.30 \\ 20 & 12.803 \\ 21 & 12.632 \\ 22 & 12.623 \\ 23 & 12.538 \\ 24 & 12.469 \\ 25 & 12.401 \\ 26 & 12.341 \\ 27 & 12.285 \\ 28 & 12.240 \\ 29 & 12.231 \\ 30 & 12.217 \\ 31 & 12.227 \\ 32 & 12.236 \\ 33 & 12.247 \\ 34 & 12.273\end{array}$




\section{APPENDIX IV}

THERMAL CONDUCTIVITIES AND CORRESPONDING DEPTHS FOR WELLS IN THE RAFT RIVER AREA, CASSIA COUNTY, IDAHO

\section{CONTENTS}

Well No.

Page

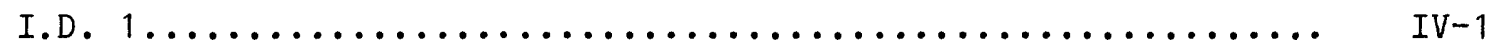

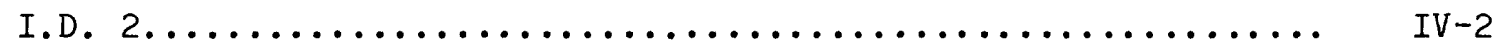

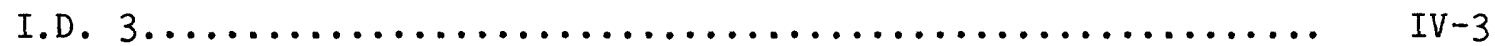

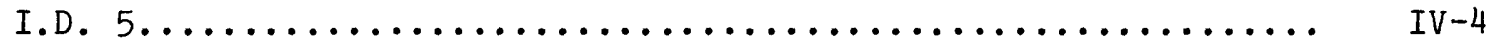


RAFT RIVER, CASSIA COUNTY, IDAHO - I.D. 1 - THERMAL CONDUCTIVITY

DEPTH CONDUCTIVITY DEPTH CONDUCTIVITY
(FT)

$\begin{array}{llll}293.0 & 1.03 & 1015.0 & 1.07 \\ 308.0 & 1.01 & 1030.0 & 1.21 \\ 319.0 & 1.07 & 1040.0 & 1.05 \\ 328.0 & 1.11 & 1055.0 & 1.43 \\ 336.0 & 1.13 & 1065.0 & 1.19 \\ 346.0 & 1.08 & 1080.0 & 1.03 \\ 362.0 & 1.04 & 1090.0 & 0.96\end{array}$

$\begin{array}{llll}373.0 & 1.13 & 1100.0 & 1.18\end{array}$

$383.0 \quad 1.12$

392.0 - 1.54

$401.0 \quad 1.07$

$407.0 \quad 1.02$

$435.0 \quad 1.22$

$444.1 \quad 1.02$

$44.1 \quad 1.01$

$475.0 \quad 1.02$

$490.0 \quad 1.02$

$504.0 \quad 1.62$

$515.0 \quad 1.02$

$537.0-0.93$

$537.0 \quad 0.92$

$547.0 \quad 1.23$

$557.0 \quad 1.00$

$568.0 \quad 0.91$

$568.0 \quad 0.94$

$579.0 \quad 1.27$

$588.0 \quad 1.02$

$599.0 \quad 1.03$

$614.0 \quad 1.25$

$624.0 \quad 1.12$

$634.0 \quad 1.01$

$644.0 \quad 1.07$

$660.0 \quad 1.07$

$670.0 \quad 0.99$

$680.0 \quad 1.06$

$690.0 \quad 1.45$

$690.0 \quad 1.49$

$700.0 \quad 1.03$

$710.0 \quad 1.08$

$727.0 \quad 1.05$

$740.0 \quad 1.04$

$\begin{array}{ll}750.0 & 0.97 \\ 750.0 & 0.96\end{array}$

$750.0 \quad 0.96$

$770.0 \quad 1.07$

$785.0 \quad 1.13$

$795.0 \quad 1.07$

$815.0 \quad 1.21$

$824.0 \quad 0.99$

$835.0 \quad 1.00$

$850.0 \quad 0.93$

$861.0 \quad 1.08$

$861.0 \quad 1.06$

$871.0 \quad 1.11$

$882.0 \quad 1.16$

$899.0 \quad 1.11$

$\begin{array}{ll}89.0 & 0.92 \\ 910.0 & 0.93\end{array}$

$920.0 \quad 0.92$

$930.0 \quad 0.91$

$940.0 \quad 1.40$

$952.0 \quad 0.98$

$962.0 \quad 0.96$

$\begin{array}{rl}972.0 & 0.96 \\ 990.0 & 0.91\end{array}$

$1000.0 \quad 1.03$

$1015.0 \quad 1.05$ 
RAFT RIVER, CASSIA COUNTY, IDAHO - 1.D. 2 - THERHAL CONOUCTIVITY DEPTH CONOUCTIVITY

$\begin{array}{ll}257.0 & 1.36 \\ 266.0 & 1.14 \\ 284.0 & 1.17 \\ 24.0 & 1.30 \\ 303.0 & 1.19 \\ 34.0 & 1.28 \\ 353.0 & 1.27 \\ 334.0 & 1.47 \\ 337.0 & 1.90 \\ 440.0 & 1.45 \\ 450.0 & 1.41 \\ 550.0 & 1.38 \\ 528.0 & 1.42 \\ 541.0 & 2.02 \\ 568.0 & 1.78 \\ 615.0 & 1.31 \\ 699.0 & 1.13 \\ 711.0 & 1.54 \\ 722.0 & 0.98 \\ 733.0 & 0.98 \\ 749.0 & 0.99 \\ 755.0 & 0.96 \\ 758.0 & 0.97 \\ 733.0 & 0.86 \\ 786.0 & 1.31\end{array}$


RAFT RIVER, CASSIA CONTY, IDAHO - I.D. 3 - THERMAL CONDUCTIVITY

DEPTH CONDUCTIVITY DEPTH CONDUCTIVITY
(FT)
$(\mathrm{H} / \mathrm{aK})$

$\begin{array}{llll}212.0 & 1.04 & 1084.0 & 1.44 \\ 224.0 & 0.97 & 1094.0 & 2.48 \\ 233.0 & 1.18 & 1105.0 & 1.22 \\ 237.8 & 1.54 & 1114.0 & 1.34 \\ 254.5 & 1.46 & 1150.0 & 1.41 \\ 266.0 & 1.37 & 1157.0 & 1.45 \\ 290.0 & 2.32 & 1165.0 & 1.46 \\ 301.0 & 1.34 & 1184.0 & 1.52 \\ 314.0 & 1.64 & 1191.0 & 1.37 \\ 351.0 & 1.25 & 1204.0 & 1.70 \\ 361.5 & 2.09 & 1206.0 & 2.04 \\ 371.0 & 2.01 & 1212.0 & 1.77 \\ 388.0 & 1.22 & 1221.0 & 1.88 \\ 404.0 & 1.30 & 1228.0 & 1.62 \\ 413.0 & 1.32 & 1235.0 & 1.23 \\ 429.0 & 1.21 & 1259.0 & 1.80 \\ 444.5 & 1.79 & 1274.0 & 1.67 \\ 467.0 & 1.29 & 1283.0 & 1.89 \\ 479.0 & 1.63 & 1288.0 & 1.85 \\ 493.0 & 1.93 & 1306.0 & 2.37 \\ 505.0 & 2.13 & 1314.0 & 1.84 \\ 519.0 & 1.46 & 1328.0 & 1.80\end{array}$

$\begin{array}{lll}519.0 & 1.46 & 1328.0\end{array}$

$543.5 \quad 1.26$

$575.0 \quad 1.25$

$586.0 \quad 1.39$

$598.0 \quad 1.81$

$627.0 \quad 2.24$

$636.5 \quad 1.47$

$650.0 \quad 2.47$

$660.0 \quad 1.86$

$672.5 \quad 2.21$

$\begin{array}{ll}685.0 & 1.97 \\ 701.0 & 2.44\end{array}$

$\begin{array}{ll}712.0 & 1.30 \\ 723.5 & 1.40\end{array}$

$739.5 \quad 1.35$

$749.0 \quad 1.26$

$761.0 \quad 1.34$

$770.0 \quad 2.31$

$790.0 \quad 1.38$

$803.0 \quad 1.76$

$814.0 \quad 1.79$

$822.0 \quad 2.02$

826.51 .81

$837.0 \quad 1.63$

$842.0 \quad 1.57$

$849.0 \quad 1.92$

$857.5 \quad 1.13$

$863.0 \quad 1.38$

$887.5 \quad 1.70$

$914.0 \quad 3.03$

$922.0 \quad 2.78$

$930.5 \quad 2.40$

$949.0 \quad 2.39$

$959.0 \quad 1.85$

$965.0 \quad 1.83$

$979.0 \quad 1.97$

$996.0 \quad 1.46$

$1010.0 \quad 1.73$

$1016.0 \quad 1.76$

$1041.0 \quad 1.05$

$1050.0 \quad 1.26$

$1056.0 \quad 1.04$

$1066.0 \quad 2.65$

$1076.0 \quad 1.43$ 
RAFT RIVER, CASSIA COUNTY, IDAHO - 1.D. 5 - THERMAL CONOUCTIVITY

\section{DEPTH COMDUCTIVITY

(FT) (H/EK)

$114.0 \quad 1.22$

$123.0 \quad 1.51$

$140.0 \quad 1.95$

$149.0 \quad 1.61$

$158.0 \quad 1.58$

$184.0 \quad 2.00$

$193.0 \quad 1.90$

$204.0 \quad 1.72$

$212.0 \quad 2.00$

224.0 - 2.07

$234.0 \quad 1.83$

$247.5 \quad 1.78$

$252.0 \quad 2.08$

$265.0 \quad 1.91$

$273.0 \quad 1.95$

$288.0 \quad 1.62$

$299.0 \quad 2.06$

$308.0 \quad 1.74$

$318.5 \quad 2.24$

$331.0 \quad 1.85$

$341.3 \quad 1.83$

$354.0 \quad 2.04$

$362.5 \quad 2.13$

$37.0 \quad 2.12$

$392.0 \quad 2.05$

$405.0 \quad 2.08$

$415.0 \quad 1.84$

$427.5 \quad 2.96$

$437.5 \quad 2.47$

$456.0 \quad 2.44$

$467.0 \quad 2.27$

$477.0 \quad 2.67$

$498.0 \quad 2.96$

$495.0 \quad 2.84$

$507.0 \quad 2.57$

$515.5 \quad 2.30$

$531.0 \quad 1.83$

$560.0 \quad 1.84$

$591.5 \quad 1.98$

$599.0 \quad 2.52$

$612.0 \quad 2.49$

$621.0 \quad 1.97$

$628.0 \quad 2.58$

$638.0 \quad 2.45$

648.0 2.11

$658.0 \quad 2.71$ 